\title{
EXCESS FOUNDRY SAND \\ CHARACTERIZATION AND EXPERIMENTAL INVESTIGATION IN CONTROLLED \\ LOW-STRENGTH MATERIAL AND \\ HOT-MIXING ASPHALT
}

Prepared for

U.S. Department of Energy

Contract No. DE-FC36-01ID13974

FINAL REPORT

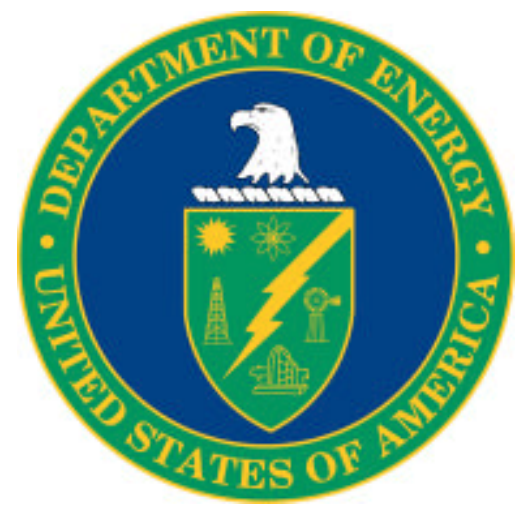

October 2004

By P. J. Tikalsky, H. U. Bahia, A. Deng and T. Snyder

PENNSTATE

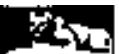

1) 55

Pennsylvania Transportation Institute

The Pennsylvania State University Transportation Research Building University Park, PA 16802-4710 (814) 865-1891 www.pti.psu.edu 
Technical Report Documentation Page

\begin{tabular}{|c|c|c|c|}
\hline \multirow{3}{*}{\multicolumn{2}{|c|}{\begin{tabular}{|l|l|} 
1. Report No. & 2. Government Accession No. \\
DE-FC36-01ID13974 & \\
4. Title and Subtitle \\
Excess Foundry Sand Characterization and Experimental Investigation \\
in Controlled Low-Strength Material and Hot-Mix Asphalt
\end{tabular}}} & \multicolumn{2}{|c|}{ 3. Recipient's Catalog No. } \\
\hline & & \multicolumn{2}{|l|}{$\begin{array}{l}\text { 5. Report Date } \\
\text { October } 2004\end{array}$} \\
\hline & & \multicolumn{2}{|c|}{ 6. Performing Organization Code } \\
\hline \multicolumn{2}{|c|}{$\begin{array}{l}\text { 7. Author(s) } \\
\text { Paul J. Tikalsky, Hussain U. Bahia, An Deng and Thomas Snyder }\end{array}$} & \multicolumn{2}{|c|}{$\begin{array}{l}\text { 8. Performing Organization Report No. } \\
\qquad 2005-01\end{array}$} \\
\hline \multirow{2}{*}{\multicolumn{2}{|c|}{$\begin{array}{l}\text { 9. Performing Organization Name and Address } \\
\text { The Pennsylvania Transportation Institute } \\
\text { Transportation Research Building } \\
\text { The Pennsylvania State University } \\
\text { University Park, PA 16802-4710 }\end{array}$}} & \multicolumn{2}{|c|}{ 10. Work Unit No. (TRAIS) } \\
\hline & & \multicolumn{2}{|c|}{$\begin{array}{l}\text { 11. Contract or Grant No. } \\
\text { DE-FC36-01ID13974 }\end{array}$} \\
\hline \multirow{3}{*}{\multicolumn{2}{|c|}{$\begin{array}{l}\text { 12. Sponsoring Agency Name and Addre } \\
\text { U.S. Department of Energy } \\
\text { Golden Field Office } \\
1617 \text { Cole Blvd., } \\
\text { Golden, CO } 80401-3393\end{array}$}} & \multicolumn{2}{|c|}{ 13. Type of Report and Period Cov } \\
\hline & & \multicolumn{2}{|c|}{$\begin{array}{l}\text { Final Report } \\
\text { July 16, } 2003 \text { - October 15, } 2004\end{array}$} \\
\hline & & \multicolumn{2}{|c|}{ 14. Sponsoring Agency Code } \\
\hline \multicolumn{4}{|c|}{$\begin{array}{l}\text { 15. Supplementary Notes } \\
\text { COTR: Debo Aichbhaumik, (303) 275-4763 }\end{array}$} \\
\hline \multicolumn{4}{|l|}{ 16. Abstract } \\
\hline \multicolumn{4}{|c|}{$\begin{array}{l}\text { This report provides technical data regarding the reuse of excess foundry sand. The report addresses three topics: a } \\
\text { statistically sound evaluation of the characterization of foundry sand, a laboratory investigation to qualify excess } \\
\text { foundry sand as a major component in controlled low-strength material (CLSM), and the identification of the best } \\
\text { methods for using foundry sand as a replacement for natural aggregates for construction purposes, specifically in } \\
\text { asphalt paving materials. The survival analysis statistical technique was used to characterize foundry sand over a } \\
\text { full spectrum of general chemical parameters, metallic elements, and organic compounds regarding bulk analysis } \\
\text { and leachate characterization. Not limited to characterization and environmental impact, foundry sand was evaluated } \\
\text { by factor analyses, which contributes to proper selection of factor and maximization of the reuse marketplace for } \\
\text { foundry sand. Regarding the integration of foundry sand into CLSM, excavatable CLSM and structural CLSM } \\
\text { containing different types of excess foundry sands were investigated through laboratory experiments. Foundry sand } \\
\text { was approved to constitute a major component in CLSM. Regarding the integration of foundry sand into asphalt } \\
\text { paving materials, the optimum asphalt content was determined for each mixture, as well as the bulk density, } \\
\text { maximum density, asphalt absorption, and air voids at } N_{\text {ini, }}, N_{\text {des, }} \text {, and } N_{\text {max. }} \text { It was found that foundry sands can be } \\
\text { used as an aggregate in hot-mix asphalt production, but each sand should be evaluated individually. Foundry sands } \\
\text { tend to lower the strength of mixtures and also may make them more susceptible to moisture damage. Finally, } \\
\text { traditional anti-stripping additives may decrease the moisture sensitivity of a mixture containing foundry sand, but not } \\
\text { to the level allowed by most highway agencies. }\end{array}$} \\
\hline $\begin{array}{l}\text { 17. Key Words } \\
\text { Foundry sand, hot-mix asphalt, con } \\
\text { aggregate, asphalt paving materials } \\
\text { analysis, moisture damage, bulk de }\end{array}$ & $\begin{array}{l}\text { olled low-strength material, } \\
\text { leachate characterization, survival } \\
\text { sity, optimum asphalt content }\end{array}$ & $\begin{array}{l}\text { 18. Distribution St } \\
\text { No restrictions. Th } \\
\text { available from the } \\
\text { Information Servic } \\
22161\end{array}$ & $\begin{array}{l}\text { nt } \\
\text { cument is } \\
\text { hal Technical } \\
\text { ingfield, VA }\end{array}$ \\
\hline $\begin{array}{llll} & \text { Security } & \text { Classif. (of this } \\
\text { report) } & & & \\
\text { Unclassified } & & & \end{array}$ & $\begin{array}{l}\text { 20. Security Classif. (of this page) } \\
\text { Unclassified }\end{array}$ & $\begin{array}{l}\text { 21. No. of Pages } \\
324\end{array}$ & 22. Price \\
\hline
\end{tabular}




\title{
EXCESS FOUNDRY SAND CHARACTERIZATION AND EXPERIMENTAL INVESTIGATION IN CONTROLLED LOW-STRENGTH MATERIAL AND HOT-MIXING ASPHALT
}

\author{
Prepared for
}

U.S. Department of Energy

Contract No. DE-FC36-01ID13974

\author{
By \\ Paul J. Tikalsky / Penn State \\ Hussain U. Bahia / University of Wisconsin \\ An Deng / Penn State \\ Thomas Snyder / University of Wisconsin
}

FINAL REPORT
Pennsylvania Transportation Institute The Pennsylvania State University
201 Transportation Research Building
University Park, PA 16802-4710

October 2004

PTI 2005-01 


\section{TABLE OF CONTENTS}

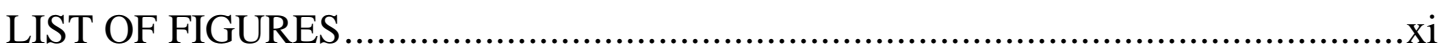

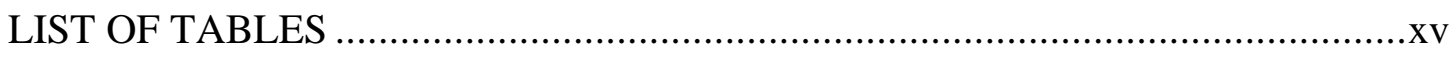

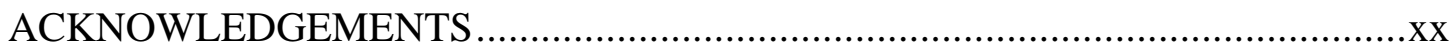

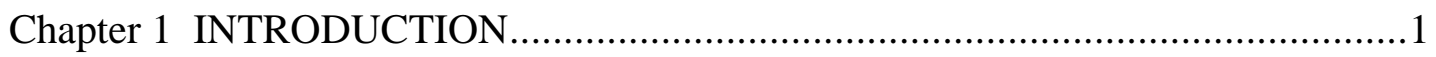

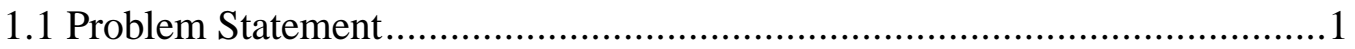

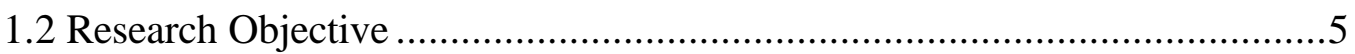

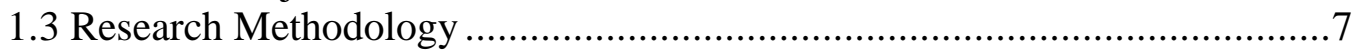

1.4 Potential Impact to Society .................................................................... 11

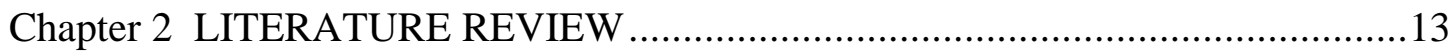

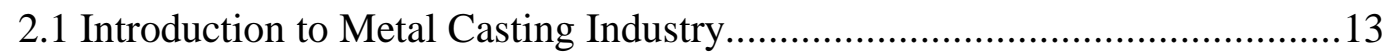

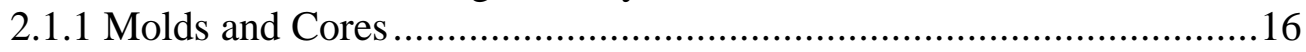

2.1.2 Virgin Foundry Sands and Excess Foundry Sands..............................18

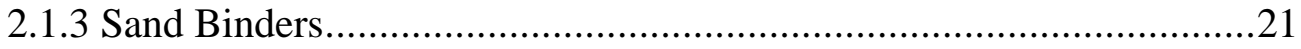

2.1.4 Sand Bonding Processes ............................................................25

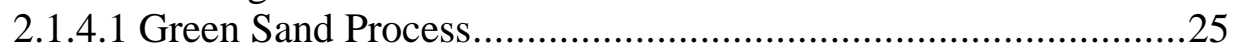

2.1.4.2 Chemical Bonding Process ....................................................22

2.1.4.3 Unbonded Systems ................................................................28

2.1.4.4 Environmental Improvements ……………............................30

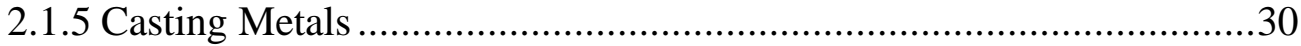

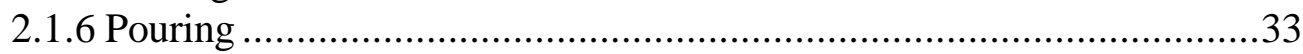

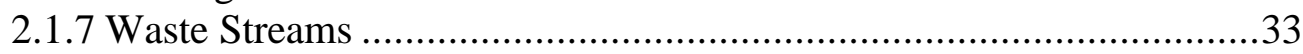

2.2 Chemical and Environmental Characterization of Excess Foundry Sands......35

2.2.1 Act and Regulations.........................................................................35

2.2.2 Chemical Analytical Techniques........................................................38

2.2.2.1 Toxicity Characteristic Leaching Procedure (TCLP) .................39

2.2.2.2 Synthetic Precipitation Leaching Procedure (SPLP) ..................40

2.2.2.3 Shake Extraction of Solid Waste with Water (ASTM D

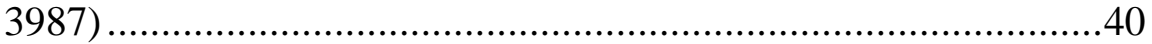

2.2.3 Research on Characterization of Excess Foundry Sands ......................41

2.2.4 Chemical Characteristics from Past Studies..........................................42

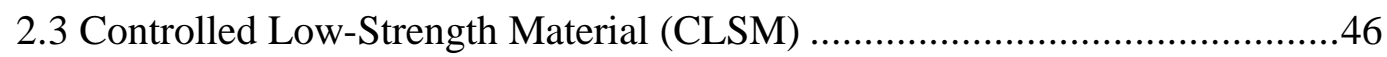

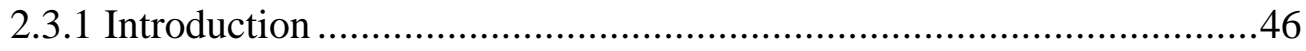

2.3.2 Specification of CLSM Containing Excess Foundry Sands ................48

2.3.3 Research of CLSM Containing Excess Foundry Sands........................51

2.4 Hot-Mixing Asphalt (HMA) ...................................................................54

2.4.1 Findings From Previous Studies at UW - Madison ..............................54

2.4.2 Overcoming Problems Faced in Previous Studies...............................57

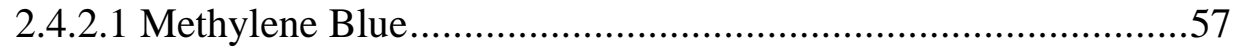

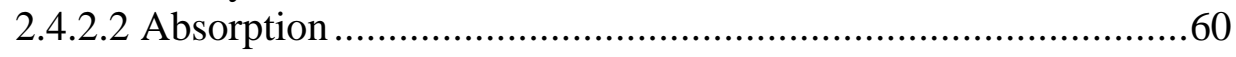


2.4.2.3 Tensile Strength Ratio ............................................................62

Chapter 3 CHARACTERIZATION DATA ........................................................64

3.1 Excess Foundry Sand Characterization System............................................64

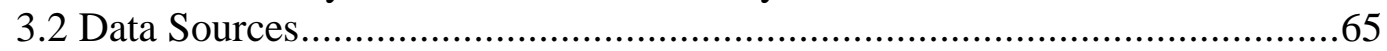

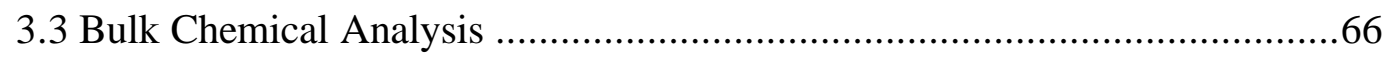

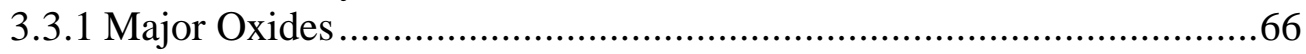

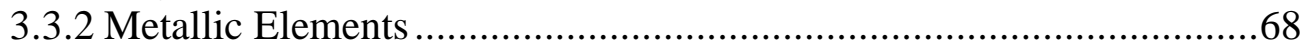

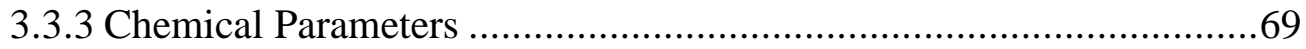

3.3.4 Organic Compounds .......................................................................

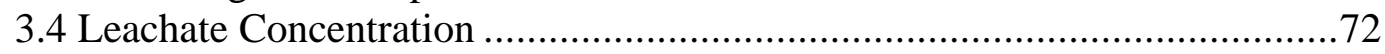

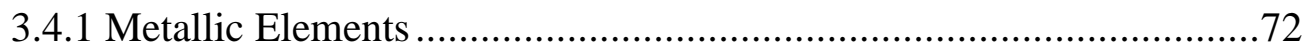

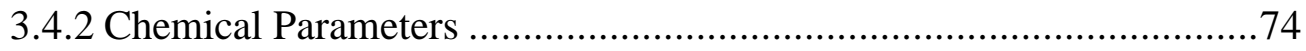

3.4.3 Organic Compounds .....................................................................74

Chapter 4 METHODOLOGY ADDRESSING CENSORED DATA..........................77

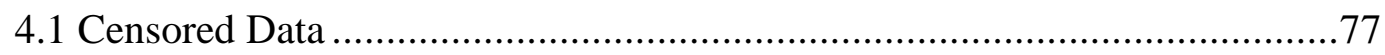

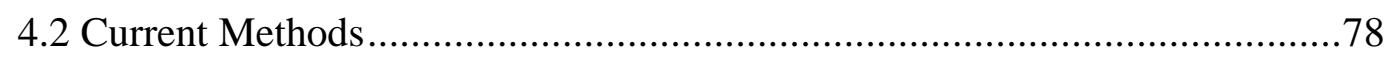

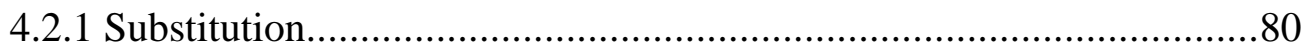

4.2.2 Regression on Order Statistics (ROS)............................................ 80

4.2.3 Maximum Likelihood Estimation (MLE) ........................................... 81

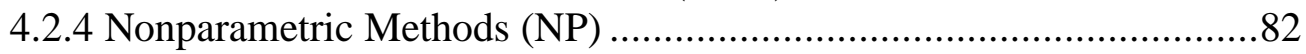

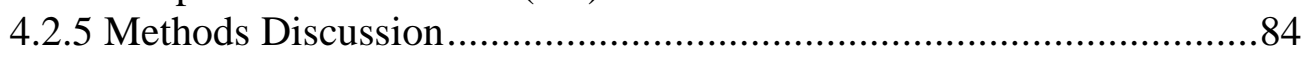

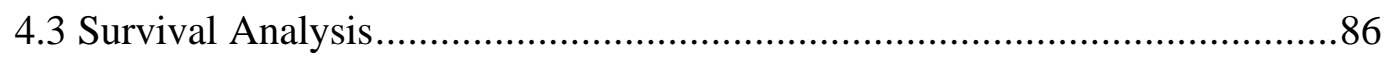

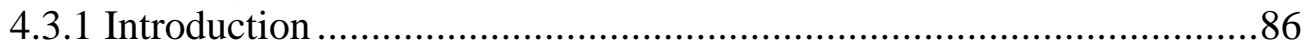

4.3.2 Cumulative Probability and Survival Probability.................................8 88

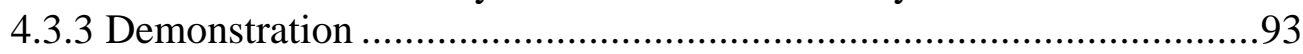

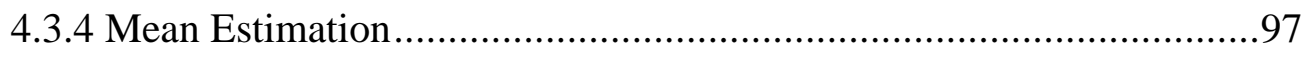

4.3.5 Estimation of Censored Data..........................................................99

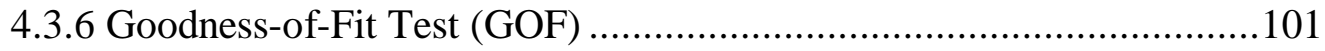

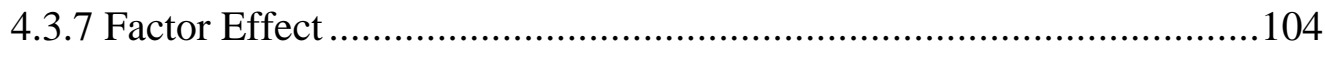

Chapter 5 CHARACTERIZATION ANALYSES AND CONCLUSIONS................107

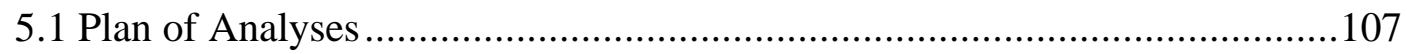

5.2 Estimated Mean for As-Received Sand ......................................................110

5.3 Factor of Metal Cast for As-received Sand ..................................................113

5.4 Estimated Mean for Sand Leachate per Leaching Protocol............................116

5.5 Factor of Metal Cast in TCLP Sand Leachate .............................................120

5.6 Impacts of Metallic Elements in Foundry Sand .........................................123

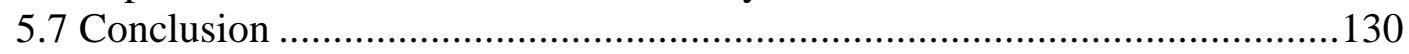

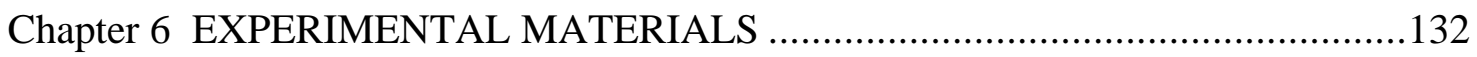

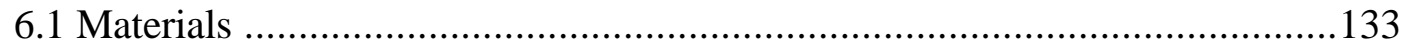

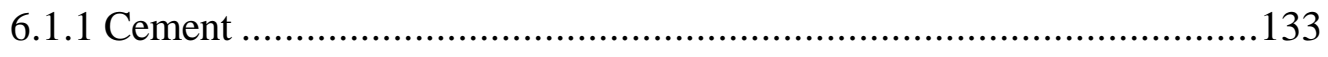




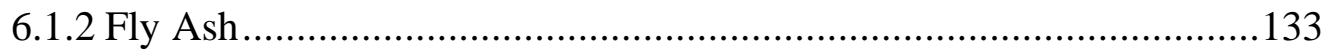

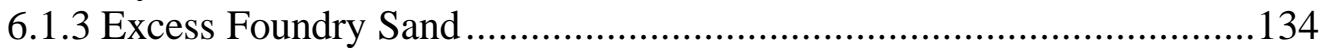

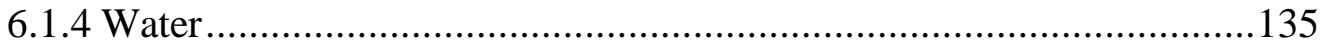

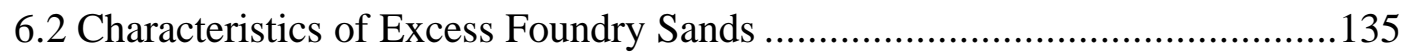

6.2.1 Bulk Density, Compaction and Void ................................................137

6.2.2 Specific Gravity and Absorption .......................................................139

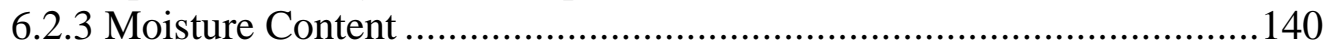

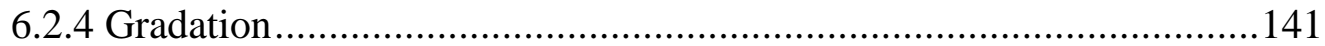

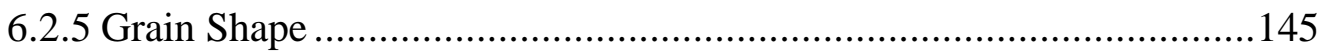

6.2.6 Organic Content and Methylene Blue Value ......................................147

6.3 Bulk Chemical Analysis of Excess Foundry Sands .....................................151

Chapter 7 EXPERIMENTS QUALIFYING CLSM USING EXCESS

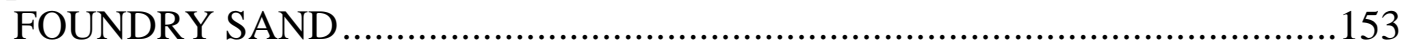

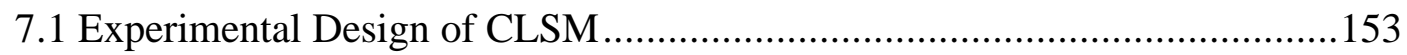

7.1.1 Executive Summary ....................................................................153

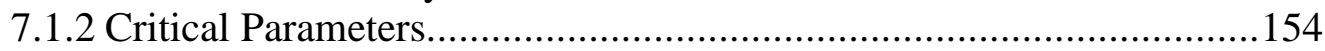

7.1.3 Formulations of Scouting CLSM …………...................................157

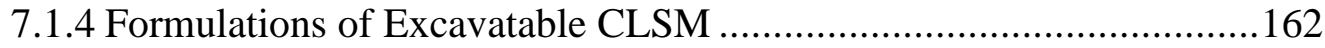

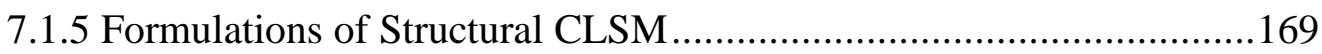

7.2 CLSM Mixing Procedure and Sample Preparation.......................................172

7.3 CLSM Testing Procedure and Results.........................................................173

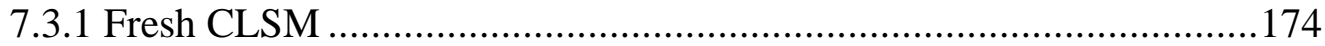

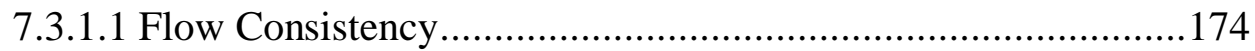

7.3.1.2 Bleeding and Settlement...........................................................174

7.3.1.3 Setting Time and Penetration Resistance ...................................178

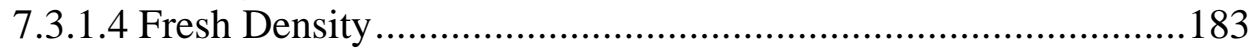

7.3.2 Hardened CLSM................................................................................190

7.3.2.1 Wet Density, Oven-Dry Density and Water Content..................190

7.3.2.2 Hydraulic Conductivity ............................................................191

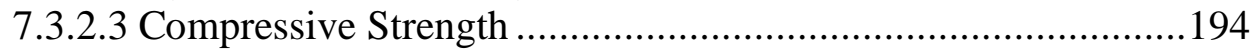

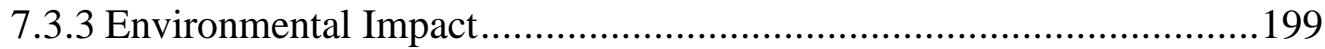

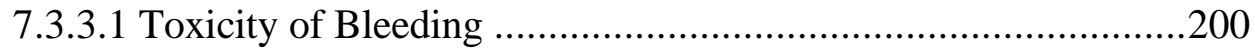

7.3.3.2 Toxicity of Hardened CLSM...................................................202

7.3.3.3 Corrosivity of Hardened CLSM ...............................................202

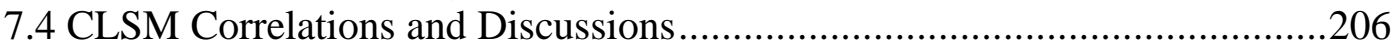

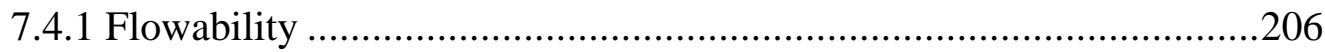

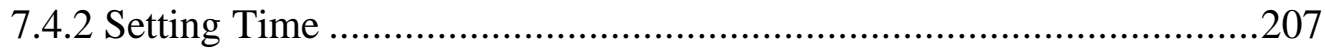

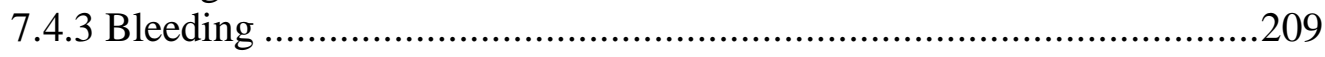

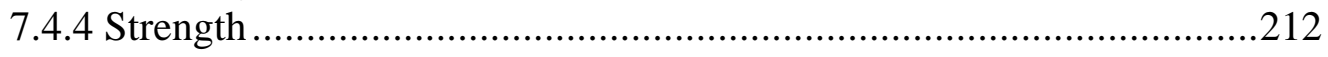

Chapter 8 EXPERIMENTS QUALIFYING HMA USING EXCESS

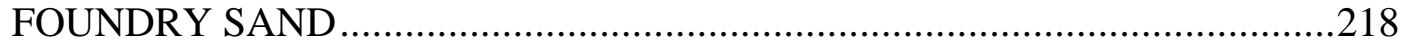

8.1 Experimental Methodology with Examples..................................................218

8.1.1 Density, Absorption and Angularity................................................219 
8.1.1.1 Aggregate Maximum Density .....................................................2.219

8.1.1.2 Bulk Density of Coarse and Fine Aggregates Using the

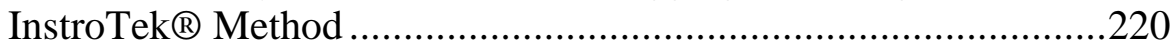

8.1.1.3 Water Absorption/Percentage of Micro Voids in Aggregates....221

8.1.1.4 Indirect Method to Determine the Apparent Density of Foundry Sand 222

8.1.1.5 Indirect Method to Determine the Bulk Density of Foundry Sand 223

8.1.1.6 Uncompacted Void Content of Fine Aggregates and Foundry Sands 223

8.1.1.7 Bulk Density of Compacted Bitumen Samples .........................224

8.1.1.8 Effective Density of Aggregates..............................................225

8.1.1.9 Asphalt Absorption of Bituminous Samples .............................222

8.1.2 Surface Area \& Average Asphalt Film Thickness .............................226

8.1.3 Densities at Different Gyrations, Energy Indices, and Indirect

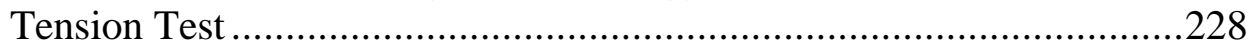

8.1.3.1 Densities at Different Gyrations ……………………............228

8.1.3.2 Paver, Roller, and Traffic Energy Indices.................................230

8.1.3.3 Strength Measured By the Indirect Tension Test ......................233

8.1.3.4 Secant Modulus Using Indirect Tension ..................................234

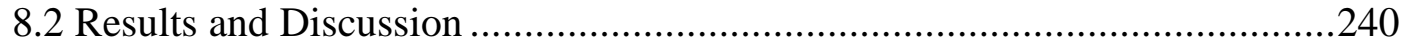

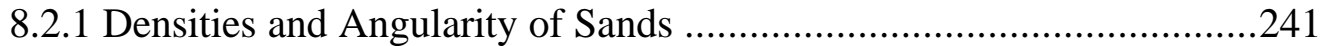

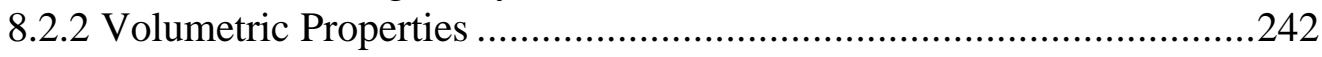

8.2.2.1 Volumetric Properties of Aggregate Blends..............................243

8.2.2.2 Volumetric Properties of HMA Mixtures ..................................246

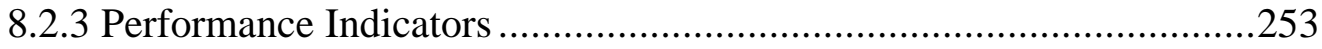

8.2.3.1 Paver, Roller and Traffic Energy Indices ..................................253

8.2.3.2 Tensile Strength and Secant Modulus Ratios.............................256

8.2.3.3 Comparison of Tensile Strength Ratio to Clay Content..............266

8.2.3.4 The Use of Anti-Stripping Agents .............................................268

Chapter 9 CONCLUSIONS AND RECOMMENDATIONS ………………........272

9.1 Characterization of Excess Foundry Sand ................................................272

9.2 Physical Properties of Excess Foundry Sand .............................................22

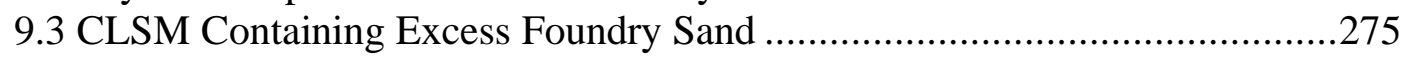

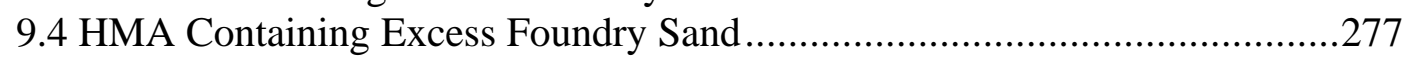

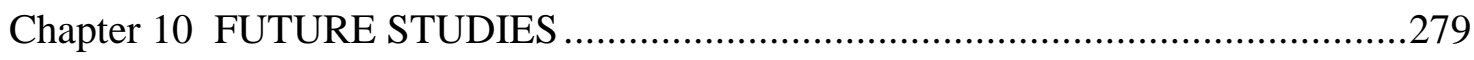

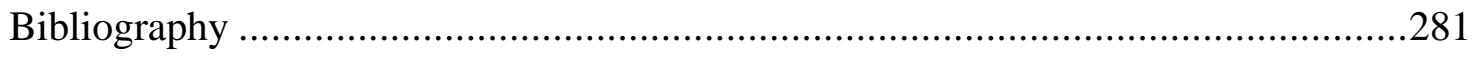

Appendix A: Glossary of Terms .....................................................................

A.1 Civil Engineering .................................................................................

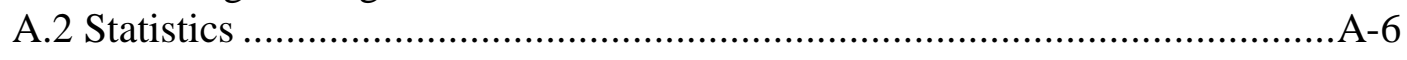


Appendix B: List of Testing Specifications (CLSM).

Appendix C: Solid Waste Hazardous Threshold, Drinking Water and

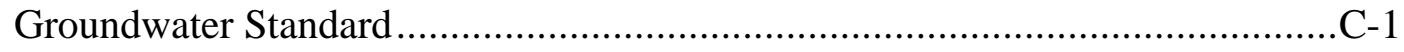

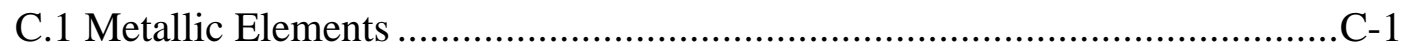

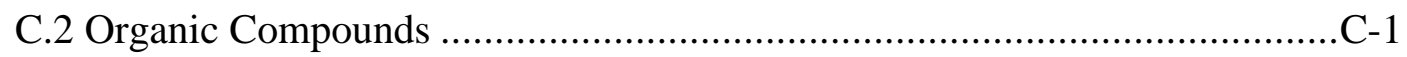

Appendix D: Bulk Analysis of Excess Foundry Sand …………………………....

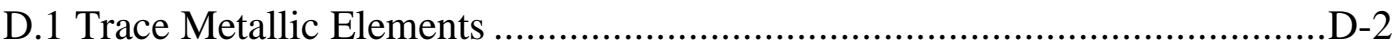

D.2 General Chemical Parameters ………………......................................

D.3 Organic Compounds .........................................................................

Appendix E: Composition of Leachate Extracted from Sand Stream........................E-1

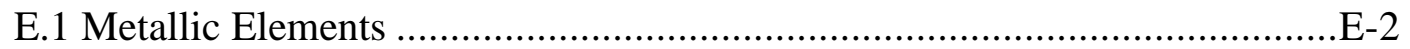

E.2 General Chemical Parameters.................................................................E-13

E.3 Organic Compounds.................................................................................

Appendix F: Survival Plot and Estimated Mean......................................................F-1

F.1 Bulk Characterization of Excess Foundry Sand …………………….........F-1

F.1.1 General Chemical Parameters ........................................................

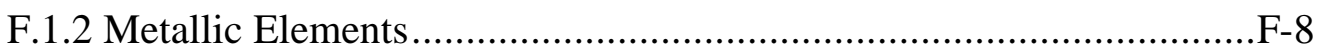

F.1.3 Organic Compounds …….......................................................F-20

F.2 Bulk Characterization of Excess Foundry Sand per Metal Cast ......................F-30

F.2.1 Metallic Elements.........................................................................F-31

F.3 Leaching Characterization of Excess Foundry Sand per Leaching

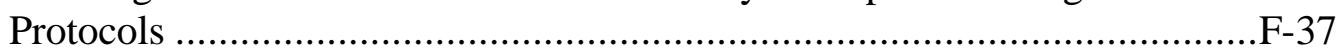

F.3.1 General Chemical Parameters ........................................................

F.3.2 Metallic Elements...................................................................

F.3.3 Organic Compounds ……………..............................................F-56

F.4 TCLP Leaching Characterization of Excess Foundry Sand per Metal Cast...F-62

F.4.1 Metallic Elements........................................................................6-63

Appendix G: Gradation and Grain Shape ............................................................

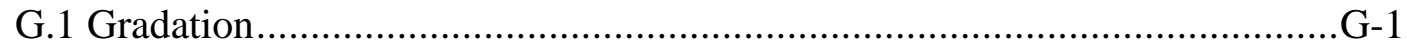

G.2 Grain Shape ………........................................................................

Appendix H: Bulk Chemical Analysis of Excess Foundry Sands in Experiments.....H-1

H.1 Organic Compounds ..............................................................................

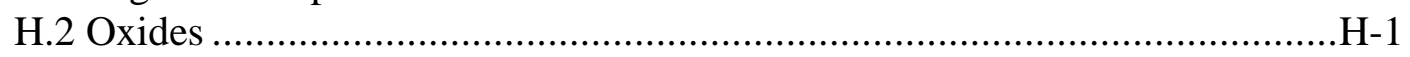

Appendix I: Setting Time and Penetration Resistance for CLSM ............................... 
I.1 Excavatable CLSM

Appendix J: Proof of Mean....................................................................................

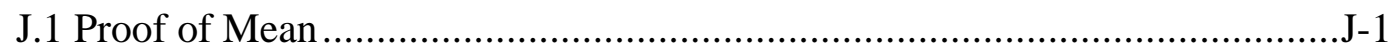

Appendix K: Flow Chart of Procedure for CLSM………………........................

K.1 Characterization of Foundry Waste Streams .............................................

K.2 Experimental Investigation of CLSM......................................................

Appendix L: Standard Specification for Excess Foundry Sand for Use in

Controlled Low-Strength Material (CLSM) ........................................................-1 


\section{LIST OF FIGURES}

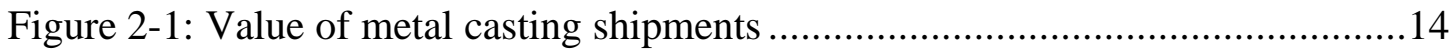

Figure 2-2: Producers's shipments of metal castings .......................................... 14

Figure 2-3: Flow diagram for typical molding sand system [Beeley 2001] ...............16

Figure 2-4: Compaction characteristics of a green sand [Webster 1980] ...................17

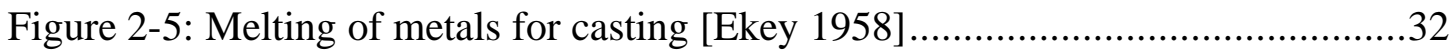

Figure 2-6: Demonstration of leachate transport model [Kimmell 1999] ...................37

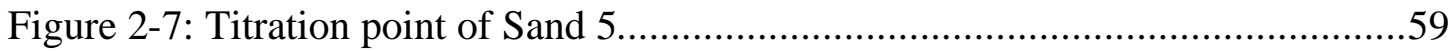

Figure 2-8: Apparent, effective and bulk densities [Ishai and Ton 1977] ...............60

Figure 2-9: Difference between packing, macro and micro voids

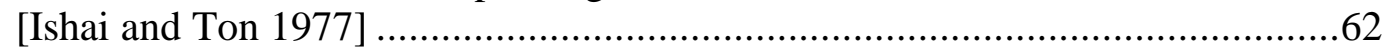

Figure 3-1: Silica content by mass in waste streams.......................................6

Figure 4-1: Demonstration of cumulative distribution function ...........................90

Figure 4-2: Demonstration of survival function .............................................93

Figure 4-3: Survival function for barium in TCLP ...........................................96

Figure 4-4: Survival function for barium in ASTM D 3987 ................................97

Figure 4-5: Comparison of survival functions for barium.................................98

Figure 4-6: Demonstration of censored data and uncensored data .........................100

Figure 4-7: Demonstration of estimation of censored data ..................................101

Figure 4-8: Comparison of fitness for barium in TCLP leachate ........................... 103

Figure 4-9: Comparison of fitness for barium in ASTM D 3987 leachate..................104

Figure 6-1: Gradation curve of excess foundry sands .................................. 142

Figure 6-2: Comparison of fines content to M.B.V. .......................................... 150

Figure 6-3: Comparison of fines content to M.B.V. for bentonite system.................151

Figure 7-1: Setting time of excavatable CLSM phase I ........................................180 
Figure 7-2: Setting time of excavatable CLSM phase II....................................181

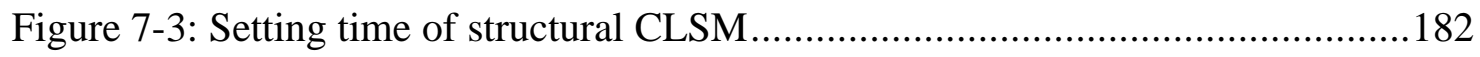

Figure 7-4: Hydraulic conductivity of excavatable CLSM phase I .........................192

Figure 7-5: Hydraulic conductivity of excavatable CLSM phase II ........................193

Figure 7-6: Strength development of scouting CLSM ......................................195

Figure 7-7: Strength development of excavatable CLSM phase I ..........................196

Figure 7-8: Strength development of excavatable CLSM phase II.........................197

Figure 7-9: Strength development of structural CLSM.......................................198

Figure 7-10: Relation between grain fineness and water content regarding

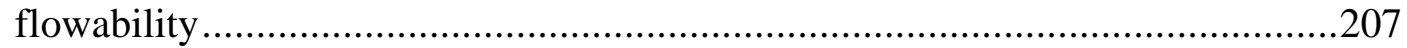

Figure 7-11: Comparison of penetration resistance ........................................209

Figure 7-12: Relation between bleeding and bleed time .................................210

Figure 7-13: Relation between bleeding and water proportion ............................211

Figure 7-14: Relation between bleed time and setting time ..............................212

Figure 7-15: Relation between 28-day compressive strength and 12-hour

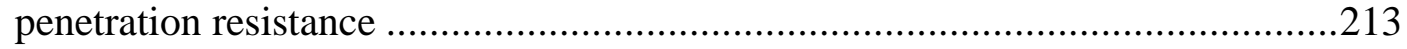

Figure 7-16: Relation between 28-day compressive strength and water-cement

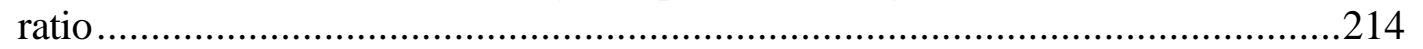

Figure 7-17: Relation between 28-day compressive strength and water-cement

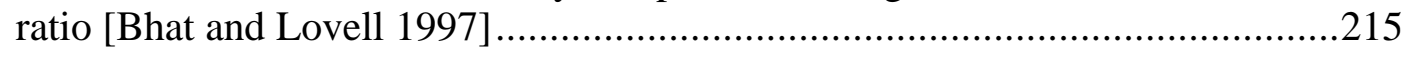

Figure 7-18: Relation between 28-day compressive strength and cement

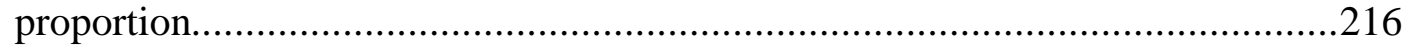

Figure 7-19: Relation between 28-day compressive strength and 90-day

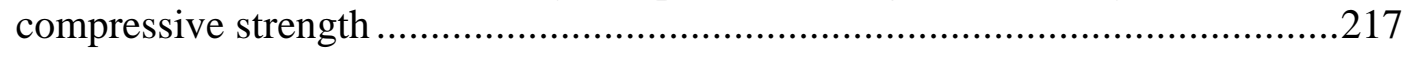

Figure 7-20: Relation between 28-day compressive strength and 180-day

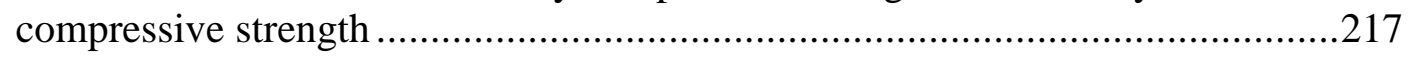

Figure 8-1: Areas from left to right are PEI, REI, TEI .....................................231

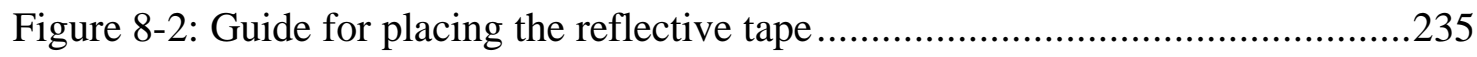


Figure 8-3: Prepared sample ready for crushing ..........................................236

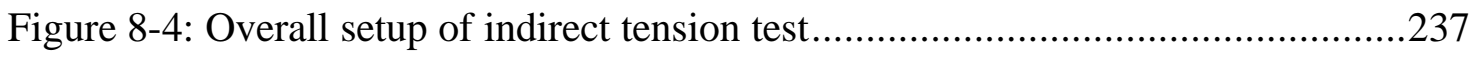

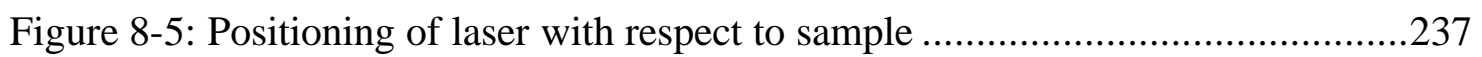

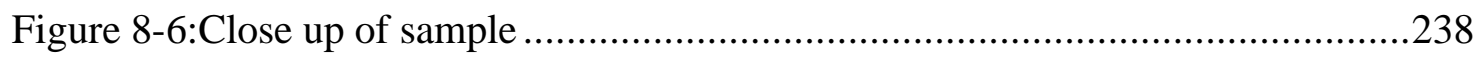

Figure 8-7: Data acquisition setup for gathering load cell and laser data ................239

Figure 8-8: Relation between voids and asphalt absorbed ................................245

Figure 8-9: Relation between voids and optimum asphalt content ........................245

Figure 8-10: Relation between optimum asphalt content and binder system.............247

Figure 8-11: Relation between the optimum asphalt content and film thickness .......249

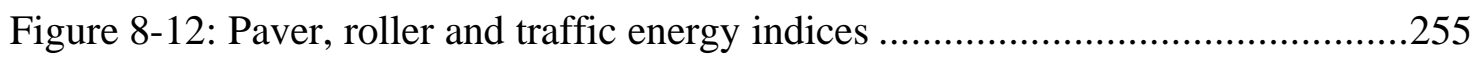

Figure 8-13: Comparison between the optimum asphalt content and the TEI ...........255

Figure 8-14: Comparison between angularity and TEI .......................................256

Figure 8-15: Strain verses stress plot for the second IDT for sand FS13 .................258

Figure 8-16: Tensile strengths with moisture conditioning ...................................261

Figure 8-17: Secant modulus with moisture conditioning .................................261

Figure 8-18: Tensile strength without moisture conditioning ................................262

Figure 8-19: Secant modulus without moisture conditioning ................................263

Figure 8-20: Comparison of tensile strength ratios.............................................264

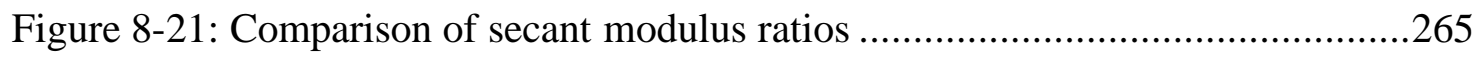

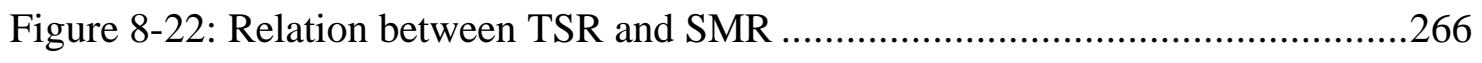

Figure 8-23: Comparison of Methylene blue value to TSR .................................267

Figure 8-24: Comparison of methylene blue value to TSR for only bentonite

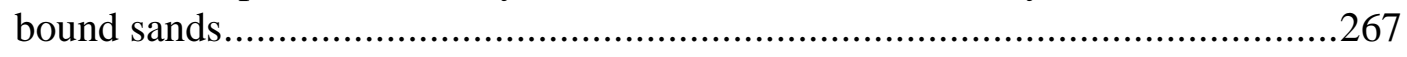

Figure 8-25: Comparison of control with and without anti-stripping additive ............268

Figure 8-26: Comparison of sand FS17 with and without anti-stripping additives .....270

Figure 8-27: Comparison of sand FS16 with and without anti-stripping additives .....271 
Figure 8-28: Comparison of sand FS05 with and without anti-stripping additive 271

Figure D-1: Bulk analysis of trace metallic elements (24 charts) ...........................

Figure D-2: Bulk analysis of general chemical parameters (17 charts) ..................... D-10

Figure D-3: Bulk analysis of organic compounds (30 charts) ...............................

Figure E-1: Leachate concentration of metallic elements per leaching protocol (23 charts)

Figure E-2: Leachate concentration of general chemical parameters (21 charts)........E-14

Figure E-3: Leachate concentration of organic compounds (37 charts) .....................

Figure F-1: Survival plots for bulk general chemical parameters (11 charts) ............F-2

Figure F-2: Survival plots for bulk metallic elements (23 charts) ............................

Figure F-3: Survival plots for bulk organic compounds (21 charts) .........................

Figure F-4: Survival plots for bulk metallic elements per metal cast (12 charts)........F-31

Figure F-5: Survival plots for general chemical parameters in leachates (16 charts)..F-37

Figure F-6: Survival plots for metallic elements in leachates (20 charts) ..................F-46

Figure F-7: Survival plots for organic compounds in TCLP leachate (13 charts).......F-56

Figure F-8: Survival plots for metallic elements in TCLP leachate per

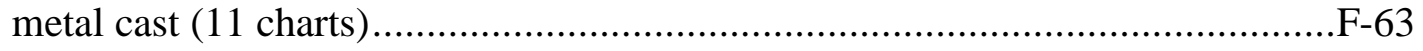

Figure G-1: Grain shape partitioned by gradation (17 charts) ................................

Figure I-1: Setting time of excavatable CLSM phase I (4 charts) ..........................

Figure I-2: Setting time of excavatable CLSM phase II (2 charts) ..........................

Figure I-3: Setting time of structural CLSM (3 charts) .................................. I-5

Figure J-1: Demonstration of probability function and survival function..................J-1

Figure K-1: Flow chart for characterization of foundry waste streams ....................

Figure K-2: Flow chart for experimental investigation of CLSM ............................. 


\section{LIST OF TABLES}

Table 2-1: Comparison of foundry base sands [Webster 1980] …………...................19

Table 2-2: Conventional binders and bonding systems [Bol'shakov and

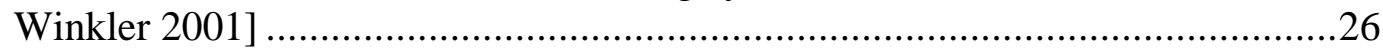

Table 2-3: Chemical bonding series [Carey and Sturtz 1995]....................................29

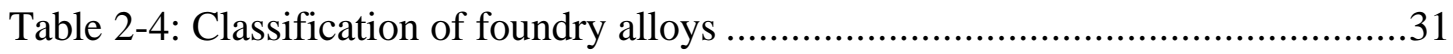

Table 2-5: Conventional description for foundry waste streams .................................34

Table 2-6: Leachability of metals from foundry sand waste [Winkler 2000] …..........44

Table 2-7: Bulk content of metals in foundry sand waste, sands and soils

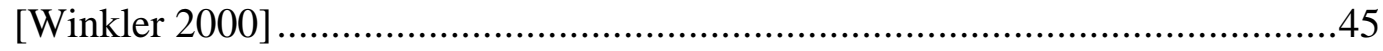

Table 2-8: Typical TCLP and SPLP results from smelting operation sludge

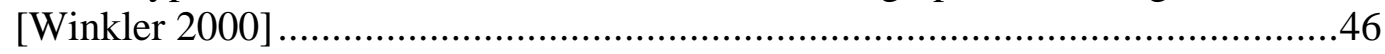

Table 2-9: Lead leachability versus total element analysis of red brass foundry sand samples and synthesized mixtures [Winkler 2000]........................46

Table 2-10: PennDOT specifications for CLSM [PennDOT 1995].............................49

Table 2-11: Iowa DOT specifications for CLSM [Iowa DOT 2004]...........................50

Table 2-12: Ohio DOT specifications for flowable fill [ODOT 1997] ........................50

Table 2-13: Starting mixtures of CLSM [Abichou et al. 1999].................................51

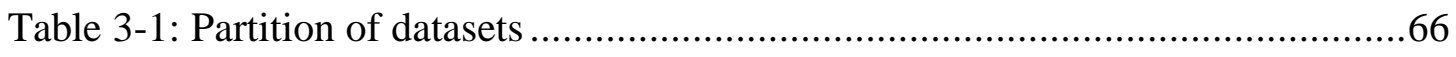

Table 3-2: Foundry sand sample chemical oxide composition [AFS 1991] ................67

Table 3-3: Metallic elements in bulk chemical analyses............................................68

Table 3-4: General chemical parameters in bulk chemical analyses ...........................69

Table 3-5: Organic compounds in bulk chemical analyses ........................................71

Table 3-6: Metallic elements in leachate analyses......................................................72

Table 3-7: General chemical parameters in leachate analyses ....................................74

Table 3-8: Organic compound in leachate analyses...................................................75 
Table 4-1: Demonstration data of arsenic in leachates....................................... 92

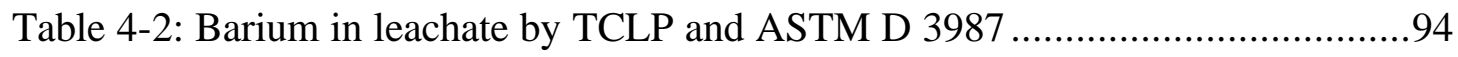

Table 4-3: Estimated cumulative function of barium in leachate by TCLP ...............95

Table 5-1: List of hypothesis tests per metal cast for as-received foundry sand .........109

Table 5-2: List of hypothesis tests per leaching protocol for sand leachates .............109

Table 5-3: List of hypothesis tests per metal cast for TCLP sand leachate................110

Table 5-4: Estimated mean for bulk chemical analysis of as-received sand (general chemical parameters) ........................................................... 111

Table 5-5: Estimated mean for bulk chemical analysis of as-received sand

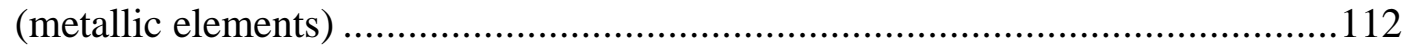

Table 5-6: Estimated mean for bulk chemical analysis of as-received sand (organic compounds)

Table 5-7: Estimated mean and factor effect for as-received sand (metallic elements)

Table 5-8: Estimated mean for sand leachate per leaching protocol (general chemical parameters).

Table 5-9: Estimated mean and factor effect for sand leachate per leaching protocol (metallic elements)

Table 5-10: Estimated mean for TCLP sand leachate (organic compounds)

Table 5-11: Estimated mean and factor effect for TCLP sand leachate (metallic elements)

Table 5-12: Metallic elements comparison between soil and excess foundry sand.....123

Table 5-13: Comparison of means and $95^{\text {th }}$ percentiles among soil, foundry sand and sand leachates

Table 5-14: Comparison of means and $95^{\text {th }}$ percentiles among soil, foundry sand and sand leachates (Cont.).

Table 5-15: Comparison of means and $95^{\text {th }}$ percentiles among soil, foundry sand and sand leachates (Cont.)

Table 5-16: Comparison of means and $95^{\text {th }}$ percentiles among soil, foundry sand and sand leachates (Cont.) 
Table 6-1: Bulk chemical composition of Type I cement and Class F fly ash

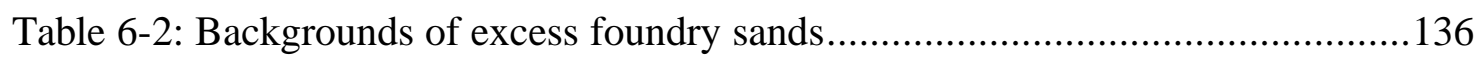

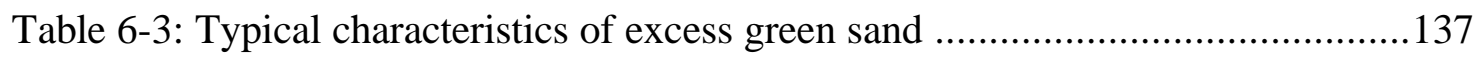

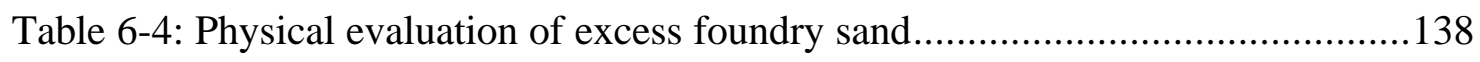

Table 6-5: Gradation evaluation of excess foundry sand ...................................144

Table 6-6: Organic Contents and Methylene Blue Values ....................................149

Table 7-1: Parameters qualifying CLSM using excess foundry sand .......................155

Table 7-2: Formulation by mass as oven-dry in scouting experiment I....................158

Table 7-3: Formulation by volume as oven-dry in scouting experiment I ................159

Table 7-4: Formulation by mass as oven-dry in scouting experiment II....................160

Table 7-5: Formulation by volume as oven-dry in scouting experiment II............... 161

Table 7-6: Formulation by mass as oven-dry in excavatable CLSM experiment

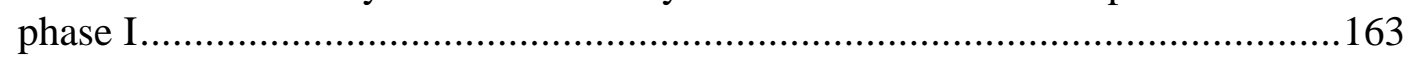

Table 7-7: Formulation by volume as oven-dry in excavatable CLSM experiment

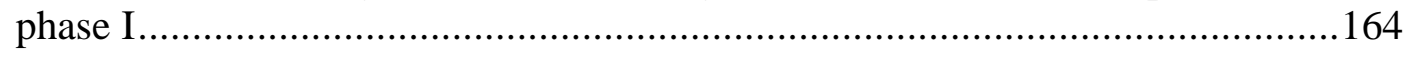

Table 7-8: Formulation by mass as oven-dry in excavatable CLSM experiment

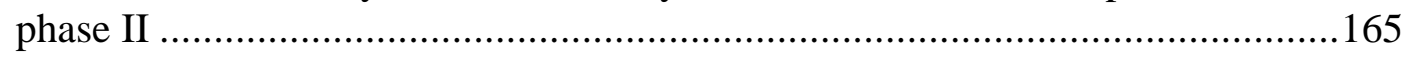

Table 7-9: Formulation by volume as oven-dry in excavatable CLSM experiment

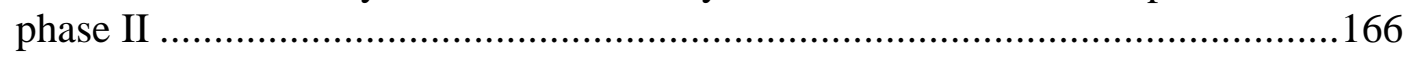

Table 7-10: Final formulation by mass as oven-dry for excavatable CLSM .............167

Table 7-11: Final formulation by volume as oven-dry for excavatable CLSM...........168

Table 7-12: Formulation by mass as oven-dry in structural CLSM experiment .........170

Table 7-13: Formulation by volume as oven-dry in structural CLSM experiment .....171

Table 7-14: Bleeding and settlement of excavatable CLSM phase I ...................... 175

Table 7-15: Bleeding and settlement of excavatable CLSM phase II......................176

Table 7-16: Bleeding and settlement of structural CLSM.................................177 
Table 7-17: Critical setting time and penetration resistance for excavatable CLSM phase I

Table 7-18: Critical setting time and penetration resistance for excavatable CLSM phase II

Table 7-19: Critical setting time and penetration resistance for structural CLSM ......186

Table 7-20: Density development of excavatable CLSM phase I ..........................187

Table 7-21: Density development of excavatable CLSM phase II ...........................188

Table 7-22: Density development of structural CLSM ...................................189

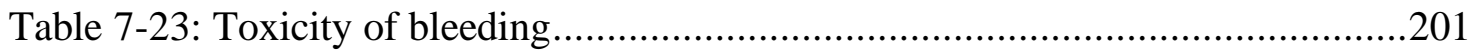

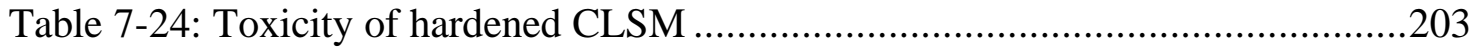

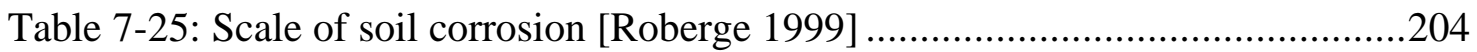

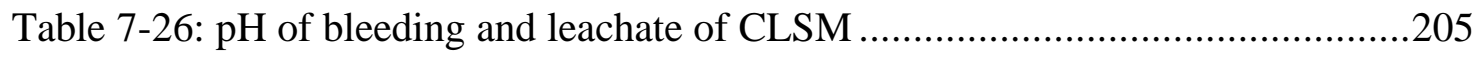

Table 7-27: Resistivity of hardened CLSM (Under Investigation) ..........................205

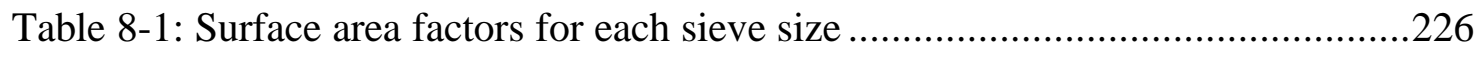

Table 8-2: Job mix formula according to percent retained..................................227

Table 8-3: Apparent and bulk densities, and angularity of sands ...........................242

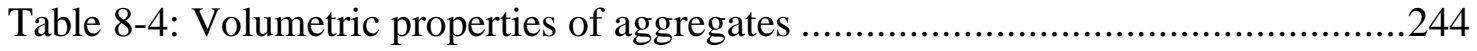

Table 8-5: Volumetric properties at optimum asphalt content .............................246

Table 8-6: Volumetric properties of HMA mixtures ........................................251

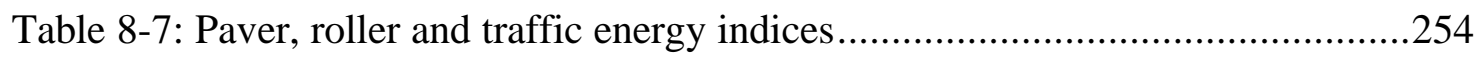

Table 8-8: Tensile strengths and secant moduli.............................................258

Table 8-9: Tensile strengths after anti-stripping agents ..................................269

Table 9-1: Starting mix of excavatable CLSM ............................................27

Table C-1: Threshold for inorganic elements ..................................................

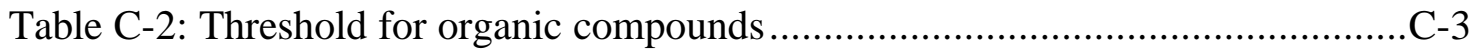

Table C-3: Threshold for organic compounds (Cont.) ....................................... 
Table G-1: Summary of oven-dry sieve analysis of excess foundry sand -

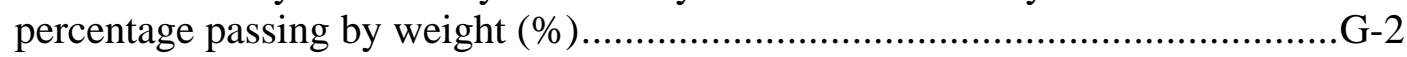

Table G-2: Summary of oven-dry sieve analysis of foundry sand percentage retained by weight $(\%)$....................................................................

Table H-1: Organic compounds of excess foundry sands ........................................-2

Table H-2: Oxides of excess foundry sands ………….......................................... 


\section{ACKNOWLEDGEMENTS}

The authors wish to express their thanks to the U.S. Department of Energy, Metal Casting Industry of the Future, for financial support of this research, and to industry coordinator Ms. Elizabeth Olenbush and the following industry and agency partners for their cooperation: American Foundrymens Society, Badger Mining, Foundry Association of Michigan, Foundry Industry Recycling Starts Today (FIRST), General Motors Corporation, Indiana Cast Metals, Kurtz Brothers, Inc., Ohio Cast Metals Association, Payne and Dolan, Pennsylvania Foundry Association, Process Recovery Corporation, U.S. Environmental Protection Agency - Region 5, and Wisconsin Cast Metals Association. 


\section{Chapter 1}

\section{INTRODUCTION}

This report is organized in 10 chapters. The first chapter includes the problem statement, research objective, and work plan of the study. Chapter 2 presents the literature review. Chapters 3 through 5 are concentrated on the characterization of foundry sand. Chapter 6 describes the characteristics of the materials and the test methods used in the laboratory studies. Chapters 7 and 8 are dedicated to the experimental investigation, results, and discussion. Chapter 9 presents conclusions based on the previous chapters, and Chapter 10 details recommendations for future studies.

\subsection{Problem Statement}

One of the most pressing problems facing the metal casting industry today is the disposal of foundry byproducts. The annual generation of foundry by-product was reported to range from 9 to 13.6 million tons in 1999 [Bol'shakov and Winkler 2001, DOE 1999]. An estimated 6-10 million tons of excess foundry sand is disposed of annually in the United States. These byproducts are generated by metal casting foundries

during the production of cast metal components. The disposal of foundry byproducts represents a significant cost for the foundry industry, whether disposal occurs in company-owned facilities or in municipal or privately owned landfills. Most of the excess foundry sands are potentially available for various reuse applications [Regan et al. 
1993]. Diversion of byproduct materials into beneficial reuse will have a substantial positive impact on foundries' bottom lines. Given the national average tipping fee of foundry byproducts to landfills ( $\$ 15-75$ per ton) [Winkler et al. 1999], including costs to store the sand, transportation, labor, and other directly related costs, there is a potential average annual savings of around $\$ 180$ million in disposal expenses with a $50 \%$ reutilization of excess foundry sand.

In the mid- to late-1980s, the era of environmental awareness and corporate liability of the foundry industry started. The binder and heavy metal residuals in foundry byproducts have attracted attention for their environmental impact, government regulations, and long-term liability. These issues compel manufacturers to make processing changes to comply with the Resource Conservation and Recovery Act (RCRA, Section 2.2.1). In addition, large numbers of landfills began to close, with the total number declining from 20,000 in the 1970 s to only 2,500 after 1997 [Foundry Management \& Technology 1996]. With the decrease in available landfill sites, landfill disposal costs are escalating due to the surcharges for transportation and landfill operation. Although the disposal of excess foundry sand can be reduced by reclamation of a greater percentage of the sand before disposal, new sands are required to supplement for shake-out loss, and sand that has degraded beyond quality standard. Degraded sand should be removed from the casting process to keep a good binding system and ensure casting quality. An ultimate solution to this issue is to beneficially reuse foundry byproducts. 
Controlled Low-Strength Material (CLSM) and Hot-Mix-Asphalt (HMA) are two growing markets that meet the basic economic and technical qualifications to address a beneficial reuse program. There are a variety of technical, economic, and environmental arguments that need to be addressed before foundry byproducts can focus on these applications.

CLSM in its simplest form is a premixed flowing soil-cement that hardens to form a strong, cohesive geotechnical product. It can be designed for nearly any strength (0.3 8.3 MPa), hydraulic conductivity $\left(10^{-2}-10^{-6} \mathrm{~cm} / \mathrm{sec}\right)$, rheological property $(100-275 \mathrm{~mm}$ slump), or setting time (2 - 24 hours). In addition, it can be mixed, pumped, and placed with standard concrete equipment.

Excess foundry sand is basically a fine mineral aggregate. More than $80 \%$ of the particles by mass are concentrated by size between $0.15-0.70 \mathrm{~mm}$, compared to $0.30-$ $4.75 \mathrm{~mm}$ for conventional fine aggregate. The AFS Grain Fineness Number (AFS GFN), a foundry industry parameter indicating the average grain size (bigger, finer), ranges between $40-90$ for most of the excess foundry sands, compared to around 40 for conventional fine aggregate. In addition, foundry sand is a uniform equidimentionalsubangular aggregate. These basic physical properties enhance the flow consistency of CLSM when excess foundry sand is incorporated into CLSM mixtures.

The reuse of foundry sand offers substantial environmental and economic benefits. The cost and environmental impact of mining and dredging virgin sand for 
CLSM, asphalt and concrete are gradually rising. These rising costs of transportation, environmental regulation, fuel and energy in mining and dredging, and the developmental cost of virgin sources of aggregate are driving the need for the reuse of natural resources. The use of CLSM and HMA containing foundry sand reduces the need for mining or dredging virgin granular aggregate. There are no costs of disposing foundry byproducts to landfills, only the shipping to local contractors. Both natural mineral resources and the urban environment are protected.

The lack of technical performance data and specifications puts use of foundry sand in the CLSM and HMA at a competitive disadvantage, not only against conventional granular materials but also against other recycled materials such as fly ash, steel mill slag, and recycled glass. The critical qualifications include the characterization of foundry sand, the engineering specifications, and technical performance standards for its CLSM and HMA applications.

The characteristics of excess foundry sands are not universally documented, particularly in regard to chemical and environmental characteristics. As a result of a wide variety of binding processes, sources of metal feed, and differences in state regulations, up to 48 inorganic elements and 43 organic elements may be evaluated by chemical extraction and testing procedures prior to its disposal or reuse. The widespread collection of the results of these characterizations is not centrally located. Systematic characterization of foundry sand needs to be addressed to ensure environmental compliance. 
The performance of CLSM and HMA containing foundry sand is not documented by highway and building standards or specifications. Detailed technical data need to be collected from CLSM and HMA containing foundry sand to validate its performance. Use guidelines pertaining to the construction and performance of CLSM and HMA need to be set up and standardized.

\subsection{Research Objective}

Overcoming technical barriers to reuse excess foundry sand in CLSM and HMA is the primary goal of this research. The research will result in substantial energy and cost savings for both foundries and user industries, as well as provide an environmental benefit at the local and national level.

A universally acceptable and open database was to be developed to address the characterization of excess foundry sand. Large numbers of datasets addressing the environmental, chemical, and physical properties of excess foundry sands were pooled from industrial partners and input into this unit for initial evaluation purposes. Follow-up datasets were added from various resources to make the data resource more robust and abundant.

Data mining research covered chemical, environmental, and physical aspects of excess foundry sand, and intended to define the variations of foundry sand characteristics, 
and how it performs as a component of CLSM and HMA. Major topics included in the research are concentration level and distribution models of individual elements, factors influencing the elements and engineering parameters. Most of the research is carried out using statistical methodology and corresponding software. The inference from this part is statistically confident and supported by nationwide data resources. It deserves to be a valuable reference for the state's characterization of excess foundry sand.

Documenting material and performance specifications of CLSM and HMA with excess foundry sand opens the door to beneficially reusing sand. The generation of materials standards (American Society for Testing and Materials [ASTM], American Association of State Highway and Transportation Officials [AASHTO]) and construction specifications is essential in order for legal entities to adopt provisions for materials that contain CLSM and HMA. Most other competitive industry byproducts, such as fly ash and slag, are documented by various technical standards for multiple engineering applications. The research eliminates the gap between excess foundry sand and other byproducts after successfully developing CLSM and HMA containing excess foundry sand.

The development of user guidelines for CLSM and HMA mixture designs finalizes the research objective. Developing educational and professional guidelines creates a standard of practice that contractors and engineers can be expected to meet. This development is essential for the specification and implementation of CLSM and HMA containing excess foundry sand. 
Broad dissemination of the dataset, guidelines, standards, and specifications will be the cornerstone of the implementation of the proposed work.

\subsection{Research Methodology}

The scope of the research includes three parts: characterization of excess foundry sand, technical qualifications of CLSM using excess foundry sand, and technical qualifications of HMA containing excess foundry sand. The first part concentrates on the chemical and environmental issues of excess foundry sand through data processing techniques, such as the toxicity level of excess foundry sand and factors influencing its toxicity. The latter two parts are experimental research in a laboratory environment that mixes CLSM and HMA samples using excess foundry sand and qualifies its performance in comparison to standard CLSM and HMA specifications.

The research was performed in stages as follows:

\section{Stage 1 - Collection of Datasets}

Datasets addressing bulk chemical analysis, extraction chemical analysis, and physical analysis of excess foundry sand were collected from regulatory independent commercial laboratories. These datasets were input into a database coded with MSAccess for convenient management. 


\section{$\underline{\text { Stage } 2 \text { - Evaluation of Datasets }}$}

Confident statistical methodologies were developed to characterize excess foundry sand from chemical, environmental, and physical standpoints. First, a data plotting technique was applied. Data plotting is a basic and straightforward technique that presents the real characterization without any assumption or data transformation. Furthermore, an innovative approach, survival analysis, was developed to address environmental monitoring data containing censored data.

\section{Stage 3 - Scouting of CLSM}

Representative excess foundry sands from nationwide foundry facilities were collected to evaluated CLSM characteristics. The selection spans two significant factors differentiating individual excess foundry sand, including metals cast and binding systems.

Prior to the initiation of the massive investigation of CLSM performance, small trial batches were conducted to probe the formulations of excavated CLSM and structural CLSM. Controlling parameters of CLSM were addressed in this stage, such as flowability consistency, bleeding, shrinkage, and strength development. The formulations were approved or improved depending on how close their performance complied with CLSM specifications.

\section{$\underline{\text { Stage } 4 \text { - Massive Excavatable CLSM }}$}

Using the recommended formulations from Stage 3 for each excess foundry sand, massive excavatable CLSMs were mixed and tested. Sufficient numbers of samples were 
prepared for performance investigation purposes, such as strength development, hydraulic conductivity, density development, and setting time. Most of the mixtures of CLSM in this stage complied with major provisions of standard CLSM specifications. In cases where some excess foundry sands did not qualify, mixes with improved formulations were prepared and investigated.

\section{$\underline{\text { Stage } 5 \text { - Massive Structural CLSM }}$}

The formulations of structural CLSM were inferred from the formulations of scouting CLSM, formulations of excavatable CLSM, and highway specification for CLSM. Investigation procedures are identical to those for excavatable CLSM.

\section{$\underline{\text { Stage } 6 \text { - Evaluation of CLSM }}$}

Factors influencing CLSM performance were discussed in this stage. Technical problems, such as the control of strength development, bleeding reduction, flow consistency improvement, and acceleration of setting time were resolved.

\section{Stage 7 - Collection of HMA Data}

The engineering properties were determined for the control sand and each of the 17 foundry sands chosen, and the aggregate mixes in which they were incorporated. These properties included gradation by mass, angularity, asphalt absorption, maximum and bulk density, clay content, and organic material content. In many cases, nontraditional methods were used to test the sands' properties because standard methods could not be applied for some foundry sands. For the maximum density, the CoreLok ${ }^{\mathrm{TM}}$ 
(InstroTek, 2001) device, built by InstroTek, was used instead of the traditional vacuum pump method. The bulk density was determined using a modified version of a testing method developed by InstroTek. It involves finding the density of an aggregate that is submerged in water but has air trapped in its surface pores. The clay content was determined by saturating the foundry sands with methylene blue dye and measuring the concentration of unabsorbed methylene blue with a spectrophotometer.

\section{Stage 8-Collection of Performance Data of HMA}

The performance data collected can be broken down into the following categories: HMA volumetric data, energy indices derived from gyratory compaction testing, indirect tensile stress and strain measurements, and moisture sensitivity testing. The performance measures are standard methods used by the asphalt pavement industry.

\section{Stage 9 - Development of Guidelines for HMA}

Based on the engineering properties of the foundry sands and the performance data collected, guidelines were developed. These guidelines describe the responsibilities of each of the foundries and the aggregate suppliers in the preparation and shipment of the sands. There are also specifications limiting the amount of foundry sand used based on its methylene blue content and binder system used. The guidelines also include specific steps that asphalt contractors should follow to produce mixtures with foundry sands of acceptable quality. 


\subsection{Potential Impact to Society}

Results from the research will aid the foundry industry in reducing costs and decrease potential energy consumption and environmental impacts. The lack of technical data is eliminated through this project, mitigating a critical barrier that makes it costly and time consuming for large foundries to develop reuse alternatives and financially infeasible for small foundries. The competitive advantage of beneficial reuse is strengthened by this research.

The energy industry is a large potential beneficiary of the research. National energy savings on the order of magnitude of 50 million MBtu will result from reducing energy otherwise expended by foundries in handling and reclaiming foundry byproducts [Tikalsky 2000]. Energy savings will also accrue from greenhouse gas emissions reductions; from avoidance of energy requirements of transportation, disposal, and construction of landfill capacity to dispose of foundry byproducts; and from substitution of foundry byproducts for construction sand and gravel as an alternative to virgin mineral extraction activities.

This research provides an environmental benefit at the local and national level. Increased beneficial reuse of excess foundry sand is an effective way to reduce emissions to the environment, conserve increasingly limited capacity, increase the amount of material that is recycled in the industry, and reduce the amount of virgin sand being mined or dredged. All of these factors result in substantial environmental benefits. 
The construction industry benefits by the introduction of CLSM and HMA containing excess foundry sand. Cost savings arise with decrease in material cost, sufficient material supplies and matched construction quality; designers are free to incorporate excess foundry sand into CLSM and HMA supported by technical documents; and constructors benefit from the less labor-intensive technique and its rapid construction. 


\section{Chapter 2}

\section{LITERATURE REVIEW}

The first part of chapter 2 gives an overview of the foundry process and explains several key concepts used in the foundry industry. This includes the difference between a mold and a core, different types of base sands, different types of binder systems, and the different kinds of fillers used. The second part of chapter 2 focuses on past studies regarding the characterization of foundry sand. The last part of chapter 2 is an overview of past studies about incorporating recycled foundry sand into CLSM and HMA.

\subsection{Introduction to Metal Casting Industry}

The scope of the metal casting industry encompasses a major segment of the U.S. economy. Its sales reached $\$ 18.3$ billion in 2001 (see Figure 2-1) and its shipments reached 12.2 million tons in 2001 (see Figure 2-2), [U.S. Census Bureau 2002]. It employs, directly and indirectly, 225,000 people. The industry's product, castings, enters into every field in which metals serve man, including but not limited to automotive, military, construction, agriculture, power generator, aerospace and atomic energy applications. 


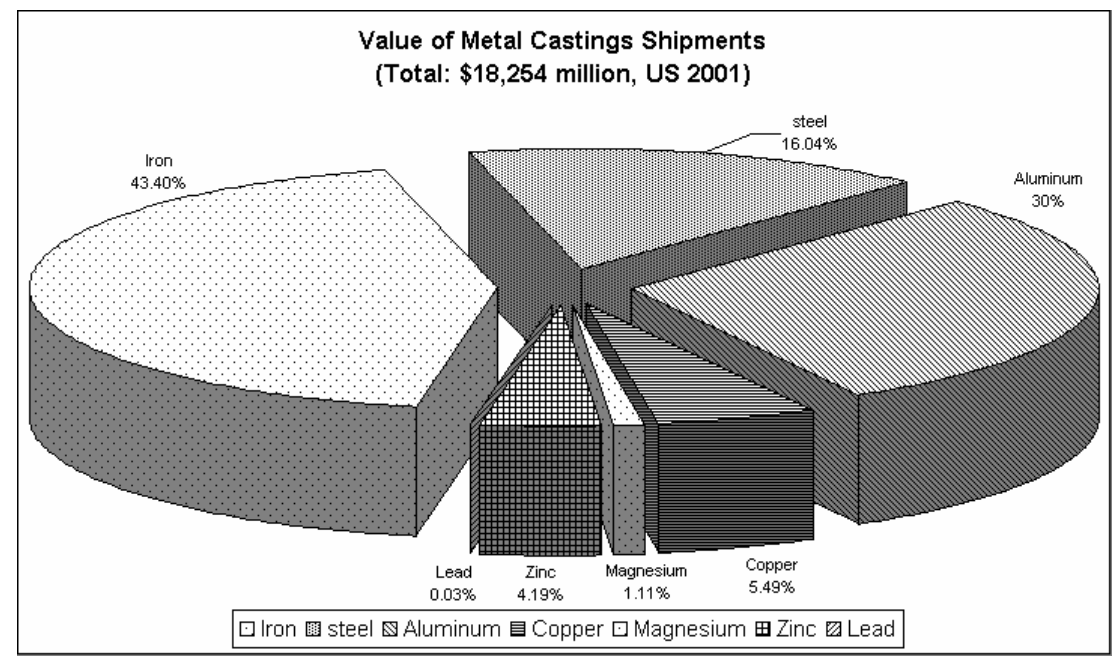

Figure 2-1: Value of metal casting shipments

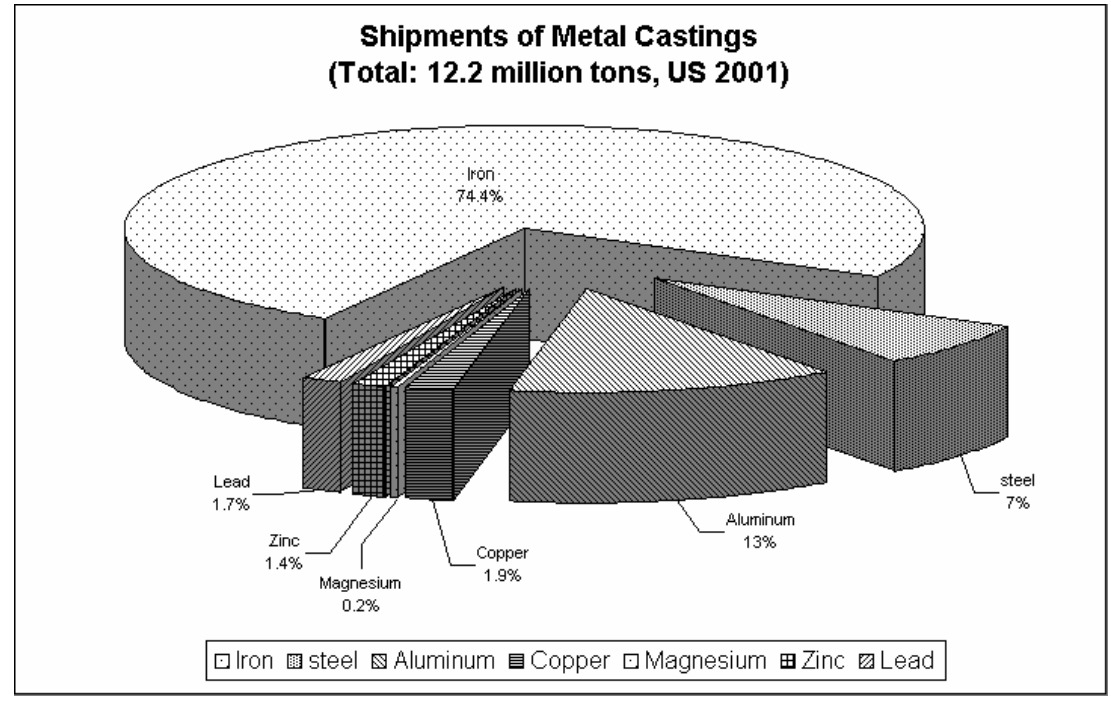

Figure 2-2: Producers' shipments of metal castings

A metal casting foundry is a commercial establishment for producing metal castings. A metal casting is a shape obtained by pouring liquid metal into a mold or cavity and allowing it to freeze and thus to take the form of the mold. In a typical foundry facility system, five fundamental steps are followed to fulfill the casting: patternmaking, 
core making, molding, melting and pouring, cleaning and reclaiming. The sequence of elements in such a system is shown in Figure 2-3.

The types of molding and core making processes generally include a sand casting process, permanent-mold process, die-casting process, centrifugal process, shell-mold process, investment process, lost foam process, plaster process, and graphite process. The sand casting process is the most popular casting process, with low cost, high efficiency, and reuse cycles. Of the total tonnage of castings produced each year, the greatest percentage is produced by sand casting [Ammen 2000]. The process consists of making molds with sand aggregate, then pouring a molten metal or alloy into them and allowing it to solidify. 


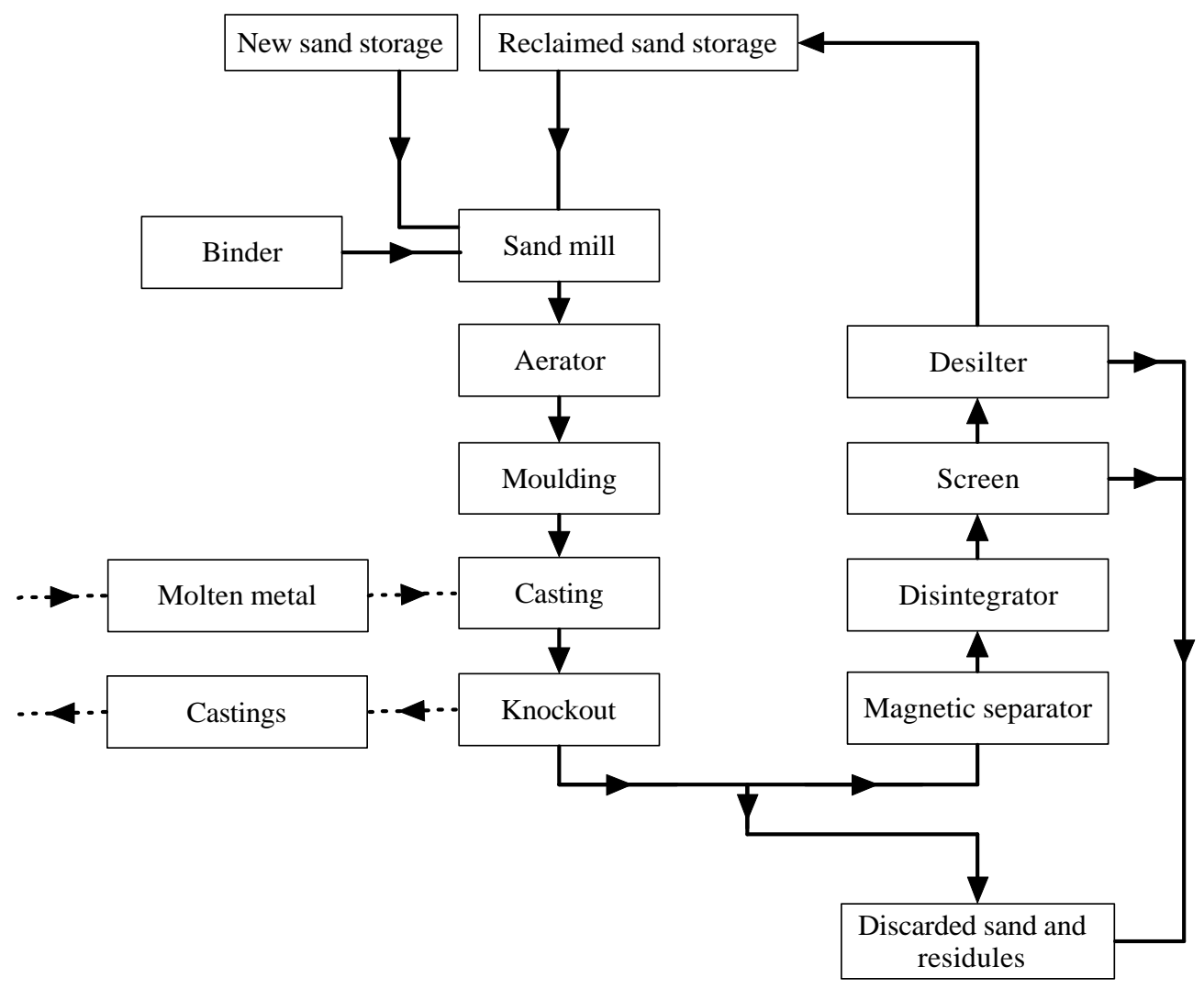

Figure 2-3: Flow diagram for typical molding sand system [Beeley 2001]

\subsubsection{Molds and Cores}

In the sand casting process, molds and cores are prepared prior to pouring melting metals. The mold is made by packing molding aggregate around a pattern, Figure 2-4. When the pattern is withdrawn, its imprint provides the mold cavity, which is ultimately filled with metal to become the casting. In general, sand molds are shaken off and reclaimed to new molds. Molds are made by various bonding systems, including green sand, organic bonding systems, and inorganic bonding systems, addressed in Section 2.1.4.1 and Section 2.1.4.2. 


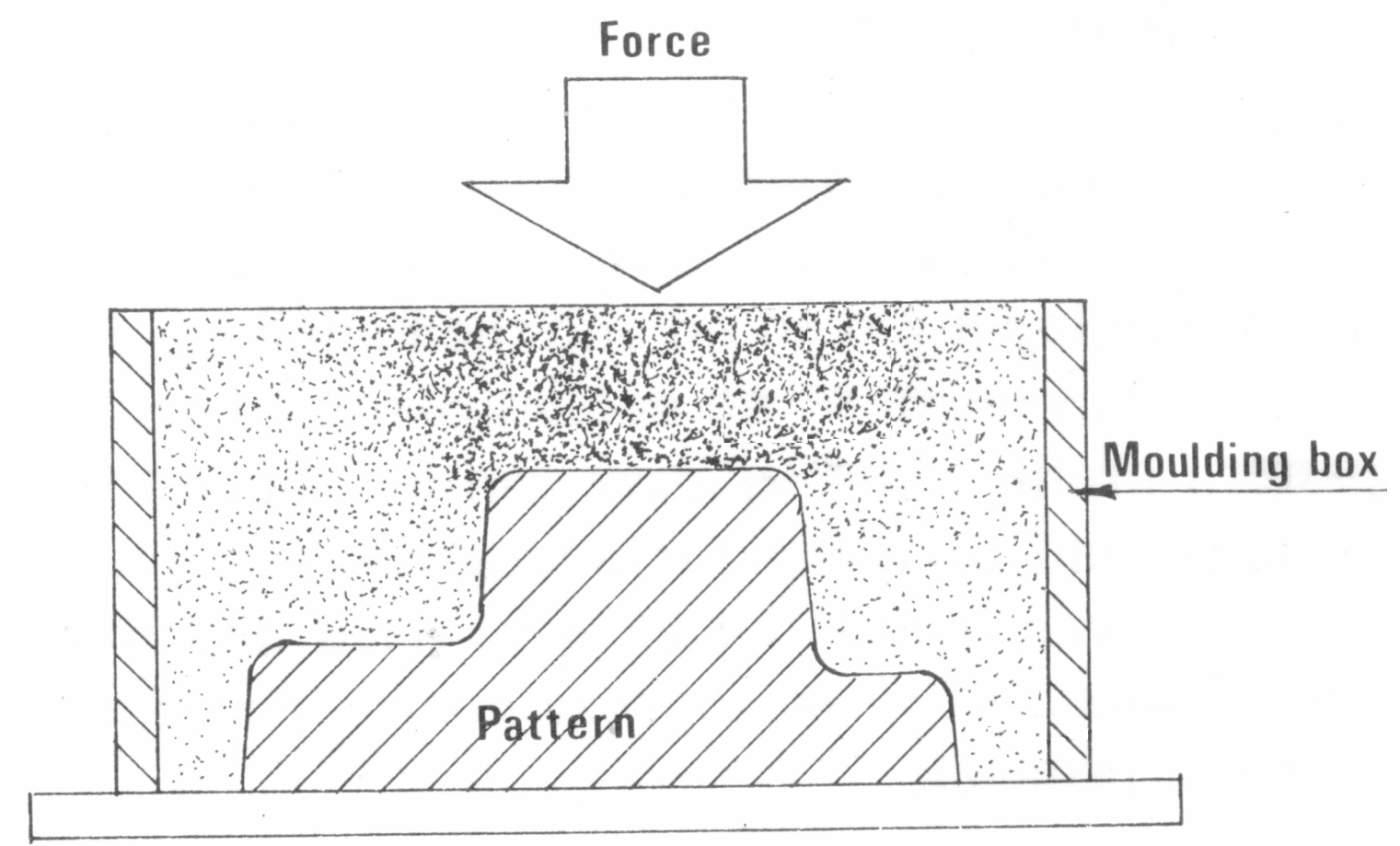

Figure 2-4: Compaction characteristics of a green sand [Webster 1980]

Cores are forms, usually made of sand, which are placed into a mold cavity to form the interior surface of a casting. The void space between the core and mold-cavity surface is what eventually becomes the casting. Cores are made by core boxes, which are filled with core sand, rammed, and struck off. The immediate example is a simple round cylinder form needed to core a hole through the hub of a wheel or bushing. Its external shape becomes the internal shape of the casting. The cores are usually made of organic and inorganic bonding systems, addressed in Section 2.1.4.2. 


\subsubsection{Virgin Foundry Sands and Excess Foundry Sands}

The most widely used aggregate for molds and cores is sand because it has the ability to absorb and transmit heat as it allows gases evolved during binder breakdown to pass between the grains; the ability of grains holding together and giving strength; and the ability to withstand high heat with moderate breaking down or fusing [Ekey 1958]. As shown in Table 2-1, there are many types of sand utilized by the foundry industry. However, because of its wide availability and relatively low cost, silica sand is the one used in most of the metal casting [Carey 1994]. Quartz and other silica minerals are the main components of silica sand. It is essentially $\mathrm{SiO}_{2}$. Silica sand is found in nature on the bottoms and banks of river, lakes, on seashores, in dry river deposits, and in substrata layers of the earth. Many of these deposits are mined and used without alteration.

The cost of new, clean, dry, clay-free sand in conjunction with enhanced environmental requirements, transport, and tipping charges has meant that greater urgency has been given to possible recovery and reuse of sands used in the molding and coremaking systems. The principal methods of sand recovery are dry scrubbing (pneumatic, mechanical, or shot blast) for clay, organic and inorganic binders, calcination

(thermal) for organic binders, and wet washing for clay and silicate binder [Granlund 1984]. 
Table 2-1: Comparison of foundry base sands [Webster 1980]

\begin{tabular}{|c|c|c|c|c|c|c|c|}
\hline & Silica & Olivine & Chromite & Zircon & $\begin{array}{l}\text { Zircon/Aluminum } \\
\text { Silicate }\end{array}$ & Staurolite & Carbon Sand \\
\hline Composition & $\begin{array}{l}\text { Varying } \\
\text { connection of } \\
\mathrm{SiO}_{4}^{4-} \text { tetrahedron }\end{array}$ & $\begin{array}{l}\mathrm{SiO}_{4}^{4-} \text { tetrahedron } \\
\text { bonded together by } \\
\mathrm{Mg}^{2+} \text { and } \mathrm{Fe}^{2+}\end{array}$ & --- & $\mathrm{ZrSiO}_{4}$ & --- & --- & Petroleum, C \\
\hline Origin & USA & $\begin{array}{l}\text { USA (WA, NC) } \\
\text { Norway }\end{array}$ & S. Africa & $\begin{array}{l}\text { USA, } \\
\text { Australia }\end{array}$ & USA (FL) & USA (FL) & Man-made \\
\hline Color & $\begin{array}{l}\text { White-Light } \\
\text { Brown }\end{array}$ & Greenish Gray & Black & White-Brown & Salt \& Pepper & Dark Brown & --- \\
\hline Hardness & $6.0-7.0$ & $6.5-7.0$ & $5.5-7.0$ & $7.0-7.5$ & $6.5-7.0$ & $6.5-7.0$ & --- \\
\hline $\begin{array}{l}\text { Dry Bulk Density } \\
\left(\mathrm{lb} / \mathrm{ft}^{3}\right)\end{array}$ & $85-100$ & $100-125$ & $155-165$ & $160-185$ & $155-168$ & $143-146$ & 64 \\
\hline Specific Gravity & $2.2-2.6$ & $3.2-3.6$ & $4.3-4.5$ & $4.4-4.7$ & $3.2-4.0$ & $3.1-3.8$ & --- \\
\hline Grain Shape & Angular/Rounded & Angular & Angular & $\begin{array}{l}\text { Rounded/ } \\
\text { Angular }\end{array}$ & Rounded & Rounded & Rounded \\
\hline $\begin{array}{l}\text { Thermal Expan. } \\
1,600^{\circ} \text { (in./in.) }\end{array}$ & 0.018 & 0.0083 & 0.005 & 0.003 & 0.005 & 0.007 & --- \\
\hline Appar. Heat Transfer & Average & Low & Very High & High & High & High & \\
\hline Fusion Point $\left({ }^{\circ} \mathrm{F}\right)$ & $2,600-3,200$ & $2,800-3,200$ & $3,200-3,600$ & $3,700-4,000$ & $3,300-3,600$ & $2,500-2,800$ & $>4,532$ \\
\hline High Temp. Reaction & Acid & basic & basic & Acid & Slightly Acid & Slightly Acid & --- \\
\hline $\begin{array}{l}\text { Wettability with } \\
\text { Molten Metal }\end{array}$ & Easily & Not Generally & Resistant & Resistant & Resistant & Resistant & --- \\
\hline Chemical Reaction & Acid-Neutral & Basic & Basic-Neutral & Acid-Neutral & Neutral & Neutral & --- \\
\hline $\begin{array}{l}\text { Grain Dist. (\# } \\
\text { Screens) }\end{array}$ & $2-5$ & $3-4$ & $4-5$ & $2-3$ & 3 & $3-4$ & --- \\
\hline AFS GFN & $25-180$ & $40-160$ & $50-90$ & $95-160$ & Approx. 80 & Approx. 70 & --- \\
\hline $\begin{array}{l}\text { Cost: } 1 \text { cheapest; } 4 \\
\text { most expensive }\end{array}$ & 1 & 2 & 3 & 4 & --- & --- & Expensive \\
\hline $\begin{array}{l}\text { Density: } 1 \text { lightest; } 4 \\
\text { heaviest }\end{array}$ & 1 & 2 & 3 & 4 & --- & --- & lightweight \\
\hline Composition & $>95 \% \mathrm{SiO}_{2}$ & $\begin{array}{l}\mathrm{SiO}_{2}(42 \%), \mathrm{MgO} \\
(50 \%), \mathrm{Fe}_{2} \mathrm{O}_{3}(7 \%)\end{array}$ & $\begin{array}{l}\mathrm{SiO}_{2}(2 \%), \mathrm{MgO} \\
(10 \%), \mathrm{Fe}_{2} \mathrm{O}_{3} \\
(25 \%), \\
\mathrm{Cr}_{2} \mathrm{O}_{3}(50 \%), \\
\mathrm{Al}_{2} \mathrm{O}_{3}(15 \%)\end{array}$ & $\begin{array}{l}\mathrm{SiO}_{2}(32 \%), \\
\mathrm{ZrO}_{2}(65 \%)\end{array}$ & --- & --- & --- \\
\hline
\end{tabular}


Sand employed in a mechanical sand preparation and handling system is called system sand. After several cycles of reclamation, sand is removed from the system and becomes excess foundry sand. Reclamation units allow foundries to reuse mold sand and thereby generate less excess sand product. Grain cracking, core sand additions, and free clay content limit the amount of reclamation to approximately $75-90 \%$; even foundries using reclaim units send sand out as a waste stream. A considerable amount of excess foundry sand, around 6-10 million tons, is discarded from facilities annually as continuous reuse of sands is followed by deterioration of its quality and workability. For example, in a closed sand system, if a 97\% yield is obtained after reclamation but the molding sand mixture can only be made with $80 \%$ reclaimed sand and $20 \%$ new sand, the excess $17 \%$ reclaimed sand must be discarded or recycled to another application.

The steady worsening of the sand mass can be physical. Reclamation causes physical changes to occur to the sand, since it smoothes and rounds the surface of the grains. Breakdown of quartz grains and accumulation of fusible fines reduce both permeability and refractoriness [Beeley 2001]. The shakeout may be a mixture of mold and core sand with different gradation which is not feasibly reclaimed due to low yields. Inorganic residuals in the pores of grains may not be removed, which results in incompatibility [Carey 1994].

The degradation can also be of a chemical nature. The surface chemistry of reclaimed sand is drastically altered by the resin, catalyst, and additives. Even if the coating is almost completely removed during every reclamation cycle, the chemistry of 
the reclaimed sand may vary somewhat with each cycle [Carey and Sturtz 1996]. The clay at or near the casting surface becomes heated and destructed to "dead." After this has occurred, milling and additions of water will not bring back the bond. Too much "dead" clay results in brittle sand, poor strip, and bad lifts. [Webster 1980].

\subsubsection{Sand Binders}

A binder is a "material added to the sand or provided by nature with the sand that imparts cohesiveness" [Ekey 1958]. Its typical mass percentage varies from $0.5 \%$ to $15 \%$ based on the base sand, binder and bonding system. Binders are categorized into three groups: clay-type binders, organic-type binders, and inorganic-type binders. The last two groups are frequently called chemical binders.

Clay-type binder, in the amount of $4-10 \%$ by weight of base sand [Bol'shakov and Winkler 2001], is used for making small, medium, and large molds. It is relatively inexpensive and of low strength. Chemical binder is usually used for small to mediumsize molds and cores and is relatively stronger with good erosion resistance. The major subdivisions of the clay family are montmorillonites (bentonites mostly used in foundry), kaolinites, and illite.

Organic binders include two major groups, resin and oil-based binders. They are combustible and are destroyed by heat [Heine 1967]. The resin is made by mixing various proportions of phenolic, furfuryl alcohol (furan), urethane and formaldehydes, 
ranging from 1.5 to $8 \%$ based on base sand [Sylvia 1972]. They are liquids or gums, natural or synthetic, where the individual molecules have the capacity to polymerize or fuse together to form very long chains. This polymerizing reaction can be triggered by certain chemical reagents or heat. The resulting chain hardens to form a powerful bond with other materials [Burns 1986].

The oils in the amount of $0.5-3 \%$ can be either natural oils, such as linseed, perilla, tung and dehydrated castor oils, or processed oils, such as unsaturated mineral oils, synthetic oil, and alkyd resin. Their mechanism of hardening is similar to resin, polymerization with or without heat.

Inorganic-type binders include sodium silicate and portland cement, which are incombustible. They are environmentally benign compared to organic binders and have low cost, low sand sensitivity, and low gas evolution. The sodium silicate, generally called water glass, refers to a three-ingredient system: silica, sodium oxide, and water. The cement-based bonding system is a mixture of sand, $8-12 \%$ high-early-strength hydraulic cement, and 4-6\% water. Inorganic-type binders develop great hardness and strength by the setting action of sodium silicate or portland cement. However, they present slow cure characteristics, and once used, they are poor to break down or reuse [Sylvia 1972, Webster 1980], which offsets their environmental advantage. 
In most processes, one or multiple additives are added to the sand for improving some special bonding or casting features, or catalyzing the binders. Examples are sea coal, wood flour, and silica flour.

\section{Sea Coal}

Sea coal is nothing more than ground bituminous coal. It helps to improve the surface finish of the molded part. During the pouring process, the sea coal swells and fills the voids between sand grains [American Foundry Society 2000]. It also helps to remove the part from the mold sand during the shakeout process. Lastly, it helps prevent the mold sand from drying out, by retaining water.

\section{Iron Oxide}

Red Oxide in the form of $\mathrm{Fe}_{2} \mathrm{O}_{3}$ is the most commonly used iron oxide [American Foundry Society 2000]. During the pouring process, the iron oxide fuses together with the silica sand and forms a plastic glaze [American Foundry Society 2000]. This helps to decrease certain casting defects like veining and metal penetration. It also helps to fill in the gaps between sand grains and increase the finish of the cast part.

\section{Soda Ash}

Sodium carbonate, a.k.a. soda ash, is usually added in very small quantities, $0.0025-0.005 \%$ of the batch weight [American Foundry Society 2000]. It is used to free up additives and inert fines so they are not clumped. It also is used to increase the $\mathrm{pH}$ of 
the molding sand as it is returned and reused through the mold system. The fermentation of cereals can decrease the $\mathrm{pH}$.

\section{Cellulose}

Cellulose, in the actual sense, is the material plants create to give their cell walls structural support, and essentially to give the whole plant structural support. Cellulose, for foundries, is any material that has been derived from plants or a plant by-product [American Foundry Society 2000]. It usually is made from corn cobs, oat hulls, wood, nut shells and rice hulls. They are processed and sized to make them suitable for foundry usage. Cellulose is more commonly used for bentonite bound mold sand, and acts like a cushioning material in much the same way as cereals and starch [American Foundry Society 2000]. As metal is poured in the mold, the silica sand grains expand due to the heat. Simultaneously, the cellulose burns up, giving the expanding sand grains the needed room to expand. Cellulose also helps to retain water and improves shakeout and flowability.

\section{Cereals \& Starches}

The foundry industry classifies cereals and starches as natural organic carbohydrates, and are produced from different grains that all behave in a similar manner when used in foundry sand [American Foundry Society 2000]. Common types of cereals and starches include corn, milo (sorghum), wheat, bran, rye, and oat flour, dextrin and corn sugar. Like cellulose, cereals and starches are used to compensate for the thermal expansion of the silica sand grains, by burning up during metal pouring. They also help 
with moisture retention and control, to increase the range of acceptable moisture levels. Finally, they allow the mold sand to be more moldable to accommodate more complicated patterns [American Foundry Society 2000].

\subsubsection{Sand Bonding Processes}

Binders need to be exposed to the appropriate process to activate cohesion between the refractory grains in the green or hardened state. Generally the processes are divided into green sand, chemical bonding process, and unbonded sand system. Combinations of binder and its bonding systems are presented in Table 2-2.

\subsubsection{Green Sand Process}

Green sand is the most widely used bonding process in the world because it is low in cost, high in performance, and environmentally benign, and the materials are reusable. It is composed of uniform high-quality refractory quartz sand (85-95\%), cohesive bentonite clay (4-10\%) as the binder, a volatile carbonaceous sea-coal additive (2-10\%) as filler to improve casting surface finish, iron oxide $(0.5-5 \%)$ for strength, and water to activate clay (2-5\%) [Bol'shakov and Winkler 2001]. It is referred to as "green" because of the moisture present and is thus distinguished from dry sand. 
Table 2-2: Conventional binders and bonding systems [Bol'shakov and Winkler 2001]

\begin{tabular}{|c|c|c|c|}
\hline & Thermosetting Processes & Self-Setting Processes & Gas-Cured Processes \\
\hline 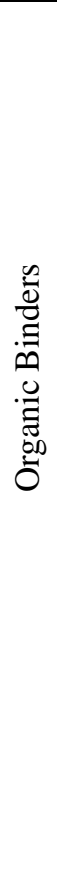 & $\begin{array}{l}\text { 1. Shell process: } \\
\text { Novolac ("shell") resin }+ \\
\text { Phenol Formaldehyde } \\
\text { 2. Hot Box processes: } \\
\text { Urea Formaldehyde } \\
\text { Phenol Formaldehyde } \\
\text { a. Novolac } \\
\text { b. Resole } \\
\text { Furan Modified } \\
\text { a. UF + FA } \\
\text { b. PF + FA } \\
\text { c. PF + UF } \\
\text { 3. Warm Box process: } \\
\text { Furan } \\
\text { a. free formaldehyde } \\
\text { b. UF or PF } \\
\text { c. sulfonic acids or } \\
\text { copper salts } \\
\text { 4. Core Oil process: } \\
\text { Oils } \\
\text { water activated cereal }\end{array}$ & $\begin{array}{l}\text { 1. Acid No-Bake processes: } \\
\text { Furan no-bake } \\
\text { a. } \mathrm{H}_{3} \mathrm{PO}_{4} \\
\text { b. TSA } \\
\text { c. BSA } \\
\text { Phenol Formaldehyde } \\
\text { a. TSA } \\
\text { b. BSA } \\
\text { c. xelenesulfonic acid } \\
\text { 2. Ester Cured processes: } \\
\text { Phenolic Resole } \\
\text { a. free phenol } \\
\text { b. free formaldehyde } \\
\text { 3. Urethane No-Bake (Amine } \\
\text { Cured) processes: } \\
\text { Alkyd Urethane } \\
\text { a. vegetable oil } \\
\text { b. polyisocyanate } \\
\text { Phenolic Urethane } \\
\text { a. pyridine derivative } \\
\text { b. polyphenyl PIC } \\
\text { Polyol Urethane }\end{array}$ & $\begin{array}{l}\text { 1. Free Radical Curing: } \\
\text { Vinyl Urethane Oligomer } \\
\text { a. } \mathrm{N}_{2}+\mathrm{SO}_{2} \\
\text { b. epoxy / } \\
\text { hydroperoxyde } \\
\text { 2. Cold Box processes: } \\
\text { Phenolic Urethane } \\
\text { Polymeric Isocyanate } \\
\text { a. TEA vapor + air } \\
\text { b. DMEA vapor }+ \text { air } \\
\text { Furan }+\mathrm{SO}_{2} \\
\text { a. methyl alcohol } \\
\text { b. MEK peroxide } \\
\text { Acrylic/Epoxy }+\mathrm{SO} \mathrm{S}_{2} \\
\text { a. hydroperoxide } \\
\text { Phenolic Resole }+ \text { Ester } \\
\text { a. glycol ethers } \\
\text { b. methylformate vapor }\end{array}$ \\
\hline 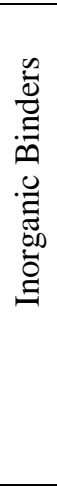 & $\begin{array}{l}\text { 1. Clay Based processes: } \\
\text { Bentonites } \\
\text { Fire Clays } \\
\text { Kaolinite }\end{array}$ & $\begin{array}{l}\text { 1. Ester Cured processes: } \\
\text { Sodium Silicate } \\
\text { a. glycerol diacetate } \\
\text { b. EGDA } \\
\text { c. glycerol triacetate } \\
\text { Ethyl Silicate } \\
\text { 2. Cement Bonding process: } \\
\text { Hydraulic Cements } \\
\text { 3. Oxide Cured process: } \\
\text { Phosphates } \\
\text { a.aluminum phosphate } \\
\text { b.magnesium oxide }\end{array}$ & $\begin{array}{l}\text { 1. CO2 Silicate process: } \\
\text { Sodium Silicate } \\
\left(\mathrm{SiO}_{2}: \mathrm{Na}_{2} \mathrm{O}\right)+\mathrm{CO}_{2}\end{array}$ \\
\hline & \multicolumn{3}{|c|}{$\begin{aligned} \text { Notes: } & \text { FA }=\text { furfuryl alcohol } \\
& \text { UF }=\text { urea formaldehyde } \\
& \text { PF }=\text { phenol formaldehyde } \\
& \text { TEA }=\text { triethylamine } \\
& \text { DMEA = dimethylethylamine }\end{aligned}$} \\
\hline
\end{tabular}

Clays used in foundries include hydrous alumina silicates, known as "bentonites," addressed in Section 2.1.3. Their properties provide cohesion and plasticity in the green state and also high strength when dried. In most of the cases, two types of bentonite, 
sodium bentonite (western-type) and calcium bentonite (southern-type), are blended to produce strong molding sands with low moisture content.

The principal properties of green sand are governed by the clay-water-silica relationship. Because of lack of electrical neutrality of montmorillonite and an unequal balance of charge at the fractured quartz surface, polarized water molecules are attracted either between the clay plates or to the quartz surface. As a result, a linkage, quartzwater-clay-water-quartz (or clay), is therefore set up throughout the molding sand [Flinn 1963, Blistan 1974]. Besides the electrostatic bonding, surface tension forces and interparticle friction bond also build the clay bonding system [Sylvia 1972, Blistan 1974].

\subsubsection{Chemical Bonding Process}

Chemical binders, addressed in Section 2.1.3, need to be exposed to the appropriate chemical bonding process to exhibit their cohesion feature. The chemical bonding process is normally classified into three groups, defined by the respective approaches to hardening, hot curing, cold setting, and gas or vapor hardening [Beeley 2001], providing a cold and hot tensile strength ranging from 50 psi to 150 psi depending on the bonding system. The most widely used bonding processes include cold box, nobake, hot box, and warm box.

Cold box core and mold making describes any binder process that uses a gas or vaporized catalyst to cure resin-coated sand while the sand is in contact with a room- 
temperature pattern. The no-bake core and mold making process is designed around a continuous mixer that blends the binder with the catalysts or coreactants as it coats the sand and dispenses the coated sand into the tooling. In the hot box coremaking process, after the sand has been coated, it is blown into a heated corebox pattern at a temperature of 450 to $550^{\circ} \mathrm{F}$. It starts to harden and form a shell-like skin. Warm box coremaking uses the same sand processing system as hot box. They differentiate in the temperature of hot pattern. The typical temperature for the warm box pattern ranges from 350 to $450^{\circ} \mathrm{F}$. The most employed combinations of binder types and bonding process are listed chronologically in Table 2-3.

\subsubsection{Unbonded Systems}

Unbonded sand systems make the mold and core without the addition of binders to hold refractory aggregates into shapes. Frozen molds, in which the main bonding action is derived from the water content of the sand, enable block molds of high strength to be produced with the aid of liquid nitrogen or carbon dioxide sprays. Another innovative unbonded process is magnetic molding, in which a magnetic granular material is held in form by a strong magnetic field. The most effective and widely adopted systems have been those in which unbonded sand is held in shape by the application of vacuum suction to the sand mass within the mold container [Beeley 2001]. 
Table 2-3: Chemical bonding series [Carey and Sturtz 1995]

\begin{tabular}{|c|c|c|}
\hline $\begin{array}{l}\text { Approx. } \\
\text { commercial } \\
\text { intro. }\end{array}$ & System & $\begin{array}{l}\text { Major resin, catalyst and mass percentage } \\
\text { based on base sand }\end{array}$ \\
\hline 1950 & Core oil & $\begin{array}{l}\text { Oil (up to } 20 \text { ingredients), cereal, water, less } \\
\text { release agent, } 2-3 \%\end{array}$ \\
\hline 1950 & Shell; liquid and flake & \begin{tabular}{|lccc}
$\begin{array}{l}\text { Phenolic } \\
\text { hexamethylenetetramine }\end{array}$ & flake & resin, \\
\end{tabular} \\
\hline 1952 & Silicate $/ \mathrm{CO}_{2}$ & Sodium silicate, water, $\mathrm{CO}_{2}, 3-5 \%$ \\
\hline 1953 & Airset oils & --- \\
\hline 1958 & $\begin{array}{l}\text { Phenolic acid catalyzed no } \\
\text { bake }\end{array}$ & --- \\
\hline 1958 & Furan acid catalyzed no bake & --- \\
\hline 1960 & Furan no bake & $\begin{array}{l}\text { Furfuryl alcohol (from corn husks, rice hulls), } \\
0.9-2.0 \%\end{array}$ \\
\hline 1962 & $\begin{array}{l}\text { Phenolic/furfuryl alcohol hot } \\
\text { box }\end{array}$ & $\begin{array}{l}\text { Phenol, furfuryl alcohol, urea, formaldehyde, } \\
\text { ammonium chloride, ammonium nitrate, } 1.5- \\
2.0 \%\end{array}$ \\
\hline 1965 & Oil urethane no bake & $\begin{array}{l}\text { Oil resin, polymeric isocyanate, amine and } \\
\text { metallic compounds, } 1-2 \%\end{array}$ \\
\hline 1967 & Phenolic $\mathrm{CO}_{2}$ cold box & --- \\
\hline 1968 & $\begin{array}{l}\text { Phenolic/urethane/amine } \\
\text { cold box }\end{array}$ & $\begin{array}{l}\text { Phenolic resin, polymeric isocyanate, amine, } \\
0.8-1.5 \%\end{array}$ \\
\hline 1968 & $\begin{array}{l}\text { Silicate ester catalyzed no } \\
\text { bake }\end{array}$ & Sodium silicate, ester catalyst, $2-4 \%$ \\
\hline 1970 & Phenolic urethane no bake & Phenolic polyol, isocyanate, amine, $0.8-1.75 \%$ \\
\hline 1977 & Furan $\mathrm{SO}_{2}$ & $\begin{array}{l}\text { Phenolic modified furan, silane, organic } \\
\text { peroxide reacted with } \mathrm{SO}_{2}\end{array}$ \\
\hline 1978 & Polyol urethane no bake & Polyol resin, isocyanate, amine \\
\hline 1978 & (Furan) warm box & $\begin{array}{l}20 \% \text { less resin than that of hot box, copper } \\
\text { salts of toluene sulfonic acid, copper chloride }\end{array}$ \\
\hline 1980 & $\begin{array}{l}\mathrm{CO}_{2} \text { cured alkaline phenolic } \\
\text { cold box }\end{array}$ & Alkaline phenolic resin, around $2.0-3.5 \%$ \\
\hline 1982 & Free radical curing $\mathrm{SO}_{2}$ & Acrylic resin, $\mathrm{SO}_{2}, 0.5-2 \%$ \\
\hline 1983 & Epoxy $\mathrm{SO}_{2}$ & Epoxy, $\mathrm{SO}_{2}, 0.5-2 \%$ \\
\hline 1984 & Phenolic ester no bake & $\begin{array}{l}\text { Phenolic resole resin, } 1.0-1.5 \% \text {, ester (organic, } \\
\text { alcohol) }\end{array}$ \\
\hline 1985 & Phenolic ester cold box & Water soluble, ester (methyl formate) \\
\hline 1992 & Alumina phosphate & --- \\
\hline
\end{tabular}




\subsubsection{Environmental Improvements}

Critical concerns pertinent to binders are their environmental and health impacts inside the workshop and at the disposal site, as some resin binder potentially poses harmful threats on the human body. Most foundry binder systems are available in a leadfree version. Foundry resin manufacturers replace lead with other dryers. The elimination of lead not only benefits workers, but has also made excess foundry sand safer and easier to reuse, reclaim and dispose. Exposure to formaldehyde has been a concern. Binder suppliers have endeavored to reduce formaldehyde content and to reduce the emission of formaldehyde during binder curing. Techniques include more efficient manufacturing processes, the use of formaldehyde scavengers, and reduction in level by improving catalyst technology [Carey and Sturtz 1996].

\subsubsection{Casting Metals}

The most common alloys used in metalcasting are shown in Table 2-4. Metals are most commonly categorized as ferrous or nonferrous.

The metalcasting industry is the "original recycler." Rather than use new or "virgin" materials as melt stock, nearly all foundries reuse scrap metals as their primary melting material. Annually, foundries consume 15-20 million tons of recycled scrap metal, giving new life to products that would otherwise go to landfills. The properties of casting metals can be improved with respect to fluidity, melting point, strength, and 
hardness by the addition of alloying elements [Ammen 2000, Sylvia 1972]. Typical metals for casting and their melting points are listed in Figure 2-5.

Table 2-4: Classification of foundry alloys

\begin{tabular}{l|l|l}
\hline \multicolumn{1}{|c|}{ Ferrous castings } & $\begin{array}{l}\text { Nonferrous } \\
\text { castings }\end{array}$ \\
\hline Cast Steel & Cast iron & Aluminum-base \\
\hline Plain carbon steel & Gray cast iron (high-carbon) & Copper-base \\
Low-alloy steel & $\begin{array}{l}\text { Ductile or nodular cast iron (spheroidal- } \\
\text { graphite) } \\
\text { High-alloy steel }\end{array}$ & $\begin{array}{l}\text { Lead-base } \\
\text { Magnesium-base }\end{array}$ \\
& $\begin{array}{l}\text { Malleable iron (annealed white iron with } \\
\text { graphite) }\end{array}$ & $\begin{array}{l}\text { Nickel-base } \\
\text { Tin-base } \\
\text { Zinc-base } \\
\end{array}$ \\
\hline
\end{tabular}

The charge materials for typical iron casting consist of pig iron, iron and steel scrap, foundry returns, lime and iron ore. Typical melting stock for steel casting includes pig iron, steel, cast iron and alloying additions. The alloying elements commonly employed for the purpose of securing steel hardenability include manganese, chromium, molybdenum, nickel, silicon, copper, vanadium, totally up to $87 \%$ by mass.

The normal metal charge of aluminum-based facility consists of clean foundry scrap (remelt), prealloyed aluminum pig and scrap of heterogeneous origin. Alloying elements, such as copper, silicon, zinc, nickel, chromium, titanium, manganese, are added as rich alloys or hardeners [Heine 1967]. 


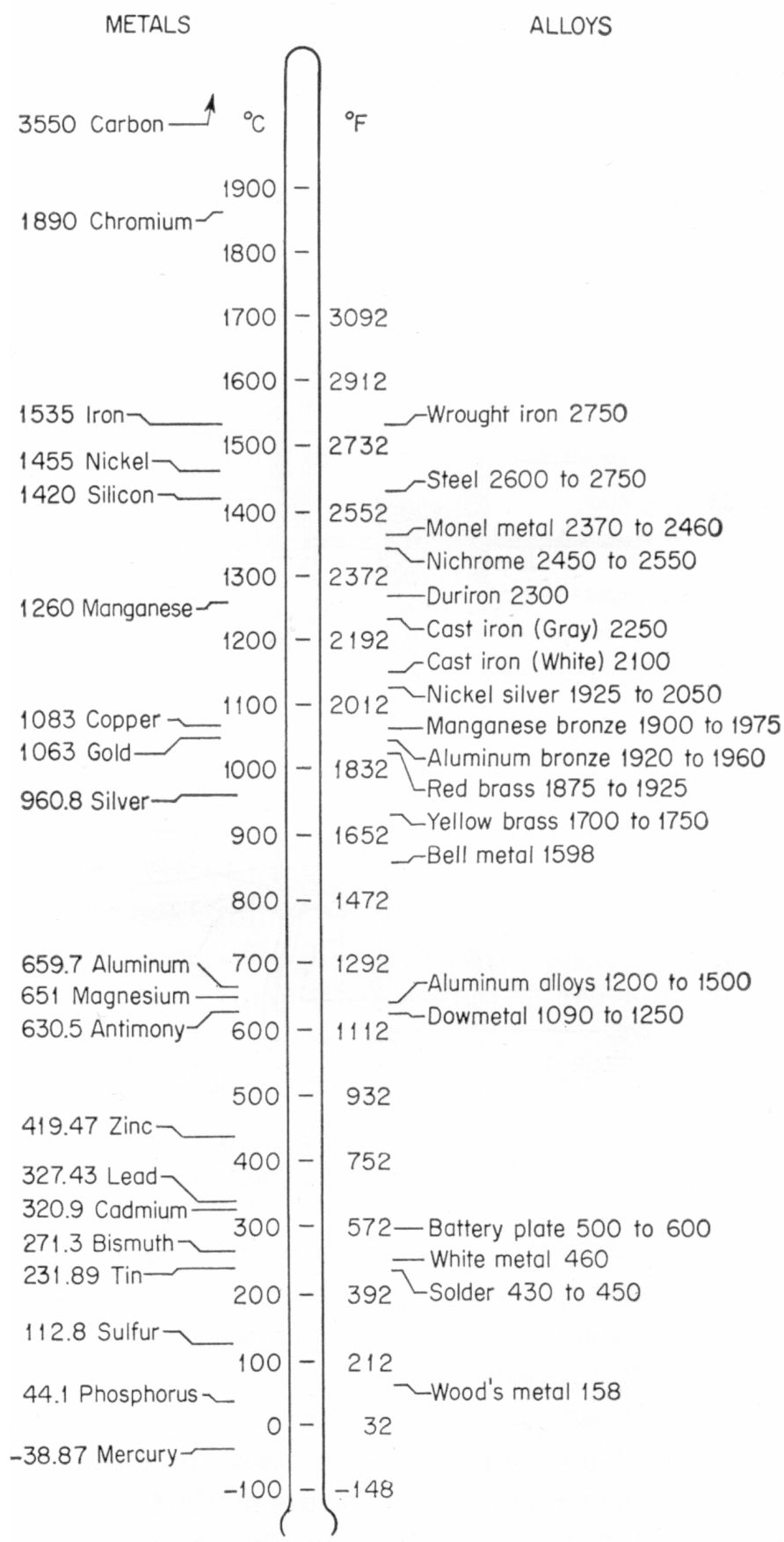

Figure 2-5: Melting of metals for casting [Ekey 1958]

The normal metal charge for copper-based facility consists of clean foundry scrap (remelt) and pig or ingot of the desired analysis. Additions, such as zinc, tin and lead, are 
made to achieve the desired analysis. Many copper-alloy castings require excellent surface finish as cast. Fines aggregate has been found to give a more desirable finish. Facing materials such as cereal, flour, plumbago, and sea coal are dusted on the moldcavity face to improve surface finish [Heine 1967].

\subsubsection{Pouring}

When metal is poured into a mold, the following events occur: (1) Free moisture is driven off the sand beginning at the mold-metal interface and either condenses in the cooler parts of the mold or evolves as steam into the atmosphere. (2) Volatile matter is driven from organic materials such as sea coal and from the residual binder on the surfaces of core sand that has entered into the system by the shakeout route. (3) As the sand heats up, the clay bonding materials are deactivated and "killed." At temperatures

above $600{ }^{\circ} \mathrm{C}\left(1,100{ }^{\circ} \mathrm{F}\right)$, combined water is driven out of the clay bond. All clays lose their combined water in parts of the molds as they reach $1,470{ }^{\circ} \mathrm{F}$ and become ineffective. (4) The sand heats up as the casting solidifies and expands. (5) When the sand is heated up to temperatures over $820^{\circ} \mathrm{C}\left(1500{ }^{\circ} \mathrm{F}\right)$, the possibility of chemical reaction between metal and sand, fusion of the sand, increases. [Heine 1967, Carey 2002].

\subsubsection{Waste Streams}

A typically foundry can generate from 8 to 40 individual waste streams, including excess molding sand, core sand, copula slag, scrubber sludge, baghouse dusts, shotblast 
fines, buffling wastes, and others [Winkler 2000]. Typically, they are classified into four waste streams depending on the nature of the waste streams: sand, dust, slag, and sludge, as listed in Table 2-5. Mold and core sands are the main ingredients of excess foundry sand and occupy up to $80 \%$ of the total waste streams. Further, their behavior is more stable than other waste streams, and environmental characteristics are gentler. Sands can often be beneficially reused in construction applications. Furthermore, the disposal expense of large quantities of excess foundry sands is a big burden on foundries. Hence, excess foundry sands are selected for beneficial reuse purposes. Dust is mainly the fine particles collected by various collectors installed in a foundry facility. The formation of slags used in metal melting and refining processes can be summarized as protection of the melt from contaminations from the furnace atmosphere and combustion products, insulation of the melt to maintain the heat in it, and an acceptor of unwanted materials as refining media to the melt [Webster 1980].

Table 2-5: Conventional description for foundry waste streams

\begin{tabular}{l|l}
\hline Waste Streams & \multicolumn{1}{c}{ Descriptions } \\
\hline Sand & $\begin{array}{l}\text { Sand, green sand, mold, shell core, oil sand, furfural alcohol, } \\
\text { recyclable sand, isocure, tank, tail, pulley, sand dropout }\end{array}$ \\
\hline Dust & $\begin{array}{l}\text { Dust, shot blast, shakeout (sand), muller, grinding, wheelabrators, } \\
\text { collector, cleanroom, blast, fume, pretreater and desulfurization, cutoff, } \\
\text { fines, finish, pagborn, carter day collector, large wet collector, sand } \\
\text { reclaimer, tailing, baghouse }\end{array}$ \\
\hline Slag & $\begin{array}{l}\text { Slag, furnace refractory, iron, ductile, melt, copula, popcorn, ladle } \\
\text { relining, crucibles/ brick }\end{array}$ \\
\hline Sludge & Sludge, copula, furnace, pugmill, cake \\
\hline
\end{tabular}




\subsection{Chemical and Environmental Characterization of Excess Foundry Sands}

Chemical and environmental characterization of excess foundry sand is a critical issue that needs to be addressed prior to wider acceptance of beneficial reuse programs. It is essential to understanding the potential for the development of beneficial applications. Each stream of material has unique characteristics that assist engineers in making a decision between beneficial reuse, reclamation or waste disposal. Chemical and environmental concerns of excess foundry sands dominate the issue of reusing them. They may contain elements or compounds that pose impacts to environments and threats to human health. The thresholds of these elements or compounds are regulated by environmental regulations. Their measurements are covered by extraction procedures and chemical analytical techniques.

\subsubsection{Act and Regulations}

The act regulating excess foundry sand as a solid waste is the Resource Conservation and Recovery Act (RCRA). RCRA was enacted in 1976 with the intent to "protect human health and the environment from the improper handling of solid waste and to encourage the conservation of natural resources." The Office of Solid Waste (OSW) of the U.S. Environmental Protection Agency (EPA) administers the RCRA program.

The Code of Federal Regulations (CFR) is a codification of the rules published in the Federal Register by executive departments and agencies of the federal government. 
The CFR is divided into 50 titles that represent broad areas subject to federal regulation, with environmental regulations contained mainly in CFR Title 40. CFR Title 40 contains the rules that EPA uses to implement the requirements of the RCRA.

The parts closely relating to the characterization of excess foundry sands in CFR Title 40 are Part 261, "Identification and Listing of Hazardous Waste," Part 141 "National Primary Drinking Water Regulations," Part 143, "National Secondary Drinking Water Regulations," and Part 264, "Standards for Owners and Operators of Hazardous Waste Treatment, Storage, and Disposal Facilities." Part 264 provides the maximum concentration of constituents that the uppermost aquifer underlying the waste management area meets for groundwater protection purposes. The thresholds corresponding to individual regulation are listed in Table C-1, Table C-2 and Table C-3 in Appendices C.1 and C.2.

A groundwater transport model is used to set regulatory levels against which concentrations in the leachate by the Toxicity Characteristics Leaching Procedure (TCLP, addressed in Section 2.2.2.1) are compared. Regulatory levels represent a backcalculation from an acceptable chronic exposure level in a receptor well, through the unsaturated and saturated zones, back to the sources, the bottom of the landfill, accounting for dilution and attenuation that is predicted to occur between bottom of the landfill and receptor well, through a groundwater transport model, as demonstrated in Figure 2-6. EPA assumes that water wells were situated $150 \mathrm{~m}(500 \mathrm{ft})$ down gradient from the landfill on which EPA based its determination of dilution and attenuation factor 
(DAF) of 100 for all of the listed toxic constituents based on the availability of chronic toxicity reference levels [EPA 1995]. The available references include Standards of National Drinking Water and Groundwater. For instance, the limit for mercury in drinking water and groundwater is $0.002 \mathrm{ppm}$. Considering the dilution and attenuation occurring to mercury when transported $150 \mathrm{~m}$ from the initial landfill leaching site to water wells or sources, the maximum allowable concentration for mercury in initial leachate ("A" in Figure 2-6) is 0.2 ppm.
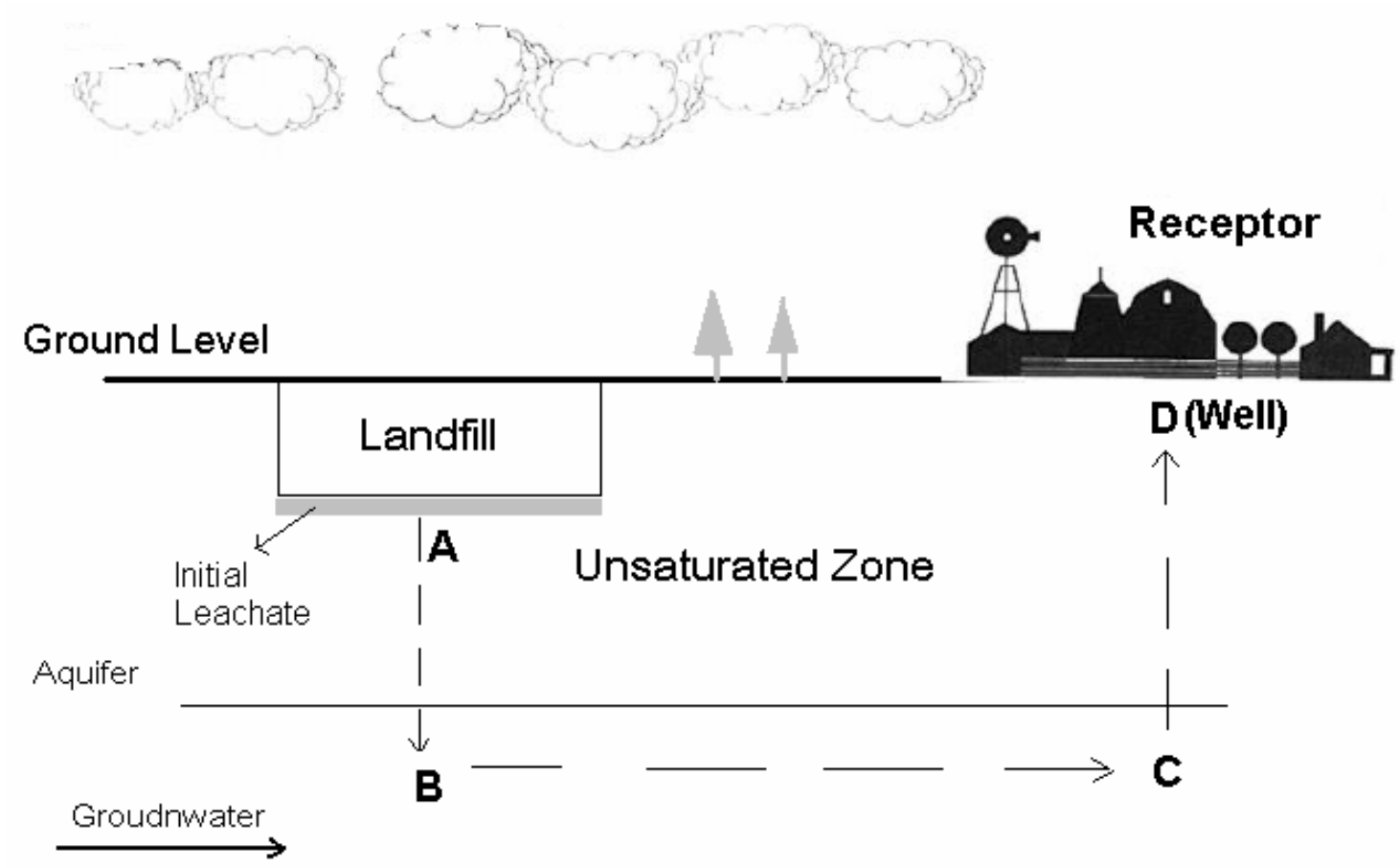

Figure 2-6: Demonstration of leachate transport model [Kimmell 1999]

Although thresholds are only specified for leachates by the Toxicity Characteristics Leaching Procedure based on the dilution and attenuation model, leaching protocol is essentially independent of the threshold values. The thresholds are determined by a transport model of leachate and available standards for groundwater and drinking water. Thresholds are basically set for any initial leachate below landfills ("A" in 
Figure 2-6). That is, a leachate from Synthetic Penetration Leaching Procedure (SPLP, Section 2.2.2.2) should transport according to the dilution and attention model as well. Thresholds for that leachate similarly comply with DAF 100. Hence, thresholds for TCLP are applicable to an initial leachate extracted by any leaching protocol. In essence, the difference among various leaching protocols is not their thresholds, but the field conditions they simulate, such as actively decomposing municipal solid waste landfills simulated by TCLP (Section 2.2.2.1) and industrial waste monofills by SPLP (Section 2.2.2.2).

\subsubsection{Chemical Analytical Techniques}

The ability to accurately detect and quantify an element or compound present in a sample, even at extremely low concentrations, is critically important in terms of assessing a waste stream for potential contamination. For example, when monitoring a disposed foundry sand, the toxicity violation of a single volatile organic compound in its leachate is often taken as evidence that the foundry sand has a direct impact on the environmental quality, while the absence of volatile organic compounds leads to a neutral assessment. The assessment as out-of-compliance could be extremely costly to the facility, and may prevent the beneficial reuse of future material.

Chemical analytical techniques are used to prepare, identify and quantify elements or compounds contained in excess foundry sand. The tasks are mainly classified into two parts: bulk chemical analysis and leachate chemical analysis. Bulk chemical 
analysis determines the compound mass percentage of solid wastes in dry or as-received condition without any extraction. Leachate chemical analysis evaluates the compound concentration level in the leachate extracted from dry or as-received solid waste samples by appropriate leaching protocols. The latter is generally selected to determine whether the disposed solid wastes are hazardous or not. The most used protocols include Toxicity Characteristics Leaching Procedure, Synthetic Precipitation Leaching Procedure, and Shake Extraction of Solid Waste With Water (ASTM D 3987). Both bulk chemical analyses and leachate concentration analyses require selecting appropriate chemical procedures to measure an element or compound. The most used procedure manuals include: "Test Methods for Evaluating Solid Waste, Physical/Chemical Methods" [EPA SW-846 2000] and "Methods for the Determination of Metals in Environmental Samples" [EPA 1991].

\subsubsection{Toxicity Characteristics Leaching Procedure}

TCLP is a leaching protocol published by EPA as Method 1311 [EPA SW-846 2000]. This leaching protocol is meant to model the leaching behavior of a material disposed in an actively decomposing municipal solid waste landfill in which carboxylic acids are formed from microbial processes, which is a particular worst-case scenario under the RCRA specification of a mismanagement scenario of wastes. If disposal conditions are different from the municipal landfill conditions, another test may better predict the actual leaching of a waste and provide better numerical estimates of leaching. In TCLP, solid samples are extracted with an acetate buffer solution. The extraction fluid 
employed is a function of the alkalinity of the solid phase of the waste. A liquid-to-solid ratio of 20:1 by weight is used for an extraction period of $18 \pm 2$ hours. After extraction the solids are filtered through a 0.6 to $0.8 \mu \mathrm{m}$ filter from the liquid extract, and analyses are conducted on the leachate to determine the elements' concentrations.

\subsubsection{Synthetic Precipitation Leaching Procedure}

SPLP is a leaching protocol published by EPA as Method 1312 [EPA SW-846 2000]. This leaching protocol was developed to simulate leaching through an industrial waste monofill under acid rain conditions. The procedure is similar to the TCLP; however, the amount of acidity used in the test is significantly less. Furthermore, an aqueous solution of nitric/sulfuric acid mixture is used in the SPLP as an extraction fluid, rather than a more aggressive buffered acetic acid in the TCLP. This procedure more closely simulates the field conditions for beneficial use in the general construction industry.

\subsubsection{Shake Extraction of Solid Waste with Water (ASTM D 3987)}

ASTM D 3987 is a neutral leaching protocol published by ASTM [ASTM D 3987 1985]. This procedure is a useful indicator in considering the potential environmental impact of foundry sand waste and its beneficial use practices. The intent of this test method is that the water extraction simulates conditions where the solid waste is the dominant factor in determining the $\mathrm{pH}$ of the extract. It uses Type IV reagent water as the 
extraction solution, which differentiates it from the acidic medium leaching protocols TCLP and SPLP.

\subsubsection{Research on Characterization of Excess Foundry Sands}

A literature review indicated that characterization of excess foundry sand can be divided into two parts, qualitative characterization and quantitive characterization. Qualitative characterization represents those evaluations concentrating on summary statistics of excess foundry sand characterization. The frequent topics are as follows:

- Is the sand hazardous in the context of toxicity regulation [Orkas 2002, Ji et al. 2001, Ham et al. 1993]?

- What is the maximum extraction level of some compounds?

- Is there any correlation between field leaching level and laboratory extraction level [Ham 1984, Kendall 2003], among compounds [Boyle 1984], or with other external factors [Ham and Boyle 1981]?

- Which waste stream leaches worse [Ham 1984, Boyle 1984]?

- What is the distribution of compounds after arbitrary assumption [Kauffman and Voigt 1999]?

- What are the chemical characteristics of spent sand across the foundries [Regan et al. 1994]? 
Quantitive characterization concentrates on deeper characteristics of excess foundry sand. Supported by abundant data resources, it employs a professional statistical methodology to look further into the data and make data more meaningful. The topics include censored data processing, estimation of mean and its confidence interval, and factorial design and analysis. Peer disciplines such as water resources, medical and biological studies, chemical studies, and soil and earth studies have conducted research on censored data. However, little work has been done to study excess foundry sand characterization at the quantitative level.

\subsubsection{Chemical Characteristics from Past Studies}

An overview of publicly available results on leachability of metals from foundry sand waste is collected and summarized in Tables 2-6 through 2-9. Random scattering around mean values is significant. The standard deviations are usually greater than the respective mean concentrations. Average, median, or maximum values are occasionally reported. Only a few projects were completed with a comprehensive statistical analysis that included the determination of a roster of statistical parameters to characterize a vast pool of analytical data [Winkler 2000].

One vital conclusion drawn from Table 2-6 is that excess foundry sand disposed of in a foundry monofill or used in highway construction leaches regulated metals well below the characteristic toxicity levels. This is usually because the constituents are either present in forms that are not soluble or are unavailable to the leaching media. Even when 
the presence of the constituents in a bulk waste stream can be traced, this does not always imply that they could readily be leached. A comparison to a mixed-waste municipal landfill indicates that the foundry sand waste leaches one to two orders of magnitude less than the typical waste in a municipal landfill [Winkler 2000]. 
Table 2-6: Leachability of metals from foundry sand waste [Winkler 2000]

\begin{tabular}{|c|c|c|c|c|c|c|c|c|c|c|c|c|c|c|}
\hline \multirow[t]{2}{*}{ Research Description } & \multirow[t]{2}{*}{ Leaching Method } & \multicolumn{7}{|c|}{ Primary Contaminants (mg/l) } & \multicolumn{5}{|c|}{ Secondary Contaminants (mg/l) } & \multirow{2}{*}{$\begin{array}{ll}* * \\
\mathrm{Ni} \\
\end{array}$} \\
\hline & & As & $\mathrm{Ba}$ & $\mathrm{Cd}$ & $\mathrm{Cr}$ & $\mathrm{Hg}$ & $\mathrm{Pb}$ & $\mathrm{Se}$ & $\mathrm{Ag}$ & $\mathrm{Cu}$ & $\mathrm{Fe}$ & $\mathrm{Mn}$ & $\mathrm{Zn}$ & \\
\hline Drinking Water Maximum Contaminant Level & Total Elem & 0.05 & 2.0 & 0.005 & 0.01 & 0.002 & $0.015^{*}$ & 0.05 & 0.1 & 1.0 & 0.3 & 0.05 & 5.0 & \\
\hline Toxicity Characteristic Level & TCLP & 5.0 & 100 & 1.0 & 5.0 & 0.2 & 5.0 & 1.0 & 5.0 & & & & & \\
\hline $\begin{array}{l}\text { Highway embankment constructed with Indiana } \\
\text { ferrous foundry waste sand. Maximum measured } \\
\text { values. }\end{array}$ & Field Leachate & & & 0.054 & - & & - & & & - & & & 0.85 & $0.0 !$ \\
\hline $\begin{array}{l}\text { Median Wisconsin field leachate values, averaged over } \\
\text { six ferrous foundry mixed waste landfills. }\end{array}$ & Field Leachate & $<.005$ & $<0.46$ & $<.001$ & $<0.02$ & $<.005$ & $<0.02$ & $<0.13$ & $<.002$ & 0.02 & 0.54 & 0.27 & 0.15 & \\
\hline $\begin{array}{l}\text { Typical Wisconsin mixed municipal solid waste } \\
\text { landfill. Maximum values. }\end{array}$ & Field Leachate & & & 0.07 & 1 & & 1.1 & & & 0.3 & & & 54 & 1.7 \\
\hline $\begin{array}{l}\text { Spent molding sands. Average over } 52 \text { Pennsylvania } \\
\text { foundries. }\end{array}$ & TCLP & 0.06 & 0.4 & 0.03 & "0.1 & 0.005 & 0.3 & 0.08 & $<<.049$ & 0.25 & 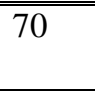 & 0.9 & 2.2 & 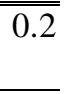 \\
\hline $\begin{array}{l}\text { Spent sand waste. Average over a cluster of } 28 \text { out of } \\
33 \text { Pennsylvania ferrous and non-ferrous foundries. }\end{array}$ & TCLP, ASTMD346 & 0.006 & 0.33 & 0.03 & 0.1 & .0005 & 0.25 & 0.03 & 0.06 & 0.2 & 28 & 0.35 & 0.4 & 0.1 \\
\hline $\begin{array}{l}\text { Mixed molding waste (sand, binder, dust, sludge). } \\
\text { Gostyn foundry, Poland. }\end{array}$ & $\begin{array}{l}\text { TCLP } \\
\text { EP }\end{array}$ & & & $\overline{-}$ & - & & $\begin{array}{l}0.25 \\
-\end{array}$ & & & $\begin{array}{l}1 \\
0.2\end{array}$ & $\begin{array}{l}13 \\
31\end{array}$ & $\begin{array}{l}1.6 \\
2.2\end{array}$ & $\begin{array}{l}1.4 \\
0.9\end{array}$ & $\begin{array}{l}7 \\
-\end{array}$ \\
\hline Wisconsin brass foundry sands + dusts. Average value. & TCLP+EP & & & & & & 11 & & & & & & & \\
\hline $\begin{array}{l}\text { Wisconsin brass foundry sands }+ \text { dusts, chemically } \\
\text { treated to convert metals into non-leaching forms. }\end{array}$ & TCLP+EP & - & - & 0.018 & - & - & 0.18 & - & - & & & & & \\
\hline $\begin{array}{l}\text { Wisconsin ferrous foundry waste sand. Average of } \\
\text { two. }\end{array}$ & EP & & & & & & & & & & 0.04 & 1.1 & & \\
\hline $\begin{array}{l}\text { Wisconsin typical ferrous foundry mixed waste } \\
\text { landfill. }\end{array}$ & EP & $<.005$ & $<0.46$ & $<.001$ & $<.003$ & & $<0.01$ & $<0.13$ & $<.002$ & $\begin{array}{l}<.00 \\
2\end{array}$ & 66 & 2.9 & 0.4 & \\
\hline $\begin{array}{l}\text { Molding sand (6\% western bentonite, } 7 \% \text { sea-coal) } \\
\text { subjected to process temperature. Maximum values. }\end{array}$ & $\begin{array}{l}3 \text { LeachingCycles, } \\
\mathrm{H}_{2} \mathrm{O}\end{array}$ & & $<<0.75$ & & $<1.1$ & & & & & 1.1 & 15 & $\begin{array}{l}<0.7 \\
5\end{array}$ & 1.2 & \\
\hline $\begin{array}{l}\text { Brown-black furan-bonded sand waste }+ \text { dust (1:1), air } \\
\text { dried. Dessau foundry, Germany. }\end{array}$ & $\begin{array}{l}\mathrm{DIN} 38414 \mathrm{~S} 4 \mathrm{H}_{2} \mathrm{O} \\
\mathrm{pH}=7.5\end{array}$ & 0.01 & & $<0.1$ & 0.07 & $<.001$ & 0.6 & & & 0.25 & & & 0.5 & $\overline{0.3}$ \\
\hline $\begin{array}{l}\text { Black-brown silicate waterglass-bonded fine sand } \\
\text { waste, air dried. Magdeburg foundry, Germany. }\end{array}$ & $\begin{array}{l}\mathrm{DIN} 38414 \mathrm{~S} 4 \mathrm{H}_{2} \mathrm{O} \\
\mathrm{pH}=10.1\end{array}$ & 0.13 & & $<0.1$ & 0.08 & $<.001$ & 0.6 & & & 0.5 & & & 1.1 & 0.6 \\
\hline $\begin{array}{l}\text { Brown-black furan-bonded sand waste }+ \text { dust (1:1), air } \\
\text { dried. Dessau foundry, Germany. }\end{array}$ & \begin{tabular}{|l|} 
DIN 38414 S7 Aqua \\
Regia
\end{tabular} & 0.45 & & $\bar{~} 0.3$ & (269 & 0.02 & 111 & & & 27 & & & 33 & $\bar{~} 6$ \\
\hline $\begin{array}{l}\text { Black-brown silicate waterglass-bonded fine sand } \\
\text { waste, air dried. Magdeburg foundry, Germany. }\end{array}$ & \begin{tabular}{|l|} 
DIN 38414 S7 Aqua \\
Regia
\end{tabular} & 0.67 & & 0.3 & 79 & $<.002$ & 4.6 & & & 8.5 & & & 17 & 16 \\
\hline
\end{tabular}

Note: See notes under Table 2-7. 
Table 2-7: Bulk content of metals in foundry sand waste, sands and soils [Winkler 2000]

\begin{tabular}{|c|c|c|c|c|c|c|c|c|c|c|c|c|c|c|}
\hline \multirow[t]{2}{*}{ Research Description } & \multirow[t]{2}{*}{ Method } & \multicolumn{7}{|c|}{ Primary Contaminants $(\mathrm{mg} / \mathrm{kg})$} & \multicolumn{5}{|c|}{ Secondary Contaminants (mg/kg) } & \multirow{2}{*}{$\begin{array}{c}* * \\
\mathrm{Ni}\end{array}$} \\
\hline & & As & $\mathrm{Ba}$ & $\mathrm{Cd}$ & $\mathrm{Cr}$ & $\mathrm{Hg}$ & $\mathrm{Pb}$ & $\mathrm{Se}$ & $\mathrm{Ag}$ & $\mathrm{Cu}$ & $\mathrm{Fe}$ & $\mathrm{Mn}$ & $\mathrm{Zn}$ & \\
\hline Drinking Water Maximum Contaminant Level & Total Elem & 0.05 & 2.0 & 0.005 & 0.01 & 0.002 & $0.015^{*}$ & 0.05 & 0.1 & 1.0 & 0.3 & 0.05 & 5.0 & \\
\hline Toxicity Characteristic Level, mg/l & TCLP & 5.0 & 100 & 1.0 & 5.0 & 0.2 & 5.0 & 1.0 & 5.0 & & & & & \\
\hline $\begin{array}{l}\text { Brown-black furan-bonded sand waste + dust (1:1), air } \\
\text { dried. Dessau foundry, Germany. }\end{array}$ & $\begin{array}{l}\text { Total Elem X- } \\
\text { ray fluo }\end{array}$ & $2<2$ & & $<2$ & 284 & $<2$ & 14 & & & 27 & & & 33 & $\overline{55}$ \\
\hline $\begin{array}{l}\text { Black-brown silicate waterglass-bonded fine sand waste, } \\
\text { air dried. Magdeburg foundry, Germany. }\end{array}$ & $\begin{array}{l}\text { Total Elem X- } \\
\text { ray fluo }\end{array}$ & $<2$ & & $<2$ & 5870 & $<2$ & 7 & & & 7 & & & 32 & 21 \\
\hline $\begin{array}{l}\text { Spent molding sands. Average over } 52 \text { Pennsylvania } \\
\text { foundries. }\end{array}$ & Total Elem & 2.3 & 24 & 2.2 & 29 & 1.5 & 49 & 2.2 & $<1.9$ & 308 & $1.5 \%$ & 108 & 246 & \\
\hline $\begin{array}{l}\text { Spent sand waste. Average over a cluster of } 30 \text { out of } 33 \\
\text { Pennsylvania ferrous and non-ferrous foundries. }\end{array}$ & Total Elem & 1.1 & 13 & 1.9 & 8.9 & 0.06 & 24 & 1.2 & 2.4 & 117 & $3.9 \%$ & 72 & 26 & 29 \\
\hline $\begin{array}{l}\text { Molding waste (sand, binder, dust, sludge). Median } \\
\text { values derived from } 14 \text { samples. Gostyn foundry, Poland }\end{array}$ & Total Elem & & & 3 & 101 & & 45 & & & 15 & $15 \%$ & 291 & 112 & 32 \\
\hline $\begin{array}{l}\text { Black spent sand piles. Samples averaged over two New } \\
\text { England iron casting foundries. }\end{array}$ & $\begin{array}{l}\text { Total Elem } \\
\text { EPA } 6010\end{array}$ & 2 & 30 & $<0.2$ & 2.8 & 0.02 & 7.5 & $<5$ & $<0.5$ & & & & & \\
\hline $\begin{array}{l}\text { Black spent sand + dust. Massachusetts iron foundry. } \\
\text { Average of } 4 \text { samples collected within } 6 \text { months. }\end{array}$ & $\begin{array}{l}\text { Total Elem } \\
\text { EPA } 6010 \\
\end{array}$ & 4.5 & 35 & 0.2 & 3.4 & 0.01 & 9 & $<5$ & $<0.5$ & & & & & \\
\hline $\begin{array}{l}\text { Florida natural soil: Candler fine sand }(96.7 \% \text { sand, } 2.5 \% \\
\text { clay, } 0.8 \% \text { silt, } 0.8 \% \text { organics, } \mathrm{pH}=6.5) \text {. }\end{array}$ & $\begin{array}{l}\text { Leaching } \\
\text { Cycles, } \mathrm{H}_{2} \mathrm{O}\end{array}$ & & & & & & & & & 0.23 & 0.20 & 0.44 & 0.08 & \\
\hline $\begin{array}{l}\text { Unprocessed sand for molding. Median values obtained } \\
\text { with } 5 \text { samples. Gostyn foundry, Poland. }\end{array}$ & Total Elem & & & 1 & - & & 6 & & & 0.4 & 970 & - & 21 & - \\
\hline U.S. sandy soils, lithosols on sandstones. Vegetation safe & Total Elem & 5.1 & 400 & & 40 & 0.08 & 17 & 0.5 & & 14 & $\begin{array}{c}1- \\
3 \%\end{array}$ & 345 & 40 & 175 \\
\hline U.S. non-contaminated soils. Maximum values. & Total Elem & 60 & 3000 & 0.7 & 1000 & 0.3 & 200 & 2 & 5 & 100 & $5 \%$ & 3000 & 300 & 300 \\
\hline
\end{tabular}

Note: A cell is blank if a parameter was not measured.

$<$ Below detection limit.

- Below unspecified detection limit.

* Action level which triggers treatment of water system if exceeded in more than $10 \%$ tap water samples.

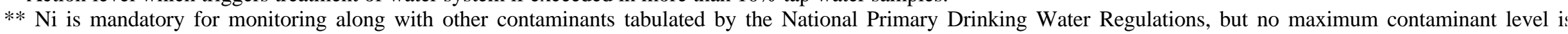

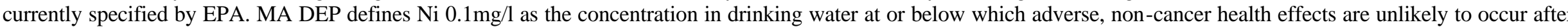
chronic (lifetime) exposure. MA DEP only indicates a potential need for further legislative action to be decided. 
Table 2-8: Typical TCLP and SPLP results from smelting operation sludge [Winkler 2000]

\begin{tabular}{l|l|l}
\hline Metal Leaching & TCLP $(\mathrm{mg} / \mathrm{L})$ & SPLP $(\mathrm{mg} / \mathrm{L})$ \\
\hline $\mathrm{Pb}$ & 570 & 1.5 \\
\hline $\mathrm{Cd}$ & 1.9 & 0.13 \\
\hline $\mathrm{Cr}$ & 5.1 & 0.9 \\
\hline
\end{tabular}

Table 2-9: Lead leachability versus total element analysis of red brass foundry sand samples and synthesized mixtures [Winkler 2000]

\begin{tabular}{|c|c|c|c|}
\hline Sample & $\begin{array}{c}\text { EP Toxicity }{ }^{a} \\
\mathrm{~Pb}, \mathrm{mg} / \mathrm{L}\end{array}$ & $\begin{array}{c}\text { Total }^{\mathbf{b}} \mathrm{Pb} \\
\mathrm{mg} / \mathrm{kg}\end{array}$ & $\begin{array}{c}\text { Ratio } \\
\text { EP Tox/Total }\end{array}$ \\
\hline Spent molding sand, Leaded brass foundry A & 70 & 1900 & 0.04 \\
\hline Spent molding sand, Leaded brass foundry B & 111 & 1100 & 0.10 \\
\hline Spent molding sand, Leaded brass foundry $\mathrm{C}$ & 35 & & \\
\hline Clean virgin sand $+\mathrm{Pb}$ fine particles $(0.1 \%)$ & 673 & 1000 & 0.67 \\
\hline Clean virgin sand $+\mathrm{PbO}(1 \%)$ & 6380 & 10000 & 0.64 \\
\hline Clean virgin sand $+\mathrm{PbSiO}_{3}(0.1 \%)$ & 318 & 1000 & 0.32 \\
\hline
\end{tabular}

a $100 \mathrm{~g}$ sample, $2000 \mathrm{~mL}$ acetic acid solution $(0.5 \mathrm{~N}), \mathrm{pH}=5.0$, agitation 24 hours.

b $1 \mathrm{~g}$ random sub-sample, total acid digestion $\left(\mathrm{HNO}_{3}+\mathrm{HCl}\right)$.

\subsection{Controlled Low-Strength Material (CLSM)}

\subsubsection{Introduction}

CLSM, also referred to as flowable fill, is a self-compacted, self-set, marginally cementitious material used primarily as a backfill in lieu of compacted backfill. It has the following features listed by American Concrete Institute (ACI) Committee 229 [ACI Committee 229 1994]: 
- Self-leveling, liquid-like materials, and self-compacting to $95-100 \%$ of the maximum unit weight with minimal effort and no vibration or tamping.

- Maximum compressive strength ${ }^{1}$ of $8 \mathrm{MPa}(1200 \mathrm{psi})$ at 28 days.

Most CLSM applications require unconfined compressive strengths of $1,400 \mathrm{kPa}$ (200 psi) or less to allow for future excavation using hand tools or light digging equipment.

CLSM necessarily contains cement and water. It may also contain fly ash, aggregate, or chemical admixtures in proportions such that the final product meets the strength and flow consistency requirements.

The main construction advantages of CLSM can be summarized as: limited labor, equipment and inspection, accelerated construction, ready placement at inaccessible locations, improved safety, and the possibility of manual excavation in the future [Abichou et al. 1999]. CLSM may be an economical alternative to placing and compacting soil in trenches and around, pipes, vaults, sewers, and manholes. There could be considerable savings of time and labor over conventional backfill methods. CLSM can be delivered in ready-mixed concrete trucks and placed by chute directly into the area to be filled or into a pump for final placement. It eliminates the need for vibrating compaction machinery on a job site, increases the speed of construction, and reduces the liability exposure for backfilling deep trenches.

\footnotetext{
${ }^{1}$ In this report, compressive strength represents unconfined compressive strength.
} 
The geotechnical advantages of CLSM include: improved flow properties, improved strength control, limited subsidence, uniform density and load, negligible impact from volume changes due to moisture levels, and low hydraulic conductivity.

The main applications of CLSM can be summarized as follows, depending on the strength control. Excavatable CLSM is used widely as backfill for utility cuts, trenches, pipes, vaults, abandoned underground pipes, culverts, tanks, mines, sewers, voids under roadways, foundations, and retaining walls. Structural filling is used widely as load support for pipe bedding, road base, bridge approaches, paving subbase, floor slab subbase, liner base, cover mats, and replacement of poor-quality soil [Adaska and Krell 1992, Naik et al. 1990, Naik and Singh 1997, Larsen 1990, Wilson 1999].

Limitations and cautions do exist for CLSM, despite its numerous advantages. CLSM is a heavy fluid material and during placement will exert high fluid pressures against any form, embankment, or walls. Placing CLSM in multiple layers may be required to control movement or shifting and prevent floating of pipes or vaults.

\subsubsection{Specification of CLSM Containing Excess Foundry Sands}

The use of CLSM on construction projects is gaining popularity, and most states have developed or are developing specifications or regulations for CLSM [Abichou et al. 1999, Regan et al. 1998, Regan et al. 1997, Regan and Voigt 1996, Kauffman et al. 1996, Van Tassel 1999]. Pennsylvania Department of Transportation (PennDOT) has 
developed extensive specifications for flowable backfills. The specifications were developed for four types of CLSM [PennDOT 1995]. Type A and Type B are CLSM where future excavation is desirable. They are typically used as backfill for utility trenches, pipe trenches and bridge abutments, and around box or arch culverts. Type C CLSM is used when future excavation is not anticipated. It is typically used to replace unsuitable soils below foundations and to fill abandoned conduits, tunnels, and mines. Type D CLSM is used for construction on areas requiring low-density backfill such as abutments over soft soils, backfill for sensitive retaining walls, vault filling, and backfill on top of buried structures. Type A and Type D CLSM contain only fly ash and cement and therefore are not considered for beneficial reuse of foundry sands. PennDOT specifications for these four CLSM are summarized in Table 2-10.

Table 2-10: PennDOT specifications for CLSM [PennDOT 1995]

\begin{tabular}{l|c|c|c|c}
\hline Component & Type A & Type B & Type C & Type D \\
\hline Cement $\left(\mathrm{Kg} / \mathrm{m}^{3}\right)$ & 60 & 30 & $89-119$ & $178-415$ \\
\hline Fly ash $\left(\mathrm{Kg} / \mathrm{m}^{3}\right)$ & 1187 & 178 & 178 & $60-237$ \\
\hline Bottom ash $\left(\mathrm{Kg} / \mathrm{m}^{3}\right)$ & 0 & 1543 & 1543 & --- \\
\hline Spread $(\mathrm{cm})$ & $15-23$ & $15-23$ & $15-23$ & $15-23$ \\
\hline Strength, 3 day $(\mathrm{kPa})$ & $>172$ & $>172$ & $>2069$ & $>276$ \\
\hline Strength, 28 day $(\mathrm{kPa})$ & $345-862$ & $345-862$ & $>5516$ & $621-2758$ \\
\hline
\end{tabular}

The flowable fill specification developed by the Iowa Department of Transportation allows the use of fine aggregate, fly ash and cement. Fine aggregate is defined as natural sand consisting of mineral aggregate particles or foundry sand from the castings of ferrous material. The basic proportioning for flowable fill mortar is presented in Table 2-11. 
Table 2-11: Iowa DOT specifications for CLSM [Iowa DOT 2004]

\begin{tabular}{l|l}
\hline \multicolumn{2}{c}{ Quantities of Dry Materials Per Cubic Yard (Cubic Meter) } \\
\hline Cement & 100 pounds $(60 \mathrm{~kg})$ \\
Fly Ash & 300 pounds $(180 \mathrm{~kg})$ \\
Fine Aggregate & 2,600 pounds $(1545 \mathrm{~kg})$ \\
\hline
\end{tabular}

The specifications of Ohio Department of Transportation refer to CLSM as lowstrength mortar backfills. They recommend the mixture design presented in Table 2-12. The Ohio specifications specifically mention the possibility of using foundry sands and require the development of alternative mixtures to meet the strength and flowability criteria. These specifications also provide detailed information about mixing and placement of flowable fills.

Table 2-12: Ohio DOT specifications for flowable fill [ODOT 1997]

\begin{tabular}{l|c|c|c|c|c|c}
\hline & \multicolumn{2}{|c|}{ Type $1^{*}$} & \multicolumn{2}{c|}{ Type 2 } & \multicolumn{2}{c}{ Type 3 } \\
\hline Cement & $\mathrm{kg} / \mathrm{m}^{3}$ & $1 \mathrm{bs} / \mathrm{yd}^{3}$ & $\mathrm{~kg} / \mathrm{m}^{3}$ & $\mathrm{lbs} / \mathrm{yd}^{3}$ & $\mathrm{~kg} / \mathrm{m}^{3}$ & $\mathrm{lbs} / \mathrm{yd}^{3}$ \\
\hline Fly Ash, Class F & 30 & 50 & 59 & 100 & 0 & 0 \\
\hline $\begin{array}{l}\text { Fly Ash, Class C } \\
* * * *\end{array}$ & 148 & 250 & $* *$ & $* *$ & 890 & 1500 \\
\hline Sand *** & 0 & 0 & 0 & 0 & 297 & 500 \\
\hline Water (Target) & 1726 & 2910 & 1436 & 2420 & 0 & 0 \\
\hline & 297 & 500 & $125-178$ & $210-300$ & 504 & 850 \\
\hline
\end{tabular}

* An air-entraining agent specifically designed for use in the low-strength mortar mixture may be added to this mix.

** Entrained air is substituted for fly ash in this mix.

*** Saturated surface dry.

****Class $\mathrm{C}$ fly ash may be substituted for Class $\mathrm{F}$ fly ash in Type 1 mixes with an approved mix design meeting the alternate mix design criteria of this specification.

Other states require mixture designs similar to the above specifications. However, they do not specifically refer to the potential beneficial reuse of foundry sand as a portion 
or a full replacement of the fine aggregate in flowable fills. In addition, it was recommend that design mixtures be developed to satisfy local and state strength and flow requirements. The mix presented in Table 2-13 could be used as a starting mixture. Cement quantity is adjusted to meet strength criteria [Abichou et al. 1999].

Table 2-13: Starting mixtures of CLSM [Abichou et al. 1999]

\begin{tabular}{l|l|l}
\hline Components & Mix I & Mix II (IDOT) \\
\hline Cement $\left(\mathrm{kg} / \mathrm{m}^{3}, \mathrm{lbs} / \mathrm{yd}^{3}\right)$ & $30 / 50$ & $59 / 100$ \\
\hline Fly Ash $\left(\mathrm{kg} / \mathrm{m}^{3}, \mathrm{lbs} / \mathrm{yd}^{3}\right)$ & $148 / 250$ & 0 \\
\hline Sand $\left(\mathrm{kg} / \mathrm{m}^{3}, \mathrm{lbs} / \mathrm{yd}^{3}\right)$ & $1726 / 2910$ & $1436 / 2420$ \\
\hline Water $\left(\mathrm{kg} / \mathrm{m}^{3}, \mathrm{lbs} / \mathrm{yd}^{3}\right)$ & \multicolumn{2}{|l}{ Adjusted to meet flowability criteria } \\
\hline
\end{tabular}

\subsubsection{Research of CLSM Containing Excess Foundry Sands}

The introduction of excess foundry sand to CLSM is a recent innovation. The early research can be traced back to the demonstration projects on flowable fill that were initiated by the Ohio Cast Metals Association (OCMA) et al. in 1993.

CLSM is typically a mixture of sand, fly ash, cement, and water. Since sand is the major component of CLSM, replacing the natural sand with foundry sand is an attractive beneficial reuse application. Research includes laboratory experiments and field construction and investigation. The main topics concentrate on the percentage of excess foundry sand substituted for fly ash or fine aggregate, monitoring performance and environmental impacts in highway construction, and developing mixture formulations. Most of the research used one to three excess foundry sands as the raw feed. The critical 
performance parameters, as proposed by this research, include flowability/work consistency, unconfined compression strength development, hydraulic conductivity, and environmental impacts.

Javed and Lovell prepared flowable fill mixtures using Class F fly ash, Type I cement, and a foundry sand [Mast 1997]. Mixture designs were tested for spread (flowability), set time, and compressive strength. It was concluded that foundry sand mixtures perform better than conventional flowable fill mixture [Joved and Lovell 1994B]. Investigations regarding the economy of foundry sand reuse, flow characteristics, hardening patterns, 28-day compressive strength, long-term strength, pore size distribution, hydraulic conductivity, $\mathrm{pH}$ of the pore water, and stress-strain characteristics were conducted, and it was concluded that flowable fill, containing up to $55.5 \%$ foundry sand, is an economic alternative to conventional compacted fills [Bhat and Lovell 1996]. In the same investigation, a step-by-step procedure for flowable mix design was developed. The CLSMs containing Class F fly ash and foundry sand from ferrous castings are environmentally benign [Joved and Lovell 1994B, Bhat and Lovell 1996, Bhat and Lovell 1997].

Naik and Singh [Naik and Singh 1997B] concluded that foundry sand can be used in flowable fills to replace up to $85 \%$ of the fly ash used in the reference mixture, and that the permeability of the flowable mixtures was affected by an increase in either the water to cementitious materials ratio or the foundry sand content. The other aspect of the study dealt with hydraulic conductivity [Naik and Singh 1997]. The hydraulic conductivity is 
important because more permeable fills have greater leachability potential. Results of the test program showed that a minimum hydraulic conductivity occurs when $30 \%$ of the fly ash is replaced with foundry sand. Flowable fills having $70 \%$ of the fly ash replaced with foundry sand do not have significantly different hydraulic conductivities. However, when $85 \%$ of the fly ash is replaced with foundry sand, the hydraulic conductivity increases dramatically. Low hydraulic conductivity is also correlated to water to cementitious material ratios ranging from 0.4 to 0.6 . Three green sands from ferrous foundries and two Class F fly ashes were used. The flow behavior, hardening characteristics, ultimate strength behavior, and permeability characteristics of flowable fill were investigated. The penetration resistance necessary to sustain workability as the fresh flowable fill hardens was determined. The $\mathrm{pH}$ of pore solution of hardened flowable fill indicated that the potential for corrosivity was low.

Additional research studies have been performed to enhance or qualify excess foundry sand applied to CLSM. The chemical characteristics of a wide spectrum of spent molding sands from Pennsylvania foundries were established, from which environmental impacts might be determined, and statistical protocols were used, including clustering and factorial analyses to address the chemical characteristics [Regan et al. 1993]. A database addressing the projects on the beneficial reuse of excess foundry sand was developed [Abichou et al. 1999]. In this database, 98 nationwide projects were categorized into applications of flowable fill, embankment and subgrade, portland cement concrete, asphalt concrete, portland cement manufacturing, landfill cover and linear system, and soil amendments. The section of flowable fill reviewed the laboratory 
research and field study on the beneficial reuse of excess foundry sand to flowable fill. Laboratory tests were performed to characterize physical and mechanical properties of foundry sands [Hiltunen et al. 1992]. The leaching of chemicals from all major foundry waste streams and leachability of materials that include the beneficially used foundry byproducts as aggregates were studied [Winkler 2000, Bol'shakov and Winkler 2001].

Research studies have been performed to demonstrate the beneficial reuse of excess foundry sand, which are not limited to CLSM. The addition of non-hazardous excess foundry sands to soil amendments for the greenhouse and nursery industries has been investigated as an environmentally sound, beneficial use option of these foundry residuals [Dunkelberger and Regan 1997]. The potential reuse of selected foundry wastes for highway construction, such as for bridge approach embankments, was performed [Lovejoy et al. 1996].

\subsection{Hot-Mixing Asphalt (HMA)}

\subsubsection{Findings From Previous Studies at UW - Madison}

The first study from the University of Wisconsin-Madison Asphalt Research Group that dealt with incorporating foundry sand into HMA was led by Ms. Edna Miller and was entitled, "Utilization of Foundry Sand in Hot Mix Asphalt" [Miller et al. 1998]. In this study, 17 foundry sands from around Wisconsin were tested for their gradation, angularity, absorption and sand equivalent value. They were also individually 
incorporated into a mix design to see how they would affect the volumetric properties of HMA. The JMF of each mix was held moderately constant to the control JMF.

It was found that foundry sands can contain between $1.0 \%$ and $16.5 \%$ of material passing the $75 \mu \mathrm{m}$ (\#200) sieve, depending on the type of binder system used. Generally, if a sand contains bentonite, there will be a larger amount of fines. The angularity of the sands ranged between 39\% and 48\%. The high angularities were attributed to rough surface texture and irregular rounded shapes. This was partly due to burnt additives adhering to the silica sand surface, as when iron oxide adheres to silica sand when used with the sodium silicate binder. The clay content was measured using the sand equivalent test, varied between $6 \%$ and $97 \%$. Finally, the percent absorption ranged between $0.3 \%$ to $6.2 \%$.

The second study completed by the University of Wisconsin - Madison Asphalt Research Group was led by Mr. Kenneth Delage [Delage et al. 2001]. This project focused more on the performance testing of the compacted samples, and not as much on the physical properties of the foundry sands, or on how they affect the volumetric properties of HMA. Five of the sands from Miller's study were used. The main performance tests used were the GLPA, the Hamburg Wheel for rutting and moisture damage, and the Superpave Shear Tester.

Delage concluded that it was difficult to measure the sand equivalency of foundry sands and suggested this could be overcome by mixing foundry sands with regular 
natural sands. It was also concluded that foundry sands cause HMA to become less sensitive to moisture damage as compared to the control. Lastly, it was concluded that foundry sands do not necessarily increase or decrease a mixture's rutting potential, but do improve fatigue performance.

The most recent study completed by the UW Asphalt Research Group was one led by Mr. Andrew Braham entitled, "The Use of Blended Recycled Foundry Sand in Hot Mix Asphalt" [Braham 2002]. In this study, five sands from Indiana and three sands from Wisconsin were used. Each sand was tested for its absorption, angularity, and percent of material passing the $75 \mu \mathrm{m}$ sieve. The sands were then mixed together to vary the levels of absorption, angularity and $\mathrm{P}-75 \mu \mathrm{m}$ to see how it would affect the volumetric properties of the mixes, energy indices, and moisture sensitivity.

It was concluded that varying the levels of the absorption, angularity and fines did not affect the performance of the HMA. Secondly, the absorption test used for aggregates does not work well for foundry sands, because of the high amount of clay found in most foundry sands. Also, the sand equivalent test could not be used for foundry sands due to the additives "clouding up" the test. Last, it appeared that foundry sands decreased the tensile strength ratio of HMA mixes, but because the $95 \%$ confidence interval was so large, the TSR values could have been negative, or exceeded $100 \%$. 


\subsubsection{Overcoming Problems Faced in Previous Studies}

Based on these three studies, it was decided that in order to be able to accurately determine the effects of foundry sand on HMA performance, a better way to measure the clay content, absorption, and moisture sensitivity would have to be developed. For the clay content, a method used by the foundry industry involving the methylene blue cation was modified and used. For absorption, a method developed by a company called Instrotek $^{\circledR}$ was modified and used. For the TSR, stricter sieving procedures were used, as well as the help of an automated saturation device. The following sections give background for each of the methods used.

\subsubsection{Methylene Blue}

The methylene blue molecule has the following chemical composition [Pike 1992]:

$$
\mathrm{C}_{16} \mathrm{H}_{18} \mathrm{~N}_{3} \mathrm{~S} . \mathrm{Cl} . \mathrm{xH}_{2} \mathrm{O}
$$

The methylene blue cation is readily absorbed by clays. Worrall [1968] stated that methylene blue was absorbed in three different stages. In the first stage, the methylene blue cations create a monolayer around the clay molecules. After this, they begin to replace all the other cations that are on the clay surface (for example, sodium cations on western bentonite and calcium ions on southern bentonite). Eventually the methylene blue cations are physically absorbed by the clay surface, but because of the large size of the MB cation, the absorption is only minor. Hang and Brindley [1970] surface area of the molecule to be about $130 ?^{2}$ with dimensions of $17.0 \times 7.6 \times 3.25 ?$. 
The foundry industry has taken advantage of clay's ability to absorb the methylene blue cation and has developed several standards to measure the clay content of foundry sands. As mentioned earlier in this chapter, due to the intense heat of the foundry process, clay can break down and lose its ability to adhere to silica sand particles. When this happens, it also loses its ability to attract methylene blue cations. By monitoring how much methylene blue a foundry sand can absorb, a foundry can determine the level of active clay left in the foundry sand. The two most common methods used are the AFS 2210-00-SS "Methylene Blue Clay Test, Ultrasonic Method, Molding Sand," and 2211-00-S, "Methylene Blue Clay Test, Boiling Method, Molding Sand" [American Foundry Society 2001].

Originally for this project, it was decided that AFS 2210-00-SS would be used to determine the methylene blue values of the foundry sands. The boiling method was more dangerous and left more room for error. The AFS 2210-00-SS method uses an ultrasonic cleaner to remove any clay from the silica sand particles and put them into solution. The solution is then stirred using a magnetic stir rod, and methylene blue dye is added by use of a burette. To check the titration point, material is removed using a glass stirring rod and dropped onto filter paper. Once a light blue halo appears, the titration point has been found, and the clay particles are assumed to be saturated.

The most difficult problem with this method is determining when the blue halo appears. Through personal contacts with Mr. Mark Nagel of the Cast Metals Institute, it 
was apparent that there is no definite blue halo, but its presence is subject to the opinion of the person running the test. Since the graduate and undergraduate students working on this project lacked the experience to determine where the blue halo occurs, a procedure was developed using a spectrophotometer. The wavelength was set at $670 \mathrm{~nm}$. It was shown by Gessner, Schmitt, and Neumann that methylene blue dye absorbs this wavelength of light most readily.

The second obstacle to overcome was to determine the level of absorbance (absorbance of $670 \mathrm{~nm}$ light) at which the sample has reached its titration point. Figure 27 shows a plot of volume of methylene blue dye solution verses absorbance. From this figure it seems that at an absorbance of about 0.2 the slope of the data changes. The point at which the slope changes was considered to be the titration point. It was assumed that the clay is no longer absorbing the methylene blue cation, but is instead going into solution.

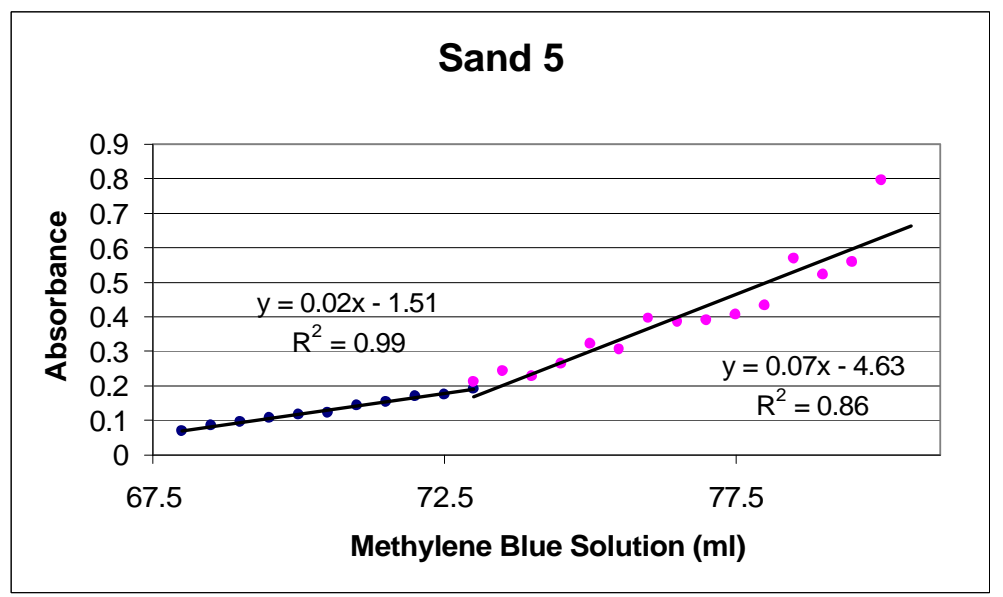

Figure 2-7: Titration point of Sand 5 


\subsubsection{Absorption}

The main purpose of the absorption test is to determine the bulk and effective density of an aggregate. The method commonly used to determine the bulk density is ASTM C127. In this method, the fine aggregates are soaked in water for 24 hours. This soaking is supposed to encourage water to penetrate the macro and micro pores of the aggregate. If the aggregate is then weighed in water, the apparent density can be determined. This method is straightforward and leaves less room for debate than finding the bulk density. The bulk density is defined as the mass of aggregate per volume of aggregate plus its micro pores. The main question then arises, "What is the defining line between a micro pore and a macro pore?" (see Figure 2-8).

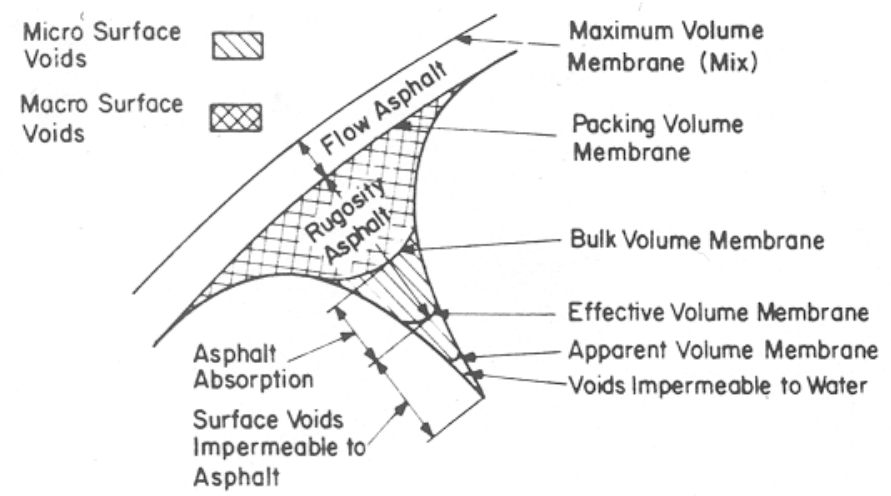

Figure 2-8: Apparent, effective and bulk densities [Ishai and Ton 1977]

According to ASTM C127, after the aggregates have been totally saturated with water, they have to become Saturated Surface Dried (SSD). SSD is when the macro pores are free of water, but the micro pores are still filled with water. To reach the saturated surface dried condition, the aggregates are very slowly dried using a fan. This process can 
take up to 6 hours. As the aggregates become drier, they are placed in a cone and tapped 25 times. Once the aggregates slump, the water is assumed to have left the macro pores, and has reached a saturated surface dry condition. Most contractors and researchers do not like this test, because it is time consuming and is not based on scientific reasoning. As Braham pointed out in his thesis [Braham 2002], the clay particles made it difficult to reach the SSD condition, because clay likes to bind silica sand particles together. So, even if the sand had reached the SSD condition, it may not have slumped due to the adhesive nature of the clay.

The InstroTek ${ }^{\circledR}$ Company developed another method to determine the dividing line between macro and micro pores. Instead of saturating the sample with water and then slowly drying the aggregates, their method first saturates the macro and micro pores with air, and fills the macro pores with water, while leaving the micro pores filled with air. This is done by placing the aggregates in water, and then quickly, but gently stirring the aggregates, so that air is removed from the packing and macro pores, but left in the micro pores (Figure 2-9). The aggregates are then weighed in water and the bulk density is determined. This method is much easier to use because it takes only a fraction of the time as ASTM C127, so it can be repeated more rapidly. Also, there is no cone and tapping hammer, which can lead to human errors. Last, and most important to this research project, the clay's adhesive properties do not cause error in determining the bulk density. Even though clay is adhesive, it should not prevent water from penetrating the packing and macro pores. 


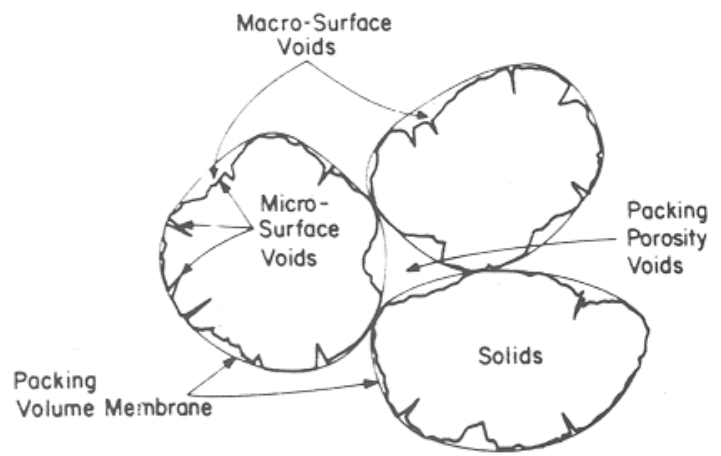

Figure 2-9: Difference between packing, macro and micro voids [Ishai and Ton 1977]

\subsubsection{Tensile Strength Ratio}

One of the important findings reported by Braham in his study report is that the error associated with the indirect tension test rendered most of the results to be invaluable, even though specifications were followed [Braham 2002]. Khosla, Birdsall, and Kawaguchi [2000] showed that the level of air voids and saturation greatly influenced the indirect tension values. They showed that as the percent air voids increased from $6 \%$ to $8 \%$, and as the level of saturation increased from $55 \%$ to $80 \%$, the TSR value decreased from $74.5 \%$ to $48.6 \%$ with Asheboro aggregates, $79.6 \%$ to $58.2 \%$ with Fountain aggregates, and $88.8 \%$ to $61.7 \%$ with Castle Hayne aggregates. So although the same aggregate, gradation, and asphalt content were used, and ASTM D4867 was followed properly, the variation associated with the TSR caused the results to be inconclusive as to whether or not an HMA mixture was susceptible to moisture damage. 
The first step that was taken to narrow the error in this study was to do a more thorough job of sieving aggregates. It was assumed that as aggregates are more carefully sieved, and sieved to more fine components, better quality control can be achieved. Typically, aggregates are only sieved down to the $2.36 \mathrm{~mm}(\# 8)$ sieve size. All material passing the $2.36 \mathrm{~mm}$ sieve is considered as one size material and is sieved no further. In this study, all the fine aggregates were sieved down to the $75 \mu \mathrm{m}$ (\#200) level. Also, the dust correction factor was taken for each aggregate size to take into account dust that was statically attached to the larger aggregates. This sieving procedure allowed a more consistent air void content to be achieved. To create a more consistent level of saturation, the Corelok $^{\mathrm{TM}}$ device was used instead of the rice pump. Since the Corelok ${ }^{\mathrm{TM}}$ device is automated, it can very consistently draw a partial (and programmable) vacuum on the compacted asphalt sample, so that a certain level of air voids become saturated. 


\section{Chapter 3}

\section{CHARACTERIZATION DATA}

In this chapter, the datasets addressing the characterization of excess foundry sand and other foundry by-products, including dust, slag, and sludge, that were collected nationwide were analyzed through charts of scatterplots. Charts of plots are more informative than lists of tables. It is an inductive procedure in which data are summarized rather than tested. Results through plotting provide patterns and support theories of how the data systems behave and provide guidance for the selection of appropriate deductive hypothesis testing procedures [Hesel and Hirsch 1992].

\subsection{Excess Foundry Sand Characterization System}

According to RCRA and federal regulations, excess foundry sand needs to be exposed to toxicity evaluation preceding its disposal or reutilization. The mandatory evaluation is on the leachate extracted by approved leaching protocols. Some samples are pre-exposed to bulk chemical analysis to evaluate whether the samples require leaching evaluation.

The leaching protocols extensively used by commercial chemical laboratories include TCLP (Section 2.2.2.1), SPLP (Section 2.2.2.2), and ASTM D 3987 (Section 2.2.2.3). The selection of leaching protocols depends on state regulations and laboratory 
facilities. The significant difference among three leaching protocols is the extraction environments they simulate, which leads to operation variation. TCLP intends to simulate an actively decomposing municipal solid waste landfill in which carboxylic acids are formed from microbial processes. SPLP intends to simulate an acid rain condition similar to an industrial waste monofill. ASTM D 3987 intends to simulate a condition where the solid waste is the dominant factor in determining the $\mathrm{pH}$ of the extract.

Not all leaching protocols have corresponding toxicity thresholds for TCLP, which forms a barrier to assess the toxic characterization of samples extracted by SPLP and ASTM D 3987. However, TCLP thresholds are reasonably applicable to evaluation of SPLP and ASTM D 3987 leachates (Section 2.2.1).

\subsection{Data Sources}

The data were collected from 180 foundries throughout the United States from the years of 1997-2001 using tests performed by independent commercial laboratories for regulatory compliance. The datasets are partitioned by waste streams, leaching protocols and metals cast in Table 3-1. In each dataset, up to 27 general chemical parameters, 24 metallic elements, and 55 organic compounds are addressed. They are managed by a database coded with Microsoft Access, which was released as a CD disk. In this study, excess foundry sand was exclusively characterized. 
Table 3-1: Partition of datasets

\begin{tabular}{|c|c|c|c|c|c|c|}
\hline & \multicolumn{3}{|c|}{ Bulk analysis } & \multicolumn{3}{|c|}{ Leachate composition } \\
\hline Sand & \multirow{4}{*}{314} & 192 & $61 \%$ & \multirow{4}{*}{650} & 343 & $53 \%$ \\
\hline Dust & & 58 & $18 \%$ & & 173 & $27 \%$ \\
\hline Slag & & 57 & $18 \%$ & & 111 & $17 \%$ \\
\hline Sludge & & 7 & $2 \%$ & & 23 & $4 \%$ \\
\hline TCLP & \multirow{3}{*}{\multicolumn{3}{|c|}{ N/A }} & \multirow{3}{*}{1163} & 543 & $47 \%$ \\
\hline SPLP & & & & & 345 & $30 \%$ \\
\hline ASTM D 3987 & & & & & 275 & $24 \%$ \\
\hline Iron-based facility & \multirow{4}{*}{263} & 187 & $71 \%$ & \multirow{4}{*}{607} & 457 & $75 \%$ \\
\hline Steel-based facility & & 30 & $11 \%$ & & 64 & $11 \%$ \\
\hline Aluminum-based facility & & 31 & $12 \%$ & & 61 & $10 \%$ \\
\hline Copper-based facility & & 15 & $6 \%$ & & 25 & $4 \%$ \\
\hline
\end{tabular}

\subsection{Bulk Chemical Analysis}

Bulk chemical analysis is the characterization of foundry sand without any extraction. Elements covered in bulk chemical analysis include those regulated by TCLP thresholds and heavily involved in the metal casting process. They are normally divided into three groups: general chemical parameters, metallic elements and organic compounds. Most of them are measured at trace level in parts per million, except for several parameters in their own standard units, such as $\mathrm{pH}$, conductivity, and moisture, etc.

\subsubsection{Major Oxides}

Major oxides are analyzed by x-ray fluorescence analysis. The American Foundry Society (AFS) conducted an investigation of oxides in excess foundry sands, with results 
presented in Table 3-2. It is indicated that silica occupies on average $88 \%$ by mass of the excess foundry sand.

Table 3-2: Foundry sand sample chemical oxide composition [AFS 1991]

\begin{tabular}{l|c}
\hline Oxides & Percentage by mass (\%) \\
\hline $\mathrm{SiO}_{2}$ & 87.91 \\
\hline $\mathrm{Al}_{2} \mathrm{O}_{3}$ & 4.70 \\
\hline $\mathrm{Fe}_{2} \mathrm{O}_{3}$ & 0.94 \\
\hline $\mathrm{CaO}$ & 0.14 \\
\hline $\mathrm{MgO}$ & 0.30 \\
\hline $\mathrm{SO}_{3}$ & 0.09 \\
\hline $\mathrm{Na}_{2} \mathrm{O}$ & 0.19 \\
\hline $\mathrm{K}_{2} \mathrm{O}$ & 0.25 \\
\hline $\mathrm{TiO}_{2}$ & 0.15 \\
\hline $\mathrm{P}_{2} \mathrm{O}_{5}$ & 0.00 \\
\hline $\mathrm{Mn}_{2} \mathrm{O}_{3}$ & 0.02 \\
\hline $\mathrm{SrO}$ & 0.03 \\
\hline TOTAL & 94.72 \\
\hline
\end{tabular}

Silica is not a regulated toxic element and is environmentally benign regardless of its concentration, except for its respirable hazard at a defined particle size. It mainly comes from the sand aggregates that are the refractory materials of casting molds and cores. Its variation in concentration spans from 36\% to $99.7 \%$ as shown in Figure 3-1.

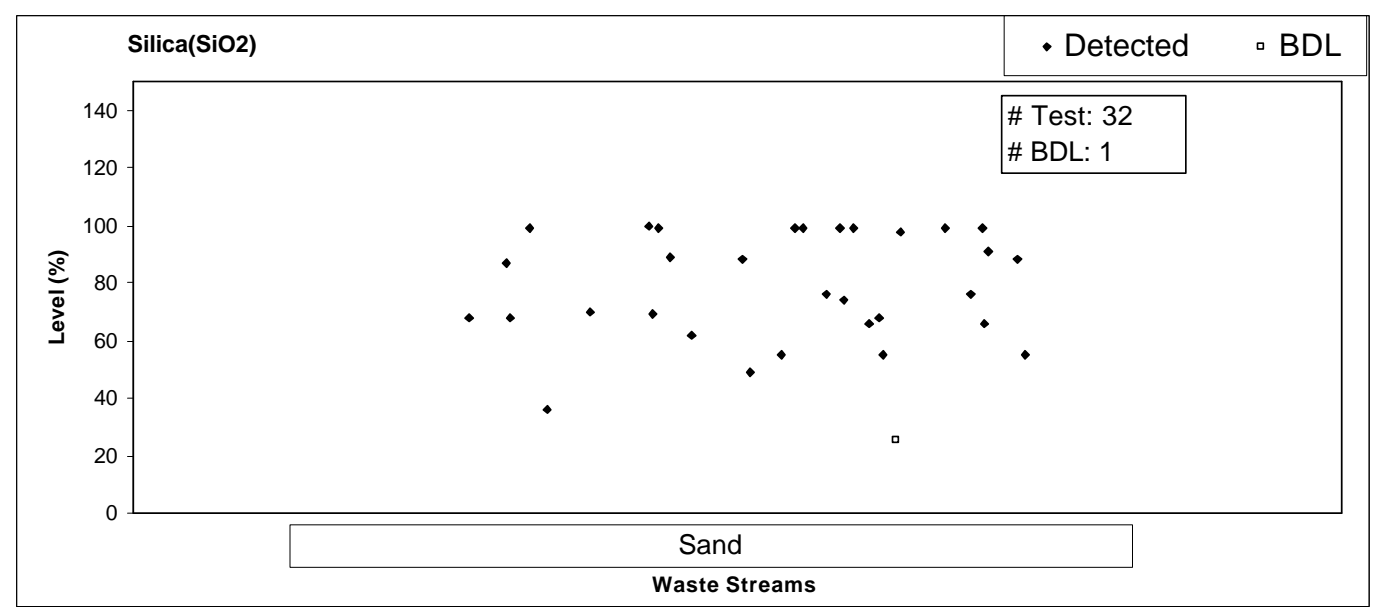

Figure 3-1: Silica content by mass in waste streams 


\subsubsection{Metallic Elements}

The term "trace element" is rather loosely used in the scientific literature to designate a number of elements that occur in natural systems in small concentrations. As defined in many dictionaries, trace elements are those chemical elements, especially metals, used by organisms in minute quantities but believed essential to their physiology. However, the term is and has been used to designate elements with no known physiological function which, when present in sufficient concentrations, may be toxic to living systems [Bradford et al. 1996].

Trace level metallic elements listed in Table 3-3 are the mostly monitored elements. They cover a wide spectrum of heavy metals that may come from various scrap feed or alloy additions, and common light metals that are also hazardous to human health if digested or inhaled in enough amounts. The compositions of these elements for collected samples are plotted in Figure D-1 in Appendix D.1.

Table 3-3: Metallic elements in bulk chemical analyses

\begin{tabular}{l|l|l|l|l}
\hline Aluminum & Antimony & Arsenic & Barium & Beryllium \\
\hline Boron & Cadmium & Calcium & Chromium & Cobalt \\
\hline Copper & Iron & Lead & Magnesium & Manganese \\
\hline Mercury & Molybdenum & Nickel & Selenium & Silicon \\
\hline Silver & Thallium & Zinc & --- & -- \\
\hline
\end{tabular}

It is indicated that the distribution of most metallic elements is consistent with the dilution theory and simulation model of environmental data, "a concentration resulting from a series of independent random dilutions tends to be lognormally distributed" [Ott 1990]. As illustrated in Figure D-1, the dominating tendency of distribution is that 
observations heavily cluster to a low level, even zero, and occurrence frequency varies inversely against concentration. Therefore, the estimated frequency curve is right skewed. However, each assumption for the distribution model of each metallic element, in spite of lognormal or normal distribution, needs to be statistically tested to make results defensible.

\subsubsection{Chemical Parameters}

General chemical parameters listed in Table 3-4 represent typical inorganic elements and chemical properties identified in general chemical characterization reports. Most of them are inorganic and nonmetallic elements. Parts of them address characteristics regulated by environmental regulations, such as reactive cyanide and reactive sulfide for reactivity, $\mathrm{pH}$ for corrosivity, and ignitable point for ignitability. The detailed distributions for these parameters are plotted in Figure D-2 in Appendix D.2.

Table 3-4: General chemical parameters in bulk chemical analyses

\begin{tabular}{l|l|l|l}
\hline Chloride & Reactive cyanide & Total cyanide & Fluoride \\
\hline Formaldehyde & Ignitable point & Hexavalent chromium & Total phenols \\
\hline Total moisture & $\begin{array}{l}\text { Total petroleum } \\
\text { hydrocarbons }\end{array}$ & $\begin{array}{l}\text { Extractable organic halides } \\
\text { (EOX) }\end{array}$ & $\begin{array}{l}\text { Total organic } \\
\text { halogens (TOX) }\end{array}$ \\
\hline pH & Total volatile solids & Oil and grease & Reactive sulfide \\
\hline Sulfide & Total volatile residue & Loss on ignition (LOI) & --- \\
\hline
\end{tabular}

The reactivity characteristic is to identify wastes that, because of their extreme instability and tendency to react violently or explode, pose a problem at all stages of the waste management process [EPA SW-846 2000]. One evaluating method is the concentration of reactive cyanide and reactive sulfide. 
For reactivity, the concentrations of reactive cyanide for foundry sands are well below the threshold of environmental regulation. The concentrations of reactive sulfide for foundry sands are well below the threshold of environmental regulation, except for one sample: sand from an aluminum-based facility. The observation is $2,075 \mathrm{ppm}$ against a reactivity threshold of $500 \mathrm{ppm}$.

The corrosivity characteristic is designed to identify wastes that might pose a hazard to human health or the environment due to their ability to mobilize toxic metals if discharged into a landfill environment, to corrode handling, storage, transportation, and management equipment, or to destroy human or animal tissue in the event of inadvertent contact [EPA SW-846 2000]. From the pH tests, it is presented that foundry sands are non-corrosive. All observations are within threshold intervals of 2-12.5. And foundry sands tend to be alkali.

The objective of the ignitability characteristic is to identify wastes that either present fire hazards under routine storage, disposal, and transportation or are capable of severely exacerbating a fire once started [EPA SW-846 2000]. Foundry sands have a flash point greater than $60{ }^{\circ} \mathrm{C}$, which is the regulated minimum of ignitability. Thus, foundry sands are non-ignitable. 


\subsubsection{Organic Compounds}

Trace-level organic elements listed in Table 3-5 represent most compounds used or potentially produced by decomposition in metal casting bonding processes. The bulk distributions of these compounds for collected foundry sand samples are plotted in Figure D-3 in Appendix D.3.

Table 3-5: Organic compounds in bulk chemical analyses

\begin{tabular}{l|l|l|l}
\hline Aroclor-1016 & Aroclor-1221 & Aroclor-1232 & Aroclor-1242 \\
\hline Aroclor-1248 & Aroclor-1254 & Aroclor-1260 & Total-PCBs \\
\hline Acenaphthylene & Anthracene & Benzene & Benzo(a)anthracene \\
\hline Benzo(b)fluornathene & Benzo(k)fluornathene & Chrysene & o-Cresol \\
\hline m_p-Cresol & 2,4-Dimethylphenol & Ethylbenzene & Fluoranthene \\
\hline Fluorene & 1-Methyl naphthalene & $\begin{array}{l}\text { 2-Methyl } \\
\text { naphthalene }\end{array}$ & $\begin{array}{l}\text { 4-chloto-3-methyl } \\
\text { Phenol }\end{array}$ \\
\hline Naphthalene & Phenanthene & Pyrene & Styrene \\
\hline Toluene & Xylene & --- & \multicolumn{1}{c}{--- } \\
\hline
\end{tabular}

Censored observations, those values below detection limits (BDL), occupy a great portion of observations for bulk organic compounds in foundry sands. Compounds with 100\% censoring include Aroclor-1016, Aroclor-1221, Aroclor-1232, Aroclor-1242, Aroclor-1248, Aroclor-1254, Aroclor-1260, and Total-PCBs. The other organic compounds have censoring fraction mostly above $50 \%$. The principal reason for a high censoring fraction is that most of the organic compounds are significantly burned out during high-temperature melting. Even if minimum organic residuals are attached to byproduct grains, they cannot be quantified by bulk chemical analyses. Due to high censoring, there is no information to infer but that these organic compounds are below reporting limits. 


\subsection{Leachate Concentration}

The criteria to determine whether a waste stream is hazardous or not depends on the characteristics of leachate extracted from waste streams using acceptable leaching protocols [40 CFR Part 261 1986]. Currently the federal regulation accepts TCLP as a leaching procedure and provides a toxicity threshold for toxicity assessment purposes. Some regulatory agencies and commercial laboratories use SPLP and ASTM D 3987 leaching procedures, as mentioned in Section 2.2.2. The leachate characterization is divided into three groups: metallic elements, general chemical parameters, and organic compounds. Most of them are measured at trace level using unit ppm or ppb.

\subsubsection{Metallic Elements}

The leachate concentrations of metallic elements listed in Table 3-6 are plotted in Figure E-1 in Appendix E.1. The $\mathrm{X}$-axis represents leaching protocols; the Y-axis represents concentrations of elements. Each point (solid or hollow) corresponds to an observation. The count of total observations and count of censored observations are listed in each chart associated with corresponding leaching protocols. The dashed line in the top of the chart indicates the toxicity threshold.

Table 3-6: Metallic elements in leachate analyses

\begin{tabular}{l|l|l|l|l}
\hline Aluminum & Antimony & Arsenic & Barium & Beryllium \\
\hline Boron & Cadmium & Chromium & Cobalt & Copper \\
\hline Iron & Lead & Magnesium & Manganese & Mercury \\
\hline Molybdenum & Nickel & Selenium & Silver & Sodium \\
\hline Thallium & Vanadium & Zinc & --- & --- \\
\hline
\end{tabular}


The EPA toxicity thresholds, presented in Table C-1 in Appendix C.1, are the standards by which foundry sands are assessed. If the TCLP leachate concentration of any metallic element, including arsenic, barium, cadmium, chromium, lead, mercury, selenium, and silver is above the corresponding toxicity threshold, the foundry sand yielding the leachate is considered hazardous [40 CFR Part 261 1986].

Although metallic elements do exist in the leachate as presented in Figure E-1 in Appendix E.1, excess foundry sand has been found to be largely environmentally benign through toxicity assessment. The concentrations of RCRA eight metallic elements, including arsenic, barium, cadmium, chromium, lead, mercury, selenium, and silver, are well below corresponding thresholds. It is suggested that excess foundry sand can be beneficially reused, posing very low environmental or human health risk.

Two observations for selenium exceeded TCLP toxicity limits. The description of the samples is "recyclable." Further classification from the metal casting facilities and chemical lab would be required to determine the nature of the observed samples.

Leaching protocol is a significant factor. TCLP tends to yield more metallic elements, particularly RCRA eight metallic elements, than SPLP and ASTM D 3987 protocols. This is because the toxicity tests are designed to simulate the worst-case conditions in a municipal landfill. The other two protocols more closely simulate beneficial reuse conditions of foundry sand, such as embankments and flowable fill. A further quantitive investigation is performed in Section 5.6. 
The distributions of metallic element concentrations in the leachate are closer to the lognormal model than normal model, which means that denser frequency is close to zero. This is consistent with the distribution tendency indicated by bulk analysis in Section 3.3.2. However, the distribution assumption requires further diagnosis.

\subsubsection{Chemical Parameters}

The leachate concentrations of general chemical parameters listed in Table 3-7 are plotted in Figure E-2 in Appendix E.2.

Table 3-7: General chemical parameters in leachate analyses

\begin{tabular}{|c|c|c|c|}
\hline Cyanide & Formaldehyde & Phenols & Initial $\mathrm{pH}$ \\
\hline Final $\mathrm{pH}$ & Fluoride & Ammonia & Hexavalent chromium \\
\hline Chloride & Nitrate & Nitrite & Oil and grease \\
\hline Sulfate & Acidity & Alkalinity & Conductivity \\
\hline $\begin{array}{l}\text { Total dissoluble } \\
\text { solid (TDS) }\end{array}$ & $\begin{array}{l}\text { Total organic } \\
\text { halide }(\mathrm{TOH})\end{array}$ & $\begin{array}{l}\text { Total petroleum } \\
\text { hydrocarbon }\end{array}$ & $\begin{array}{l}\text { Chemical oxygen } \\
\text { demand (COD) }\end{array}$ \\
\hline Total sulfide & --- & --- & --- \\
\hline
\end{tabular}

\subsubsection{Organic Compounds}

The leachate concentrations of organic compounds listed in Table 3-8 are plotted in Figure E-3 in Appendix E.3. According to RCRA, 34 organic compounds are regulated regarding the toxicity of solid waste by federal regulations [40 CFR Part 261 1986], as presented in Tables C-2 and C-3 in Appendix C.2. As with metallic elements, a violation of any organic compound results in the assessment that samples are hazardous and cannot 
be disposed to traditional landfills or reused in construction. In this investigation of foundry sands, 23 out of 37 organic compounds are regulated as presented in Figure E-3. All observations are well below toxicity thresholds and foundry sands are non-hazardous based on regulated organic compounds.

Table 3-8: Organic compound in leachate analyses

\begin{tabular}{l|l|l}
\hline Compounds with 100\% censoring & \\
\hline Cresol_para & Pentachlorophenol & Methylene chloride \\
\hline Cresol_total & Pyridine & 1_1_1-Trichloroethane \\
\hline Dinitrotoluene (2_4)_total & Trichlorophenol (2_4_5) & Vinyl chloride \\
\hline Hexachloro-1_3-butadienel & Trichlorophenol (2_4_6) & m_p Xylene \\
\hline Hexachlorobenzene & Carbon tetrachloride,total & Chlorobenzene \\
\hline Hexachloroethane & Fluoranthene & Chloroform \\
\hline Nitrobenzene & Methyl ethyl ketone & $\begin{array}{l}\text { Dichlorobenzene (1,4 } \\
\text { total) }\end{array}$ \\
\hline 1_2-Dichloroethane & 1_1-Dichloroethylene & \\
\hline Compounds with censored and uncensored data (censoring percentage) \\
\hline Acetone (0\%) & Phenanthrene (75\%) & Benzene (96\%) \\
\hline Toluene (55\%) & Ethyl benzene (75\%) & $\begin{array}{l}\text { Tetrachloroethylene } \\
(96 \%)\end{array}$ \\
\hline Styrene (69\%) & $\begin{array}{l}\text { 2_4-Dimethylphenol } \\
\text { (84\%) }\end{array}$ & Cresol_ortho (85\%) \\
\hline Naphthalene (71\%) & Xylene_total (85\%) & $\begin{array}{l}\text { Methyl isobutkl ketone } \\
(88 \%)\end{array}$ \\
\hline Trichloroethylene (73\%) & Cresol_meta (92\%) & \\
\hline
\end{tabular}

It is presented in Figure E-3 that censored observations, which are marked by hollow squares, occupy a great portion of the observations. In Table 3-8, 23 out of 37 typical organic compounds are $100 \%$ below detection limits in sand leachate. Seven out of the remaining 14 compounds are more than $80 \%$ censored; these are cresol_m, cresol_o, benzene, 2_4-Dimethylphenol, tetrachloroethylene, xylene_total and methyl isobutkl ketone. Compounds with a censoring fraction less than $80 \%$ include acetone, ethyl benzene, naphthalene, phenanthrene, styrene, toluene, and trichloroethylene. Those 
23 organic compounds with $100 \%$ censoring are suggested to be eliminated from regulatory requirements, as inappropriate testing keeps foundry sand from beneficial reuse.

The phenomenon of high censoring for organic compounds is consistent with the high censoring that observed in the bulk analysis of organic compounds. The same reasoning can be used to explain that most of the organic compounds are significantly burned out during high-temperature melting, and even if minimum organic residuals are attached to by-product grains, they are difficult to dissolve or extracted and quantified in the leachate analyses. Due to high censoring, there is no information to infer but that these organic compounds are below reporting limits. 


\section{Chapter 4}

\section{METHODOLOGY ADDRESSING CENSORED DATA}

This chapter addresses a statistical methodology to evaluate characterization data that are highly nested with incomplete observations. Starting with survival analysis, this method estimates summary statistics and hypothesis testing with sound confidence.

\subsection{Censored Data}

More often than not, environmental monitoring data consist of a mixture of results that can and cannot be quantified precisely. The portion of nonquantifiable observations, reported as below limits or "not detected," is referred to as "censored data" for statistical purposes.

In chemical analyses, the instrument detector provides the electronic signals to register the mass to determine the concentration. The lower the concentration is, the more difficult it is to clearly distinguish the signals from other electronic background noise. Conceptually there has to be some limit, below which it is not possible to determine if there is detection or not. In that concept, some numerical standard is brought in to specify the limitation of the instrument. 
One important standard is called the "Method Detection Limit" (MDL), which is approved by the U.S. EPA. MDL is defined as the minimum concentration of a substance that can be measured and reported with $99 \%$ confidence, where the analyte concentration is greater than zero and is determined from analysis of a sample in a given matrix containing the analyte [40 CFR Part 136 Appendix B 1986]. The derived terms of detection limits that may exist in chemical reports include "Quantity Detection Limit" (QDL) and "Low Detection Limit" (LDL).

In the database of this research program, datasets may contain observations censored at multiple reporting limits for a parameter. This occurs frequently as limits are lowered over time at a single laboratory or when data having different censoring limits are pooled from multiple laboratories [Hesel 1990]. Environmental monitoring data usually are right skewed, and sometimes very highly skewed. This is especially true for data close to zero that include censored data, because the lower bound of zero ensures a right skew [Hesel 1990].

\subsection{Current Methods}

In general, if the data were not censored, they could be used to determine the parameters of a Probability Density Function (PDF) and Cumulative Distribution Function (CDF) to diagnose the best-fit distribution model of uncensored data, and then PDF and distribution models could be used to predict any characteristics of waste streams. Data on environmental chemicals are often modeled with the lognormal PDF 
[Ott 1990]. Lognormal PDF eliminates the risk of estimating negative concentrations and predicts mostly low concentrations except for a long tail to the right, simulating contaminated situations [Science Advisory Board 1995]. Weibull PDF is another option that has the same feature with the additional advantage that it can be integrated analytically to produce a $\mathrm{CDF}$ that enables convenient estimates of the occurrence of specific concentrations [Mackay and Paterson 1984].

However, the nature of frequently censored environmental data impedes the application of conventional statistical procedures. The data of interest are not complete. Censored observations represent ranges rather than a number. Censoring ranges from 0 to 1 depending on the composition of samples and quantitive level of instruments. It is not applicable to run conventional statistical programs on data containing quantified and nonquantifiable data, and it is not suitable to substitute arbitrarily for nonquantifiable data. Multiple censoring limits in a data pool further complicate the issue.

A flag system is recommended to annotate those censored data to facilitate data processing [Mikel 2001]. EPA has recommended that laboratories be asked to provide uncensored data on all water samples with measurements near or below the limit of detection [EPA 1991]. However, for those censored data in the research reported herein, there are no quantitative values provided but the reporting limits. A methodology needs to be developed to address censored data. In 2001, a literature search was conducted for EPA to identify articles that discuss detection and quantitative limit approaches 
[DynCorp 2001]. A total of 181 publications were identified in this search. The main approaches are discussed in the followed sections.

\subsubsection{Substitution}

EPA has traditionally substituted zero or one-half the reporting limits for censored data and computed the average concentration in the usual way. Its assumption of equal precision or zero fill-ins is demonstrably false, but the method is computationally simple and often adequate for most practical purposes if the quantifiable frequency is $80 \%$ or more [Gibbons and Coleman 2001, Hesel 1990]. With the advent of convenient software, there appears to be no reason to use simple substitutions.

\subsubsection{Regression on Order Statistics (ROS)}

Hashumoto and Trussell were the first to suggest normal regression for the problem of estimation of censored water quality data [Hashimoto and Trussell 1983]. A straight line is fit to the normal scores of the order statistics for the uncensored observations and then to fill in values extrapolated from the straight line for the observations below the detection limit. Distribution parameters of mean and standard deviation were determined by Gilliom and Hesel [Gilliom and Hesel 1986]. First, the mean and standard deviation are computed by using the half detection limits, then the censored data are replaced by random variates selected from a right-truncated normal distribution with derived mean and standard deviation. The mean and standard deviation 
are recomputed as the estimated parameters for total population. This method is modified by more robust approaches, which combine uncensored data with censored data extrapolated, assuming a distributional shape fitted by maximum likelihood estimation (MLE) or probability plot procedures, in order to compute estimates of summary statistics [Hesel and Cohn 1988]. In a similar way, censored data are replaced by the expected value of the normal order statistics with the mean and standard deviation estimated from the noncensored data. The process is repeated until there is no significant change in the mean and standard deviation in the total data set [Gleit 1985].

\subsubsection{Maximum Likelihood Estimation}

Maximum likelihood estimation uses both uncensored and censored observations to compute statistics for the entire data set. It calculates the estimated mean and standard deviation by adjusting downward the statistics of the uncensored observations as a function of the amount of censoring in the data set.

The earliest and most used method was published in two papers by Cohen [Cohen 1959, Cohen 1961]. This method gives the following maximum likelihood estimators for censored data, Equation 4.1 and Equation 4.2, in terms of a tabulated function of two arguments:

$$
\begin{array}{ll}
m_{M L}=m^{*}-\left(m^{*}-x_{0}\right) f(g, h) & \text { Equation 4.1 } \\
s_{M L}^{2}=s^{* 2}+\left(m^{*}-x_{0}\right)^{2} f(g, h) & \text { Equation 4.2 }
\end{array}
$$


Where: $m_{M L}=$ estimated value of the population mean by maximum likelihood

$$
\begin{aligned}
& s_{M L}^{2}=\text { estimated value of the population variance by maximum likelihood } \\
& m^{*}=\text { sample mean for uncensored values } \\
& s^{*}=\text { sample standard deviation for uncensored values } \\
& x_{0}=\text { detection limit } \\
& g=\frac{s^{* 2}}{\left(m^{*}-x_{0}\right)^{2}} \\
& h=\text { fraction of censored data } \\
& f=\text { Refer to tables developed by Cohen }
\end{aligned}
$$

Persson and Rootzen [1977] developed two sets of estimators that are somewhat simpler to compute, called restricted maximum likelihood estimators. However, these methods are limited to single censoring data, which are not popular in environmental monitoring data.

\subsubsection{Nonparametric Methods (NP)}

ROS and MLE are parametric methods, because their validity depends on knowing the population distribution function, and any hypothesis test or confidence interval estimation is based on the assumption that the population distribution function is known, or known except for some unknown parameters. Nonparametric methods do not assume a particular population probability distribution, and are therefore valid for data from any population with any probability distribution, which can remain unknown. These 
methods use ranks that may be considered preferable to the actual data. If the numbers assigned to the observations have no meaning by themselves but attain meaning only in an ordinal comparison with the other observations, the numbers contain no more information than the ranks contain [Conover 1999].

A number of studies have been published comparing these methods and showing alternative algorithms for solving the censoring problem [Gleit 1985, Gilliom and Hesel 1986, Kroll and Stedinger 1996, Gibbons and Coleman 2001]. These studies have consistently found the ROS and MLE procedures superior to simple substitution methods. ROS and MLE perform about equally well under low to moderate censoring, while MLE has some advantage when the censoring fraction is high (up to 80\%). As expected, the efficiency of the estimators decreases with the degree of censoring and the effect is more pronounced on the estimate of the variance versus the mean.

Even with an agreed-upon censoring point, there is considerable controversy regarding the appropriate method or methods for incorporating the censored data in computing summary statistics, resting hypothesis, and computing interval estimates. This is not at all surprising, since the correct choice of method depends on both the degree of censoring (e.g., $20 \%$ versus $80 \%$ nondetects) and the type of application (e.g., computing the mean versus computing a prediction limit from data that are a mixture of quantifiable and nonquantifiable observations), as well as ease of use. Additionally, the controversy can be fueled by an inclination toward a particular favorable outcome [Gibbons and Coleman 2001]. 
Although various methods for processing censored data are available, none of them will perform better than the analogous method for uncensored data, and the method for censored data will probably be more complicated [Porter, Ward and Bell 1988].

When censoring is low (i.e., less than 50\%) these methods are sufficiently robust to estimate the parameters [Gibbons and Coleman 2001]. When censoring is higher than $50 \%$, none of the methods discussed above works well, and due to the validity of assumptions taken by most methods, an alternative strategy must be employed.

\subsubsection{Methods Discussion}

Ignoring censored data is the worst procedure because it loses the left tail information, which occupies much in the case of left-censored data. Replacing those censored data with any arbitrary number, such as zero, a tenth of the reporting limits, half of the reporting limits, and the report limits, is theoretically unsupportable and results in unpredictable significant bias. Competitive substitutions may include the mean or median of those uncensored data below the reporting limits. However, it is difficult to qualify this substitution, too. Essentially the mean or median of the uncensored data below a reporting limit is not the unbiased estimate of the reporting limit. Methods using any kind of substitution should be avoided.

An assumption of many current methods is that the measurements are continuous and normally distributed, or can be suitably transformed to approximate a normal 
distribution. In the context of foundry sand characterization applications there are two special problems. First, measurements are nested within laboratory and sand background. The data from a laboratory on a sample may be normally distributed; however, each sampling location may have a different mean and variance, offsetting the measurements from one location to another. A test of normality for the composite will generally yield a rejection of the hypothesis that the data are normally distributed.

Second, the presence of censored observations will generally produce rejection of the normality hypothesis regardless of whether or not the quantifiable observations are normally distributed. One solution is simply to ignore the censored data and test the assumption of normality on uncensored data. When detection frequency is high (say $90 \%$ or more), this may produce reasonable results. If the censoring is high, ignoring the nondetects can be misleading because it eliminates the lower tail of the distribution and can be therefore falsely reject the hypothesis of normality. On the other hand, including the nonquantifiable observations at the detection limit introduces a spike in the distribution that can also, incorrectly, cause the rejection of normality [Gibbons and Coleman 2001].

Both MLE and ROS assume that the true concentrations in all samples come from a common population that is lognormally distributed. Violation of this assumption can lead to invalid applications of a statistical technique. The decisions and conclusions derived from incorrectly used statistics can be expensive. For example, incorrect use of a statistical limit may lead to excessively conservative or unsafe conclusions, such as 
remediation of a clean part of the site, or no remediation at a contaminated part of the site. The first conservative conclusion will result in an unnecessary cleanup, whereas the second unsafe conclusion may cause a threat to human health and the environment. It is likely that the availability of new and improved statistical software has also increased the misuse of statistical techniques [Singh et al. 1997].

When a statistical method is based on assumptions that appear to be invalid or impossible to verify, nonparametric methods have been become essential tools in the statistical analysis workshop. It does not depend on any distribution assumption and making more efficient use of the data, when parametric methods are inappropriately applied. The immediate application to censoring data is survival analysis.

\subsection{Survival Analysis}

In this section, survival analysis is discussed and applied to process environmental censored data. The main topics include estimated mean and its confidence interval, the hypothesis tests, and distribution model analysis.

\subsubsection{Introduction}

Survival analysis addresses the right-censored observations, namely the time to "death." The time may be observable, say "death," or unobservable, say "loss." The Kaplan-Meier Estimator (KME) [Conover 1999] is a widely used method in this field. It 
uses the fact that if "death" occurs after time $\mathrm{x}$, then "death" also occurs after all time prior to $\mathrm{x}$. For example, 3 of 10 timing belts survive 85,000 miles, and the other 7 timing belts break prior to 85,000 miles. The probability of surviving 85,000 miles $P$ ( $X$ > $85,000)=3 / 10=0.3$. If a timing belt survives 85,000 miles, it definitely survived 80,000 miles. The joint probability of surviving both 85,000 miles and 80,000 miles is $P$ ( $X$ > $85,000, X>80,000)=3 / 10=0.3$. Hence, the probability of timing belts surviving 85,000 miles is equal to the probability of timing belt surviving both 85,000 miles and 80,000 miles. Equations 4.3 and 4.4 provide general reasoning.

$$
\text { Call } x_{0}<x_{1}<x_{2} \text {, }
$$

then $P\left(X>x_{1}\right)=P\left(X>x_{1}, X>x_{0}\right)=P\left(X>x_{1} \mid X>x_{0}\right) \cdot P\left(X>x_{0}\right) \quad$ Equation 4.3

$$
P\left(X>x_{2}\right)=P\left(X>x_{2}, X>x_{1}\right)=P\left(X>x_{2} \mid X>x_{1}\right) \cdot P\left(X>x_{1}\right) \quad \text { Equation } 4.4
$$

Where: $P\left(X>x_{1}\right)=$ the probability of variable $X$ greater than observation $x_{1}$

$$
\begin{aligned}
P\left(X>x_{1}, X>x_{0}\right)= & \text { joint probability of variable } X \text { greater than } \\
& \text { observation } x_{1} \text { and } x_{0} \\
P\left(X>x_{1} \mid X>x_{0}\right)= & \text { the probability of variable } X \text { greater than } \\
& \text { observation } x_{1} \text { given variable } X \text { greater than } \\
& \text { observation } x_{0} \text {, a conditional probability }
\end{aligned}
$$

In real censored data, $P\left(X>x_{0}\right)$ can be determined, and $P\left(X>x_{i}\right)$ and $P\left(X>x_{i-1}\right)$ are frequently correlated. However, $P\left(X>x_{i} \mid X>x_{i-1}\right)$ needs to be 
estimated because the probability of survival of $x_{i}$ is a future event that has not yet been reached.

\subsubsection{Cumulative Probability and Survival Probability}

In environmental data, data are generally left-censored, rather than right-censored data in survival analysis. This means that data are reported below reporting limits or quantifiable values. Two examples help differentiate right-censored and left-censored data.

Example 1 (right-censored data)

Eight fanbelts are tested on cars. The mileages are recorded. At the conclusion of testing, four have broken with mileages (in thousands of miles) of 77, 47, 81, and 56. The other four are unbroken with mileages of $62,60,43$, and 71 . The latter four data are censored. They present nothing but the information that their mileages are larger than 62 , 60,43 , and 71 , respectively.

Example 2 (left-censored data)

The concentrations of arsenic in 8 solutions are tested by 8 chemical labs. Four have detected values (in ppm) of $0.1,0.03,0.25$, and 0.07 . The other four are reported below method limits of $0.2,0.04,0.1$ and 0.01 . The latter four data are censored. They present nothing but the information that their arsenic concentrations are less than 0.2 , $0.04,0.1$, and 0.01 , respectively. 
The fact used for probability reasoning for left-censored data is similar but in reverse to that for right-censored data. If the concentration of a sample is below $x$, then the concentration is also below all limits or values above $x$. Here is the detailed reasoning:

Call $n$ observations in descending order, $x_{0}>x_{1}>x_{2}>\ldots \ldots>x_{(n-1)}$, then

$$
\begin{gathered}
P\left(X<x_{0}\right)=1 \\
P\left(X<x_{1}\right)=P\left(X<x_{0}, X<x_{1}\right)=P\left(X<x_{1} \mid X<x_{0}\right) \cdot P\left(X<x_{0}\right) \\
P\left(X<x_{2}\right)=P\left(X<x_{1}, X<x_{2}\right)=P\left(X<x_{2} \mid X<x_{1}\right) \cdot P\left(X<x_{1}\right) \\
\cdots \cdots \\
P\left(X<x_{(n-1)}\right)=P\left(X<x_{(n-2)}, X<x_{(n-1)}\right)=P\left(X<x_{(n-1)} \mid X<x_{(n-2)}\right) \\
\cdot P\left(X<x_{(n-2)}\right)
\end{gathered}
$$$$
\text { Equation } 4.5
$$$$
\text { Equation 4.6 }
$$$$
\text { Equation } 4.7
$$

In these equations, $P\left(X<x_{i} \mid X<x_{(i-1)}\right)$ is a conditional probability, which represents the probability of $X$ less than $x_{i}$ given that $X$ is less than $x_{(i-1)}$. It is estimated using Equation 4.9. $P\left(X<x_{i}\right)$ is estimated using Equation 4.10, which is obtained by repetitive replacements of the above equations.

$$
\begin{aligned}
& \hat{P}_{i}=\left(\text { Number of samples below } x_{i}\right) /\left(\text { Number of samples below } x_{(i-1)}\right) \\
& \hat{P}\left(x_{i}\right)=\hat{P}\left(X<x_{i}\right)=\hat{P}_{1} \cdot \hat{P}_{2} \cdot \hat{P}_{3} \cdots \cdots \hat{P}_{i}=\prod_{0<j \leq i} \hat{P}_{j}
\end{aligned}
$$


So, the cumulative distribution function $P(x)=P(X<x)$ is estimated by Equations 4.11 through 4.14 , which are similar to the KME. It is an increasing step function that takes steps at observed data. It is demonstrated in Figure 4-1. The function estimates the probability less than a value. For instance, a cumulative probability for the concentration of arsenic is $\hat{P}(0.3)=\hat{P}(X<0.3)=0.49$ in Figure 4-1. It indicates that the population probability of the arsenic concentration less than 0.3 is estimated as $49 \%$, or equally, that the $49 \%$ quantile of population concentration is estimated as 0.3 .

$$
\begin{array}{lll}
\hat{P}(x)=\hat{P}(X<x)=1 & \text { for } x>x_{0}, & \text { Equation 4.11 } \\
\hat{P}(x)=\hat{P}(X<x)=\prod_{x(i) \geq x} \hat{P}_{i} & \text { for } x_{(n-1)} \leq x \leq x_{0}, & \text { Equation 4.12 } \\
\hat{P}(x)=\hat{P}(X<x)=0, & \text { for } x<x_{(n-1)}, x_{(n-1)} \text { is uncensored } & \text { Equation 4.13 } \\
\hat{P}(x)=\hat{P}(X<x) \in\left[0, \hat{P}\left(x_{(n-1)}\right)\right], \text { for } x<x_{(n-1)}, x_{(n-1)} \text { is censored } & \text { Equation 4.14 }
\end{array}
$$

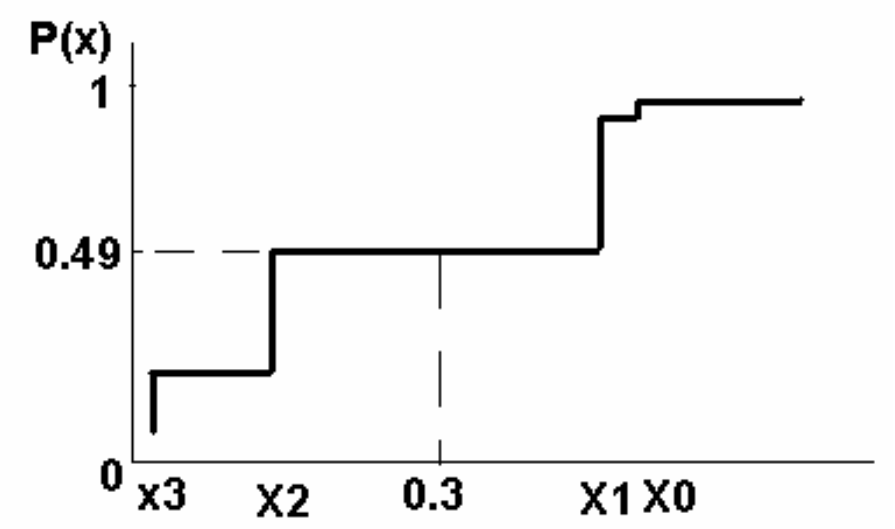

Figure 4-1: Demonstration of cumulative distribution function

The facts used for the reasoning above are the following: if the concentration of a sample is below $x$, then the concentration is also below all limits or values above $x$. It is inappropriate to use the fact that if the concentration of a sample is above $x$, then the concentration is also above all limits or values less than $x$, which is applicable to right- 
censored data. The main reason for rejecting the second fact is the nature of left censoring.

Call $n$ observations in descending order, $x_{0}>x_{1}>x_{2}>\ldots . .>x_{(n-1)}$, and parts of them are left-censored. At first, $P\left(X>x_{(n-1)}\right)$ cannot be estimated as 1 , for there are chances that parts of the left-censored data have real values less than $x_{(n-1)}$. Secondly, Equation 4.15 is inferred; however, its conditional probability $P\left(X>x_{(i+1)} \mid X>x_{i}\right)$ cannot be estimated. The number of observations known to be greater than $x_{i}$ and $x_{(i+1)}$ is uncountable. Some observations are censored at limits greater than $x_{i}$ and $x_{(i+1)}$; however, their real values are not meant to be greater than $x_{i}$ or $x_{(i+1)}$. Counting these observations produces bias to an extent that depends on the nature behind these censored observations.

$$
P\left(X>x_{i}\right)=P\left(X>x_{(i+1)}, X>x_{i}\right)=P\left(X>x_{(i+1)} \mid X>x_{i}\right) \cdot P\left(X>x_{i}\right) \quad \text { Equation 4.15 }
$$

Here is an example to further support the rejection. Consider an assumed arsenic concentration in leachates, Table $4-1$. To estimate cumulative probability $P(X<0.01)$, it is known that $P(X<0.01) \geq 10 \%$. Ninety percent of observations place censors at 0.5 , and the uncensored data are well below 0.5. These uncensored data may indicate by their own weight that the real values of those 0.5 -censored observations are well below 0.5 or within the range $0-0.01$. Hence, the probability of observations less than 0.01 is increased more than $10 \%$. If using the rejected fact, it is seriously biased to estimate $P(X>0.01)$ as $90 \%$ or $(1-10 \%)$, for those 90 censored observations may actually be partly or wholly 
less than 0.01 according to their censoring nature and 10 quantified observations. This possibility results in a decrease of $P(X>0.01)$.

Table 4-1: Demonstration data of arsenic in leachates

\begin{tabular}{l|l|l}
\hline Concentrations & Observations & Left censoring \\
\hline $0-0.01$ & 10 & No \\
\hline 0.5 & 90 & Yes \\
\hline
\end{tabular}

The arithmetic to calculate the CDF of left-censored data can be executed using a Microsoft-Excel spreadsheet, discussed in Section 4.3.3. However, relying on Excel to process left-censored data is time consuming and yields relatively sparse statistical information. Important statistical parameters, including standard error, confidence interval etc., are not provided. And sometimes it is more important to know the percentage of variable exceeding a limit in environmental assessment. For instance, given a pool of datasets and a corresponding threshold, the probability of the data exceeding the threshold is investigated. A professional statistical software package called Mini Tab can handle this concern with ease. Its program for left-censored data outputs survival probability $S(X)$ rather than cumulative probability $P(X)$.

Survival probability $S(X)$ represents the probability that a variable exceeds a limit, $S\left(x_{0}\right)=P\left(X>x_{0}\right)$ in Section 4.3.1. The relation between the factors of cumulative probability $P(X)$ and survival probability $S(X)$ is presented by Equation 4.16. Hence, the probability determination is simple. The survival function is a decreasing step function, as demonstrated in Figure 4-2. It only decreases at quantified observations. It is 
indicated that the probability of the variable greater than 0.3 is estimated as $32 \%$, or equally, that the $32 \%$ quantile of population concentration is estimated as 0.3 .

$$
P(X)+S(X)+p(X)=1
$$

Equation 4.16

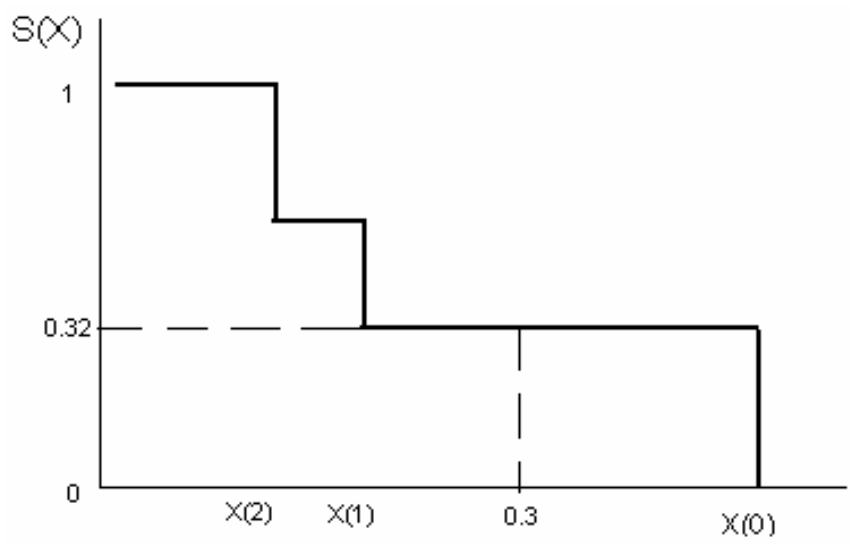

Figure 4-2: Demonstration of survival function

\subsubsection{Demonstration}

An actual example to demonstrate survival analysis is presented in this section. Two datasets addressing the concentrations of barium in the leachate extracted from aluminum-based excess foundry sands by TCLP and ASTM D 3987 protocols are illustrated. Raw data are presented in Table 4-2. The raw data were fed into the spreadsheet of MS-Excel and coded arithmetically according to the survival analysis discussed in Section 4.3.2. Results are presented in Table 4-3. Regarding the cumulative distribution of barium in the TCLP leachate, it is estimated that $100 \%$ of concentrations are below $10 \mathrm{ppm}, 94.4 \%$ of concentrations are below $1.8 \mathrm{ppm}$, the percentile corresponding to $1 \mathrm{ppm}$ is $88.9 \%$, and so on. 
Table 4-2: Barium in leachate by TCLP and ASTM D 3987

\begin{tabular}{|c|c|c|c|}
\hline \multicolumn{2}{|l|}{ TCLP } & \multicolumn{2}{|l|}{ ASTM D 3987} \\
\hline Test Result (ppm) & Censoring & Test Result (ppm) & Censoring \\
\hline 10 & Yes & 0.47 & No \\
\hline 10 & Yes & 0.33 & No \\
\hline 1.8 & $\mathrm{No}$ & 0.31 & $\mathrm{No}$ \\
\hline 1.62 & No & 0.18 & No \\
\hline 1 & Yes & 0.17 & No \\
\hline 0.7 & No & 0.14 & No \\
\hline 0.54 & No & 0.126 & No \\
\hline 0.39 & No & 0.089 & $\mathrm{No}$ \\
\hline 0.36 & No & 0.04 & No \\
\hline 0.33 & $\mathrm{No}$ & 0.033 & $\mathrm{No}$ \\
\hline 0.32 & No & 0.01 & Yes \\
\hline 0.31 & No & 0.01 & Yes \\
\hline 0.3 & Yes & 0.01 & Yes \\
\hline 0.2 & Yes & & \\
\hline 0.2 & Yes & & \\
\hline 0.2 & Yes & & \\
\hline 0.185 & $\mathrm{No}$ & & \\
\hline 0.115 & No & & \\
\hline 0.0783 & No & & \\
\hline 0.0251 & No & & \\
\hline
\end{tabular}


Table 4-3: Estimated cumulative function of barium in leachate by TCLP

\begin{tabular}{l|l|l|l|l|l}
\hline $\begin{array}{l}\text { Observations } \\
(\mathrm{ppm})\end{array}$ & Censoring & Numerator & Denominator & $P_{i}$ & $P(X)$ \\
\hline 10 & Yes & 20 & 20 & 1 & 1 \\
\hline 10 & Yes & 19 & 19 & 1 & 1 \\
\hline 1.8 & No & 17 & 18 & 0.944 & 0.944 \\
\hline 1.62 & No & 16 & 17 & 0.941 & 0.889 \\
\hline 1 & Yes & 16 & 16 & 1.000 & 0.889 \\
\hline 0.7 & No & 14 & 15 & 0.933 & 0.830 \\
\hline 0.54 & No & 13 & 14 & 0.929 & 0.770 \\
\hline 0.39 & No & 12 & 13 & 0.923 & 0.711 \\
\hline 0.36 & No & 11 & 12 & 0.917 & 0.652 \\
\hline 0.33 & No & 10 & 11 & 0.909 & 0.593 \\
\hline 0.32 & No & 9 & 10 & 0.900 & 0.533 \\
\hline 0.31 & No & 8 & 9 & 0.889 & 0.474 \\
\hline 0.3 & Yes & 8 & 8 & 1.000 & 0.474 \\
\hline 0.2 & Yes & 7 & 7 & 1.000 & 0.474 \\
\hline 0.2 & Yes & 6 & 6 & 1.000 & 0.474 \\
\hline 0.2 & Yes & 5 & 5 & 1.000 & 0.474 \\
\hline 0.185 & No & 3 & 4 & 0.750 & 0.356 \\
\hline 0.115 & No & 2 & 3 & 0.667 & 0.237 \\
\hline 0.0783 & No & 1 & 2 & 0.500 & 0.119 \\
\hline 0.0251 & No & 0 & 1 & 0.000 & 0.000 \\
\hline
\end{tabular}

MiniTab was used to evaluate the data in the calculation of survival function. The survival function for barium concentration in sand TCLP leachate is presented in Figure 4-3. From the survival function, it is interpreted that the maximum quantified observation is $1.8 \mathrm{ppm}$. Its minimum observation is close to zero, say $0+$. Ninety percent of actual concentrations are between 0 and $0.7 \mathrm{ppm}$. The median is around $0.3 \mathrm{ppm}$. In addition, any quantile corresponding to a concerned probability and any percentile corresponding to a concerned value are estimated by interpreting the X-axis or Y-axis of crossed point in the survival function. For instance, the probability of concentration above $1 \mathrm{ppm}$ is around 0.11 . The quantile for a survival probability of $5 \%$ is around 1.8 
$\mathrm{ppm}$. The probability of concentration less than or equal to $0.54 \mathrm{ppm}$ is estimated to be 0.8296 , which is obtained by subtracting 0.1704 from 1 .

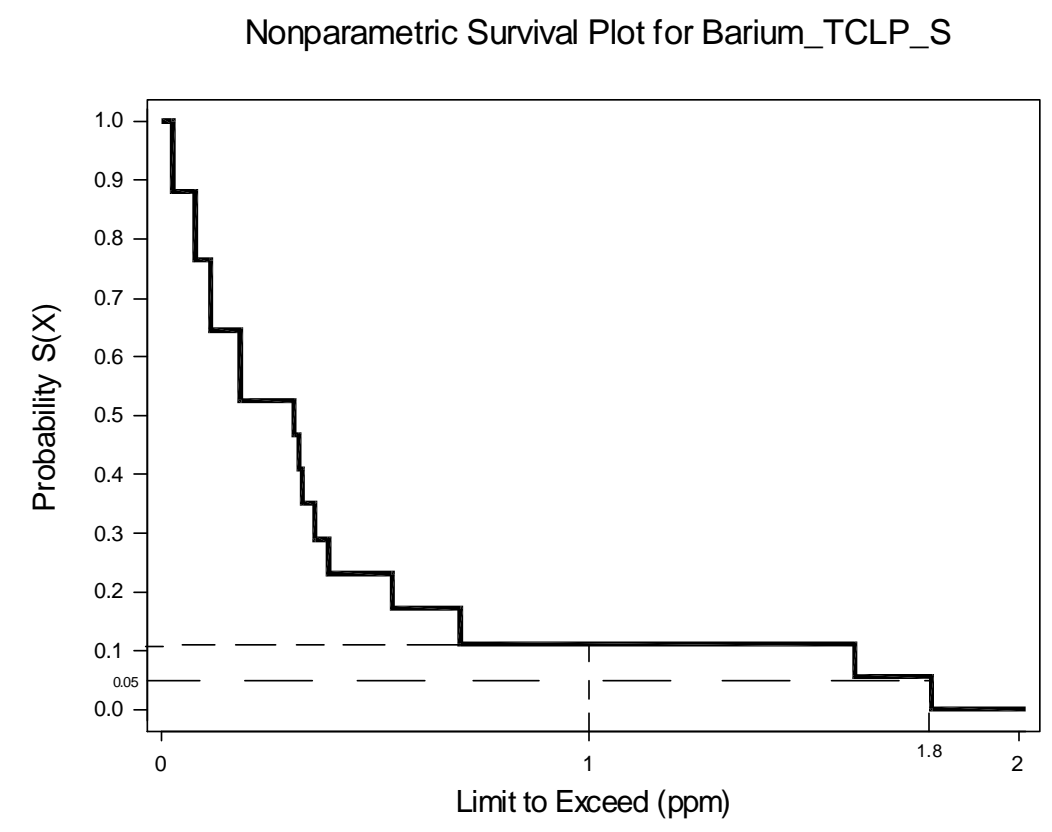

Figure 4-3: Survival function for barium in TCLP

Equation 4.16 is verified through the combination of survival function $S(X)$ in Figure 4-3 and cumulative function $P(X)$ in Table 4-3. For example, when $x=0.31$ ppm, $P(x<0.31)$ is equal to $0.474 ; p(x=0.31)=P(x<0.32)-P(x<0.31)=0.0593$; and $S(x>0.31)$ is equal to 0.4667 . The sum of three factors is 1 .

Using the same procedure as for TCLP, the survival function for barium in ASTM D 3987 is presented in Figure 4-4. 
Nonparametric Survival Plot for Barium_ASTM_S

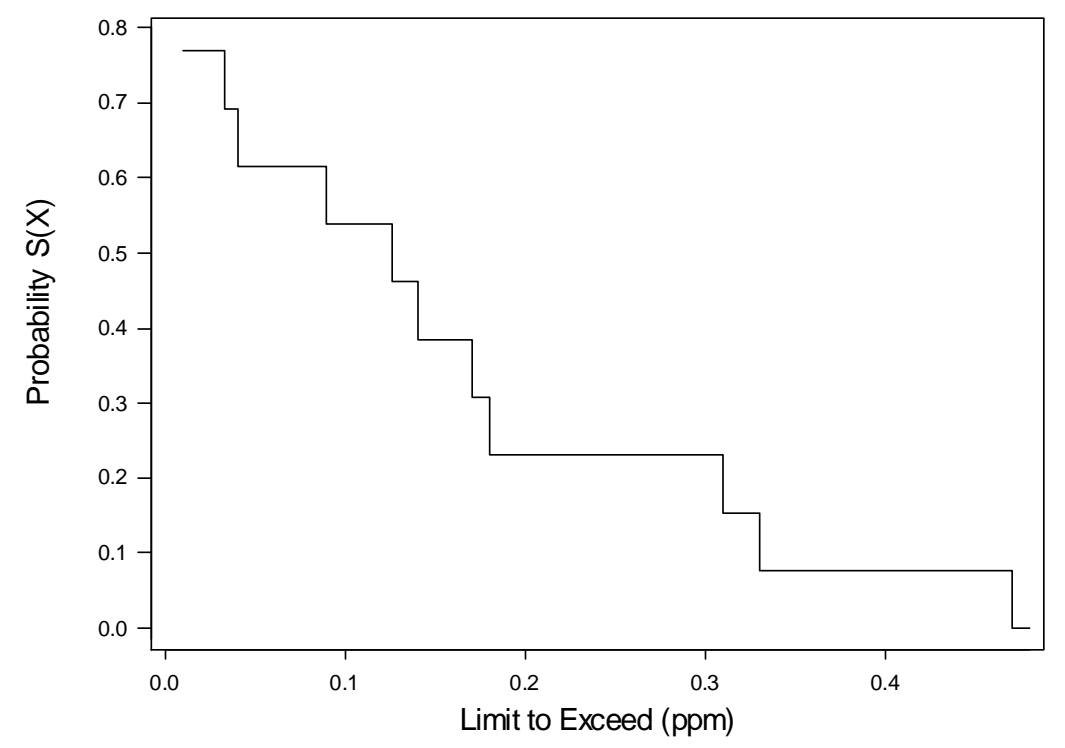

Figure 4-4: Survival function for barium in ASTM D 3987

\subsubsection{Mean Estimation}

The mean of a nonnegative random variable is equal to the area under the corresponding survival function [Kaplan and Meier 1958], which is proven in Appendix J.1. The survival functions of barium in TCLP and ASTM D 3987 leachate are presented in the same chart in Figure 4-5. It is indicated that the estimated mean of barium concentration in TCLP leachate is greater than the estimated mean of barium concentration in ASTM D 3987 leachate by the comparison of their areas under individual survival function. 


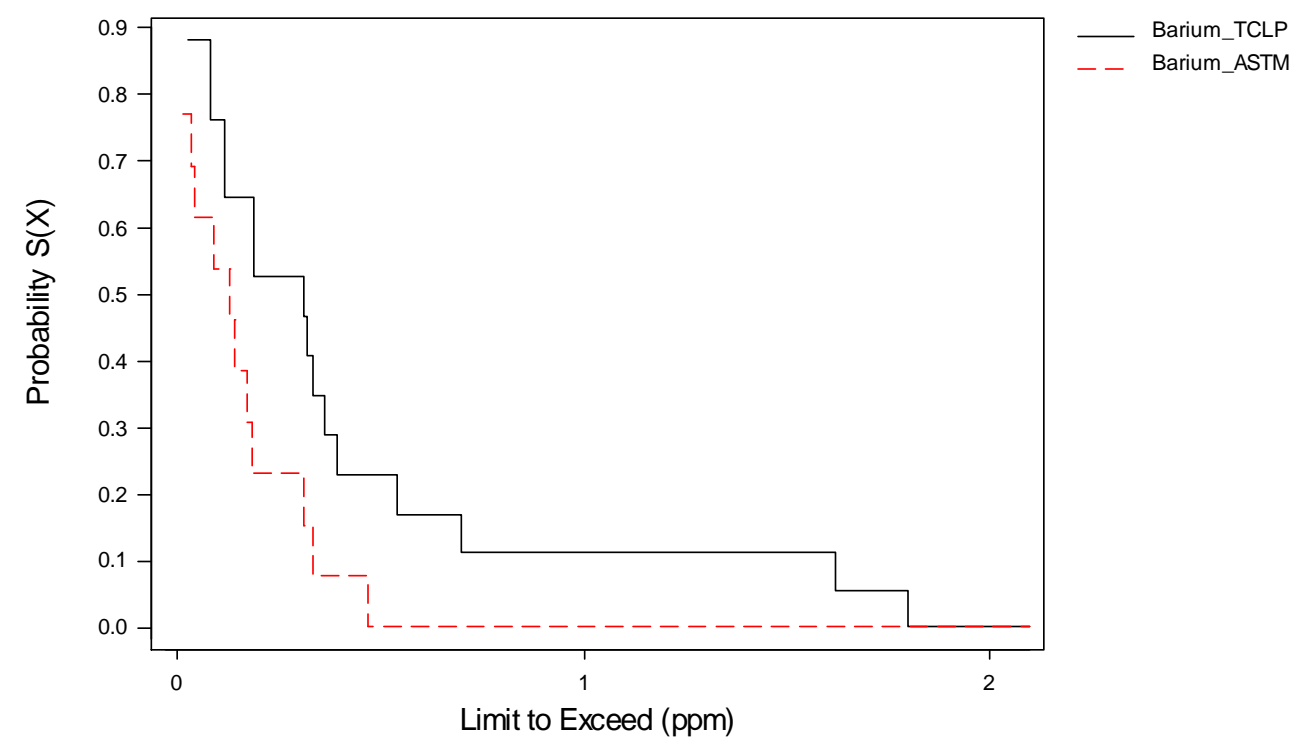

Figure 4-5: Comparison of survival functions for barium

The CDF is not determined at every value, as indicated in Equation 4.14. When $X$ is less than the minimum observation $x_{(n-1)}$ and $x_{(n-1)}$ is censored, its cumulative probability $\hat{P}(x)$ is expressed as $\hat{P}(X<x) \in\left[0, \hat{P}\left(x_{(n-1)}\right)\right]$. Kaplan and Meier regarded the results as unbiased if $\hat{P}(x)$ is "completed" by following $x<x(n-1)$ to $x(n-1)$ [Kaplan and Meier 1958]. Then, survival function is "completed" similarly. Hence, the estimated mean for left-censored is calculable.

By summing the area of partitioned rectangular slices, the estimated mean for barium concentration in TCLP leachate is:

$$
\begin{aligned}
\hat{\mu} & =(0.025-0)(1)+(0.0783-0.025)(0.88)+\ldots+(1.62-0.7)(0.111)+(1.8-1.62)(0.056) \\
& =0.410 \mathrm{ppm}
\end{aligned}
$$


The estimated mean for barium concentration in ASTM D 3987 leachate is:

$$
\begin{aligned}
\hat{\mu} & =(0.033-0)(0.77)+(0.04-0.033)(0.69)+\ldots+(0.33-0.31)(0.15)+(0.47-0.33)(0.077) \\
& =0.145 \mathrm{ppm}
\end{aligned}
$$

The confidence interval for estimated mean is determined by calculating the area under survival functions of $95 \%$ confidence level.

\subsubsection{Estimation of Censored Data}

Censored data impede the application of most conventional statistical methodologies such as regression analysis, analysis of variance (ANOVA), and multivariate analysis of variance (MAVOVA) because the data for these methods need to be complete, quantified or categorized. This issue can be resolved by estimating censored data using survival analysis. Furthermore, estimating censored data helps discover the nature of foundry waste streams, which is hidden by censoring. In this section, a simple method is presented to estimate the censored data.

In Figure 4-6, 30 observations (solid diamonds) are quantified and 10 observations (hollow squares) are censored at 5. It is intended to estimate the mean of those 10 censored observations based on the other 30 quantified observations. First, estimate the mean $m$ of the whole 40 observations using survival analysis (Section 4.3.4). Second, calculate arithmetically the summary $s$ of those 30 quantified observations. Finally, treat the estimated mean for those 10 censored observations as

unknown variable $\bar{x}$, and $\bar{x}$ is arithmetically determined by Equation 4.17. In this case, 
$m$ is estimated as 1.371 using survival analysis, and $s$ is equal to 43.750 . Thus, $\bar{x}$ is equal to 1.109 . It is discovered that $\bar{x}$ is equal to the estimated mean of quantified observations less than censoring limit 5. Hence, the mean estimation of censored data is uniquely determined by those quantified data less than its censoring limit.

$$
\frac{10 \bar{x}+s}{40}=m
$$

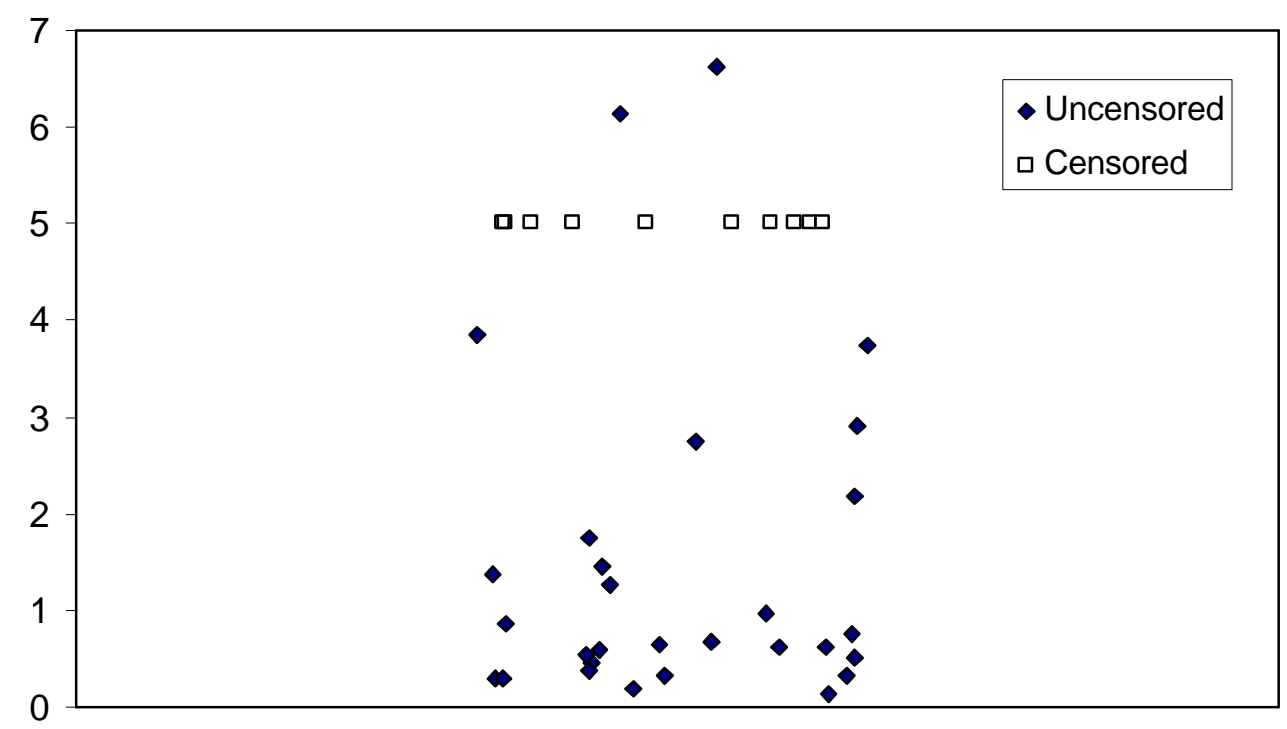

Figure 4-6: Demonstration of censored data and uncensored data

The finding that estimation of censored data is determined by quantified observations below its censoring is demonstrated in Figure 4-7. Figure 4-7 presents observations on 15 samples by three labs. Instruments of these three labs have different reporting limits, which results in different censoring fractions on observations. Lab1 has the greatest censoring fraction. Its results only present 3 quantified observations and 12 censored observations. Lab2 lowers its instrument reporting limit and presents 11 
quantified observations and 4 censored observations. Instrument of Lab3 has the capacity of quantifying every sample. In the estimation of censored observations from Lab2, quantified observations from Lab3 are referred to. Four censored observations from Lab2 are estimated by four quantified observations below Lab2's reporting limit.

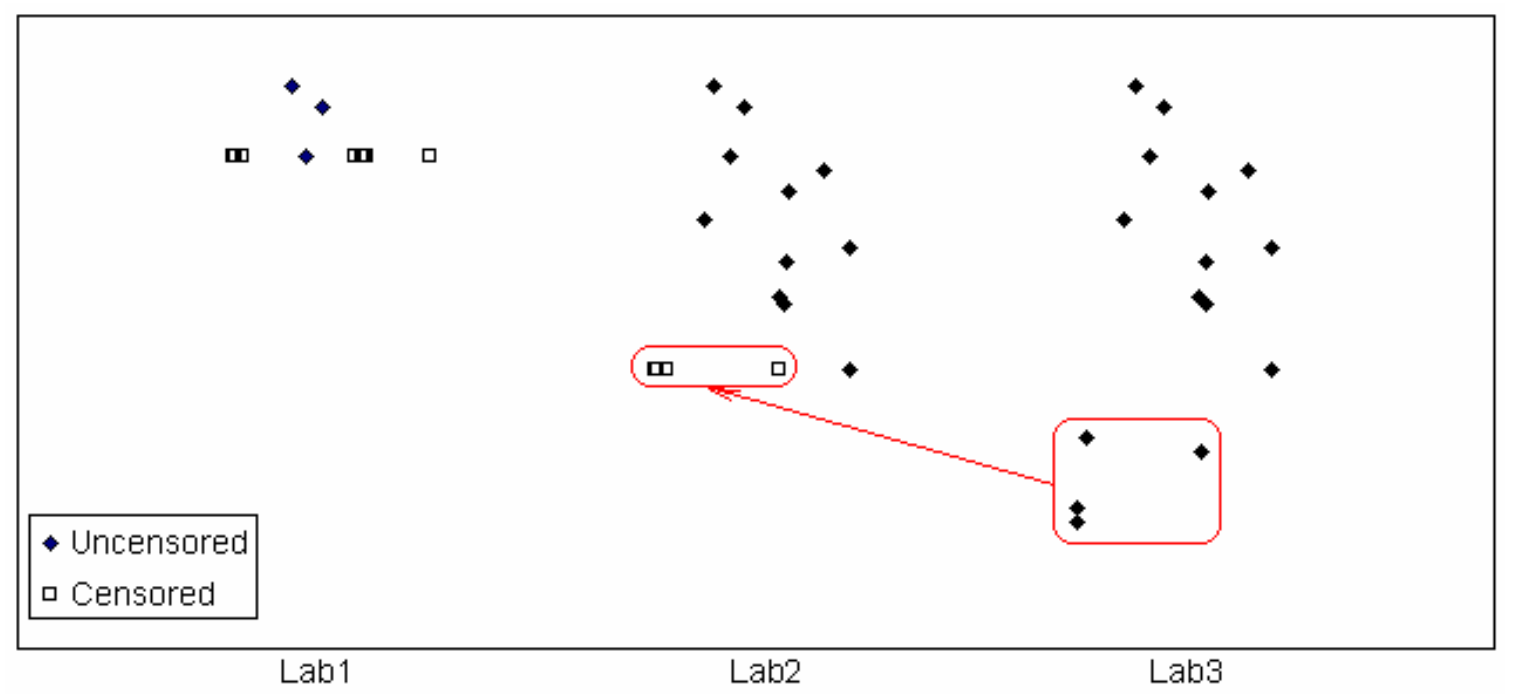

Figure 4-7: Demonstration of estimation of censored data

\subsubsection{Goodness-of-Fit Test (GOF)}

There are numerous distribution diagnosis methods available, but the methods applied to censored data are limited. Ignoring the nonquantifiable data can be misleading because it eliminates the lower tail of the distribution and can therefore falsely reject the hypothesis of normality or any distribution assumption. A method testing normality in censored samples is reviewed by Gibbons and Coleman [Gibbons and Coleman 2001]. The chi-square goodness-of fit (GOF) can test any distribution based on cumulative function and binned data only if the sample size is large enough [Conover 1999]. The 
Kolmogorov-Smirnov (KS) test is a nonparametric test that can be used to evaluate the fit of any hypothesized distribution [Conover 1999]. One of its limitations is that the parameters of the hypothesized distribution are assumed to be known, but actually partly unavailable. And the KS test is not valid if the parameters of the hypothesized distribution are estimated from the dataset [Gilbert 1987]. It is a method based on CDF. Another approach based on CDF is the Anderson-Darling (AD) test, which is modified from the KS test and focuses on the weight of tails [Stephens 1974]. It tests the distribution assumptions of normal, lognormal, extreme-value, Weibull, etc. This is done using probability plots for those distributions. Using the $\mathrm{AD}$ test, conventional distribution models can be compared to infer which distribution is the most appropriate.

Probability plots are based on a scheme that plots the quantified observations (or a transformation of the quantified observations) on the $\mathrm{x}$-axis versus the estimated cumulative probabilities $P$ on the y-axis. Transformations of both the $\mathrm{x}$ and y data are needed to ensure that the plotted $\mathrm{y}$ values are a linear function of the plotted $\mathrm{x}$ values. To help assess the linearity of the plotted data, a fitted line is also drawn on the probability plots. The probability plots for concentration of barium in TCLP leachate are presented in Figure 4-8. Four mostly used and approximated distribution models, including Weibull, lognormal, normal, and logistic, are fitted and compared. It is indicated that the lognormal-based distribution fits the data pool better than distributions of normal, Weibull, and logistic $(1.052<1.099<1.883<2.150)$. Its probability plot is closest to a straight line. However, MiniTab does not provide P-value for the GOF test. Hence, it is 
unknown whether the lognormal model fits the concentration of barium in TCLP leachate with confidence or not.

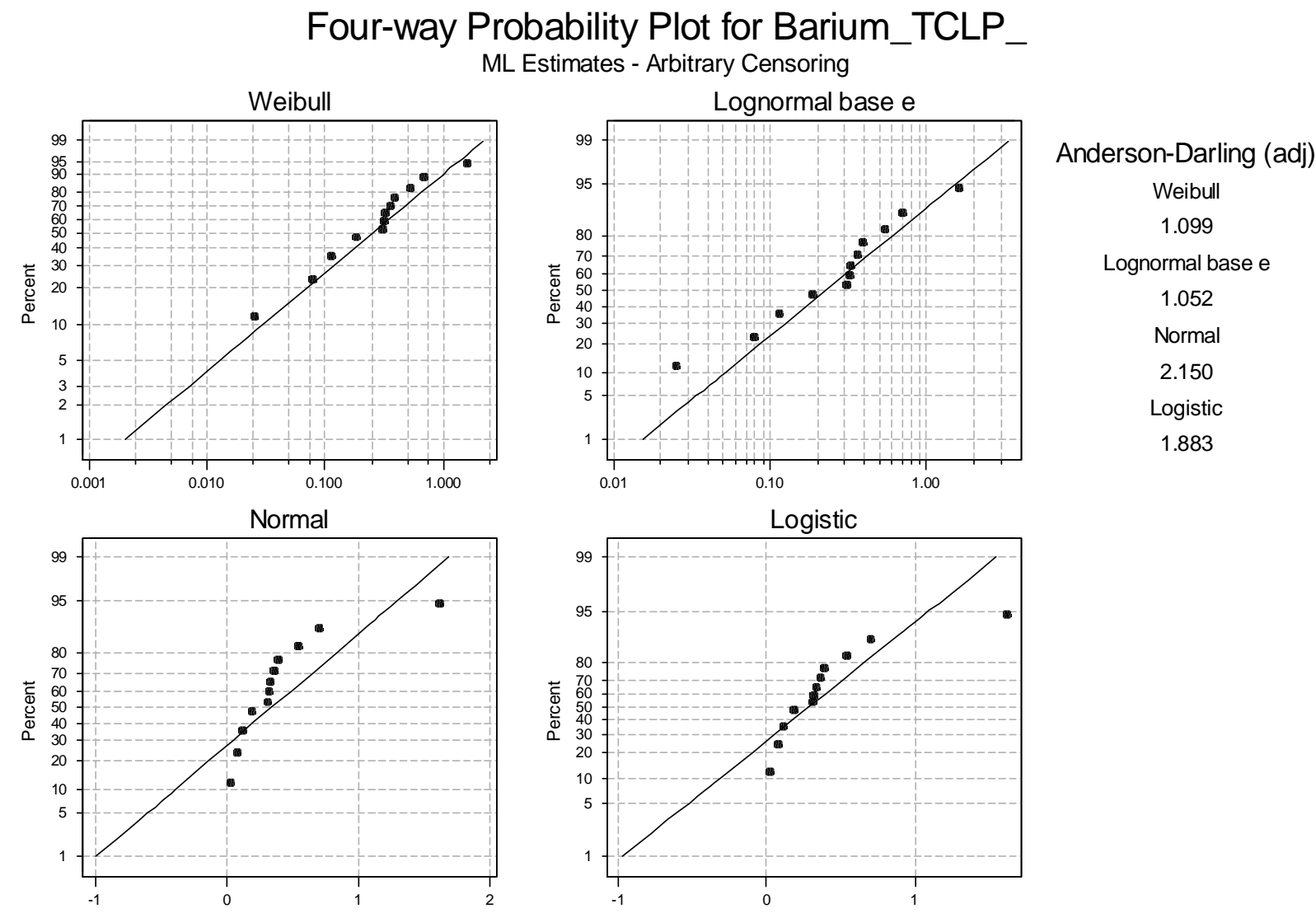

Figure 4-8: Comparison of fitness for barium in TCLP leachate

The same fitting procedures are conducted for concentration of barium in ASTM D 3987 leachate. The probability plots are presented in Figure 4-9. It is discovered that Weibull distribution most closely fits the data $(1.668<1.803<1.854<1.873)$. Hence, the best fitting model is not always lognormal for environmental monitoring data. It is changeable, which depends essentially on the distribution nature of the data pool. In practice, the distributions of environmental data are rarely if ever known [Hesel and 
Hirsch 1992]. To assume any distribution model without diagnosing its fitness may result in less sound conclusions.

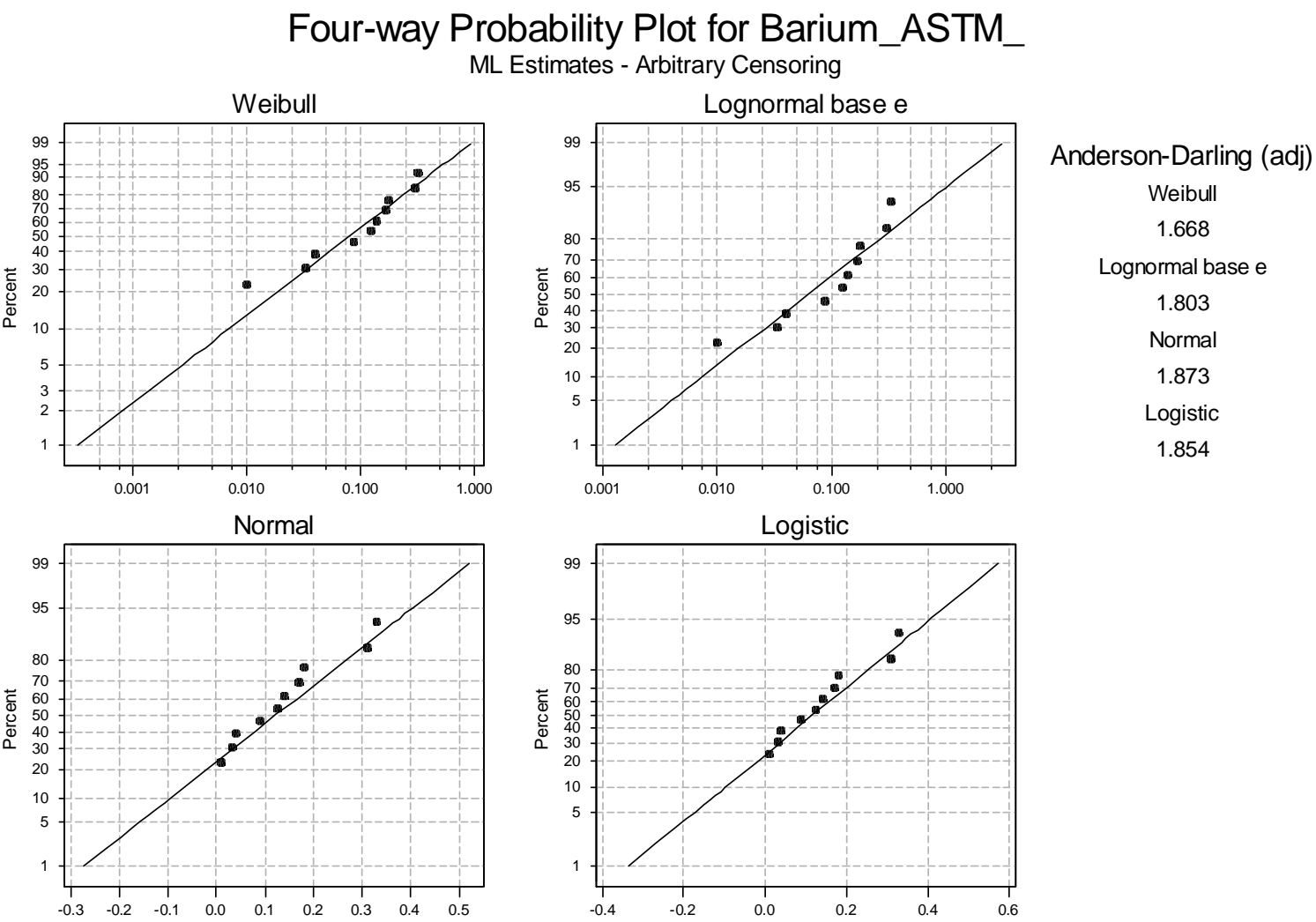

Figure 4-9: Comparison of fitness for barium in ASTM D 3987 leachate

\subsubsection{Factor Effect}

Although the comparisons of critical points, including estimated means or median, of two independent sample groups are different, it is not determinate to conclude that two groups are significantly different. A further statistical procedure is performed to test whether these two sample groups are from two different populations; whether the two 
distribution functions associated with two populations are identical; or whether the factor effect between the two groups of data is significant.

The two-sample hypotheses can be tested using KS test and Cramer-von Mises (CM) test based on the survival function [Conover 1999]. But they are not figured out for censored data. And there is no commercial statistical software package handling a twosample test of censored data. Alternatively, the testing procedures can be fulfilled by Microsoft Excel spreadsheet coding using the methodology of the KS or CM test.

In this research, the KS test is employed to compare data pools and to evaluate factor effect, for $\mathrm{KS}$ is more related to $\mathrm{CDF}$ and survival function. In the applications of KS test, two CDFs of two data groups are presented in a chart. The maximum vertical distance between two CDFs is regarded as the test statistic for the two-sided test. Although the KS test was developed for CDF, the maximum vertical distance between two survival functions is identical to the maximum vertical distance between corresponding CDFs. Hence, the KS test is applied directly to survival functions. Generally, test confidence level $\alpha$ is selected as 0.05 . If the test statistic is greater than the 1- $\alpha$ quantile for a two-sided test, this goes against the hypothesis that two data groups tend to present identical results or that the factor is not significant at $\alpha$ level. If the test statistic is less than the 1- $\alpha$ quantile for a two-sided test, it is concluded that there is no significant evidence against the hypothesis, and that hypothesis is accepted. Another test used is a one-sided test that employs the maximum one-way vertical 
distance as the test statistic. Its testing criteria are identical to the two-sided test. However, this test evaluates whether one data pool is greater than another.

The test procedure on two data groups is demonstrated. One data group is the concentration of barium in TCLP leachate, and the other data group is the concentration of barium in ASTM D 3987 leachate, presented in Table 4-3. Their survival functions are presented in Figure 4-5. The spreadsheet calculation indicates that the maximum vertical distance between two survival functions is 0.3128. Referring to "Table of Quantiles of the Smirnov Test Statistic for Two Samples of Different Size” [Conover 1999], the 95\% quantile for the two-sided test is $7 / 15$, which is greater than the test statistic 0.3128 . By interpolation, the $p$ value is estimated to be larger than 0.20 . A $p$ value close to zero signals that null hypothesis is false, and typically that a difference is very likely to exist. Large $p$ values closer to 1 imply that there is no detectable difference for the sample groups. A $p$ value of 0.05 is a typical threshold used in industry to evaluate the null hypothesis. Therefore, hypothesis $\mathrm{H}_{0}$ that two data groups have an identical distribution function or concentration is accepted at the 0.05 level. Leaching protocols of TCLP and ASTM D 3987 are not significant factors differentiating concentrations of barium in leachates. 


\section{Chapter 5}

\section{CHARACTERIZATION ANALYSES AND CONCLUSIONS}

This chapter concentrates on the characterization of foundry sand through both bulk analysis and leaching analysis. The main technique is the survival function, which is discussed in Chapter 4. Major topics includes survival mean estimation, sample group comparison, and environmental impact assessment.

\subsection{Plan of Analyses}

Using the survival function for left-censored data (discussed in Section 4.3.2, demonstrated in Section 4.3.3) and mean estimation for left-censored data (discussed in Section 4.3.4), the survival probability function and estimated mean for general chemical parameters, inorganic elements, and organic compounds are presented in Sections F.1 through F.4 in Appendix F. The main factors considered in the factorial design include leaching protocol and metal cast. The effect of factors, or statistical comparison of the cumulative distribution of elements using survival technique, is evaluated using the methodology discussed in Section 4.3.7. Parameters to be analyzed include all chemicals in bulk chemical analysis and leachate characterizing analysis. It covers up to 27 general chemical parameters, up to 24 metallic elements and up to 55 organic compounds. 
By the presentation of survival probability plot and estimated mean, the characterization of foundry sand is delineated. First, the presentation addresses all elements and compounds regarding the chemical and environmental characteristics of foundry sands. These parameters not only cover those parameters specified by federal and state regulation; they also span the environmental concerns encountered in any beneficial reuse application of foundry by-product. Second, the barrier to looking into the distribution of each parameter is eliminated. The censoring issue regarding almost all parameters is addressed by the introduction of survival theory. The characterization of excess foundry sand is addressed with statistical confidence. Distribution for an individual parameter is more easily interpreted and located, including minimum, maximum, mean, and any percentile. A reliable and hands-on methodology addressing the censored data is set up. Finally, the factors of leaching protocol and metal cast are considered. Two to four survival plots with one factor effect are presented in a chart. Statistical tests are performed to evaluate the significance of the factor. For instance, it is estimated whether TCLP leaches out more arsenic from sand than SPLP or ASTM D 3987. This kind of hypothesis test is a more general form of location alternative than the statement that samples differ only by a critical point (mean or median) [Conover 1999]. It can be solved by the KS test.

The factor tests are diverse. In evaluation of leaching characterization, two factors are considered, leaching protocol (3 treatments) and metal cast (4 treatments), and up to $12(3 \times 4=12)$ treatments exist. Their pairwise combination is up to $C_{12}^{2}=66$. Thus, up to 66 comparisons are targeted to be evaluated on up to 76 variables. Not all 66 comparisons 
are required in this research. Comparison interest is focused on comparisons of leaching protocols and metals cast. The list of hypothesis tests for selected pairwise factors is presented in Tables 5-1, 5-2, and 5-3.

Table 5-1: List of hypothesis tests per metal cast for as-received foundry sand

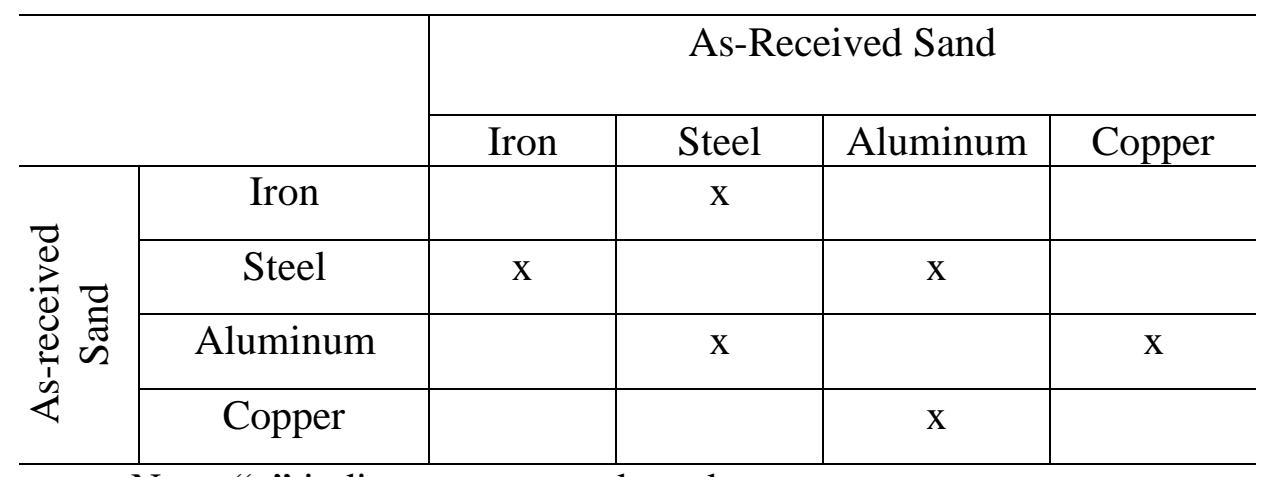

Note: "x" indicates tests conducted.

Table 5-2: List of hypothesis tests per leaching protocol for sand leachates

\begin{tabular}{|c|c|c|c|c|}
\hline & \multicolumn{3}{|c|}{ Sand Leachate } \\
\hline & & TCLP & SPLP & ASTM D3987 \\
\hline \multirow{3}{*}{ 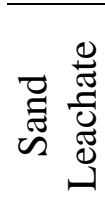 } & TCLP & $\bar{A}$ & $\mathrm{x}$ & \\
\hline & SPLP & $\mathrm{x}$ & & $\mathrm{x}$ \\
\hline & ASTM D3987 & & $\mathrm{x}$ & \\
\hline
\end{tabular}


Table 5-3: List of hypothesis tests per metal cast for TCLP sand leachate

\begin{tabular}{|c|c|c|c|c|c|}
\hline & & \multicolumn{4}{|c|}{ TCLP Sand Leachate } \\
\hline & & Iron & Steel & Aluminum & Copper \\
\hline \multirow{4}{*}{ 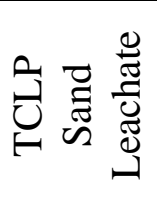 } & Iron & & $\mathrm{X}$ & & \\
\hline & Steel & $\mathrm{x}$ & & $X$ & \\
\hline & Aluminum & & $\mathrm{x}$ & & $\mathrm{x}$ \\
\hline & Copper & & & $X$ & \\
\hline
\end{tabular}

Note: " $\mathrm{x}$ " indicates tests conducted.

\subsection{Estimated Mean for As-Received Sand}

The estimated means for the bulk chemical analysis of sand are presented in Table 5-4 (general chemical parameters), Table 5-5 (metallic elements) and Table 5-6 (organic compounds). Further characterization in distribution shape and percentile for individual parameter scan be referred to corresponding charts grouped in Figure F-1 (general chemical parameters) in Appendix F.1.1, Figure F-2 (metallic elements) in Appendix F.1.2, and Figure F-3 (organic compounds) in Appendix F.1.3. 
Table 5-4: Estimated mean for bulk chemical analysis of as-received sand (general chemical parameters)

\begin{tabular}{l|l}
\hline Parameters & $\begin{array}{l}\text { Estimated Mean (Unit: ppm, expect } \\
\text { LOI, moisture, } \mathrm{pH})\end{array}$ \\
\hline Silica & 77.2 \\
\hline LOI, \% & 2.54 \\
\hline Moisture, \% & 1.27 \\
\hline Oil and grease & 1744 \\
\hline Total petroleum hydrocarbon & 223.52 \\
\hline Formalhyde & 9.21 \\
\hline Phenol & 21.45 \\
\hline pH & 8.11 \\
\hline Total reactive sulfide & 37.02 \\
\hline Total volatile residual & 20995 \\
\hline Total volatile solid & 1.90 \\
\hline
\end{tabular}


Table 5-5: Estimated mean for bulk chemical analysis of as-received sand (metallic elements)

\begin{tabular}{l|l}
\hline Parameters & Estimated Mean $(\mathrm{ppm})$ \\
\hline Aluminum & 3326 \\
\hline Antimony & 4.34 \\
\hline Arsenic & 0.86 \\
\hline Barium & 14.95 \\
\hline Beryllium & 0.08 \\
\hline Boron & 38.53 \\
\hline Cadmium & 0.22 \\
\hline Calcium & 3309 \\
\hline Chromium & 114.03 \\
\hline Hexavalent chromium & 0.08 \\
\hline Copper & 103.60 \\
\hline Iron & 10911 \\
\hline Lead & 15.72 \\
\hline Magnesium & 1881 \\
\hline Manganese & 257 \\
\hline Mercury & 0.04 \\
\hline Molybdenum & 38.84 \\
\hline Nickel & 107.94 \\
\hline Selenium & 0.64 \\
\hline Silicon & 1772 \\
\hline Silver & 0.47 \\
\hline Thallium & 0.43 \\
\hline Zinc & 102.48
\end{tabular}


Table 5-6: Estimated mean for bulk chemical analysis of as-received sand (organic compounds)

\begin{tabular}{l|l|l|l|l}
\hline Parameter & Acenaphthylene & Anthracene & Benzene & $\begin{array}{l}\text { Benzo- } \\
\text { a)anthracene }\end{array}$ \\
\hline $\begin{array}{l}\text { Estimated } \\
\text { Mean }(\mathrm{ppm})\end{array}$ & 0.10 & 0.37 & 0.12 & 0.01 \\
\hline \hline Parameter & $\begin{array}{l}\text { Benzo- } \\
\text { (b)fluornathene }\end{array}$ & $\begin{array}{l}\text { Benzo- } \\
\text { (k)fluornathene }\end{array}$ & Chrysene & o-Cresol \\
\hline $\begin{array}{l}\text { Estimated } \\
\text { Mean (ppm) }\end{array}$ & 0.01 & 0.002 & 0.027 & 2.722 \\
\hline \hline Parameter & $\mathrm{m} \_$p-Cresol & $\begin{array}{l}2,4- \\
\text { Dimethylphenol }\end{array}$ & Ethylbenzene & Fluoranthene \\
\hline $\begin{array}{l}\text { Estimated } \\
\text { Mean (ppm) }\end{array}$ & 1.119 & 1.194 & 0.046 & 0.306 \\
\hline \hline Parameter & Fluorene & $\begin{array}{l}1-\text { Methyl } \\
\text { naphthalene }\end{array}$ & $\begin{array}{l}2-\text {-Methyl } \\
\text { naphthalene }\end{array}$ & Naphthalene \\
\hline $\begin{array}{l}\text { Estimated } \\
\text { Mean (ppm) }\end{array}$ & 0.037 & 0.328 & 0.406 & 0.435 \\
\hline \hline Parameter & Phenanthene & Pyrene & Styrene & Toluene \\
\hline $\begin{array}{l}\text { Estimated } \\
\text { Mean (ppm) }\end{array}$ & 1.604 & 0.047 & 7.796 & 0.045 \\
\hline Parameter & Xylene & --- & --- & --- \\
\hline $\begin{array}{l}\text { Estimated } \\
\text { Mean (ppm) }\end{array}$ & 0.061 & --- & --- & -- \\
\hline
\end{tabular}

\subsection{Factor of Metal Cast for As-received Sand}

The estimated mean and factor effect of metal cast for metallic elements from asreceived sand are presented in Table 5-7. Major considered comparisons include "concentrations of elements in sand from iron-based facility versus concentrations of elements in sand from steel-based facility"; "concentrations of elements in sand from a steel-based facility versus concentrations of elements in sand from an aluminum-based facility"; and "concentrations of elements in sand from an aluminum-based facility versus concentrations of elements in sand from a copper-based facility." Further comparison and 
characterization in distribution shape and percentile for individual parameters can be referred to corresponding charts grouped in Figure F-4 in Appendix F.2.1.

Based on the statistical comparison of the cumulative distribution of elements using survival technique presented in Table 5-7, there is no significant evidence against the hypotheses that sand from iron-based facilities and sand from steel-based facilities tend to present similar amounts of aluminum, arsenic, barium, boron, calcium, chromium, copper, iron, lead, manganese, and nickel. Sand from iron-based facilities tends to present more zinc than sand from steel-based facilities. There is no significant evidence against the hypotheses that sand from steel-based facilities and sand from aluminum-based facilities tend to present similar amounts of aluminum, arsenic, barium, calcium, chromium, and copper. Sand from steel-based facilities tends to present more iron, manganese, and nickel and less zinc than sand from aluminum-based facilities. There is no significant evidence against the hypotheses that sand from aluminum-based facilities and sand from copper-based facilities tend to present similar amounts of aluminum, arsenic, barium, calcium, chromium, iron, and manganese. Sand from aluminum-based facilities tends to present less copper, lead, nickel, and zinc than sand from copper-based facilities. Sands from iron-based facilities and steel-based facilities tend to present less copper and zinc than sand from copper-based facilities. Sand from iron-based facilities tends to present less lead than sand from copper-based facilities. There is no significant evidence against the hypotheses that sand from steel-based facilities and sand from copper-based facilities tend to present similar amounts of lead. 
Table 5-7: Estimated mean and factor effect for as-received sand (metallic elements)

\begin{tabular}{|c|c|c|c|c|c|}
\hline \multirow[t]{2}{*}{ Parameter } & $\begin{array}{l}\text { Iron- } \\
\text { based }\end{array}$ & $\begin{array}{l}\text { Steel- } \\
\text { based }\end{array}$ & $\begin{array}{l}\text { Aluminum- } \\
\text { based }\end{array}$ & $\begin{array}{l}\text { Copper- } \\
\text { based }\end{array}$ & \multirow[t]{2}{*}{$\begin{array}{l}\text { Statistical comparison of the cumulative distribution of elements using } \\
\text { survival technique }\end{array}$} \\
\hline & \multicolumn{4}{|c|}{ Unit: ppm } & \\
\hline Aluminum & 1327 & 1576 & 12931 & 1190 & $\begin{array}{l}\text { "Iron=Steel", } p>0.20 ; \text { "Steel=Aluminum", } p>0.20 \\
\text { "Aluminum=Copper", } p>0.15 ;\end{array}$ \\
\hline Arsenic & 0.76 & 6 & 0.34 & 0.39 & $\begin{array}{l}\text { "Iron=Steel", } p>0.20 ; \text { "Steel=Aluminum", } p>0.20 \\
\text { "Aluminum=Copper", } p>0.20\end{array}$ \\
\hline Barium & 24.31 & 7.77 & 6.53 & 3.97 & $\begin{array}{l}\text { "Iron=Steel", } p=0.17 ; \text { "Steel=Aluminum", } p=0.2+; \\
\text { "Aluminum=Copper", } p=0.14 ;\end{array}$ \\
\hline Boron & 9.86 & 276.2 & & & "Iron=Steel", $p>0.20$ \\
\hline Calcium & 4842 & 1366 & 927 & 2481 & $\begin{array}{l}\text { "Iron=Steel", } p>0.20 ; \text { "Steel=Aluminum", } p>0.20 \\
\text { "Aluminum=Copper", } p>0.20\end{array}$ \\
\hline Chromium & 16.23 & 1664.1 & 4.63 & 3.21 & $\begin{array}{l}\text { "Iron=Steel", } p>0.20 ; \text { "Steel=Aluminum", } p=0.2+\text {; } \\
\text { "Aluminum=Copper", } p>0.20 \text {; }\end{array}$ \\
\hline Copper & 30.32 & 404.73 & 17.68 & 653.2 & $\begin{array}{l}\text { "Iron=Steel", } p>0.20 \text {; "Steel=Aluminum", } p=0.20 ; \\
\text { "Aluminum<Copper", } p=0.008 \text {; "Iron }<\text { Copper", } p=0.005 ; \\
\text { "Steel }<\text { Copper", } p=0.01+;\end{array}$ \\
\hline Iron & 11163 & 60819 & 1898 & 1986 & $\begin{array}{l}\text { "Iron=Steel", } p>0.20 ; \text { "Steel }>\text { Aluminum", } p=0.02 \text {; } \\
\text { "Aluminum=Copper", } p>0.20 \text {; }\end{array}$ \\
\hline Lead & 19.81 & 8.42 & 3.62 & 88.6 & $\begin{array}{l}\text { "Iron=Steel", } p>0.20 ; \text { " "Steel=Aluminum", } p=0.20 \\
\text { "Aluminum<Copper", } p=0.008 \text {; "Iron }<\text { Copper", } p=0.008 ; \\
\text { "Steel=Copper", } p>0.20\end{array}$ \\
\hline Manganese & 483 & 523 & 26.66 & 14.33 & $\begin{array}{l}\text { "Iron=Steel", } p>0.20 ; \text { "Steel }>\text { Aluminum", } p=0.02 \\
\text { "Aluminum=Copper", } p=0.2+\end{array}$ \\
\hline Nickel & 6.85 & 1664.6 & 44.17 & 9.86 & $\begin{array}{l}\text { "Iron=Steel", } p=0.20 ; \text {;"Steel>Aluminum", } p=0.04 \text {; } \\
\text { "Aluminum<Copper", } p=0.04\end{array}$ \\
\hline Zinc & 131 & 64.1 & 31.6 & 691.2 & $\begin{array}{l}\text { "Iron }>\text { Steel", } p<0.05 \text {; "Steel<Aluminum", } p=0.04 ; \\
\text { "Aluminum<Copper", } p=0.008 \text {; "Iron }<\text { Copper", } p<0.005 ; \\
\text { "Steel }<\text { Copper", } p=0.01+;\end{array}$ \\
\hline
\end{tabular}

Note: A $p$ value close to zero, or $p<0.05$, signals a significant factor effect. 
It is inferred that metal cast in the facilities is not a significant factor regarding most metallic elements in as-received excess foundry sand. The exceptions are lead, copper, and zinc, which are frequently of greater composition in as-received sand from copper-based facilities than from other alloy facilities. The greater composition for these three metallic elements is possibly caused by the relatively substantial addition of lead, copper, and zinc (up to 30\%) as alloys in copper-based facilities.

\subsection{Estimated Mean for Sand Leachate per Leaching Protocol}

The estimated mean for parameters in sand leachate is presented in Table 5-8 (general chemical parameters), Table 5-9 (metallic elements), and Table 5-10 (organic compounds). Hypothesis tests in regard to leaching protocol on metallic elements are presented in Table 5-9. Further characterization in distribution shape and percentile for individual parameters can be referred to corresponding charts grouped in Figure F-5 (general chemical parameters) in Appendix F.3.1, Figure F-6 (metallic elements) in Appendix F.3.2, and Figure F-7 (organic compounds) in Appendix F.3.3.

The estimated mean and leaching protocol effect for metallic elements in sand leachates are presented in Table 5-9. Considered comparisons include "concentrations of elements in TCLP leachate versus concentrations of elements in SPLP leachate"; "concentrations of elements in SPLP leachate versus concentrations of elements in ASTM D 3987 leachate"; and "concentrations of elements in TCLP leachate versus concentrations of elements in ASTM D 3987 leachate." 
Table 5-8: Estimated mean for sand leachate per leaching protocol (general chemical parameters)

\begin{tabular}{l|l|l}
\hline \multirow{2}{*}{ Parameter } & TCLP & ASTM \\
\cline { 2 - 3 } & \multicolumn{2}{|c}{ Unit: $\mathrm{ppm}$} \\
\hline Acidity & \multicolumn{2}{|c}{11.8} \\
\hline Alkalinity & & 50 \\
\hline Ammonia & & 4.511 \\
\hline Chemical oxygen demand & 439 \\
\hline Chloride & & 8.003 \\
\hline Cyanide & & 0.003 \\
\hline Fluoride & & 0.734 \\
\hline Formalhyde & 0.332 & \\
\hline Nitrate & & 1.266 \\
\hline Nitrite & & 0.021 \\
\hline Oil and grease & & 14.700 \\
\hline Phenol & 0.154 & 0.992 \\
\hline Sulfate & & 56.596 \\
\hline Total dissolved solid & & 347 \\
\hline Total oxygen halogen & & 6.221 \\
\hline Total petroleum hydrocarbon & & 2.047 \\
\hline
\end{tabular}


Table 5-9: Estimated mean and factor effect for sand leachate per leaching protocol (metallic elements)

\begin{tabular}{|c|c|c|c|c|}
\hline \multirow[t]{2}{*}{ Parameter } & TCLP & SPLP & ASTM D 3987 & \multirow{2}{*}{$\begin{array}{l}\text { Statistical comparison of the cumulative distribution of elements using } \\
\text { survival technique }\end{array}$} \\
\hline & \multicolumn{3}{|c|}{ Unit: ppm } & \\
\hline Aluminum & 1.785 & 2.698 & 2.094 & “TCLP $\neq$ SPLP”, $p<0.01 ;$ "SPLP=ASTM", $p=0.1-;$ \\
\hline Antimony & & 0.083 & & \\
\hline Arsenic & 0.031 & 0.001 & 0.003 & "TCLP>SPLP", $p=0.025$; "SPLP<ASTM", $p<0.005$; \\
\hline Barium & 0.639 & 0.388 & 0.173 & “TCLP>SPLP”, $p<0.005$; "SPLP>ASTM", $p<0.005 ;$ \\
\hline Boron & & 0.236 & & \\
\hline Cadmium & 0.004 & --- & 0.0003 & "TCLP=ASTM", $p>0.20$ \\
\hline Chromium & 0.042 & 0.002 & 0.007 & "TCLP=SPLP", $p>0.20$; "SPLP=ASTM", $p>0.20$; \\
\hline $\begin{array}{l}\text { Hexavalent } \\
\text { chromium }\end{array}$ & & & 0.008 & \\
\hline Copper & 0.521 & 0.061 & 0.087 & “TCLP=SPLP”, $p>0.20$; “SPLP=ASTM", $p>0.20$ \\
\hline Iron & 61.78 & 6.095 & 1.245 & “TCLP>SPLP”, $p<0.025$; “SPLP>ASTM”, $p<0.005$ \\
\hline Lead & 0.222 & 0.009 & 0.008 & “TCLP>SPLP”, $p=0.01 ;$ "SPLP=ASTM", $p>0.20$ \\
\hline Manganese & 1.009 & 0.215 & 0.061 & “TCLP>SPLP”, $p<0.005$; “SPLP=ASTM", $p>0.20$ \\
\hline Mercury & 0.0002 & --- & 0.0002 & “TCLP<ASTM", $p=0.005$ \\
\hline Molybdenum & & 0.233 & & \\
\hline Nickel & 0.183 & 0.029 & 0.006 & “TCLP>SPLP”, $p<0.005$; “SPLP=ASTM", $p>0.20$ \\
\hline Selenium & 0.041 & --- & 0.002 & "TCLP>ASTM", $p=0.02$ \\
\hline Silver & 0.004 & & & \\
\hline Sodium & & & 15.043 & \\
\hline Vanadium & & & 0.003 & \\
\hline Zinc & 1.006 & 0.264 & 0.177 & “TCLP>SPLP”, $p<0.005$; “SPLP>ASTM", $p<0.025$ \\
\hline
\end{tabular}

Note: A $p$ value close to zero, or $p<0.05$, signals a significant factor effect. 
Table 5-10: Estimated mean for TCLP sand leachate (organic compounds)

\begin{tabular}{l|l|l|l|l}
\hline Parameter & Cresol_m & Cresol_O & Acetone & Benzene \\
\hline $\begin{array}{l}\text { Estimated } \\
\text { Mean }(\mathrm{ppm})\end{array}$ & 22.33 & 46.40 & 265.5 & 1.46 \\
\hline \hline Parameter & $\begin{array}{l}\text { 2_4- } \\
\text { Dimethylphenol }\end{array}$ & $\begin{array}{l}\text { Ethyl } \\
\text { benzene }\end{array}$ & Naphthalene & Phenanthrene1 \\
\hline $\begin{array}{l}\text { Estimated } \\
\text { Mean (ppm) }\end{array}$ & 30.59 & 5.78 & 71.69 & 6.99 \\
\hline \hline Parameter & Styrene & Toluene & $\begin{array}{l}\text { Trichloro- } \\
\text { ethylene }\end{array}$ & Xylene_total \\
\hline $\begin{array}{l}\text { Estimated } \\
\text { Mean (ppm) }\end{array}$ & 57.30 & 51.92 & 7.23 & 3.73 \\
\hline \hline Parameter & $\begin{array}{l}\text { Methyl isobutkl } \\
\text { ketone }\end{array}$ & --- & --- & --- \\
\hline $\begin{array}{l}\text { Estimated } \\
\text { Mean (ppm) }\end{array}$ & 50.5 & --- & --- & --- \\
\hline
\end{tabular}

Based on the statistical comparison of the cumulative distribution of elements using survival technique presented in Table 5-9, TCLP sand leachate tends to present more arsenic, barium, iron, lead, manganese, nickel, and zinc than SPLP sand leachate. TCLP sand leachate tends to present less phenol and mercury, and more selenium than ASTM D 3987 sand leachate. TCLP sand leachate tends to present a different amount of aluminum from SPLP sand leachate. There is no significant evidence against the hypotheses that TCLP sand leachate and SPLP sand leachate tend to present similar amounts of chromium and copper. There is no significant evidence against the hypotheses that TCLP sand leachate and ASTM D 3987 sand leachate tend to present similar amounts of cadmium. SPLP sand leachate tends to present less arsenic and more barium, iron, and zinc than ASTM D 3987 sand leachate. There is no significant evidence against the hypotheses that SPLP sand leachate and ASTM D 3987 sand leachate tend to 
present similar amounts of aluminum, chromium, copper, lead, manganese, and nickel. It is inferred that TCLP is more aggressive on sand than SPLP and ASTM D 3987.

\subsection{Factor of Metal Cast in TCLP Sand Leachate}

The estimated mean and factor effect of metal cast for metallic elements in TCLP sand leachate are presented in Table 5-11. Major considered comparisons include "concentrations of elements in TCLP sand leachate from an iron-based facility versus concentrations of elements in TCLP sand leachate from a steel-based facility"; "concentrations of elements in TCLP sand leachate from a steel-based facility versus concentrations of elements in TCLP sand leachate from an aluminum-based facility"; and "concentrations of elements in TCLP sand leachate from an aluminum-based facility versus concentrations of elements in TCLP sand leachate from a copper-based facility." Further comparison in distribution, shape, and percentile for individual parameters can be referred to corresponding charts grouped in Figure F-8 in Appendix F.4.1. 
Table 5-11: Estimated mean and factor effect for TCLP sand leachate (metallic elements)

\begin{tabular}{|c|c|c|c|c|c|}
\hline \multirow[t]{2}{*}{ Parameter } & $\begin{array}{l}\text { Iron- } \\
\text { based }\end{array}$ & $\begin{array}{l}\text { Steel- } \\
\text { based }\end{array}$ & $\begin{array}{l}\text { Aluminum- } \\
\text { based }\end{array}$ & $\begin{array}{l}\text { Copper- } \\
\text { based }\end{array}$ & \multirow[t]{2}{*}{$\begin{array}{l}\text { Statistical comparison of the cumulative distribution of elements using } \\
\text { survival technique }\end{array}$} \\
\hline & \multicolumn{4}{|c|}{ Unit: ppm } & \\
\hline Aluminum & 1.793 & 4.239 & 0.816 & 1.393 & $\begin{array}{l}\text { "Iron=Steel", } p=0.15 ; \text {;"Steel<Aluminum", } p<0.017 \text {; } \\
\text { "Aluminum=Copper", } p>0.20 ;\end{array}$ \\
\hline Barium & 0.672 & 0.582 & 0.413 & 0.589 & $\begin{array}{l}\text { "Iron=Steel", } p>0.20 ; \text { "Steel=Aluminum", } p>0.20 \\
\text { "Aluminum=Copper", } p>0.20\end{array}$ \\
\hline Cadmium & 0.003 & 0.009 & --- & --- & "Iron=Steel", $p>0.20$ \\
\hline Chromium & 0.044 & 0.063 & --- & --- & "Iron=Steel", $p=0.05+;$ \\
\hline Iron & 106.7 & 23.8 & 3.44 & & "Iron=Steel", $p=0.2+;$ "Steel=Aluminum", $p>0.20$ \\
\hline Lead & 0.123 & 0.023 & 0.174 & 0.73 & $\begin{array}{l}\text { "Iron=Steel", } p=0.15 ; \text {;'Steel=Aluminum", } p>0.20 ; \\
\text { "Aluminum<Copper", } p=0.035 \text {; "Iron }<\text { Copper", } p=0.005 \text {; } \\
\text { "Steel<Copper", } p=0.008 ;\end{array}$ \\
\hline Manganese & 1.409 & 1.307 & 0.447 & --- & "Iron=Steel", $p>0.20$; "Steel=Aluminum", $p>0.20$ \\
\hline Mercury & 0.0004 & 0.0006 & 0.0005 & --- & "Iron=Steel", $p>0.20$; "Steel=Aluminum", $p>0.20$ \\
\hline Nickel & 0.198 & 0.455 & 0.071 & --- & "Iron=Steel", $p=0.15 ;$ "Steel $>$ Aluminum", $p>0.035$ \\
\hline Selenium & 0.042 & 0.021 & --- & --- & "Iron=Steel", $p=0.20$ \\
\hline Zinc & 0.785 & 0.318 & 0.715 & --- & "Iron=Steel", $p>0.20$; "Steel=Aluminum", $p>0.20$ \\
\hline
\end{tabular}

Note: A $p$ value close to zero, or $p<0.05$, signals a significant factor effect. 
Based on the statistical comparison of the cumulative distribution of elements using survival technique presented in Table 5-11, there is no significant evidence against the hypotheses that TCLP sand leachate from iron-based facilities and TCLP sand leachate from steel-based facilities tend to present similar amounts of aluminum, barium, cadmium, chromium, iron, lead, manganese, mercury, nickel, selenium, and zinc. There is no significant evidence against the hypotheses that TCLP sand leachate from steelbased facilities and TCLP sand leachate from aluminum-based facilities tend to present similar amounts of barium, iron, lead, manganese, mercury, and zinc. TCLP sand leachate from steel-based facilities tends to present less aluminum and more nickel than TCLP sand leachate from aluminum-based facilities. There is no significant evidence against the hypotheses that TCLP sand leachate from aluminum-based facilities and TCLP sand leachate from copper-based facilities tend to present similar amounts of aluminum and barium. TCLP sand leachate from aluminum-based facilities tends to present less lead than TCLP sand leachate from copper-based facilities.

It is inferred that metal cast in the facilities is not a significant factor regarding most metallic elements in TCLP sand leachate. One of the exceptions is lead, which is frequently of greater concentration in TCLP sand leachate from copper-based facilities than in TCLP sand leachate from other alloy facilities. This finding is consistent with the finding mentioned in Section 5.3 that lead is frequently of greater composition in asreceived sand from copper-based facilities than from other alloy facilities. 


\subsection{Impacts of Metallic Elements in Foundry Sand}

When environmental problems related to trace elements in soils or groundwater are discovered, there has been a tendency for the public to identify the most visible industry first without proper technical assessment of other possible environmental or natural causes [Letey et al. 1986]. To defeat the arbitrary judgment, a comparison of background concentrations of trace metallic elements in soils of a U.S. state [Bradford et al. 1996] to characterization of foundry sand and sand leachates is presented in Table 513 through Table 5-16.

The comparison between background concentration of soil and average bulk analysis of foundry sand suggests the degree of environmental impact waste streams may pose. The comparison is summarized in Table 5-12, supported by data in Tables 5-13 through 5-16. While excess foundry sands contain higher levels of metallic elements (aluminum, copper, and iron), the concentrations of most regulated metallic elements are less than or in the same level as those of soil. This illustrates that excess foundry sands do not pose greater threats to the environment than soil.

Table 5-12: Metallic elements comparison between soil and excess foundry sand

\begin{tabular}{l|l}
\hline Foundry sand > Soil & $\begin{array}{l}\text { aluminum, antimony, copper, iron, molybdenum, and silicon, } \\
\text { selenium }\end{array}$ \\
\hline Foundry sand = Soil & $\begin{array}{l}\text { boron, cadmium, chromium, magnesium, nickel, lead, silver, } \\
\text { thallium, zinc }\end{array}$ \\
\hline Foundry sand < Soil & arsenic, barium, beryllium, calcium, mercury, manganese \\
\hline
\end{tabular}


Listed in Tables 5-13 through 5-16, TCLP leachate threshold [40 CFR Part 261 1986] is the only available federal regulation evaluating hazardous characteristics of solid wastes. Full thresholds cover toxicity, reactivity, ignitability, and corrosivity (Section 3.3.3) [EPA SW-846 2000]. A typical question in the reuse of excess foundry sand is the impact of metallic elements in the sand to the environment. To assess its toxicity, excess foundry sand is exposed to the TCLP leaching procedure, and its leachate, which may seep into the surrounding environment and potentially pose a threat to human beings, is chemically analyzed. The results are compared against corresponding toxicity thresholds. If all metallic elements are within corresponding TCLP thresholds, excess foundry sand is nonhazardous and has the potential to be reused. A violation of any metallic element leads to the judgment that excess foundry sand is hazardous and should be disposed of in a special landfill.

Characterization data for any metallic elements are dispersed. Hence, only comparisons of estimated means and associated distributions to thresholds are convincible. There may exist significant tail exceeding thresholds. To prove statistically that foundry sand is well below thresholds, the $95^{\text {th }}$ percentile is presented for each metallic element. If sand has its $95^{\text {th }}$ percentile below thresholds, it is statistically sound to infer that the sand is nonhazardous.

Tables 5-13 through 5-16 present worst-case comparisons between TCLP thresholds and $95^{\text {th }}$ percentiles of TCLP sand leachate to assess the toxicity and impact of foundry sand on the condition of an actively decomposing municipal solid- waste landfill. 
Alternative comparisons were conducted between TCLP thresholds and SPLP or ASTM D 3987 leachate level, approved in Section 2.2.1. The comparison between TCLP thresholds and $95^{\text {th }}$ percentiles of SPLP sand leachate is to assess the toxicity and impact of excess foundry sand in condition of industrial waste monofills, and the comparison between TCLP thresholds and $95^{\text {th }}$ percentiles of ASTM D 3987 sand leachate is to assess the toxicity and impact of excess foundry sand in the condition of neutral extraction. It is indicated that the sand leachate from any leaching protocol has its $95^{\text {th }}$ percentiles of metallic element concentrations well less than thresholds. Thus, excess foundry sand is generally not hazardous.

In Tables 5-13 through 5-16, additional comparison between TCLP thresholds and $95^{\text {th }}$ percentiles of bulk analyses of as-received sand contributes to saving of toxicity evaluation, because if bulk analyses of waste streams are within thresholds there is no requirement for further leaching evaluation. Comparisons indicate that sand has its $95^{\text {th }}$ percentiles of metallic element compositions well less than thresholds, except for lead and chromium. It is suggested that the full spectrum of metallic elements need not be exposed to leaching analysis. The selection relies on observed concentration of metallic elements. 
Table 5-13: Comparison of means and $95^{\text {th }}$ percentiles among soil, foundry sand and sand leachates

\begin{tabular}{|c|c|c|c|c|c|c|c|}
\hline & & Silver (Ag) & Aluminum $(\mathrm{Al})$ & Arsenic (As) & Boron (B) & Barium $(\mathrm{Ba})$ & Beryllium $(\mathrm{Be})$ \\
\hline \multirow[t]{2}{*}{ Soil } & Mean & 0.8 & 7.3 & 3.5 & 19 & 509 & 1.28 \\
\hline & Standard deviation & 1.43 & 1.7 & 2.5 & 15 & 210 & 0.52 \\
\hline \multicolumn{2}{|c|}{ TCLP leachate threshold } & 5 & --- & 5 & --- & 100 & --- \\
\hline $\begin{array}{l}\text { As-received } \\
\text { foundry by- } \\
\text { products (mean / } \\
95^{\text {th }} \text { percentile) }\end{array}$ & Sand & $0.47 / 0.21$ & $3326 / 4300$ & $0.86 / 1.6$ & $38.53 / 88.9$ & $14.95 / 28$ & $0.08 / 0.229$ \\
\hline \multirow{4}{*}{$\begin{array}{l}\text { As-received } \\
\text { excess foundry } \\
\text { sand (mean } / 95^{\text {th }} \\
\text { percentile) }\end{array}$} & Iron-based facilities & --- & $1327 / 3800$ & $0.76 / 1.8$ & $9.86 / 39$ & $24.31 / 52$ & --- \\
\hline & Steel-based facilities & --- & $1576 / 1200$ & $6 / 0.56$ & $276.2 / 29$ & $7.77 / 18$ & -- \\
\hline & Aluminum-based facilities & --- & $12931 / 15000$ & $0.34 / 0.67$ & --- & $6.53 / 12$ & --- \\
\hline & Copper-based facilities & --- & $1190 / 480$ & $0.39 / 0.49$ & --- & $3.97 / 4.51$ & --- \\
\hline $\begin{array}{l}\text { TCLP leachate } \\
\text { (mean } / 95^{\text {th }} \\
\text { percentile) }\end{array}$ & Sand & $0.004 / 0.008$ & $1.785 / 4.51$ & $0.031 / 0.07$ & --- & $0.639 / 2.4$ & --- \\
\hline $\begin{array}{l}\text { SPLP leachate } \\
\left(\text { mean } / 95^{\text {th }}\right. \\
\text { percentile) }\end{array}$ & Sand & --- & $2.698 / 12$ & $0.001 / 0.002$ & $0.236 / 0.89$ & $0.388 / 1.9$ & --- \\
\hline $\begin{array}{l}\text { ASTM D } 3987 \\
\text { leachate (mean / } \\
95^{\text {th }} \text { percentile) }\end{array}$ & Sand & --- & 2.094 / 6.6 & $0.003 / 0.005$ & --- & $0.173 / 0.6$ & --- \\
\hline \multirow{4}{*}{$\begin{array}{l}\text { TCLP leachate } \\
\text { sand (mean } / 95^{\text {th }} \\
\text { percentile) }\end{array}$} & Iron-based facilities & --- & $1.793 / 11$ & --- & --- & $0.672 / 2.4$ & --- \\
\hline & Steel-based facilities & --- & $4.239 / 21$ & -- & --- & $0.582 / 1.48$ & -- \\
\hline & Aluminum-based facilities & --- & $0.816 / 3.5$ & --- & --- & $0.413 / 1.62$ & --- \\
\hline & Copper-based facilities & --- & $1.393 / 2.7$ & --- & --- & $0.589 / 1.68$ & --- \\
\hline
\end{tabular}


Table 5-14: Comparison of means and $95^{\text {th }}$ percentiles among soil, foundry sand and sand leachates (Cont.)

\begin{tabular}{|c|c|c|c|c|c|c|c|}
\hline & & Calcium $(\mathrm{Ca})$ & Cadmium $(\mathrm{Cd})$ & Chromium $(\mathrm{Cr})$ & Copper $(\mathrm{Cu})$ & Iron $(\mathrm{Fe})$ & Mercury (Hg) \\
\hline \multirow[t]{2}{*}{ Soil } & Mean & 14466 & 0.36 & 122 & 28.7 & 3.7 & 0.26 \\
\hline & Standard deviation & 10703 & 0.31 & 223 & 19.3 & 1.6 & 0.21 \\
\hline \multicolumn{2}{|c|}{ TCLP leachate threshold } & $\begin{array}{ll}-- \\
--\end{array}$ & 1 & 5 & $\begin{array}{ll}-- \\
--\end{array}$ & --- & 0.2 \\
\hline $\begin{array}{l}\text { As-received } \\
\text { foundry by- } \\
\text { products (mean / } \\
95^{\text {th }} \text { percentile) }\end{array}$ & Sand & $3309 / 11000$ & $0.22 / 0.25$ & $114.03 / 65$ & $103.6 / 350$ & $10911 / 25000$ & $0.04 / 0.06$ \\
\hline \multirow{4}{*}{$\begin{array}{l}\text { As-received } \\
\text { excess foundry } \\
\text { sand (mean / } \\
95^{\text {th }} \text { percentile) }\end{array}$} & Iron-based facilities & $4842 / 12200$ & --- & $16.23 / 67$ & $30.32 / 154$ & $11163 / 25000$ & --- \\
\hline & Steel-based facilities & $1366 / 3600$ & +-- & $1664.1 / 5.6$ & $404.73 / 7.4$ & $60819 / 340000$ & $\begin{array}{ll}-- \\
--\end{array}$ \\
\hline & Aluminum-based facilities & $927 / 2100$ & --- & $4.63 / 19.3$ & $17.68 / 64.3$ & $1898 / 4600$ & --- \\
\hline & Copper-based facilities & $2481 / 278$ & --- & $3.21 / 4.82$ & $653.2 / 1860$ & 1986 / 5750 & --- \\
\hline $\begin{array}{l}\text { TCLP leachate } \\
\left(\text { mean } / 95^{\text {th }}\right. \\
\text { percentile) }\end{array}$ & Sand & --- & $0.004 / 0.018$ & $0.042 / 0.11$ & $0.521 / 0.4$ & $61.78 / 285$ & $0.0002 / 0.00004$ \\
\hline $\begin{array}{l}\text { SPLP leachate } \\
\left(\text { mean } / 95^{\text {th }}\right. \\
\text { percentile) }\end{array}$ & Sand & --- & --- & $0.002 / 0.003$ & $0.061 / 0.18$ & $6.095 / 30$ & --- \\
\hline $\begin{array}{l}\text { ASTM D } 3987 \\
\text { leachate (mean / } \\
95^{\text {th }} \text { percentile) }\end{array}$ & Sand & --- & $0.0003 / 0.0004$ & $0.007 / 0.018$ & $0.087 / 0.033$ & $1.245 / 5.02$ & $0.0002 / 0.0003$ \\
\hline \multirow{4}{*}{$\begin{array}{l}\text { TCLP leachate } \\
\text { sand (mean / } \\
95^{\text {th }} \text { percentile) }\end{array}$} & Iron-based facilities & --- & $0.003 / 0.012$ & $0.044 / 0.11$ & --- & $106.7 / 285$ & $0.0004 / 0.002$ \\
\hline & Steel-based facilities & --- & $0.009 / 0.06$ & $0.063 / 0.39$ & --- & $23.8 / 72.2$ & $0.0006 / 0.004$ \\
\hline & Aluminum-based facilities & --- & --- & --- & --- & $3.44 / 4.23$ & $0.0005 / 0.001$ \\
\hline & Copper-based facilities & --- & --- & --- & --- & --- & --- \\
\hline
\end{tabular}


Table 5-15: Comparison of means and $95^{\text {th }}$ percentiles among soil, foundry sand and sand leachates (Cont.)

\begin{tabular}{|c|c|c|c|c|c|c|c|}
\hline & & Magnesium (Mg) & Manganese (Mn) & Molybdenum (Mo) & Sodium $(\mathrm{Na})$ & Nickel (Ni) & $\overline{\text { Lead }(\mathrm{F}}$ \\
\hline \multirow[t]{2}{*}{ Soil } & Mean & 9923 & 646 & 1.3 & 15838 & 57 & 23.9 \\
\hline & Standard deviation & 5356 & 285 & 1.5 & 9309 & 80 & 13.8 \\
\hline \multicolumn{2}{|c|}{ TCLP leachate threshold } & --- & --- & --- & --- & --- & 5 \\
\hline $\begin{array}{l}\text { As-received } \\
\text { foundry by- } \\
\text { products (mean / } \\
95^{\text {th }} \text { percentile) }\end{array}$ & Sand & $1881 / 5240$ & $257 / 480$ & --- & --- & $107.94 / 58$ & $15.72 /$ \\
\hline \multirow{4}{*}{$\begin{array}{l}\text { As-received } \\
\text { excess foundry } \\
\text { sand (mean } / 95^{\text {th }} \\
\text { percentile) }\end{array}$} & Iron-based facilities & --- & $483 / 548$ & --- & --- & $6.85 / 29$ & $19.81 /$ \\
\hline & Steel-based facilities & --- & $523 / 2800$ & --- & --- & $1664.6 / 12.3$ & $8.42 / 3$ \\
\hline & Aluminum-based facilities & --- & 26.66 / 109 & --- & --- & $44.17 / 3$ & $3.62 / 1$ \\
\hline & Copper-based facilities & --- & $14.33 / 33$ & --- & --- & $9.86 / 17.8$ & $88.6 / 6$ \\
\hline $\begin{array}{l}\text { TCLP leachate } \\
\left(\text { mean } / 95^{\text {th }}\right. \\
\text { percentile) }\end{array}$ & Sand & --- & $1.009 / 5.72$ & --- & --- & $0.183 / 0.875$ & $0.222 /$ \\
\hline $\begin{array}{l}\text { SPLP leachate } \\
\left(\text { mean } / 95^{\text {th }}\right. \\
\text { percentile })\end{array}$ & Sand & --- & $0.215 / 0.928$ & $0.233 / 0.1$ & --- & $0.029 / 0.06$ & $0.009 /$ \\
\hline $\begin{array}{l}\text { ASTM D } 3987 \\
\text { leachate (mean / } \\
95^{\text {th }} \text { percentile) }\end{array}$ & Sand & --- & $0.061 / 0.19$ & --- & $15.043 / 56.3$ & $0.006 / 0.026$ & $0.008 /$ \\
\hline \multirow{4}{*}{$\begin{array}{l}\text { TCLP leachate } \\
\text { sand (mean } / 95^{\text {th }} \\
\text { percentile) }\end{array}$} & Iron-based facilities & --- & $1.409 / 7.03$ & --- & --- & $0.198 / 0.875$ & $0.123 /$ \\
\hline & Steel-based facilities & --- & $1.307 / 4.31$ & --- & --- & $0.455 / 1.28$ & $0.023 /$ \\
\hline & Aluminum-based facilities & --- & $0.447 / 2.04$ & --- & --- & $0.071 / 0.439$ & $0.14 / 0$ \\
\hline & Copper-based facilities & --- & -- & --- & --- & --- & $0.73 / 2$ \\
\hline
\end{tabular}


Table 5-16: Comparison of means and $95^{\text {th }}$ percentiles among soil, foundry sand and sand leachates (Cont.)

\begin{tabular}{|c|c|c|c|c|c|c|}
\hline & & Antimony (Sb) & Selenium $(\mathrm{Se})$ & Silicon $(\mathrm{Si})$ & \begin{tabular}{|l|} 
Vanadium $(\mathrm{V})$ \\
\end{tabular} & Zinc $(\mathrm{Zn})$ \\
\hline \multirow[t]{2}{*}{ Soil } & Mean & 0.6 & 0.058 & 29.4 & 112 & 149 \\
\hline & Standard deviation & 0.39 & 0.084 & 4.6 & 53 & 32 \\
\hline \multicolumn{2}{|c|}{ TCLP leachate threshold } & --- & 1 & --- & --- & --- \\
\hline $\begin{array}{l}\text { As-received } \\
\text { foundry by- } \\
\text { products (mean / } \\
95^{\text {th }} \text { percentile) }\end{array}$ & Sand & $4.34 / 31$ & $0.64 / 0.49$ & $1772 / 1410$ & --- & $102.48 / 216$ \\
\hline \multirow{4}{*}{$\begin{array}{l}\text { As-received } \\
\text { excess foundry } \\
\text { sand (mean / } 95^{\text {th }} \\
\text { percentile) }\end{array}$} & Iron-based facilities & --- & --- & --- & --- & $131 / 42$ \\
\hline & Steel-based facilities & --- & --- & --- & --- & $64.1 / 7.4$ \\
\hline & Aluminum-based facilities & --- & --- & --- & --- & $31.6 / 99$ \\
\hline & Copper-based facilities & --- & --- & --- & --- & $691.2 / 620$ \\
\hline $\begin{array}{l}\text { TCLP leachate } \\
\text { (mean / 95 } \\
\text { percentile) }\end{array}$ & Sand & --- & $0.041 / 0.1$ & --- & --- & $1.006 / 5.29$ \\
\hline $\begin{array}{l}\text { SPLP leachate } \\
\text { (mean / 95 } \\
\text { percentile) }\end{array}$ & Sand & $0.083 / 0.46$ & --- & --- & --- & $0.264 / 0.84$ \\
\hline $\begin{array}{l}\text { ASTM D } 3987 \\
\text { leachate (mean / } \\
95^{\text {th }} \text { percentile) }\end{array}$ & Sand & --- & $0.002 / 0.0026$ & --- & $0.003 / 0.0043$ & $0.177 / 0.41$ \\
\hline \multirow{4}{*}{$\begin{array}{l}\text { TCLP leachate } \\
\text { sand (mean } / 95^{\text {th }} \\
\text { percentile) }\end{array}$} & Iron-based facilities & $\begin{array}{ll}-- \\
--\end{array}$ & $0.042 / 0.191$ & $\begin{array}{c}-- \\
--\end{array}$ & --- & $0.785 / 2.54$ \\
\hline & Steel-based facilities & --- & $0.021 / 0.07$ & --- & --- & $0.318 / 0.52$ \\
\hline & Aluminum-based facilities & $\begin{array}{c}-- \\
--\end{array}$ & --- & $\begin{array}{ll}-- \\
--\end{array}$ & --- & $0.715 / 0.65$ \\
\hline & Copper-based facilities & --- & --- & --- & --- & --- \\
\hline
\end{tabular}




\subsection{Conclusions}

Based on the statistical comparison of the cumulative distribution of elements using survival technique rather than a location (such as mean, maximum, or any quantile) comparison, the following conclusions are summarized for the characterization of foundry sands. Further comparisons in shape and scale of survival function for individual elements/compounds/parameters can be referred to corresponding charts in Appendices F.1 through F.4.

TCLP is more aggressive on sand than SPLP and ASTM D 3987. SPLP is most reprehensive for fill applications.

For as-received foundry sand, metal cast in the facilities is not a significant factor regarding most metallic elements. The exceptions are lead, copper, and zinc, which are frequently of greater composition in sand from copper-based facilities than from other alloy facilities.

For TCLP sand leachate, metal cast in the facilities is not a significant factor regarding most metallic elements. The exception is lead, which is frequently of greater concentration in TCLP sand leachate from copper-based facilities than in TCLP sand leachate from other alloy facilities. 
In comparison with soil, excess foundry sands contain more metallic elements that are frequently and heavily used in the casting process than soil, including aluminum, copper, and iron. However, its compositions of most regulated metallic elements are less than or in the same level as those of soil. Hence, excess foundry sands do not pose more threats to the environment than soil.

Sand leachate has its $95^{\text {th }}$ percentiles of metallic element concentrations well less than TCLP thresholds. Thus, foundry sands are generally not hazardous.

Comparisons indicate that foundry sand has its $95^{\text {th }}$ percentiles of metallic element bulk analyses well less than TCLP thresholds, except for lead and chromium. Hence, it is not necessary to expose sand to a leaching protocol and measure the full spectrum of metallic elements. 


\section{Chapter 6}

\section{EXPERIMENTAL MATERIALS}

In this chapter, materials used in CLSM and HMA programs are introduced. Materials include cement, fly ash, aggregate, water, and excess foundry sand.

In the CLSM program, materials selected include Type I portland cement, Class F fly ash, excess foundry sand, and water. The selection was inferred from the specification of CLSM containing cement, fly ash, and bottom ash/coarse aggregate/fine aggregate developed by Pennsylvania Department of Transportation (PennDOT) [PennDOT 1995], and previous studies on this topic.

In the HMA program, aggregates from a local asphalt pavement contractor were used. They can be classified as a crushed limestone. The natural sand (lake sand) used was supplied by a local aggregate supplier. The control gradation used for this research project was designed by the same contractor.

Seventeen different foundry sands were used in this study. The sands came from 11 different states in the United States: Illinois, Indiana, Massachusetts, Michigan, North Carolina, Ohio, Pennsylvania, Texas, Virginia, West Virginia, and Wisconsin. Variables in excess foundry sand include metal cast, binder, and binding process. No other 
variables, such as additives of silica fume and slag, were considered for the purpose of focusing on variation of excess foundry sands.

\subsection{Materials}

\subsubsection{Cement}

The cement used in this research conforms to AASHTO M 85, "Standard Specification for Portland Cement," for Type I/II designation and was manufactured by ESSROC. The chemical composition of the cement is shown in Table 6-1. Cement was kept the same throughout experimental investigation.

\subsubsection{Fly Ash}

The fly ash used in this research originated from Mineral Solutions, Inc., which is a provider approved for transportation material sources. It is a Class F fly ash, and its bulk chemical analysis is presented in Table 6-1. Fly ash was kept the same throughout the experimental investigation. 
Table 6-1: Bulk chemical composition of Type I cement and Class F fly ash

\begin{tabular}{|c|c|c|}
\hline Component (Oxide) & Capitol Cement & Min. Solutions Fly Ash \\
\hline & \multicolumn{2}{|c|}{ Percentage by Mass } \\
\hline $\mathrm{Al}$ & 5.04 & 20.40 \\
\hline $\mathrm{B}$ & $<0.005$ & 0.06 \\
\hline $\mathrm{Ba}$ & 0.06 & 0.05 \\
\hline $\mathrm{Ca}$ & 64.00 & 2.66 \\
\hline $\mathrm{Co}$ & 0.01 & 0.01 \\
\hline $\mathrm{Cr}$ & 0.01 & 0.01 \\
\hline $\mathrm{Fe}$ & 3.00 & 12.70 \\
\hline $\mathrm{K}$ & 1.04 & 1.67 \\
\hline $\mathrm{Mg}$ & 3.25 & 0.39 \\
\hline $\mathrm{Mn}$ & 0.04 & 0.02 \\
\hline Mo & $<0.005$ & $<0.005$ \\
\hline $\mathrm{Na}$ & 0.40 & 0.68 \\
\hline $\mathrm{Ni}$ & $<0.005$ & $<0.005$ \\
\hline $\mathrm{Si}$ & 19.50 & 57.00 \\
\hline $\mathrm{Sr}$ & 0.13 & 0.06 \\
\hline $\mathrm{Ti}$ & 0.21 & 0.32 \\
\hline $\mathrm{V}$ & $<0.005$ & 0.02 \\
\hline $\mathrm{Zn}$ & 0.01 & 0.01 \\
\hline $\mathrm{F}$ & $<0.005$ & $<0.005$ \\
\hline $\mathrm{Cl}$ & 0.01 & $<0.005$ \\
\hline $\mathrm{NO}_{2}$ & $<0.005$ & $<0.005$ \\
\hline $\mathrm{NO}_{3}$ & $<0.005$ & $<0.005$ \\
\hline $\mathrm{PO}_{4}$ & $<0.005$ & $<0.005$ \\
\hline $\mathrm{SO}_{4}$ & 2.26 & 0.99 \\
\hline Total & 100.14 & 98.69 \\
\hline Moist. & 0.04 & 0.08 \\
\hline LOI (1000) & 1.17 & 1.64 \\
\hline
\end{tabular}

\subsubsection{Excess Foundry Sand}

In the database, information from 180 foundry facilities was collected, which spans a wide variety of backgrounds identified by metals cast, binders, and binding systems. Using statistical sampling, 17 facilities were randomly selected as sources of 
excess foundry sands. Their backgrounds are presented in Table 6-2. Their physical properties and chemical compositions are listed in Sections 6.2 and 6.3. As indicated in Table 6-2, iron-based facilities using bentonite and green sand are the most used combination in the foundry industry.

\subsubsection{Water}

External water is required to produce CLSM mixtures. Potable tap water at approximate room temperature $\left(23^{\circ} \mathrm{C}\right)$ was used for mixing.

\subsection{Characteristics of Excess Foundry Sands}

The physical characteristics of foundry sands, such as particle size distribution, grain fineness number, and grain surface characteristics, are important determinants of flowability, compacted density, and strength in reuse applications [Carey and Sturtz 1995]. Excess foundry sands are not as pure as virgin silica sand after repetitive exposures to inorganic or organic bonding processes, high-temperature contact, and mechanical reclamations, which is demonstrated by the characteristics of green sand presented in Table 6-3 [Winkler et al. 1999]. Investigations of physical characteristics of excess foundry sand not only affect its reuse, but recognize the difference among waste streams as well. 
Table 6-2: Backgrounds of excess foundry sands

\begin{tabular}{|c|c|c|c|c|c|}
\hline $\mathrm{SN}$ & Sand origin & Metal Cast & Binder & Fillers & Binding System \\
\hline FS01 & Lake sand & Ductile iron & $\begin{array}{l}\text { Bentonite (Mold) + Phenolic } \\
\text { Urethane (Core) }\end{array}$ & $\begin{array}{l}\text { Sea Coal, Starch, Peanut Hulls, etc. } \\
\text { (Mold) + Iron Oxide (Core) }\end{array}$ & Green Sand \\
\hline FS02 & Silica sand & Gray and ductile iron & Furfurl Alcohol (Mold) & None & --- \\
\hline FS03 & Silica sand & Gray and ductile iron & $\begin{array}{l}\text { Bentonite (Mold) + Phenolic } \\
\text { Urethane (Core) }\end{array}$ & Sea Coal (Mold) +None (Core) & Green Sand + Cold Box/Shell \\
\hline FS04 & Silica sand & Gray and ductile iron & $\begin{array}{l}\text { Bentonite }(\text { Mold })++ \text { Phenolic } \\
\text { Urethane }\end{array}$ & Sea Coal & Green Sand \\
\hline FS05 & Silica sand & Grey iron & $\begin{array}{l}\text { Bentonite (Mold) + Phenolic } \\
\text { Urethane (Core) }\end{array}$ & Sea Coal (Mold) + None (Core) & $\begin{array}{l}\text { Green Sand (Mold) + Shell/Cold } \\
\text { Box/No-Bake (Core) }\end{array}$ \\
\hline FS06 & Sand & Gray iron & $\begin{array}{l}\text { Bentonite }(\text { Mold })+\text { Sodium } \\
\text { Silicate (Core) }\end{array}$ & $\begin{array}{l}\text { Sea Coal, Cereal, Soda Ash } \\
\text { (Molding) + None (Core) }\end{array}$ & Green Sand + Sodium Silicate \\
\hline FS07 & Lake sand, Silica & Gray iron & Bentonite (Mold) & Sea Coal, Flocarb & Green Sand \\
\hline FS08 & Lake sand & Gray and ductile iron & $\begin{array}{l}\text { Bentonite (Mold) + Phenolic } \\
\text { Urethane Cold Box + Phenol } \\
\text { Formaldehyde Hot Box }\end{array}$ & Sea Coal, Klean Kast, Carbonate & Green Sand \\
\hline FS09 & Silica sand & Iron & $\begin{array}{l}\text { Bentonite }+ \text { Phenolic Urethane } \\
\text { Cold Box }+ \text { Phenol Formaldehyde } \\
\text { Hot Box }\end{array}$ & Sea Coal + Fuse Silica & Green Sand \\
\hline FS10 & Lake sand & Aluminum & Sodium Silicate & Corn Flour & --- \\
\hline FS11 & Lake sand & Aluminum & $\begin{array}{l}\text { Bentonite (Mold) + Phenolic } \\
\text { Urethane (Core) }\end{array}$ & None (Mold, Core) & $\begin{array}{l}\text { Green Sand (Mold)+ Isocure } \\
(\text { Core })\end{array}$ \\
\hline FS12 & Silica sand & Aluminum & Phenolic Urethane & None & No-Bake \\
\hline FS13 & Silica sand & Gray and ductile iron & $\begin{array}{l}\text { Bentonite (Mold)+ Phenolic } \\
\text { Urethane (Cold Box) }\end{array}$ & Sea Coal, Corn Flour & Green Sand \\
\hline FS14 & Silica sand & Brass/Bronze/Aluminum & $\begin{array}{l}\text { Bentonite (Mold) + Phenolic } \\
\text { Urethane Cold Box }\end{array}$ & $\begin{array}{l}\text { Sea Coal, Cellulose, Soda Ash, } \\
\text { Gilsonite }\end{array}$ & Green Sand \\
\hline FS15 & Sand & Gray and ductile iron & Bentonite (Mold)+Furan+Oil & Sea Coal & Green Sand+Shell \\
\hline FS16 & Silica sand & Steel & $\begin{array}{l}\text { Bentonite (Mold)+ Phenolic } \\
\text { Urethane }\end{array}$ & Corn Flour & Green Sand \\
\hline FS17 & Silica sand & Steel & Sodium Silicate (Mold + Core) & None & No-bake \\
\hline
\end{tabular}


Table 6-3: Typical characteristics of excess green sand

\begin{tabular}{l|l}
\hline Components & Property Range \\
\hline Sand & $70-80 \%$ \\
\hline Water & $2-4 \%$ \\
\hline Clay & $5-15 \%$ \\
\hline Additives & $2-5 \%$ \\
\hline Moisture & $0-4 \%$ \\
\hline Carbon Loss on Ignition & $0.2-8 \%$ \\
\hline pH & $3-12$ \\
\hline AFS-Grain Fineness No. & $40-150$ \\
\hline$\%$ Fines (Passes 200 Mesh Sieve) & $1-12 \%$ (higher for brass) \\
\hline Density & $1.0-1.6 \mathrm{~g} / \mathrm{cm} 3$ (zircon: $2.6 \mathrm{~g} / \mathrm{cm} 3)$ \\
\hline
\end{tabular}

\subsubsection{Bulk Density, Compaction and Void}

Bulk density is necessary for use in selecting proportions of mixture, and it is used for determining mass/volume relationship for conversions in purchase agreements [ASTM C 29 1997]. Void represents the space between particles in foundry sand mass not occupied by solid particles. It estimates the space that should be filled by other materials when mixing together. The testing specification for this investigation is ASTM C29/C29M-97, "Standard Test Method for Bulk Density (Unit Weight) and Voids in Aggregate." In the investigation, the compaction was conducted by rodding procedure, in which compaction is fulfilled with 25 strokes of the tamping rod by 3 layers. Testing results are presented in Table 6-4. It is indicated that the distribution for variables is normal, and corresponding distribution parameters are provided. 
Table 6-4: Physical evaluation of excess foundry sand

\begin{tabular}{|c|c|c|c|c|c|c|c|c|c|}
\hline FS ID & \begin{tabular}{|l|} 
Loose Bulk \\
Density as \\
Received \\
$\left(\mathrm{kg} / \mathrm{m}^{3}\right)$
\end{tabular} & $\begin{array}{l}\text { Loose } \\
\text { Bulk } \\
\text { Density as } \\
\text { Oven-dry } \\
\left(\mathrm{kg} / \mathrm{m}^{3}\right) \\
\end{array}$ & $\begin{array}{l}\text { Compact Bulk } \\
\text { Density as } \\
\text { Oven-dry by } \\
\text { Rodding } \\
\left(\mathrm{kg} / \mathrm{m}^{3}\right)\end{array}$ & $\begin{array}{l}\text { Void for } \\
\text { Loose } \\
\text { Sand as } \\
\text { Oven-dry, } \\
\%\end{array}$ & $\begin{array}{l}\text { Void for } \\
\text { Compact } \\
\text { Sand as } \\
\text { Oven-dry, } \\
\%\end{array}$ & $\begin{array}{l}\text { Bulk } \\
\text { Specific } \\
\text { Gravity }\end{array}$ & $\begin{array}{l}\text { Specific } \\
\text { Gravity } \\
\text { (SSD) }\end{array}$ & $\begin{array}{l}\text { Absorption } \\
(\%)\end{array}$ & $\begin{array}{l}\text { Moisture } \\
\text { Content as } \\
\text { Received (\%) }\end{array}$ \\
\hline & \multicolumn{5}{|c|}{ ASTM C 29 / C 29M } & \multicolumn{3}{|c|}{ ASTM C 128} & ASTM C 566 \\
\hline FS01 & 1458 & 1289.17 & 1432.08 & 48.39 & 42.66 & 2.503 & 2.579 & 3.029 & 1.73 \\
\hline FS02 & 1666.39 & 1381.67 & 1576.25 & 49.13 & 41.97 & 2.722 & 2.732 & 0.381 & 0.29 \\
\hline FS03 & 1383 & 1285.00 & 1412.50 & 46.91 & 41.64 & 2.425 & 2.481 & 2.312 & 3.17 \\
\hline FS04 & 1466.9 & 1422.92 & 1530.42 & 39.99 & 35.46 & 2.376 & 2.446 & 2.944 & 1.42 \\
\hline FS05 & 1415 & 1369.58 & 1519.58 & 43.01 & 36.76 & 2.408 & 2.449 & 1.688 & 1.96 \\
\hline FS06 & 1314 & 1095.00 & 1238.33 & 56.90 & 51.26 & 2.546 & 2.592 & 1.807 & 1.70 \\
\hline FS07 & 1518 & 1303.33 & 1435.83 & 48.73 & 43.52 & 2.547 & 2.587 & 1.564 & 0.00 \\
\hline FS08 & 1378.1 & 1288.75 & 1393.33 & 45.86 & 41.47 & 2.385 & 2.465 & 3.348 & 2.03 \\
\hline FS09 & 1518 & 1429.17 & 1545.42 & 44.66 & 40.16 & 2.588 & 2.616 & 1.092 & 1.62 \\
\hline FS10 & 1491 & 1447.92 & 1606.25 & 44.87 & 38.84 & 2.632 & 2.645 & 0.523 & 3.50 \\
\hline FS11 & 1606 & 1328.75 & 1441.67 & 48.68 & 44.32 & 2.594 & 2.617 & 0.860 & 4.08 \\
\hline FS12 & 1650 & 1505.00 & 1651.67 & 44.01 & 38.55 & 2.693 & 2.714 & 0.787 & 0.64 \\
\hline FS13 & 1580 & 1522.08 & 1670.83 & 42.19 & 36.54 & 2.638 & 2.635 & 0.718 & 0.00 \\
\hline FS14 & 1544 & 1389.58 & 1557.08 & 43.86 & 37.10 & 2.480 & 2.583 & 4.148 & 0.14 \\
\hline FS15 & 1103 & 1052.08 & 1201.25 & 55.68 & 49.40 & 2.379 & 2.470 & 3.834 & 4.08 \\
\hline FS16 & 1478 & 1162.50 & 1312.08 & 53.83 & 47.88 & 2.523 & 2.601 & 3.093 & 1.02 \\
\hline FS17 & 1572 & 1553.75 & 1715.00 & 40.94 & 34.81 & 2.636 & 2.667 & 1.171 & 4.85 \\
\hline Min & 1103 & 1052 & 1201 & 39.99 & 34.81 & 2.38 & 2.45 & 0.38 & 0.00 \\
\hline $\operatorname{Max}$ & 1666 & 1554 & 1715 & 56.90 & 51.26 & 2.72 & 2.73 & 4.15 & 4.85 \\
\hline Average & 1479 & 1343 & 1484 & 46.92 & 41.31 & 2.53 & 2.58 & 1.96 & 1.90 \\
\hline $\begin{array}{l}\text { Standard } \\
\text { Deviation } \\
(S D)\end{array}$ & 137 & 142 & 146 & 4.92 & 4.85 & 0.11 & 0.09 & 1.23 & 1.54 \\
\hline Normality & Yes, $p=0.35$ & Yes, $p=0.48$ & Yes, $p=0.79$ & $Y e s, p=0.44$ & Yes, $p=0.45$ & Yes, $p=0.5$ & Yes, $p=0.13$ & Yes, $p=0.17$ & Yes, $p=0.21$ \\
\hline
\end{tabular}




\subsubsection{Specific Gravity and Absorption}

Bulk specific gravity (saturated surface dry, SSD) is the ratio of the mass of a volume of a material-including the mass of water within the pores in the material (but excluding the voids between particles) — at a stated temperature, to the mass of an equal volume of distilled water at a stated temperature. Absorption values are used to calculate change in the weight of an aggregate due to water absorbed in the pore spaces within the constituent particles, compared to the dry condition, when it is deemed that the aggregate has been in contact with water long enough to satisfy most of the absorption potential [ASTM C 128 1993]. Specific gravity is an aggregate characteristic generally used for calculation of the volume occupied by the foundry sand aggregate in CLSM that is proportioned or analyzed on an absolute volume basis. It is also used in the computation of voids in aggregate and the determination of moisture in aggregate by displacement in water [ASTM C 70 1994].

The testing specification for specific gravity and absorption is ASTM C128 “Standard Test Method for Density, Relative Density (Specific Gravity), and Absorption of Fine Aggregate." The results are presented in Table 6-4. The variation in specific gravity of excess foundry sands has been attributed to the variability in fines and additive contents in different samples. Reported values of absorption are found to vary widely, which can be attributed to the presence of binders and additives [Joved and Lovell 1994]. 
The variation of both variables are verified to be normal distributed, Table 6-4. Corresponding parameters are presented in Table 6-4.

\subsubsection{Moisture Content}

Most binder systems need water as solvent or catalyst to activate various inorganic and organic binder raw materials. Although the melting temperature is high, it decreases significantly along the dimensions of molding. After the refractory process, a low water content is retained in the excess foundry sand. Ignoring this part of water leads to the inaccuracy of CLSM formulation. The water content is treated as a source of water in this research and is considered when CLSM formulations are made. The testing specification for water content is ASTM C566-97, "Standard Test Method for Total Evaporable Moisture Content of Aggregate by Drying." In this investigation, samples are kept in a ventilated oven at a temperature of $110{ }^{\circ} \mathrm{C}$ until each sample's weight is constant [ASTM C 566 1997].

The results of the water content investigation are presented in Table 6-4. They ranged from 0 to $4.85 \%$, which may depend on the bonding process and stocking methods. Water contents of excess foundry sands are normally distributed, as indicated in Table 6-4. 


\subsubsection{Gradation}

Gradation has an important effect upon flowable consistency, compaction and strength. Particularly in the application of CLSM, compaction is its pronounced feature. It is shown that the true sphere of a given size when perfectly packed occupies $74 \%$ of the total volume. Greater compaction can be obtained by filling the remaining voids with particles of decreasing size order [Webster 1980]. Evaluating the gradation of excess foundry sand is an essential step toward integrating excess foundry sands into CLSM.

Gradation was investigated by sieve analysis and visualized by the gradation curve. The testing specification for sieve analysis is AFS 1105-00 "Sieve Analysis (Particle Size Determination of Sand)." It was conducted by separating the grains into 10 segments by letting a representative sample pass through a series of screens with openings that get increasingly smaller; plotting individual weight of the fraction to form an $x / y$ graph of particle size versus individual weight will give a bell-shaped curve that represents the grain distribution (by size) for that sand [AFS 1105 2000].

The quantified results of sieve analysis are presented in Tables G-1 and G-2 in Appendix G.1. Corresponding gradation curves are plotted in Figure 6-1. The gradation curves show distributions of particle sizes. It is indicated that gradation of excess foundry sand is dominated by a common shape. Although grain sizes span from $3.5 \mathrm{~mm}$ down to zero, the size is highly concentrated, with $90 \%$ in the narrow range of $0.15 \mathrm{~mm}(5 \%$ quantile) to $0.80 \mathrm{~mm}(95 \%$ quantile $)$. And in this range, particles are 


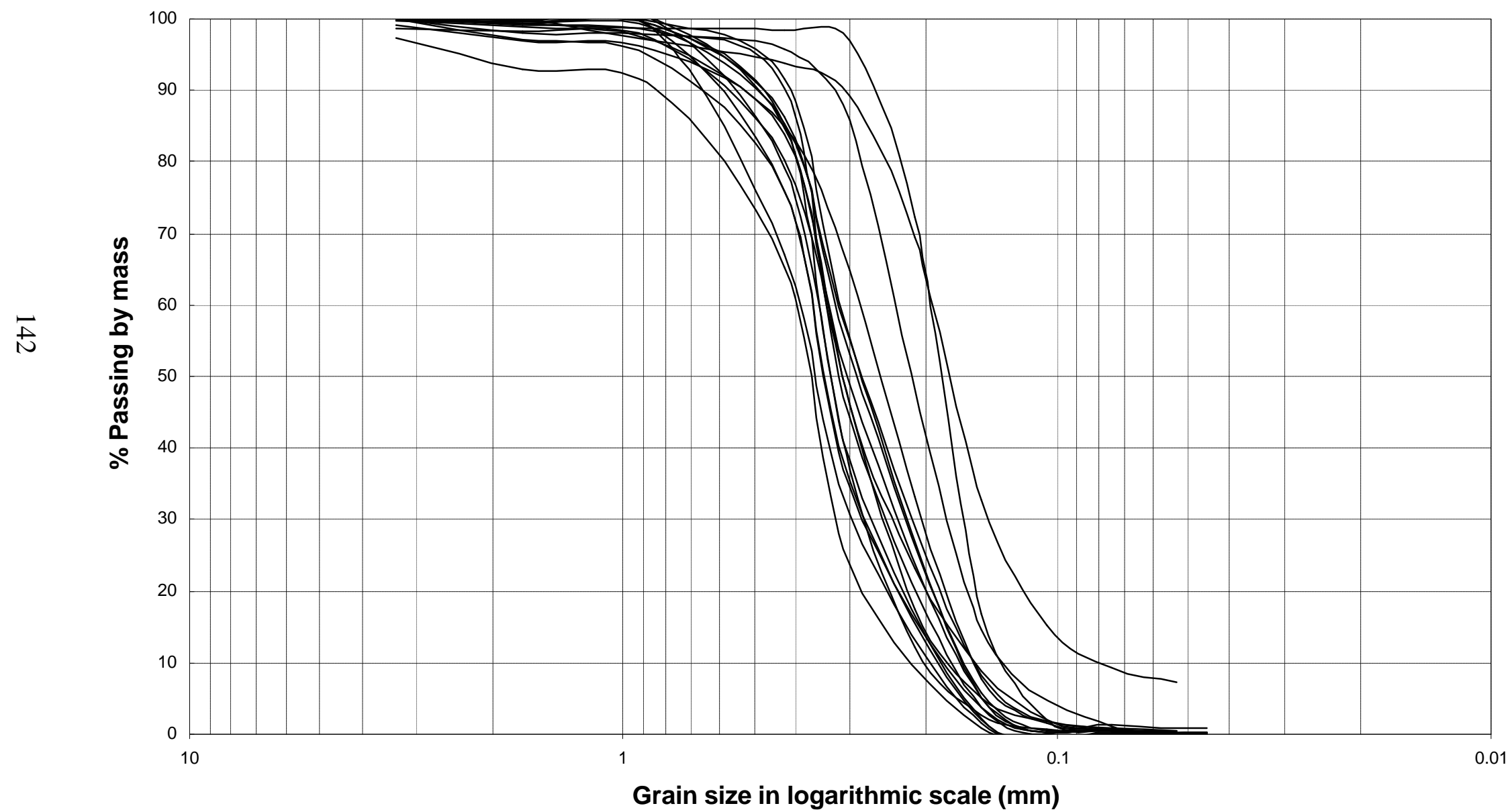

Figure 6-1: Gradation curve of excess foundry sands 
distributed by size. Ninety percent by mass of foundry sands are uniformly distributed along size $0.15-0.80 \mathrm{~mm}$. The remaining $10 \%$ of the particles are extended two-way. The grain size distribution of excess foundry sand is more uniform and finer than conventional CLSM fine sand. While the fineness of spent foundry sand contributes to limited segregation of flowable fill, there is a need to investigate the impact of alternative gradations to permit wider use of excess foundry sand for this application.

Table 6-5 presents parameters describing the gradation characteristics of sand. $\mathrm{D}_{10}, \mathrm{D}_{30}, \mathrm{D}_{50}, \mathrm{D}_{60}$ represents the particle sizes corresponding to $10 \%, 30 \%, 50 \%$, and $60 \%$ finer in the gradation curve. Uniformity coefficient $C_{u}, D_{60}$ divided by $D_{10}$, and coefficient of gradation $\mathrm{C}_{\mathrm{z}}$, square of $\mathrm{D}_{30}$ divided by product of $\mathrm{D}_{60}$ and $\mathrm{D}_{10}$, are used to characterize gradation. By these two terms, foundry sands are classified as very uniform $\left(\mathrm{C}_{\mathrm{u}}<5\right)$ and well graded $\left(1<\mathrm{C}_{\mathrm{z}}<3\right)$ aggregates. The long-established AFS Grain Fineness Number (AFS GFN), a measure of average grain size, is based on the number of openings per inch of a sieve that would just pass the average size calculated from the sieving analysis [Beeley 2001].

Steel castings typically use a very coarse sand grain size with a low grain fineness number, such as 45-55 AFS GFN, because they have a lot of gas to get rid of in a hurry. Iron castings use a slightly finer sand, 55-70 AFS GFN. Nonferrous castings are even finer, 65-90 AFS GFN. The AFS GFN investigated on the 17 foundry sands according to AFS 1106-00-S, "Grain Fineness Number, AFS GFN, Calculation," are presented in Table 6-5. It is consistent with the variation of foundry background. 
Table 6-5: Gradation evaluation of excess foundry sand

\begin{tabular}{|c|c|c|c|c|c|c|c|c|}
\hline FS ID & $\begin{array}{l}\mathrm{D}_{50} \\
(\mathrm{~mm})\end{array}$ & $\begin{array}{l}\mathrm{D}_{10} \\
(\mathrm{~mm})\end{array}$ & $\begin{array}{l}\mathrm{D}_{30} \\
(\mathrm{~mm})\end{array}$ & $\begin{array}{l}\mathrm{D}_{60} \\
(\mathrm{~mm})\end{array}$ & $\begin{array}{l}\text { Size } 200(0.075 \mathrm{~mm}) \text { passing } \\
\text { percentage by weight }(\%)\end{array}$ & $\mathrm{C}_{\mathrm{u}}$ & $\mathrm{C}_{\mathrm{z}}$ & AFS GFN \\
\hline & \multicolumn{4}{|c|}{ Quantiles of Gradation } & ASTM C117 & $\frac{D_{60}}{D_{10}}$ & $\frac{D_{30}^{2}}{D_{60} \times D_{10}}$ & AFS 1106 \\
\hline FS01 & 0.347 & 0.180 & 0.278 & 0.377 & 0.482 & 2.094 & 1.136 & 50.19 \\
\hline FS02 & 0.345 & 0.186 & 0.276 & 0.376 & 0.078 & 2.021 & 1.089 & 49.45 \\
\hline FS03 & 0.331 & 0.182 & 0.265 & 0.358 & 0.172 & 1.966 & 1.081 & 51.69 \\
\hline FS04 & 0.225 & 0.124 & 0.182 & 0.246 & 1.186 & 1.980 & 1.091 & 76.92 \\
\hline FS05 & 0.284 & 0.156 & 0.220 & 0.320 & 0.493 & 2.051 & 0.968 & 61.26 \\
\hline FS06 & 0.379 & 0.209 & 0.319 & 0.409 & 0.000 & 1.953 & 1.186 & 41.97 \\
\hline FS07 & 0.284 & 0.165 & 0.224 & 0.316 & 0.655 & 1.917 & 0.967 & 60.28 \\
\hline FS08 & 0.304 & 0.167 & 0.236 & 0.340 & 0.502 & 2.035 & 0.978 & 56.28 \\
\hline FS09 & 0.290 & 0.164 & 0.227 & 0.326 & 0.522 & 1.996 & 0.966 & 59.18 \\
\hline FS10 & 0.316 & 0.173 & 0.247 & 0.343 & 1.345 & 1.979 & 1.030 & 56.68 \\
\hline FS11 & 0.366 & 0.193 & 0.296 & 0.401 & \begin{tabular}{|l|}
0.077 \\
\end{tabular} & 2.076 & 1.132 & 46.92 \\
\hline FS12 & 0.187 & 0.130 & 0.165 & 0.198 & 0.369 & 1.526 & 1.054 & 69.99 \\
\hline FS13 & 0.313 & 0.155 & 0.238 & 0.249 & 0.515 & 1.601 & 1.467 & 51.62 \\
\hline FS14 & 0.179 & \begin{tabular}{|l|}
0.079 \\
\end{tabular} & 0.143 & 0.196 & \begin{tabular}{|l|}
9.209 \\
\end{tabular} & 2.479 & 1.318 & 90.36 \\
\hline FS15 & 0.338 & 0.198 & 0.275 & 0.368 & 0.414 & 1.861 & 1.040 & 45.66 \\
\hline FS16 & 0.259 & 0.156 & 0.204 & 0.287 & 0.726 & 1.843 & 0.936 & 54.71 \\
\hline FS17 & 0.315 & 0.175 & 0.251 & 0.351 & 0.928 & 2.006 & 1.024 & 48.68 \\
\hline $\min$ & 0.1791 & 0.079 & 0.143 & 0.196 & 0.0000 & 1.526 & 0.936 & 41.974 \\
\hline $\max$ & 0.3789 & 0.209 & 0.319 & 0.409 & 9.2088 & 2.479 & 1.467 & 90.363 \\
\hline average & 0.2978 & 0.164 & 0.238 & 0.321 & 1.0396 & 1.964 & 1.086 & 57.166 \\
\hline$S D$ & 0.0576 & 0.0311 & 0.0465 & 0.0654 & 2.1372 & 0.2039 & 0.1370 & 12.2559 \\
\hline Normality & $\begin{array}{l}\text { Yes, } \\
p=0.19\end{array}$ & $\begin{array}{l}Y e s, \\
p=0.14\end{array}$ & $\begin{array}{l}\text { Yes, } \\
p=0.89\end{array}$ & $\begin{array}{l}\text { Yes, } \\
p=0.53\end{array}$ & $\begin{array}{l}\text { No, } p=0+\text { or Yes without } \\
\text { FS14, } p=0.23\end{array}$ & $\begin{array}{l}\text { No, } \\
p=0.007\end{array}$ & $\begin{array}{l}\text { No, } \\
p=0.02\end{array}$ & $\begin{array}{l}\text { No, } p=0.03, \text { or } \\
\text { Yes without } \\
\text { FS14, } p=0.32\end{array}$ \\
\hline
\end{tabular}


Aggregates finer than $0.075 \mathrm{~mm}$ sieve can be separated from larger particles much more efficiently and completely by wet sieving than through the use of dry sieving [ASTM C 117 1995]. Therefore, this wet sieving approach of ASTM C 117, "Standard Test Method for Materials Finer than $75-\mu \mathrm{m}$ (No. 200) Sieve in Mineral Aggregates by Washing," was selected to determine the concentration of particles finer than $0.075 \mathrm{~mm}$.

The results are shown in Table 6-5. In general, less than $1.345 \%$ by mass of foundry sands passes $0.075 \mathrm{~mm}$, except for sand from copper-based facilities, which use finer aggregate for finer finishing.

The normality tests for each variable are presented in Table 6-5. It is assumed that quantiles for gradations of sands are normally distributed, which include $\mathrm{D}_{10}, \mathrm{D}_{30}, \mathrm{D}_{50}$, and $\mathrm{D}_{60}$. This assumption is verified by gradation curves presented in Figure 6-1, in which horizontal lines are plotted across the cluster of curves, and the $\mathrm{x}$ values of these crossed points are normally distributed. However, the rest of the variables are not normalized very well. Mass percentage passing size 200 and AFS GFN would be normalized only if data of sand FS14 were eliminated. Sand FS14 is from a copper-based facility that uses finer particles than the other facilities. Gradation coefficients $C_{u}$ and $C_{z}$ are not normally distributed.

\subsubsection{Grain Shape}

Grain shape of virgin sand is determined by its geological origin [Beeley 2001]. Four different basic shapes are recognizable for foundry sand: round, sub-angular, 
angular, and compound or composite [AFS 1107 2000]. The characteristics may be readily observed under a microscope. Grain shape is important with respect to flowability and strength. Round sands give superior flowability to angular sands. And angular sands yield higher strength than round sands do. However, round grain has the lowest surface area and requires the least amount of cementitious material. Most lake sands are in this shape. Angular sands have grains with edges that form acute angles (less than 90 degrees). They are poor for making a good working consistency, for angular particles lock up and neither slide nor compact as well as round ones do. They are made from crushed rock. Compound sands have grains that are stacked together. They look like popcorn balls. They do not flow well, and when they do break apart, there are a lot of fines.

Sand was screened into 10 segments as a result of sieve analysis in Section 6.2.4. The shape of each segment was investigated according to Standard AFS 1107, "Grain Shape Classification." The shape observation for each segment is mingled with corresponding gradation results and presented in Figure G-1 in Appendix G.2.

In sands with sizes covering $90 \%$ excess foundry sands, $0.15-0.80 \mathrm{~mm}$ (Section 6.2.4), subangular and round grains occupy more than $50 \%$ by mass. It was discovered that as the size increases, the shape tends to be compound or round. If the size decreases, the shape tends to be angular or jagged. It was observed that the compound grains were composed of subangular and round grains once they were crushed under a microscope. Therefore, subangular and round dominate the shape of sands. The spherical shape of 
excess foundry sand particles contributes to superior flowable characteristics of flowable fill. However, increased particle fineness and sphericity result in lower strength-bearing capacity of the hardened flowable fill [Larsen 1990].

\subsubsection{Organic Content and Methylene Blue Value}

The organic content was determined using AASHTO Standard Procedure T 26786, with one modification. Instead of being burned for 6 hours, the samples were burned for 24 hours to ensure thorough combustion of the organic components.

The following procedure originated from AFS 2210-00-S standard, "Methylene Blue Clay Test, Ultrasonic Method, Molding Sand" [American Foundry Society 2001]. Instead of using the spot test on filter paper to determine the titration point, a spectrophotometer was used. For our study, a Milton Roy Spectronic 301, Item: 335401 was used to measure the "\% absorbed" to determine the level of blueness. The titration equipment, HM-58, was purchased from the Gilson Company, along with the methylene blue dye, HMA-78. The methylene blue test was run a total of three times. The first two times were to get a general idea of where the methylene blue titration point was, the last time was to get a more exact value.

Each sand was tested for its organic content, and methylene blue value (MBV). The organic content is the amount of material burned off at $455 \mathrm{C}$ for a period of 24 
hours. The $95 \%$ confidence interval is also shown for the organic content. The confidence intervals were calculated by Equation 6.1 .

$$
95 \% \text { C.I. }=\frac{t \times S . D .}{\sqrt{n}}
$$

S.D. = Standard deviation of the measurements

$\mathrm{t}=$ Student's $\mathrm{t}$ distribution $=3.18$

$\mathrm{n}=$ Number of measurements per sand $=4$

All of the confidence intervals were very tight for the organic contents. The organic material mostly comes from sea coal, but can also come from carbohydrates that are added to the sand, or from organic-based binder systems. Since all of the organic contents are under $5 \%$, and since the mix is only made up of $10 \%$ foundry sand, the organic content does not seem to be a significant contributor to the performance of the mix.

The methylene blue value is an indicator of the amount of clay that is in a foundry sand or aggregate. It is the ratio of the milligrams of methylene blue dye that a gram of foundry sand can absorb. It is measured using a spectrophotometer. The $95 \%$ C.I. is also shown for the control and three sands in Table 6-6. For these, the student's t-distribution is 4.30 since the number of measurements was limited to 3 per sand. The confidence interval seems to vary more widely for the MBV as compared to the organic content, since it ranges from 0.3 to 5.3. The MBV was also recorded for the foundry sands after they were tested for their organic contents. 
Table 6-6: Organic Contents and Methylene Blue Values

\begin{tabular}{c|c|c|c}
\hline Sand No. & \% O.C. & $\begin{array}{c}\text { M.B.V. (mg } \\
\text { M.B./gram F.S.) }\end{array}$ & $\begin{array}{c}\text { M.B.V. After } \\
\text { LOI @ 455 EC }\end{array}$ \\
\hline \hline Control (P0.60) & N.R. & $1.9 \pm 0.3$ & N.R. \\
\hline FS01 & $2.5 \pm 0.1$ & $31.4 \pm 5.3$ & 30.5 \\
\hline FS02 & $4.7 \pm 0.1$ & 0.8 & 0.8 \\
\hline FS03 & $3.8 \pm 0.1$ & 27.3 & 28.1 \\
\hline FS04 & $2.9 \pm 0.1$ & 22.8 & 23.2 \\
\hline FS05 & $4.7 \pm 0.1$ & 28.1 & 29.6 \\
\hline FS06 & $1.3 \pm 0.1$ & 1.3 & 0.8 \\
\hline FS07 & $2.5 \pm 0.1$ & 22.4 & 23.8 \\
\hline FS08 & $3.3 \pm 0.1$ & $27.5 \pm 0.4$ & 23.5 \\
\hline FS09 & $1.3 \pm 0.1$ & 10.1 & 8.9 \\
\hline FS10 & $0.2 \pm 0.1$ & 0.8 & 0.6 \\
\hline FS11 & $0.7 \pm 0.1$ & 21.0 & 19.6 \\
\hline FS12 & $1.2 \pm 0.1$ & 0.4 & 0.8 \\
\hline FS13 & $1.2 \pm 0.1$ & 0.8 & 0.6 \\
\hline FS14 & $1.6 \pm 0.1$ & $3.2 \pm 1.8$ & 2.5 \\
\hline FS15 & $2.1 \pm 0.1$ & 30.2 & 28.2 \\
\cline { 1 - 1 } FS16 & $1.6 \pm 0.1$ & 36.3 & 34.1 \\
\hline FS17 & $0.2 \pm 0.1$ & 1.4 & 0.8
\end{tabular}

According to the Green Sand Additives [American Foundry Society 2000] handbook, the temperature of destruction for southern bentonite ranges between 315-390 $\mathrm{EC}$, and for western bentonite, ranges between 650-815 EC. Since the LOI test was run at $455 \mathrm{EC}$, it was believed all the southern bentonite would be destroyed, thus allowing a measure of the western bentonite. But from the values shown in Table 6-6, it appears the MBV hardly changes. It is possible that during the foundry process the southern bentonite was already destroyed, or the values given in the AFS handbook are not completely accurate. The other possibility is that the level of southern bentonite in the sand is lower than the accuracy of the test. Either way, measuring the MBV of the foundry sand after it has been tested for LOI does not seem to be a good method to use to determine the southern bentonite content. 
The methylene blue value was also determined for pure southern and western bentonite. They were very high compared to the foundry sands, with southern bentonite showing a MBV of $391 \mathrm{mg} / \mathrm{g}$ and western bentonite showing a MBV of $385 \mathrm{mg} / \mathrm{g}$. Sand FS16 has the highest MBV of $36.3 \mathrm{mg} / \mathrm{g}$. If clay has a typical MBV of about $388 \mathrm{mg} / \mathrm{g}$, this means the clay content of sand FS16 would lay at around 9.4\%. Figure 6-2 shows the comparison between the fines content and the methylene blue value of each of the foundry sands. There is a general correlation between the two, which shows that as the fines content increases, so does the MBV. Since clay is a fine material, this seems intuitive. Figure 6-3 shows a comparison between the fines content and MBV for foundry sands that contain bentonite. The higher $\mathrm{R}^{2}$ value further verifies that the clay content is related to the fines content.

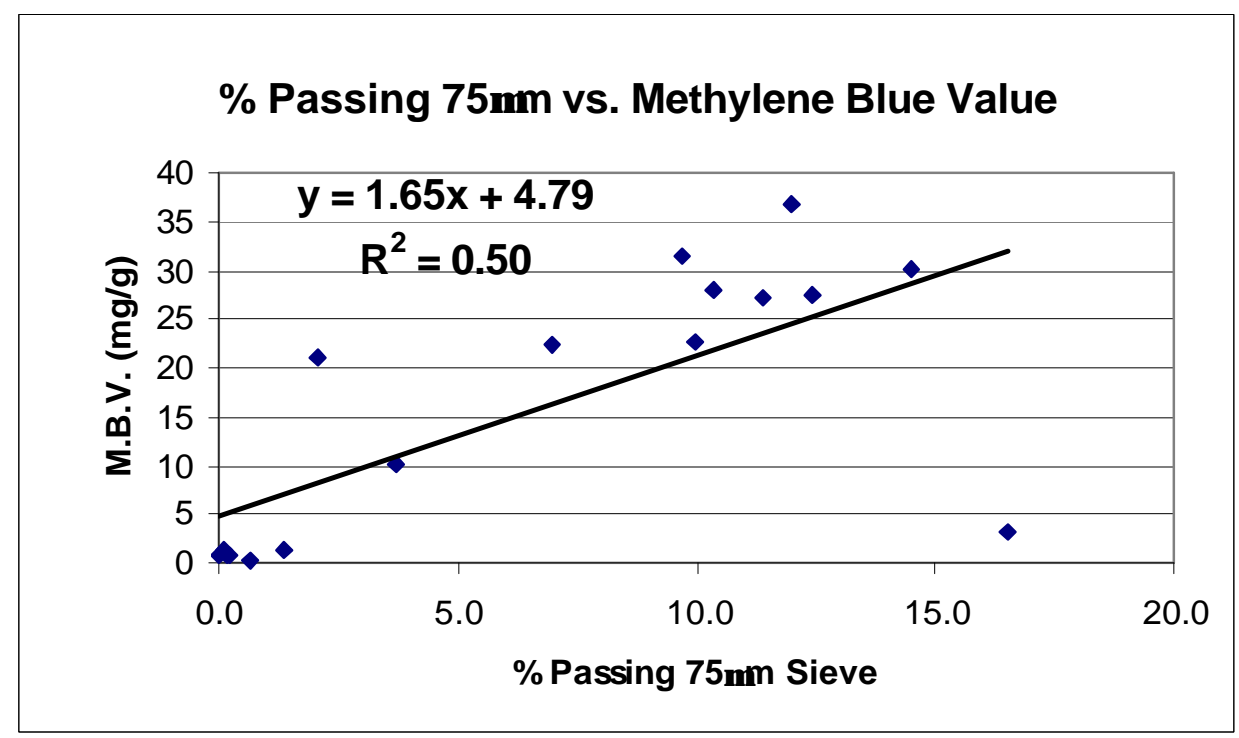

Figure 6-2: Comparison of fines content to M.B.V. 


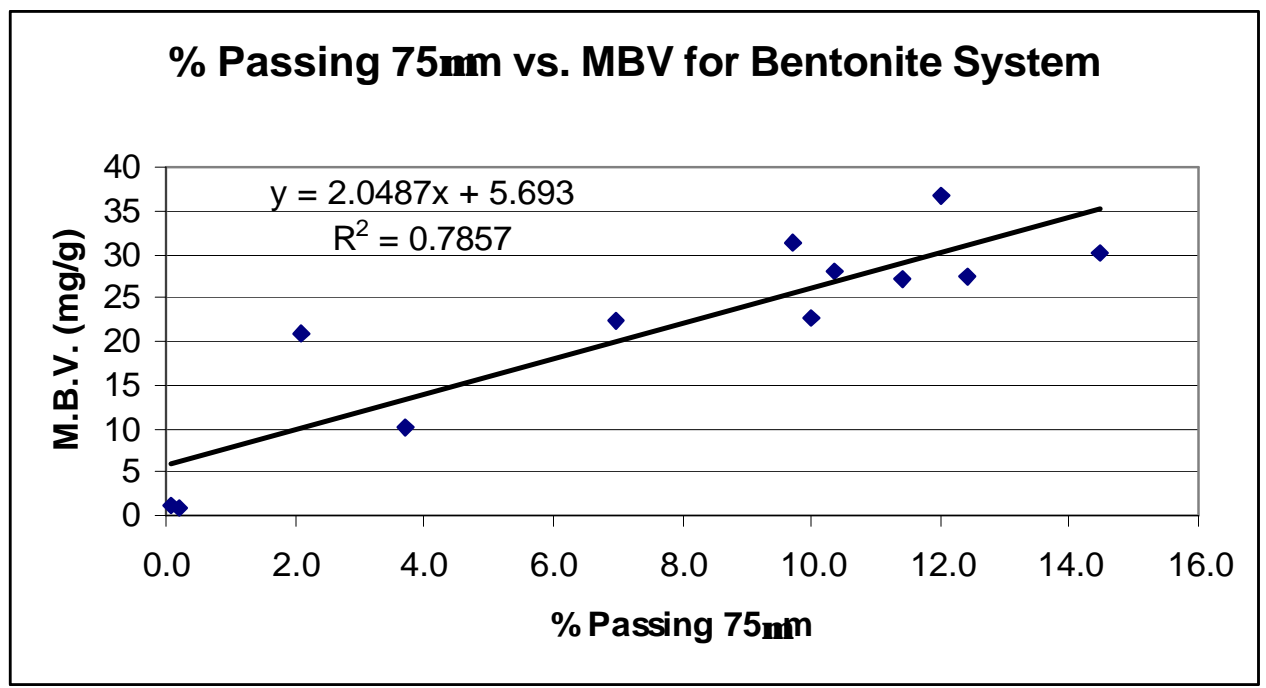

Figure 6-3: Comparison of fines content to M.B.V. for bentonite system

\subsection{Bulk Chemical Analysis of Excess Foundry Sands}

Bulk chemical analysis was conducted on 17 sands to evaluate the concentrations of elements that are regulated. The toxicity evaluation is avoided if the bulk chemical analysis is within the toxicity threshold.

EXYGEN, a commercial analytical chemical laboratory, performed the bulk chemical analysis according to EPA testing methods and X-ray fluorescence methods. The elements investigated include oxides and organic compounds. Results are presented in Table H-1 for organic compounds in Appendix H.1 and Table H-2 for oxides in Appendix H.2. For the organic compounds, the frequent quantified compounds include ocresol, m,p-cresol, cresol-total, 2,4-dimethylphenol, phenanthrene, and acetone. Two observations for naphthalene and one observation for methyl ethyl ketone were reported 
much greater than observations of the other samples. Further investigation is desired regarding these unusual observations. For the oxides, FS14 is identified as containing more $\mathrm{CaO}$ and $\mathrm{MgO}$ and less $\mathrm{SiO}_{2}$ than the other excess foundry sands. FS10 is identified as containing more $\mathrm{SO}_{3}, \mathrm{MgO}$, and $\mathrm{CaO}$ than the other sands. 


\section{Chapter 7}

\section{EXPERIMENTS QUALIFYING CLSM USING EXCESS FOUNDRY SAND}

In this chapter, an experimental program was conducted to characterize the performance of CLSM containing excess foundry sands, and to provide technical data qualifying CLSM using excess foundry sands.

\subsection{Experimental Design of CLSM}

\subsubsection{Executive Summary}

Flowable fill mixtures are usually designed on the basis of compressive strength, generally after 28 days of ambient temperature curing, but sometimes on the basis of longer-term (90 days or more) strength. They are designed to have high flowability during placement and to develop limited strength, typically between $340 \mathrm{kPa}(50 \mathrm{psi})$ and $1,400 \mathrm{kPa}$ (200 psi) at 28 days, which is sufficient to support traffic without settling, yet can be readily excavated and gain limited strength after that [PCA 1990].

Fist of all, scouting mixtures were conducted to produce limited excavatable CLSM samples. The behavior of the CLSM was tested and observed, and the formulations were improved for excavatable CLSM. Using the modified formulations, an initial batch of excavatable CLSM was mixed using all 17 sands. Their construction behavior and geotechnical performance were tested and observed. If the parameters of 
CLSM attain the specified requirements of CLSM by ACI 229, no further excavatable CLSM is needed. Otherwise, mixtures were redesigned and tested until critical performances were attained. The design of structural CLSM was based on the design of excavatable CLSM and PennDOT specification for Type C CLSM. Identical experimental mixing and testing were performed. For this research, water was added until the desired flowability was achieved according to the flowability test of ASTM D 610397, "Standard Test Method for Flow Consistency of Controlled Low Strength Material (CLSM)."

\subsubsection{Critical Parameters}

The integration of excess foundry sand to CLSM is mainly qualified by its competitive performance against general CLSM containing fine aggregates. The performances are compared using construction and geotechnical properties. A summary of the critical parameters is listed in Table 7-1. Corresponding experimental programs, including sampling and testing procedures, are listed as well.

Flow consistency testing determines the fluidity and monitors the CLSM capacity in self-leveling, self-compaction, and workability in the fresh condition. Flowability is a critical parameter in flowable fill design because it ensures that all void space in the designed cavity is filled by the flowable fill material [Mast 1997]. Bleed tests were used to measure the relative quantity of mixing water that will bleed from freshly mixed CLSM. Bleeding is the discharges of CLSM to surrounding environments, which 
possibly will pose environmental concerns. Its volume influences the assessment of environmental impact resulting from CLSM.

Table 7-1: Parameters qualifying CLSM using excess foundry sand

\begin{tabular}{|c|c|c|c|}
\hline & Parameters & Samples or Containers & Standard \\
\hline \multirow{6}{*}{ 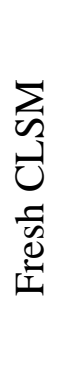 } & Fresh Density & $1-\phi 15.24$ x $12.7 \mathrm{~cm}$ steel measurement & ASTM D 6023 \\
\hline & Flow Consistency & $1-\phi 7.6 \times 15.2 \mathrm{~cm}$ open-ended cylinder & ASTM D 6103 \\
\hline & Bleeding & $1-\phi 10.2$ x $20.4 \mathrm{~cm}$ cylinder & ASTM C 232 \\
\hline & Settlement & $4-\phi 10.2 \times 20.4 \mathrm{~cm}$ cylinders & ASTM C 827 \\
\hline & $\begin{array}{l}\text { Setting Time and } \\
\text { Penetration Resistance }\end{array}$ & $\begin{array}{l}1-23.5 \times 23.5 \times 8.7 \mathrm{~cm} \text { or } 30.5 \times 30.5 \times \\
8.7 \mathrm{~cm} \text { container }\end{array}$ & ASTM C 403 \\
\hline & Toxicity of Bleeding & Up to $1,500 \mathrm{ml}$ bleeding & EPA SW-486 \\
\hline \multirow{6}{*}{ 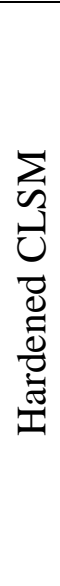 } & $\begin{array}{l}\text { Density }(3,7,14,28,90, \\
\text { and } 180 \text { day) }\end{array}$ & $4-\phi 10.2$ x $20.4 \mathrm{~cm}$ cylinders & ------ \\
\hline & $\begin{array}{l}\text { Oven-Dry Density (180 } \\
\text { day) }\end{array}$ & 1 or $2-\phi 10.2 \times 20.4 \mathrm{~cm}$ cylinders & ASTM C 567 \\
\hline & $\begin{array}{l}\text { Hydraulic Conductivity } \\
\text { ( } 28 \text { day) }\end{array}$ & $1-\phi 10.2 \times 10.2 \mathrm{~cm}$ metal proctor mold & ASTM D 2434 \\
\hline & $\begin{array}{l}\text { Compressive Strength ( } 3 \text {, } \\
7,14,28,90, \text { and } 180 \\
\text { day) }\end{array}$ & $18-\phi 10.2$ × $20.4 \mathrm{~cm}$ cylinders & ASTM D 4832 \\
\hline & $\begin{array}{l}\text { Toxicity of CLSM ( } 28 \\
\text { day) }\end{array}$ & $1-\phi 10.2 \times 20.4 \mathrm{~cm}$ cylinder & EPA SW-486 \\
\hline & Resistivity & $1-23.5 \times 9 \times 8.7 \mathrm{~cm}$ container & ASTM G57 \\
\hline
\end{tabular}

Settlement testing determines the change in height of a cylindrical specimen from the time of placement until its final set. It provides a means for comparing the relative settlement or expansion of cementitious mixtures. It is particularly applicable to grouting, patching, and form-filling operations where the objective is to completely fill a cavity or other defined space with a freshly mixed cementitious mixture that will continue to fill the same space at the time of hardening. Generally, CLSM presents settlement to an extent that depends on the water bleed, particle gradation and hydration of cementitious materials. 
Since the setting of CLSM is a gradual process, any definition of time of setting must necessarily be arbitrary. The times required for the CLSM to reach specified values of resistance to penetration are used to define times of setting. It provides a measure of the effects of variables, such as foundry sand variation, grain gradation and shape, and water-cement ratio upon the hardening properties of CLSM.

Hydraulic conductivity determines the coefficient of permeability by a constanthead method using tap water through hardened CLSM. It establishes representative values of coefficient of permeability of CLSM placed as embankments or backfills. This parameter is useful in the evaluation of environmental impact and corrosivity of CLSM. Less hydraulic conductivity helps mitigate leaching and stagnate ion movement.

CLSM is typically used as a backfill material around structures, particularly in confined or limited spaces. Compressive strength testing is performed to assist in the design of the mixture and to serve as a control technique during construction. Mixture design is typically based on 28-day strengths and construction control tests performed 3 , 7, 14, 90, and 180 days after placement.

Bleeding and leachate from CLSM are the two discharges of CLSM that pose potential environmental impact. In this research program, they are collected and analyzed for toxicity evaluation of CLSM. 


\subsubsection{Formulations of Scouting CLSM}

The formulations presented in Section 7.1.3, 7.1.4, and 7.1.5 are calculated by mass and by volume on solid materials at oven-dry condition. They were worked out through a back-calculation procedure based on measurements. These measurements include mass and specific gravity of raw materials, water content of foundry sands, and fresh density of CLSM. Simple substitution of foundry sand for standard fine aggregate in current CLSM specifications produces errors due to significant difference in gradation and specific gravity of the two materials.

Scouting experiments included two parts: scouting experiment I tending to probe the lower bound of formulations, and scouting experiment II tending to probe the upper bound of formulations. Eleven sands were used in the scouting experiments. The formulations by mass and by volume for scouting experiment I are summarized in Table 7-2 and Table 7-3, respectively. Corresponding formulations for scouting experiment II are summarized in Table 7-4 and Table 7-5. Water addition in the mixing

proportion complies with normal distribution, $N(405,65)$ for scouting experiment I and $N(406,61)$ for scouting experiment II, which indicates the water addition amount. The fresh densities of two scouting experiments are normally distributed as well, $N$ (1920, $138)$ and $N(1930,136)$, respectively. 
Table 7-2: Formulation by mass as oven-dry in scouting experiment I

\begin{tabular}{l|l|l|l|l|l|l|l|l|l}
\hline FS ID & \multicolumn{4}{|c|}{ Bulk Proportion $\left(\mathrm{Unit}_{\left.\mathrm{kg} / \mathrm{m}^{3}\right)}\right.$} & \multicolumn{3}{c}{ Percentage by Weight, \% } \\
\hline & Cement & Fly Ash & Sand & Water & Total & Cement & Fly Ash & Sand & Water \\
\hline FS01 & 29 & 263 & 862 & 543 & 1697 & 2 & 16 & 51 & 32 \\
\hline FS02 & 45 & 401 & 1332 & 339 & 2116 & 2 & 19 & 63 & 16 \\
\hline FS03 & 38 & 338 & 907 & 481 & 1764 & 2 & 19 & 51 & 27 \\
\hline FS04 & 38 & 343 & 991 & 436 & 1808 & 2 & 19 & 55 & 24 \\
\hline FS05 & 40 & 362 & 1005 & 428 & 1835 & 2 & 20 & 55 & 23 \\
\hline FS06 & 49 & 440 & 1137 & 365 & 1991 & 2 & 22 & 57 & 18 \\
\hline FS07 & 41 & 372 & 1130 & 397 & 1940 & 2 & 19 & 58 & 20 \\
\hline FS08 & 43 & 383 & 1036 & 402 & 1863 & 2 & 21 & 56 & 22 \\
\hline FS09 & 44 & 400 & 1196 & 367 & 2007 & 2 & 20 & 60 & 18 \\
\hline FS10 & 49 & 444 & 1303 & 315 & 2111 & 2 & 21 & 62 & 15 \\
\hline FS11 & 43 & 383 & 1176 & 383 & 1984 & 2 & 19 & 59 & 19 \\
\hline Min. & 29 & 263 & 862 & 315 & 1697 & 2 & 16 & 51 & 15 \\
\hline Max. & 49 & 444 & 1332 & 543 & 2116 & 2 & 22 & 63 & 32 \\
\hline Avg. & 42 & 375 & 1098 & 405 & 1920 & 2 & 19 & 57 & 21 \\
\hline SD & 5.64 & 50.53 & 152.03 & 65.09 & 137.51 & 0.00 & 1.57 & 3.95 & 5.04 \\
\hline
\end{tabular}

* Mass of raw material per unit of fresh CLSM 
Table 7-3: Formulation by volume as oven-dry in scouting experiment I

\begin{tabular}{|c|c|c|c|c|c|c|c|c|c|c|c|c|}
\hline \multirow[t]{2}{*}{ FS ID } & \multicolumn{3}{|c|}{$\begin{array}{l}\text { Bulk Volume as Oven-dry, } \\
\mathrm{m}^{3} / \mathrm{m}^{3 *}\end{array}$} & \multicolumn{5}{|c|}{ Percentage by Bulk Volume as Oven-dry, $\%$} & \multicolumn{4}{|c|}{$\begin{array}{l}\text { Percentage by Absolute Volume in } \\
\text { CLSM, \% }\end{array}$} \\
\hline & Cement & Fly Ash & Sand & Water & Cement & Fly Ash & Sand & Water & Cement & Fly Ash & Sand & Water \\
\hline FS01 & 0.03 & 0.20 & 0.67 & 0.54 & 1.8 & 13.9 & 46.5 & 37.8 & 0.9 & 10.3 & 34.4 & 54.3 \\
\hline FS02 & 0.04 & 0.30 & 0.96 & 0.34 & 2.4 & 18.4 & 58.6 & 20.6 & 1.4 & 15.7 & 49.0 & 33.9 \\
\hline FS03 & 0.03 & 0.26 & 0.71 & 0.48 & 2.3 & 17.3 & 47.8 & 32.6 & 1.2 & 13.3 & 37.4 & 48.1 \\
\hline FS04 & 0.03 & 0.26 & 0.70 & 0.44 & 2.4 & 18.2 & 48.9 & 30.6 & 1.2 & 13.5 & 41.7 & 43.6 \\
\hline FS05 & 0.04 & 0.27 & 0.73 & 0.43 & 2.4 & 18.6 & 49.9 & 29.1 & 1.3 & 14.2 & 41.8 & 42.8 \\
\hline FS06 & 0.04 & 0.33 & 1.04 & 0.36 & 2.4 & 18.7 & 58.4 & 20.5 & 1.6 & 17.3 & 44.7 & 36.5 \\
\hline FS07 & 0.04 & 0.28 & 0.87 & 0.40 & 2.3 & 17.8 & 54.8 & 25.1 & 1.3 & 14.6 & 44.4 & 39.7 \\
\hline FS08 & 0.04 & 0.29 & 0.80 & 0.40 & 2.5 & 18.9 & 52.4 & 26.2 & 1.4 & 15.1 & 43.4 & 40.2 \\
\hline FS09 & 0.04 & 0.30 & 0.84 & 0.37 & 2.5 & 19.6 & 54.1 & 23.7 & 1.4 & 15.7 & 46.2 & 36.7 \\
\hline FS10 & 0.04 & 0.34 & 0.90 & 0.31 & 2.7 & 21.1 & 56.4 & 19.7 & 1.6 & 17.4 & 49.5 & 31.5 \\
\hline FS11 & 0.04 & 0.29 & 0.89 & 0.38 & 2.4 & 18.2 & 55.5 & 24.0 & 1.4 & 15.0 & 45.3 & 38.3 \\
\hline Min. & 0.03 & 0.20 & 0.67 & 0.31 & 1.8 & 13.9 & 46.5 & 19.7 & 0.9 & 10.3 & 34.4 & 31.5 \\
\hline Max. & 0.04 & 0.34 & 1.04 & 0.54 & 2.7 & 21.1 & 58.6 & 37.8 & 1.6 & 17.4 & 49.5 & 54.3 \\
\hline$A v g$. & 0.04 & 0.28 & 0.83 & 0.41 & 2.4 & 18.3 & 53.0 & 26.4 & 1.3 & 14.7 & 43.4 & 40.5 \\
\hline$S D$ & 0.005 & 0.038 & 0.118 & 0.065 & 0.220 & 1.753 & 4.229 & 5.669 & 0.196 & 1.983 & 4.529 & 6.509 \\
\hline
\end{tabular}


Table 7-4: Formulation by mass as oven-dry in scouting experiment II

\begin{tabular}{l|l|l|l|l|l|l|l|l|l}
\hline FS ID & \multicolumn{4}{|c|}{ Bulk Proportion $^{*}\left(\mathrm{unit}_{\mathrm{kg}} / \mathrm{m}^{3}\right)$} & \multicolumn{4}{c}{ Percentage by Weight, \% } \\
\hline & Cement & Fly Ash & Sand & Water & Total & Cement & lly Ash & Sand & Water \\
\hline FS01 & 66 & 299 & 947 & 483 & 1795 & 4 & 17 & 53 & 27 \\
\hline FS02 & 88 & 395 & 1268 & 351 & 2102 & 4 & 19 & 60 & 17 \\
\hline FS03 & 74 & 334 & 859 & 491 & 1758 & 4 & 19 & 49 & 28 \\
\hline FS03 & 73 & 242 & 915 & 505 & 1735 & 4 & 14 & 53 & 29 \\
\hline FS04 & 81 & 363 & 1012 & 406 & 1862 & 4 & 20 & 54 & 22 \\
\hline FS05 & 77 & 346 & 922 & 457 & 1802 & 4 & 19 & 51 & 25 \\
\hline FS06 & $\mathbf{1 4 4}$ & 433 & 1025 & 381 & 1984 & 7 & 22 & 52 & 19 \\
\hline FS06 & $\mathbf{1 9 2}$ & 431 & 973 & 388 & 1984 & 10 & 22 & 49 & 20 \\
\hline FS07 & 81 & 362 & 1060 & 416 & 1919 & 4 & 19 & 55 & 22 \\
\hline FS08 & 82 & 369 & 956 & 428 & 1835 & 4 & 20 & 52 & 23 \\
\hline FS09 & 91 & 409 & 1178 & 355 & 2034 & 4 & 20 & 58 & 17 \\
\hline FS10 & $\mathbf{1 4 7}$ & 440 & 1198 & 325 & 2110 & 7 & 21 & 57 & 15 \\
\hline FS10 & $\mathbf{1 9 9}$ & 447 & 1168 & 318 & 2131 & 9 & 21 & 55 & 15 \\
\hline FS11 & 86 & 386 & 1149 & 379 & 1999 & 4 & 19 & 57 & 19 \\
\hline Min. & 66 & 242 & 859 & 318 & 1735 & 4 & 14 & 49 & 15 \\
\hline Max. & 199 & 447 & 1268 & 505 & 2131 & 10 & 22 & 60 & 29 \\
\hline Avg. & 106 & 375 & 1045 & 406 & 1932 & 5 & 19 & 54 & 21 \\
\hline SD & 45.15 & 58.12 & 126.44 & 60.61 & 135.79 & 2.12 & 2.07 & 3.29 & 4.67 \\
\hline
\end{tabular}

* Mass of raw material per unit of fresh CLSM. 
Table 7-5: Formulation by volume as oven-dry in scouting experiment II

\begin{tabular}{|c|c|c|c|c|c|c|c|c|c|c|c|c|}
\hline \multirow[t]{2}{*}{ FS ID } & \multicolumn{4}{|c|}{ Bulk Volume as Oven-dry, $\mathrm{m}^{3} / \mathrm{m}^{3 *}$} & \multicolumn{4}{|c|}{$\begin{array}{l}\text { Percentage by Bulk Volume as } \\
\text { Oven-dry, \% }\end{array}$} & \multicolumn{4}{|c|}{$\begin{array}{l}\text { Percentage by Absolute Volume in } \\
\text { CLSM, \% }\end{array}$} \\
\hline & Cement & Fly Ash & Sand & Water & Cement & Fly Ash & Sand & Water & Cement & Fly Ash & Sand & Water \\
\hline FS01 & 0.06 & 0.23 & 0.73 & 0.48 & 3.9 & 15.1 & 48.9 & 32.2 & 2.1 & 11.7 & 37.8 & 48.3 \\
\hline FS02 & 0.08 & 0.30 & 0.92 & 0.35 & 4.7 & 18.2 & 55.8 & 21.4 & 2.8 & 15.5 & 46.6 & 35.1 \\
\hline FS03 & 0.07 & 0.25 & 0.67 & 0.49 & 4.4 & 17.1 & 45.2 & 33.2 & 2.4 & 13.1 & 35.4 & 49.1 \\
\hline FS03 & 0.06 & 0.18 & 0.71 & 0.50 & 4.4 & 12.5 & 48.7 & 34.5 & 2.3 & 9.5 & 37.7 & 50.5 \\
\hline FS04 & 0.07 & 0.28 & 0.71 & 0.41 & 4.9 & 18.8 & 48.6 & 27.7 & 2.6 & 14.3 & 42.6 & 40.6 \\
\hline FS05 & 0.07 & 0.26 & 0.67 & 0.46 & 4.7 & 17.9 & 46.1 & 31.3 & 2.4 & 13.6 & 38.3 & 45.7 \\
\hline FS06 & 0.13 & \begin{tabular}{|l|}
0.33 \\
\end{tabular} & 0.94 & 0.38 & 7.2 & 18.5 & 52.8 & 21.5 & 4.6 & 17.0 & 40.3 & 38.1 \\
\hline FS06 & 0.17 & 0.33 & $\mathbf{0 . 8 9}$ & 0.39 & 9.6 & 18.4 & 50.2 & 21.9 & 6.1 & 16.9 & 38.2 & 38.8 \\
\hline FS07 & 0.07 & 0.27 & 0.81 & 0.42 & 4.5 & 17.4 & 51.7 & 26.4 & 2.6 & 14.2 & 41.6 & 41.6 \\
\hline FS08 & 0.07 & 0.28 & 0.74 & 0.43 & 4.8 & 18.4 & 48.8 & 28.1 & 2.6 & 14.5 & 40.1 & 42.8 \\
\hline FS09 & 0.08 & 0.31 & 0.82 & 0.36 & 5.1 & 19.7 & 52.5 & 22.6 & 2.9 & 16.1 & 45.5 & 35.5 \\
\hline FS10 & 0.13 & 0.33 & 0.83 & 0.33 & 8.0 & 20.6 & 51.2 & 20.1 & 4.7 & 17.3 & 45.5 & 32.5 \\
\hline FS10 & 0.18 & 0.34 & 0.81 & 0.32 & 10.7 & 20.7 & 49.2 & 19.4 & 6.3 & 17.6 & 44.4 & 31.8 \\
\hline FS11 & 0.08 & \begin{tabular}{|l|}
0.29 \\
\end{tabular} & 0.86 & 0.38 & 4.7 & 18.1 & 53.7 & 23.5 & 2.7 & 15.1 & 44.3 & 37.9 \\
\hline Min. & 0.06 & 0.18 & 0.67 & 0.32 & 3.9 & 12.5 & 45.2 & 19.4 & 2.1 & 9.5 & 35.4 & 31.8 \\
\hline Max. & 0.18 & 0.34 & 0.94 & 0.50 & 10.7 & 20.7 & 55.8 & 34.5 & 6.3 & 17.6 & 46.6 & 50.5 \\
\hline Avg. & 0.09 & 0.28 & 0.79 & 0.41 & 5.8 & 18.0 & 50.2 & 26.0 & 3.4 & 14.7 & 41.3 & 40.6 \\
\hline$S D$ & 0.041 & 0.045 & 0.090 & 0.059 & 2.155 & 2.112 & 2.911 & 5.207 & 1.434 & 2.292 & 3.561 & 6.061 \\
\hline
\end{tabular}

* Bulk volume of raw material per unit of fresh CLSM. 


\subsubsection{Formulations of Excavatable CLSM}

Investigation of the formulation of excavatable CLSM took a multi-phase procedure. Due to the number of selected foundry sands and the variations in individual properties, each CLSM formulation was continuously redesigned to comply with standardized performances. The formulations by mass and by volume for phase I are summarized in Table 7-6 and Table 7-7, respectively. Corresponding formulations for phase II are summarized in Table 7-8 and Table 7-9. The water addition in the mixture proportions is normally distributed, $N(412,55)$ for phase I and $N(378,56)$ for phase II, which indicates water addition amount. The fresh densities are normally distributed as well, $N(1919,93)$ and $N(1947,80)$, respectively. All the parameters correlate soundly with corresponding parameters in the scouting experiments.

In summary of two phases of excavatable experiments, formulations by mass and by volume of final excavatable CLSM are presented in Table 7-10 and Table 7-11, respectively. Cement, which significantly influences the strength of CLSM, varies its

proportion due to the variation of foundry sand. In fresh CLSM, both water and sand occupy more than a third by absolute volume (water averaged $40 \%$, and sand averaged $43 \%)$. 
Table 7-6: Formulation by mass as oven-dry in excavatable CLSM experiment phase I

\begin{tabular}{l|l|l|l|l|l|l|l|l|l}
\hline FS ID & \multicolumn{4}{|l|}{ Bulk Proportion ${ }^{*}\left(\right.$ unit: $\left.\mathrm{kg} / \mathrm{m}^{3}\right)$} & \multicolumn{3}{|c}{ Percentage by Weight, \% } \\
\hline & Cement & Fly Ash & Sand & Water & Total & Cement & lly Ash & Sand & Water \\
\hline FS01 & 66 & 355 & 970 & 448 & 1839 & 3.6 & 19.3 & 52.8 & 24.4 \\
\hline FS02 & 73 & 394 & 1264 & 343 & 2074 & 3.5 & 19.0 & 61.0 & 16.5 \\
\hline FS03 & 63 & 340 & 876 & 504 & 1784 & 3.5 & 19.1 & 49.1 & 28.3 \\
\hline FS04 & 70 & 379 & 1055 & 405 & 1910 & 3.7 & 19.9 & 55.3 & 21.2 \\
\hline FS05 & 69 & 371 & 988 & 445 & 1872 & 3.7 & 19.8 & 52.8 & 23.8 \\
\hline FS06 & 83 & 448 & 1059 & 364 & 1954 & 4.2 & 22.9 & 54.2 & 18.6 \\
\hline FS07 & 65 & 352 & 1031 & 428 & 1876 & 3.5 & 18.8 & 55.0 & 22.8 \\
\hline FS08 & 67 & 360 & 934 & 460 & 1822 & 3.7 & 19.8 & 51.3 & 25.3 \\
\hline FS09 & 74 & 400 & 1151 & 355 & 1979 & 3.7 & 20.2 & 58.1 & 17.9 \\
\hline FS10 & 81 & 436 & 1180 & 345 & 2042 & 4.0 & 21.4 & 57.8 & 16.9 \\
\hline FS11 & 66 & 358 & 1066 & 440 & 1931 & 3.4 & 18.5 & 55.2 & 22.8 \\
\hline FS12 & 65 & 353 & 1156 & 359 & 1933 & 3.4 & 18.3 & 59.8 & 18.6 \\
\hline FS13 & 75 & 405 & 1218 & 349 & 2048 & 3.7 & 19.8 & 59.5 & 17.1 \\
\hline FS14 & 72 & 389 & 1154 & 392 & 2006 & 3.6 & 19.4 & 57.5 & 19.5 \\
\hline FS15 & 74 & 400 & 818 & 489 & 1782 & 4.2 & 22.5 & 45.9 & 27.5 \\
\hline FS16 & 64 & 345 & 975 & 464 & 1849 & 3.5 & 18.7 & 52.8 & 25.1 \\
\hline Min. & 63 & 340 & 818 & 343 & 1782 & 3.4 & 18.3 & 45.9 & 16.5 \\
\hline Max. & 83 & 448 & 1264 & 504 & 2074 & 4.2 & 22.9 & 61.0 & 28.3 \\
\hline Avg. & 70 & 380 & 1056 & 412 & 1919 & 3.7 & 19.8 & 54.9 & 21.6 \\
\hline SD & 5.97 & 32.03 & 125.63 & 54.68 & 93.23 & 0.25 & 1.35 & 4.08 & 3.88 \\
\hline
\end{tabular}

* Mass of raw material per unit of fresh CLSM. 
Table 7-7: Formulation by volume as oven-dry in excavatable CLSM experiment phase I

\begin{tabular}{|c|c|c|c|c|c|c|c|c|c|c|c|c|}
\hline \multirow[t]{2}{*}{ FS ID } & \multicolumn{4}{|c|}{ Bulk Volume as Oven-dry, $\mathrm{m}^{3} / \mathrm{m}^{3 *}$} & \multicolumn{4}{|c|}{$\begin{array}{l}\text { Percentage by Bulk Volume as } \\
\text { Oven-dry, } \%\end{array}$} & \multicolumn{4}{|c|}{ Percentage by Absolute Volume, $\%$} \\
\hline & Cement & Fly Ash & Sand & Water & Cement & Fly Ash & Sand & Water & Cement & Fly Ash & Sand & Water \\
\hline FS01 & 0.06 & 0.27 & 0.75 & 0.45 & 3.8 & 17.6 & 49.3 & 29.3 & 2.1 & 14.0 & 38.9 & 45.0 \\
\hline FS02 & 0.06 & 0.30 & 0.92 & 0.34 & 4.0 & 18.4 & 56.5 & 21.2 & 2.4 & 15.7 & 47.2 & 34.8 \\
\hline FS03 & 0.06 & 0.26 & 0.68 & 0.50 & 3.7 & 17.2 & 45.5 & 33.7 & 2.0 & 13.1 & 35.4 & 49.5 \\
\hline FS04 & 0.06 & 0.29 & 0.74 & 0.41 & 4.1 & 19.2 & 49.6 & 27.1 & 2.2 & 14.6 & 43.5 & 39.7 \\
\hline FS05 & 0.06 & 0.28 & 0.72 & 0.45 & 4.0 & 18.6 & 47.8 & 29.5 & 2.1 & 14.2 & 40.1 & 43.5 \\
\hline FS06 & 0.07 & 0.34 & 0.97 & 0.36 & 4.2 & 19.4 & 55.5 & 20.9 & 2.7 & 17.9 & 42.4 & 37.1 \\
\hline FS07 & 0.06 & 0.27 & 0.79 & 0.43 & 3.7 & 17.3 & 51.3 & 27.7 & 2.1 & 14.0 & 40.8 & 43.1 \\
\hline FS08 & 0.06 & 0.27 & 0.73 & 0.46 & 3.9 & 18.0 & 47.8 & 30.4 & 2.1 & 14.0 & 38.6 & 45.4 \\
\hline FS09 & 0.07 & 0.30 & 0.81 & 0.36 & 4.3 & 19.8 & 52.7 & 23.2 & 2.4 & 16.0 & 45.4 & 36.2 \\
\hline FS10 & 0.07 & 0.33 & 0.82 & 0.35 & 4.6 & 21.1 & 52.2 & 22.1 & 2.6 & 17.3 & 45.3 & 34.9 \\
\hline FS11 & 0.06 & 0.27 & 0.80 & 0.44 & 3.7 & 17.2 & 51.0 & 28.0 & 2.1 & 13.9 & 40.6 & 43.5 \\
\hline FS12 & 0.06 & 0.27 & 0.77 & 0.36 & 4.0 & 18.4 & 52.9 & 24.7 & 2.2 & 14.6 & 45.3 & 37.9 \\
\hline FS13 & 0.07 & 0.31 & 0.81 & 0.33 & 4.4 & 20.2 & 53.5 & 21.9 & 2.4 & 16.2 & 47.6 & 33.8 \\
\hline FS14 & 0.06 & 0.29 & 0.83 & 0.39 & 4.0 & 18.6 & 52.6 & 24.8 & 2.2 & 14.8 & 45.1 & 37.9 \\
\hline FS15 & 0.07 & 0.30 & 0.78 & 0.49 & 4.0 & 18.5 & 47.5 & 29.9 & 2.3 & 15.5 & 33.9 & 48.3 \\
\hline FS16 & 0.06 & 0.26 & 0.84 & 0.46 & 3.5 & 16.1 & 51.8 & 28.6 & 2.0 & 13.5 & 38.4 & 46.1 \\
\hline Min. & 0.06 & 0.26 & 0.68 & 0.33 & 3.5 & 16.1 & 45.5 & 20.9 & 2.0 & 13.1 & 33.9 & 33.8 \\
\hline Max. & 0.07 & 0.34 & 0.97 & 0.50 & 4.6 & 21.1 & 56.5 & 33.6 & 2.7 & 17.9 & 47.6 & 49.5 \\
\hline$A v g$. & 0.06 & 0.29 & 0.80 & 0.41 & 4.0 & 18.5 & 51.1 & 26.4 & 2.2 & 14.9 & 41.8 & 41.0 \\
\hline$S D$ & 0.005 & 0.024 & 0.073 & 0.056 & 0.286 & 1.273 & 3.007 & 3.837 & 0.206 & 1.364 & 4.109 & 5.102 \\
\hline
\end{tabular}

* Bulk volume of raw material per unit of fresh CLSM. 
Table 7-8: Formulation by mass as oven-dry in excavatable CLSM experiment phase II

\begin{tabular}{l|l|l|l|l|l|l|l|l|l}
\hline FS ID & \multicolumn{3}{|l|}{ Bulk Proportion ${ }^{*}\left(\mathrm{unit}_{\mathrm{kg}} \mathrm{m}^{3}\right.$ ) } & \multicolumn{4}{l}{ Percentage by Weight, \% } \\
\hline & Cement & Fly Ash & Sand & Water & Total & Cement & Fly Ash & Sand & Water \\
\hline FS05 & 37 & 334 & 972 & 474 & 1817 & 2.0 & 18.4 & 53.5 & 26.1 \\
\hline FS06 & 64 & 429 & 1094 & 378 & 1965 & 3.2 & 21.8 & 55.7 & 19.3 \\
\hline FS08 & 41 & 370 & 998 & 395 & 1803 & 2.3 & 20.5 & 55.4 & 21.9 \\
\hline FS09 & 45 & 404 & 1207 & 320 & 1976 & 2.3 & 20.4 & 61.1 & 16.2 \\
\hline FS10 & 32 & 463 & 1212 & 329 & 2036 & 1.6 & 22.8 & 59.5 & 16.2 \\
\hline FS13 & 37 & 394 & 1242 & 348 & 2020 & 1.8 & 19.5 & 61.5 & 17.2 \\
\hline FS14 & 15 & 398 & 1182 & 394 & 1990 & 0.8 & 20.0 & 59.4 & 19.8 \\
\hline FS16 & 94 & 361 & 1020 & 426 & 1901 & 4.9 & 19.0 & 53.7 & 22.4 \\
\hline FS17 & 43 & 387 & 1118 & 423 & 1972 & 2.2 & 19.6 & 56.7 & 21.5 \\
\hline FS17 & 25 & 444 & 1225 & 291 & 1984 & 1.2 & 22.4 & 61.7 & 14.7 \\
\hline Min. & 15 & 334 & 972 & 291 & 1803 & 0.8 & 18.4 & 53.5 & 14.7 \\
\hline Max. & 94 & 463 & 1242 & 474 & 2036 & 4.9 & 22.8 & 61.7 & 26.1 \\
\hline Avg. & 43 & 398 & 1127 & 378 & 1947 & 2.2 & 20.5 & 57.8 & 19.5 \\
\hline SD & 21.99 & 39.05 & 101.52 & 56.10 & 80.28 & 1.15 & 1.46 & 3.20 & 3.53 \\
\hline
\end{tabular}

* Mass of raw material per unit of fresh CLSM. 
Table 7-9: Formulation by volume as oven-dry in excavatable CLSM experiment phase II

\begin{tabular}{|c|c|c|c|c|c|c|c|c|c|c|c|c|}
\hline \multirow[t]{2}{*}{ FS ID } & \multicolumn{4}{|c|}{ Bulk Volume as Oven-dry, $\mathrm{m}^{3} / \mathrm{m}^{3 *}$} & \multicolumn{4}{|c|}{$\begin{array}{l}\text { Percentage by Bulk Volume as } \\
\text { Oven-dry, } \%\end{array}$} & \multicolumn{4}{|c|}{ Percentage by Absolute Volume, $\%$} \\
\hline & Cement & Fly Ash & Sand & Water & Cement & Fly Ash & Sand & Water & Cement & Fly Ash & Sand & Water \\
\hline FS05 & 0.03 & 0.25 & 0.71 & 0.47 & 2.2 & 17.2 & 48.3 & 32.3 & 1.2 & 12.9 & 39.5 & 46.4 \\
\hline FS06 & 0.06 & 0.32 & 1.00 & 0.38 & 3.2 & 18.5 & 56.8 & 21.5 & 2.0 & 16.9 & 43.1 & 38.0 \\
\hline FS08 & 0.04 & 0.28 & 0.77 & 0.39 & 2.4 & 18.8 & 52.2 & 26.6 & 1.3 & 14.9 & 43.1 & 40.6 \\
\hline FS09 & 0.04 & 0.31 & 0.84 & 0.32 & 2.6 & 20.2 & 55.9 & 21.2 & 1.5 & 16.5 & 48.6 & 33.4 \\
\hline FS10 & 0.03 & 0.35 & 0.84 & 0.33 & 1.8 & 22.7 & 54.2 & 21.3 & 1.0 & 18.3 & 47.7 & 33.0 \\
\hline FS13 & 0.03 & 0.30 & 0.82 & 0.35 & 2.2 & 20.0 & 54.6 & 23.3 & 1.2 & 15.7 & 48.0 & 35.2 \\
\hline FS14 & 0.01 & 0.30 & 0.85 & 0.39 & 0.9 & 19.3 & 54.5 & 25.3 & 0.5 & 15.2 & 46.2 & 38.2 \\
\hline FS16 & 0.08 & 0.27 & 0.88 & 0.43 & 5.0 & 16.5 & 52.9 & 25.7 & 3.0 & 14.2 & 40.4 & 42.5 \\
\hline FS17 & 0.04 & 0.29 & 0.72 & 0.42 & 2.6 & 19.9 & 48.8 & 28.7 & 1.4 & 15.0 & 41.9 & 41.8 \\
\hline FS17 & 0.02 & 0.34 & 0.79 & 0.29 & 1.5 & 23.4 & 54.9 & 20.3 & 0.8 & 18.6 & 49.6 & 31.0 \\
\hline Min. & 0.01 & 0.25 & 0.71 & 0.29 & 0.9 & 16.5 & 48.3 & 20.3 & 0.5 & 12.9 & 39.5 & 31.0 \\
\hline Max. & 0.08 & 0.35 & 1.00 & 0.47 & 5.0 & 23.4 & 56.8 & 32.3 & 3.0 & 18.6 & 49.6 & 46.4 \\
\hline$A v g$. & 0.04 & 0.30 & 0.82 & 0.38 & 2.4 & 19.7 & 53.3 & 24.6 & 1.4 & 15.8 & 44.8 & 38.0 \\
\hline$S D$ & 0.020 & 0.031 & 0.084 & 0.055 & 1.104 & 2.159 & 2.831 & 3.859 & 0.695 & 1.782 & 3.647 & 4.888 \\
\hline
\end{tabular}

* Bulk volume of raw material per unit of fresh CLSM. 
Table 7-10: Final formulation by mass as oven-dry for excavatable CLSM

\begin{tabular}{l|l|l|l|l|l|l|l|l|l}
\hline FS ID & \multicolumn{3}{|l}{ Bulk Proportion ${ }^{*}\left(\mathrm{unit} \mathrm{kg} / \mathrm{m}^{3}\right)$} & \multicolumn{3}{|c}{ Percentage by Weight, \% } \\
\hline & Cement & Fly Ash & Sand & Water & Total & Cement & Fly Ash & Sand & Water \\
\hline FS01 & 66 & 355 & 970 & 448 & 1839 & 3.6 & 19.3 & 52.8 & 24.4 \\
\hline FS02 & 73 & 394 & 1264 & 343 & 2074 & 3.5 & 19.0 & 61.0 & 16.5 \\
\hline FS03 & 63 & 340 & 876 & 504 & 1784 & 3.5 & 19.1 & 49.1 & 28.3 \\
\hline FS04 & 70 & 379 & 1055 & 405 & 1910 & 3.7 & 19.9 & 55.3 & 21.2 \\
\hline FS05 & 37 & 334 & 972 & 474 & 1817 & 2.0 & 18.4 & 53.5 & 26.1 \\
\hline FS06 & 64 & 429 & 1094 & 378 & 1965 & 3.2 & 21.8 & 55.7 & 19.3 \\
\hline FS07 & 65 & 352 & 1031 & 428 & 1876 & 3.5 & 18.8 & 55.0 & 22.8 \\
\hline FS08 & 41 & 370 & 998 & 395 & 1803 & 2.3 & 20.5 & 55.4 & 21.9 \\
\hline FS09 & 45 & 404 & 1207 & 320 & 1976 & 2.3 & 20.4 & 61.1 & 16.2 \\
\hline FS10 & 32 & 463 & 1212 & 329 & 2036 & 1.6 & 22.8 & 59.5 & 16.2 \\
\hline FS11 & 66 & 358 & 1066 & 440 & 1931 & 3.4 & 18.5 & 55.2 & 22.8 \\
\hline FS12 & 65 & 353 & 1156 & 359 & 1933 & 3.4 & 18.3 & 59.8 & 18.6 \\
\hline FS13 & 37 & 394 & 1242 & 348 & 2020 & 1.8 & 19.5 & 61.5 & 17.2 \\
\hline FS15 & 74 & 400 & 818 & 489 & 1782 & 4.2 & 22.5 & 45.9 & 27.5 \\
\hline FS16 & 94 & 361 & 1020 & 426 & 1901 & 4.9 & 19.0 & 53.7 & 22.4 \\
\hline FS17 & 25 & 444 & 1225 & 291 & 1984 & 1.2 & 22.4 & 61.7 & 14.7 \\
\hline Min. & 25 & 334 & 818 & 291 & 1782 & 1.2 & 18.3 & 45.9 & 14.7 \\
\hline Max. & 94 & 463 & 1264 & 504 & 2074 & 4.9 & 22.8 & 61.7 & 28.3 \\
\hline Avg. & 57.3 & 383.1 & 1075.4 & 398.6 & 1914.4 & 3.0 & 20.0 & 56.0 & 21.0 \\
\hline SD & 18.81 & 37.77 & 133.64 & 63.79 & 92.02 & 1.02 & 1.55 & 4.58 & 4.25 \\
\hline
\end{tabular}

* Mass of raw material per unit of fresh CLSM. 
Table 7-11: Final formulation by volume as oven-dry for excavatable CLSM

\begin{tabular}{|c|c|c|c|c|c|c|c|c|c|c|c|c|}
\hline \multirow[t]{2}{*}{ FS ID } & \multicolumn{4}{|c|}{ Bulk Volume as Oven-dry, $\mathrm{m}^{3} / \mathrm{m}^{3 *}$} & \multicolumn{4}{|c|}{$\begin{array}{l}\text { Percentage by Bulk Volume as } \\
\text { Oven-dry, } \%\end{array}$} & \multicolumn{4}{|c|}{ Percentage by Absolute Volume, $\%$} \\
\hline & Cement & Fly Ash & Sand & Water & Cement & Fly Ash & Sand & Water & Cement & Fly Ash & Sand & Water \\
\hline FS01 & 0.06 & 0.27 & 0.75 & 0.45 & 3.8 & 17.6 & 49.3 & 29.3 & 2.1 & 14.0 & 38.9 & 45.0 \\
\hline FS02 & 0.06 & 0.30 & 0.92 & 0.34 & 4.0 & 18.4 & 56.5 & 21.2 & 2.4 & 15.7 & 47.2 & 34.8 \\
\hline FS03 & 0.06 & 0.26 & 0.68 & 0.50 & 3.7 & 17.2 & 45.5 & 33.7 & 2.0 & 13.1 & 35.4 & 49.5 \\
\hline FS04 & 0.06 & 0.29 & 0.74 & 0.41 & 4.1 & 19.2 & 49.6 & 27.1 & 2.2 & 14.6 & 43.5 & 39.7 \\
\hline FS05 & 0.03 & 0.25 & 0.71 & 0.47 & 2.2 & 17.2 & 48.3 & 32.3 & 1.2 & 12.9 & 39.5 & 46.4 \\
\hline FS06 & 0.06 & 0.32 & 1.00 & 0.38 & 3.2 & 18.5 & 56.8 & 21.5 & 2.0 & 16.9 & 43.1 & 38.0 \\
\hline FS07 & 0.06 & 0.27 & 0.79 & 0.43 & 3.7 & 17.3 & 51.3 & 27.7 & 2.1 & 14.0 & 40.8 & 43.1 \\
\hline FS08 & 0.04 & 0.28 & 0.77 & 0.39 & 2.4 & 18.8 & 52.2 & 26.6 & 1.3 & 14.9 & 43.1 & 40.6 \\
\hline FS09 & 0.04 & 0.31 & 0.84 & 0.32 & 2.6 & 20.2 & 55.9 & 21.2 & 1.5 & 16.5 & 48.6 & 33.4 \\
\hline FS10 & 0.03 & 0.35 & 0.84 & 0.33 & 1.8 & 22.7 & 54.2 & 21.3 & 1.0 & 18.3 & 47.7 & 33.0 \\
\hline FS11 & 0.06 & 0.27 & 0.80 & 0.44 & 3.7 & 17.2 & 51.0 & 28.0 & 2.1 & 13.9 & 40.6 & 43.5 \\
\hline FS12 & 0.06 & 0.27 & 0.77 & 0.36 & 4.0 & 18.4 & 52.9 & 24.7 & 2.2 & 14.6 & 45.3 & 37.9 \\
\hline FS13 & 0.03 & 0.30 & 0.82 & 0.35 & 2.2 & 20.0 & 54.6 & 23.3 & 1.2 & 15.7 & 48.0 & 35.2 \\
\hline FS15 & 0.07 & 0.30 & 0.78 & 0.49 & 4.0 & 18.5 & 47.5 & 29.9 & 2.3 & 15.5 & 33.9 & 48.3 \\
\hline FS16 & 0.08 & 0.27 & 0.88 & 0.43 & 5.0 & 16.5 & 52.9 & 25.7 & 3.0 & 14.2 & 40.4 & 42.5 \\
\hline FS17 & 0.02 & 0.34 & 0.79 & 0.29 & 1.5 & 23.4 & 54.9 & 20.3 & 0.8 & 18.6 & 49.6 & 31.0 \\
\hline Min. & 0.02 & 0.25 & 0.68 & 0.29 & 1.5 & 16.5 & 45.5 & 20.3 & 0.8 & 12.9 & 33.9 & 31 \\
\hline Max. & 0.08 & 0.35 & 1 & 0.5 & 5 & 23.4 & 56.8 & 33.7 & 3 & 18.6 & 49.6 & 49.5 \\
\hline$A v g$. & 0.051 & 0.291 & 0.805 & 0.399 & 3.244 & 18.819 & 52.088 & 25.863 & 1.838 & 15.213 & 42.850 & 40.119 \\
\hline$S D$ & 0.017 & 0.029 & 0.080 & 0.064 & 0.997 & 1.951 & 3.374 & 4.189 & 0.600 & 1.682 & 4.703 & 5.689 \\
\hline
\end{tabular}

\footnotetext{
* Bulk volume of raw material per unit of fresh CLSM.
} 


\subsubsection{Formulations of Structural CLSM}

Formulations of structural CLSM were based on the formulations in scouting experiments (Section 7.1.3), formulations of excavatable CLSM experiments (Section 7.1.4), and specifications for PennDOT Type C flowable fill (Table 2-10). The formulations of structural CLSM are summarized in Table 7-12 and Table 7-13. Water addition is normally distributed, $N(405,67)$, which is close to the distribution of water addition for excavatable CLSM, $N(399,64)$. Thus, water addition is not significantly correlated with type of CLSM. It may be determined exclusively by cement content, or sand gradation and shape. Fresh density of structural CLSM is normally distributed, $N(1922,109)$, which is close to the distribution of excavatable CLSM, $N(1914,92)$. This is primarily true because the major difference between excavatable CLSM and structural CLSM is the proportion of cement, and the percentage by mass of cement is relatively low, $3 \%$ and $12 \%$, respectively. 
Table 7-12: Formulation by mass as oven-dry in structural CLSM experiment

\begin{tabular}{l|l|l|l|l|l|l|l|l|l}
\hline FS ID & \multicolumn{3}{|l}{ Bulk Proportion ${ }^{*}\left(\right.$ unit: $^{\mathrm{kg}} / \mathrm{m}^{3}$ ) } & \multicolumn{4}{l}{ Percentage by Weight, \% } \\
\hline & Cement & Fly Ash & Sand & Water & Total & Cement & Fly Ash & Sand & Water \\
\hline FS01 & 215 & 291 & 779 & 488 & 1773 & 12 & 16 & 44 & 28 \\
\hline FS02 & 257 & 347 & 1113 & 338 & 2055 & 13 & 17 & 54 & 16 \\
\hline FS03 & 227 & 306 & 763 & 491 & 1787 & 13 & 17 & 43 & 27 \\
\hline FS04 & 235 & 318 & 873 & 447 & 1874 & 13 & 17 & 47 & 24 \\
\hline FS05 & 214 & 321 & 914 & 396 & 1845 & 12 & 17 & 50 & 21 \\
\hline FS06 & 231 & 389 & 974 & 401 & 1995 & 12 & 20 & 49 & 20 \\
\hline FS07 & 230 & 310 & 909 & 428 & 1877 & 12 & 17 & 48 & 23 \\
\hline FS08 & 135 & 303 & 802 & 504 & 1744 & 8 & 17 & 46 & 29 \\
\hline FS09 & 168 & 378 & 1104 & 311 & 1960 & 9 & 19 & 56 & 16 \\
\hline FS10 & 124 & 450 & 1175 & 320 & 2068 & 6 & 22 & 57 & 15 \\
\hline FS11 & 257 & 347 & 992 & 371 & 1967 & 13 & 18 & 50 & 19 \\
\hline FS12 & 247 & 333 & 1085 & 348 & 2013 & 12 & 17 & 54 & 17 \\
\hline FS13 & 206 & 371 & 1164 & 329 & 2071 & 10 & 18 & 56 & 16 \\
\hline FS15 & 277 & 374 & 732 & 471 & 1854 & 15 & 20 & 39 & 25 \\
\hline FS16 & 435 & 284 & 790 & 433 & 1941 & 22 & 15 & 41 & 22 \\
\hline Min. & 124 & 284 & 732 & 311 & 1744 & 6 & 15 & 39 & 15 \\
\hline Max. & 435 & 450 & 1175 & 504 & 2071 & 22 & 22 & 57 & 29 \\
\hline Avg. & 230.5 & 341.5 & 944.6 & 405.1 & 1921.6 & 12.1 & 17.8 & 48.9 & 21.2 \\
\hline SD & 71.30 & 44.71 & 154.68 & 66.73 & 108.77 & 3.56 & 1.78 & 5.69 & 4.71 \\
\hline
\end{tabular}

* Mass of raw material per unit of fresh CLSM. 
Table 7-13: Formulation by volume as oven-dry in structural CLSM experiment

\begin{tabular}{|c|c|c|c|c|c|c|c|c|c|c|c|c|}
\hline \multirow[t]{2}{*}{ FS ID } & \multicolumn{4}{|c|}{ Bulk vVlume as Oven-dry, $\mathrm{m}^{3} / \mathrm{m}^{3 *}$} & \multicolumn{4}{|c|}{$\begin{array}{l}\text { Percentage by Bulk Volume as Oven- } \\
\text { dry, } \%\end{array}$} & \multicolumn{4}{|c|}{ Percentage by Absolute Volume, \% } \\
\hline & Cement & Fly Ash & Sand & Water & Cement & Fly Ash & Sand & Water & Cement & Fly Ash & Sand & Water \\
\hline FS01 & 0.19 & 0.22 & 0.61 & 0.49 & 13 & 15 & 40 & 32 & 7 & 12 & 32 & 50 \\
\hline FS02 & 0.23 & 0.26 & 0.81 & 0.34 & 14 & 16 & 49 & 21 & 8 & 14 & 42 & 35 \\
\hline FS03 & 0.20 & 0.23 & 0.59 & 0.49 & 13 & 15 & 39 & 32 & 7 & 12 & 32 & 49 \\
\hline FS04 & 0.21 & 0.24 & 0.61 & 0.45 & 14 & 16 & 41 & 30 & 7 & 12 & 36 & 44 \\
\hline FS05 & 0.19 & 0.24 & 0.67 & 0.40 & 13 & 16 & 45 & 26 & 7 & 13 & 39 & 41 \\
\hline FS06 & 0.20 & 0.29 & 0.89 & 0.40 & 11 & 16 & 50 & 22 & 7 & 15 & 38 & 40 \\
\hline FS07 & 0.20 & 0.24 & 0.70 & 0.43 & 13 & 15 & 45 & 27 & 7 & 12 & 36 & 44 \\
\hline FS08 & 0.12 & 0.23 & 0.62 & 0.50 & 8 & 16 & 42 & 34 & 4 & 12 & 34 & 50 \\
\hline FS09 & 0.15 & 0.29 & 0.77 & 0.31 & 10 & 19 & 51 & 21 & 6 & 16 & 45 & 33 \\
\hline FS10 & 0.11 & 0.34 & 0.81 & 0.32 & 7 & 22 & 51 & 20 & 4 & 18 & 45 & 33 \\
\hline FS11 & 0.23 & 0.26 & 0.75 & 0.37 & 14 & 16 & 46 & 23 & 8 & 14 & 39 & 38 \\
\hline FS12 & 0.22 & 0.25 & 0.72 & 0.35 & 14 & 16 & 47 & 23 & 8 & 14 & 42 & 36 \\
\hline FS13 & 0.18 & 0.28 & 0.77 & 0.33 & 12 & 18 & 49 & 21 & 7 & 15 & 45 & 33 \\
\hline FS15 & 0.25 & 0.28 & 0.70 & 0.47 & 14 & 17 & 41 & 28 & 9 & 14 & 30 & 46 \\
\hline FS16 & 0.38 & 0.22 & 0.68 & 0.43 & 22 & 13 & 40 & 25 & 14 & 11 & 31 & 44 \\
\hline Min. & 0.11 & 0.22 & 0.59 & 0.31 & 7 & 13 & 39 & 20 & 4 & 11 & 30 & 33 \\
\hline Max. & 0.38 & 0.34 & 0.89 & 0.5 & 22 & 22 & 51 & 34 & 14 & 18 & 45 & 50 \\
\hline$A v g$. & 0.2 & 0.3 & 0.7 & 0.4 & 12.8 & 16.4 & 45.1 & 25.7 & 7.3 & 13.6 & 37.7 & 41.1 \\
\hline$S D$ & 0.06 & 0.03 & 0.09 & 0.07 & 3.38 & 2.06 & 4.32 & 4.64 & 2.29 & 1.88 & 5.27 & 6.22 \\
\hline
\end{tabular}




\subsection{CLSM Mixing Procedure and Sample Preparation}

After a mixture formulation was established, mixing and sample preparation were performed using the following procedures:

Step 1: Proportioning and blending dry materials.

The dry materials (cement, fly ash, foundry sands) were weighed according to formulations presented in Sections 7.1.3, 7.1.4, and 7.1.5. They were added into a 0.057

$\mathrm{m}^{3}$ steel concrete mixer by sequences of $1 / 3$ foundry sand, $1 / 2$ fly ash, $1 / 2$ cement, $1 / 3$ foundry sand, $1 / 2$ fly ash, 1/2 cement, and $1 / 3$ foundry sand. The blending of these dry materials lasted 10-15 minutes prior to the addition of water.

Step 2: Measuring and adding water.

After proportioning and blending of the dry materials, water was weighed and prepared according to the corresponding formulation of the individual batch. It was divided into four equal parts. Initially, two parts were added to the dry mixture. The blending of moist materials lasted 5-10 minutes. The remaining water was added in small increments followed by additional flow consistency measurements until a flow greater than $20.3 \mathrm{~cm}$ was achieved [ASTM D 6103 1997]. Once this point was reached, any remaining water or supplemented water was weighed and subtracted or added to the original formulation. 
Step 3: Filling molds and performing fresh CLSM tests.

After thorough mixing, all cylinders or containers were filled with fresh CLSM and its fresh properties were tested for parameters listed in Table 7-1. Samples in cylinders were made in accordance with ASTM C 192 except that the samples were not rodded or vibrated.

Step 4: Curing samples.

After placement, cylinder samples for strength tests were placed in an open area with air contact and temperature at $23{ }^{\circ} \mathrm{C}$ for one day. After that period, the forms were removed and the CLSM cylinders were placed into a curing chamber $\left(23^{\circ} \mathrm{C}, 100 \% \mathrm{RH}\right)$ until the date of testing. The steel proctor mold containing CLSM for hydraulic conductivity testing was placed into curing chamber immediately after it was cast.

\subsection{CLSM Testing Procedure and Results}

To characterize the behavior of CLSM both in fresh and hardened condition, critical parameters listed in Table 7-1 were selected to investigate. The performances on these parameters determine whether foundry sands are qualified as a major component integrated into CLSM matrix. The investigation was divided into two parts, performances of fresh CLSM and performances of hardened CLSM. 


\subsubsection{Fresh CLSM}

\subsubsection{Flow Consistency}

The workability of the CLSM was determined by its flow consistency in accordance with ASTM D 6103, "Standard Test Method for Flow Consistency of Controlled Low Strength Material." This procedure involves a bottomless, plastic, cylindrical mold resting on a smooth, non-absorbent, metal surface. The mold was filled with fresh CLSM and vertically removed over a 10-second interval. The diameter of the resulting spread of fresh CLSM was measured to determine the flow consistency [ASTM D 6103 1997, Van Tassel 1999]. In this experiment, water was added to each mixture until the spread diameter was greater than $20.3 \mathrm{~cm}$ to ensure that the fresh CLSM was self-leveling and self-compacting.

\subsubsection{Bleeding and Settlement}

The volume of water collected on the surface of the fresh CLSM cylinders $(\phi 10.2$ x $20.4 \mathrm{~cm}$ ) was taken as a measurement of bleeding until the bleeding stops. The settlement was determined by measuring the change in height from fresh CLSM cylinders ( $\phi 10.2 \times 20.4 \mathrm{~cm})$ to hardened CLSM cylinders. Both measurements were performed on those cylinder samples for strength measurements. The results of bleeding and settlement of excavatable CLSM phase I and II, and structural CLSM based on $\phi 10.2 \times 20.4 \mathrm{~cm}$ cylinders, are summarized in Table 7-14, Table 7-15, and Table 7-16, respectively. 
Table 7-14: Bleeding and settlement of excavatable CLSM phase I

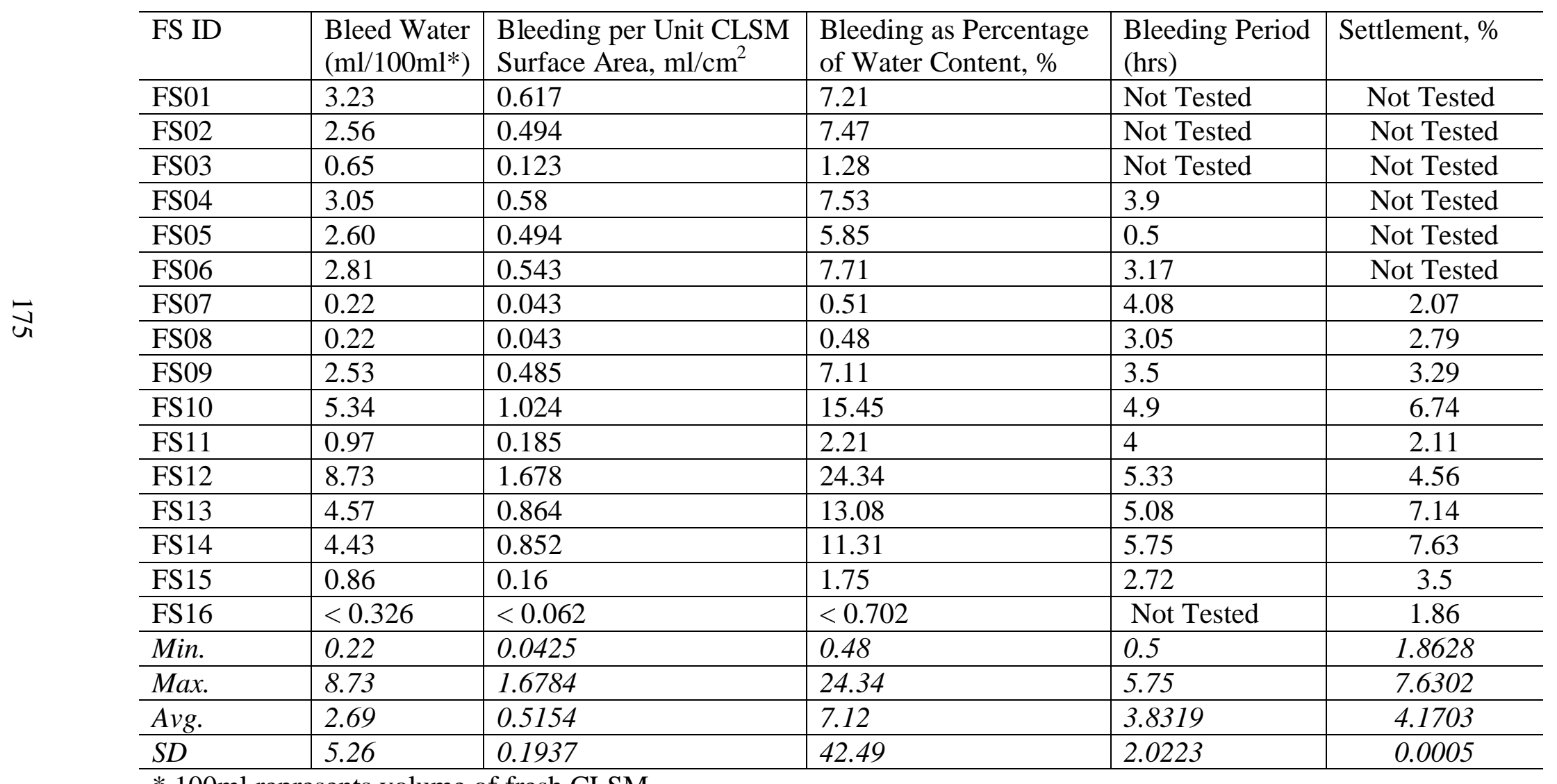

* $100 \mathrm{ml}$ represents volume of fresh CLSM. 
Table 7-15: Bleeding and settlement of excavatable CLSM phase II

\begin{tabular}{ll|l|l|l|l|l}
\hline FS ID & $\begin{array}{l}\text { Bleed Water } \\
\left(\mathrm{ml} / 100 \mathrm{ml}^{*}\right)\end{array}$ & $\begin{array}{l}\text { Bleeding per Unit } \\
\text { CLSM Surface Area, } \\
\mathrm{ml} / \mathrm{cm}^{2}\end{array}$ & $\begin{array}{l}\text { Bleeding as Percentage } \\
\text { of Water Content, \% }\end{array}$ & $\begin{array}{l}\text { Bleeding } \\
\text { Period (hrs) }\end{array}$ & $\begin{array}{l}\text { Settlement, } \\
\%\end{array}$ \\
\hline$\vec{\sigma}$ & 0.326 & 0.062 & 0.689 & 4.00 & 2.07 \\
\hline FS05 & 3.874 & 0.753 & 10.237 & 3.38 & 8.19 \\
\hline FS06 & 0.225 & 0.043 & 0.572 & 2.87 & 3.48 \\
\hline FS09 & 2.449 & 0.469 & 7.644 & 3.12 & 3.69 \\
\hline FS10 & 2.900 & 0.555 & 2.900 & 5.67 & 4.16 \\
\hline FS13 & 5.754 & 1.086 & 16.553 & 3.55 & 9.42 \\
\hline FS14 & 5.078 & 0.963 & 5.078 & 6.77 & Not Tested \\
\hline FS16 & 0.000 & 0.000 & 0.000 & 0 & 0.08 \\
\hline FS17 & 3.965 & 0.740 & 9.367 & 2.75 & 6.19 \\
\hline FS17 & 6.146 & 1.148 & 20.947 & 3.00 & 6.14 \\
\hline Min. & 0 & 0 & 0 & 0 & 0.08 \\
\hline Max. & 6.146 & 1.148 & 20.947 & 6.77 & 9.42 \\
\hline Avg. & 3.1 & 0.6 & 7.4 & 3.5 & 4.8 \\
\hline SD & 2.30 & 0.43 & & 1.80 & 2.95 \\
\hline
\end{tabular}

* 100ml represents volume of fresh CLSM. 
Table 7-16: Bleeding and settlement of structural CLSM

\begin{tabular}{l|l|l|l|l|l}
\hline FS ID & $\begin{array}{l}\text { Bleed Water } \\
\left(\mathrm{ml} / 100 \mathrm{ml}^{*}\right)\end{array}$ & $\begin{array}{l}\text { Bleeding per Unit CLSM } \\
\text { Surface Area, ml/cm }\end{array}$ & $\begin{array}{l}\text { Bleeding as Percentage } \\
\text { of Water Content, } \%\end{array}$ & $\begin{array}{l}\text { Bleeding Period } \\
\text { (hrs })\end{array}$ & $\begin{array}{l}\text { Settlement, } \\
\%\end{array}$ \\
\hline FS01 & 0.260 & 0.049 & 0.533 & 2.62 & 2.88 \\
\hline FS02 & 2.049 & 0.395 & 6.160 & 2.95 & 1.59 \\
\hline FS03 & 0.712 & 0.136 & 1.465 & 2.50 & 3.38 \\
\hline FS04 & 0.519 & 0.099 & 1.150 & 2.42 & 2.11 \\
\hline FS05 & 2.734 & 0.518 & 6.719 & 3.82 & 5.03 \\
\hline FS06 & 0.000 & 0.000 & 0.000 & 0 & 2.02 \\
\hline FS07 & 0.692 & 0.136 & 1.661 & 3.13 & 1.29 \\
\hline FS08 & 0.128 & 0.025 & 0.255 & 1.33 & 3.00 \\
\hline FS09 & 0.964 & 0.185 & 3.171 & 2.18 & 1.67 \\
\hline FS10 & 4.308 & 0.827 & 13.633 & 4.17 & 4.38 \\
\hline FS11 & 0.778 & 0.148 & 2.088 & 2.57 & 2.29 \\
\hline FS12 & 2.569 & 0.494 & 7.456 & 3.43 & 1.24 \\
\hline FS13 & 4.245 & 0.802 & 13.035 & 3.73 & 4.24 \\
\hline FS15 & 1.387 & 0.259 & 2.887 & 2.75 & 3.60 \\
\hline FS16 & 1.043 & 0.197 & 2.476 & 7.67 & -2.65 \\
\hline FS17 & 0.940 & 0.173 & 2.776 & 3.25 & 2.66 \\
\hline Min. & 0 & 0 & 13.633 & 1.33 & -2.65 \\
\hline Max. & 4.308 & 0.827 & 4.091563 & 7.67 & 5.03 \\
\hline Avg. & 1.458 & 0.277688 & 4.240767 & 3.234667 & 2.420625 \\
\hline SD & 1.356676 & 0.258653 & & 1.420849 & 1.766056 \\
\hline
\end{tabular}

* $100 \mathrm{ml}$ represents volume of fresh CLSM. 
The investigation indicates that the bleed volume ranges between 0 and $8.73 \%$ based on the volume of fresh CLSM. It generally lasts 0-7 hours after first contact with water. As a result of bleeding, settlement resulting from fresh CLSM to solid CLSM ranged from $0.08 \%$ to $9.42 \%$. Furthermore, two-tailed correlation testing indicates that bleeding and settlement are fairly positive-correlated for excavatable $(r=0.728)$ and the correlation is significant $(p=0.001)$. Fifty-three percent of the variation in settlement is related to bleeding. The rest of the variation may involve the gradation of sand particles. For structural CLSM, bleeding and settlement are not correlated $(r=0.396, p=0.129)$. Bleeding is moderately positive-correlated with bleeding period $(r=0.469, p=0.028)$ for excavatable CLSM. That is, more bleeding tends to last longer. However for structural CLSM, bleeding and bleeding period are not correlated $(r=0.324, p=0.239)$. It was discovered that moderate bleeding for FS16 takes the longest period to drain. If FS16 were eliminated, bleeding and bleeding period would heavily correlate for the remaining structural CLSM $(r=0.827, p=0+)$.

\subsubsection{Setting Time and Penetration Resistance}

The setting time, also referred to as hardening time, was evaluated according to ASTM C 403, "Standard Test Method for Time of Setting of Concrete Mixtures by Penetration Resistance" [ASTM C 403 1999]. In this experiment, a rectangular, wood water-tight mold was filled with fresh CLSM. The drainage was allowed only through the top surface of the materials. Any free bleeding water was removed before each measurement of penetration resistance. The container was tilted slightly to aid the 
collection of the bleeding by a pipette. After at least 2 hours had elapsed from the first addition of water, the CLSM penetration resistance was measured using a $19.35 \mathrm{~cm}^{2}$, $6.45 \mathrm{~cm}^{2}, 3.23 \mathrm{~cm}^{2}$, or $1.61 \mathrm{~cm}^{2}$ steel penetration needle, depending on the resistance development. From that point onward, the penetration resistance was measured at 1 to 4 hour intervals until penetration resistance was over $344 \mathrm{kN} / \mathrm{m}^{2}$ (50 psi), which is defined as the initial set penetration resistance.

The presentations of setting time versus penetration resistance of excavatable CLSM phase I and II are summarized in Figure I-1 and Figure I-2 in Appendix I.1. Corresponding presentations for structural CLSM are summarized in Figure I-3 in Appendix I.2. All test results are summarized in Figure 7-1, Figure 7-2, and Figure 7-3. The horizontal dashed lines represent $344 \mathrm{kPa}$ (50 psi), $689 \mathrm{kPa}(100 \mathrm{psi}), 1,379$ (200 psi), and 2,758 $\mathrm{kPa}$ (400 psi), respectively, which are thresholds for critical loads, such as human body loads or vehicle loads. This indicates that the penetration resistance starts to develop after 3-5 hours following water addition. The resistance development is not uniform for CLSM with varying foundry sands. Structural CLSM develops penetration resistance more quickly than excavatable CLSM. 


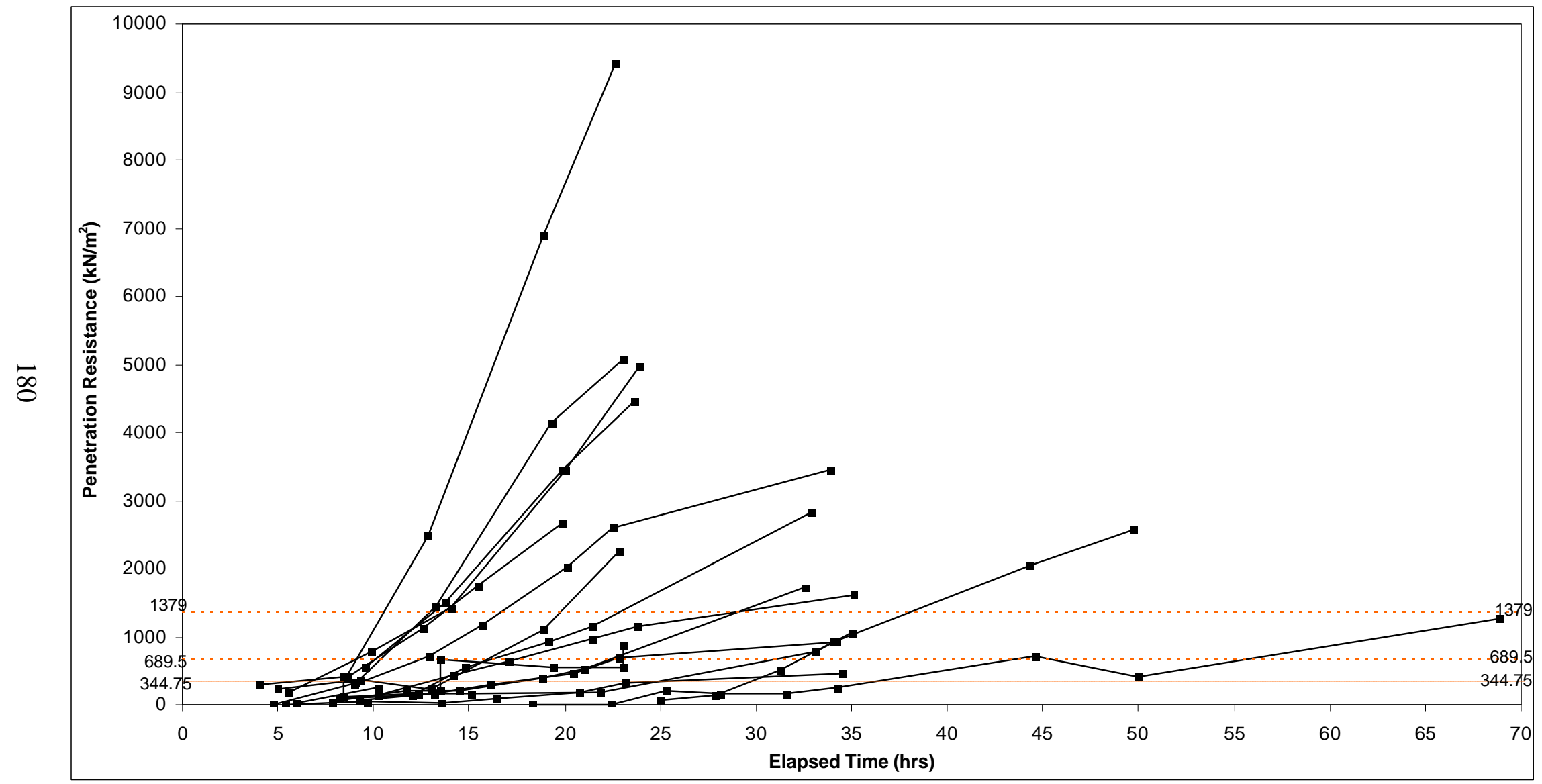

Figure 7-1: Setting time of excavatable CLSM phase I 


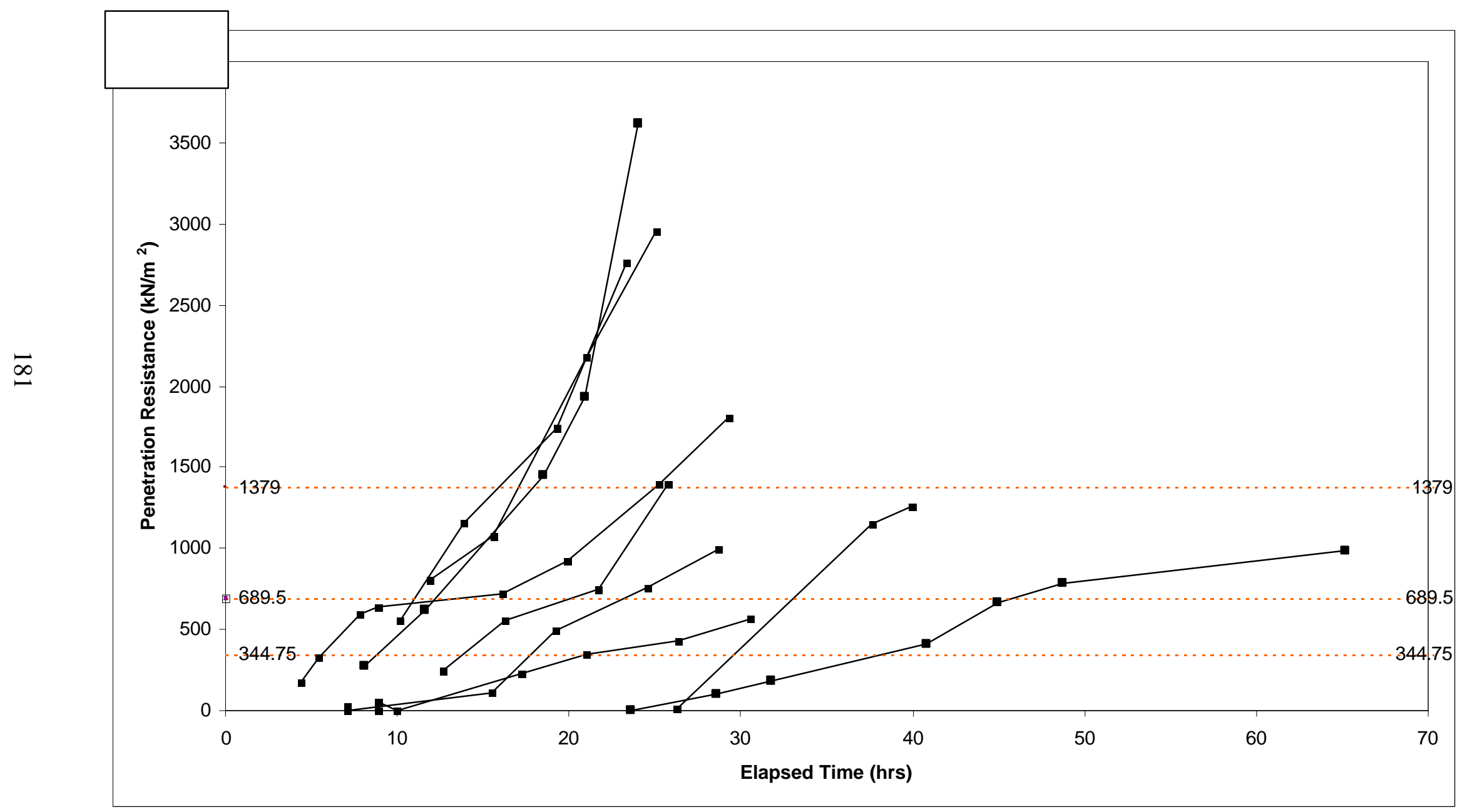

Figure 7-2: Setting time of excavatable CLSM phase II 


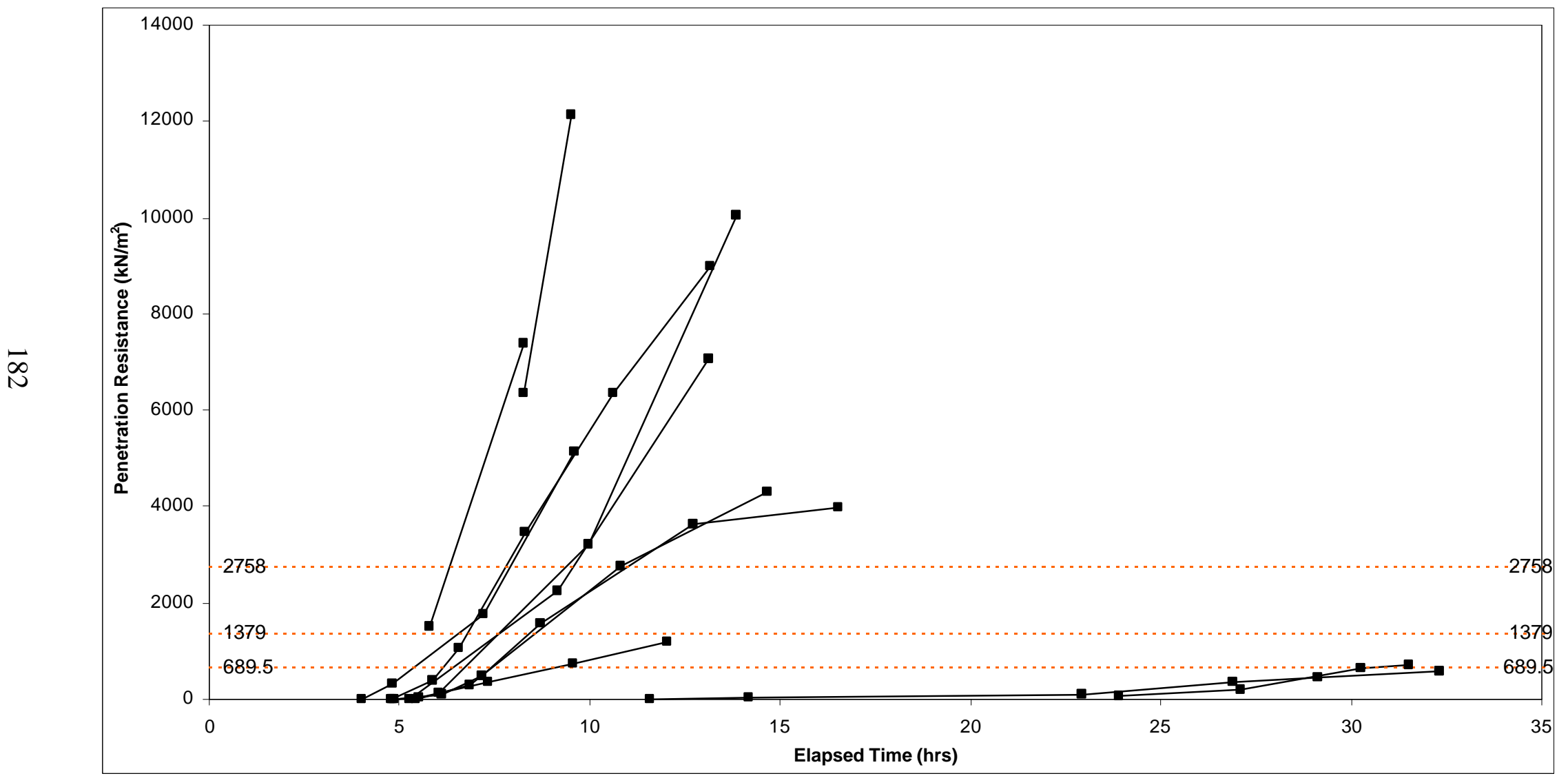

Figure 7-3: Setting time of structural CLSM 
Interpolation was used to estimate the elapsed time corresponding to critical penetration resistances of $344 \mathrm{kPa}(50 \mathrm{psi})$ and 689 (100psi) and penetration resistances corresponding to elapsed time of $6,12,18$, and 24 hours. The results are presented in Table 7-17, Table 7-18, and Table 7-19. It is indicated that the time to attain $344 \mathrm{kPa}$, which is defined as the initial set penetration resistance or bearing capacity for human body loads, ranges from 6.73 to 36.23 hours for excavatable CLSM phase I, 5.65 to 38.06 hours for excavatable CLSM phase II, and 4.89 to 28.19 hours for structural CLSM.

\subsubsection{Fresh Density}

Fresh CLSM was placed into a calibrated volume container to compute the fresh density. The results are presented in Table 7-20 and Table 7-21 for excavatable CLSM, and Table 7-22 for structural CLSM to compare its corresponding hardened density. 
Table 7-17: Critical setting time and penetration resistance for excavatable CLSM phase I

\begin{tabular}{|c|c|c|c|c|c|c|}
\hline \multirow[t]{2}{*}{ FS ID } & \multicolumn{2}{|c|}{ Elapsed Time (hrs) } & \multicolumn{4}{|c|}{ Penetration Resistances $\left(\mathrm{kN} / \mathrm{m}^{2}\right)$} \\
\hline & $\begin{array}{l}\text { Attaining } 344 \\
\mathrm{kN} / \mathrm{m}^{2} \text { (50 psi) }\end{array}$ & $\begin{array}{l}\text { Attaining } 689 \\
\mathrm{kN} / \mathrm{m}^{2} \text { (100 psi) }\end{array}$ & 6 hrs elapse & 12 hrs elapse & 18 hrs elapse & 24 hrs elapse \\
\hline FS01 & 13.1 & 17.8 & 21 & 267 & 704 & 1158 \\
\hline FS02 & 6.7 & 9.2 & 186 & 1104 & 2764 & 4964 \\
\hline FS03 & 25.0 & --- & 0 & 60 & 121 & 334 \\
\hline FS04 & 8.7 & 10.2 & --- & 1094 & 2857 & 4468 \\
\hline FS05 & 17.1 & 23.1 & --- & 136 & 377 & 708 \\
\hline FS06 & 8.4 & 14.7 & 272 & 558 & 766 & 905 \\
\hline FS07 & 9.4 & 12.8 & --- & 610 & 1611 & 2707 \\
\hline FS08 & 8.3 & 10.2 & --- & 1024 & 2278 & 3533 \\
\hline FS09 & 9.3 & 10.4 & 96 & 1108 & 3558 & 5297 \\
\hline FS10 & 24.9 & 31.5 & --- & 156 & 170 & 297 \\
\hline FS11 & 13.4 & 16.5 & --- & 145 & 814 & 1536 \\
\hline FS12 & 13.7 & 16.1 & --- & 89 & 975 & 2596 \\
\hline FS13 & 8.5 & 9.2 & --- & 2085 & 6265 & 10353 \\
\hline FS14 & 36.2 & 44.3 & 0 & 0 & 0 & 110 \\
\hline FS15 & 17.8 & 22.6 & 0 & 208 & 353 & 831 \\
\hline FS16 & 29.8 & 32.5 & 0 & 0 & 0 & 76 \\
\hline Min. & 6.7 & 9.2 & 0 & 0 & 0 & 76 \\
\hline Max. & 36.2 & 44.3 & 272 & 2085 & 6265 & 10353 \\
\hline Avg. & 15.6 & 18.7 & 72 & 540 & 1476 & 2492 \\
\hline$S D$ & 8.9 & 10.4 & 105 & 591 & 1706 & 2744 \\
\hline
\end{tabular}

Note: $1 \mathrm{kN} / \mathrm{m}^{2}=0.145 \mathrm{psi}$. 
Table 7-18: Critical setting time and penetration resistance for excavatable CLSM phase II

\begin{tabular}{|c|c|c|c|c|c|c|}
\hline \multirow[t]{2}{*}{ FS ID } & \multicolumn{2}{|c|}{ Elapsed Time (hrs) } & \multicolumn{4}{|c|}{ Penetration Resistances $\left(\mathrm{kN} / \mathrm{m}^{2}\right)$} \\
\hline & $\begin{array}{l}\text { Attaining } 344 \\
\mathrm{kN} / \mathrm{m}^{2} \text { (50 psi) }\end{array}$ & $\begin{array}{l}\text { Attaining } 689 \\
\mathrm{kN} / \mathrm{m}^{2} \text { (100 psi) }\end{array}$ & 6 hrs elapse & 12 hrs elapse & 18 hrs elapse & 24 hrs elapse \\
\hline FS05 & 21.0 & 34.4 & 0 & 63 & 251 & 391 \\
\hline FS06 & 5.7 & 13.7 & 386 & 670 & 821 & 1282 \\
\hline FS08 & 17.8 & 23.2 & 0 & 74 & 369 & 731 \\
\hline FS09 & 13.8 & 20.1 & --- & 197 & 613 & 1112 \\
\hline FS13 & --- & 10.4 & --- & 800 & 1575 & 2752 \\
\hline FS14 & 38.1 & 45.8 & 0 & 0 & 0 & 7 \\
\hline FS16 & 29.6 & 33.0 & 0 & 0 & 0 & 7 \\
\hline FS17 & 8.9 & 11.0 & --- & 858 & 1604 & 2915 \\
\hline FS17 & 8.8 & 12.2 & 75 & 661 & 1387 & 3613 \\
\hline Min. & 5.6 & 10.4 & 0 & 0 & 0 & 7 \\
\hline Max. & 38.1 & 45.8 & 386 & 858 & 1604 & 3613 \\
\hline$A v g$. & 17.9 & 22.6 & 77 & 369 & 735 & 1424 \\
\hline$S D$ & 11.2 & 12.6 & 154 & 368 & 648 & 1344 \\
\hline
\end{tabular}


Table 7-19: Critical setting time and penetration resistance for structural CLSM

\begin{tabular}{|c|c|c|c|c|c|c|}
\hline \multirow[t]{2}{*}{ FS ID } & \multicolumn{2}{|c|}{ Elapsed Time (hrs) } & \multicolumn{4}{|c|}{ Penetration Resistances $\left(\mathrm{kN} / \mathrm{m}^{2}\right)$} \\
\hline & $\begin{array}{l}\text { Attaining } 344 \\
\mathrm{kN} / \mathrm{m}^{2}(50 \mathrm{psi})\end{array}$ & $\begin{array}{l}\text { Attaining } 689 \\
\mathrm{kN} / \mathrm{m}^{2}(100 \mathrm{psi})\end{array}$ & 6 hrs elapse & 12 hrs elapse & 18 hrs elapse & 24 hrs elapse \\
\hline FS01 & 6.8 & 7.5 & 110 & 3277 & --- & --- \\
\hline FS03 & 6.9 & 7.5 & 121 & 3235 & --- & --- \\
\hline FS04 & 6.3 & 6.8 & 138 & 6779 & --- & --- \\
\hline FS05 & 5.9 & 6.5 & 419 & 5695 & --- & --- \\
\hline FS06 & 26.7 & 35.9 & 0 & 7 & 62 & 162 \\
\hline FS07 & 5.8 & 6.2 & 501 & 7781 & --- & --- \\
\hline FS08 & 7.2 & 9.2 & 128 & 1186 & --- & --- \\
\hline FS09 & 4.9 & 5.5 & 1027 & 8542 & --- & --- \\
\hline FS10 & 28.2 & 31.5 & 0 & 0 & 0 & 48 \\
\hline FS11 & --- & 5.4 & 2026 & 16125 & --- & --- \\
\hline FS12 & --- & --- & 0 & 23642 & --- & --- \\
\hline Min. & 4.9 & 5.4 & 0 & 0 & 0 & 48 \\
\hline Max. & 28.2 & 35.9 & 2026 & 23642 & 62 & 162 \\
\hline$A v g$. & 11.0 & 12.2 & 447 & 6933 & 31 & 105 \\
\hline$S D$ & 9.4 & 11.4 & 637 & 7243 & 44 & 80 \\
\hline
\end{tabular}

Note: $1 \mathrm{kN} / \mathrm{m}^{2}=0.145$ psi. 
Table 7-20: Density development of excavatable CLSM phase I

\begin{tabular}{l|l|l|l|l|l|l|l}
\hline FS ID & Fresh Density $\left(\mathrm{kg} / \mathrm{m}^{3}\right)$ & Hardened Density $\left(\mathrm{kg} / \mathrm{m}^{3}\right)$ & \multicolumn{4}{l}{} \\
\hline & 0 day & 3 day & 7 day & 14 day & 28 day & 90 day & 180 day \\
\hline FS01 & 1838.8 & 1833.1 & 1834.6 & 1823.9 & 1814.1 & --- & 1862.6 \\
\hline FS02 & 2073.6 & 2037.2 & 2041.5 & 2036.7 & 2029.7 & --- & 2093.2 \\
\hline FS03 & 1783.6 & 1769.5 & 1775.6 & 1775.8 & 1774.1 & --- & 1795.4 \\
\hline FS04 & 1909.9 & 1923.7 & 1924.6 & 1925.3 & 1917.1 & 1895.5 & 1946.2 \\
\hline FS05 & 1872.5 & 1881.9 & 1879.3 & 1879.1 & 1882.3 & 1891.6 & 1891.2 \\
\hline FS06 & 1954.0 & 2016.3 & 2022.9 & 2034.0 & 2039.4 & 2055.7 & 2045.4 \\
\hline FS07 & 1876.1 & 1890.0 & 1889.2 & 1892.4 & 1901.4 & 1912.3 & 1919.6 \\
\hline FS08 & 1821.7 & 1846.5 & 1850.0 & 1854.6 & 1857.9 & 1862.1 & 1869.0 \\
\hline FS09 & 1979.5 & 2001.4 & 2013.1 & 2022.1 & 2025.7 & 2032.7 & 2044.2 \\
\hline FS10 & 2042.3 & 2097.2 & 2098.5 & 2107.2 & 2119.6 & 2129.4 & 2162.5 \\
\hline FS11 & 1931.1 & 1939.4 & 1936.8 & 1942.8 & 1948.1 & 1955.7 & 1961.0 \\
\hline FS12 & 1933.0 & 1940.4 & 1934.7 & 1952.7 & 1968.7 & 1996.2 & 2010.0 \\
\hline FS13 & 2048.4 & 2124.2 & 2132.1 & 2134.0 & 2140.0 & 2149.1 & 2158.7 \\
\hline FS14 & 2006.2 & 2054.0 & 2055.0 & 2063.3 & 2071.3 & 2080.6 & 2082.8 \\
\hline FS15 & 1781.6 & 1805.4 & 1807.8 & 1811.4 & 1816.7 & 1820.5 & 1822.0 \\
\hline FS16 & 1848.7 & 1818.9 & 1818.9 & 1835.5 & 1844.6 & 1857.1 & 1865.8 \\
\hline Min. & 1781.6 & 1769.5 & 1775.6 & 1775.8 & 1774.1 & 1820.5 & 1795.4 \\
\hline Max. & 2073.6 & 2124.2 & 2132.1 & 2134.0 & 2140.0 & 2149.1 & 2162.5 \\
\hline Avg. & 1918.8 & 1936.2 & 1938.4 & 1943.2 & 1946.9 & 1972.2 & 1970.6 \\
\hline SD & 93.4 & 109.1 & 110.4 & 111.7 & 113.9 & 109.4 & 117.7 \\
\hline Not $1 \mathrm{~kg}$ & 3 & & &
\end{tabular}

Note: $1 \mathrm{~kg} / \mathrm{m}^{3}=1.685 \mathrm{lb} / \mathrm{yd}^{3}$. 
Table 7-21: Density development of excavatable CLSM phase II

\begin{tabular}{|c|c|c|c|c|c|c|c|c|c|}
\hline \multirow[t]{2}{*}{ FS ID } & \multirow{2}{*}{$\begin{array}{l}\text { Fresh } \\
\text { Density } \\
\left(\mathrm{kg} / \mathrm{m}^{3}\right)\end{array}$} & \multicolumn{6}{|c|}{ Hardened Density $\left(\mathrm{kg} / \mathrm{m}^{3}\right)$} & \multirow{2}{*}{$\begin{array}{l}\text { Oven-dry } \\
\text { Density } \\
\left(\mathrm{kg} / \mathrm{m}^{3}\right)\end{array}$} & \multirow{2}{*}{$\begin{array}{l}\text { Void } \\
31 \%\end{array}$} \\
\hline & & 3 day & 7 day & 14 day & 28 day & 90 day & 180 day & & \\
\hline SFS05 & 1817.3 & 1829.0 & 1830.7 & 1833.3 & 1835.0 & 1836.4 & 1837.0 & 1398.3 & $18 \%$ \\
\hline SFS06 & 1965.5 & 2038.9 & 2039.6 & 2040.0 & 2041.7 & 2052.1 & 2060.0 & 1748.1 & $32 \%$ \\
\hline SFS08 & 1803.0 & 1843.3 & 1845.7 & 1848.0 & 1849.8 & 1852.9 & 1853.5 & 1407.3 & $19 \%$ \\
\hline SFS09 & 1976.0 & 2016.4 & 2018.3 & 2021.1 & 2022.9 & 2027.0 & 2028.1 & 1701.6 & $17 \%$ \\
\hline SFS10 & 2036.4 & --- & 2044.6 & 2055.4 & 2064.5 & 2080.9 & 2086.0 & 1787.6 & $14 \%$ \\
\hline SFS13 & 2020.1 & 2118.6 & 2120.7 & 2125.1 & 2128.1 & 2135.5 & 2143.9 & 1874.2 & $20 \%$ \\
\hline SFS14 & 1990.3 & --- & --- & --- & 2076.8 & 2099.5 & 2113.4 & 1756.5 & $27 \%$ \\
\hline SFS16 & 1900.6 & 1852.9 & 1860.4 & 1878.5 & 1892.0 & 1911.5 & 1915.8 & 1503.0 & $17 \%$ \\
\hline SFS17 & 1971.6 & 2039.3 & 2046.0 & 2060.7 & 2068.1 & 2075.7 & 2083.7 & 1787.7 & $31 \%$ \\
\hline SFS17 & 1984.3 & 2062.0 & 2063.7 & 2071.0 & 2074.9 & 2082.1 & 2074.3 & --- & --- \\
\hline Min. & 1803.0 & 1829.0 & 1830.7 & 1833.3 & 1835.0 & 1836.4 & 1795.4 & 1398.3 & $14 \%$ \\
\hline Max. & 2036.4 & 2118.6 & 2120.7 & 2125.1 & 2128.1 & 2135.5 & 2143.9 & 1874.2 & $32 \%$ \\
\hline$A v g$. & 1946.5 & 1975.0 & 1985.5 & 1992.6 & 2005.4 & 2004.5 & 1992.4 & 1662.7 & $22 \%$ \\
\hline$S D$ & 80.3 & 114.4 & 108.8 & 108.8 & 105.5 & 107.8 & 110.2 & 178.3 & 0.067 \\
\hline
\end{tabular}

Note: $1 \mathrm{~kg} / \mathrm{m}^{3}=1.685 \mathrm{lb} / \mathrm{yd}^{3}$. 
Table 7-22: Density development of structural CLSM

\begin{tabular}{|c|c|c|c|c|c|c|c|c|c|}
\hline \multirow[t]{2}{*}{ FS ID } & \multirow{2}{*}{$\begin{array}{l}\text { Fresh Density } \\
\left(\mathrm{kg} / \mathrm{m}^{3}\right)\end{array}$} & \multicolumn{6}{|c|}{ Hardened Density $\left(\mathrm{kg} / \mathrm{m}^{3}\right)$} & \multirow{2}{*}{$\begin{array}{l}\text { Oven-dry } \\
\text { Density }\left(\mathrm{kg} / \mathrm{m}^{3}\right) \\
180 \text { day }\end{array}$} & \multirow[t]{2}{*}{ Void } \\
\hline & & 3 day & 7 day & 14 day & 28 day & 90 day & 180 day & & \\
\hline FS01 & 1773.4 & 1801.7 & 1805.7 & 1825.1 & 1837.6 & 1852.5 & 1859.3 & 1406.1 & $32 \%$ \\
\hline FS02 & 2055.1 & 2071.0 & 2075.0 & 2089.6 & 2098.5 & 2112.1 & 2118.0 & --- & --- \\
\hline FS03 & 1787.3 & 1833.4 & 1853.7 & 1858.8 & 1863.5 & 1876.0 & 1883.1 & 1450.6 & $30 \%$ \\
\hline FS04 & 1873.9 & 1889.6 & 1892.6 & 1910.3 & 1919.8 & 1933.6 & 1942.6 & 1571.4 & $24 \%$ \\
\hline FS05 & 1844.8 & 1919.8 & 1927.1 & 1933.8 & 1938.4 & 1947.4 & 1952.4 & 1596.0 & $22 \%$ \\
\hline FS06 & 1994.5 & 2019.5 & 2024.2 & 2025.9 & 2026.9 & 2037.8 & 2057.4 & --- & --- \\
\hline FS07 & 1877.3 & 1896.5 & 1901.4 & 1904.5 & 1910.6 & 1922.3 & 1928.1 & 1533.7 & $26 \%$ \\
\hline FS08 & 1744.2 & 1795.7 & 1800.7 & 1804.3 & 1809.6 & 1814.7 & 1817.9 & 1340.2 & $36 \%$ \\
\hline FS09 & 1960.4 & 1990.9 & 1994.1 & 1997.3 & 2003.0 & 2011.0 & 2014.8 & --- & --- \\
\hline FS10 & 2068.4 & 2126.1 & 2133.0 & 2136.9 & 2140.7 & 2143.7 & 2133.4 & --- & --- \\
\hline FS11 & 1966.9 & 2001.7 & 2009.2 & 2012.9 & 2016.8 & 2024.9 & 2030.1 & 1712.9 & $19 \%$ \\
\hline FS12 & 2013.4 & 2023.7 & 2032.9 & 2042.7 & 2047.3 & 2062.6 & 2068.8 & 1776.5 & $16 \%$ \\
\hline FS13 & 2070.7 & 2135.3 & 2138.8 & 2147.0 & 2151.9 & 2156.6 & --- & --- & --- \\
\hline FS15 & 1854.3 & 1899.7 & 1901.2 & 1926.2 & 1940.0 & 1949.2 & 1952.4 & 1549.2 & $26 \%$ \\
\hline FS16 & 1941.3 & 1851.7 & 1856.9 & 1871.3 & 1881.3 & 1890.7 & 1890.3 & --- & --- \\
\hline FS17 & 2042.9 & 2091.5 & 2100.1 & 2103.7 & 2111.3 & 2121.7 & 2105.0 & --- & --- \\
\hline Min. & 1744.2 & 1795.7 & 1800.7 & 1804.3 & 1809.6 & 1814.7 & 1817.9 & 1340.2 & $16 \%$ \\
\hline Max. & 2070.7 & 2135.3 & 2138.8 & 2147.0 & 2151.9 & 2156.6 & 2133.4 & 1776.5 & $36 \%$ \\
\hline Avg. & 1929.3 & 1959.2 & 1965.4 & 1974.4 & 1981.1 & 1991.1 & 1983.6 & 1548.5 & $26 \%$ \\
\hline$S D$ & 109.3 & 113.2 & 112.8 & 110.7 & 109.4 & 108.5 & 99.8 & 139.3 & 0.062 \\
\hline
\end{tabular}

Note: $1 \mathrm{~kg} / \mathrm{m}^{3}=1.685 \mathrm{lb} / \mathrm{yd}^{3}$. 


\subsubsection{Hardened CLSM}

\subsubsection{Wet Density, Oven-Dry Density and Water Content}

At the time of testing, four solid CLSM cylinders were weighed and their density was calculated. On the 180th day, 1-2 solid cylinders were oven-dried at a temperature of $105{ }^{\circ} \mathrm{C}$ until there was no mass decrease. Oven-dry density was calculated based on these cylinders. Water content measures the ratio of water by mass in hardened CLSM. It is based on the difference between the wet density and the oven-dry density. The results of density and water content for excavatable CLSM phases I and II and structural CLSM are summarized in Table 7-20, Table 7-21, and Table 7-22, respectively.

It is indicated that the density of solid CLSM increases slightly with time if cured under moist conditions, which means external water is absorbed into the CLSM matrix if available. The density is normally distributed regarding various foundry sands, except for the density measured in excavatable CLSM phase II experiments. There is no more than one-third mass occupied by water in CLSM after 180 days of moisture curing. The difference in water content between excavatable CLSM and structural CLSM is not significant, $22 \%$ for excavatable CLSM versus $26 \%$ for structural CLSM. Both of them are normally distributed. 


\subsubsection{Hydraulic Conductivity}

The hydraulic conductivity tests were carried out in accordance with ASTM D 2434, "Standard Test Method for Permeability of Granular Soils (Constant Head)" on cylindrical samples ( $\phi 10.2 \times 10.2 \mathrm{~cm})$ at a curing time of 28 days. The hydraulic gradient was kept around $15 \mathrm{~m} / \mathrm{m}$. In general, water seepage lasted 24 hours. Results of hydraulic conductivity of excavatable CLSM phases I and II are presented in Figure 7-4 and Figure 7-5, respectively. It is indicated that the hydraulic conductivity of excavatable CLSM is typically in the range of $3 \times 10^{-6}$ to $4 \times 10^{-7} \mathrm{~cm} / \mathrm{sec}$, which is comparable to the hydraulic conductivity of sandy to silty clay. 


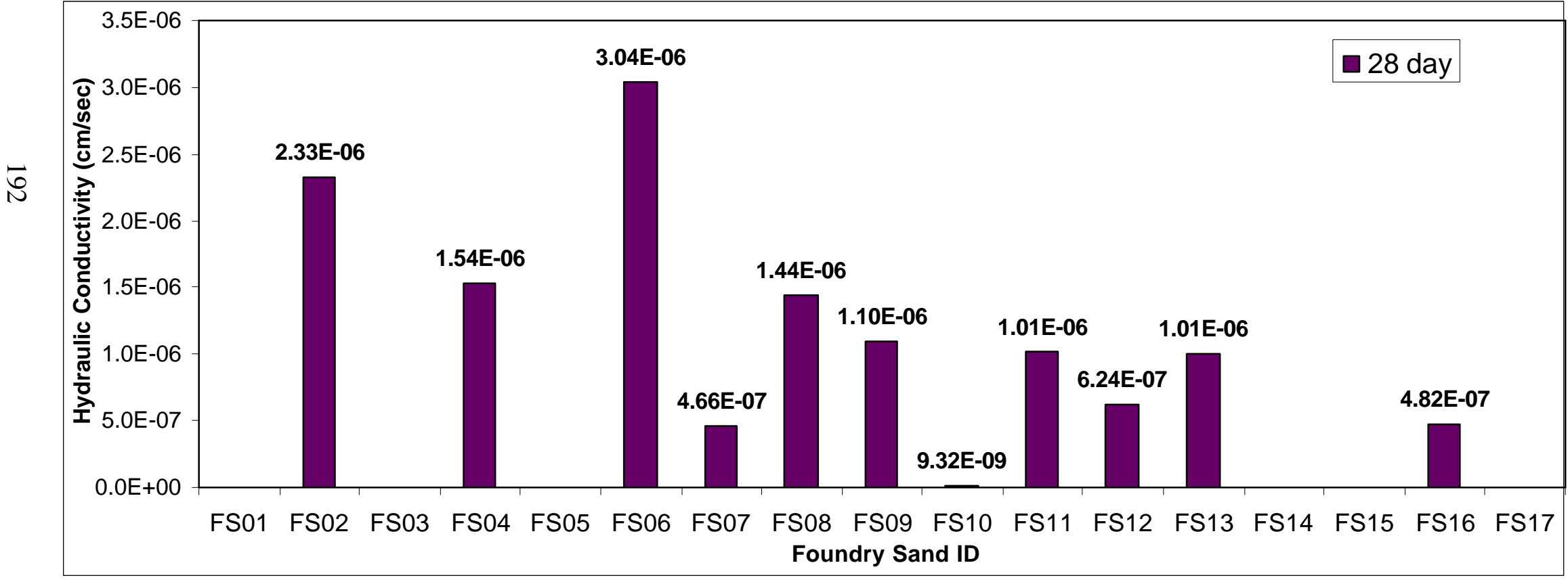

Figure 7-4: Hydraulic conductivity of excavatable CLSM phase I 


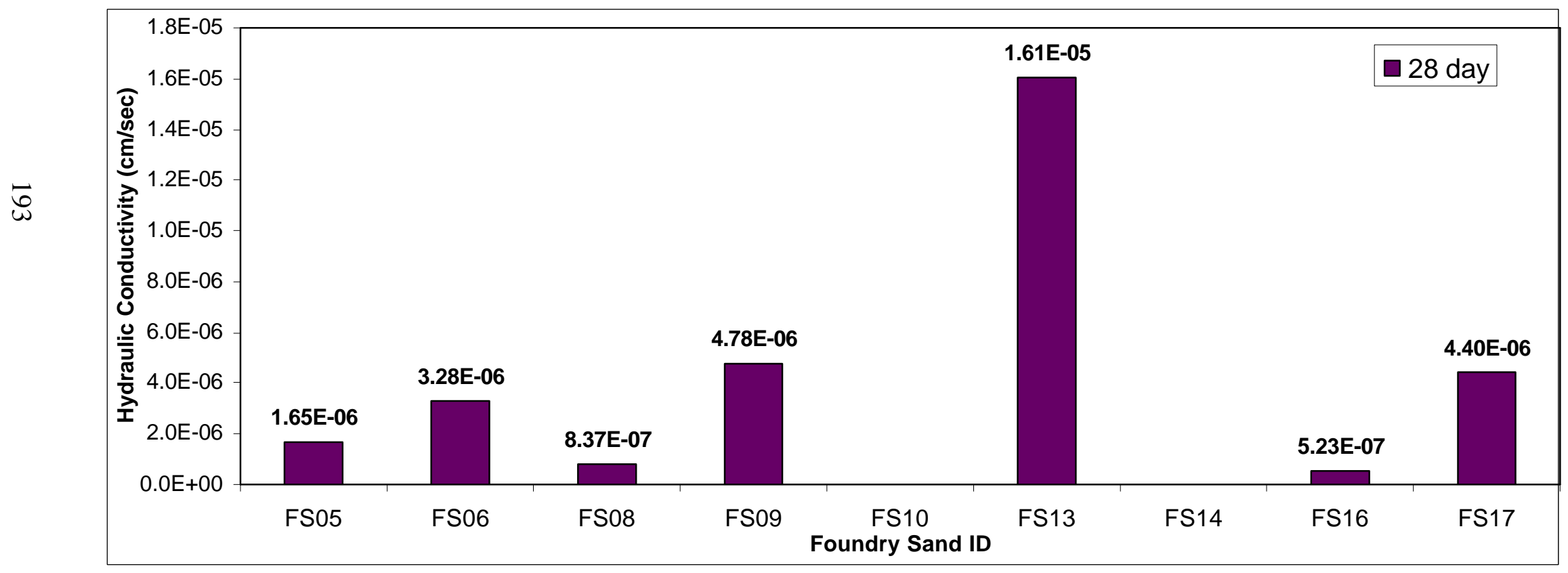

Figure 7-5: Hydraulic conductivity of excavatable CLSM phase II 


\subsubsection{Compressive Strength}

The unconfined compressive strength of CLSM cylinders $(\phi 10.2 \times 20.4 \mathrm{~cm})$ was conducted at curing date: 3, 7, 14, 28, 90, and 180 days. At least three cylinders were measured for a curing time by compression machine. If the variation in strength was not acceptable, another 1-2 cylinders were supplemented to obtain acceptable variance. Strength is defined as the average strength of cylinders measured. The strength developments of the scouting experiment, excavatable CLSM phases I and II and structural CLSM are presented in Figure 7-6, Figure 7-7, Figure 7-8, and Figure 7-9, respectively. 

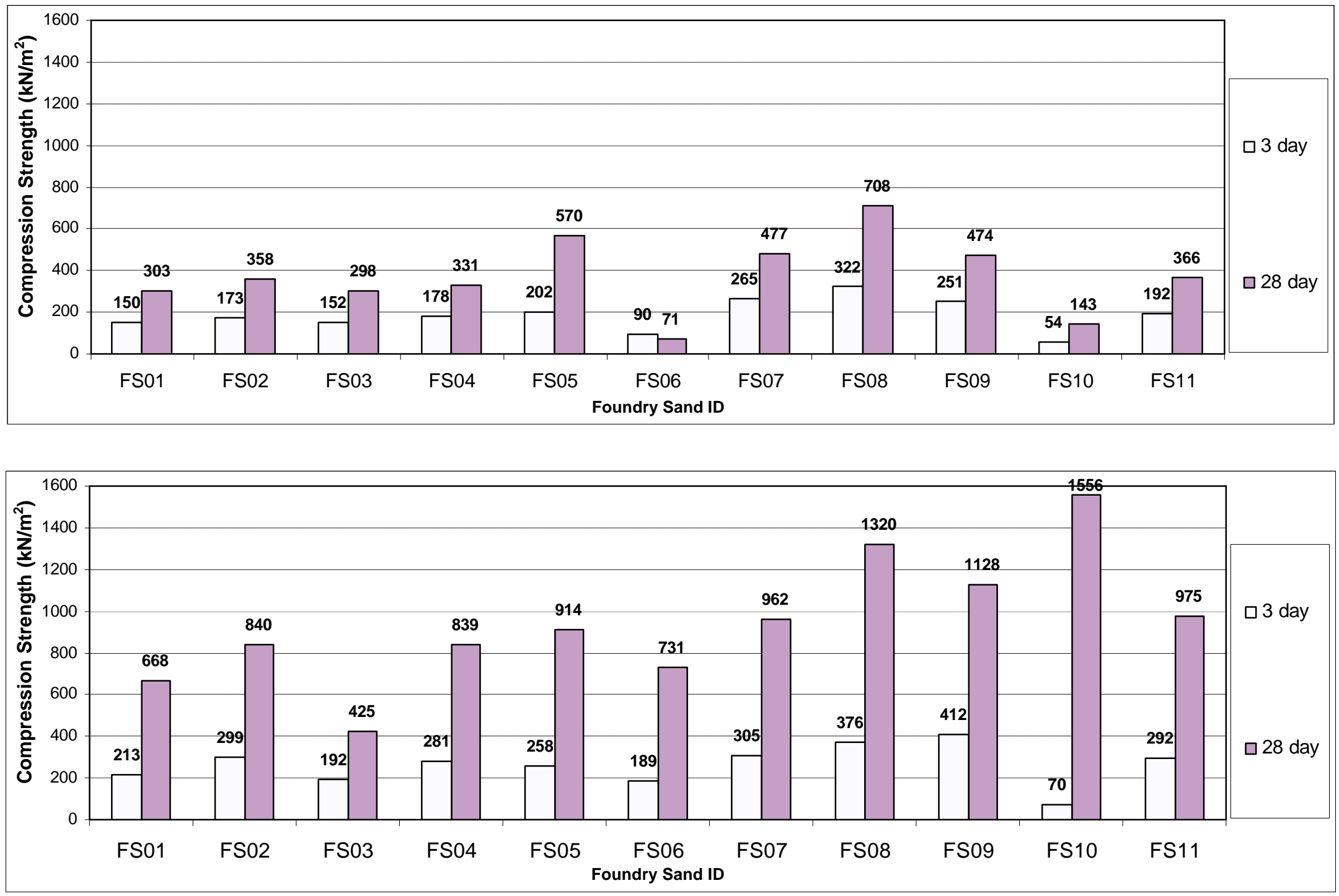

Figure 7-6: Strength development of scouting CLSM 

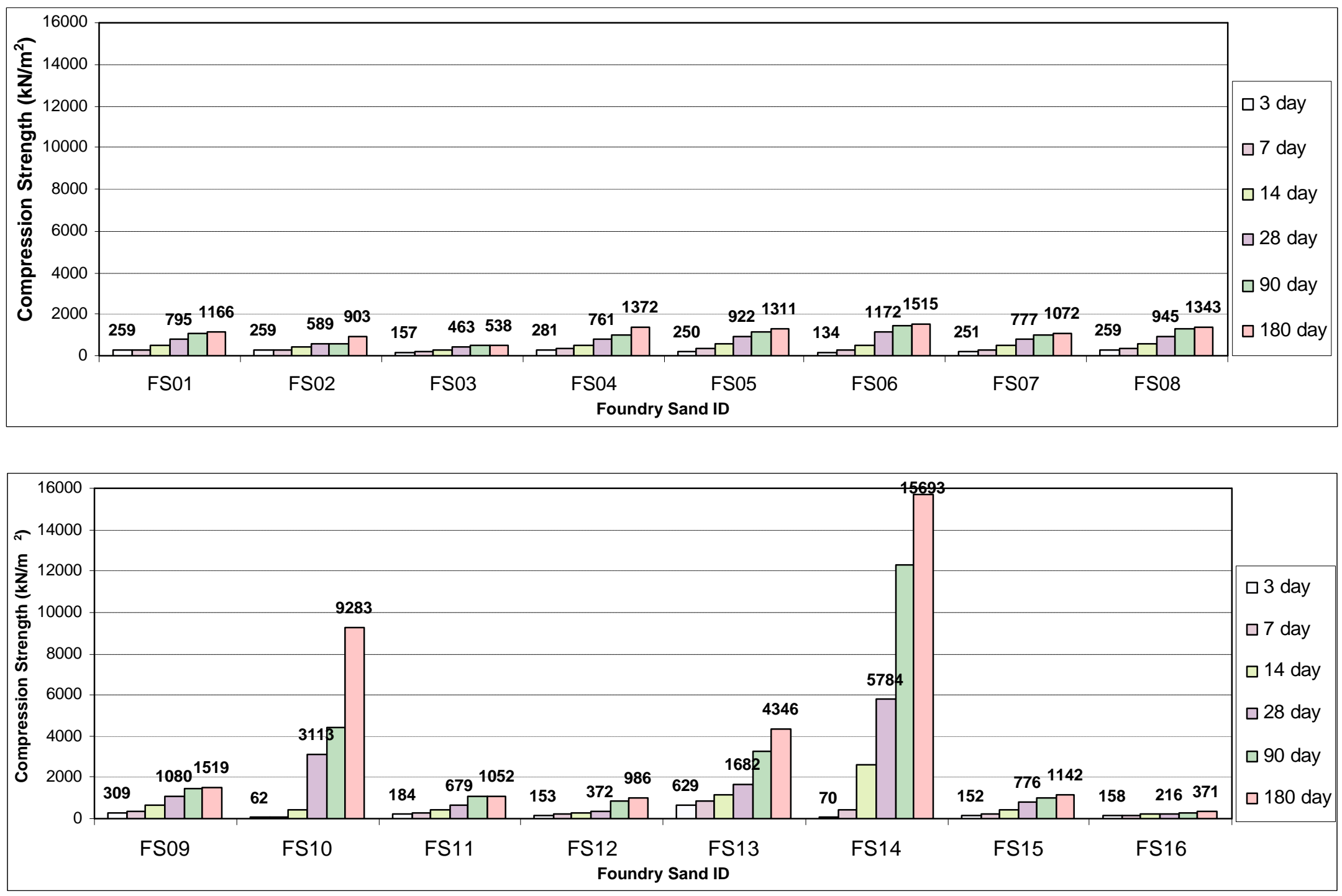

Figure 7-7: Strength development of excavatable CLSM phase I 

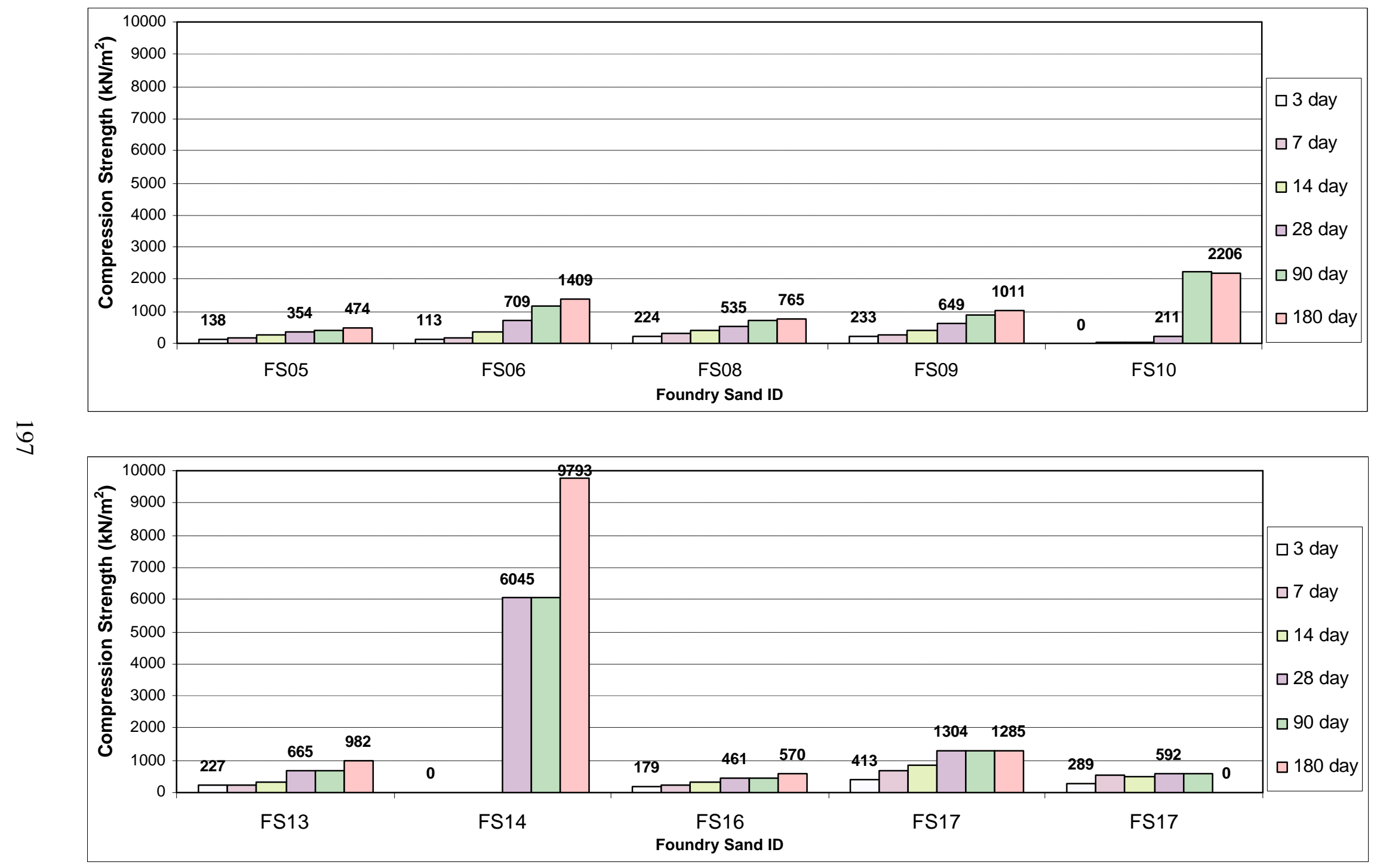

Figure 7-8: Strength development of excavatable CLSM phase II 

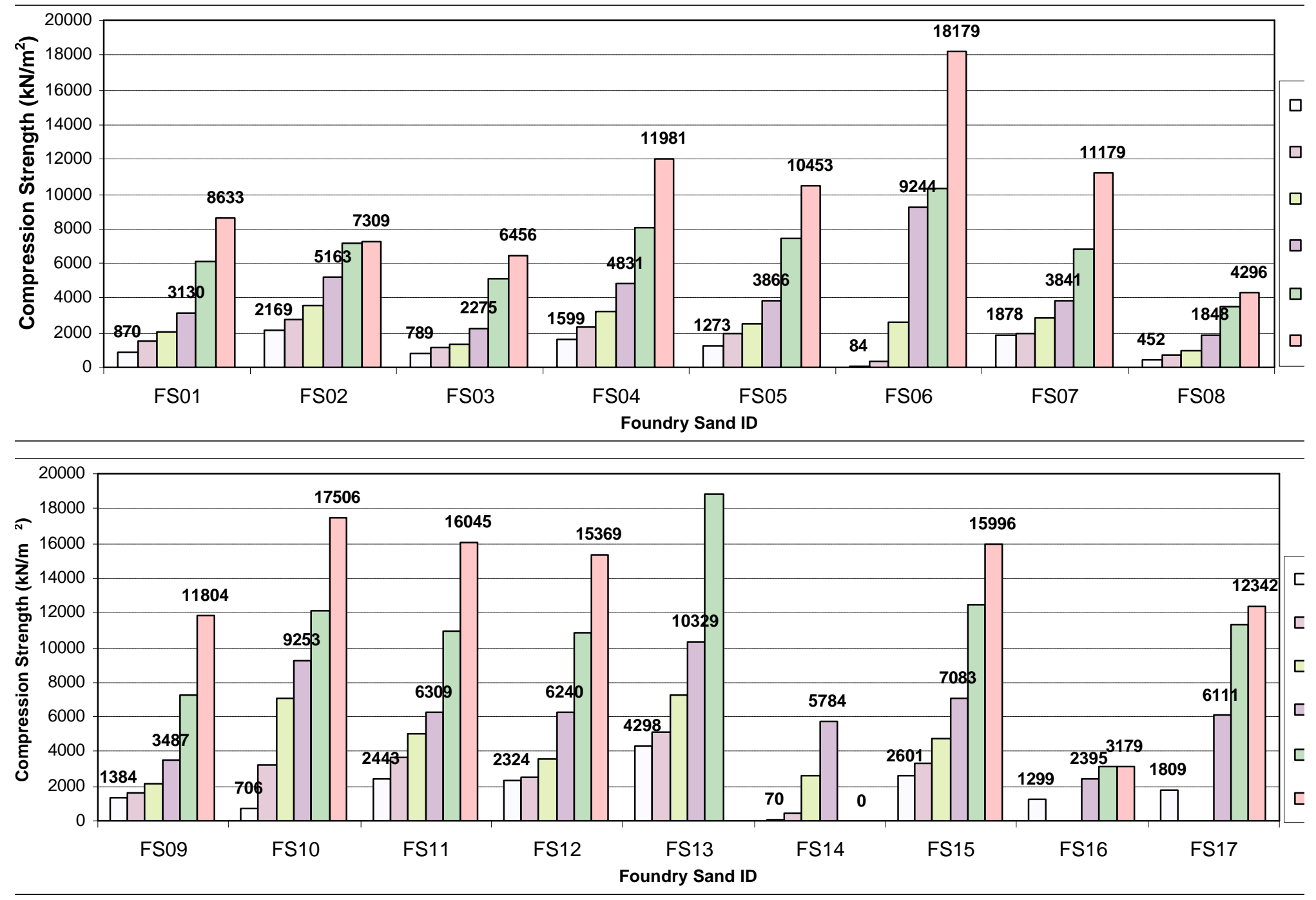

Figure 7-9: Strength development of structural CLSM 
Excavatable CLSM requires a compressive strength greater than $170 \mathrm{kPa}(25 \mathrm{psi})$ at 3 days and in the range of $345-862 \mathrm{kPa}(50-125 \mathrm{psi})$ at 28 days [PennDOT 1995]. It is indicated that in scouting experiments, the objective of probing specifications for CLSM with a strength of $345 \mathrm{kPa}$ or $487 \mathrm{kPa}$ at 28 days was achieved. The configured formulations for phase I excavatable CLSM indicate most excess foundry sands performing well as a component in CLSM. For those not attaining the strength requirement, supplemented mixings were conducted in the phase II experiment. After the two-phase investigation, the final formulations, listed in Table 7-10 and Table 7-11, were determined for 16 of 17 excess foundry sands in the application of excavatable CLSM. FS14 is not suitable for excavatable CLSM due to its high strength at 28 days and later (Figure 7-7 and Figure 7-8). Its cement proportion is the least in the phase II investigation, $15 \mathrm{~kg} / \mathrm{m}^{3}$ (Table 7-8) versus average proportion of $57 \mathrm{~kg} / \mathrm{m}^{3}$ (Table 7-10); however, its 28-day compressive strength is 6,045 $\mathrm{kPa}$ in Figure 7-8. This phenomenon is likely due to the $\mathrm{CaO}$ content and least content in $\mathrm{SiO}_{2}(\mathrm{Table} \mathrm{H}-2)$, finest grains (Table 6-5), and too many particles passing sieve 200 (Table 6-5) among selected excess foundry sands. Although 16 out of 17 sands are qualified as a major component in CLSM, their variation in formulation and performance should be concerned. Examination on a source-specific basis is required when sands are reused.

\subsubsection{Environmental Impact}

The environmental impact of using foundry sand is a necessary issue that needs to be addressed before these materials are integrated into CLSM specifications. The ability 
of the CLSM matrix to bind metallic elements and organic compounds affects the potential toxicity of CLSM using foundry sands. There are two discharges to environmental surroundings from the CLSM matrix, the bleed water of CLSM when it is placed, and the leachate from CLSM when it is extracted.

Based on the review of bulk chemical analyses of excess foundry sands discussed in Section 6.3, four top-risk sands, FS02, FS12, FS13, and FS16 were selected to evaluate the environmental impact of their CLSM, which was mixed in the phase I excavatable CLSM experiment.

\subsubsection{Toxicity of Bleeding}

The bleed water of the fresh CLSM was collected and sent to a commercial laboratory for chemical analysis and toxicity evaluation. Results are presented in Table 723. It is indicated that minimal amounts of metallic elements exist in the bleed water, and the levels are well below the RCRA toxicity thresholds. Hence, bleeding of CLSM poses little or no hazardous impact to the environment. 
Table 7-23: Toxicity of bleeding

\begin{tabular}{|c|c|c|c|c|c|c|}
\hline & & FS02 & FS12 & FS13 & FS16 & $\begin{array}{l}\text { TCLP } \\
\text { Toxicity } \\
\text { Threshold }\end{array}$ \\
\hline Arsenic & \multirow{8}{*}{ 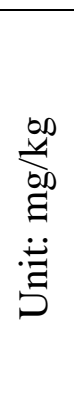 } & .0733 & .0183 & .0314 & .378 & 5 \\
\hline Barium & & .62 & .505 & .278 & .289 & 100 \\
\hline Cadmium & & .0064 & .0064 & .0077 & .0102 & 1 \\
\hline Chromium & & \begin{tabular}{|l|}
.0758 \\
\end{tabular} & .0485 & .189 & .681 & 5 \\
\hline Lead & & .0267 & .0231 & .0137 & .0936 & 5 \\
\hline Mercury & & $<<.0002$ & $<.0002$ & $<.0002$ & $<.0002$ & 0.2 \\
\hline Selenium & & .1 & .0317 & .034 & .0269 & 1 \\
\hline Silver & & .0006 & $<.0003$ & $<.0003$ & .002 & 5 \\
\hline Acetone & \multirow{20}{*}{ 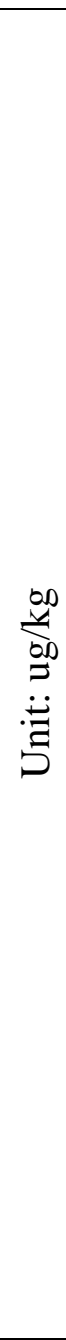 } & --- & 41 & 56 & 1540 & --- \\
\hline Benzene & & \begin{tabular}{|l}
-- \\
\end{tabular} & $<5$ & $<5$ & $<5$ & 500 \\
\hline $\begin{array}{l}\text { Carbon } \\
\text { Tetrachloride }\end{array}$ & & --- & $<5$ & $<5$ & $<5$ & 500 \\
\hline Chlorobenzene & & \begin{tabular}{|l|}
-- \\
\end{tabular} & $<5$ & $<5$ & $<5$ & 100000 \\
\hline Chloroform & & --- & $<5$ & $<5$ & $<5$ & 6000 \\
\hline $\begin{array}{l}1,4- \\
\text { Dichlorobenzene }\end{array}$ & & \begin{tabular}{|l|}
--- \\
\end{tabular} & $<5$ & $<5$ & $<5$ & 7500 \\
\hline $\begin{array}{l}1,2- \\
\text { Dichloroethane }\end{array}$ & & \begin{tabular}{|l}
--- \\
\end{tabular} & $<5$ & $<5$ & $<5$ & 500 \\
\hline $\begin{array}{l}1,1- \\
\text { Dichloroethene }\end{array}$ & & \begin{tabular}{|l}
--- \\
\end{tabular} & $<5$ & $<5$ & $<5$ & 700 \\
\hline Ethyl benzene & & $\begin{array}{ll}-- \\
\end{array}$ & $<5$ & $<5$ & $<5$ & \\
\hline $\begin{array}{l}\text { Methyl ethyl } \\
\text { ketone }\end{array}$ & & \begin{tabular}{|l|}
-- \\
\end{tabular} & $<10$ & $<10$ & $<10$ & 200000 \\
\hline $\begin{array}{l}\text { Methylene } \\
\text { chloride }\end{array}$ & & \begin{tabular}{|l|}
--- \\
\end{tabular} & $<5$ & $<5$ & $<5$ & --- \\
\hline Naphthalene & & \begin{tabular}{|l|}
-- \\
\end{tabular} & 619 & 180 & 115 & --- \\
\hline Styrene & & \begin{tabular}{|l}
-- \\
\end{tabular} & $<5$ & $<5$ & $<5$ & --- \\
\hline Tetrachloroethene & & $-1-$ & $<5$ & $<5$ & $<5$ & 700 \\
\hline Toluene & & \begin{tabular}{|l|}
-- \\
\end{tabular} & $<5$ & $<5$ & $<5$ & --- \\
\hline $\begin{array}{l}\text { 1,1,1- } \\
\text { Trichloroethane }\end{array}$ & & \begin{tabular}{|l}
--- \\
\end{tabular} & $<5$ & $<5$ & $<5$ & --- \\
\hline Trichloroethene & & \begin{tabular}{|l}
-- \\
\end{tabular} & $<5$ & $<5$ & $<5$ & 500 \\
\hline Vinyl chloride & & --- & $<10$ & $<10$ & $<10$ & 200 \\
\hline M, P-Xylene & & ---- & $<5$ & $<5$ & $<5$ & --- \\
\hline Xylene-total & & $-1-$ & $<10$ & $<10$ & $<10$ & --- \\
\hline
\end{tabular}




\subsubsection{Toxicity of Hardened CLSM}

CLSM of four top-risk sands, FS02, FS12, FS13, and FS16 were sent to the commercial chemical laboratory for leaching toxicity evaluation. The results are presented in Table 7-24. It is indicated that minimal amounts of arsenic, barium, and chromium were detected in the leachate, but the levels are well below the toxicity thresholds. As with the bleed water, the leachate from CLSM containing foundry sand posed no significant hazard to the environment.

\subsubsection{Corrosivity of Hardened CLSM}

Corrosivity is designed to identify materials that potentially pose a hazard to human health or the environment due to their ability to mobilize toxic metals if discharged into a landfill environment; to corrode handling, storage, transportation, and management equipment; or to destroy human or animal tissue in the event of inadvertent contact [EPA SW-846 2000]. Corrosion can occur when water or leachate water reacts with metal plumbing. It may eventually cause leaky pipes, faucets, hot water heaters, or other plumbing fixtures. A solid waste exhibits the characteristic of corrosivity if a representative sample of the waste has the property that it is aqueous (leachate) and has a $\mathrm{pH}$ less than or equal to 2 or greater than or equal to 12.5 , as determined by a $\mathrm{pH}$ meter using Method 9040 in "Test Methods for Evaluating Solid Waste, Physical/Chemical Methods," EPA Publication SW-846 [40 CFR Part 261 1986]. However, this range is not applicable to the corrosion or passivity of all materials. For example, higher $\mathrm{pH}$ values provide passivity to exposed steel but may corrode glassy materials. 
Table 7-24: Toxicity of hardened CLSM

\begin{tabular}{|c|c|c|c|c|c|c|}
\hline & & FS02 & FS12 & FS13 & FS16 & $\begin{array}{l}\text { TCLP } \\
\text { Toxicity } \\
\text { Threshold }\end{array}$ \\
\hline Arsenic & \multirow{8}{*}{ 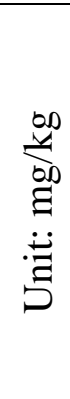 } & .0572 & $<.05$ & $<.5$ & $<.05$ & 5 \\
\hline Barium & & .0784 & .291 & .338 & $<.01$ & 100 \\
\hline Cadmium & & $<.01$ & $<.01$ & $<.01$ & $<.01$ & 1 \\
\hline Chromium & & .025 & .0609 & .0722 & $<.01$ & 5 \\
\hline Lead & & $<.03$ & $<.03$ & $<.03$ & $<.03$ & 5 \\
\hline Mercury & & $<.0002$ & $<.0002$ & $<.0002$ & $<.0002$ & 0.2 \\
\hline Selenium & & $<.05$ & $<.05$ & $<.05$ & $<.05$ & 1 \\
\hline Silver & & $<.05$ & $<.05$ & $<.05$ & $<.05$ & 5 \\
\hline Acetone & \multirow{20}{*}{ 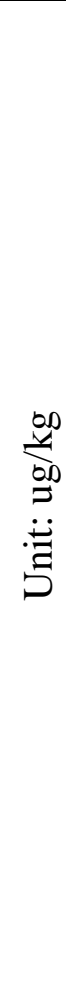 } & 86 & 86 & 100 & 115 & --- \\
\hline Benzene & & $<25$ & $<25$ & $<25$ & $<25$ & 500 \\
\hline Carbon Tetrachloride & & $<25$ & $<25$ & $<25$ & $<25$ & 500 \\
\hline Chlorobenzene & & $<25$ & $<25$ & $<25$ & $<25$ & 100000 \\
\hline Chloroform & & $<25$ & $<25$ & $<25$ & $<25$ & 6000 \\
\hline 1,4-Dichlorobenzene & & $<25$ & $<25$ & $<25$ & $<25$ & 7500 \\
\hline 1,2-Dichloroethane & & $<25$ & $<25$ & $<25$ & $<25$ & 500 \\
\hline 1,1-Dichloroethene & & $<25$ & $<25$ & $<25$ & $<25$ & 700 \\
\hline Ethyl benzene & & $<25$ & $<25$ & $<25$ & $<25$ & --- \\
\hline Methyl ethyl ketone & & $<50$ & $<50$ & $<50$ & $<50$ & 200000 \\
\hline Methylene chloride & & $<25$ & $<25$ & $<25$ & $<25$ & --- \\
\hline Naphthalene & & $<25$ & 616 & 527 & $<25$ & --- \\
\hline Styrene & & $<25$ & $<25$ & $<25$ & $<25$ & --- \\
\hline Tetrachloroethene & & $<25$ & $<25$ & $<25$ & $<25$ & 700 \\
\hline Toluene & & $<25$ & $<25$ & $<25$ & $<25$ & --- \\
\hline 1,1,1-Trichloroethane & & $<25$ & $<25$ & $<25$ & $<25$ & --- \\
\hline Trichloroethene & & $<25$ & $<25$ & $<25$ & $<25$ & 500 \\
\hline Vinyl chloride & & $<50$ & $<50$ & $<50$ & $<50$ & 200 \\
\hline M, P-Xylene & & $<25$ & $<25$ & $<25$ & $<25$ & --- \\
\hline Xylene-total & & $<25$ & $<25$ & $<25$ & $<25$ & --- \\
\hline
\end{tabular}

Factors affecting the potential for soil corrosion include water content, degree of aeration, $\mathrm{pH}$, resistivity, redox potential, chloride level, sulfate level, and microbiologically influenced corrosion (MIC) [Roberge 1999]. As for soil, its resistivity parameter is very widely used in practice and generally considered to be the dominant 
variable in the absence of microbial activity. The generally adopted corrosion severity ratings are presented in Table 7-25. In the investigation of corrosivity of CLSM, which acts as a replacement of compacted soil, this severity rating is accepted as thresholds for resistivity evaluation of CLSM.

Table 7-25: Scale of soil corrosion [Roberge 1999]

\begin{tabular}{l|l}
\hline Soil Resistivity $(\mathrm{ohm} \mathrm{cm})$ & Corrosivity Rating \\
\hline$>20,000$ & Essentially non-corrosive \\
\hline 10,000 to 20,000 & Mildly corrosive \\
\hline 5,000 to 10,000 & Moderately corrosive \\
\hline 3,000 to 5,000 & Corrosive \\
\hline 1,000 to 3,000 & Highly corrosive \\
\hline$<1,000$ & Extremely corrosive \\
\hline
\end{tabular}

Not limited to resistivity, comprehensive aspects of corrosivity regarding CLSM include the $\mathrm{pH}$ of its bleeding and leachate. The bleeding is a single-phase media, whose $\mathrm{pH}$ is determined by EPA 150.1, "pH Electrometric." The $\mathrm{pH}$ of leachate from CLSM was measured in terms of EPA SW-846. Both of them were measured by the commercial laboratory. The corrosivity of solid CLSM was measured using ASTM G 57, "Standard Test Method for Field Measurement of Soil Resistivity Using the Wenner Four-Electrode Method" [ASTM G 57 2001].

Results of $\mathrm{pH}$ for bleeding and leachate of top-risk CLSM are presented in Table 7-26. The resistivity of solid CLSM is presented in Table 7-27. It is indicated that bleeding and leachate are alkaline and pose no corrosivity threat. The higher values of $\mathrm{pH}$ in the bleed water are primarily due to the calcium hydroxide released from the hydration of portland cement. Hydrate portland cement has a pH value between 12.6 and 13.0. Due to the relatively low hydraulic conductivity compared to background media, the core of 
CLSM keep dry mostly. Hence, the embedded metal pipe would not be exposed to media of high resistivity.

Table 7-26: $\mathrm{pH}$ of bleeding and leachate of CLSM

\begin{tabular}{l|l|l|l|l|l|l}
\hline & FS02 & FS12 & FS13 & FS16 & $\begin{array}{l}\text { Corrosivity } \\
\text { Threshold }\end{array}$ & $\begin{array}{l}\text { Testing } \\
\text { Method }\end{array}$ \\
\hline Bleeding & 12.9 & 12.9 & 12.9 & 13.1 & --- & EPA 150.1 \\
\hline Leachate & 10.5 & 10.9 & 11.5 & 11.7 & $2-12.5$ & $\begin{array}{l}\text { SW-846 } \\
9040\end{array}$ \\
\hline
\end{tabular}

Table 7-27: Resistivity of hardened CLSM (Under Investigation)

\begin{tabular}{l|c|c}
\hline \multirow{2}{*}{ FS ID } & Wet at 28 Day & Oven-dry at 28 Day \\
\cline { 2 - 3 } & \multicolumn{2}{|c}{ Unit (kom cm) } \\
\hline FS01 & 4.2 & $*$ \\
\hline FS02 & 20 & $*$ \\
\hline FS03 & 10 & $*$ \\
\hline FS04 & 8.6 & $*$ \\
\hline FS05 & 5.1 & $*$ \\
\hline FS06 & 5.8 & $*$ \\
\hline FS07 & 8.0 & $*$ \\
\hline FS08 & 5.1 & $*$ \\
\hline FS09 & 32 & $*$ \\
\hline FS10 & 25 & $*$ \\
\hline FS11 & 15 & $*$ \\
\hline FS12 & 8.5 & $*$ \\
\hline FS13 & 12 & $*$ \\
\hline FS14 & 4.2 & $*$ \\
\hline FS15 & 7.6 & -- \\
\hline FS17 & 7.6 & -- \\
\hline Min. & 4.2 & -- \\
\hline Max. & 32 & -- \\
\hline Avg. & 11.17 & $*$ \\
\hline SD & 8.04 & \\
\hline
\end{tabular}

*: Exceeding the capacity of instrument. 


\subsection{CLSM Correlations and Discussions}

In this section, based on the experiment results, correlations among critical parameters of CLSM are addressed.

\subsubsection{Flowability}

The flow behavior of CLSM is an important property to control the workability, and therefore it is essential to understand critical factors affecting this behavior. The water is mainly responsible for flow. The amount of water required to produce flow depends mainly on the nature of the sand and the proportion of fly ash and sand in the mixture [Bhat and Lovell 1997]. The correlation of gradation of particles and water content is presented in Figure 7-10. Grain fineness indicates the grain size in the CLSM matrix contributed by fly ash and foundry sand. It was calculated through multiplying AFS GFN by individual component proportion. The AFS GFN of fly ash is assumed to be 200. The greater the grain fineness, the finer is the average grain size. A point represents a mixture that attains a flow spread of $23 \mathrm{~cm}(9$ in). It is indicated that the finer the average grain size, the less water is required to attain the specified flow criteria for both excavatable CLSM and structural CLSM. For excavatable and structural CLSM, the fineness degree is negatively correlated with water proportion $(r=-0.764, p=0+; r=-$ $0.825, p=0+)$. The general absolute volume of water in the fresh CLSM matrix ranges from $30 \%$ to $50 \%$ to provide working flowability of CLSM. 


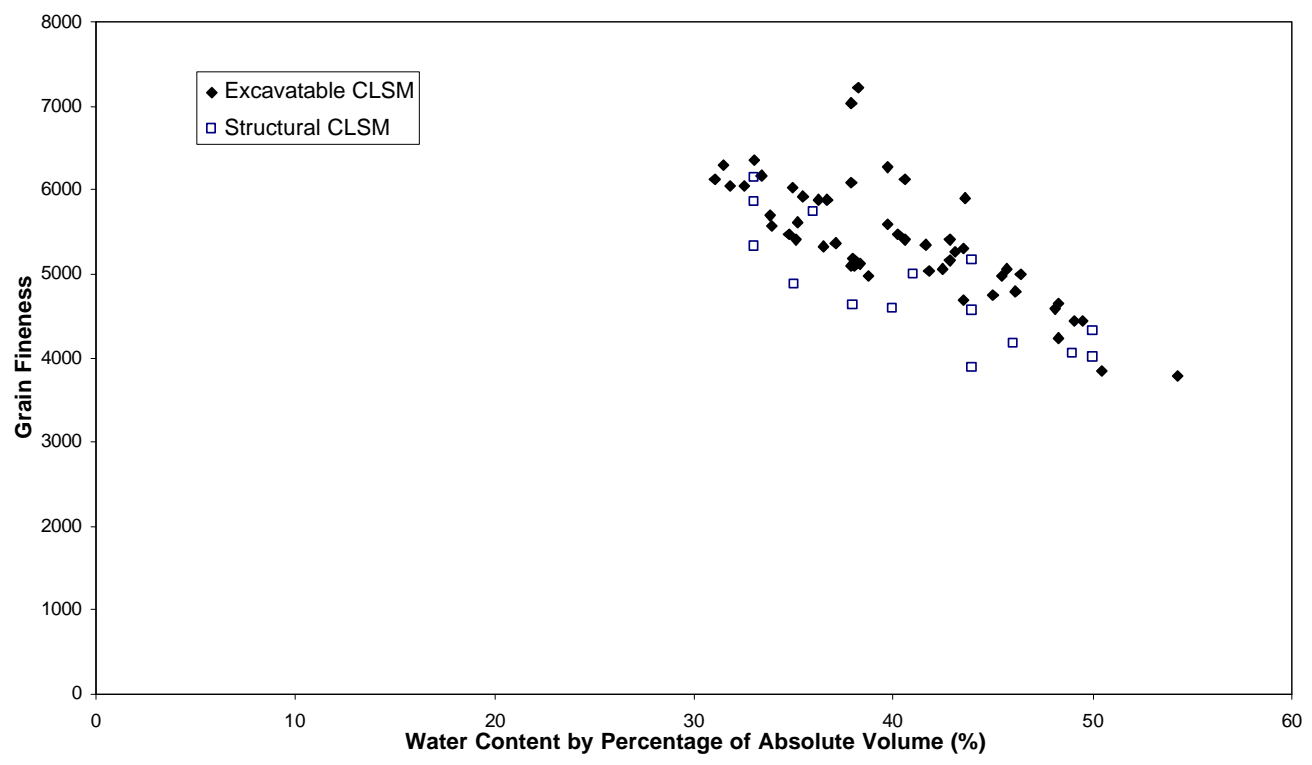

Figure 7-10: Relation between grain fineness and water content regarding flowability

\subsubsection{Setting Time}

The early age strength is important to support foot traffic and allow further loading. Generally, the flowable fill is considered to have hardened if it can support foot traffic. The short-term strength is evaluated by measuring the development of penetration resistance using a mortar penetrometer. As the hydration of cement begins, the penetration resistance increases with time [Bhat and Lovell 1997]. It is indicated from Figure 7-1, Figure 7-2, and Figure 7-3 that the development of penetration resistance is diverse and with no apparent general trend, although it increases with time. Since the proportions of mixtures are roughly at the same level for excavatable CLSM and structural CLSM, variation of excess foundry sand results in the scattering of penetration resistance. 
The comparison of penetration resistance is presented in Figure 7-11. Each point corresponds to a mixture. It is indicated that the variation in setting time of excavatable CLSM is greater than that of structural CLSM, in regard to penetration resistance of both $344 \mathrm{kPa}$ and $689 \mathrm{kPa}$. The time to attain a penetration resistance of $344 \mathrm{kPa}(50 \mathrm{psi})$ is regarded as the initial setting time. This is the level of resistance to support foot traffic without substantial settlement. The initial setting time for extractable CLSM starts from 6.7 hours and averages 15-20 hours. The penetration resistance gain from $344 \mathrm{kPa}$ to 688 $\mathrm{kPa}$ requires an additional 2-3 hours for extractable CLSM. These two sets of setting times are heavily positive-correlated $(r=0.971, p=0+)$. The initial setting time of structural CLSM is concentrated on 5-7 hours, which is contributed by the increased proportion of cement. And it requires less time, about 1 hour, to double penetration resistance. Hence, penetration resistance gain is more pronounced for structural CLSM than for excavatable CLSM. And for structural CLSM, the time to attain $344 \mathrm{kPa}$ and time to attain $688 \mathrm{kPa}$ are heavily positive-correlated $(r=0.991, p=0+)$. It is also inferred from the comparison that in the case of excavatable CLSM, foundry sand controls the initial penetration resistance, and in the other case of structural CLSM, cement dominates the initial penetration resistance. 


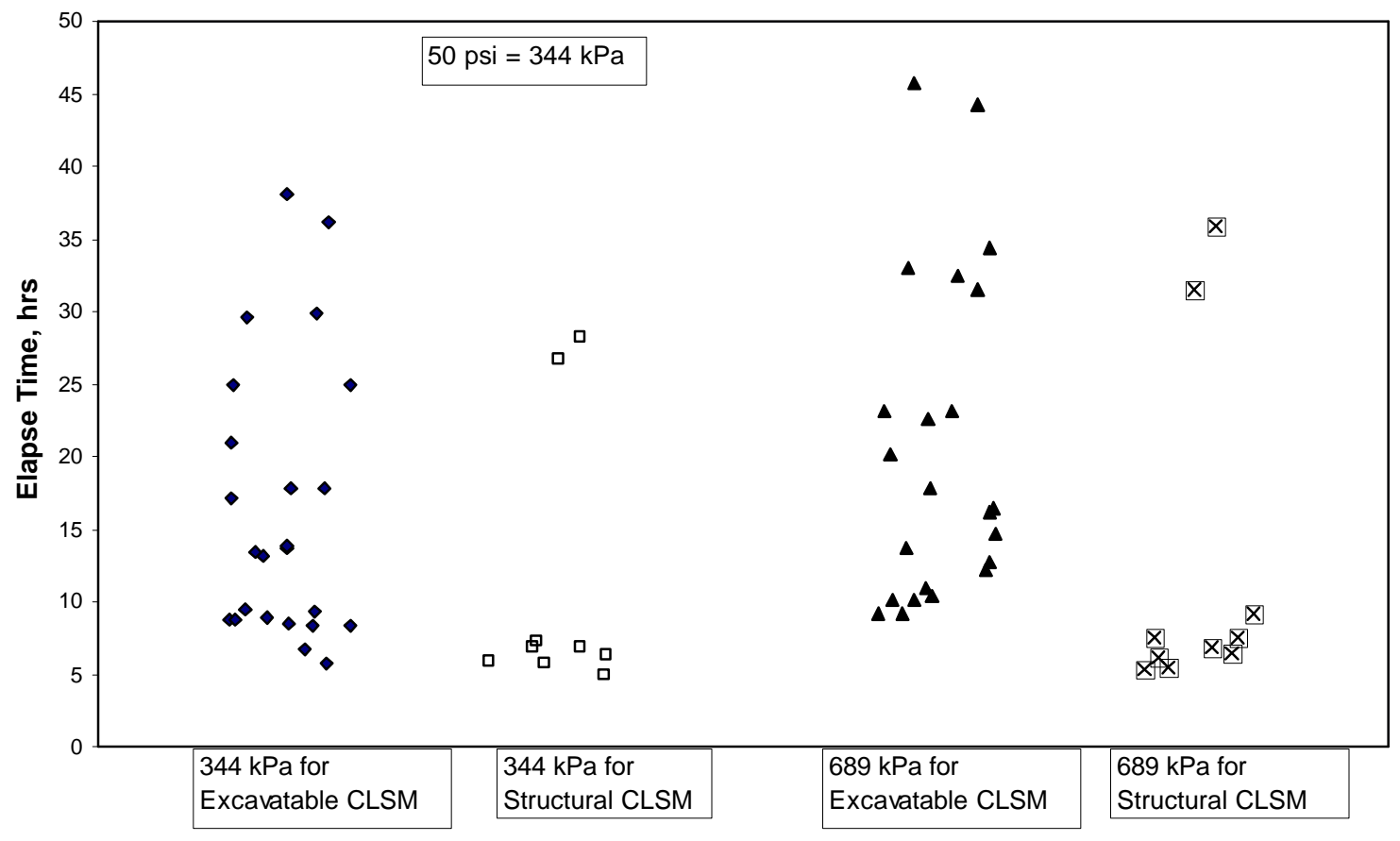

Figure 7-11: Comparison of penetration resistance

\subsubsection{Bleeding}

Bleeding is another consideration in the design of CLSM. The phenomenon of bleeding is common to all flowable fill matrices. Bleeding helps the particles contact and enhances strength development. It is expected that a higher amount of bleeding will result in a more compact structure and a lower void ratio in the material, and thereby would give a higher frictional strength. However, excessive bleeding is not desirable because it is more often a sign of poor mixture optimization. Mixtures that are not optimized will result in segregation. It will result in excessive initial subsidence of the surface after placement [Bhat and Lovell 1997]. 
The relationship between cumulative bleeding and bleed time is presented in Figure 7-12. As pointed out in Section 7.3.1.2, bleeding is moderately positive-correlated ( $r=0.469, p=0.028)$ with bleed time for excavatable CLSM and strongly correlated ( $r=0.827, p=0+$ ) for the structural CLSM, excluding FS16. Bleeding of excavatable CLSM is slightly more in volume and longer in elapse than that of structural CLSM because these formulations contain more water and less cement to minimize strength development. Bleeding of CLSM normally takes 2-5 hours, with volume up to $6 \%$ of fresh CLSM.

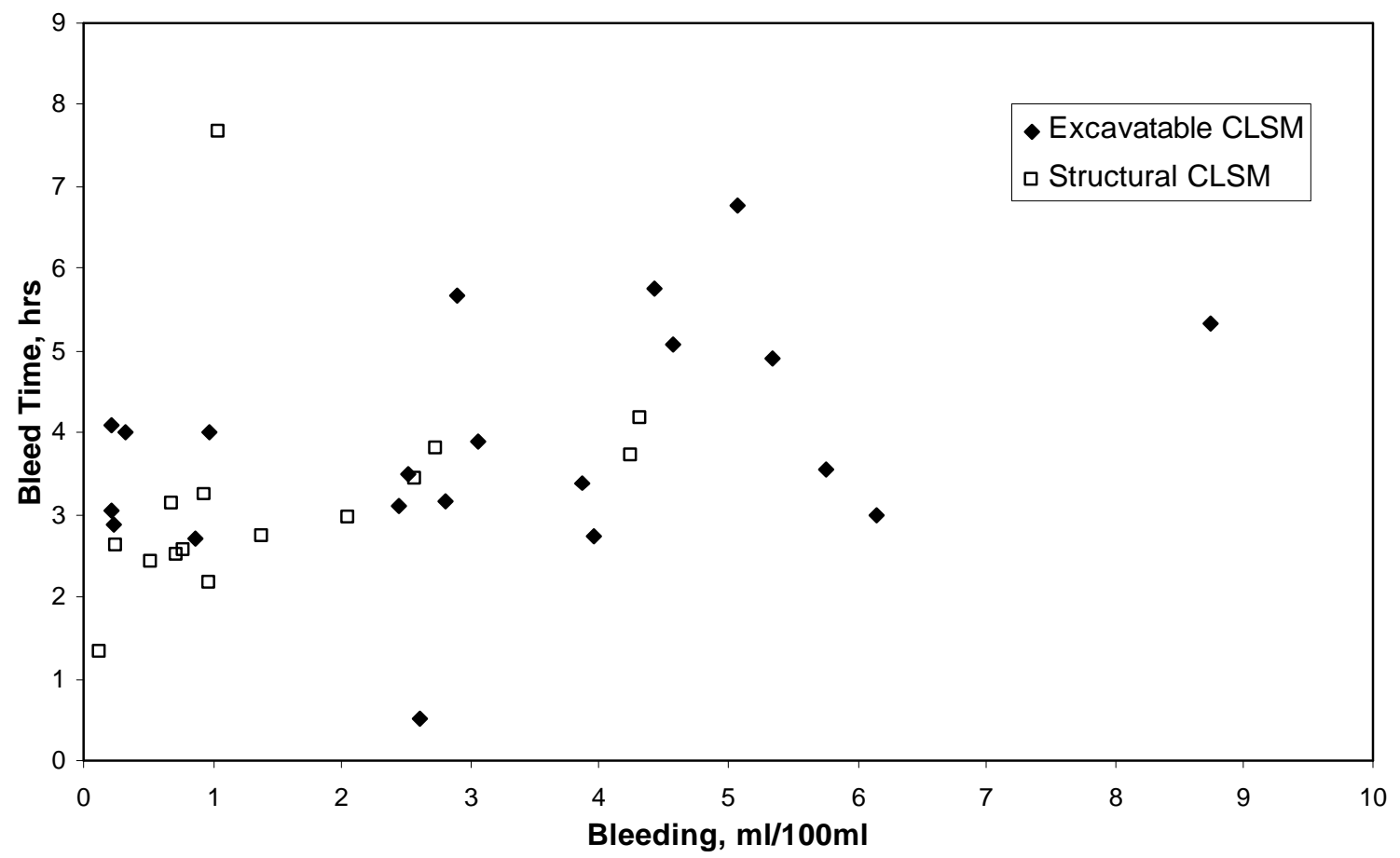

Figure 7-12: Relation between bleeding and bleed time

The relation of bleeding to water proportion is presented in Figure 7-13. The quantity of cement in structural CLSM does not influence water proportion significantly compared to that of excavatable CLSM. This indicates that water addition is exclusively 
controlled by flowability, which is closely related to grain fineness (Section 7.4.1). However, as hydration proceeds less water is available for bleed in mixtures with higher cement contents.

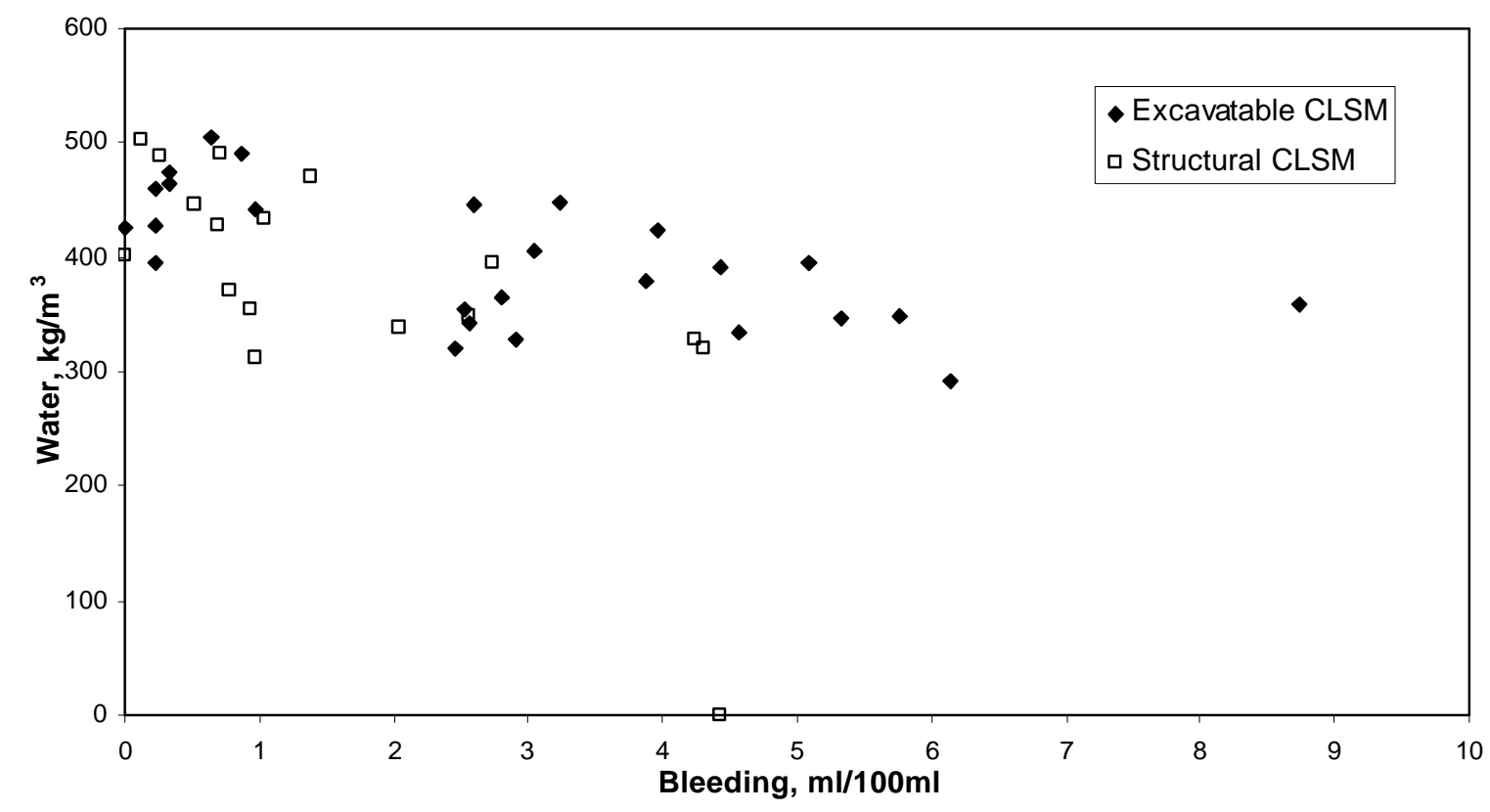

Figure 7-13: Relation between bleeding and water proportion

The relation between bleed time and setting time to attain $344 \mathrm{kPa}$ penetration resistance is presented in Figure 7-14. The time to attain $344 \mathrm{kPa}$ penetration resistance does not correlate with bleed time. 


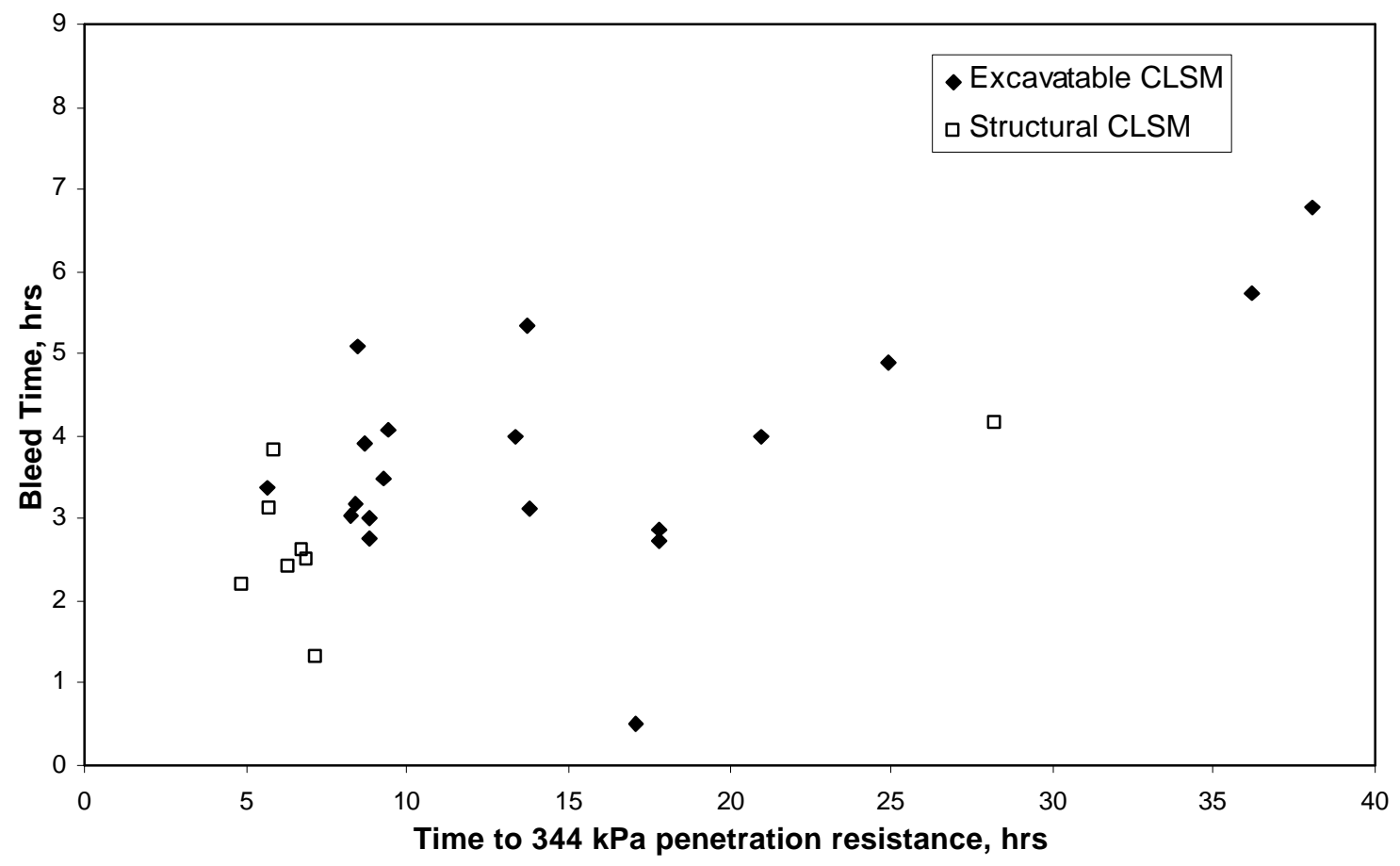

Figure 7-14: Relation between bleed time and setting time

\subsubsection{Strength}

When the CLSM is placed, and particles do not have much internal friction or cohesion, the shear strength is almost zero. As the excess water bleeds out, particles tend to settle and establish interparticle shear strength. The bond strength gained through cement hydration contributes further to the strength increment. The strength of CLSM during hardening can be thought of as having two components: frictional strength and a bond strength which is somewhat similar to cohesion in soils [Bhat and Lovell 1997]. 
The relation between 12-hour penetration resistance and 28-day compressive strength is presented in Figure 7-15. There is no significant correlation between 12-hour penetration resistance and 28-day compressive strength for excavatable CLSM ( $r=0.2$, $p=0.385$ ). For structural CLSM, after excluding two outliers, there is a correlation between 12-hour penetration resistance and 28-day compressive strength ( $r=0.892, p=$ 0.001). It is suggested that increases in cement proportion enhance the correlation of high early penetration resistance and long-term compressive strength.

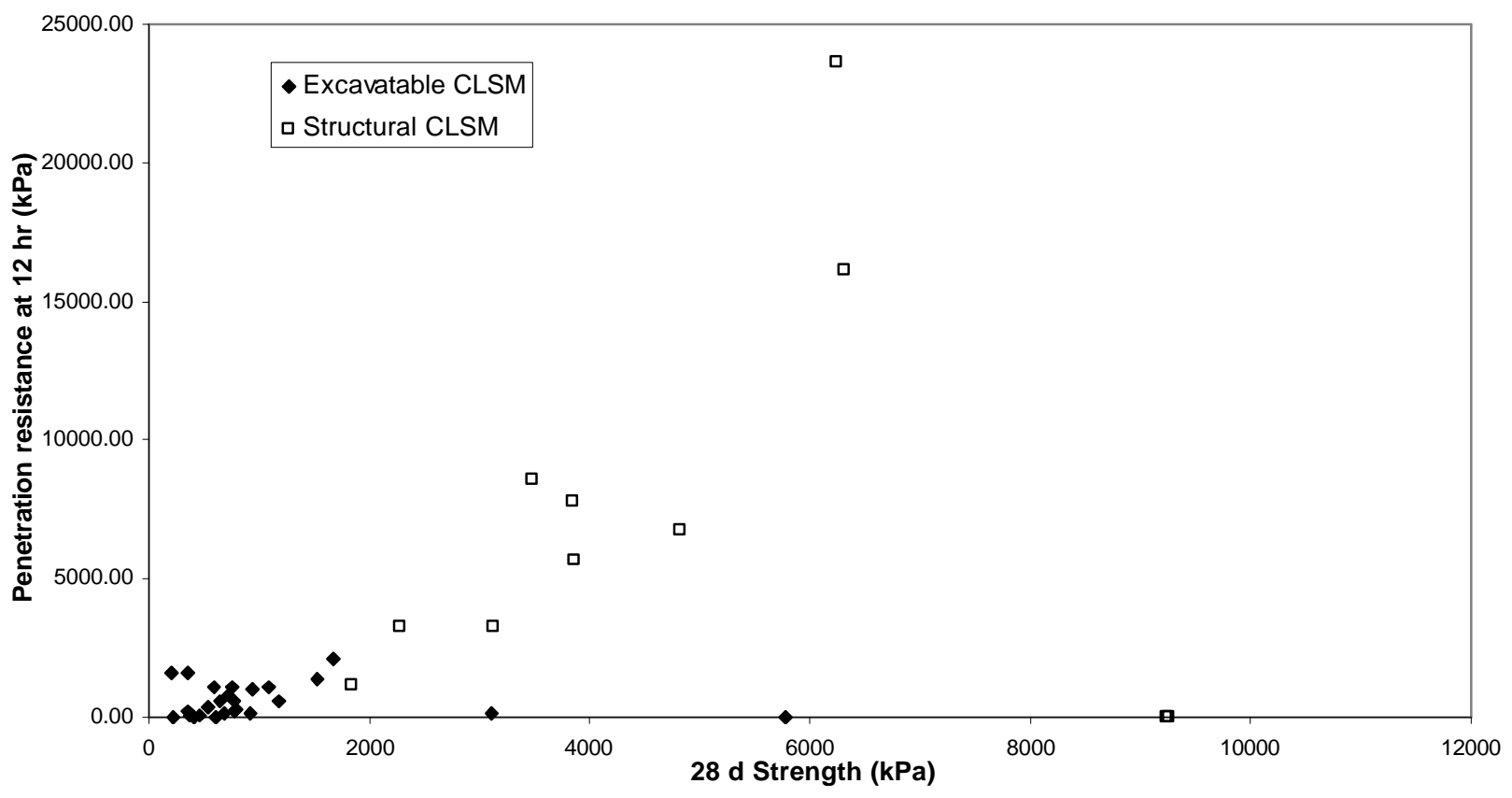

Figure 7-15: Relation between 28-day compressive strength and 12-hour penetration resistance

The relation between water-cement ratio and 28-day unconfined compressive strength is presented in Figure 7-16. It coincides with the curve tendency suggested in Figure 7-17, which was suggested by Bhat and Lovell [Bhat and Lovell 1997]. The 
variation in Figure 7-16 suggests that 28-day strength varies inversely with water-cement ratio, and that the variation of water-cement ratio needed for excavatable CLSM is greater than that for structural CLSM.

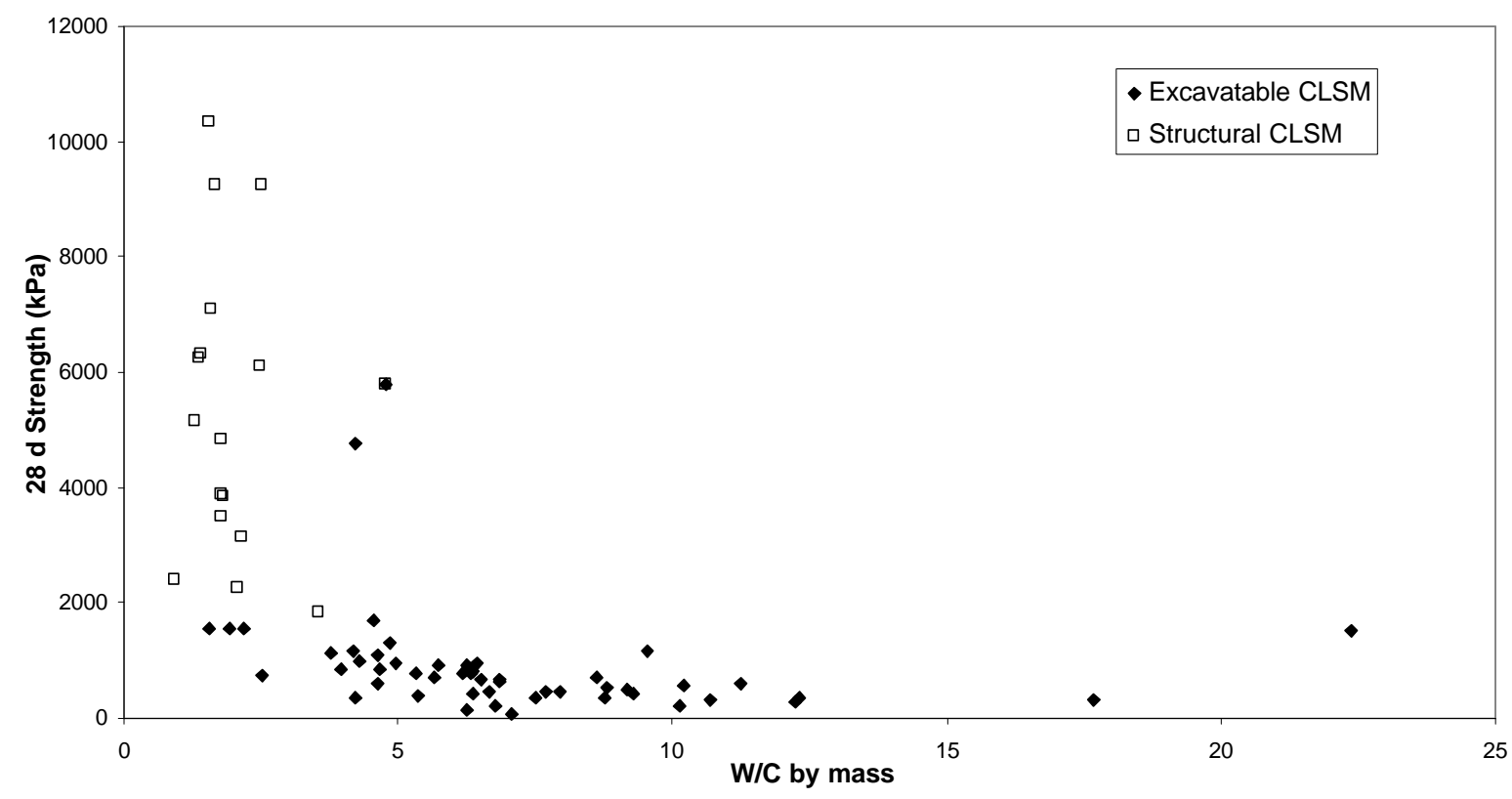

Figure 7-16: Relation between 28-day compressive strength and water-cement ratio 


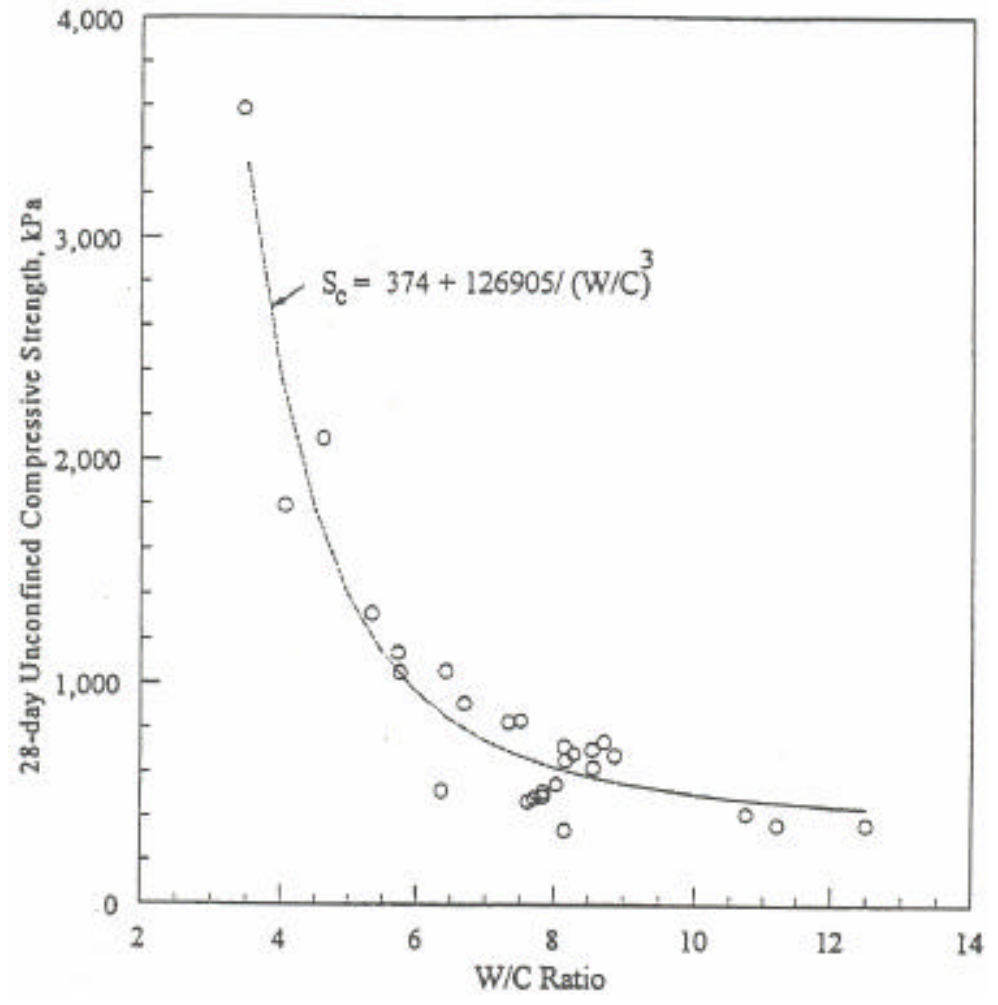

Figure 7-17: Relation between 28-day compressive strength and water-cement ratio [Bhat and Lovell 1997]

The relation between 28-day compressive strength and cement proportion is presented in Figure 7-18. It is indicated that increment in cement proportion contributes much to strength gain. For either class of CLSM, no significant regression line can be approximated. However, there is a general relationship between cement proportion and 28-day compressive strength. 


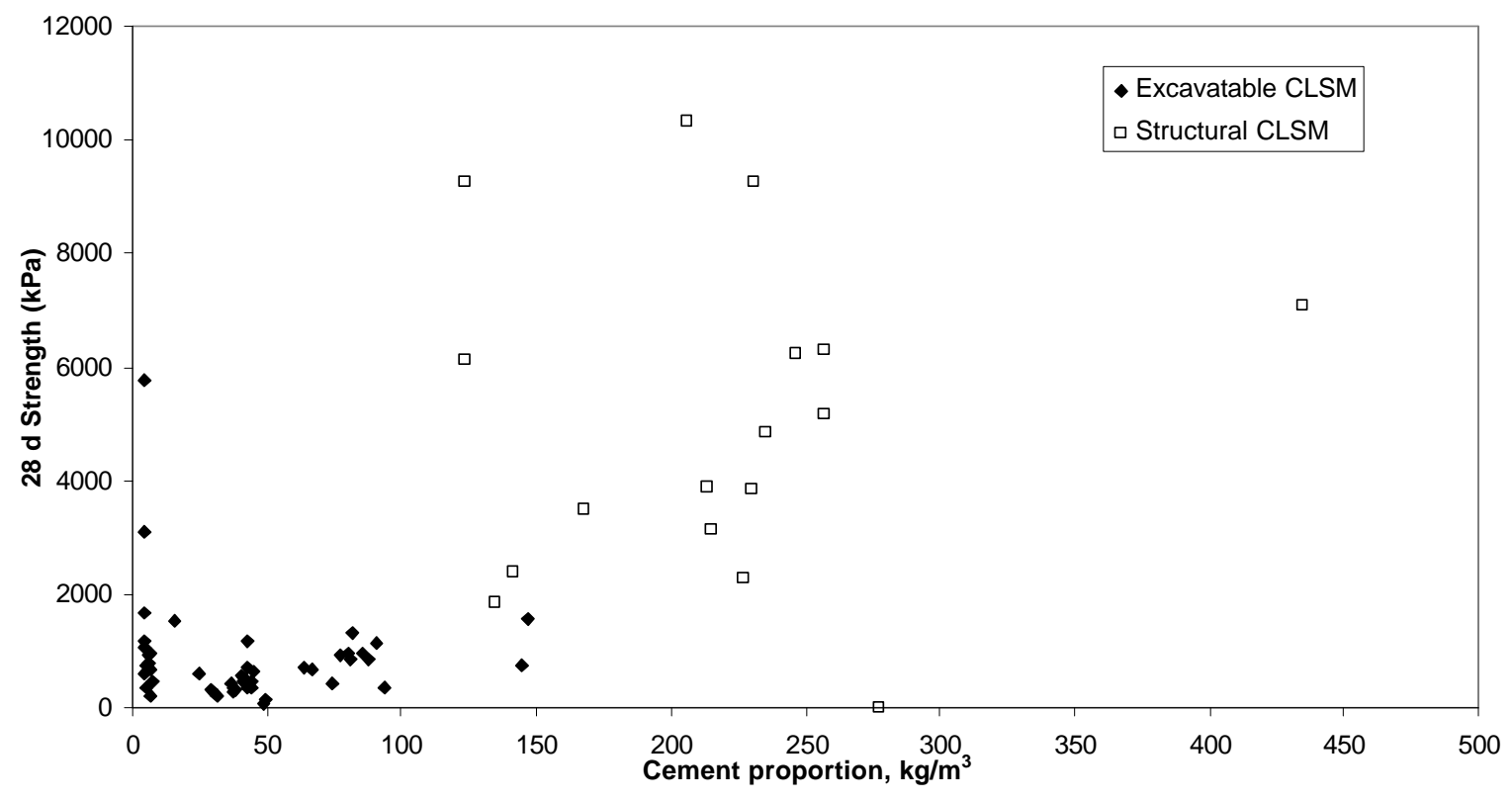

Figure 7-18: Relation between 28-day compressive strength and cement proportion

The relation between 28-day compressive strength and 90-day compressive strength is presented in Figure 7-19. It is indicated that the correlation between 28-day compressive strength and 90-day compressive strength is significant $(r=0.932, p=0+$ for excavatable CLSM, and $r=0.927, p=0+$ for structural CLSM). The relation of 28day compressive strength and 180-day compressive strength is presented in Figure 7-20. It is indicated that the correlation between 28-day compressive strength and 180-day compressive strength is significant $(r=0.92, p=0+$ for excavatable CLSM, and $r=$ 0.51, $p=0.036$ for structural CLSM). Hence, the long-term strength of CLSM can be estimated based on 28-day strength with statistical confidence. 


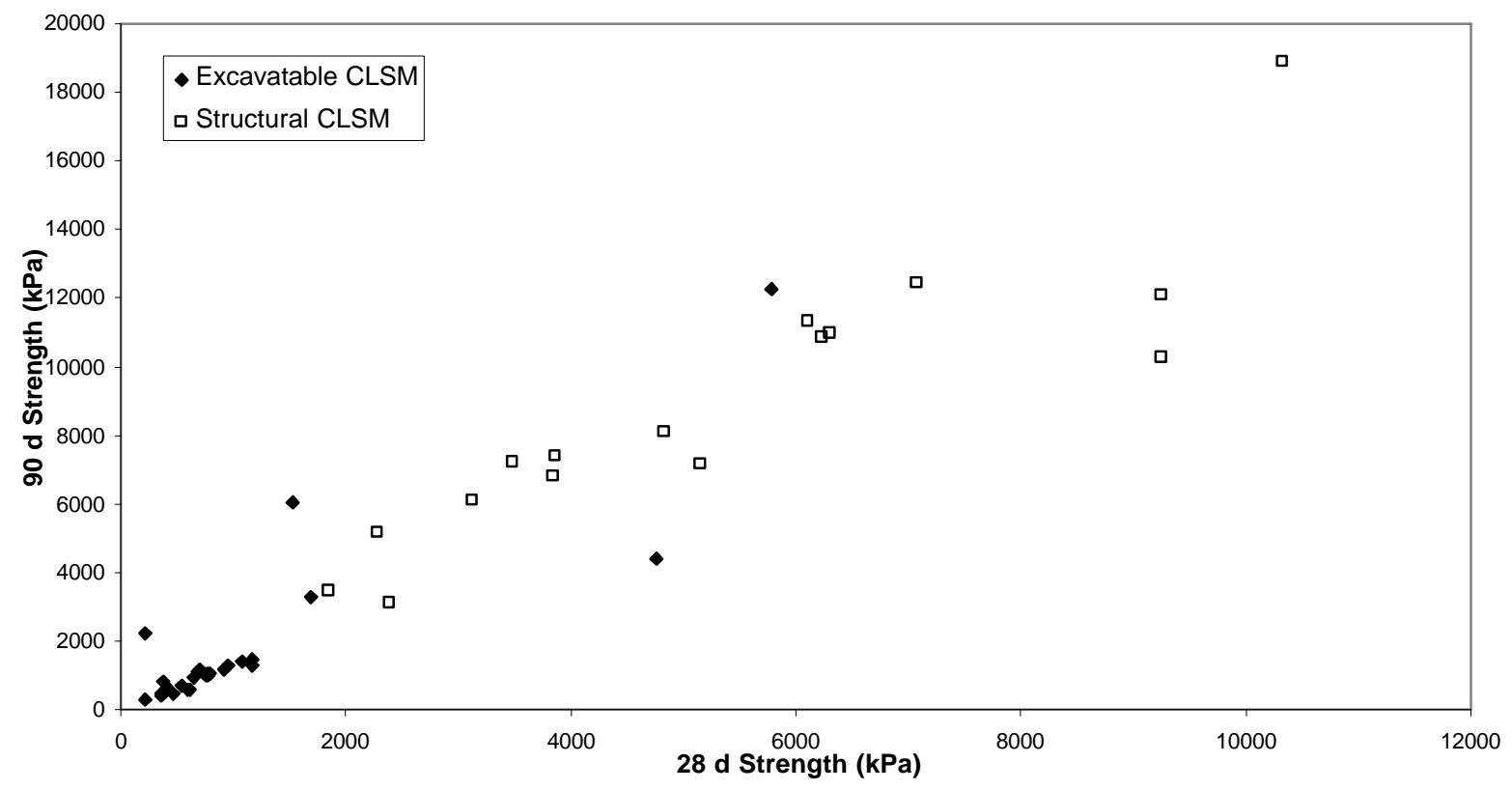

Figure 7-19: Relation between 28-day compressive strength and 90-day compressive strength

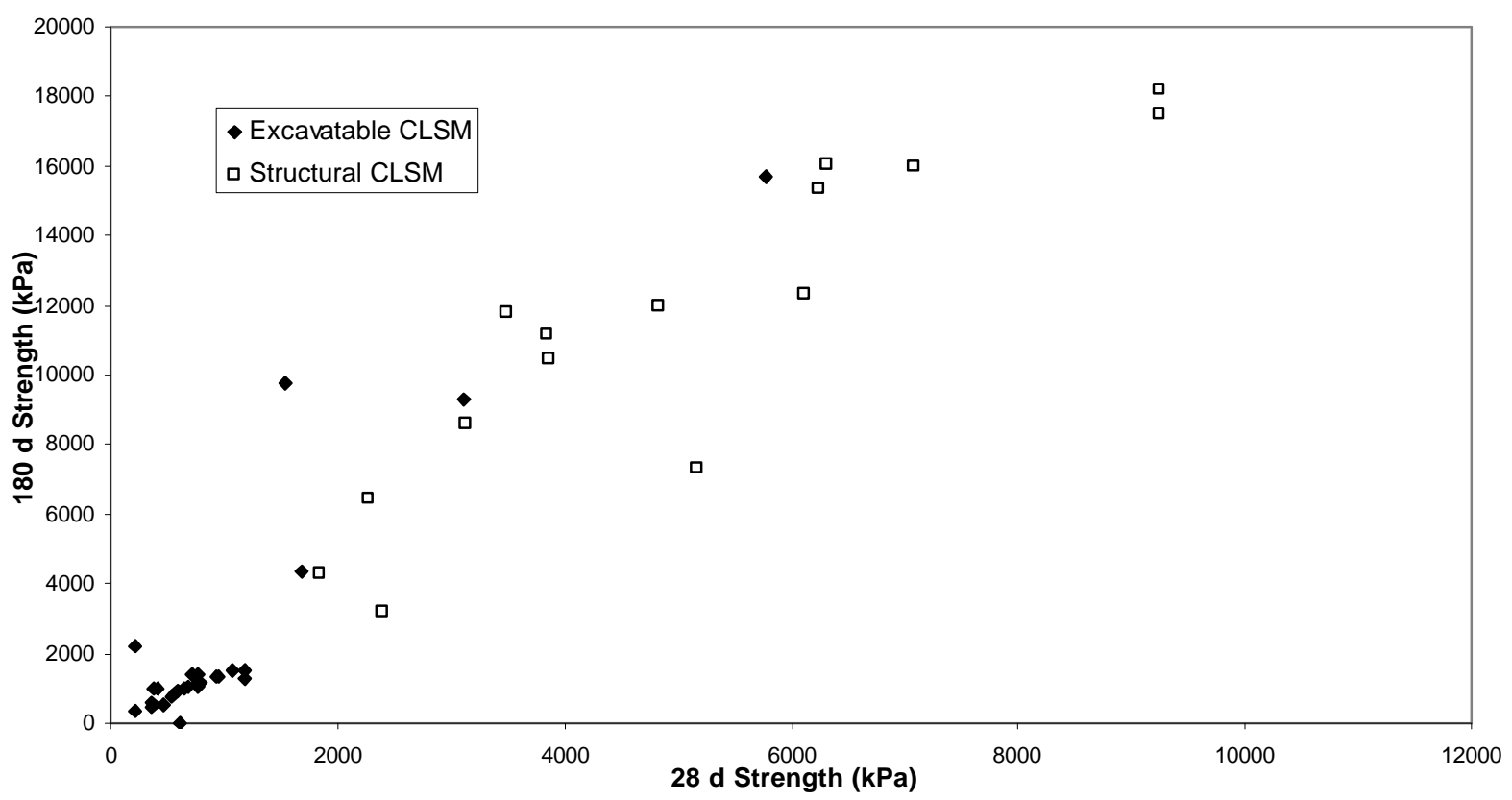

Figure 7-20: Relation between 28-day compressive strength and 180-day compressive strength 


\section{Chapter 8}

\section{EXPERIMENTS QUALIFYING HMA USING EXCESS FOUNDRY SAND}

Seventeen identical foundry sands were incorporated into an HMA mixture at a level of $10 \%$ by mass. The aggregates used came from a limestone quarry in close vicinity to the city of Madison, WI. The control gradation was a low-volume, fine gradation. The job mix formula for each of the 17 mixes was kept the same as the control job mix formula. Volumetric properties of the mixes were determined according to Superpave mix design criteria. The mixtures were also tested for moisture sensitivity through the use of the indirect tension test. The following sections give a summary of the findings pertaining to each measurement collected in the study.

\subsection{Experimental Methodology with Examples}

The purpose of this section is to explain the methods used to collect the data and analyze results of this study, including the calculation of the maximum and bulk density of aggregates, angularity of aggregates, the maximum density of loose mix, the bulk specific gravity of compacted asphalt mix samples, and the absorption of asphalt into aggregates, the calculations used for the volumetric properties, the energy indices, the indirect tension test results, and the rutting results. 


\subsubsection{Density, Absorption and Angularity}

A relatively new instrument for measuring density called the CoreLok ${ }^{\mathrm{TM}}$ device, created by a company called Instrotek ${ }^{\circledR}$, is becoming popular for used among state DOTs and asphalt contractors. This allows air to be removed from an aggregate or asphalt sample and then the sample is sealed in a bag. The sample can then be placed in water and weighed or the bag can be cut open and the sample completely saturated with water. The device can also be used to find the quantity of surface pores on an aggregate. This allows a more scientific method to determine the absorption of aggregates compared to methods used previously to measure indirectly the saturated surface dry volumetric properties.

\subsubsection{Aggregate Maximum Density}

The maximum density of the aggregates, foundry sands, and aggregate-bitumen mixtures were determined according to the procedure outlined in ASTM D6857-03 "Standard Test Method for Maximum Specific Gravity and Density of Bituminous Paving Mixtures Using Automatic Vacuum Sealing Method" [ASTM D 6857 2003]. Equation 8.1 illustrates the steps needed to calculate the maximum density.

$$
\rho_{M D}=\frac{M_{S}}{\frac{M_{S}+M_{Y}+M_{C}-M_{I W}}{\rho_{W}}-\frac{M_{Y}}{\rho_{Y}}-\frac{M_{C}}{\rho_{C}}}
$$

Where, $\mathrm{M}_{\mathrm{C}}=$ Mass of Channel Bag $=23.9 \mathrm{~g}$

$$
\mathrm{M}_{\mathrm{Y}}=\text { Mass of Large Yellow Bag }=51.0 \mathrm{~g}
$$




$$
\begin{aligned}
& M_{\mathrm{S}}=\text { Mass of Sample }=1,798.8 \mathrm{~g} \\
& \mathrm{M}_{\mathrm{IW}}=\text { Mass of Sample, Channel Bag, and Yellow Bag in Water }=1,149.3 \mathrm{~g} \\
& \rho_{\mathrm{W}}=\text { Density of Water }=0.997 \mathrm{~g} / \mathrm{ccm} \\
& \rho_{\mathrm{C}}=\text { Density of Channel Bag }=0.985 \mathrm{~g} / \mathrm{ccm} \\
& \rho_{\mathrm{Y}}=\text { Density of Yellow Bag }=0.918 \mathrm{~g} / \mathrm{ccm} \\
& \rho_{\mathrm{MD}}=\text { Maximum Density of Sample }=2.7812 \mathrm{~g} / \mathrm{ccm}
\end{aligned}
$$

\subsubsection{Bulk Density of Coarse and Fine Aggregates Using the InstroTek ${ }^{\circledR}$ Method}

The procedure used to determine the bulk density of aggregates and aggregatefoundry sand mixes is the same as outlined in the AggPlus ${ }^{\mathrm{TM}}$ System manual [nstroTek 2002], which is given with the CoreLok ${ }^{\mathrm{TM}}$ device, for the bulk density of coarse aggregates, with the following exception:

1) After placing the lid on the volumeter and filling the volumeter with water as directed using the syringe, place the filled volumeter in the water bath on the padded weigh basket.

2) Record the mass, once the mass reading has stabilized.

Originally, the Gravity Suit Software [InstroTek 2001] was used to perform the calculations. Due to erroneous results, a second method was developed to determine the bulk density of the foundry sands.

The calculation of the bulk density of the sample is according to Equation 8.2. 


$$
\rho_{B D}=\frac{M_{S} \rho_{W}}{M_{S}-M_{S I W}-M_{B I W}}
$$

where: $\mathrm{M}_{\mathrm{BIW}}=$ Mass of Volumeter and Lid in Water $=1749.7 \mathrm{~g}$

$$
\begin{aligned}
& M_{\mathrm{S}}=\text { Mass of Sample }=1806.1 \mathrm{~g} \\
& \mathrm{M}_{\mathrm{SIW}}=\text { Mass of Sample, Volumeter and Lid in Water }=2877.2 \mathrm{~g} \\
& \rho_{\mathrm{W}}=\text { Density of Water }=0.997 \mathrm{~g} / \mathrm{ccm} \\
& \rho_{\mathrm{BD}}=\text { Bulk Density of Sample }=2.6535 \mathrm{~g} / \mathrm{ccm}
\end{aligned}
$$

\subsubsection{Water Absorption/Percentage of Micro Voids in Aggregates}

There is no testing procedure used to determine the percentage of micro voids. The percentage of micro voids is determined by a calculation based on the maximum and bulk densities of aggregate samples. The percentage absorption is usually calculated using the mass of fluid absorbed divided by the mass of aggregates. However, it is

recognized that a volume percentage can also be calculated. Equation 8.3 and Equation 8.4 can be used to determine the percentage of micro voids per aggregate sample.

$$
\begin{array}{rr}
\text { \%Voids }=\left[1-\frac{\rho_{B}}{\rho_{M}}\right] \times 100 & \text { Equation 8.3 } \\
\% A b s .=\left[\frac{\rho_{W}}{\rho_{B}}-\frac{\rho_{W}}{\rho_{M}}\right] \times 100 & \text { Equation } 8.4 \\
\rho_{\mathrm{M}}=\text { Maximum Density }=2.7812 \mathrm{~g} / \mathrm{ccm} & \\
\rho_{\mathrm{B}}=\text { Bulk Density }=2.6535 \mathrm{~g} / \mathrm{ccm} &
\end{array}
$$


$\rho_{\mathrm{W}}=$ Density of Water $=0.997 \mathrm{~g} / \mathrm{ccm}$

$\%$ Voids $=$ Volume of Micro Voids Divided By Volume of Aggregate $=4.59 \%$

$\%$ Abs. $=$ Mass of Water Able to be Absorbed Divided By Mass of Aggregate $=$ $1.73 \%$

\subsubsection{Indirect Method to Determine the Apparent Density of Foundry Sand}

One of the most difficult tasks is to determine the apparent specific gravity of foundry sands. The difficulty is related to the fact that some sands have a tendency to absorb and retain water. While running the apparent and bulk density tests, a film was observed to be covering the foundry sand, possibly preventing the water from penetrating the sand, and seemed to be due to surface tension between the water and the sand. It was thought that by mixing the sand with larger aggregates, the film or skin due to the surface tension could be disrupted and allow water to penetrate the sand more thoroughly. Unfortunately, due to lack of time, data could not be gathered using the indirect method, and the results from the direct method could not be compared to those of the indirect method. The indirect method includes using Equation 8.5.

$$
\rho_{F . S .}=\frac{P_{F . S .}}{\frac{1}{\rho_{S}}-\frac{P_{A}}{\rho_{A}}}
$$

where, $\rho_{\mathrm{A}}=$ Apparent Density of Aggregates $=2.7812 \mathrm{~g} / \mathrm{ccm}$

$\rho_{\mathrm{S}}=$ Apparent Density of Sample w/FS14 $=2.7758 \mathrm{~g} / \mathrm{ccm}$

$\mathrm{P}_{\mathrm{F} . \mathrm{S} .}=$ Mass Fraction of FS14 in Sample $=0.1$ 


$$
\begin{aligned}
& \mathrm{P}_{\mathrm{A}}=\text { Mass Fraction of Aggregate in Sample }=0.9 \\
& \rho_{\text {F.S. }}=\text { Apparent Density of FS14 }=2.7279 \mathrm{~g} / \mathrm{ccm}
\end{aligned}
$$

\subsubsection{Indirect Method to Determine the Bulk Density of Foundry Sand}

As mentioned in the previous section, the direct methods may include error due to the surface tension between the sand and the water. The following procedure could be used to determine the bulk density of foundry sands. The indirect method includes using Equation 8.6.

$$
\rho_{F . S .}=\frac{P_{F . S .}}{\frac{1}{\rho_{S}}-\frac{P_{A}}{\rho_{A}}}
$$

Equation 8.6

where, $\rho_{\mathrm{A}}=$ Bulk Density of Aggregates $=2.6535 \mathrm{~g} / \mathrm{ccm}$

$$
\begin{aligned}
& \rho_{\mathrm{F} . \mathrm{S} .}=\text { Bulk Density of Sample Containing FS } 14=2.6161 \mathrm{~g} / \mathrm{ccm} \\
& \mathrm{P}_{\mathrm{F} . \mathrm{S} .}=\text { Mass Fraction of FS14 = } 0.1 \\
& \mathrm{P}_{\mathrm{A}}=\text { Mass Fraction of Aggregates }=0.9 \\
& \rho_{\text {F.S. }}=\text { Bulk Density of F.S. } \# 1=2.3299 \mathrm{~g} / \mathrm{ccm}
\end{aligned}
$$

\subsubsection{Uncompacted Void Content of Fine Aggregates and Foundry Sands}

The angularity of sands is determined using the ASTM standard C1252-98 "Standard Test Method for Uncompacted Void Content of Fine Aggregate (as Influenced by Particle Shape, Surface Texture, and Grading)." The procedure includes Equation 8.7. 


$$
U_{V}=\frac{V-M / D_{s b}}{V} \times 100
$$

where $\rho_{\mathrm{sb}}=$ Bulk Density of FS14 $=2.3299 \mathrm{~g} / \mathrm{ccm}$

$\mathrm{V}=$ Volume of Cylinder $=100 \mathrm{ccm}$

$\mathrm{M}=$ Mass of Loose Fine Aggregates to Fill Cylinder $=137.7 \mathrm{~g}$

$\mathrm{U}_{\mathrm{V}}=$ Uncompacted Void Content $=40.9 \%$

\subsubsection{Bulk Density of Compacted Bitumen Samples}

The bulk density of compacted asphalt mixture samples is measured using the ASTM standard procedure D6752-02a, "Bulk Specific Gravity and Density of Compacted Bituminous Mixtures Using Automatic Vacuum Sealing Method.” This procedure is also used for mixtures containing foundry sands. Equation 8.8 is used to calculate the bulk density,

$$
\rho_{B D}=\frac{M_{S}}{\frac{M_{S}+M_{Y}-M_{I W}}{\rho_{W}}-\frac{M_{Y}}{\rho_{Y}}}
$$

where, $M_{S}=$ Mass of Compacted Bitumen Sample $=4941.1 \mathrm{~g}$

$$
\begin{aligned}
& M_{Y}=\text { Mass of Yellow Bag }=44.7 \mathrm{~g} \\
& M_{I W}=\text { Mass of Sample and Bag in Water }=2912.1 \\
& \rho_{\mathrm{Y}}=\text { Density of Yellow Bag }=0.918 \mathrm{~g} / \mathrm{ccm} \\
& \rho_{\mathrm{W}}=\text { Density of Water }=0.997 \mathrm{~g} / \mathrm{ccm} \\
& \rho_{\mathrm{BD}}=\text { Bulk Density of Sample }=2.4325 \mathrm{~g} / \mathrm{ccm}
\end{aligned}
$$




\subsubsection{Effective Density of Aggregates}

The effective density of aggregate samples is determined using Equation 8.9.

$$
\rho_{E}=\frac{1-P_{A . S .}}{\frac{1}{\rho_{M}}-\frac{P_{A . S .}}{\rho_{\text {A.S. }}}}
$$

Equation 8.9

where, $\rho_{\mathrm{M}}=$ Maximum Density of Bituminous Sample $=2.5207 \mathrm{~g} / \mathrm{ccm}$

$$
\begin{aligned}
& \rho_{\mathrm{W}}=\text { Density of Water }=0.997 \mathrm{~g} / \mathrm{ccm} \\
& \rho_{\text {A.S. }}=\text { Density of Asphalt }=1.0228 \mathrm{~g} / \mathrm{ccm} \\
& P_{\text {A.S. }}=\text { Mass Fraction of Asphalt }=0.052 \\
& \rho_{\mathrm{E}}=\text { Effective Density of Aggregate }=2.7409 \mathrm{~g} / \mathrm{ccm}
\end{aligned}
$$

\subsubsection{Asphalt Absorption of Bituminous Samples}

The asphalt absorption of asphalt mixtures is calculated using Equation 8.10 and Equation 8.11.

$$
\begin{gathered}
\% A b s_{._{1}}=\frac{\left(\rho_{E f f}-\rho_{B}\right) \rho_{A . S .}}{\rho_{A p p}^{2}\left(\frac{1}{1-P_{A . S .}}-1\right)} \times 100 \\
\% A b s_{._{2}}=\frac{\left(\rho_{E f f}-\rho_{B}\right) \rho_{A . S .}}{\rho_{A p p}^{2}} \times 100
\end{gathered}
$$

where, $\rho_{\text {App }}=$ Apparent Density of Control Aggregates $=2.7812 \mathrm{~g} / \mathrm{ccm}$

$$
\begin{aligned}
& \rho_{\text {Eff }}=\text { Effective Density of Control Aggregate }=2.7464 \mathrm{~g} / \mathrm{ccm} \\
& \rho_{B}=\text { Bulk Density of Control Aggregates }=2.6523 \mathrm{~g} / \mathrm{ccm}
\end{aligned}
$$




$$
\begin{aligned}
& \rho_{\text {A.S. }}=\text { Density of Asphalt }=1.0228 \mathrm{~g} / \mathrm{ccm} \\
& \mathrm{P}_{\text {A.S. }}=\text { Mass Fraction of Asphalt }=0.055 \\
& \% \text { Abs. } 1_{1}=\text { Absorbed Asphalt Per Total Asphalt }=21.4 \% \\
& \% \text { Abs. } .2=\text { Absorbed Asphalt Mass Per Aggregate Mass }=1.24 \%
\end{aligned}
$$

\subsubsection{Surface Area \& Average Asphalt Film Thickness}

The asphalt film thickness was determined using a method described in Chapter 3 of the book, "Hot Mix Asphalt Materials, Mixture Design and Construction" [Roberts et al 1996]. According to this method, the surface area of all aggregate blends used should be the same, because the gradation was kept constant and the surface area calculation is totally dependent on gradation. Each sieve size is allocated a surface area factor. This means that for every pound of material retained on that particular sieve, it will have a certain surface area regardless of mineralogy or surface texture. Table 8-1 includes the surface area factors in both English and SI units.

Table 8-1: Surface area factors for each sieve size

\begin{tabular}{c|c|c}
\hline Sieve Size & S.A. Factor $\mathbf{f t}^{2} / \mathbf{l b}$. & S.A. Factor $\mathbf{~}^{2} / \mathbf{k g}$ \\
\hline \hline P25.00-R19.00 $\mathbf{~ m ~}$ & 2 & 0.41 \\
\hline P19.00-R12.50 mm & 2 & 0.41 \\
\hline P12.50-R9.50 mm & 2 & 0.41 \\
\hline P9.50-R4.75 mm & 2 & 0.41 \\
\hline P4.75-R2.36 mm & 2 & 0.41 \\
\hline P2.36-R1.18 mm & 4 & 0.82 \\
\hline P1.18-R0.60 mm & 8 & 1.64 \\
\hline P0.60-R0.30 mm & 14 & 2.87 \\
\hline P0.30-R0.15 mm & 30 & 6.15 \\
\hline P0.15-R0.075 mm & 60 & 12.29 \\
\hline P0.075 $\mathbf{~ m m ~}$ & 160 & 32.78 \\
\hline
\end{tabular}


Using the gradation of the aggregate blend used in this study (as shown in Table 8-2), the total surface area is calculated by multiplying the percentage retained on each sieve by the surface area factors listed in Table 8-1. The surface area is calculated to be $3.33 \mathrm{~m}^{2} / \mathrm{kg}$ of aggregates.

Table 8-2: Job mix formula according to percent retained

\begin{tabular}{c|c}
\hline Sieve Size & \% Retained \\
\hline \hline P25.00-R19.00 $\mathbf{~ m m}$ & 0.0 \\
\hline P19.00-R12.50 mm & 5.6 \\
\hline P12.50-R9.50 mm & 10.4 \\
\hline P9.50-R4.75 mm & 17.5 \\
\hline P4.75-R2.36 mm & 17.6 \\
\hline P2.36-R1.18 mm & 12.8 \\
\hline P1.18-R0.60 mm & 9.1 \\
\hline P0.60-R0.30 mm & 10.8 \\
\hline P0.30-R0.15 mm & 7.6 \\
\hline P0.15-R0.075 mm & 3.6 \\
\hline P0.075 $\mathbf{~ m m ~}$ & 5.0
\end{tabular}

To determine the average asphalt film thickness for the mixture, Equation 8.12 could be used.

$$
T_{A}=\frac{P_{A . S .}\left(1-A b s_{.1}\right)}{\rho_{\text {A.C. }}\left(1-P_{A . S .}\right) S . A . \times 1000}
$$

$\mathrm{P}_{\mathrm{A} . \mathrm{S} .}=$ Mass Fraction of Asphalt $=0.055$

$\%$ Abs. $1=$ Absorbed Asphalt per Total Asphalt $=21.4 \%$

$\rho_{\mathrm{AC}}=$ Density of Asphalt $=1.0228 \mathrm{~g} / \mathrm{ccm}$

S.A. $=$ Surface Area $=3.33 \mathrm{~m}^{2} / \mathrm{kg}$

$\mathrm{T}_{\mathrm{A}}=$ Average Asphalt Film Thickness $=1.343 \mathrm{E}^{-5} \mathrm{~m}=13.43 \mu \mathrm{m}$ 


\subsubsection{Densities at Different Gyrations, Energy Indices, and Indirect Tension Test}

\subsubsection{Densities at Different Gyrations}

The optimum asphalt content is the asphalt content that gives $4 \%$ air voids, when a 5,000 g HMA sample is compacted using a Superpave Gyratory Compactor. The samples were mixed at a temperature of $145 \mathrm{EC}$, and cured and compacted at a temperature of 135 EC. The AASHTO Standard Procedure T 312-01 was followed to mix and compact the samples, as well as the calculations used to determine the $\% \mathrm{Gmm}$ at each gyration. Since all the samples were E-3 mixes, $\mathrm{N}_{\text {ini }}$ was at 7 gyrations, $\mathrm{N}_{\text {des }}$ was at 75 gyrations, and $\mathrm{N}_{\max }$ was at 115 gyrations. All samples used to determine the optimum asphalt content were compacted to 115 gyrations. This means the volume correction factor required in the standard procedure was calculated at 115 gyrations. To show the specific steps for calculations of densities, Equation 8.13 is used.

$$
D_{\max -\text { theo. }}=\frac{4000 M}{\pi D^{2} H_{\max }}
$$

$\mathrm{M}=$ Mass of puck after 115 gyrations $=4959.1 \mathrm{~g}$

$\mathrm{H}_{\text {ini }}=$ Height of sample at the $7^{\text {th }}$ gyration as reported by the Superpave Gyratory Compactor $(\mathrm{SGC})=125.7 \mathrm{~mm}$

$$
\begin{aligned}
& \mathrm{H}_{\text {des }}=\text { Height of sample at the } 75^{\text {th }} \text { gyration as reported by } \mathrm{SGC}=116.9 \mathrm{~mm} \\
& \mathrm{H}_{\max }=\text { Height of sample at the } 115^{\text {th }} \text { gyration as reported by the } \mathrm{SGC}=115.7 \mathrm{~mm} \\
& \mathrm{D}=\text { Diameter of sample, constant }=150 \mathrm{~mm} \\
& \mathrm{D}_{\text {Theo. }}=\text { Theoretical Density at } 115^{\text {th }} \text { gyration }=2.4255 \mathrm{~g} / \mathrm{ccm} \\
& \mathrm{D}_{\text {bulk }}=\text { Bulk density at } 115 \text { gyrations }=2.4273 \mathrm{~g} / \mathrm{ccm}
\end{aligned}
$$




$$
C . F .=\frac{D_{\text {bulk }}}{D_{\text {Theo }}}
$$

C.F.$=$ Correction Factor $=1.00075$

The theoretical density should be calculated for each gyration and then multiplied by the correction factor to get the actual density. We can see that the theoretical density and that determined by the Corelock device are very similar.

$\mathrm{D}_{\mathrm{Max}}=$ Maximum density $=2.5134 \mathrm{~g} / \mathrm{ccm}$

$$
\% D_{\text {Max }} @ N_{M a x}=\frac{D_{B u l k}}{D_{\text {Max }}} \times 100
$$

$\% \mathrm{D}_{\mathrm{Max}} @ \mathrm{~N}_{\mathrm{Max}}=$ Percent of maximum density at 115 gyrations $=96.57 \%$

$$
D_{\text {ini-theo }}=\frac{4000 M}{\pi D^{2} H_{i n i}}
$$

$\mathrm{D}_{\text {ini-theo }}=$ Theoretical density at 7 gyrations $=2.2325 \mathrm{~g} / \mathrm{ccm}$

$$
\% D_{\max } @ N_{i n i}=\frac{D_{\text {ini-theo }} \times C . F .}{D_{\text {Max }}} \times 100
$$

$\% \mathrm{D}_{\max } @ \mathrm{~N}_{\mathrm{ini}}=$ Percent of maximum density at 7 gyrations $=89.0 \%$

$$
D_{\text {des-theo }}=\frac{4000 M}{\pi D^{2} H_{d e s}}
$$

$D_{\text {des-theo }}=$ Theoretical density at 75 gyrations $=2.4006 \mathrm{~g} / \mathrm{ccm}$

$$
\% D_{\max } @ N_{d e s}=\frac{D_{\text {des-theo }} \times C . F .}{D_{\text {Max }}} \times 100
$$

$\% \mathrm{D}_{\max } @ \mathrm{~N}_{\mathrm{des}}=$ Percent of maximum density at 75 gyrations $=95.6 \%$ 
It can be seen that the percent of maximum density at 7, 75, and 115 gyrations are $89.0 \%, 95.6 \%$, and $96.6 \%$, respectively.

\subsubsection{Paver, Roller, and Traffic Energy Indices}

The paver, roller, and traffic energy indices (PEI, REI, and TEI) are construction and rutting performance indicators based on the densification curve created during compaction. Since the height at each gyration is recorded and the correction factor at the $115^{\text {th }}$ gyration is known, the percent of maximum density can be calculated at each gyration. A plot of gyrations versus the percent of maximum density creates a curve called the densification curve.

For an E-3 mix, a mix designed to carry up to 3 million ESALs, the area under the densification curve from the first gyration to the $7^{\text {th }}$ gyration, minus the area below the horizontal line created by the percent maximum density at the $0^{\text {th }}$ gyration, is the PEI. The area under the densification curve from the $7^{\text {th }}$ gyration to the point where the percent of maximum density reaches $92 \%$ minus the area below the horizontal line

created by the percent maximum density at the $7^{\text {th }}$ gyration, is the REI. Last, the area under the densification curve from $92 \%$ of maximum density to $98 \%$ of maximum density minus the area below the horizontal line created by the $92 \%$ maximum density line, is the TEI. Figure 8-1 shows these areas. 


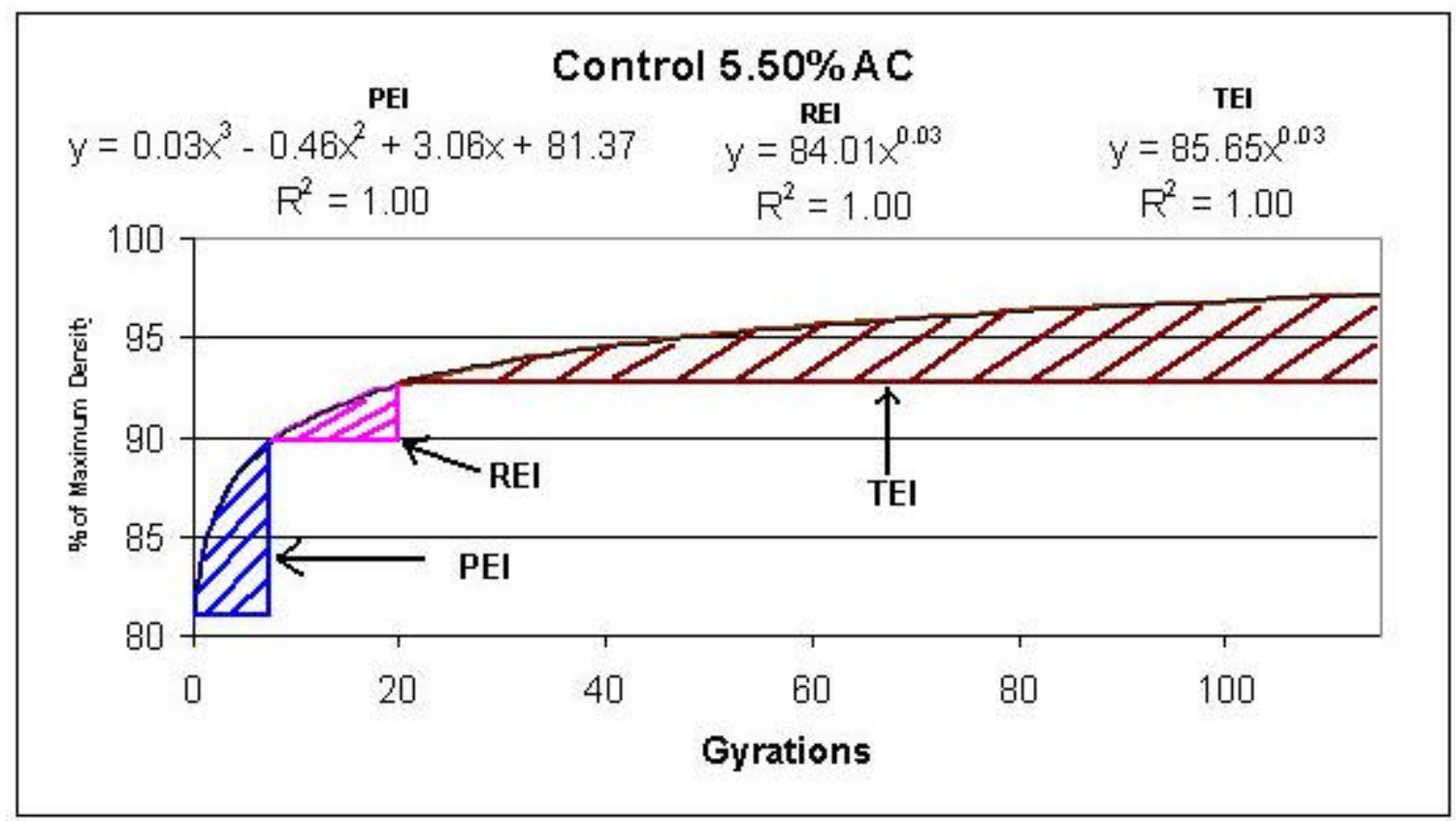

Figure 8-1: Areas from left to right are PEI, REI, TEI

The following is a procedure for how to calculate the PEI, REI, and TEI.

\section{PEI}

1) Using Excel, plot the gyrations versus the $\%$ of maximum density.

2) Fit a curve to the points including and between the $0^{\text {th }}$ and $7^{\text {th }}$ gyrations for the PEI.

3) Increase the degree of the fit curve until the $R^{2}$ value is above 0.99 .

4) Integrate the curve from the $0^{\text {th }}$ to $7^{\text {th }}$ gyration and subtract away the area created by the rectangle whose width is that from the $0^{\text {th }}$ gyration to the $7^{\text {th }}$ gyration and whose height is that from $0 \%$ of maximum density to the percent of maximum density at the $0^{\text {th }}$ gyration. 


\section{REI}

1) Using Excel, plot the gyrations versus the \% of maximum density.

2) Fit a curve to the points including and between the $7^{\text {th }}$ gyration and the lowest gyration that gives at least $92 \%$ of the maximum density.

3) Increase the degree of the fit curve until the $R^{2}$ value is above 0.99 .

4) Using the fit equation, find the gyration to the nearest hundredth of a gyration, that gives $92 \%$ of maximum density.

5) Integrate the curve from the $7^{\text {th }}$ gyration to the gyration that was calculated to the nearest hundredth.

6) Subtract away the area created by the rectangle whose width is that from the $7^{\text {th }}$ gyration to the gyration at $92 \%$ of maximum density and whose height is that from $0 \%$ of maximum density to $92 \%$ of maximum density.

\section{TEI}

1) Using Excel, plot the gyrations versus the \% of maximum density.

2) Fit a curve to the points including and between the lowest gyration that gives at least $92 \%$ of the maximum density, and the $115^{\text {th }}$ gyration.

3) Increase the degree of the fit curve until the $R^{2}$ value is above 0.99 .

4) Using the fit equation, find the gyration, to the nearest hundredth of a gyration, that gives $98 \%$ of maximum density.

5) Integrate the curve from the calculated gyration that will give $92 \%$ of maximum density, to the calculated gyration that will give $98 \%$ of maximum density.

6) Subtract away the area created by the rectangle whose width is that from the calculated gyration that will give $92 \%$ of maximum density to the calculated gyration that 
will give $98 \%$ of maximum density, and whose height is that from $0 \%$ of maximum density to $98 \%$ of maximum density.

\subsubsection{Strength Measured by the Indirect Tension Test}

The ASTM D4867 standard procedure was used to prepare samples and test for indirect tension strength and modulus before and after conditioning in water. The test is also used to measure the moisture sensitivity of each of the mixes. In the standard procedure, only vertical deflection is measured. In this study, the horizontal deflection on the face of the sample was also measured through use of a laser and reflective tape, a technique commonly used with the direct tension test of asphalt binders. The details of the procedure used are as follows:

1) Compact a 5,000 g sample to $7 \%$ air voids, plus or minus $1 \%$.

2) After it has cooled, the bulk density of the sample should be determined.

3) The sample should then be placed in the volumeter used for rice tests and covered completely with water.

4) Using the Corelock device, the sample should be saturated so that between $55 \%$ and $80 \%$ of the air voids are filled with water. Since the Corelock is automated, it gives a more repeatable level of saturation.

5) By knowing the density of the water and the percent air voids, the theoretical mass at $55 \%$ and $80 \%$ saturation can be calculated. After the sample has been saturated, 
it should be removed and dried by rolling it on a damp towel, so that it is saturated surface dried.

6) If the sample is below the $55 \%$ saturation point, the sample should be subjected to a more intense vacuum, which can be adjusted on the Corelock device. If the sample is between the two saturation points, it is ready to be submerged in the hot water bath for 24 hours. If the sample is above the $80 \%$ saturation point, it should be disposed of.

\subsubsection{Secant Modulus Using Indirect Tension}

Data to determine the secant modulus were gathered during the indirect tension test using horizontal displacement measurements. To measure horizontal deflection, reflective tape was placed on the face of the sample, approximately $5 \mathrm{~cm}$ away from each other. Figure 8-2 shows the paper guide/mask used to position the pieces of reflective tape. The diameter of the guide is the same as the diameter of the sample, $150 \mathrm{~mm}$. The two vertical slits are used to position the pieces of reflective tape, and the horizontal slit is used to make a white paint mark on the sample to better align the laser. The horizontal slit is meant to go through the center of the face of the sample, and each vertical slit is 25 $\mathrm{mm}$ from the center. 


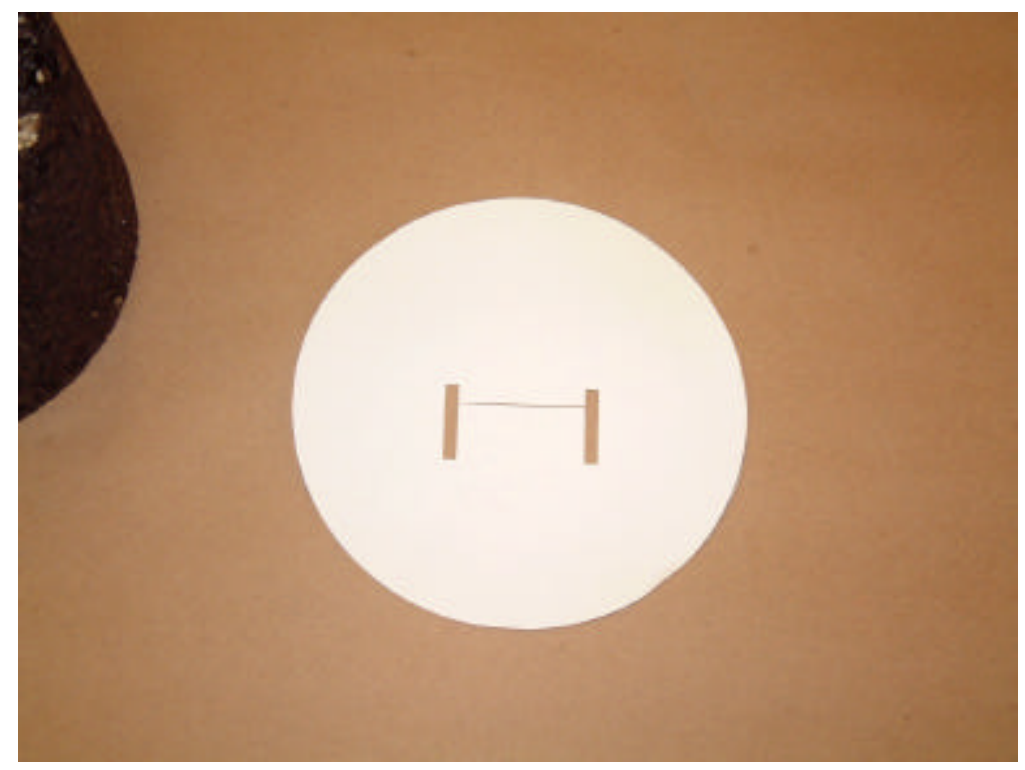

Figure 8-2: Guide for placing the reflective tape

Figure 8-3 shows what a prepared sample looks like. The two vertical strips are the reflective tape, and the horizontal strip is white paint. The sample should be prepared with reflective tape and white paint after it has already been placed in the hot water bath for 24 hours and cooled off in room temperature water for one hour. If they are prepared before, the reflective tape will come off in the water. 


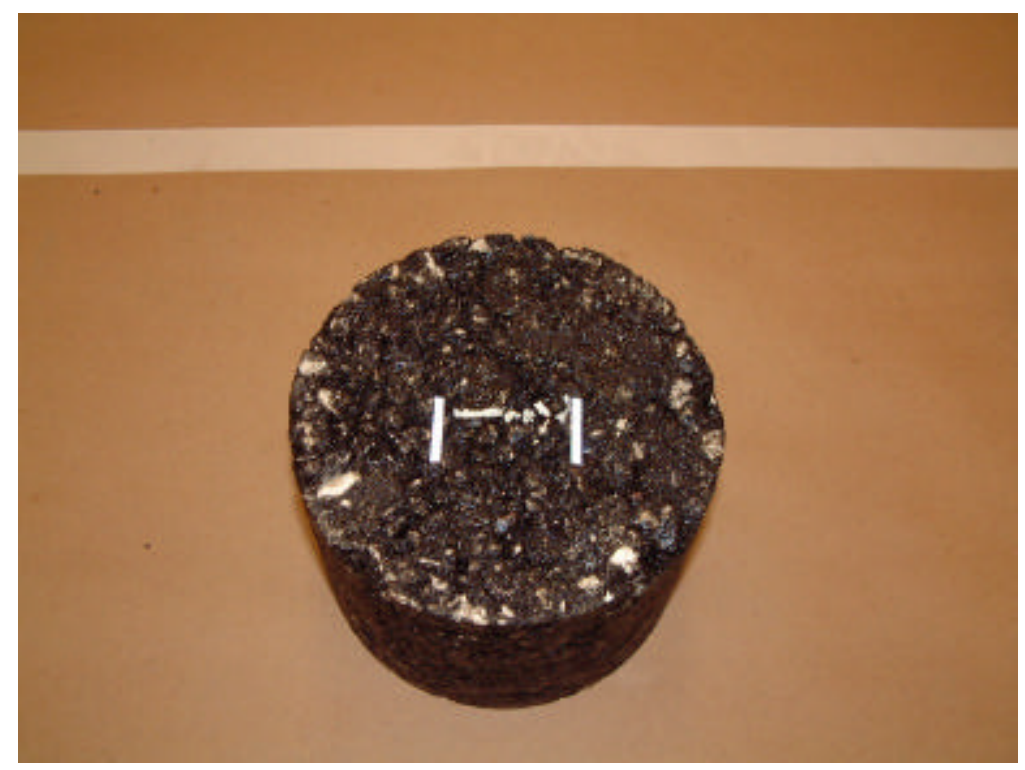

Figure 8-3: Prepared sample ready for crushing

Figures 8-4 through 8-6 show the positioning of the laser with respect to the sample. For the laser used, the face of the sample was placed $254 \mathrm{~mm}$ from the face of the laser. The laser was also balanced using the tripod setup, so that it was level with the ground. The sample was then positioned so that the beam from the laser was shining on and parallel to the white painted strip on the sample, as shown in Figure 8-6. 


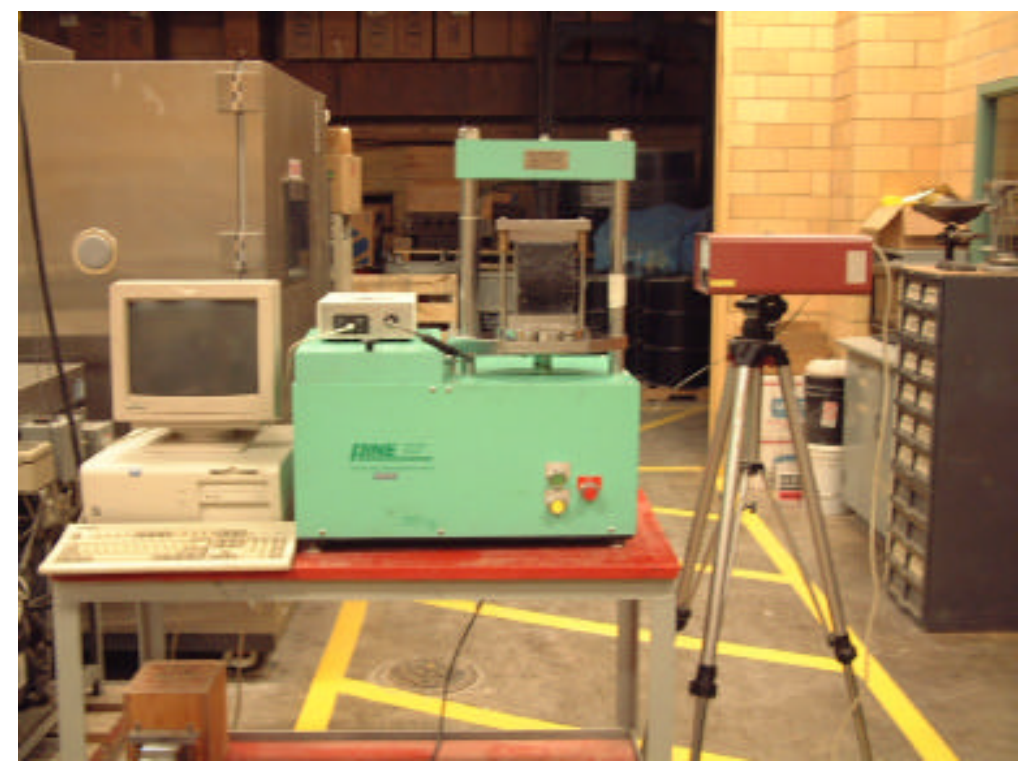

Figure 8-4: Overall setup of indirect tension test

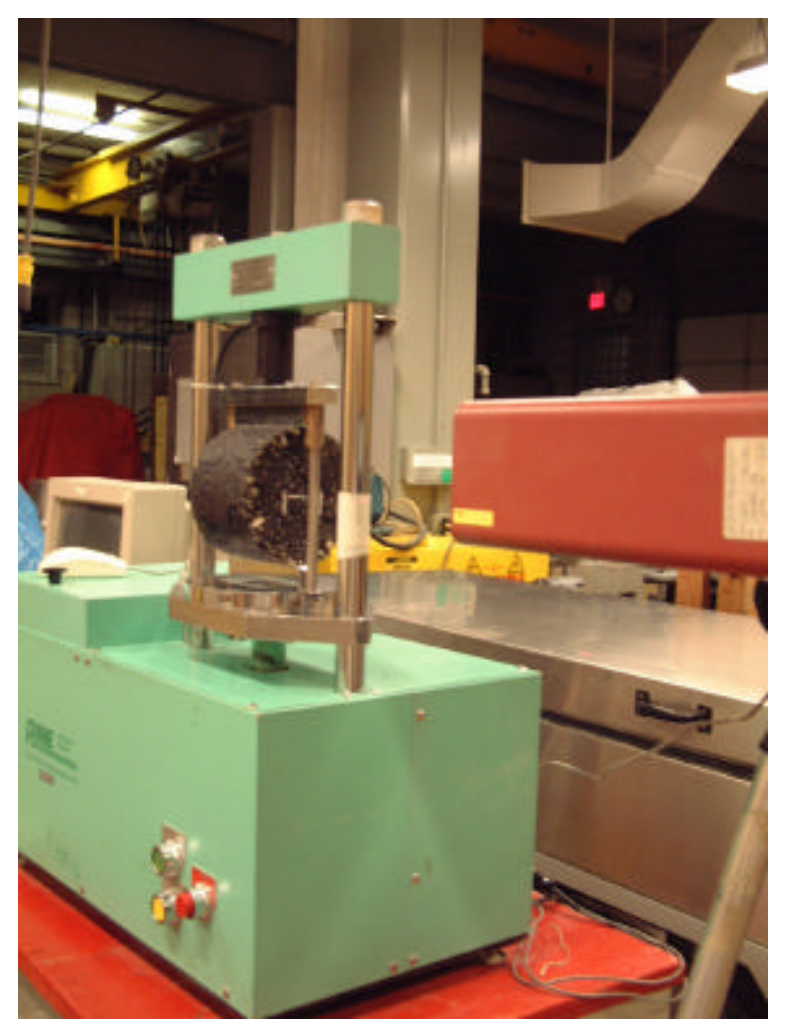

Figure 8-5: Positioning of laser with respect to sample 


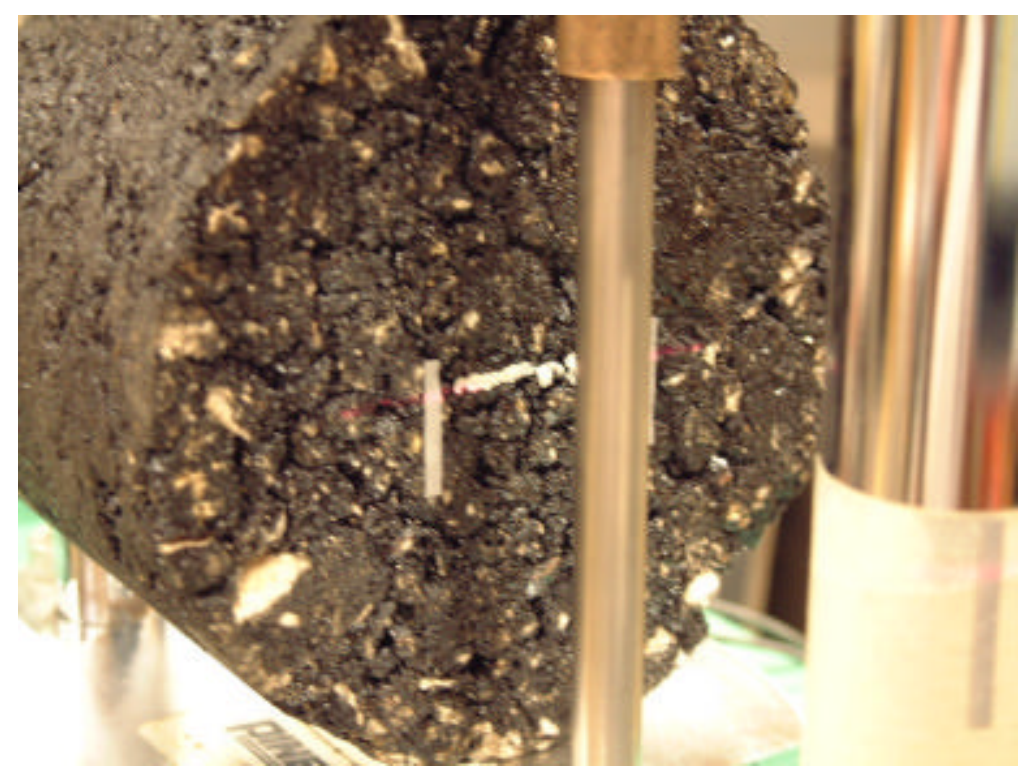

Figure 8-6:Close up of sample

While the sample is being crushed, the laser extensiometer measures the distance between the two pieces of reflective tape and sends the data to the computer. A USBbased data acquisition system is also recording the voltage from the load cell and sending the data to the computer. A LabVIEW program was written to record the data and store them as a text file. The data acquisition setup can be seen in Figure 8-7. The horizontal stress is calculated using the equation found in ASTM Designation D4867 and is presented in Equation 8.14. 


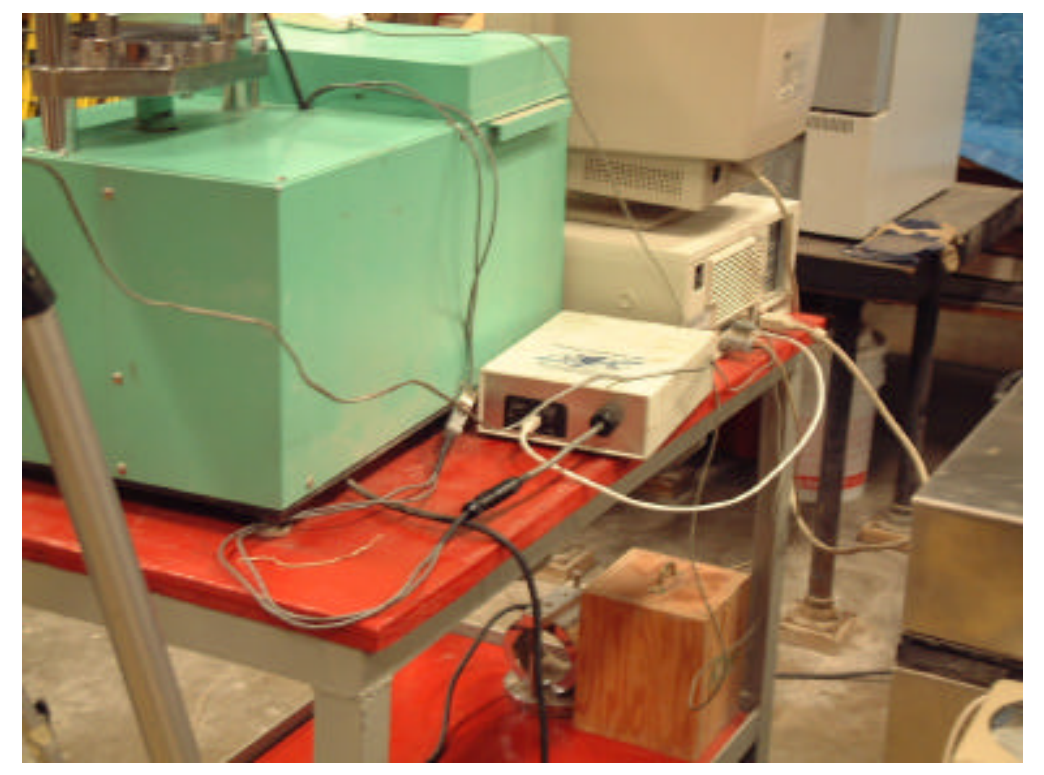

Figure 8-7: Data acquisition setup for gathering load cell and laser data

$$
\sigma=\frac{2000 P}{\pi t D}
$$

$\sigma=$ Horizontal stress $(\mathrm{kPa})$

$\mathrm{P}=$ Force applied $(\mathrm{N})$

$\mathrm{t}=$ Sample height $(\mathrm{mm})$

$\mathrm{D}=$ Sample diameter $(\mathrm{mm})$

The strain is calculated in Equation 8.15.

$$
\varepsilon=\frac{L_{C}-L_{O}}{D}
$$

Equation 8.15

$\varepsilon=$ Strain

$\mathrm{L}_{\mathrm{C}}=$ Current distance between pieces of reflective tape $(\mathrm{mm})$ 
$\mathrm{L}_{\mathrm{O}}=$ Original distance between pieces of reflective tape $(\mathrm{mm})$

$\mathrm{D}=$ Diameter of sample $(\mathrm{mm})$

The secant modulus is the ratio of the maximum stress to the strain when the maximum stress occurs, as presented in Equation 8.16.

$$
E_{\text {Secant }}=\frac{\sigma_{\operatorname{Max}}}{\varepsilon_{@ M a x}}
$$

Equation 8.16

$\mathrm{E}_{\text {Secant }}=$ Secant modulus

$\sigma_{\text {Max }}=$ Maximum tensile stress $=$ Tensile strength

$\varepsilon_{@ \text { Max }}=$ Strain at which the maximum tensile stress occurs

\subsection{Results and Discussion}

Section 8.1 describes the methodology and procedures used to determine selected physical properties of the foundry sands and aggregates, as well as the volumetric properties and performance indicators for Hot Mix Asphalt (HMA) produced in the laboratory. In this section, the results from these tests are shown along with the possible conclusions available from these results. These include the apparent and bulk densities of the sands and aggregates, as well as the difference between using the direct and indirect method for determining the bulk density on the angularity of the foundry sand; the volumetric properties of the HMA mixes; and an attempt to relate the physical properties of the foundry sands to the volumetric properties of the HMA, as well as to relate the physical and volumetric properties to the performance indicators. 


\subsubsection{Densities and Angularity of Sands}

Two different methods, a direct and indirect method, were used to determine the apparent and bulk density of the foundry sands used in this study. When using the direct method, it was noticed that a film was developing on the foundry sand and was not allowing the water to penetrate its voids. To overcome this an indirect method was developed, but due to time constraints the method could not be tested.

Results are presented in Table 8-3. The data for the 1/4" screenings, manufactured sand, and natural sand came directly from the contractor who supplied the aggregates and asphalt mix design. The control (P0.60) is all the control material passing the $0.6 \mathrm{~mm}$ sieve. A better comparison could be made between the control aggregates and the foundry sand if the P0.60 material were separated from the total control mix and tested in a similar manner as the foundry sands. From Table 8-3, the angularities of the foundry sands appear to be higher when compared to the natural sand. Sand FS13 had the lowest angularity of 43.1, which is higher than the natural sand. Sand FS06 had the highest angularity of 57.6, which is a very high angularity. This can be explained because the sand has a very low fines content, which means there are no fines to fill the packing voids (Figure 2-9). Since there are no fines to fill the packing voids, the packing density decreases, thus increasing the angularity. The remaining sands appear to have reasonable angularities comparable to the manufactured sand. Generally, manufactured sands are

desirable because they promote aggregate interlock and help resist against rutting. 
Because of their high angularities, foundry sands would be a good replacement of natural sands in HMA.

Table 8-3: Apparent and bulk densities, and angularity of sands

\begin{tabular}{l|l|l|l}
\hline Sand No. & $\begin{array}{l}\text { Apparent } \\
\text { Density }(\mathrm{g} / \mathrm{ccm})\end{array}$ & $\begin{array}{l}\text { Bulk Density } \\
(\mathrm{g} / \mathrm{ccm})\end{array}$ & $\begin{array}{l}\text { Angularity } \\
(\%)\end{array}$ \\
\hline \hline 1/4" Screenings & N.R. & 2.699 & 50.5 \\
\hline Man. Sand & N.R. & 2.660 & 48.9 \\
\hline Natural Sand & N.R. & 2.684 & 41.5 \\
\hline Control (P0.60) & 2.766 & 2.757 & 45.5 \\
\hline FS01 & 2.760 & 2.415 & 45.4 \\
\hline FS02 & 2.779 & 2.698 & 47.5 \\
\hline FS03 & 2.764 & 2.448 & 50.5 \\
\hline FS04 & 2.759 & 2.502 & 47.3 \\
\hline FS05 & 2.776 & 2.488 & 48.1 \\
\hline FS06 & 2.778 & 2.615 & 57.6 \\
\hline FS07 & 2.763 & 2.529 & 47.5 \\
\hline FS08 & 2.763 & 2.470 & 48.2 \\
\hline FS09 & 2.763 & 2.569 & 46.3 \\
\hline FS10 & 2.779 & 2.628 & 44.8 \\
\hline FS11 & 2.776 & 2.593 & 46.2 \\
\hline FS12 & 2.769 & 2.578 & 43.7 \\
\hline FS13 & 2.776 & 2.591 & 43.1 \\
\hline FS14 & 2.784 & 2.64 & 47.9 \\
\hline FS15 & 2.752 & 2.545 & 54.4 \\
\hline FS16 & 2.775 & 2.644 & 47.7 \\
\hline FS17 & 2.771 & 2.669 & 46.7 \\
\hline & & &
\end{tabular}

\subsubsection{Volumetric Properties}

In this section the results from the volumetric analysis for each of the mixes, including the control and the 17 mixes that had $10 \%$ foundry sand incorporated into them, are presented. The volumetric data include the optimum asphalt content, maximum and bulk densities of mixtures at optimum asphalt content, effective density of mixtures, 
and average asphalt film thickness. In several cases, the $95 \%$ confidence intervals are given.

\subsubsection{Volumetric Properties of Aggregate Blends}

Each aggregate blend is considered to have three different densities, an apparent density, an effective density, and a bulk density. The apparent density is the mass of the aggregate divided by the volume of just the aggregate material and the impermeable voids. The bulk density is the mass of the aggregate divided by the volume of the aggregate, impermeable voids, and microvoids of the aggregate. The effective density is the mass of the aggregate divided by the volume of the aggregate, impermeable voids, and microvoids not filled with asphalt. So in the case where all the voids are filled with asphalt, the effective density would be the same as the apparent density. Table 8-4 shows a summary of these properties. Also shown in the table is the volume of voids per volume of aggregate. This gives an indication of the level of voids in the aggregate. The last column in the table shows the maximum amount of water that can be absorbed into the aggregate per mass of aggregate. This is more commonly known as the percent absorption value. 
Table 8-4: Volumetric properties of aggregates

\begin{tabular}{l|c|c|c|c|c}
\hline $\begin{array}{l}\text { Sand } \\
\text { No. }\end{array}$ & $\begin{array}{c}\text { Apparent } \\
\text { Density } \\
\text { (g/ccm) }\end{array}$ & $\begin{array}{l}\text { Effective } \\
\text { Density @ } \\
\text { Opt. A.C. } \\
\text { (g/ccm) }\end{array}$ & $\begin{array}{l}\text { Bulk } \\
\text { Density } \\
\text { (g/ccm) }\end{array}$ & $\begin{array}{l}\text { Vol. } \\
\text { Void/Vol. } \\
\text { Agg. (\%) }\end{array}$ & $\begin{array}{l}\text { M. } \\
\text { Water/M } \\
\text { Agg. (\%) }\end{array}$ \\
\hline \hline Control & 2.781 & 2.746 & 2.652 & $4.9 \%$ & $1.7 \%$ \\
\hline FS01 & 2.752 & 2.717 & 2.627 & $4.7 \%$ & $1.7 \%$ \\
\hline FS02 & 2.771 & 2.748 & 2.652 & $4.5 \%$ & $1.6 \%$ \\
\hline FS03 & 2.756 & 2.716 & 2.628 & $4.9 \%$ & $1.8 \%$ \\
\hline FS04 & 2.751 & 2.726 & 2.648 & $3.9 \%$ & $1.4 \%$ \\
\hline FS05 & 2.767 & 2.724 & 2.645 & $4.6 \%$ & $1.7 \%$ \\
\hline FS06 & 2.770 & 2.741 & 2.656 & $4.3 \%$ & $1.5 \%$ \\
\hline FS07 & 2.754 & 2.725 & 2.612 & $5.5 \%$ & $2.0 \%$ \\
\hline FS08 & 2.755 & 2.710 & 2.611 & $5.5 \%$ & $2.0 \%$ \\
\hline FS09 & 2.755 & 2.725 & 2.653 & $3.8 \%$ & $1.4 \%$ \\
\hline FS10 & 2.770 & 2.739 & 2.678 & $3.4 \%$ & $1.2 \%$ \\
\hline FS11 & 2.768 & 2.723 & 2.649 & $4.5 \%$ & $1.6 \%$ \\
\hline FS12 & 2.760 & 2.733 & 2.662 & $3.7 \%$ & $1.3 \%$ \\
\hline FS13 & 2.768 & 2.741 & 2.677 & $3.4 \%$ & $1.2 \%$ \\
\hline FS14 & 2.776 & 2.741 & 2.616 & $6.1 \%$ & $2.2 \%$ \\
\hline FS15 & 2.743 & 2.718 & 2.621 & $4.7 \%$ & $1.7 \%$ \\
\hline FS16 & 2.766 & 2.733 & 2.668 & $3.7 \%$ & $1.3 \%$ \\
\hline FS17 & 2.769 & 2.743 & 2.647 & $4.6 \%$ & $1.7 \%$ \\
\hline
\end{tabular}

As seen in Table 8-4, the apparent densities of aggregate blends with foundry sand seem to be lower than the control, but the differences are marginal. The same can be said for the effective and bulk densities. Sands FS07, FS08, and FS14 seem to have a higher amount of permeable voids, with sand FS14 having 6.1\% permeable voids, sand FS08, 5.5\% and sand FS07, 5.5\%. This is also reflected in the last column. Generally it is thought that the greater the number of pores, or the greater the amount of water the aggregate can absorb, the more asphalt can be absorbed. From Figure 8-8, one can see there is a good correlation between the voids and the asphalt absorbed, with it being stated that as the percent voids increases, so does the percent asphalt absorbed. It might also be assumed that the optimum asphalt content would increase if the voids were to 
increase, but from Figure 8-9, it can be seen there is no correlation between them. The optimum asphalt contents are listed in Table 8-5.

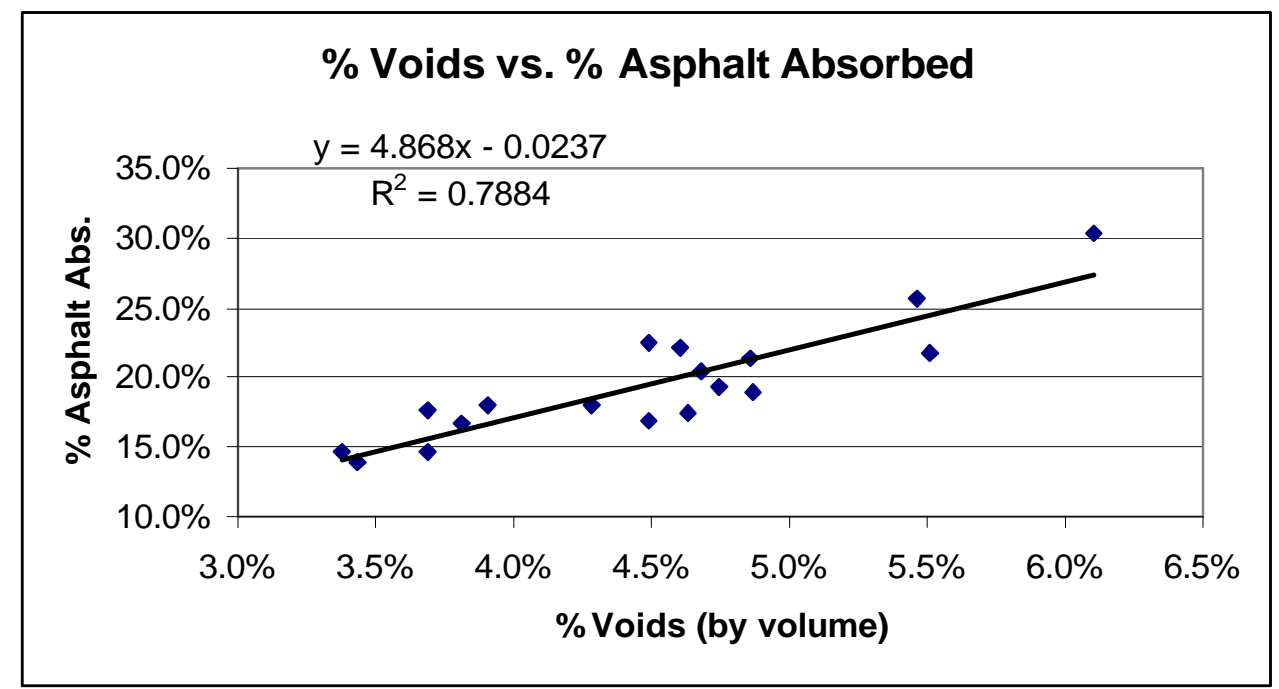

Figure 8-8: Relation between voids and asphalt absorbed

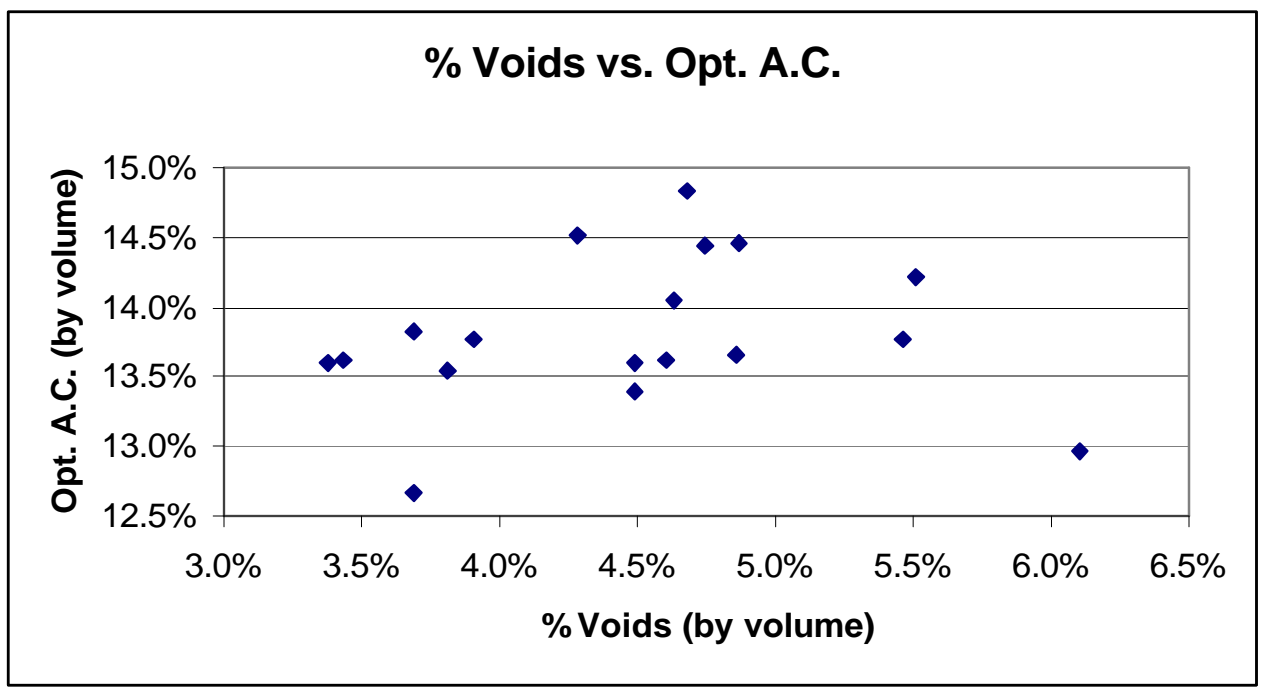

Figure 8-9: Relation between voids and optimum asphalt content 
Table 8-5: Volumetric properties at optimum asphalt content

\begin{tabular}{|c|c|c|c|c|c|c|c|c|}
\hline Column & 1 & 2 & 3 & 4 & 5 & 6 & 7 & 8 \\
\hline Sand No. & $\begin{array}{l}\text { Opt. } \\
\text { A.C. (by } \\
\text { mass) }\end{array}$ & $\begin{array}{l}\text { Opt. A.C. } \\
\text { (by } \\
\text { volume) }\end{array}$ & $\begin{array}{l}\text { M. } \\
\text { Water/M. } \\
\text { Agg. @ } \\
\text { Opt. AC }\end{array}$ & $\begin{array}{l}\text { Vol. } \\
\text { Voids/Vol. } \\
\text { Agg. @ Opt. } \\
\text { A.C. }\end{array}$ & $\begin{array}{l}\text { Vol } \\
\text { Asphalt/Vol. } \\
\text { Voids (\%) }\end{array}$ & $\begin{array}{l}\text { M. Asphalt } \\
\text { Abs./M. } \\
\text { Agg. }\end{array}$ & $\begin{array}{l}\text { Vol Asphalt } \\
\text { Abs/Vol } \\
\text { Asphalt Total } \\
\text { (\%) }\end{array}$ & $\begin{array}{l}\text { Film } \\
\text { Thickness } \\
(\mu \mathrm{m})\end{array}$ \\
\hline Control & $5.5 \%$ & $13.7 \%$ & $0.5 \%$ & $1.3 \%$ & $73.9 \%$ & $4.2 \%$ & $21.4 \%$ & 13.4 \\
\hline FS01 & $5.9 \%$ & $14.4 \%$ & $0.5 \%$ & $1.3 \%$ & $73.0 \%$ & $4.5 \%$ & $19.4 \%$ & 14.8 \\
\hline FS02 & $5.4 \%$ & $13.4 \%$ & $0.3 \%$ & $0.8 \%$ & $81.8 \%$ & $4.6 \%$ & $22.5 \%$ & 13.0 \\
\hline FS03 & $5.9 \%$ & $14.5 \%$ & $0.5 \%$ & $1.5 \%$ & $69.7 \%$ & $4.3 \%$ & $18.9 \%$ & 14.9 \\
\hline FS04 & $5.6 \%$ & $13.8 \%$ & $0.3 \%$ & $0.9 \%$ & $76.9 \%$ & $4.5 \%$ & $17.9 \%$ & 14.3 \\
\hline FS05 & $5.7 \%$ & $14.1 \%$ & $0.6 \%$ & $1.6 \%$ & $65.5 \%$ & $3.9 \%$ & $17.5 \%$ & 14.6 \\
\hline FS06 & $5.9 \%$ & $14.5 \%$ & $0.4 \%$ & $1.1 \%$ & $75.2 \%$ & $4.6 \%$ & $18.0 \%$ & 15.1 \\
\hline FS07 & $5.6 \%$ & $13.8 \%$ & $0.4 \%$ & $1.1 \%$ & $79.9 \%$ & $4.6 \%$ & $25.6 \%$ & 12.9 \\
\hline FS08 & $5.8 \%$ & $14.2 \%$ & $0.6 \%$ & $1.6 \%$ & $70.4 \%$ & $4.2 \%$ & $21.8 \%$ & 14.1 \\
\hline FS09 & $5.5 \%$ & $13.6 \%$ & $0.4 \%$ & $1.1 \%$ & $71.7 \%$ & $4.1 \%$ & $16.6 \%$ & 14.2 \\
\hline FS10 & $5.5 \%$ & $13.6 \%$ & $0.4 \%$ & $1.1 \%$ & $66.6 \%$ & $3.8 \%$ & $13.9 \%$ & 14.7 \\
\hline FS11 & $5.5 \%$ & $13.6 \%$ & $0.6 \%$ & $1.7 \%$ & $63.2 \%$ & $3.6 \%$ & $17.0 \%$ & 14.2 \\
\hline FS12 & $5.1 \%$ & $12.7 \%$ & $0.4 \%$ & $1.0 \%$ & $72.6 \%$ & $3.8 \%$ & $17.6 \%$ & 13.0 \\
\hline FS13 & $5.5 \%$ & $13.6 \%$ & $0.3 \%$ & $1.0 \%$ & $71.3 \%$ & $4.1 \%$ & $14.7 \%$ & 14.6 \\
\hline FS14 & $5.2 \%$ & $13.0 \%$ & $0.5 \%$ & $1.3 \%$ & $79.2 \%$ & $4.2 \%$ & $30.2 \%$ & 11.2 \\
\hline FS15 & $6.1 \%$ & $14.8 \%$ & $0.3 \%$ & $0.9 \%$ & $80.1 \%$ & $5.1 \%$ & $20.4 \%$ & 15.2 \\
\hline FS16 & $5.6 \%$ & $13.8 \%$ & $0.4 \%$ & $1.2 \%$ & $66.5 \%$ & $3.9 \%$ & $14.6 \%$ & 14.9 \\
\hline FS17 & $5.5 \%$ & $13.6 \%$ & $0.3 \%$ & $0.9 \%$ & $79.7 \%$ & $4.5 \%$ & $22.1 \%$ & 13.3 \\
\hline
\end{tabular}

\subsubsection{Volumetric Properties of HMA Mixtures}

The data appearing in Table 8-5 show that including foundry sand in mixtures can either raise or lower the optimum asphalt content. The optimum asphalt content for the control is $5.5 \%$, with the lowest asphalt content being $5.1 \%$, and the highest content being 6.1\%. Typically, bentonite will cause the optimum asphalt content to increase [Braham 2002]. This is because bentonite has a very large surface area, and thus would require more asphalt to coat its surface. Sodium silicate appears to have little or no effect on the optimum asphalt content, and phenols generally lower it. This is shown in Figure 8-10. 
But in reality, if a contractor were to use foundry sand for actual production, there would be a sand hump at around the $0.30 \mathrm{~mm}(\# 50)$ sieve. This sand hump would most likely cause an increase in asphalt content no matter what binder system was used in the foundry sand. A phenolic bound sand would cause less of an increase in the optimum asphalt content, where as a bentonite bound sand would cause a greater increase in the optimum asphalt content.

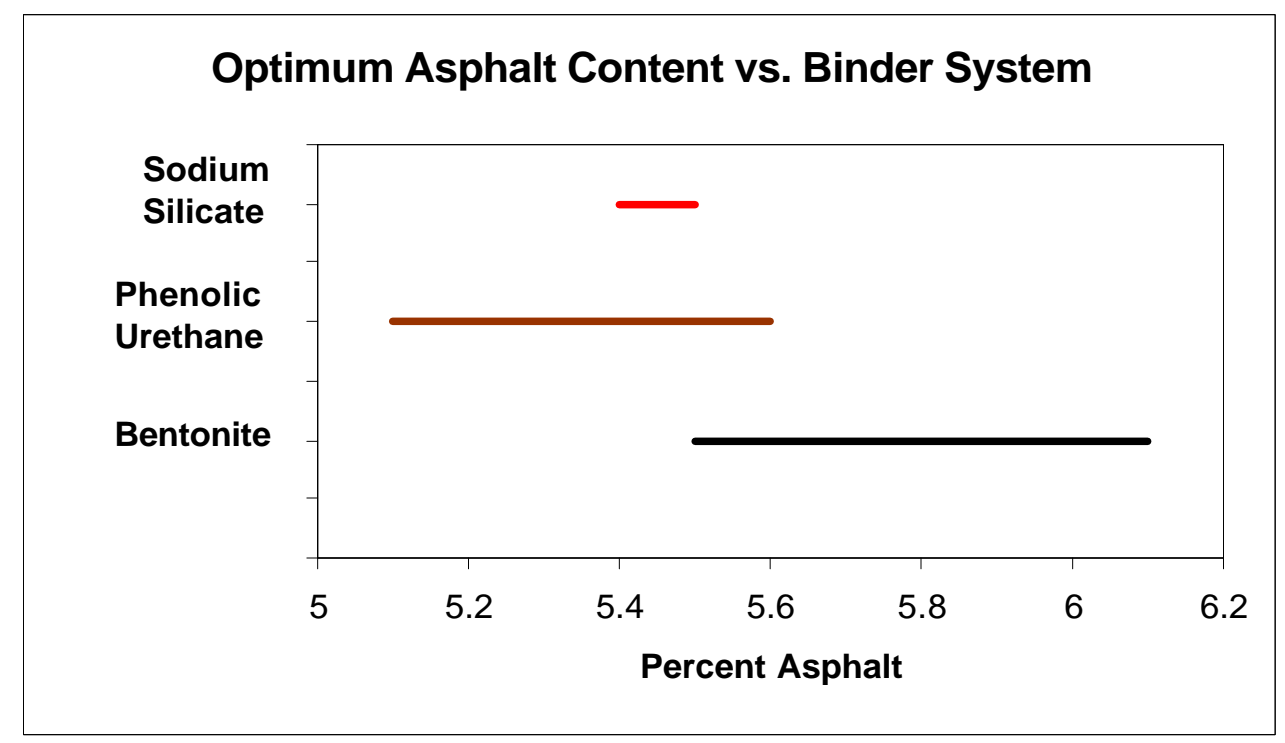

Figure 8-10: Relation between optimum asphalt content and binder system

Columns 3 and 4 of Table 8-5 show the level of asphalt penetration into the voids of the aggregate. Column 3 shows the mass of water that could be absorbed into the voids as a percentage of the mass of the aggregate. Column 4 shows the volume of the voids that have not been penetrated to the volume of the aggregate. Surprisingly, asphalt is consistently not able to penetrate the remaining $1 \%$ of the voids (column 4). For 
example, looking at the percentage of total voids of sands FS14 and FS16 in Table 8-4 (column 4), one would find them to be $6.1 \%$ and $3.7 \%$. Then looking at the remaining voids after asphalt has been added at its optimum asphalt content, the remaining voids in sands FS14 and FS16 are 1.3 and 1.2\% (Table 8-5, column 4). This trend is consistent for all the sands. On the other hand, the level of penetration with respect to the total amount of voids is not consistent. Column 5 shows the volume of asphalt absorbed compared to the total volume of the voids, or the percentage of voids that have been filled with asphalt. So, for the control, $73.9 \%$ of the total voids in the aggregate have been filled with asphalt. The remaining $26.1 \%$ of the voids are still filled with air.

Columns 6 and 7 are more for the asphalt contractor, and help to predict the amount of asphalt lost due to asphalt absorption. Column 6 shows the mass of asphalt absorbed compared to the mass of the aggregate. This number would be useful for the contractor because it allows quick calculation of the amount of asphalt lost based on the tonnage of aggregates. Aggregate bins used at HMA facilities are equipped with load sensors that continuously report the mass of aggregate being added to the mix. This allows the operator to control the gradation of the final HMA product. Column 7 shows a very similar concept, but stated in terms of the total asphalt added. So, based on columns 6 and 7, for the control mix, for every ton of aggregate, $42 \mathrm{~kg}$ of asphalt would be absorbed, or for every ton of asphalt added, $210 \mathrm{~kg}$ will be lost due to absorption. Based on column 7, sands FS14 and FS16 should probably be avoided because they take up a lot of asphalt in the voids rather than coating the aggregates. 
Column 8 of Table 8-5 shows the average asphalt film thickness estimated for each mixture. It is the ratio of the volume of asphalt not absorbed to the surface area of the mixture. The surface area calculations are based on average surface areas for aggregates based on their size. It is believed that a mix should not have too low of a film thickness, or it will not maintain its durability. At the same time, the film thickness should not be too high, or the film will act more like a lubricant and cause the pavement to rut. Figure 8-11 shows that as the optimum asphalt content increases, the film thickness also increases. This only makes sense, because as more asphalt is added, the film thickness should increase.

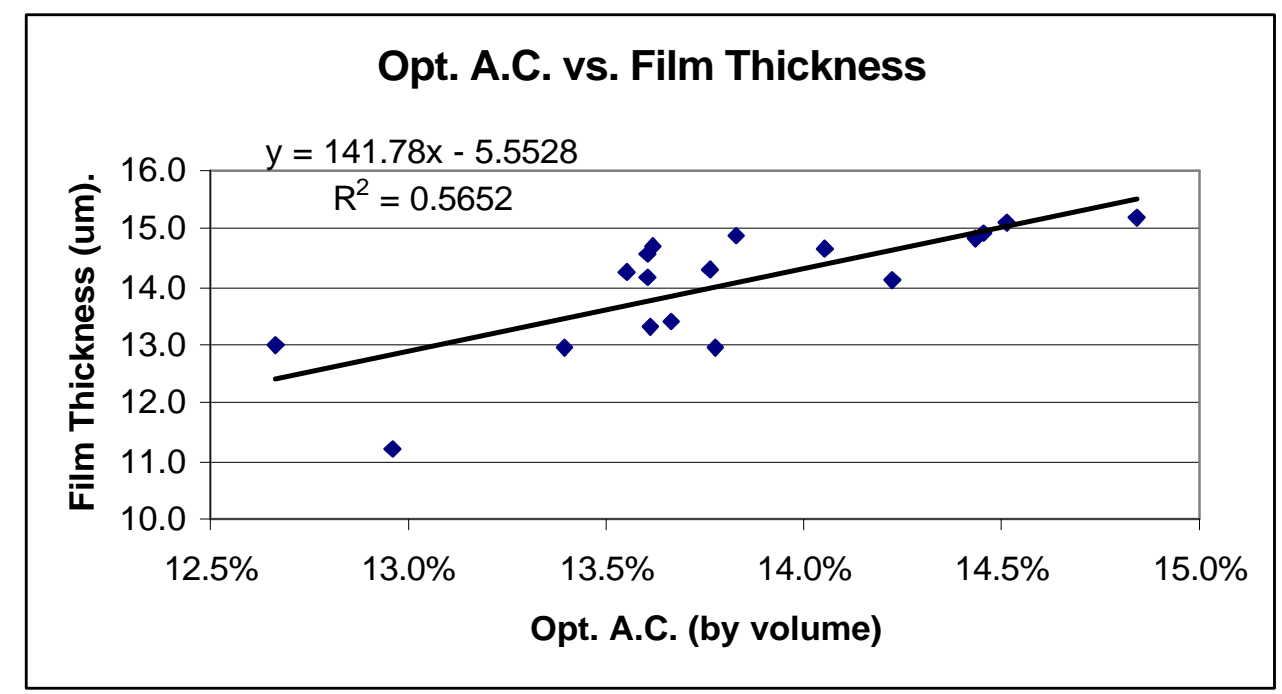

Figure 8-11: Relation between the optimum asphalt content and film thickness

In order to show the accuracy and repeatability of the data due to elaborate sieving procedures, good quality control and making use of the Corelok ${ }^{\mathrm{TM}}$ device, the maximum and bulk densities are listed in the first and second columns of data of Table 86. For the control, and sands FS01, FS03, FS08, FS09, and FS14, the 95\% confidence 
intervals were calculated using Equation 8.16 with three replications. One can see that the average $95 \%$ confidence interval for the maximum density is \pm 0.007 , and \pm 0.008 for the bulk density. Because of these tight confidence intervals, replicates often were not run when determining the optimum asphalt content. According to Superpave procedure, when determining the optimum asphalt content, the optimum asphalt content is first guessed based on experience. Duplicates are created at this asphalt content as well as duplicates at $0.5 \%$ above and $0.5 \%$ and $1.0 \%$ below the first guess. Based on these tests, a plot of asphalt content versus air voids in the mix at $\mathrm{N}_{\mathrm{des}}$ is created. Based on this plot, the true optimum asphalt content is chosen, and duplicates are run to verify. The whole process takes 10 samples. Since more time was spent on sieving and batching together very uniform gradations, replicates did not need to be run. Initially the optimum asphalt content was estimated to be $5.5 \%$ for each of the mixes. Based on the volumetric data from the first estimate, a second optimum asphalt content was estimated. In some cases only two samples needed to be created in order to find the optimum asphalt content. This greatly reduced on the time needed for mixing and compacting, and the amount of asphalt and aggregates needed. 
Table 8-6: Volumetric properties of HMA mixtures

\begin{tabular}{l|c|c|c|c|c}
\hline $\begin{array}{l}\text { Sand } \\
\text { No. }\end{array}$ & $\begin{array}{l}\text { Maximum } \\
\text { Density (g/ccm) }\end{array}$ & $\begin{array}{l}\text { Bulk Density @ } \\
\mathbf{N}_{\max }(\mathbf{g} / \mathbf{c c m})\end{array}$ & $\begin{array}{l}\mathbf{\% G}_{\mathbf{m m}} @ \\
\mathbf{N}_{\mathbf{i n i}}\end{array}$ & $\begin{array}{l}\mathbf{\% G}_{\mathbf{m m}} @ \\
\mathbf{N}_{\text {des }}\end{array}$ & $\begin{array}{l}\mathbf{\% G}_{\mathbf{m m}} @ \\
\mathbf{N}_{\mathbf{m a x}}\end{array}$ \\
\hline \hline Control & $2.513 \pm 0.003$ & $2.430 \pm 0.007$ & $89.0 \pm 0.2$ & $95.7 \pm 0.2$ & $96.7 \pm 0.2$ \\
\hline FS01 & $2.475 \pm 0.004$ & $2.392 \pm 0.009$ & $88.9 \pm 0.3$ & $95.6 \pm 0.3$ & $96.6 \pm 0.3$ \\
\hline FS02 & 2.526 & 2.441 & 89.3 & 95.7 & 96.6 \\
\hline FS03 & $2.474 \pm 0.002$ & $2.396 \pm 0.015$ & $88.9 \pm 0.4$ & $95.8 \pm 0.5$ & $96.8 \pm 0.5$ \\
\hline FS04 & 2.502 & 2.415 & 89.0 & 95.6 & 96.5 \\
\hline FS05 & 2.489 & 2.391 & 88.9 & 95.6 & 96.6 \\
\hline FS06 & 2.501 & 2.415 & 89.0 & 95.6 & 96.6 \\
\hline FS07 & 2.500 & 2.416 & 89.2 & 95.7 & 96.6 \\
\hline FS08 & $2.474 \pm 0.002$ & $2.389 \pm 0.008$ & $88.5 \pm 0.4$ & $95.6 \pm 0.4$ & $96.6 \pm 0.4$ \\
\hline FS09 & $2.497 \pm 0.016$ & $2.418 \pm 0.007$ & $89.2 \pm 0.5$ & $95.9 \pm 0.5$ & $96.9 \pm 0.5$ \\
\hline FS10 & 2.515 & 2.432 & 89.4 & 95.7 & 96.6 \\
\hline FS11 & 2.502 & 2.423 & 89.1 & 95.9 & 96.8 \\
\hline FS12 & 2.526 & 2.4390 & 89.3 & 95.7 & 96.6 \\
\hline FS13 & 2.517 & 2.434 & 89.3 & 95.8 & 96.7 \\
\hline FS14 & $2.521 \pm 0.012$ & $2.433 \pm 0.002$ & $89.2 \pm 0.5$ & $95.6 \pm 0.5$ & $96.5 \pm 0.4$ \\
\hline FS15 & 2.476 & 2.397 & 88.8 & 95.8 & 96.8 \\
\hline FS16 & 2.506 & 2.416 & 88.7 & 95.4 & 96.4 \\
\hline FS17 & 2.518 & 2.434 & 88.6 & 95.6 & 96.7 \\
\hline
\end{tabular}

The last three columns show the percent of maximum density at $\mathrm{N}_{\text {ini }}, \mathrm{N}_{\mathrm{des}}$, and $\mathrm{N}_{\max }$. Again the $95 \%$ confidence intervals are shown for the percent of maximum densities. The confidence intervals were calculated using a different method than Equation 8.16 and involved the propagation of error. For the percent of maximum density at $\mathrm{N}_{\text {ini }}, \mathrm{N}_{\text {des }}$, and $\mathrm{N}_{\max }$, the confidence interval was calculated using Equation 8.17:

$$
\begin{gathered}
95 \% \text { C.I. }=\frac{G \times t \times \sqrt{\left(\frac{S . D \cdot_{1}}{H}\right)^{2}+\left(\frac{S . D \cdot \cdot_{2}}{M}\right)^{2}+2\left(\frac{S . D \cdot 3}{G_{m m}}\right)^{2}+2\left(\frac{S . D_{\cdot}}{G_{m b}}\right)^{2}}}{\sqrt{n}} \\
\mathrm{G}=\text { The percent of maximum density at } \mathrm{N}_{\mathrm{ini}}, \mathrm{N}_{\mathrm{des}} \text {, or } \mathrm{N}_{\max } \\
\mathrm{t}=\text { Student's t-distribution }=2.57 \text { when } \mathrm{n}=6
\end{gathered}
$$$$
\text { Equation } 8.17
$$ 
S.D. $1=$ Standard deviation of heights at $\mathrm{N}_{\text {ini }}, \mathrm{N}_{\text {des }}$, or $\mathrm{N}_{\max }$

$\mathrm{H}=$ Average height at $\mathrm{N}_{\text {ini }}, \mathrm{N}_{\text {des }}$, or $\mathrm{N}_{\max }$

S.D. $2=$ Standard deviation of the masses of the gyrated samples

$\mathrm{M}=$ Average mass of the gyrated samples

S.D. 3 = Standard deviation of the maximum density at the optimum asphalt content

$\mathrm{G}_{\mathrm{mm}}=$ Average maximum density at the optimum asphalt content

S.D. $4=$ Standard deviation of the bulk density at $\mathrm{N}_{\text {ini }}, \mathrm{N}_{\mathrm{des}}$, or $\mathrm{N}_{\max }$

$\mathrm{G}_{\mathrm{mb}}=$ Average bulk density at $\mathrm{N}_{\mathrm{ini}}, \mathrm{N}_{\mathrm{des}}$, or $\mathrm{N}_{\max }$

$\mathrm{n}=$ number of samples $=6$, there are three maximum densities and three bulk densities.

So, it can be seen from the confidence intervals that the process is under control and the data are reliable.

According to Superpave specifications, the percent of maximum density at $\mathrm{N}_{\text {ini }}$ should be under $89 \%$. In column three of Table $8-6$, all of the data are at or below $89 \%$, when taking into account that the average $95 \%$ confidence interval is $0.4 \%$. Also, according to Superpave specifications, the percent of maximum density at $\mathrm{N}_{\mathrm{des}}$ should be at $96 \%$. With an average $95 \%$ confidence interval of $0.4 \%$, all of the data in column five meet this criteria except for sand FS16. The optimum asphalt could have been raised one or two tenths of a percent. For most asphalt contractors, this information is quite important, since they are allowed to produce HMA with a range $0.5 \%$ above or below the 
optimum asphalt content. Last, the specification for the percent of maximum density at $\mathrm{N}_{\max }$ is that it must be below $98 \%$. Clearly the data in the last column show that all the mixes meet this specification. The average $95 \%$ confidence interval for the percent of maximum density at $\mathrm{N}_{\max }$ is $0.4 \%$. Overall, it can be said that mixes with foundry sand can meet volumetric specification requirements without a problem and with relatively small changes in asphalt content.

\subsubsection{Performance Indicators}

\subsubsection{Paver, Roller and Traffic Energy Indices}

The paver, roller, and traffic energy indices were calculated from the densification curves and are reported in Table 8-7. The 95\% confidence intervals are also shown. They were calculated using Equation 8.16. The paver energy index is constant at about 39.0 with an average $95 \%$ confidence interval of 0.9 . The roller energy index varied between 29.4 (sand FS8) and 15.4 (sand FS13). The average 95\% confidence interval is 9.2. Because the confidence interval is so large it is difficult to notice any significant trends in the REI. The TEI varies between 750.3 (sand FS12) and 439.6 (sand FS15). The average $95 \%$ confidence interval is 69 . Figure $8-12$ shows that because of the large confidence interval, it is not clear how foundry sand affects this measure. All of the sands perform in the same range as the control. Figure 8-13 shows a plot of the optimum asphalt content versus the TEI. It is commonly known that as the asphalt content is increased above the optimum asphalt content, it is more prone to rut. So it would be 
natural to reason that as the asphalt content increases, the TEI decreases. From Figure 813 , this does not seem to be the case, since the $\mathrm{R}^{2}$ value is small. One other reason for the TEI to be small is due to low angularity. It is believed that the more angular the particles are, the more they will interlock and be resistant against rutting. Figure 8-14 shows that this is not the case for these sands.

Table 8-7: Paver, roller and traffic energy indices

\begin{tabular}{c|c|c|c}
\hline Sand No. & PEI & REI & TEI \\
\hline \hline Control & $39.0 \pm 1.4$ & $21.4 \pm 8.7$ & $670.9 \pm 68.1$ \\
\hline FS01 & $38.8 \pm 0.4$ & $23.0 \pm 9.0$ & $679.7 \pm 76.9$ \\
\hline FS02 & 39.5 & 16.6 & 730.2 \\
\hline FS03 & $38.8 \pm 1.0$ & $22.1 \pm 9.9$ & $609.7 \pm 132.3$ \\
\hline FS04 & 39.1 & 21.0 & 717.2 \\
\hline FS05 & 39.0 & 22.7 & 692.6 \\
\hline FS06 & 39.7 & 21.8 & 719.8 \\
\hline FS07 & 38.6 & 18.4 & 698.0 \\
\hline FS08 & $38.7 \pm 0.5$ & $29.4 \pm 9.7$ & $650.3 \pm 63.5$ \\
\hline FS09 & $39.4 \pm 0.9$ & $17.2 \pm 5.1$ & $631.4 \pm 59.0$ \\
\hline FS10 & 38.9 & 15.4 & 707.0 \\
\hline FS11 & 39.1 & 19.2 & 619.2 \\
\hline FS12 & 39.7 & 16.9 & 750.3 \\
\hline FS13 & 39.3 & 17.2 & 684.4 \\
\hline FS14 & $38.8 \pm 0.9$ & $16.3 \pm 12.6$ & $748.0 \pm 13.9$ \\
\hline FS15 & 39.2 & 23.4 & 439.6 \\
\hline FS16 & 39.2 & 27.0 & 735.4 \\
\hline FS17 & 39.1 & 27.4 & 640.8 \\
\hline
\end{tabular}




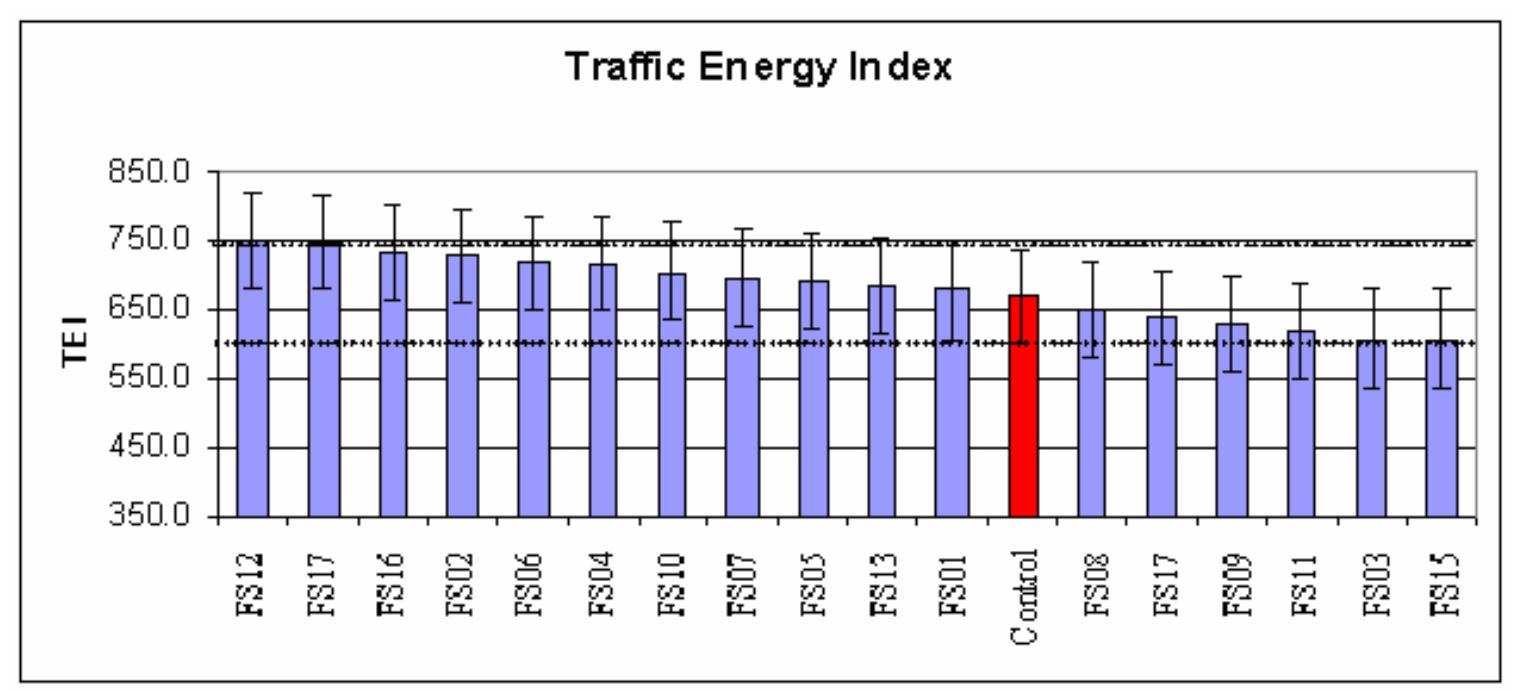

Figure 8-12: Paver, roller and traffic energy indices

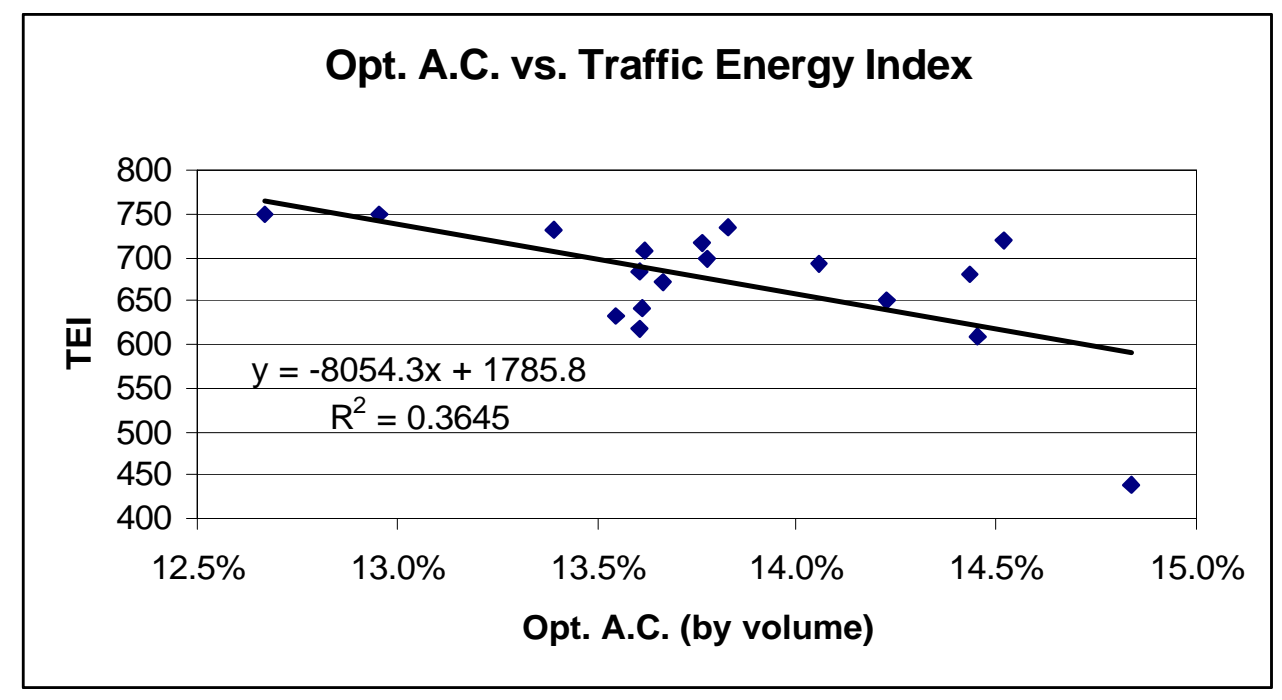

Figure 8-13: Comparison between the optimum asphalt content and the TEI 


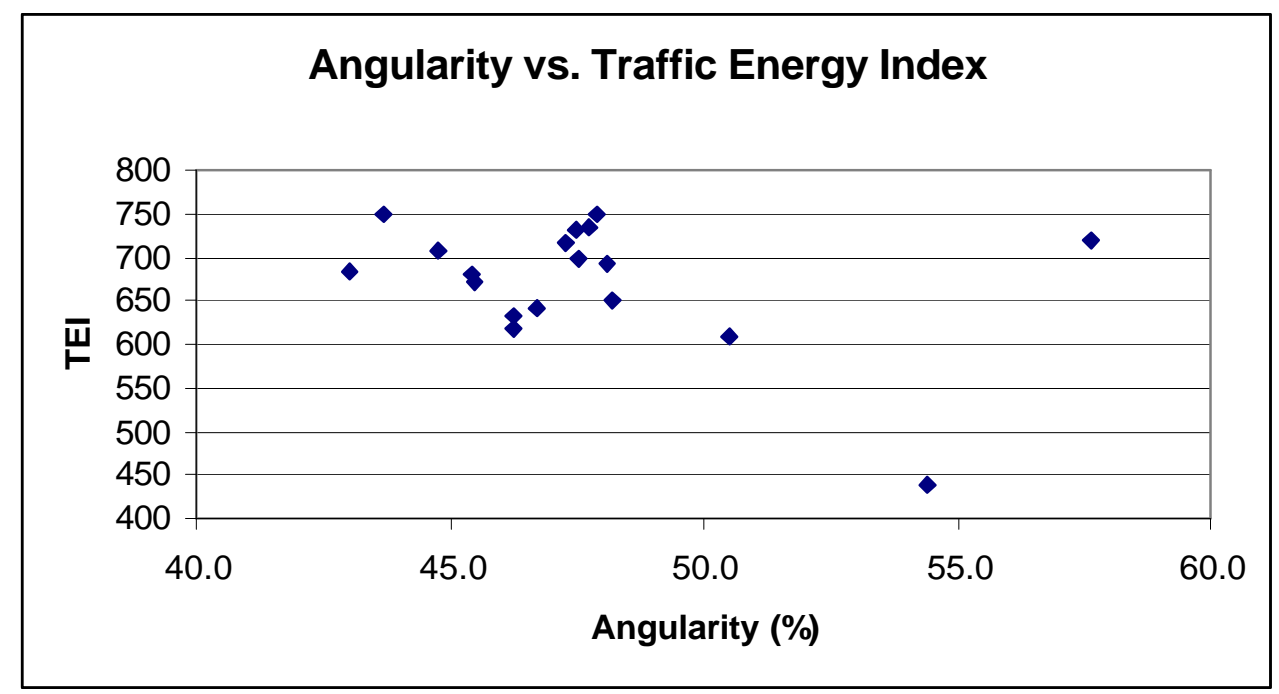

Figure 8-14: Comparison between angularity and TEI

\subsubsection{Tensile Strength and Secant Modulus Ratios}

Braham [2002] showed that mixtures containing foundry sand tend to be more susceptible to moisture damage. In this study, all of the mixtures, the control mixture plus the 17 other mixtures each containing $10 \%$ foundry sand, were tested for moisture susceptibility. This was done using the indirect tension test, according to ASTM standard procedure D4867. For this test, six samples are compacted using the Superpave Gyratory Compactor to $7 \%$ air voids. Three of these six samples are saturated with water, so that about $65 \%$ of the air voids are filled with water. They are then placed in a $60 \mathrm{EC}$ water bath for 24 hours. After the 24 hours, the samples are cooled to $25 \mathrm{EC}$ and tested in indirect tension. The tensile strengths measured are shown in Table 8-8 and are expressed in $\mathrm{kPa}$. The horizontal deflection across the face of the sample was measured using a laser measuring device. A typical plot of strain versus stress is shown in 
Figure 8-15. This plot is for the second indirect tension test sample mixture produced with sand FS13. It was not moisture conditioned. From the strain and stress data, the secant modulus is calculated as the slope of the line connecting the origin to the maximum stress point. The tensile strengths and secant moduli for samples that were moisture conditioned are shown in columns 1 and 4 of Table 8-8. The strengths are in $\mathrm{kPa}$ and the moduli are in MPa. Also, for simplicity, the standard deviations are shown, and not the $95 \%$ confidence intervals. Columns 2 and 5 show the tensile strength and secant moduli of the samples that were not moisture conditioned. Column 3 shows the ratios of the tensile strengths, hence the term "tensile strength ratio," while column 6 shows the ratios of the secant moduli. The tensile strength ratios are shown with a $95 \%$ confidence interval. Equations 8.18 and 8.19 were used to calculate this confidence interval. 
Table 8-8: Tensile strengths and secant moduli

\begin{tabular}{l|c|c|c|c|c|c}
\hline $\begin{array}{l}\text { Sand } \\
\text { No. }\end{array}$ & $\begin{array}{l}\text { Tensile } \\
\text { Strength w/ } \\
\text { M.C. (kPa) }\end{array}$ & $\begin{array}{l}\text { Tensile } \\
\text { Strength w/o } \\
\text { M.C. (kPa) }\end{array}$ & $\begin{array}{l}\text { Tensile } \\
\text { Strength } \\
\text { Ratio (\%) }\end{array}$ & $\begin{array}{l}\text { Secant } \\
\text { Modulus w/ } \\
\text { M.C. (MPa) }\end{array}$ & $\begin{array}{l}\text { Secant } \\
\text { Modulus w/o } \\
\text { M.C. (MPa) }\end{array}$ & $\begin{array}{l}\text { Secant } \\
\text { Modulus } \\
\text { Ratio (\%) }\end{array}$ \\
\hline \hline Control & $596.7 \pm 74.4$ & $884.7 \pm 8.4$ & $67.4 \pm 9.8$ & $96.7 \pm 12.2$ & $207.5 \pm 51.5$ & $46.6 \pm 25.4$ \\
\hline FS01 & $322.1 \pm 21.4$ & $710.9 \pm 16.5$ & $45.3 \pm 3.7$ & $27.4 \pm 4.7$ & $150.8 \pm 10.8$ & $18.2 \pm 3.5$ \\
\hline FS02 & $484.0 \pm 13.9$ & $738.7 \pm 29.3$ & $65.5 \pm 3.8$ & $70.1 \pm 14.8$ & $221.7 \pm 65.9$ & $31.6 \pm 12.1$ \\
\hline FS03 & $349.3 \pm 20.3$ & $694.1 \pm 14.1$ & $50.3 \pm 3.6$ & $31.8 \pm 10.4$ & $183.2 \pm 75.7$ & $17.4 \pm 9.6$ \\
\hline FS04 & $489.4 \pm 9.8$ & $789.1 \pm 62.7$ & $62.0 \pm 5.9$ & $88.0 \pm 18.5$ & $216.5 \pm 35.5$ & $40.6 \pm 13.4$ \\
\hline FS05 & $348.7 \pm 11.6$ & $774.4 \pm 21.8$ & $45.0 \pm 2.4$ & $29.8 \pm 4.6$ & $164.6 \pm 19.9$ & $18.1 \pm 3.7$ \\
\hline FS06 & NA & $789.3 \pm 41.9$ & NA & NA & $149.4 \pm 23.1$ & NA \\
\hline FS07 & $409.8 \pm 27.2$ & $750.5 \pm 38.5$ & $54.6 \pm 5.3$ & $44.9 \pm 5.9$ & $152.7 \pm 9.2$ & $29.4 \pm 4.5$ \\
\hline FS08 & $378.4 \pm 10.5$ & $696.5 \pm 28.5$ & $54.3 \pm 3.1$ & $31.8 \pm 3.7$ & $129.5 \pm 9.8$ & $24.5 \pm 3.6$ \\
\hline FS09 & $478.3 \pm 9.7$ & $684.1 \pm 19.6$ & $69.9 \pm 2.8$ & $68.8 \pm 7.7$ & $189.0 \pm 33.4$ & $36.4 \pm 8.0$ \\
\hline FS10 & $416.3 \pm 26.2$ & $815.6 \pm 17.0$ & $51.0 \pm 3.9$ & $100.8 \pm 28.9$ & $251.0 \pm 10.9$ & $40.2 \pm 12.2$ \\
\hline FS11 & $463.4 \pm 30.3$ & $758.0 \pm 18.4$ & $61.1 \pm 5.2$ & $79.3 \pm 7.2$ & $173.4 \pm 27.2$ & $45.7 \pm 8.7$ \\
\hline FS12 & $535 \pm 48$ & $721.7 \pm 20.5$ & $74.1 \pm 8.1$ & $87.9 \pm 9.3$ & $186.3 \pm 33.9$ & $47.2 \pm 7.9$ \\
\hline FS13 & $556.6 \pm 22.6$ & $782.7 \pm 10.8$ & $71.1 \pm 3.5$ & $81.5 \pm 17.0$ & $223.3 \pm 45.4$ & $36.5 \pm 11.1$ \\
\hline FS14 & $704.8 \pm 11.5$ & $798.7 \pm 9.9$ & $88.2 \pm 2.1$ & $130.9 \pm 26.1$ & $186.6 \pm 25.8$ & $70.1 \pm 17.9$ \\
\hline FS15 & $440.5 \pm 49.4$ & $703.5 \pm 44.1$ & $62.6 \pm 9.1$ & $66.1 \pm 10.6$ & $118.8 \pm 17.5$ & $55.6 \pm 12.7$ \\
\hline FS16 & $358.1 \pm 0.4$ & $696.7 \pm 20.1$ & $51.4 \pm 1.8$ & $48.5 \pm 5.0$ & $170.5 \pm 24.5$ & $28.4 \pm 6.2$ \\
\hline FS17 & $89.4 \pm 6.3$ & $780.4 \pm 18.0$ & $11.5 \pm 1.0$ & $9.8 \pm 2.2$ & $137.0 \pm 5.8$ & $7.2 \pm 1.7$ \\
\hline
\end{tabular}

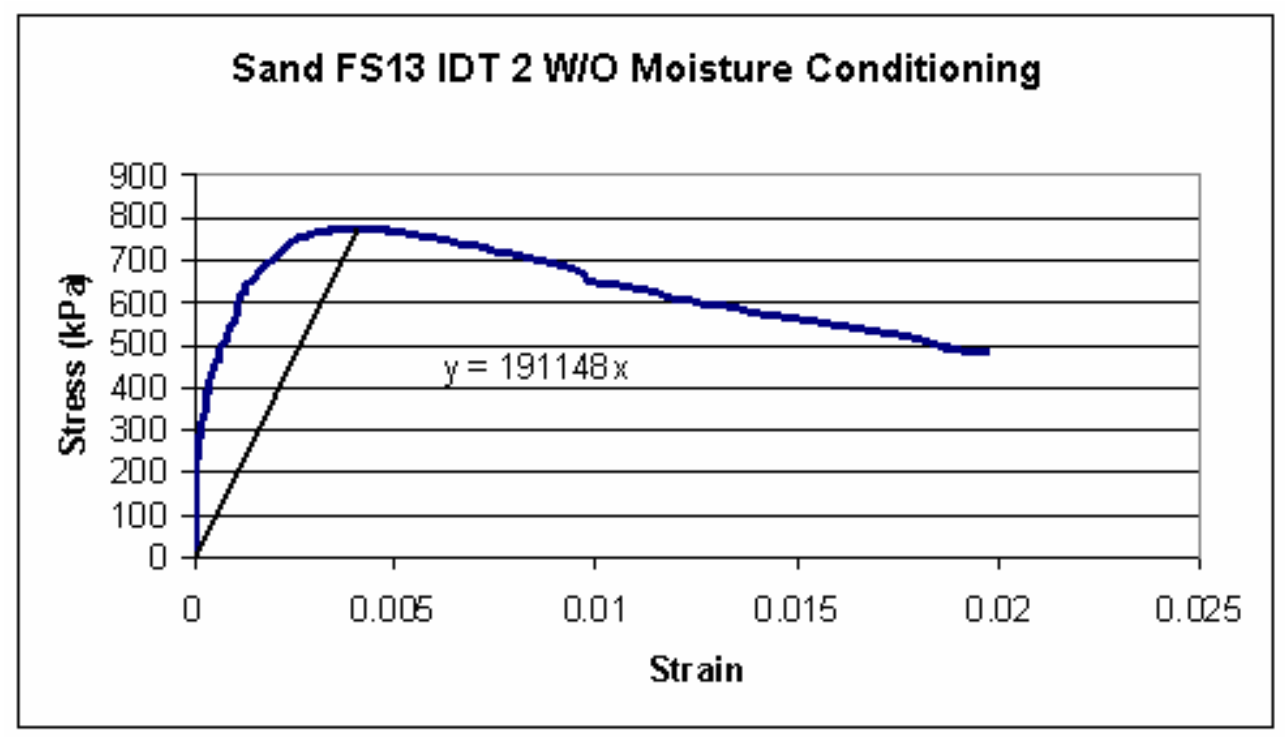

Figure 8-15: Strain versus stress plot for the second IDT for sand FS13 


$$
\operatorname{Var}=\left[\frac{X}{Y}\right]^{2} \times\left[\frac{S_{X}^{2}}{n_{X} X^{2}}+\frac{S_{Y}^{2}}{n_{Y} Y^{2}}\right]
$$

Equation $\mathbf{8 . 1 8}$

$$
95 \% \text { C.I. }=2 \sqrt{\operatorname{Var}}
$$

Equation 8.19

Var $=$ Variance of TSR

$\mathrm{X}=$ Average tensile strengths for moisture conditioned

$\mathrm{Y}=$ Average tensile strengths for not moisture conditioned

$\mathrm{S}_{\mathrm{X}}=$ Standard deviation of moisture conditioned tensile strengths

$\mathrm{S}_{\mathrm{Y}}=$ Standard deviation of not moisture conditioned tensile strengths

$\mathrm{n}_{\mathrm{X}}=$ Number of moisture conditioned samples

$\mathrm{n}_{\mathrm{Y}}=$ Number of not moisture conditioned samples

$95 \%$ C.I. $=95 \%$ confidence interval

Equations 8.18 and 8.19 were taken from a master's thesis written by Seemab Ahmad [Ahmad 1998]. His thesis topic was to evaluate procedures for assessing moisture damage of asphalt pavements in Wisconsin. The 95\% confidence interval for the secant modulus ratio was calculated in more of a traditional way, similar to Equation 8.17, and is presented in Equation 8.20. 


$$
95 \% \text { C.I. }=\frac{X}{Y} \times t \times \frac{\sqrt{\left(\frac{S_{X}}{X}\right)^{2}+\left(\frac{S_{Y}}{Y}\right)^{2}}}{\sqrt{n}}
$$

$\mathrm{X}=$ Average secant modulus of moisture conditioned samples

$\mathrm{Y}=$ Average secant modulus of not moisture conditioned samples

$\mathrm{t}=$ Student's $\mathrm{t}$-distribution $=2.57$

$\mathrm{S}_{\mathrm{X}}=$ Standard deviation of moisture conditioned samples

$\mathrm{S}_{\mathrm{Y}}=$ Standard deviation of not moisture conditioned samples

$\mathrm{n}=$ Total number of samples $=6$

A comparison between the tensile strength and secant modulus with moisture conditioning is shown graphically in Figures 8-16 and 8-17. The average 95\% confidence interval for the average conditioned tensile strengths is $57.3 \mathrm{kPa}$ and $27.6 \mathrm{MPa}$ for the average conditioned secant modulus, and are shown in Figures 8-16 and 8-17. Figure 816 shows that only one sand increased the conditioned tensile strength, while two sands showed no change and the remaining sands decreased the tensile strength. This shows that the majority of foundry sands decrease the conditioned tensile strengths, which means they will most likely cause asphalt mixtures to be more susceptible to moisture damage. But at the same time, foundry sands should be tested on a case by case basis, since not all foundry sands encourage moisture damage. Although the error bars are much greater for the conditioned secant modulus (Figure 8-17), the same trend can be seen. For the conditioned secant modulus, 11 sands show no change in modulus, with the remaining 5 sands falling below the control. 


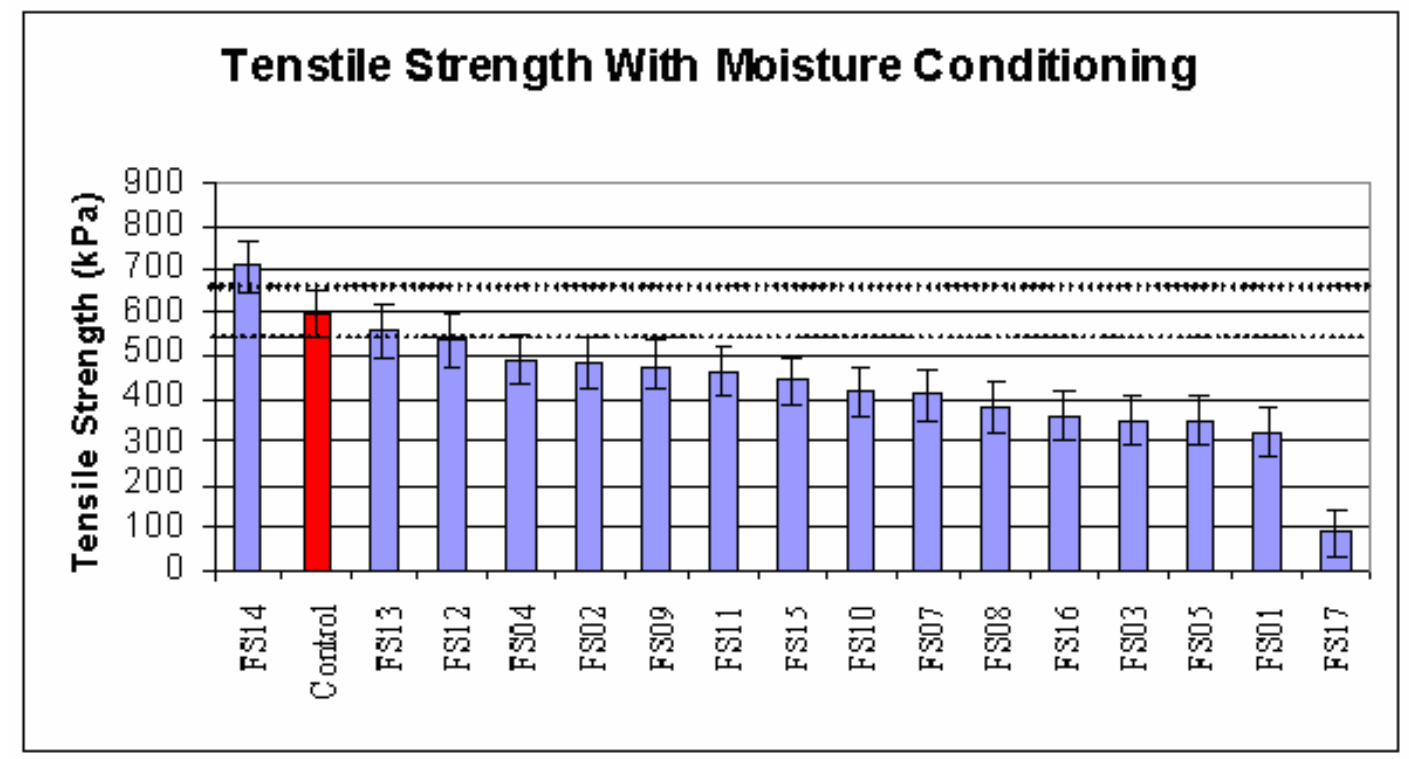

Figure 8-16: Tensile strengths with moisture conditioning

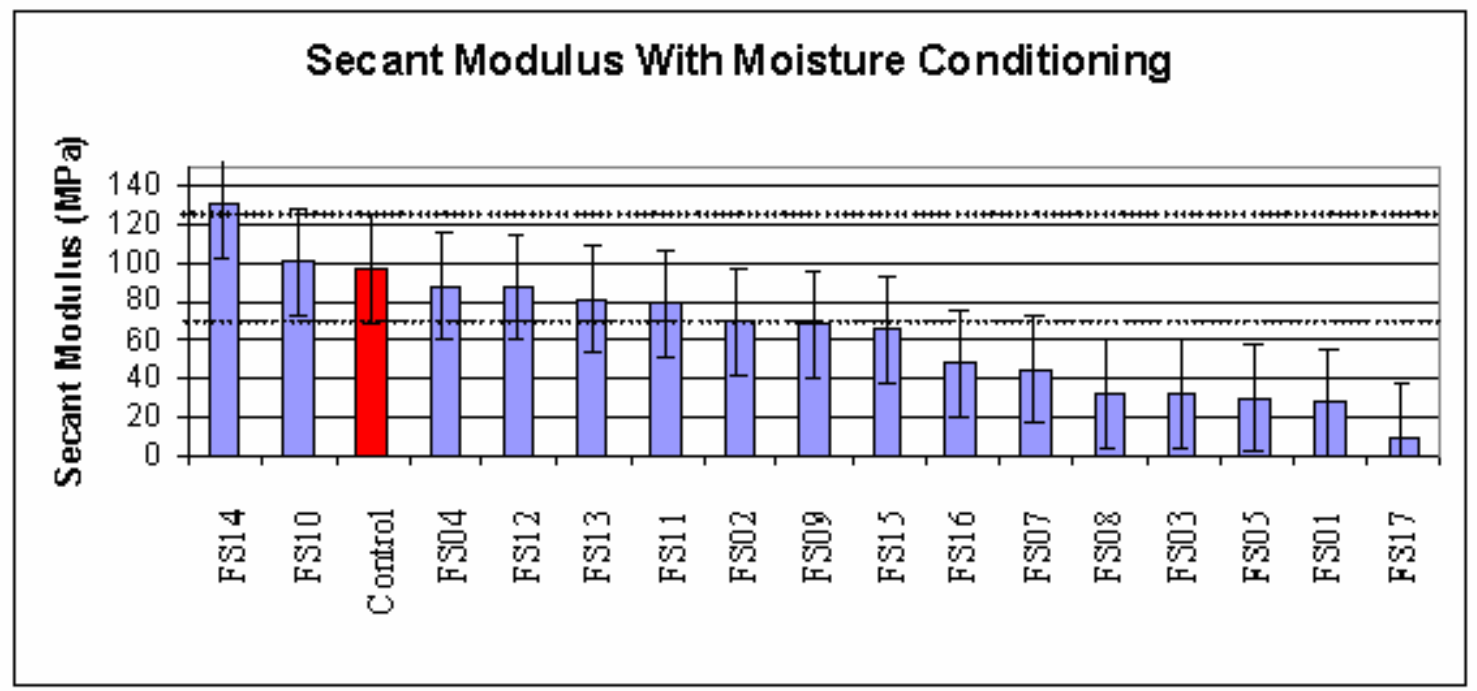

Figure 8-17: Secant modulus with moisture conditioning

A similar trend is found in Figure 8-18 and Figure 8-19. Here the tensile strengths and secant moduli for unconditioned samples are plotted with respect to each other. The average $95 \%$ confidence interval was calculated for the unconditioned tensile 
strengths $(60.2 \mathrm{kPa})$ and for the unconditioned secant modulus $(72.5 \mathrm{MPa})$. For the unconditioned tensile strengths, the control has the highest average unconditioned tensile strength, with six sands falling within its 95\% confidence interval. The remaining 11 sands fall below. This suggests that foundry sands, on average, decrease the unconditioned tensile strength, and thus the durability of asphalt mixtures. For the secant modulus, it is difficult to tell the effect of foundry sands because of the large standard deviation. From inspection of the average unconditioned secant moduli, foundry sand appears to decrease the modulus and thus the modulus of asphalt mixtures.

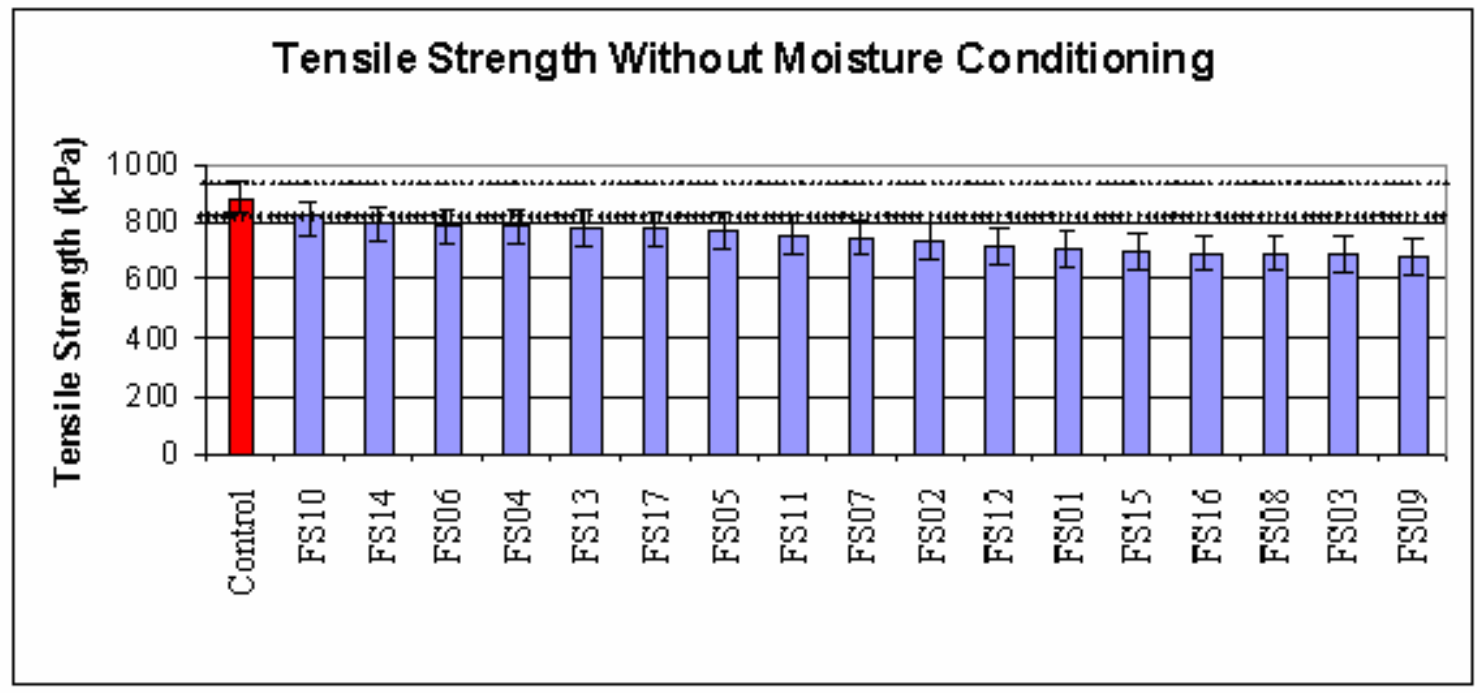

Figure 8-18: Tensile strength without moisture conditioning 


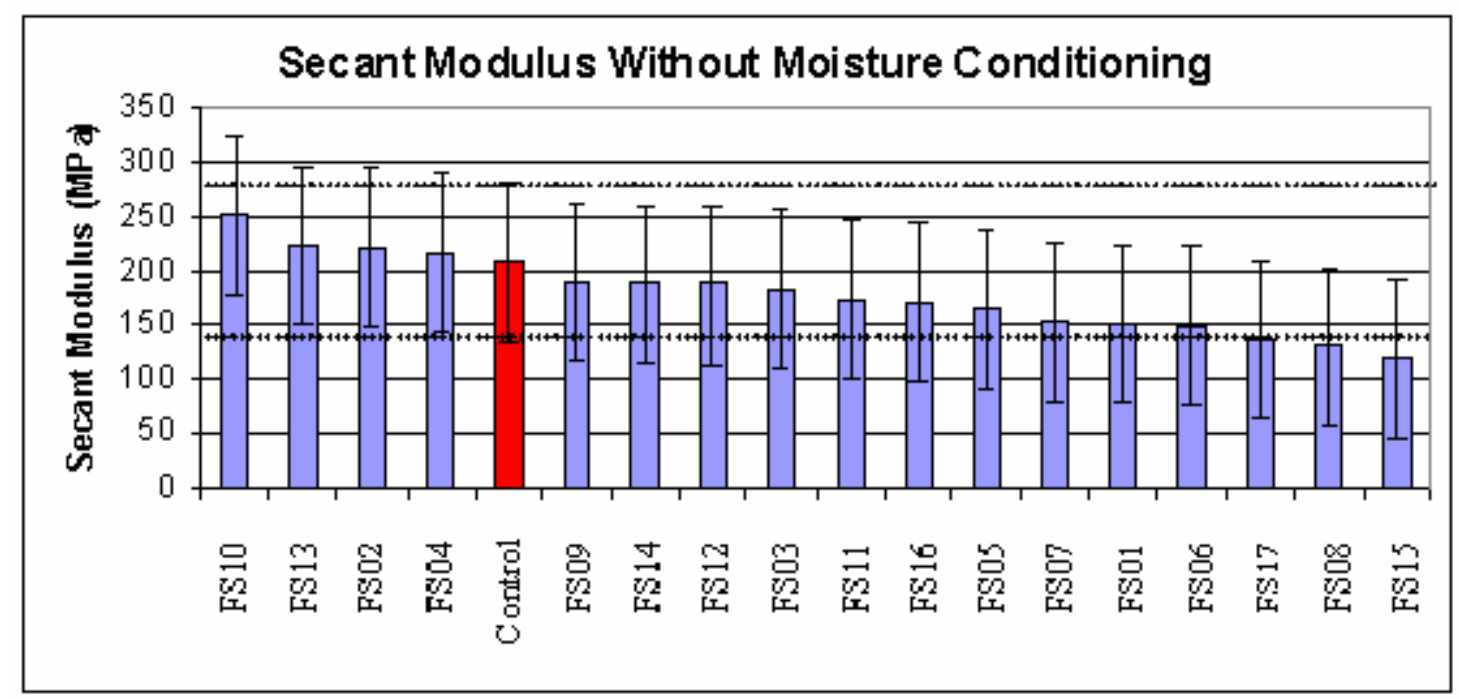

Figure 8-19: Secant modulus without moisture conditioning

The data shown in Figure 8-20 and Figure 8-21 confirm that moisture conditioning causes the tensile strengths to decrease to a level that is unacceptable to most state departments of transportation. In Wisconsin, the minimum TSR allowed is $70 \%$, meaning that moisture conditioning can decrease the tensile strength by no more than $30 \%$ of the unconditioned value. It can be seen from Table 8-6 that the control mix does not meet the Wisconsin DOT specifications. According to the data sheet that was provided by the contractor, the average tensile strength ratio was $76.7 \%$. This was a mix that was designed in September 2000, and was used with aggregates that were crushed and sieved during this same time. On the other hand, it was not until the summer of 2002 that the aggregates were gathered and testing initiated for the optimum asphalt content, asphalt absorption, tensile strength ratios, etc. Because there was a lag between the time the mix was designed and when it was used for this project, the aggregates most likely 
changed, not only in gradation but also in surface chemistry and other characteristics. Also, the asphalt binder used in 2000 was not the same binder used in 2002. These factors could have led to a decrease in the tensile strength ratio. Regardless of this, it is still possible to determine how foundry sands affect HMA performance by comparing to the control.

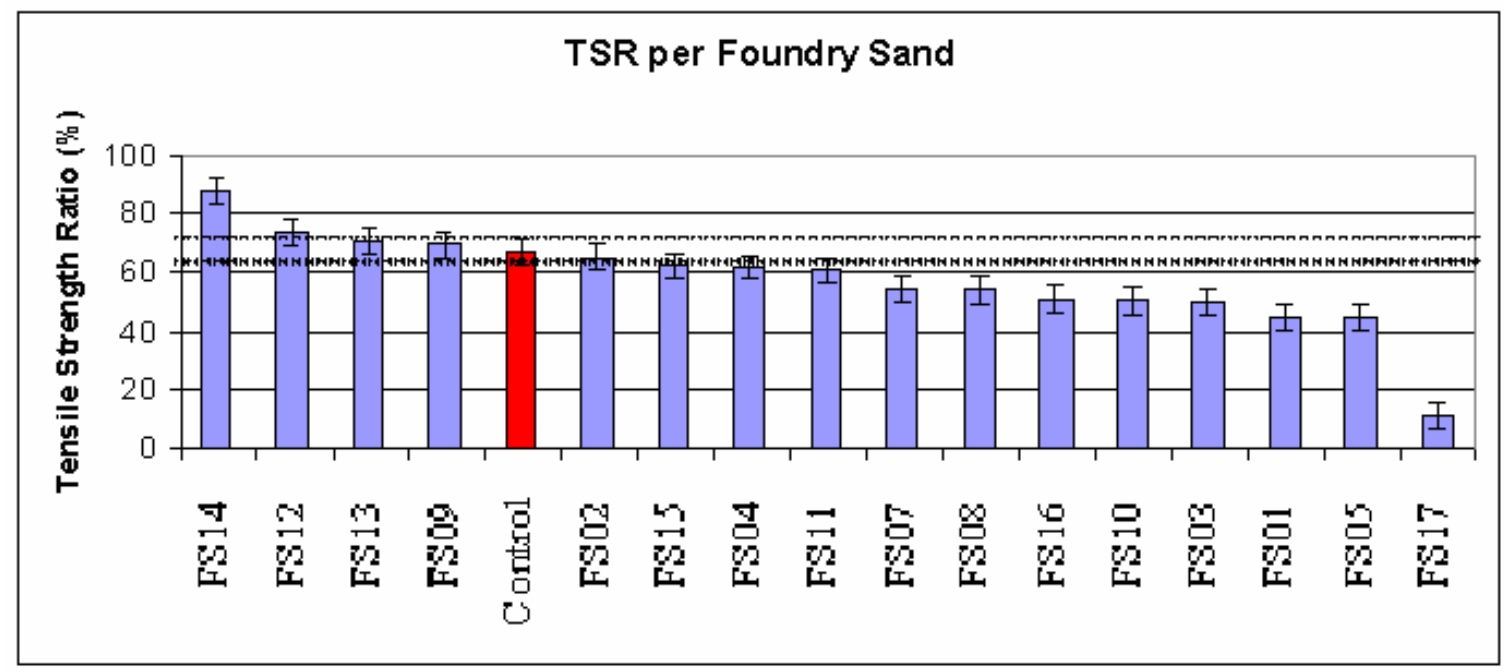

Figure 8-20: Comparison of tensile strength ratios 


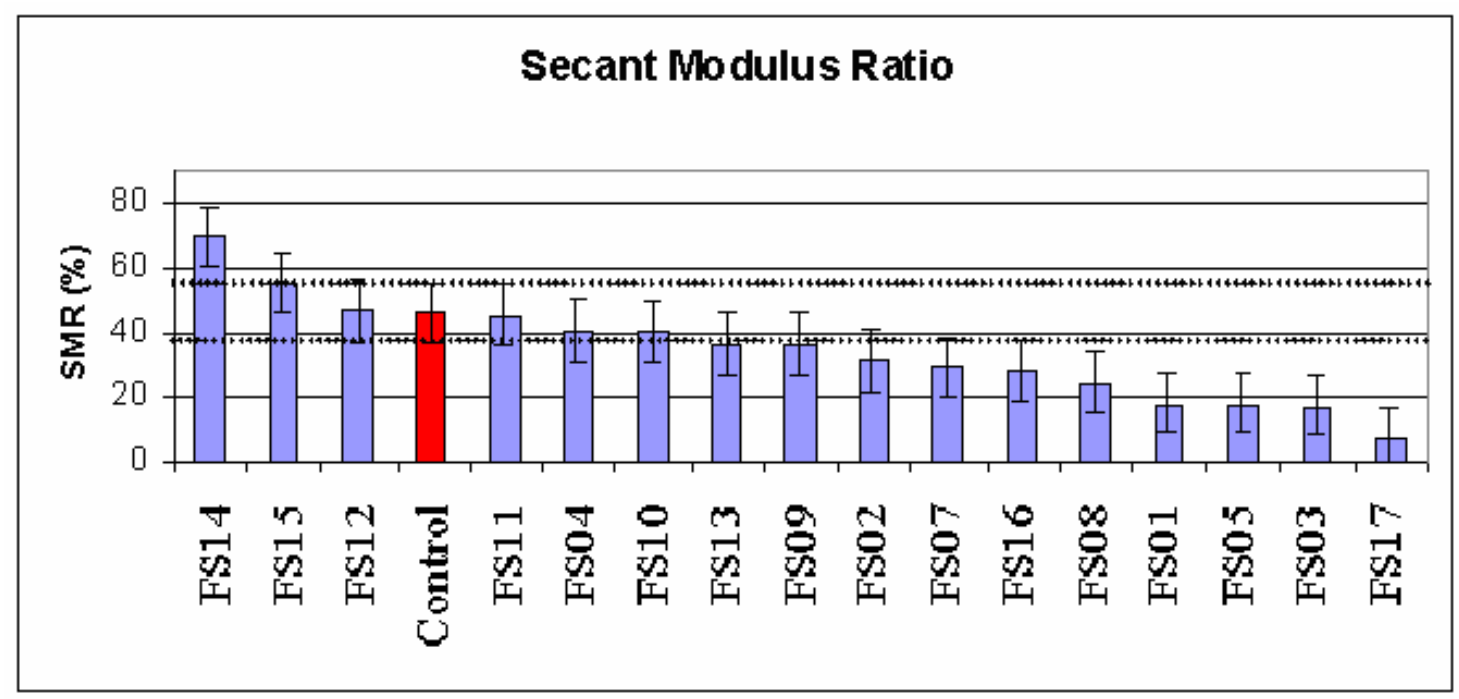

Figure 8-21: Comparison of secant modulus ratios

Of the 17 sands, only one, sand FS14, increased the TSR above the 95\% confidence interval. It had a TSR of $88.2 \%$. Applying the average $95 \%$ confidence interval of $4.4 \%$, this yields a $95 \%$ certainty that the TSR is between $92.6 \%$, and $83.8 \%$. For the control, the confidence band is $71.8 \%$ and $63 \%$. This confidence band overlaps the $95 \%$ confidence band of seven of the other sands: FS02, FS04, FS09, FS11, FS12, FS13, and FS15. The remaining nine sands all fell below the control confidence band. Sand FS06 performed so poorly that its moisture conditioned tensile strength could not be recorded. During the 24-hour soak in hot water, all three samples became so soft they crumbled under their own weight. Figure 8-22 shows there is a good correlation between the TSR and SMR, with the trend line having an $\mathrm{R}^{2}$ of 0.70 . Most of the discrepancy between the two could most likely be due to errors in measuring the displacement on the face of the asphalt sample. 


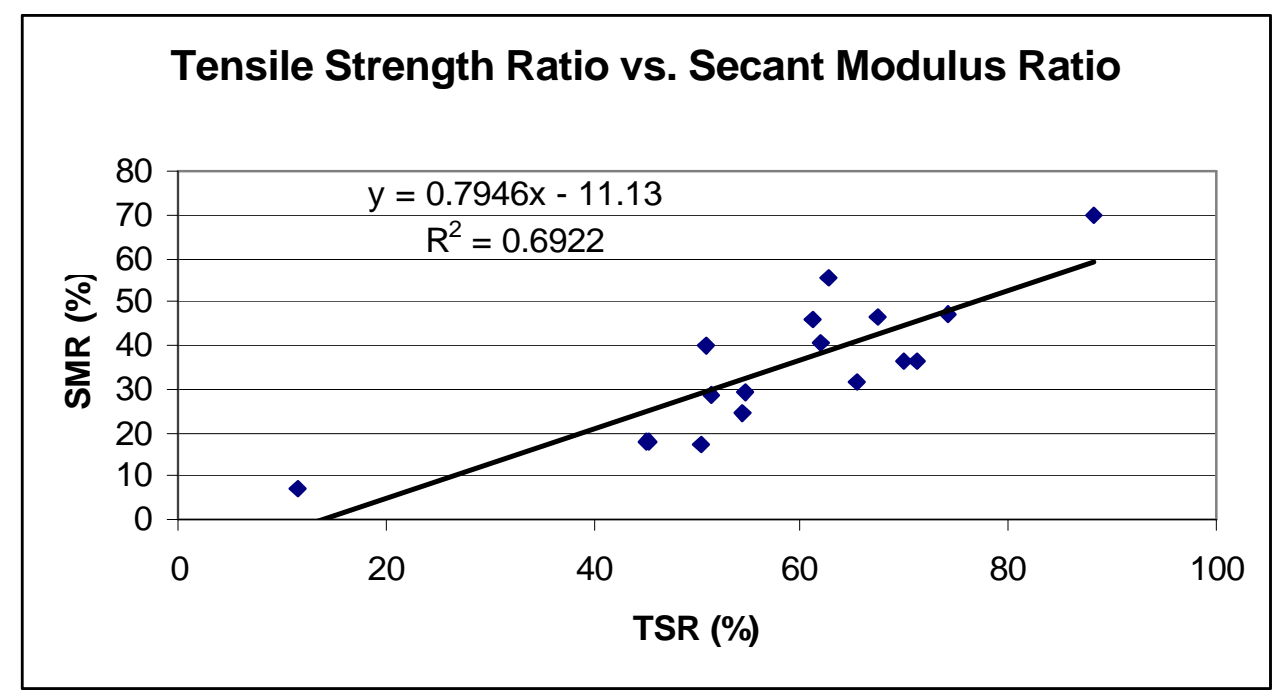

Figure 8-22: Relation between TSR and SMR

\subsubsection{Comparison of Tensile Strength Ratio to Clay Content}

Since it is well known that clay causes mixes to be more susceptible to moisture damage, the methylene blue value was plotted versus the TSR. This is shown in Figure 8-23. From looking at the $\mathrm{R}^{2}$ value, there does not seem to be a correlation between these two values. The main reason for this is because of sand FS17. It has a low MBV and a low TSR value. Since it is bound with sodium silicate and not bentonite, the MBV should be low. But this does not explain why the TSR value is low. Sands FS06 and FS10 were also bound with sodium silicate. As mentioned before, the mixture with sand FS06 was too soft to be tested, and the mixture with sand FS10 has a TSR value of $51 \%$. Since all three sands performed worse than the control, two of them failing drastically, it can be said that sodium silicate is very detrimental to HMA moisture susceptibility performance. In light of this, only the sands that use bentonite as a binder 
system were plotted versus the TSR. This is shown in Figure 8-24. When doing this, there is a better correlation between the MBV and TSR, with the linear trend line having an $\mathrm{R}^{2}$ of 0.63 . It can be generally stated that as the MBV increases, the TSR decreases.

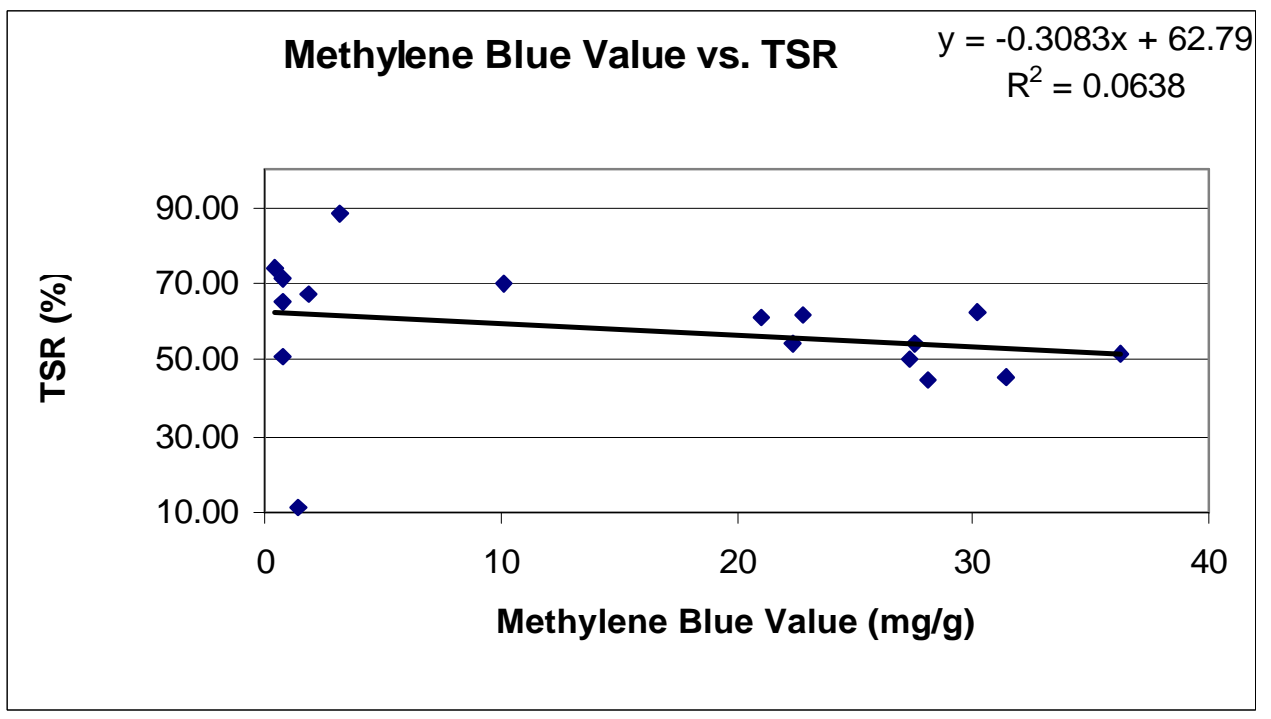

Figure 8-23: Comparison of Methylene blue value to TSR

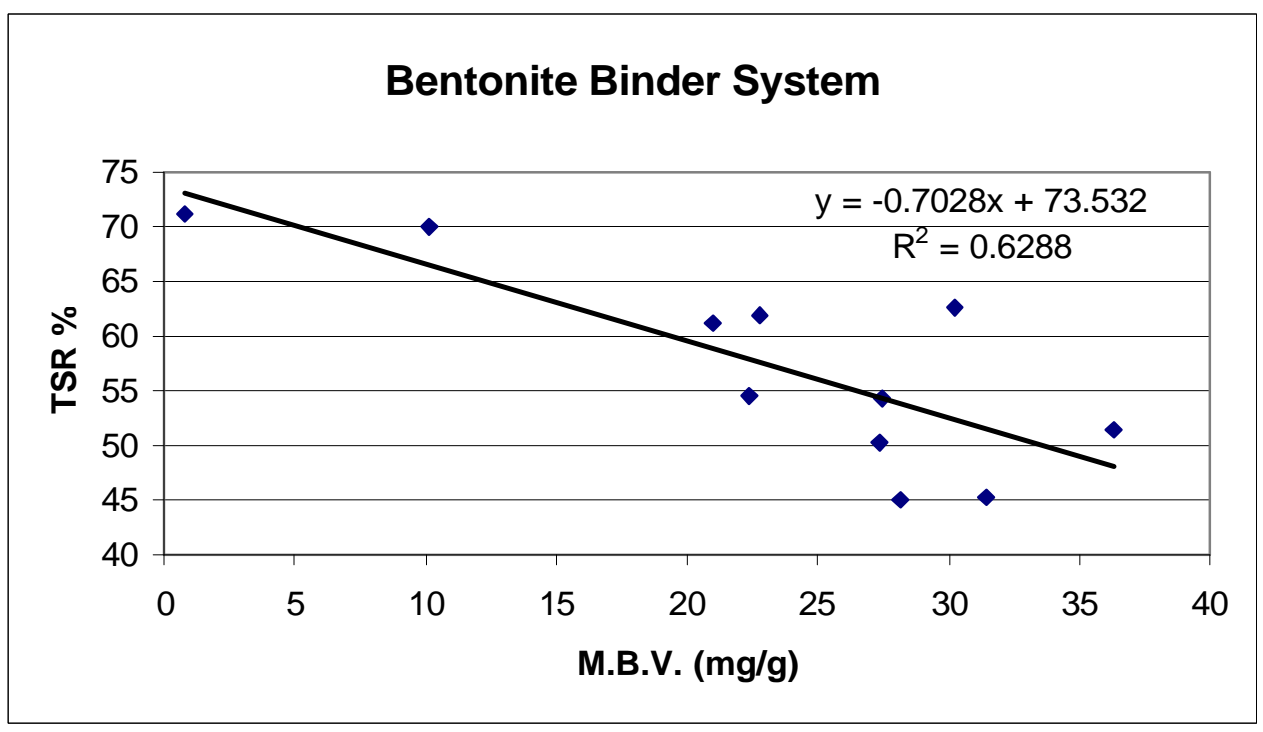

Figure 8-24: Comparison of methylene blue value to TSR for only bentonite bound sands 


\subsubsection{The Use of Anti-Stripping Agents}

In order to correct for the low TSR values, two different anti-stripping additives were added separately to four of the different mixes. The first anti-stripping additive was a liquid additive that was added to the asphalt binder at a level of $0.5 \%$ by mass of the total asphalt binder. The second additive was hydrated lime. It was added in at $2 \%$ of the total aggregate mass. Most contractors prefer to use the liquid additive because it is easier to use and more economical. Lime can be burdensome because the aggregates have to be wet before the lime is added and the job mix formula has to be changed. Since both of these materials are commonly used in practice, both were used for the study. First, the liquid anti-stripping additive, called Morelife 3300, was added to the control mixture at a level of $0.5 \%$ of the asphalt mass. This caused the TSR to increase from $67.4 \%$ to $94.3 \%$. This verifies that the Morelife 3300 was working properly. This is shown graphically in Figure 8-25 and in table form in Table 8-9.

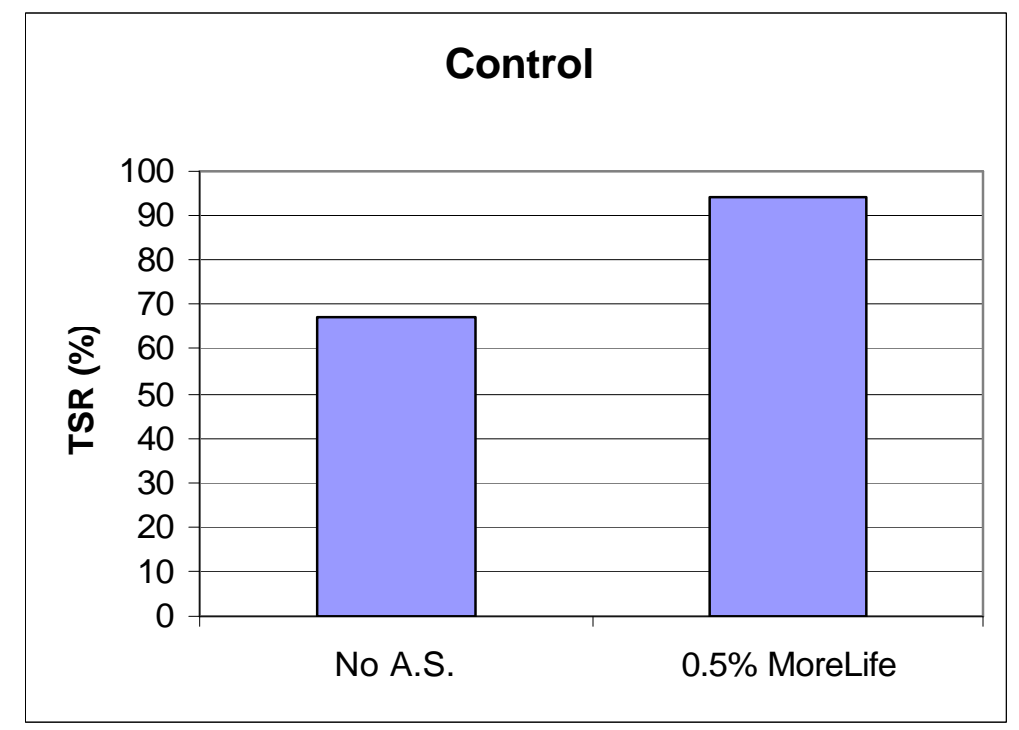

Figure 8-25: Comparison of control with and without anti-stripping additive 
Table 8-9: Tensile strengths after anti-stripping agents

\begin{tabular}{c|c|c|c}
\hline Sand No. & $\begin{array}{l}\text { Tensile Strength } \\
\text { w/ M.C. (kPa) }\end{array}$ & $\begin{array}{l}\text { Tensile Strength } \\
\text { w/o M.C. (kPa) }\end{array}$ & $\begin{array}{l}\text { Tensile Strength } \\
\text { Ratio (\%) }\end{array}$ \\
\hline \hline Control @ 0.5\% ML & $675.1 \pm 27.1$ & $715.8 \pm 28.9$ & $94.3 \pm 6.2$ \\
\hline FS17 @ 0.5\% ML & $160.9 \pm 17.4$ & $749.0 \pm 36.1$ & $21.5 \pm 2.9$ \\
\hline FS17 @ 1\% ML & $282.6 \pm 16.1$ & $811.9 \pm 11.1$ & $34.8 \pm 2.9$ \\
\hline FS17 @ 2\% Lime & $311.8 \pm 11.1$ & $842.1 \pm 11.1$ & $37.0 \pm 2.0$ \\
\hline FS05 @ 0.5\% ML & $391.2 \pm 28.2$ & $669.3 \pm 19.2$ & $58.4 \pm 5.2$ \\
\hline FS16 @ 0.5\% ML & $369.7 \pm 9.7$ & $670.5 \pm 23.5$ & $55.1 \pm 2.8$ \\
\hline FS16 @ 1.0\% ML & $414.6 \pm 14.4$ & $717.3 \pm 37.5$ & $57.8 \pm 5.1$ \\
\hline FS16 @ 2\% Lime & $460.1 \pm 13.1$ & $789.8 \pm 31.5$ & $58.3 \pm 4.0$ \\
\hline
\end{tabular}

Morelife was also added to sand FS17, since it was the worst performing sand that could be tested. At a level of $0.5 \%$, the TSR was doubled to $21.5 \%$ from the original $11.5 \%$. Since $21.5 \%$ was still well below the minimum of $75 \%$ ( $75 \%$ is the minimum when an anti-stripping agent is used), the level of Morelife was increased to $1 \%$. This allowed the TSR to triple from its original value to $34.8 \%$. Last, lime was used at a level of $2 \%$. This caused the TSR to triple also to a value of 37\%. This is shown in Figure 826. However, although there was a great improvement in TSR values, they remained well below the acceptance level. The same procedure was followed for sand FS16, but the same drastic improvements were not seen. After the addition of $0.5 \%$ of Morelife 3300, the TSR only increased from 51.4 to 55.1 . After the addition of $1.0 \%$ Morelife, it increased to 57.8\%. And again, when lime was added at a level of $2 \%$, the TSR increased to 57.8\%. This is shown in Figure 8-27. Due to lack of time and materials, sand FS05 was only tested using 0.5\% Morelife, shown in Figure 8-28. The TSR value was increased from $45 \%$ to $58.4 \%$ but was still below the acceptance limit. So it can be 
said that even though anti-stripping additives used at the $0.5-1.0 \%$ concentration increase the TSR, they may not be able to increase them enough for the HMA mixture to be accepted by most state DOTs.

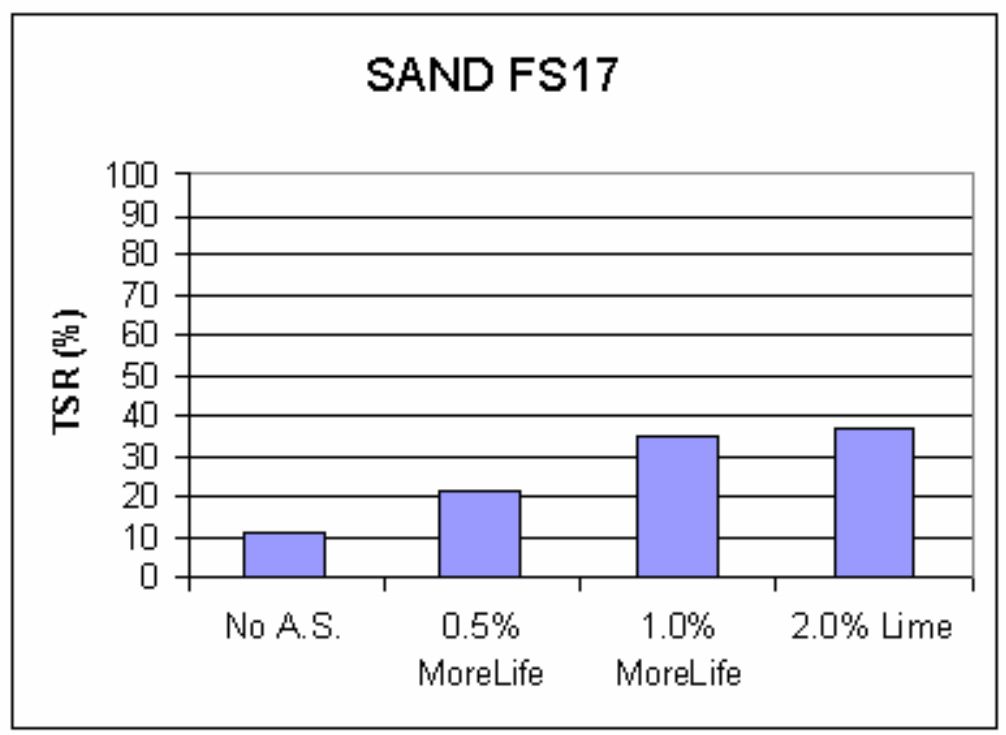

Figure 8-26: Comparison of sand FS17 with and without anti-stripping additives 


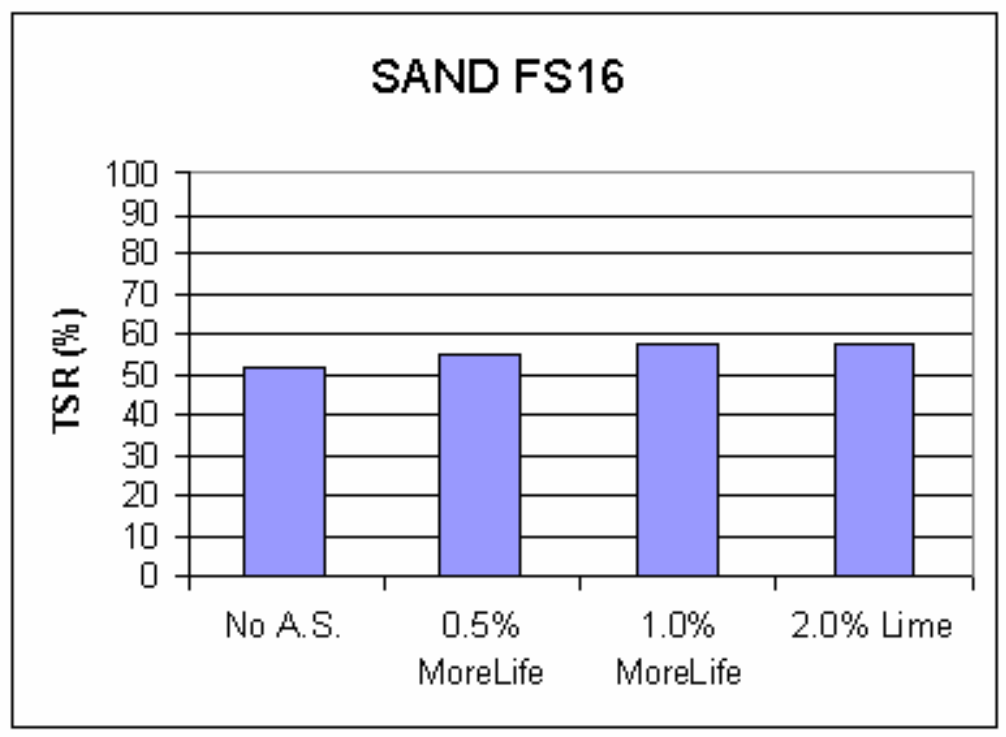

Figure 8-27: Comparison of sand FS16 with and without anti-stripping additives

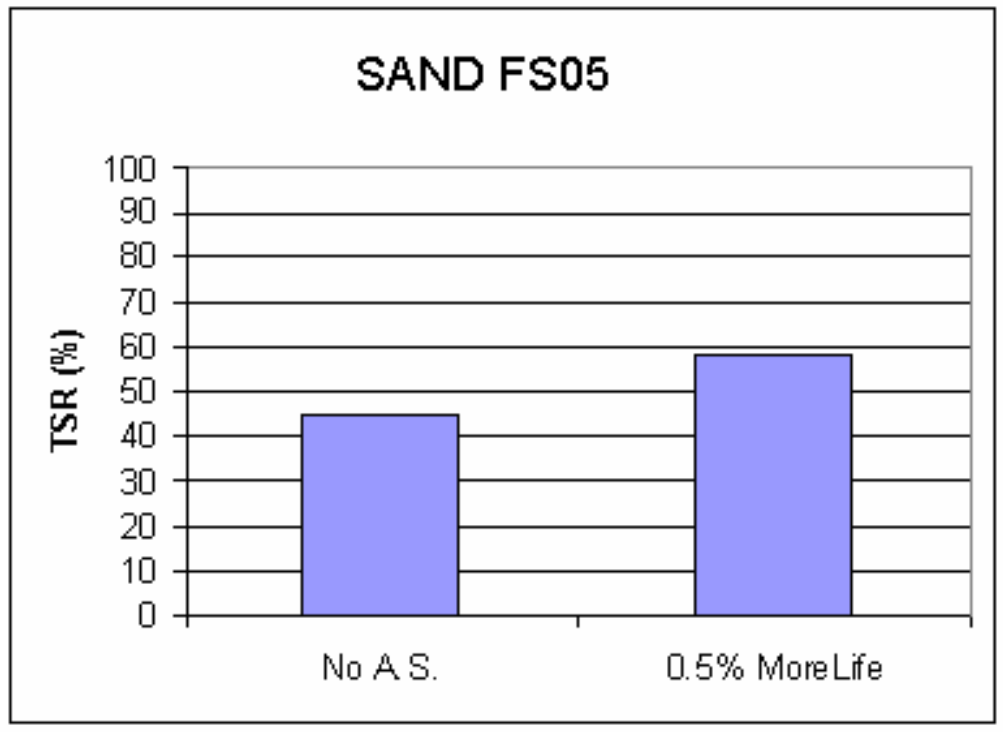

Figure 8-28: Comparison of sand FS05 with and without anti-stripping additives 


\section{Chapter 9}

\section{CONCLUSIONS AND RECOMMENDATIONS}

Based on the chemical characterization of foundry by-products and its experimental investigation in controlled low-strength materials and hot-mix asphalt, the following conclusions and recommendations are presented.

\subsection{Characterization of Excess Foundry Sand}

- Survival analysis processes the censored data characterizing foundry sands without any distribution assumption, which better matches the nature of data than other parametric methodologies.

- For as-received foundry sand, metal type cast in the facilities is not a significant factor regarding most metallic elements. The exceptions are lead, copper, and zinc, which are frequently of greater concentration in sand from copper-based facilities than from other alloy facilities.

- $\quad$ TCLP is more aggressive on sand than SPLP and ASTM D 3987. SPLP is most representative for fill applications.

- For TCLP sand leachate, metal cast in the facilities is not a significant factor regarding most metallic elements. One of the exceptions is lead, which is frequently of greater concentration in TCLP sand leachate from 
copper-based facilities than in TCLP sand leachate from other alloy facilities.

- Excess foundry sand does not pose a greater threat to the environment than soil. The concentrations of most regulated metallic elements are less than or in the same level as those of soil. In comparison with soil, excess foundry sands contain more metallic elements that are frequently and heavily used in the casting process than soil, such ash aluminum, copper, and iron.

- Leachates from foundry sand by typical leaching protocols, including TCLP, SPLP, and ASTM D 3987, have $95^{\text {th }}$ percentiles of metallic element concentrations well below the TCLP thresholds. Thus, foundry sands are generally not hazardous.

- Comparisons indicate that $95^{\text {th }}$ percentiles of bulk metallic element composition in sand are well below the TCLP thresholds, except for lead and chromium. Hence, it is not necessary to leachate and measure the full spectrum of metallic elements in sand.

- Most of the organic compounds are significantly burned out during hightemperature melting. Twenty-three of 37 typical organic compounds are $100 \%$ below detection limits in sand leachate. Seven of the remaining 14 compounds are more than $80 \%$ censored; these are cresol_m, cresol_o, benzene, 2_4-Dimethylphenol, tetrachloroethylene, xylene_total, and methyl isobutkl ketone. Compounds with censoring fractions less than 80\% include: acetone, ethyl benzene, naphthalene, phenanthrene, styrene, 
toluene, and trichloroethylene. Those 23 organic compounds with $100 \%$ censoring are suggested to be eliminated from regulatory requirements, as inappropriate testing keeps foundry sand from beneficial reuse.

\subsection{Physical Properties of Excess Foundry Sand}

- The angularity of the sands varied from 43.1 to 57.6 , with the control P0.6 being measured at 45.5, and the natural sand being reported by the contractor as 41.5. This shows that foundry sands have average to high angularities as compared to the control, and thus could promote aggregate interlock.

- The methylene blue values varied from 0.8 to $36.3 \mathrm{mg} / \mathrm{g}$, showing that foundry sands contain varying amounts of clay, with some sands not containing any clay. If the methylene blue value is above $10 \mathrm{mg} / \mathrm{g}$, the foundry sand will most likely lead to an increase in moisture damage susceptibility and will need to be washed before use.

- The organic contents ranged from $0.2 \%$ to $4.7 \%$ and were due to organic additives like sea coal, cereals, and starches. Since the level of organics is so low, they are not considered significant to the performance of the HMA mixtures. 


\subsection{CLSM Containing Excess Foundry Sand}

- CLSM containing excess foundry sands can be a high-quality component of CLSM mixtures. It presents equivalent construction behavior and geotechnical performance with an identical design effort. Its design is generally accomplished by trial batching to attain the required performances of critical parameters, which are listed in Table 7-1.

- Water proportion and grain fineness are two main factors influencing the flowability. It is indicated that the finer the average grain size, the less water is required to attain specified flow criteria for both excavatable CLSM and structural CLSM. The general absolute volume of water in the fresh CLSM matrix ranges from $30 \%$ to $50 \%$ to provide working flowability of CLSM.

- Measurements of penetration resistance at early ages help compare the hardening behavior of different mixtures. For excavatable CLSM, the main factor affecting penetration resistance is found to be the factor of foundry sand. For structural CLSM, the penetration resistance is mainly dependent on the cement proportion.

- The initial setting time for extractable CLSM starts from 6.7 hours and averages $15-20$ hours. The initial setting time of structural CLSM is concentrated at about 5-7 hours, which is contributed by the increased proportion of cement and can be accelerated or retarded with chemical 
admixtures. The increase in cement proportion enhances the correlation of high early-penetration resistance and long-term compressive strength.

- Cumulative bleeding and bleed time are not significantly correlated. Bleeding of excavatable CLSM is slightly more in volume and longer in elapse time than that of structural CLSM. Bleeding of CLSM normally takes 2-5 hours, with volume up to $6 \%$ of fresh CLSM. Foundry sand has a significant influence on bleeding.

- Water addition is exclusively controlled by flowability, which is closely related to grain fineness.

- Water-cement ratio influences 28-day strength, although the relation is nonlinear. Water-cement ratios of 2 and 5-6 are suggested starting points for design excavatable CLSM and structural CLSM, respectively. The 28day strength varies inversely with the water-cement ratio. An increase in cement proportion contributes substantially to strength gain. After 28 days, CLSM continues to gain strength. The long-term strength can be estimated by 28 -day strength.

- The initial trial mixture design of excavatable CLSM containing excess foundry sands is presented in Table 9-1. 
Table 9-1: Starting mix of excavatable CLSM

\begin{tabular}{l|l|l|l|l|l}
\hline & Bulk Proportion ${ }^{*}\left(\mathrm{Unit} \mathrm{kg} / \mathrm{m}^{3}\right)$ \\
\hline & Cement & Fly Ash & Sand & Water & Total \\
\hline Min. & 25 & 334 & 818 & 291 & 1782 \\
\hline Max. & 94 & 463 & 1264 & 504 & 2074 \\
\hline Avg. & 57.3 & 383.1 & 1075.4 & 398.6 & 1914.4 \\
\hline SD & 18.81 & 37.77 & 133.64 & 63.79 & 92.02 \\
\hline \multicolumn{7}{c}{ Mass of raw material per unit of fresh CLSM. }
\end{tabular}

\subsection{HMA Containing Excess Foundry Sand}

- The optimum asphalt contents ranged from 5.1 to $6.1 \%$, with the control being 5.5\%. The differences in asphalt content are minor, thus foundry sands are not expected to significantly change the optimum asphalt content of mixtures.

- Volumetrics: The percent of maximum density at $\mathrm{N}_{\text {ini }}$ for most of the HMA mixtures was at or below $89 \%$, with several being slightly above $89 \%$. The several that were above $89 \%$ were only above by a couple tenths of a percent. For the percent of maximum density at $\mathrm{N}_{\max }$, all the mixes were below 98\%. This shows that mixtures containing foundry sand can meet volumetric design specifications.

- Energy Indices: Due to the unavoidable error associated with calculating the energy indices from the densification curves, the effects of foundry sand on rutting performance could not be determined. It can only be concluded that the effect of foundry sand is within the experimental error of the measurements. 
- Moisture Damage Susceptibility: Seven of the 17 foundry sands did not alter the TSR of the mixture, one sand increased the TSR, and the remaining sands decreased the TSR. It was noticed that as the bentonite content increased, the TSR decreased. Also, sodium silicate seems to be extremely detrimental to HMA. Asphalt mixes should always be tested for their moisture susceptibility when foundry sands are used.

- Anti-Stripping Additives: Anti-stripping additives successfully increased the TSR of the control but were unable to increase the TSR of the mixtures containing foundry sands. Other methods may have to be used to increase TSR values of mixtures containing foundry sands.

- Overall Effects of Sand on Performance: Although all sands could be incorporated successfully in acceptable asphalt mix designs, based on collective review of the results, it is concluded that sands can vary significantly in their effects on performance. Effects can be either favorable or unfavorable, thus each sand needs to be treated as a unique source of aggregate.

- Asphalt Contactors: The two major obstacles to overcome are sodium silicate and bentonite. If the sand contains sodium silicate, it should be rejected. If it contains bentonite, consider washing the material. 


\section{Chapter 10 \\ FUTURE STUDIES}

The following future studies are suggested following the research reported herein:

- Continued dataset collection is needed to support the characterization of excess foundry sand. Due to improvements in the binding system and changes in the casting process, the characterization of foundry sand may vary over time. Ongoing updating of datasets would permit monitoring of the characterization trend over time. Tracking the trend would give the foundry industry an indication of the environmental impacts of their by-products.

- Survival function resolves the issue of left-censored data, which frequently exist in the environmental or chemical monitoring data. This kind of data is not restricted to the field of foundry by-products characterization. Correlating fields include characterization of water, air, and soil. All left-censored data in these fields can be analyzed by survival technique to estimate survival function, mean, and percentile and to make comparisons or do hypothesis testing.

- Excess foundry sand can be potentially reused in applications other than CLSM. It is not only reused solely in fills, embankments, road base, and barriers, but also integrated as a component for manufactured materials, such as concrete, portland cement, manufactured soils, and amendments. The byproducts of iron foundry copula slag are being considered as a material usable 
in cement kilns if iron foundry slag has similar chemistries to steel blast furnace slag.

- Only one source of aggregate was used for HMA research. A survey should be conducted to determine the areas that produce the largest amounts of foundry sand. Aggregate samples from quarries used by asphalt contractors in these areas should be taken and tested with foundry sands from these same areas.

- The indirect method for determining the apparent and bulk densities should be further developed. The direct method may give erroneous results, and the direct method should be verified with the indirect method.

- Other methods should be used to correct for decreased TSR values in HMA research. These include using other anti-stripping additives, washing the fines out of the sands, and exposing the sands to high temperatures to burn off organic material and destroy the clay structure. 


\section{Bibliography}

AFS 1105 (2000), Sieve Analysis (Particle Size Determination of Sand), Mold \& Core Test Handbook ( $3^{\text {rd }}$ edition), American Foundry Society, IL

AFS 1106 (2000), Grain Fineness Number, AFS GFN, Mold \& Core Test Handbook (3 ${ }^{\text {rd }}$ edition), American Foundry Society, IL

Ahmad, Seemab (1998), Evaluation of Procedures for Assessing Moisture Damage of Asphalt Mixes in Wisconsin, Thesis submitted for Master of Science Degree. Civil and Environmental Engineering Department. University of Wisconsin at Madison.

American Foundry Society (2000), Green Sand Additives, Second Edition, Des Plaines, Illinois.

American Foundry Society (2001), Mold \& Core Test Handbook,. Third Edition, Des Plaines, Illinois. Pp. 2.19-2.22.

AFS 1107 (2000), Grain Shape Classification, Mold \& Core Test Handbook ( $3^{\text {rd }}$ edition), American Foundry Society, IL

ASTM C 29 (1997), Standard Test Method for Bulk Density (Unit Weight) and Voids in Aggregate, ASTM International, PA

ASTM C 70 (1994), Standard Test Method for Surface Moisture in Fine Aggregate, ASTM International, PA

ASTM C 117 (1995), Standard Test Method for Materials Finer than 75- $\mu$ m (No. 200) Sieve in Mineral Aggregates by Washing, ASTM International, PA

ASTM C 125 (2000), Standard Terminology Relating to Concrete and Concrete Aggregates, ASTM International, PA

ASTM C 128 (1993), Standard Test Method for Density, Relative Density (Specific Gravity), and Absorption of Fine Aggregate, ASTM International, PA

ASTM C 192 (2002), Standard Practice for Making and Curing Concrete Test Specimens in the Laboratory, ASTM International, PA

ASTM C 403 (1999), Standard Test Method for Time of Setting of Concrete Mixtures by Penetration Resistance, ASTM International, PA 
ASTM C 566 (1997), Standard Test Method for Total Evaporable Moisture Content of Aggregate by Drying, ASTM International, PA

ASTM D 653 (1997), Standard Terminology relating to Soil, Rock, and Contained Fluids, ASTM International, PA

ASTM D 6857 (2003), Standard Test Method for Maximum Specific Gravity and Density of Bituminous Paving Mixtures Using Automatic Vacuum Sealing Method, ASTM International, PA

ASTM D 3987 (1985), Standard Test Method for Shake Extraction of Solid Waste with Water, ASTM International, PA

ASTM D 6103 (1997), Standard Test Method for Flow Consistency of Controlled Low Strength Material (CLSM), ASTM International, PA

ASTM G 57 (2001), Standard Test Method for Field Measurement of Soil Resistivity Using the Wenner Four-Electrode Method, ASTM International, PA

EPA (1991), Methods for the Determination of Metals in Environmental Samples, EPA/600/4-91/010, US Environmental Protection Agency, Washington DC

EPA SW-846 (2000), Test Methods For Evaluating Solid Waste Physical Chemical Methods, US Environmental Protection Agency, Washington DC

40 CFR Part 136 Appendix B (1986), Definition and Procedure for the Determination of the Method Detection Limit -- Revision 1.11, Appendix B to Part 136, 1986

40 CFR Part 141 (1986), National Primary Drinking Water Regulations, Part 141, Code of Federal Regulations, National Archives and Records Administration, Washington DC

40 CFR Part 143 (1986), National Secondary Drinking Water Regulations, Part 143, Code of Federal Regulations, National Archives and Records Administration, Washington DC

40 CFR Part 261 (1986), Identification and Listing of Hazardous Waste, Part 261, Code of Federal Regulations, National Archives and Records Administration, Washington DC

40 CFR Part 264 (1986), Standard for Owners and Operators of hazardous Waste Treatment, Storage, and Disposal, Part 264, Code of Federal Regulations, National Archives and Records Administration, Washington DC

Abichou, T. H, Benson, C. H., Edil, T. B. (1999), Beneficial Reuse of Foundry Byproducts, A Database Prepared for Wisconsin Cast Metals Association et al. 
ACI Committee 229 (1994), Controlled Low Strength Materials, Concrete International, v. 16, n. 7 , p. $55-64$

Adaska, W. S., and Krell, W. C. (1992), Bibliography on Controlled Low-Strength Materials (CLSM), Concrete International, v. 14, n. 10, p. 42-43

AFS (1991), Alternative Utilization of Foundry Waste Sand. Final Report (Phase I), American Foundry Society, IL

Ammen, C. W. (2000), Metal Casting, McGraw-Hill

Beeley, P. (2001), Foundry Technology, Butterworth-Heinemann

Bhat, S. and Lovell, C. (1996), Use of Coal Combustion Residue and Waste Foundry Sands in Flowable Fill, Final Report for Project No. FHWA/IN/JHRP-96/2, School of Engineering, Purdue University, IN

Bhat, S. T., and Lovell, C. W. (1996), Design of Flowable Fill: Waste Foundry Sand as a Fine Aggregate, Paper No. 961066, Transportation Research Board, $75^{\text {th }}$ Annual Meeting, Washington, DC

Bhat, S. and Lovell, C. (1997), Flowable Fill Using Waste Foundry Sand: A Substitute for Compacted or Stabilized Soil, ASTM Special Technical Publication, v. 1275, Proceedings of the 1997 Symposium on Testing Soil Mixed with Waste or Recycled Materials, Jan 16-17 1997, New Orleans, LA

Blistan D. D. (1974), Transmission Scanning Electron Microscopy of Green Foundry Sands: A Feasibility Study, A Master Thesis of The Pennsylvania State University, PA

Bol'shakov, A. A., and Winkler E. S. (2001), Environmental Stability of Foundry ByProducts and Materials Made with Them, Report Presented to Chelsea Center for Recycling and Economic Development, MA

Boyle, W. C. (1984), AFS Research Program on Foundry Solid Wastes at the University of Wisconsin, Foundry Solid Waste Disposal Conference, The Pennsylvania State University, PA

Bradford, G. R., Chang, A. C., Page, A. L., Bakhtar1, D., Frampton, J. A., and Wright, H. (1996), Background Concentrations of Trace and Major Elements in California Soils, Kearney Foundation Special Report, Kearney Foundation of Soil Science, Division of Agriculture and Natural Resources, University of California, CA

Braham, A. (2002), The Use of Blended Recycled Foundry Sand In Hot Mix Asphalt, Thesis submitted for Masters of Science, Civil and Environmental Engineering Department, University of Wisconsin at Madison. 
Burns, T. A. (1986), Foundryman's Handbook, $9^{\text {th }}$ Edition, Pergamon Press

Carey, P. (1994), Sand/Binders/Sand Preparation/Coremaking, Foundry Management \& Technology, v. 122, n. 1, p. D3-D12.

Carey, P. R., and Sturtz, G. (1995), Sand Binder Systems Part IV Urethane Binders, Foundry Management \& Technology, v. 123, n. 6, p. 25-29

Carey, P. R. and Sturtz G. (1996), Sand Binder Systems Part XIII-Silicate CO2, Silicate No-Bake, CO2 Cured Alkaline Phenolic, Foundry Management \& Technology, v. 124 , n. 4 , p. $74-82$

Carey, P. R. (2002), Sand/Binders/Sand Preparation \& Coremaking, Foundry Management \& Technology, v. 130, n. 1, p. 39-52

Cohen, A. C. (1959), Simplified Estimators for the Normal Distribution When Samples Are Single Censored or Truncated, Technometrics, n.1, p.217-237

Cohen, A. C. (1961), Tables for Maximum Likelihood Estimates: Singly Truncated and Singly Censored Samples, Technometrics, n.3, p.535-541

Conover, W. J. (1999), Practical Nonparametric Statistics, 3rd Edition, John Wiley \$ Sons, Inc.

Delage, K.P., Bahia, H.U., Widjaja, M., Romero, P., Harman, T.P. (2001), Performance Testing of Hot Mix Asphalt Produced with Recycled Foundry Sand, Transportation Research Board, $80^{\text {th }}$ Annual Meeting, Paper No. 01-2844.

DOE (1999), Energy and Environmental Profile of the Metalcasting Industry. U.S. Department of Energy. Office of Industrial Technologies, Washington, DC, Report (1999).

Dunkelberger, J., and Regan, R. (1997), Evaluation of Spent Foundry Sand to Growing Mix Amendments: Potential Beneficial Use Option, Proceedings of AFS 101 ${ }^{\text {st }}$ Casting Congress, Seattle, WA..

DynCorp (2001), Literature Search Regarding Detection and Quantitation Limit Approaches, DynCorp

Ekey, D.C. (1958), Introduction to Foundry Technology, McGraw-Hill

EPA (1991), Site Characterization for Surface Remediation, EPA/600/4-91/026/, US Environmental Protection Agency, Office of Research and Development, Washington DC 
EPA (1995), Applicability of the Toxicity Characteristic Leaching Procedure to Mineral Processing Wastes, Office of Solid Waste, US Environmental Protection Agency, Washington DC

Flinn, Richard A. (1963), Fundamentals of Metal Casting, Addison-wesley Publishing Company, Inc.

Foundry Management \& Technology (1996), Environment/Health and Safety, v. 124, n. 1, p. J2-J5

Gessner, F., Schmitt, C.C., Neuman, M.G. (1994), Time-Dependent Spectrophotometric Study of the Interaction of Basic Dyes with Clays. 1. Methylene Blue and Neutral Red on Montmorrillonite and Hectorite". Langmuir. Vol. 10. pp. 3779-3753.

Gibbons, R. D., and Coleman, D. E. (2001), Statistical Methods for Detection and Qualification of Environmental Contamination, John Wiely \& Sons, Inc.

Gilbert, R. O. (1987), Statistical Methods for Environmental Pollution Monitoring, Van Nostrand Reinhold COmpany, NY

Gilliom, R. J.; and Helsel, D. R. (1986), Estimation of Distributional parameters for Censored Trace Level Water Quality Data (Estimation Techniques), Water Resources Research, v. 22, p. 135-146

Gleit, A. (1985), Estimation for Small Normal Data Sets with Detection Limits, Environmental Science and Technology, v. 19, n. 12, p. 1201-1206

Granlund, M. (1984), Reclamation of Foundry Waste Sand, Foundry Solid Waste Disposal Conference, The Pennsylvania State University, PA

Ham, R. K. (1984), Recent University of Wisconsin Studies on the Leaching Characteristics of Ferrous Foundry Wastes, Foundry Solid Waste Disposal Conference, The Pennsylvania State University, PA

Ham, R. K., and Boyle, W. C. (1981), Leachability of Foundry Process Solid Wastes, Journal of the Environmental Engineering Division, v. 107, n. 1, p. 155-170

Ham, R. K., Boyle, W. C., Engroff, E. C., and Fero, R. L. (1993), Organic Compounds in Ferrous Foundry Process Waste Leachates, Journal of Environmental Engineering, v. 119, n. 1, p. 34-55

Hang, Pham Thi, Brindley, G.W. (1970), Methylene Blue absorption by Clay Minerals. Determination of Surface Areas and Cation Exchange Capacities, Clay and Clay Minerals. Vol. 18. pp. 203-212. 
Hashimoto, L. K.; and Trussell, R. R. (1983), Evaluating Water Quality Data Near the Detection Limit, Proceedings of the Annual Conference of the American Water Association, p. 1021

Heine, R. W. (1967), Principles of Metal Casting, McGraw-Hill Book Company

Hesel, D. (1990), Less Than Obvious, Environmental Science and Technology, v. 24, n. 12 , p. 1766-1774

Hesel, D. R., and Hirsch R. M. (1992), Statistical Methods in Water Resources, Studies in Environmental Science 49, Elsevier Science Publishers B.V.

Hesel, D., and Cohn, T. (1988), Estimation of Descriptive Statistics for Multiply Censored Water Quality Data, Water Resources Research, v. 24, p. 1997-2004

Hiltunen,. D., Roque, R., and Wu. X. (1992), Beneficial Reuse of Foundry Residual Wastes in Pennsylvania: Physical Characterization of Materials, Environmental Resources Research Institute, The Pennsylvania State University, PA,

InstroTek (2001), Gravity Suite Software. InstroTek®, Inc. Raleigh, NC. 2001.

InstroTek (2002), Determination of Percent Absorption and Specific Gravity of Coarse and Fine Aggregates Using the AggPlusTM System”. Revision 3. InstroTek®, Inc. Raleigh, NC. March 2002

Iowa DOT (2004), Standard Specifications with GS-01006 Revisions, Section 2506 Flowable Mortar, State Iowa Department of Transportation

Ishai, I., Tons, E. (1977), Concept and Test Method for a Unified Characterization of the Geometric Irregularity of Aggregate Particles, Journal of Testing and Evaluation. Journal of Testing and Evaluation, JTEVA, Vol. 5, No. 1. pp. 3-15.

Javed, S. and Lovell, C. W. (1994), Use of Foundry Sand in Highway Construction, Joint Highway Research Project No. C-36-50N, Department of Civil Engineering, Purdue University, IN

Javed S., and Lovell C. W. (1994B), Use of Waste Foundry Sand in Civil Engineering, Transportation Research Record, No. 1486, p. 109-113, Transportation Research Board, Washington DC

Ji, S., Wan, L., and Fan, Z. (2001), The Toxic Compounds and Leaching Characteristics of Spent Foundry Sands, Water, Air, and Soil Pollution, v. 132, n. 3, p. 347-364

Johnson, C. K. (1981), Phenols in Foundry Waste Sand, Modern Casting, American Foundry Society, IL, 
Kaplan, E. L., and Meier, P. (1958), Nonparametric Estimation From Incomplete Observations, Journal of the American Statistical Association, v. 53, n. 282, p. $457-481$

Kauffman, P., Regan, R.W., and Voigt, R.C. (1996), Survey of State Regulations for Beneficial Use of Foundry Residuals, AFS Transactions, v. 104, American Foundry Society, IL

Kauffman, P. J., and Voigt, R. C. (1999), A Comparison of the Normal and Lognormal Distributions to Analyze TCLP Results for Beneficial Use Permits, AFS Transactions, v. 107, American Foundry Society, IL

Kendall, D. S. (2003), Toxicity Characteristic Leaching Procedure and Iron Treatment of Brass Foundry Waste, Environmental Science and Technology, v. 37, n. 2, p. 367371

Khosla, N.P., Birdsall, B.G., and Kawaguchi, S. (2000), Evaluation of Moisture Susceptibility of Asphalt Mixtures, Transportation Research Record 1728. Paper No. 00-1070 pp. 43-51.

Kimmell, T. A. (1999), Original Purpose of the Toxicity Characteristic Leaching Procedure (TCLP), US EPA 1999 Public Meeting Development of New Waste Leaching Procedures, US Environmental Protection Agency, Washington DC

Kroll, C.N., and Stedinger, J.R. (1996), Estimation of Moments and Quantiles Using Censored data, Water Resources Research, v. 32, n. 4, p. 1005-1012

Larsen, R. L. (1990), Sound Uses of CLSM in the Environment, Concrete International, v. 12 , n. 7 , p. 26-29.

Letey, J., C. Roberts, M. P., and Vasek, C. (1986), An Agricultural Dilemma: Drainage Water and Toxics Disposal in the San Joaquin Valley. Division of Agriculture and Natural Resources Publications, Special Publication 3319, University of California, CA

Lovejoy, M., Ham, R., Traeger P., Hippe J., and Boylc W. (1996), Evaluation of Selected Foundry Wastes for Highway Construction, $19^{\text {th }}$ International Madison Waste Conderence, WA

Mackay, D. and S. Paterson (1984), Spatial concentration distributions, Environmental Science and Technology, v. 18, n. 7, p. 207a-214b

Mast, G. (1997), Field Demonstration of Highway Embankment Using Waste Foundry Sand, A Master Thesis From Purdue University, IN 
Mikel, D. (2001), Guidance for EPA Supersites Investigators, US Environmental Protection Agency, Quality Assurance Working Group

Miller, E., Khatri, A., Winter, M., Bahia, H.U., Benson, C. (1998), Utilization of Foundry Sand in Hot Mix Asphalt, Final Report, UW-Madison, Submitted to The University-Industry Relations.

MNR (1992), Mineral Aggregate Conservation - Reuse and Recycling, Report prepared by John Emery Geotechnical Engineering Limited for Aggregate and Petroleum Resources Section, Ontario Ministry of Natural Resources, Queen's Printer for Ontario

MOEE. (1993), Spent Foundry Sand - Alternative Uses Study, Report prepared by John Emery Geotechnical Engineering Limited for Ontario Ministry of the Environment and Energy and the Canadian Foundry Association, Queen's Printer for Ontario

Naik, T. R., Ramme, B. W., and Kolbeck, H. J. (1990), Filling Abandoned Underground Facilities with CLSM Fly Ash Slurry, Concrete International, v. 12, n. 7, p. 19-25.

Naik, T. R., and Singh, S. S. (1997), Flowable Slurry Containing Foundry Sands, Journal of Materials in Civil Engineering

Naik, T., Singh, S. (1997), Permeability of Flowable Slurry Materials Containing Foundry Sand and Fly Ash, Journal of Geotechnical and Geoenvironmental Engineering, n. 5, p. 446-452

ODOT (1997), 1997 Construction and Material Specification Ohio, Item 613: Low Strength Mortar Fill, State of Ohio Department of Transportation

Orkas, J. (2002), Re-use of Foundry Sand in Scandinavia, $12^{\text {th }}$ AFS International Environmental, Health \& Safety Conference, Lake Buena Vista, FL, p. 433-440

Ott, W. R. (1990), A Physical Explanation of the Lognormality of Pollutant Concentrations, Journal of The Air Waste Management Association, v. 40, p. 1378-1383

PCA (1990), Cementitious Grouts and Grouting, Portland Cement Association, IL

PennDOT (1995), Special Provision Item 9220-Flowable Backfill, State Pennsylvania Department of Transportation

Persson, T., and Rootzen, H. (1977), Simple and Highly Efficient Estimators for a Type I Censored Normal Sample, Biometrika, v. 64, p. 123-128,

Pike, D.C. (1992), Methodologies For Assessing the Variability of Fines in Sands Used for Concretes and Mortars, Thesis submitted for the degree of Doctor of 
Philosophy, Postgraduate Research Institute for Sedimentology, University of Reading.

Porter, P. S., Ward, R. C., and Bell, H. F. (1988), The Detection Limit, Environmental Science and Technology, v. 24, p. 856-861

Regan, R.W., Heaney, M., Dunkelberger, J.D. (1997), Beneficial Use Options: Customer's Perspective, Modern Casting, p. 45-47, August, 1997

Regan, R., Paletski, W., and Massell R. (1993), Environmental Issues: Beneficial Use of Spent Foundry Sand From Multiple Sources, $48^{\text {th }}$ Purdue Industrial Waste Conference Proceeding, IN, pp. 91-100

Regan, R.W., Tikalsky, P.J., and Voigt, R.C. (1998), Beneficial Use of Fine Foundry Aggregate: Three Promising Options, AFS Transactions, American Foundry Society, IL

Roberts, F.L., Kandhal, P.S., Brown, E.R., Lee, D.Y., Kennedy, T.W. (1996), Hot Mix Asphalt Materials, Mixture Design and Construction,. Second Edition. National Center for Asphalt Technology. NAPA Education Foundation, Lanham, Maryland.

Regan, R.W. and Voigt, R.C. (1996), Working with the Regulators for the Beneficial Use of Foundry Residuals, Proceedings of the $28^{\text {th }}$ Mid-Atlantic Industrial Waste Conference, Buffalo, NY

Regan, R., Voigt, R., Paletski, W., and Massell, R. (1994), Chemical Characterizations of Spent Molding Sands: Environmental Issues, AFS Transactions, v. 102, p. 749756, American Foundry Society, IL

Roberge, P. R. (1999), Handbook of Corrosion Engineering, McGraw-Hill Science Advisory Board (1995), Science Advisory Board (SAB) Review of Issues Related to the Regulation of Arsenic in Drinking Water, Science Advisory Board

Singh, A. K., Singh, A., and Engelhardt, M. (1997) The Lognormal Distribution in Environmental Applications, EPA Technology Support Center Issue, EPA/600/R97/006, Office of Research and Development, US Environmental Protection Agency, Washington DC

Stenphens, M. A. (1974), EDF Statistics for Goodness of Fit and Some Comparisons, Journal of the American Statistical Association, v. 69, n. 347, p. 730-737

Sylvia, G. J. (1972), Cast Metals Technology, Addison-Wesley Publishing Company 
Tikalsky, P. J. (2000), Development of Technical Data to Validate Performance of Foundry Byproducts in Hot-mix Asphalt and Controlled Low-strength Material, Research Proposal Prepared in Response to Solicitation No. DE-PS07-00ID13862

US Census Bureau. (2002). "Iron and Steel Castings: 2001”. Current Industrial Reports, MA331A(01)-1. US Census Bureau, Washington, DC

US Census Bureau. (2002). "Nonferrous Castings: 2001”. Current Industrial Reports, MA331E. US Census Bureau, Washington, DC

Van Tassel, E. L. (1999), Integration of Recycled and Co-product Materials in Controlled Low-Strength Material, A Master Thesis from The Pennsylvania State University, PA

Webster, P. D. (1980) Fundamentals of Foundry Technology, Portcullis Press Ltd

Wilson, J. (1999), Flowable Fill as Backfill for Bridge Abutments, Final Report to Wisconsin Department of Transportation, Report \# WI-16-99, 1999

Winkler, E. S. (2000), Characterization of Foundry Sand Waste, Technical Report \#31, Chelsea Center for Recycling and Economic Development, University of Massachusetts, MA

Winkler, E., Kosanovic B., Genovese, T., and Roth, I. (1999), A Survey of Foundry Participation in the Massachusetts Beneficial Use Determination Process, The Center for Energy Efficiency and Renewable Energy, University of Massachusetts Amherst, MA

Worrall, W.E. (1968), Clays - Their Nature, Origin and General Properties, London. Maclaren and Sons. 


\section{Appendix A}

\section{Glossary of Terms}

\section{A.1 Civil Engineering}

Absorption - the process by which a liquid is drawn into and tends to fill permeable pores in a porous solid body; also, the increase in mass of a porous solid body resulting from the penetration of a liquid into its permeable pores. [ASTM C 125 2000].

AFS Grain Fineness Number (AFS GFN) - an estimate of the average sieve size of a sand sample [AFS 1106 2000].

Aggregate - granular material, such as sand, gravel, crushed stone, or iron blast-furnace slag, used with a cementing medium to form hydraulic-cement concrete or mortar [ASTM C 125 2000].

Attenuation - reduction of amplitude with time or distance [ASTM D 653 1997].

ASTM D 3987, Standard Test Method for Shake Extraction of Solid Waste with Water a leaching protocol designed for leaching of solid waste to obtain an aqueous solution to be used to determine the materials leached under the specified testing conditions [ASTM D 3987 1985]. 
Bleeding - the autogenous flow of mixing water within, or its emergence from, newly placed concrete or mortar caused by the settlement of the solid materials within the mass, also called water gain [ASTM C 125 2000].

Bulk density (replaces the deprecated term "unit weight") - the mass of a unit volume of bulk aggregate material (the unit volume includes the volume of the individual particles and the volume of the voids between the particles) [ASTM C 1252000 ].

Bulk specific gravity - the ratio of the mass of a volume of a material (including the permeable and impermeable voids in the material, but excluding the voids between particles of the material) at a stated temperature to the mass of an equal volume of distilled water at a stated temperature [ASTM C 125 2000].

Bulk specific gravity (saturated surface dry, SSD) - the ratio of the mass of a volume of a material including the mass of water within the pores in the material (but excluding the voids between particles) at a stated temperature, to the mass of an equal volume of distilled water at a stated temperature [ASTM C 125 2000].

Cementitious material (hydraulic) - an inorganic material or a mixture of inorganic materials that sets and develops strength through chemical reaction with water by formation of hydrates, and is capable of doing so under water [ASTM C 125 2000]. 
Compressive strength - the load per unit area at which an unconfined cylindrical specimen of soil or rock will fail in a simple compression test [ASTM D 653 1997].

Consistency - the relative mobility or ability to flow of fresh concrete, mortar, or grout [ASTM C 125 2000].

Controlled low-strength material (CLSM) - a mixture of soil or aggregates, cementitious material, fly ash, water and sometimes chemical admixtures, that hardens into a material with a higher strength that the soil, but less than $8,400 \mathrm{kPa}$ (1200 psi); used as a replacement for compacted backfill, CLSM can be placed as a slurry, a mortar, or a compacted material and typically has strength of 350 to $700 \mathrm{kPa}$ (50 to $100 \mathrm{psi}$ ) for most applications [ASTM D 6103 1997].

Curing - action taken to maintain moisture and temperature conditions in a freshly placed cementitious mixture to allow hydraulic cement hydration and (if applicable) pozzolanic reactions to occur so that the potential properties of the mixture may develop [ASTM C 125 2000].

Fine aggregate - aggregate passing the $3 / 8$-in $(9.5-\mathrm{mm})$ sieve and almost entirely passing the 4.75-mm (No. 4) sieve and predominantly retained on the $75-\mu$ m (No. 200) sieve; or the portion of an aggregate passing the $4.75-\mathrm{mm}$ (No. 4) sieve and retained on the 75- $\mu$ m (No. 200) sieve [ASTM C 125 2000]. 
Flow consistency (refer to Consistency in this section)

Fly ash - the finely divided residue that results from the combustion of ground or powdered coal and that is transported by flue gases from the combustion zone to the particle removal system [ASTM C 125 2000].

Gradation (grain-size distribution) - the proportions by mass of a soil or fragmented rock distributed in specified particle-size ranges [ASTM D 6103 1997].

Hydraulic conductivity - the volume of water at the existing kinematics viscosity that will move in a unit time under a unit hydraulic gradient through a unit area measured at right angles to the direction of flow [ASTM D 6103 1997].

Initial set - a degree of stiffening of a grout mixture generally stated as an empirical value indicating the time in hours and minutes that is required for a mixture to stiffen sufficiently to resist the penetration of a weighted test needle [ASTM D 6103 1997].

Resource Conservation and Recovery Act (RCRA) - legislation enacted in 1976 by Congress with the intent to "protect human health and the environment from the improper handling of solid waste and to encourage the conservation of natural resources."

Resistivity - the relative ability of a medium to carry electrical currents [ASTM G 57 2001]. 
Sand - fine aggregate resulting from natural disintegration and abrasion of rock or processing of completely friable sandstone [ASTM C 125 2000].

Segregation - the unintentional separation of the constituents of concrete or particles of an aggregate, causing a lack of uniformity in their distribution [ASTM C 125 2000].

Setting - the process, due to chemical reactions, occurring after the addition of mixing water, that results in a gradual development of rigidity of a cementitious mixture [ASTM C 125 2000].

Setting settlement / shrinkage - a reduction in volume of grout prior to the final set of cement caused by bleeding, by the decrease in volume due to the chemical combination of water with cement, and by syneresis [ASTM D 6103 1997].

Synthetic Penetration Leaching Protocol (SPLP) - a leaching protocol designed to determine the mobility of both organic and inorganic analytes present in liquids, soils, and wastes [EPA SW-846 2000].

Toxicity Characteristics Leaching Procedure (TCLP) - a leaching protocol designed to determine the mobility of both organic and inorganic analytes present in liquid, solid, and multiphasic wastes [EPA SW-846 2000]. 
Time of setting - the elapsed time from the addition of mixing water to cementitious mixture until the mixture reaches a specified degree of rigidity as measured by specific procedure [ASTM C 125 2000].

Void - the space between particles in an aggregate mass not occupied by solid mineral matter [ASTM C 29 1997].

Water-cement ratio, $w / c$ - the ratio of the mass of water exclusive only of that absorbed by the aggregates, to the mass of portland cement in concrete, mortar, or grout, stated as a decimal [ASTM C 125 2000].

Water-cementitious material ratio, $w / c m$ - the ratio of the mass of water, exclusive only of that absorbed by the aggregates, to the mass of cementitious material (hydraulic) in concrete, mortar, or grout, stated as a decimal [ASTM C 125 2000].

\section{A.2 Statistics}

Alternative hypothesis, $\mathrm{H}_{1}$ - a statement of what a statistical hypothesis test is set up to establish.

Conditional probability, $P(\mathbf{A} \mid \mathbf{B})$ - the probability that event A will occur given that event B has already occurred. 
Confidence interval - an estimated range of values that is likely to include an unknown population parameter, the estimated range being calculated from a given set of sample data.

Confidence level - the probability value (1- $\alpha)$ associated with a confidence interval.

Correlation coefficient - a number between -1 and 1 that measures the degree to which two variables are linearly related.

Cumulative distribution function - a function giving the probability that the random variable $X$ (discrete and continuous) is less than or equal to $x$.

Factor - a controlled independent variable for an experiment whose levels (treatments) are set by the experimenter.

Hypothesis test - used to test the two hypotheses and to accept one of them with significance level.

Kolmogorov-Smirnov test - used to test whether or not a single sample of data is consistent with a specified distribution function; or to test whether or not two samples of data come from the same distribution. 
Nonparametric test - used in place of its parametric counterparts when certain assumptions about the underlying population are questionable.

Null hypothesis, $\mathrm{H}_{0}$ - represents a theory that has been put forward, either because it is believed to be true or because it is to be used as a basis for argument, but has not been proven.

One-sided test - a statistical hypothesis test in which the values for which null hypothesis is rejected are located entirely in one tail of the probability distribution.

Outlier - an observation in a data set that is far removed in value from the others in the data set, unusually large or small compared to the others.

$P$-Value - the probability of wrongly rejecting the null hypothesis if it is in fact true in a statistical hypothesis test.

Parameter - a value, usually unknown (and which therefore has to be estimated), used to represent a certain population characteristic.

Percentile - a value that divides a sample of data into one hundred groups containing (as far as possible) equal numbers of observations; for example, $30 \%$ of the data values lie below the 30th percentile. 
Population - any entire collection of people, animals, plants or things from which data may be collected. It is the entire group which is of interest and about which conclusions are to be drawn.

Probability - a quantitative description of the likely occurrence of a particular event.

Probability density function - a function that can be integrated to obtain the probability that a continuous random variable takes a value in a given interval.

Probability distribution - a list of probabilities associated with each of its possible values for a discrete random variable. It is also sometimes called the probability function or the probability mass function.

Quantile - a set of "cut points" that divide a sample of data into groups containing (as far as possible) equal numbers of observations.

Sample - a group of units selected from a larger group (the population); by studying the sample it is hoped that valid conclusions can be drawn about the larger group.

Sample mean - an estimator available for estimating the population mean. 
Significance level - a fixed probability of wrongly rejecting the null hypothesis $\mathrm{H}_{0}$, if it is in fact true in a statistical hypothesis test; the significance level is usually denoted by $\alpha$ and chosen to be 0.05 (or equivalently, 5\%).

Skewness - asymmetry in the distribution of the sample data values; values on one side of the distribution tend to be further from the "middle" than values on the other side.

Standard deviation - a measure of the spread or dispersion of a set of data.

Survival function - the probability that a variable $X$ takes on a value greater than a number $x$.

Test statistic - a quantity calculated from the sample of data. Its value is used to decide whether or not the null hypothesis should be rejected in hypothesis test.

Treatment - a condition or action administered to experimental units to compare its significance or effect; for example, a cornfield is divided into four plots, and each plot is "treated" with a different fertilizer to see which produces the most corn.

Two-sided test - a statistical hypothesis test in which the values for which null hypothesis is rejected are located in both tails of the probability distribution. 


\section{Appendix B}

\section{List of Testing Specifications (CLSM)}

AFS 1105 Sieve Analysis (Particle Size Determination of Sand)

AFS 1106 Grain Fineness Number, AFS GFN, Calculation

AFS 1107 Grain Shape Classification

ASTM C 29 Standard Test Method for Bulk Density (Unit Weight) and Voids in Aggregate

ASTM C 117 Standard Test Method for Materials Finer than 75- $\mu$ m (No. 200) Sieve in Mineral Aggregates by Washing

ASTM C 128 Standard Test Method for Density, Relative Density (Specific Gravity), and Absorption of Fine Aggregate

ASTM C 192 Standard Practice for Making and Curing Concrete Test Specimens in the Laboratory

ASTM C 232 Standard Test Methods for Bleeding of Concrete

ASTM C 403 Standard Test Method for Time of Setting of Concrete Mixtures by Penetration Resistance

ASTM C 566 Standard Test Method for Total Evaporable Moisture Content of Aggregate by Drying

ASTM C 567 Standard Test Method for Determining Density of Structural Lightweight Concrete

ASTM C 827 Standard Test method for Change in Height at Early-ages of Cylindrical Specimens from Cementitious Mixtures

ASTM D 2434Standard Test Method for Permeability of Granular Soils (Constant Head)

ASTM D 4832 Standard Test Method for Preparation and Testing of Controlled LowStrength Material (CLSM) Test Cylinders

ASTM D 5971 Standard Practice for Sampling Freshly Mixed Controlled Low-Strength Material 
ASTM D 6023 Standard Test Method for Unit Weight, Yield, Cement Content and Air Content (Gravimetric) of Controlled Low- Strength Material (CLSM)

ASTM D 6103 Standard Test Method for Flow Consistency of Controlled Low- Strength Material (CLSM)

ASTM G57 Standard Test Method for Field Measurement of Soil Resistivity Using the Wenner Four-Electrode Method 



\section{Appendix C \\ Solid Waste Hazardous Threshold, Drinking Water and Groundwater Standard}

\section{C.1 Metallic Elements}

The thresholds regarding metallic elements of hazardous materials [EPA SW-846 2000, 40 CFR Part 261 1986], standard of drinking water [40 CFR Part 141 1986, 40 CFR Part 143 1986], and standard of groundwater [40 CFR Part 264 1986] are presented in Table C-1.

\section{C.2 Organic Compounds}

The thresholds regarding organic compounds of hazardous materials [EPA SW846 2000, 40 CFR Part 261 1986], standard of drinking water [40 CFR Part 141 1986, 40 CFR Part 143 1986], and standard of groundwater [40 CFR Part 264 1986] are presented in Table C-2 and Table C-3. 
Table C-1: Threshold for inorganic elements

\begin{tabular}{|c|c|c|c|c|}
\hline \multirow{2}{*}{ Measuring Method } & \multicolumn{2}{|c|}{ Hazardous Material } & Drinking Water & Ground Water \\
\hline & \multicolumn{2}{|c|}{ TCLP/EPA SW-846 } & Total Analysis & Total Analysis \\
\hline & \multirow{2}{*}{\multicolumn{2}{|c|}{--- }} & Unit: $\mathrm{mg} / \mathrm{L}$ & \\
\hline Aluminum & & & $0.05-0.2$ & --- \\
\hline Antimony & \multirow{8}{*}{ 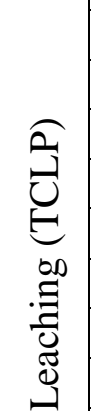 } & --- & 0.006 & --- \\
\hline Arsenic & & 5.0 & 0.01 & 0.05 \\
\hline Barium & & 100.0 & 2.0 & 1.0 \\
\hline Beryllium & & --- & 0.004 & --- \\
\hline Cadmium & & 1.0 & 0.005 & 0.01 \\
\hline Chromium & & 5.0 & 0.1 & 0.05 \\
\hline Copper & & --- & 1.0 & --- \\
\hline Iron & & --- & 0.3 & --- \\
\hline Manganese & \multirow{7}{*}{ 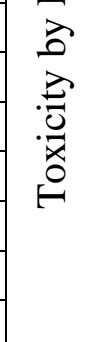 } & --- & 0.05 & --- \\
\hline Mercury & & 0.2 & 0.002 & 0.002 \\
\hline Lead & & 5.0 & 0.015 & 0.05 \\
\hline Selenium & & 1.0 & 0.05 & 0.01 \\
\hline Silver & & 5.0 & 0.1 & 0.05 \\
\hline Thallium & & --- & 0.002 & --- \\
\hline Zinc & & --- & 5 & --- \\
\hline Reactive Cyanide & & 250 & --- & --- \\
\hline Reactive Sulfide & & 500 & --- & --- \\
\hline Ignitability & & $60^{\circ} \mathrm{C}$ & --- & --- \\
\hline $\mathrm{pH}$ for Corrosivity & & $2 \sim 12.5$ & --- & --- \\
\hline Free Cyanide & & --- & 0.2 & --- \\
\hline Fluoride & & --- & 4.0 & --- \\
\hline Nitrate & & --- & 10 & --- \\
\hline Nitrite & & --- & 1 & --- \\
\hline
\end{tabular}


Table C-2: Threshold for organic compounds

\begin{tabular}{|c|c|c|c|}
\hline & $\begin{array}{l}\text { Hazardous } \\
\text { Material }\end{array}$ & $\begin{array}{l}\text { Drinking } \\
\text { Water }\end{array}$ & $\begin{array}{l}\text { Ground } \\
\text { Water }\end{array}$ \\
\hline \multirow[t]{2}{*}{ Measuring Method } & $\begin{array}{l}\text { TCLP/EPA SW- } \\
846\end{array}$ & $\begin{array}{l}\text { Total } \\
\text { Analysis }\end{array}$ & $\begin{array}{l}\text { Total } \\
\text { Analysis }\end{array}$ \\
\hline & \multicolumn{3}{|c|}{ Unit: ug/L } \\
\hline Alachlor & --- & 2 & --- \\
\hline Atrazine & --- & 3 & --- \\
\hline Benzene & 500 & 5 & --- \\
\hline Benzo(a)pyrene & --- & 0.2 & --- \\
\hline Carbofuran & --- & 40 & --- \\
\hline Carbon tetrachloride & 500 & 5 & --- \\
\hline Chlordane & 30 & 2 & --- \\
\hline Chlorobenzene & 100000 & 100 & --- \\
\hline Chloroform & 6000 & --- & --- \\
\hline o-Cresol & 200000 & --- & --- \\
\hline m-Cresol & 200000 & --- & --- \\
\hline p-Cresol & 200000 & --- & --- \\
\hline Cresol & 200000 & --- & --- \\
\hline 2,4-D acid & 10000 & 70 & 100 \\
\hline Dalapon & --- & 200 & --- \\
\hline $\begin{array}{l}\text { 1,2-Dibromo-3-chloropropane } \\
\text { (DBCP) }\end{array}$ & --- & 0.2 & --- \\
\hline o-Dichlorobenzene & & 600 & --- \\
\hline p-Dichlorobenzene & 7500 & 75 & --- \\
\hline 1,2-Dichloroethane & 500 & 5 & --- \\
\hline 1,1-Dichloroethylene & 700 & 7 & --- \\
\hline cis-1, 2-Dichloroethylene & --- & 70 & --- \\
\hline trans-1,2-Dichloroethylene & --- & 100 & --- \\
\hline Dichloromethane & --- & 5 & --- \\
\hline 1,2-Dichloropropane & --- & 5 & --- \\
\hline 2,4-Dinitrotoluene & 130 & & --- \\
\hline Di(2-ethylhexyl)adipate & --- & 400 & --- \\
\hline Di(2-ethylhexyl)phthalate & --- & 6 & --- \\
\hline Dinoseb & --- & 7 & --- \\
\hline Dioxin $(2,3,7,8$-TCDD) & --- & 0.00003 & --- \\
\hline Diquat & --- & 20 & --- \\
\hline
\end{tabular}


Table C-3: Threshold for organic compounds (Cont.)

\begin{tabular}{|c|c|c|c|}
\hline & Hazardous Material & Drinking Water & Ground Water \\
\hline Measuring Method & TCLP/EPA SW-846 & Total Analysis & Total Analysis \\
\hline & \multicolumn{3}{|c|}{ Unit: ug/L } \\
\hline Endothall & --- & 100 & --- \\
\hline Endrin & 20 & 2 & 0.2 \\
\hline Ethylbenzene & --- & 700 & --- \\
\hline Ethylene dibromide & --- & 0.05 & --- \\
\hline Glyphosate & --- & 700 & --- \\
\hline Heptachlor & 8 & 0.4 & --- \\
\hline Heptachlor epoxide & --- & 0.2 & --- \\
\hline Hexachlorobenzene & 130 & 1 & --- \\
\hline Hexachlorobutadiene & 500 & --- & --- \\
\hline Hexachloroethane & 3000 & --- & --- \\
\hline Hexachloropentadiene & & 50 & --- \\
\hline Lindane & 400 & 0.2 & 4 \\
\hline Methoxychlor & 10000 & 40 & 100 \\
\hline Methyl ethyl ketone & 200000 & --- & --- \\
\hline Nitrobenzene & 2000 & --- & --- \\
\hline Oxamyl (Vydate) & --- & 200 & --- \\
\hline $\begin{array}{l}\text { Polychlorinated biphenyls } \\
\text { (PCBs) }\end{array}$ & --- & 0.5 & --- \\
\hline Pentrachlorophenol & 100000 & 1 & --- \\
\hline Picloram & & 500 & --- \\
\hline Pyridine & 5000 & --- & --- \\
\hline Simazine & --- & 4 & --- \\
\hline Styrene & --- & 100 & --- \\
\hline Tetrachloroethylene & 700 & 5 & --- \\
\hline Toluene & & 1000 & --- \\
\hline Toxaphene & 500 & 3 & 5 \\
\hline 2,4,5-TP (Silvex) & 1000 & 50 & 10 \\
\hline 1,2,4-Trichlorobenzene & --- & 70 & --- \\
\hline 1,1,1-Trichloroethane & --- & 200 & --- \\
\hline 1,1,2-Trichloroethane & --- & 5 & --- \\
\hline Trichloroethylene & 500 & 5 & --- \\
\hline 2, 4, 5-Trichlorophenol & 400000 & --- & --- \\
\hline 2, 4, 6-Trichlorophenol & 2000 & --- & --- \\
\hline 2, 4, 5-TP (Silvex) & 1000 & --- & --- \\
\hline Vinyl chloride & 200 & 2 & --- \\
\hline Xylenes, total & --- & 10000 & --- \\
\hline
\end{tabular}




\section{Appendix D}

\section{Bulk Analysis of Excess Foundry Sand}

In this appendix, the composition of excess foundry sand is shown element by element, including 24 metallic elements, 17 general chemical parameters, and 30 organic compounds at the trace level. The datasets represented in this appendix are typical of those in the foundry industry. The matrix of the analysis is essentially the waste stream, not the leachate from the waste stream. The leachate-based composition is presented in Appendix E.

The notes to individual charts are defined as follows:

- The number following "\# Tests" represents total observations for sand stream;

- The number following “\# BDL" represents total censored observations for sand stream;

- The solid diamond legend “..”” represents an uncensored observation;

- The hollow square legend "o" represents a censored observation;

- The horizontal dashed line represents a hazardous threshold. 


\section{D.1 Trace Metallic Elements}

The following series of 24 charts, grouped into Figure D-1, presents the bulk analysis distribution for 24 trace metallic elements in individual waste stream.
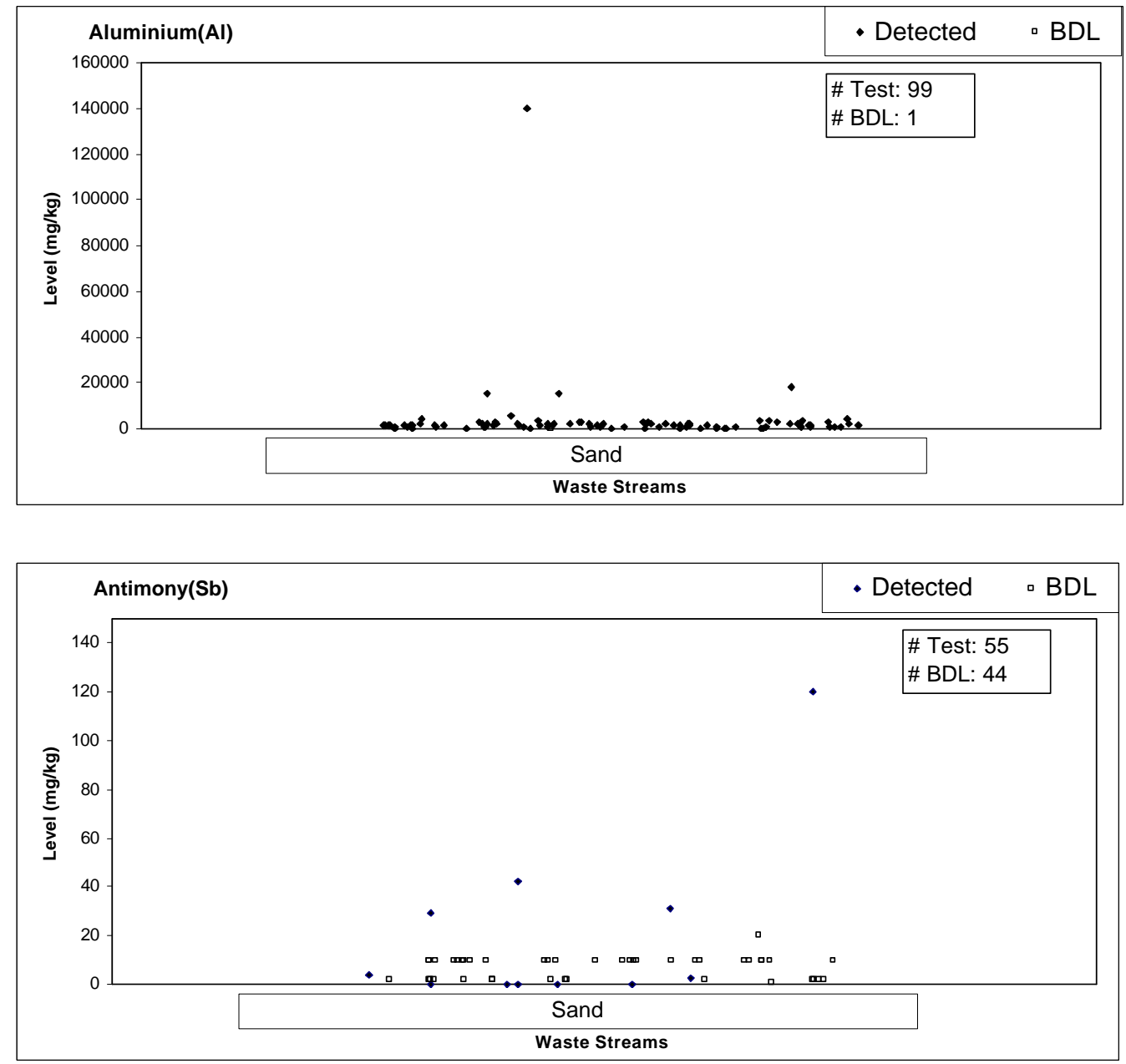

D- 2 

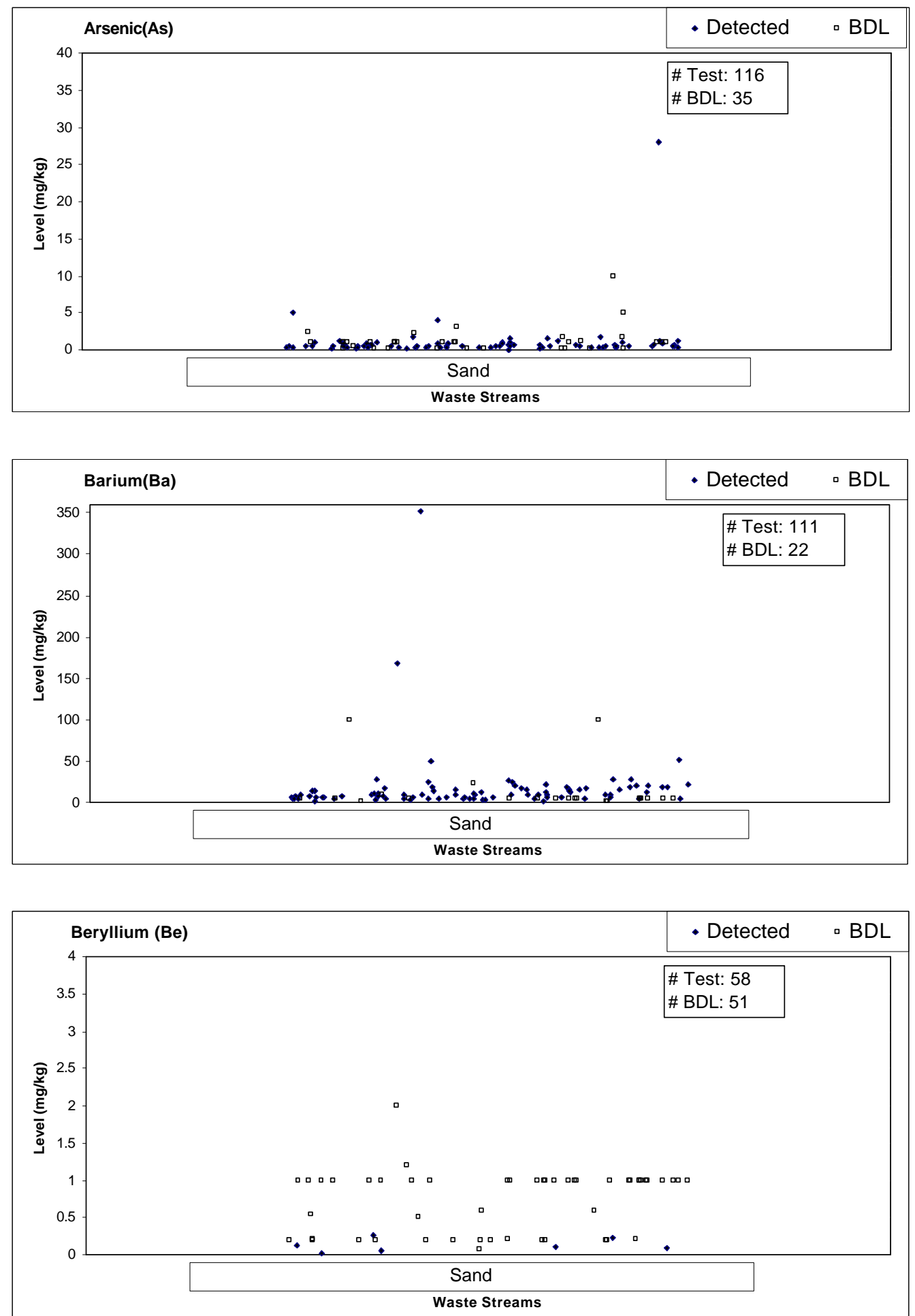

D- 3 

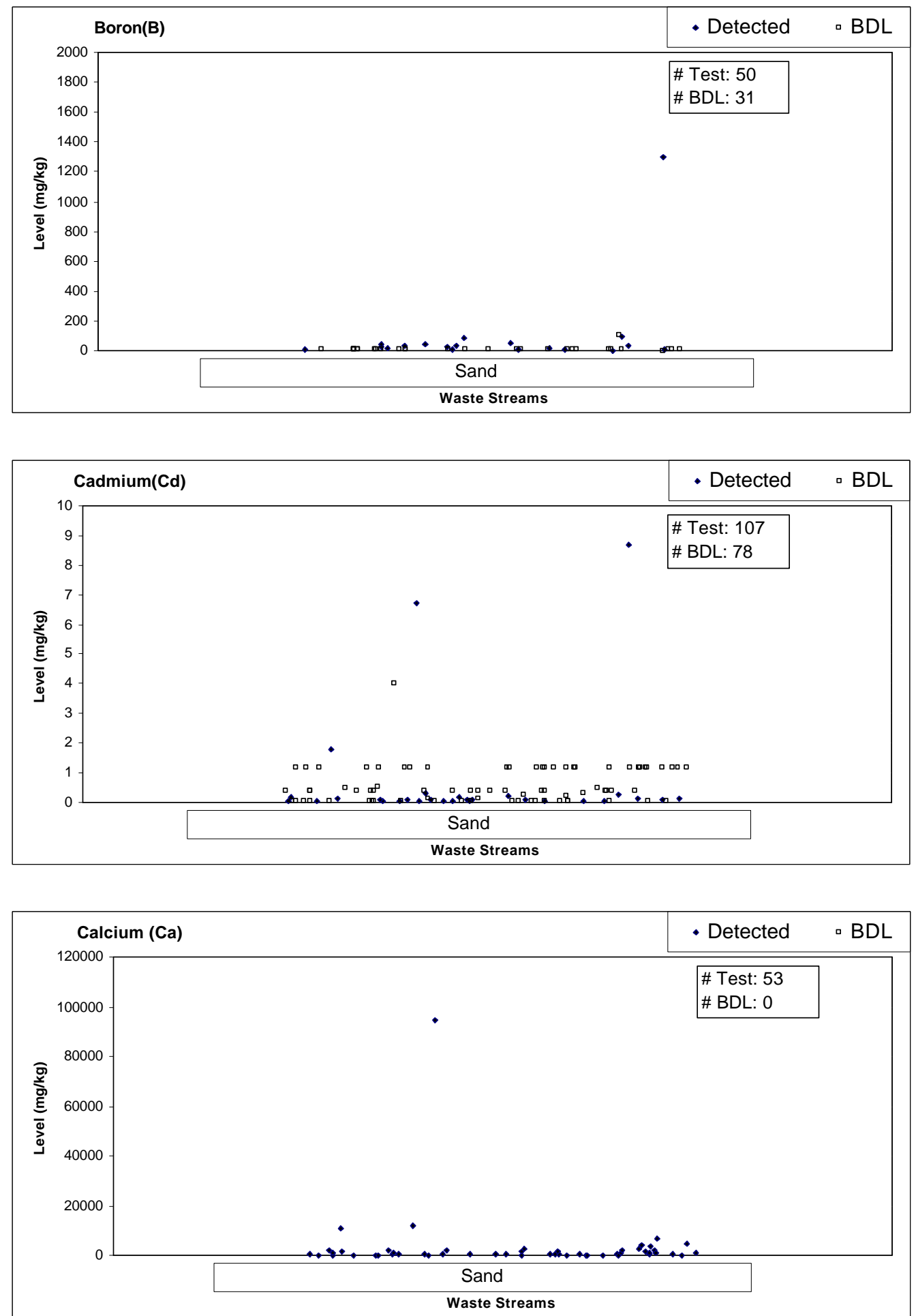

D- 4 

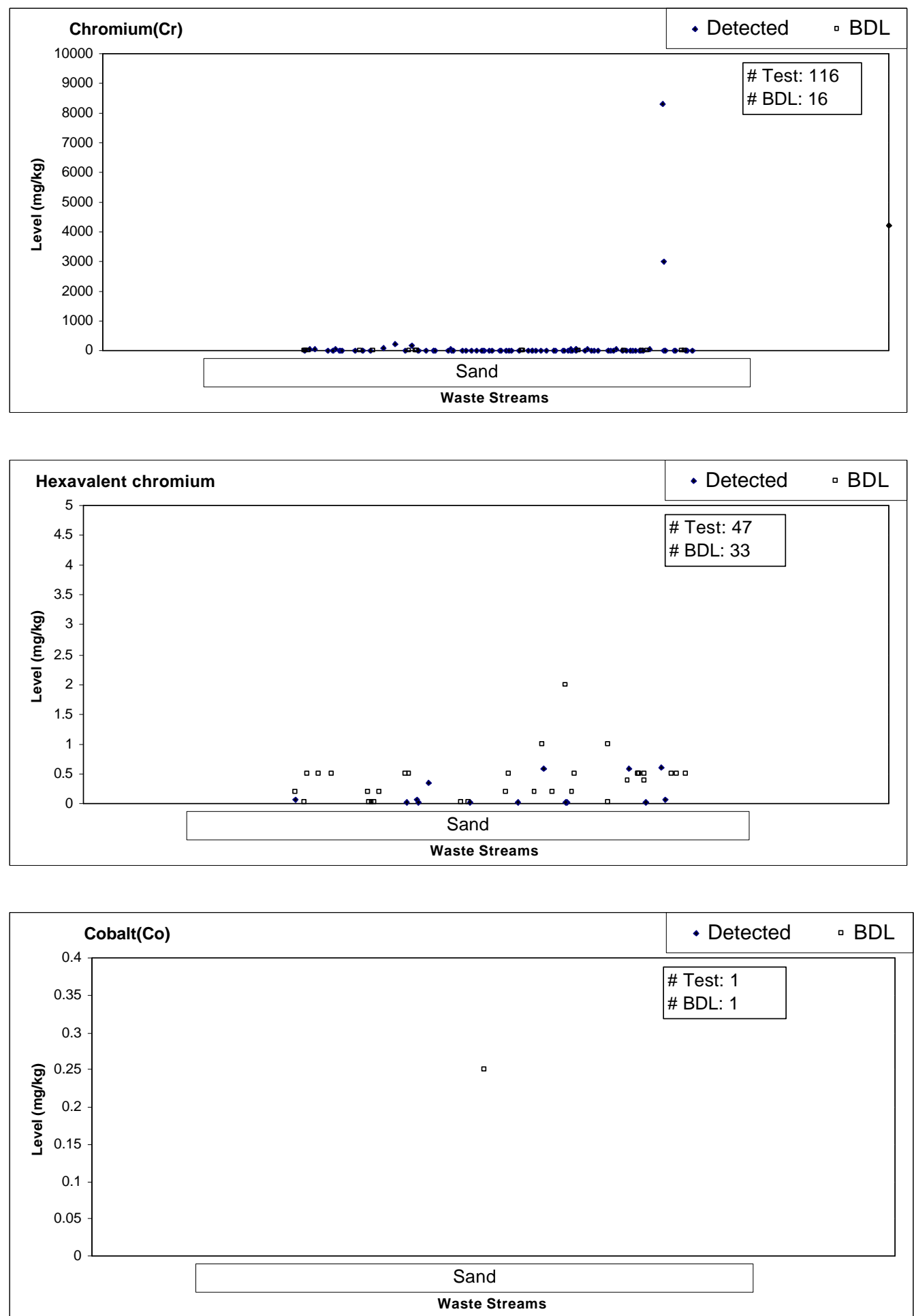

D- 5 

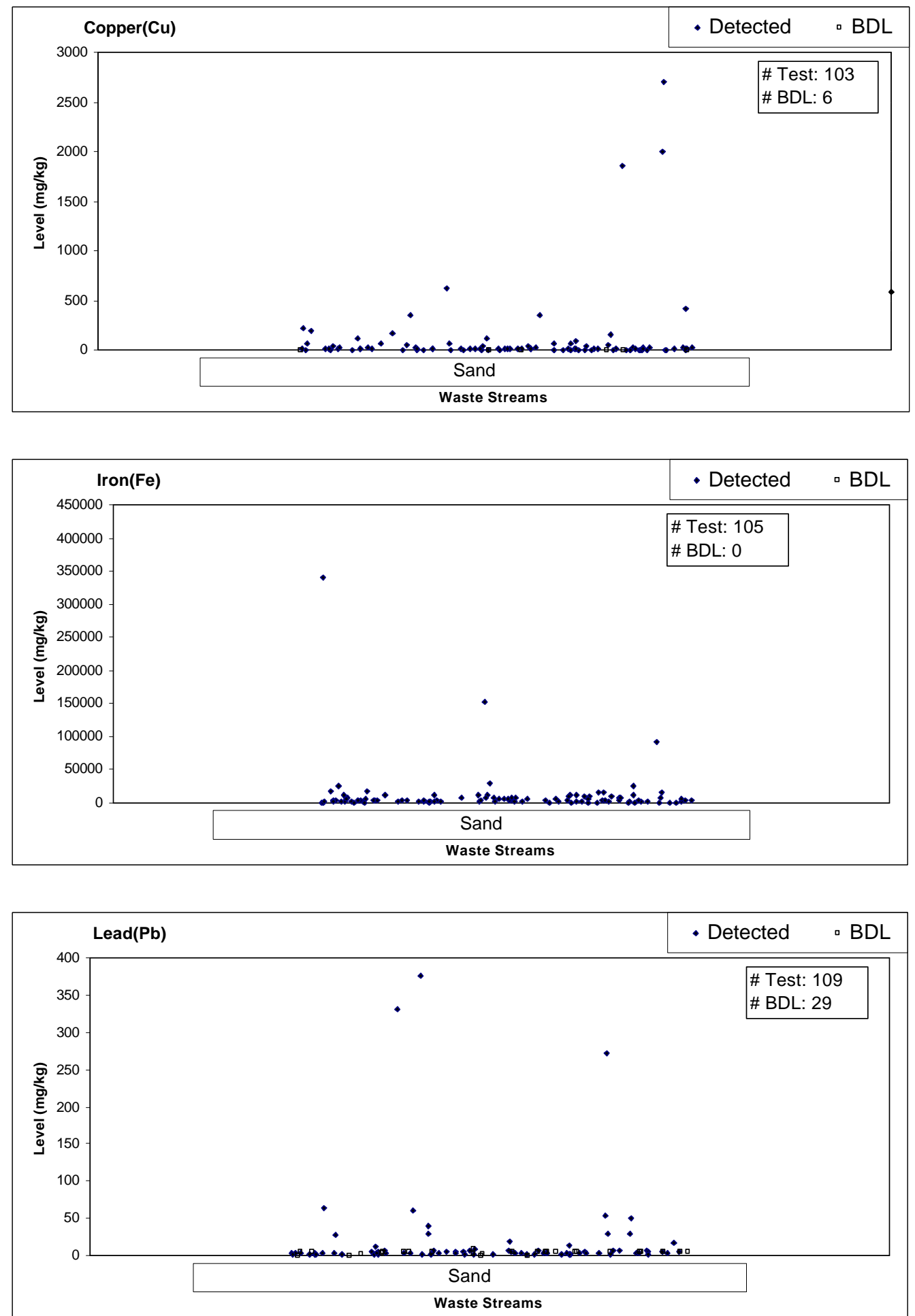

D- 6 

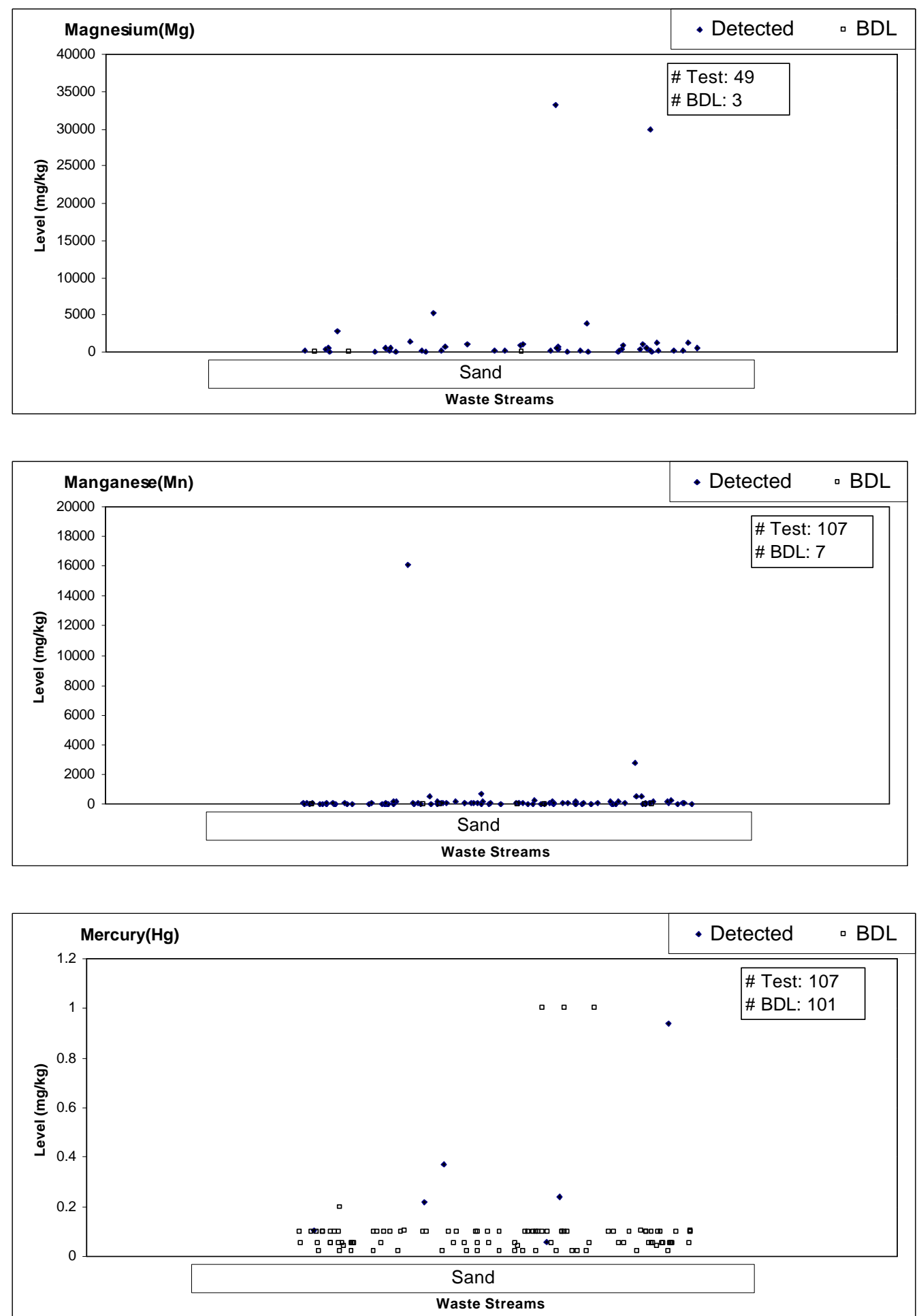

D- 7 

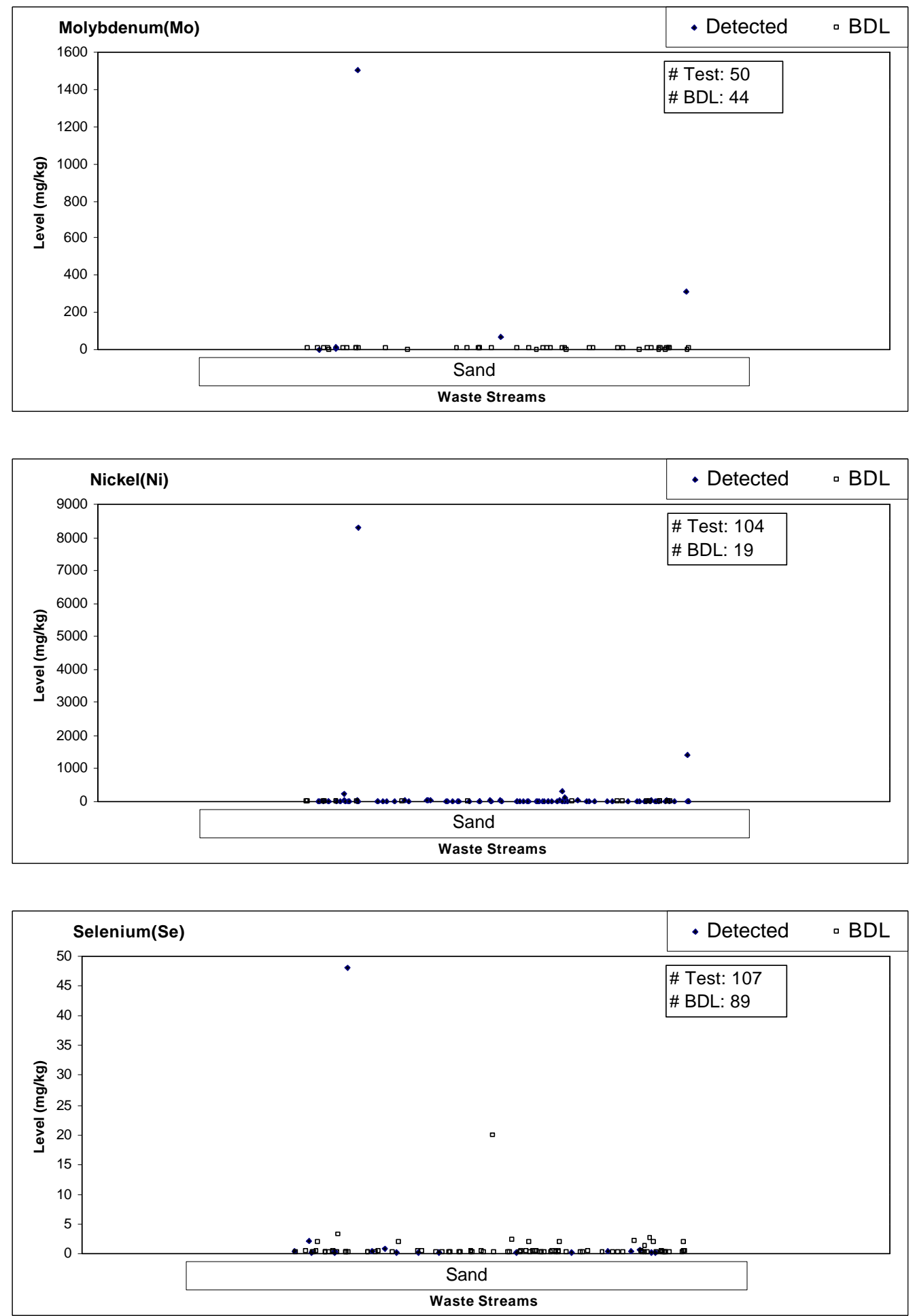

D- 8 

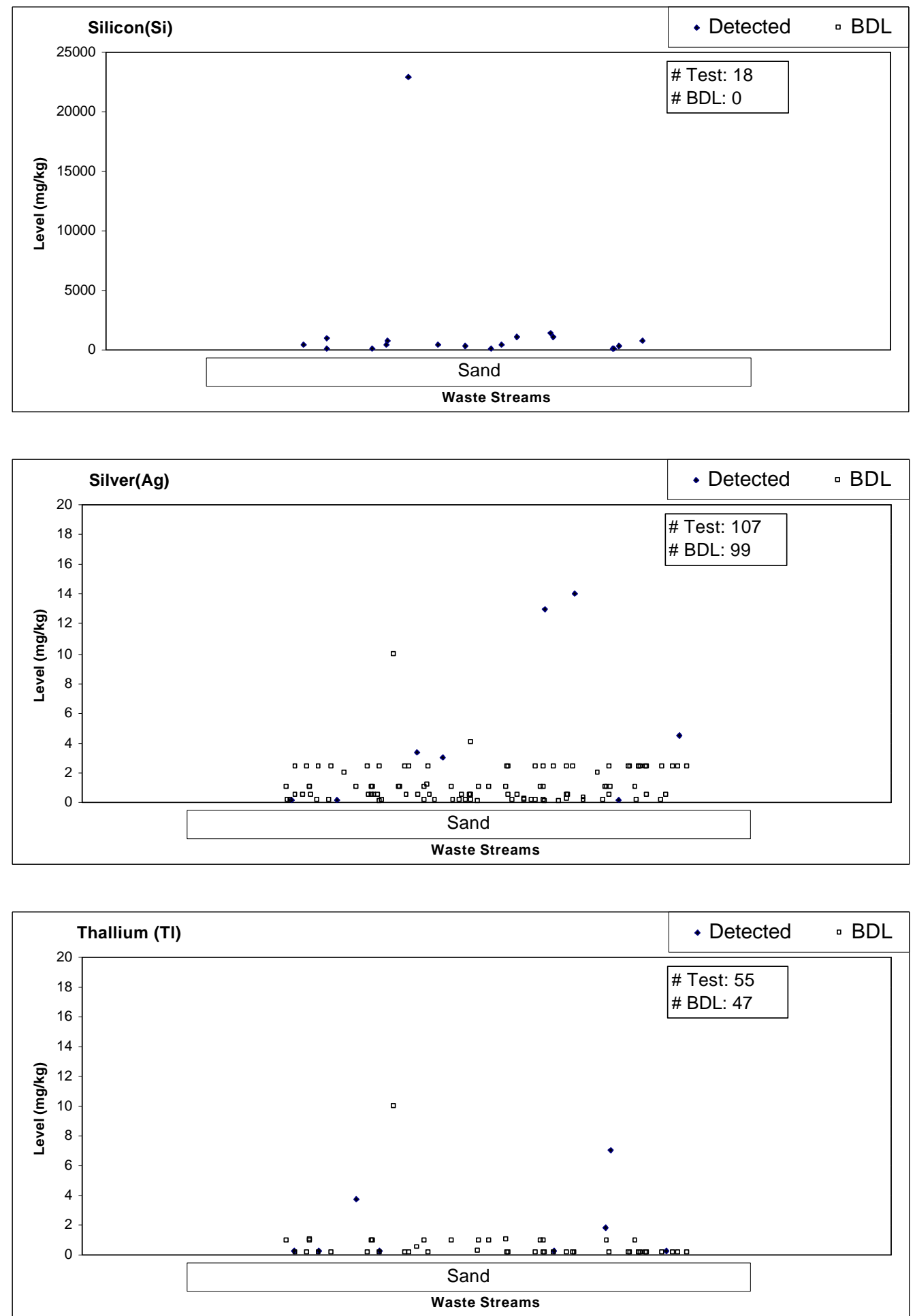

D- 9 


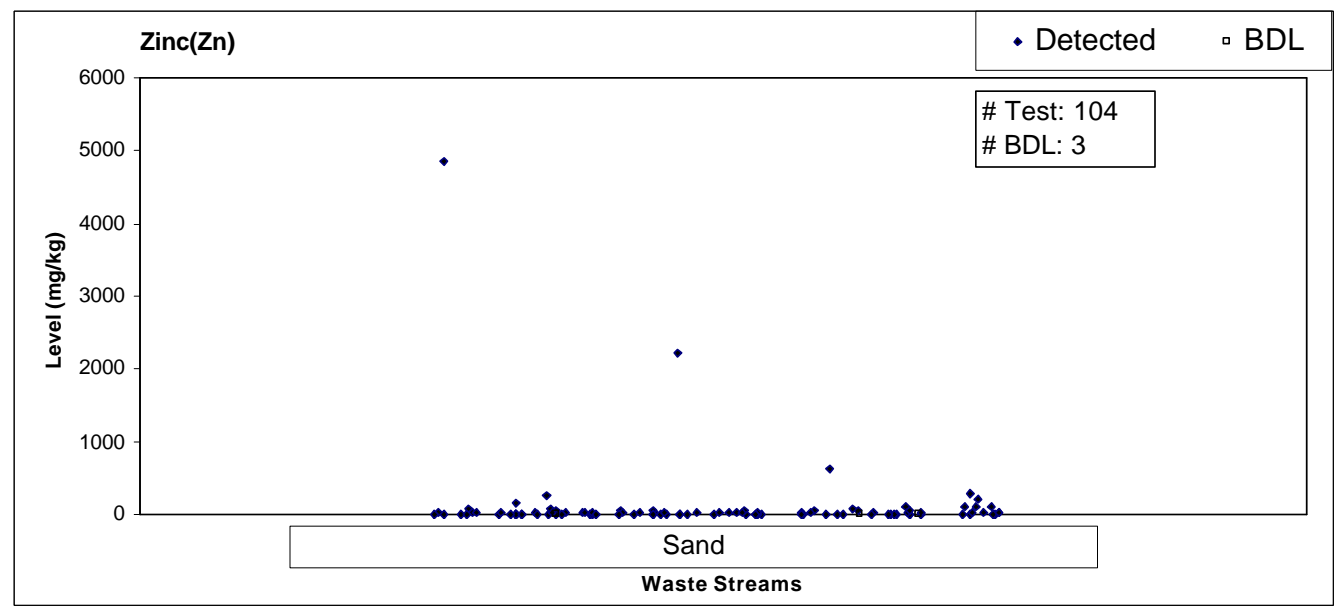

Figure D-1: Bulk analysis of trace metallic elements (24 charts)

\section{D.2 General Chemical Parameters}

Following a series of 17 charts, grouped into Figure D-2, present the bulk analysis distribution for 17 general chemical parameters in individual waste stream.

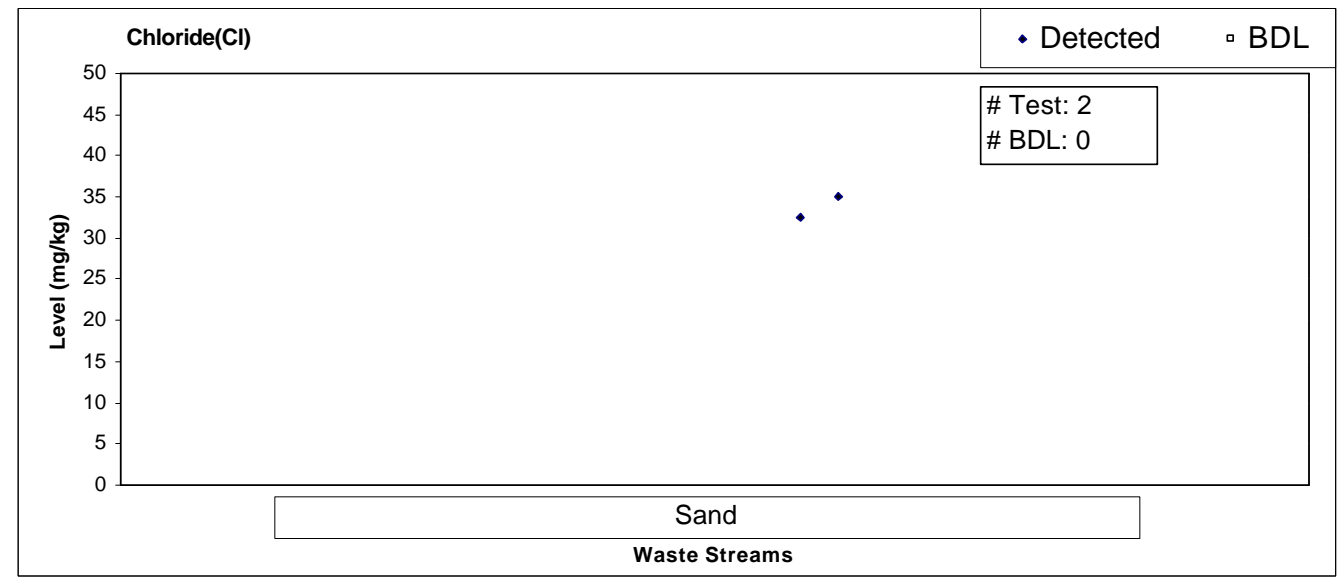



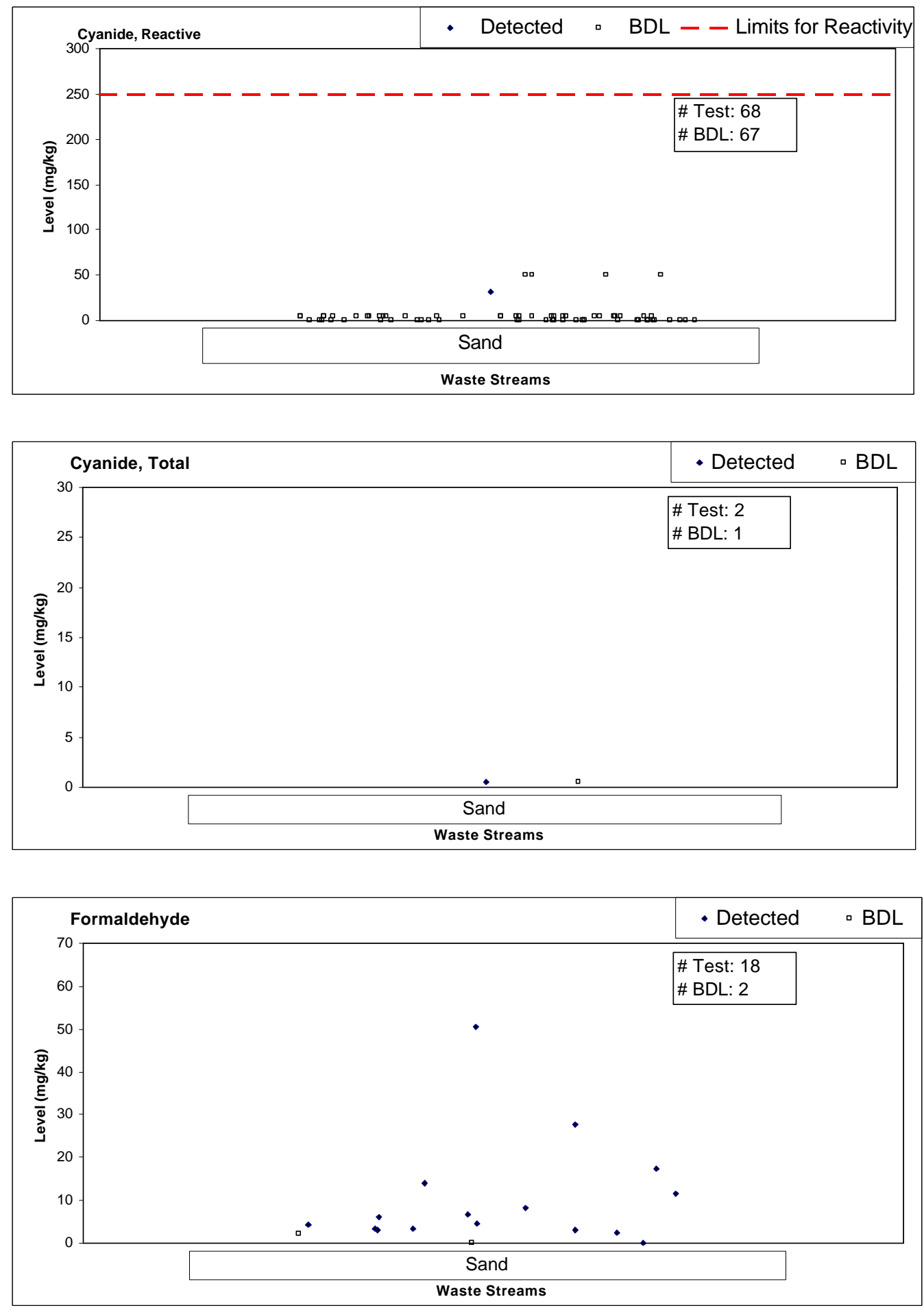

D-11 

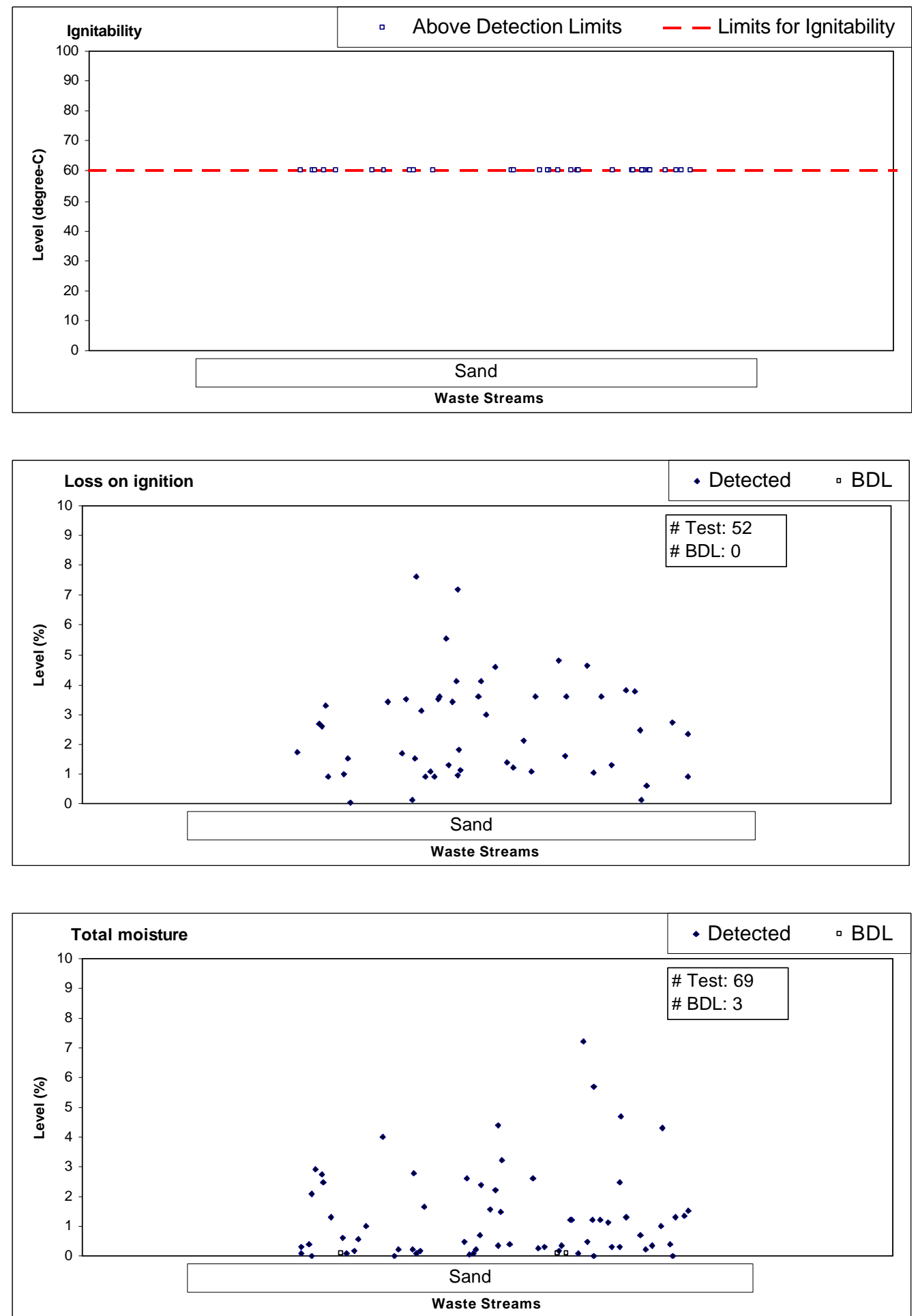

D-12 

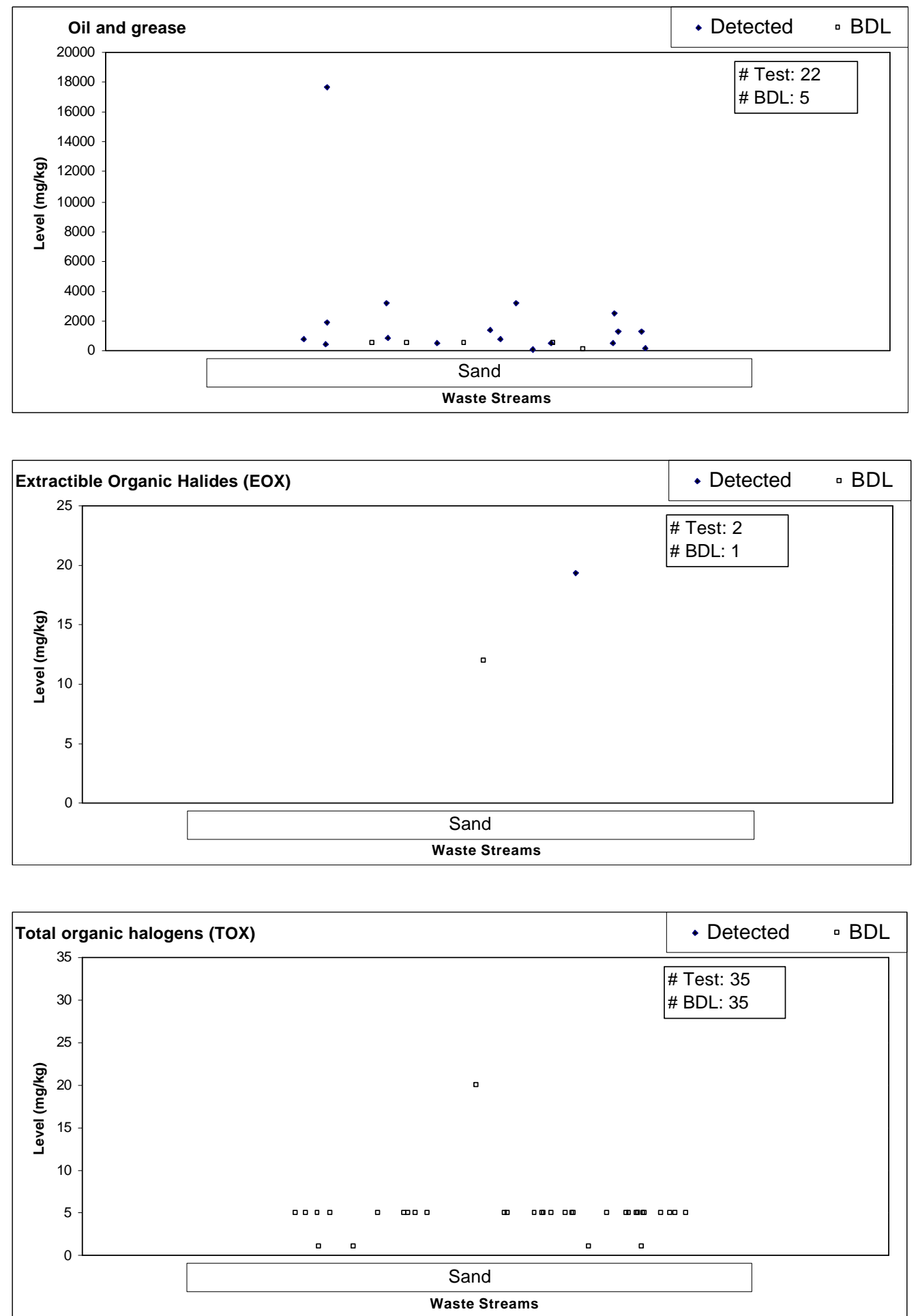

D-13 

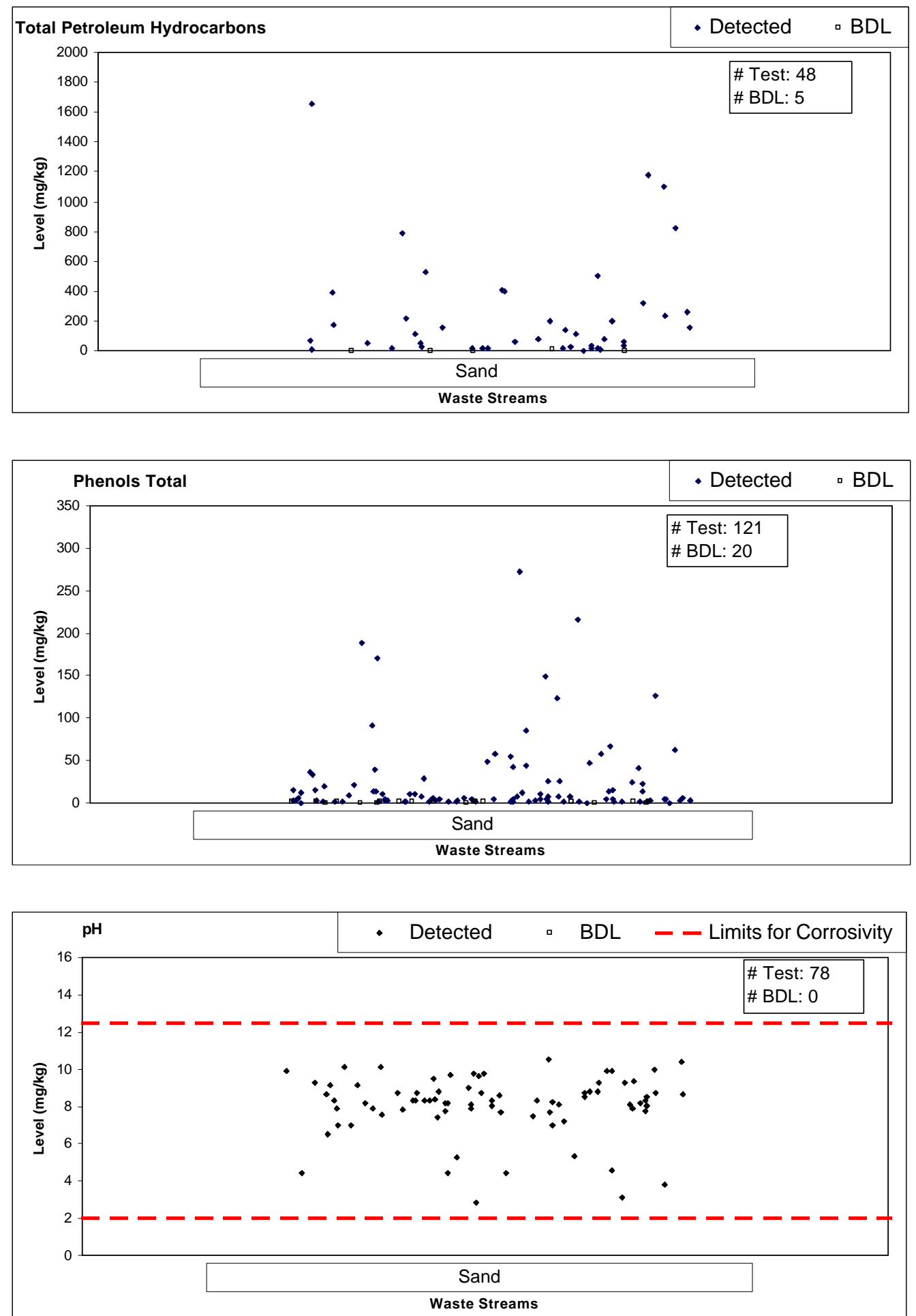

D-14 

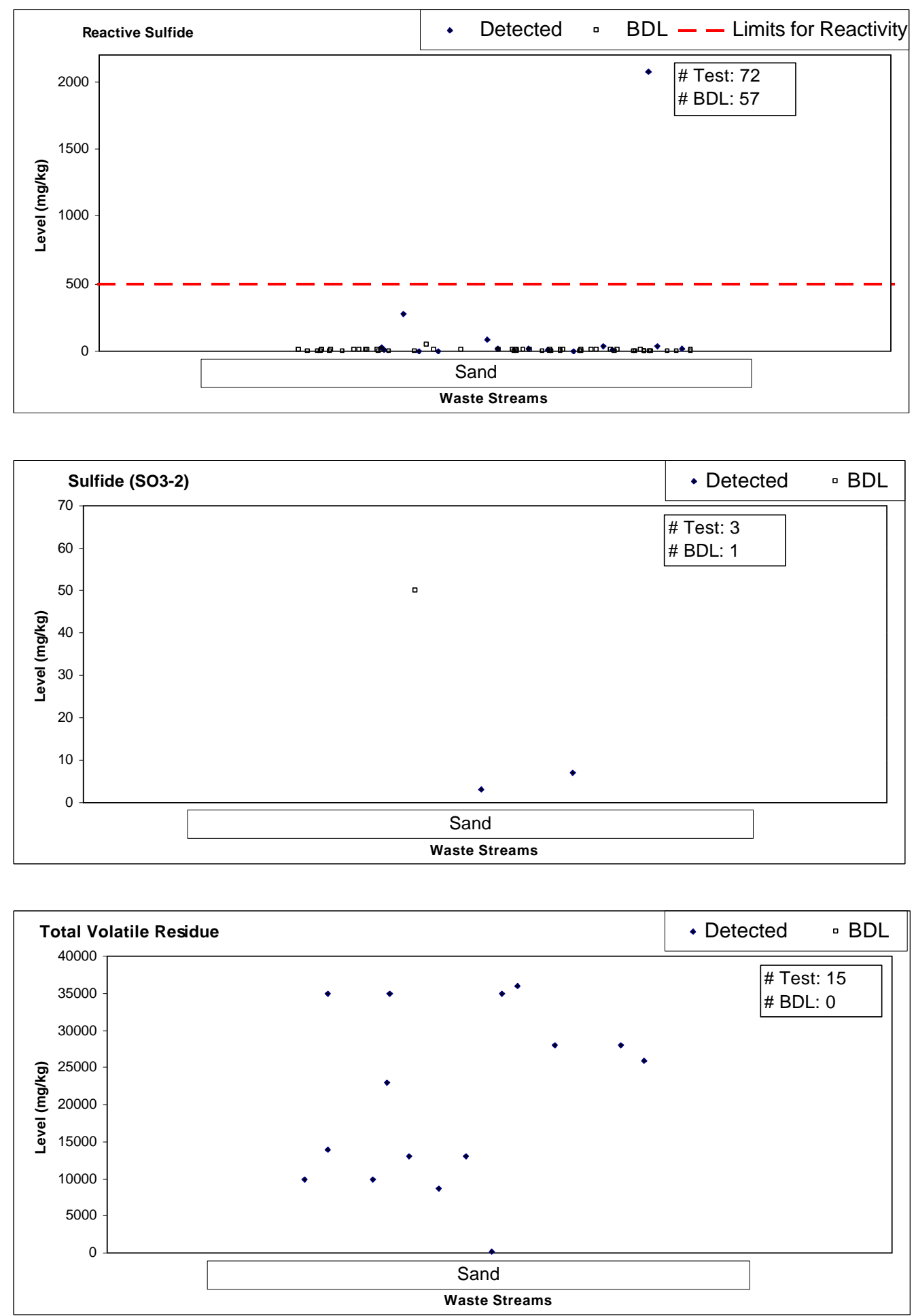

\section{D-15}




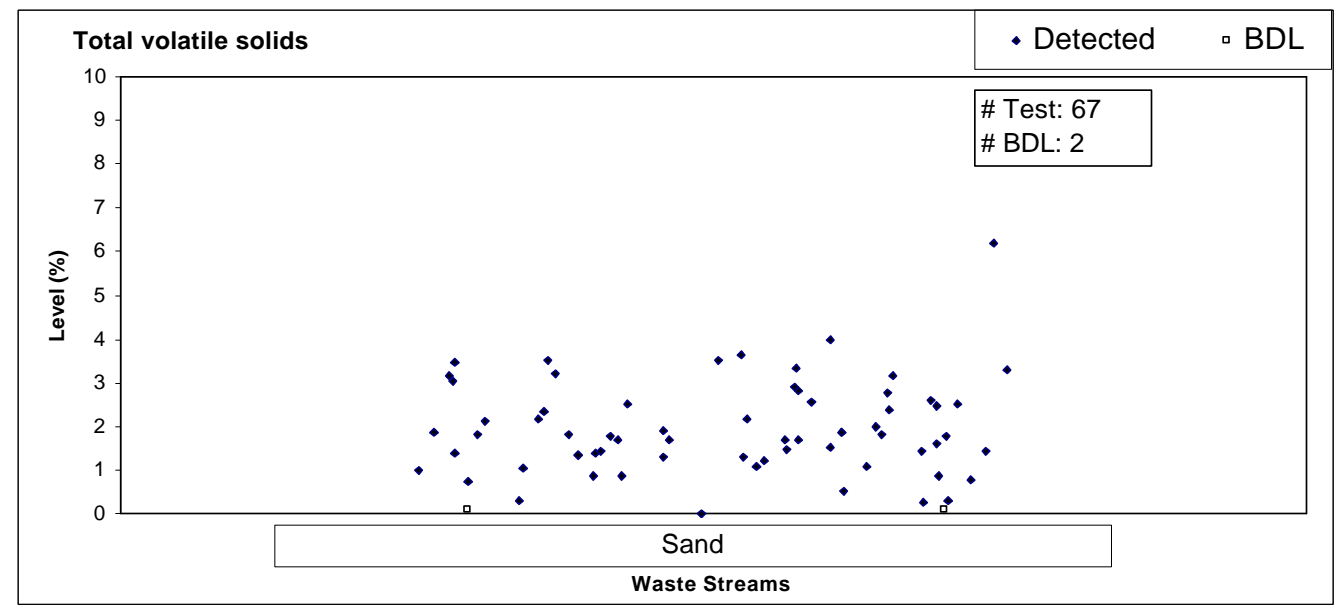

Figure D-2: Bulk analysis of general chemical parameters (17 charts)

\section{D.3 Organic Compounds}

Following a series of 30 charts, grouped into Figure D-3, present the bulk analysis distribution for 30 organic compounds in individual waste stream.

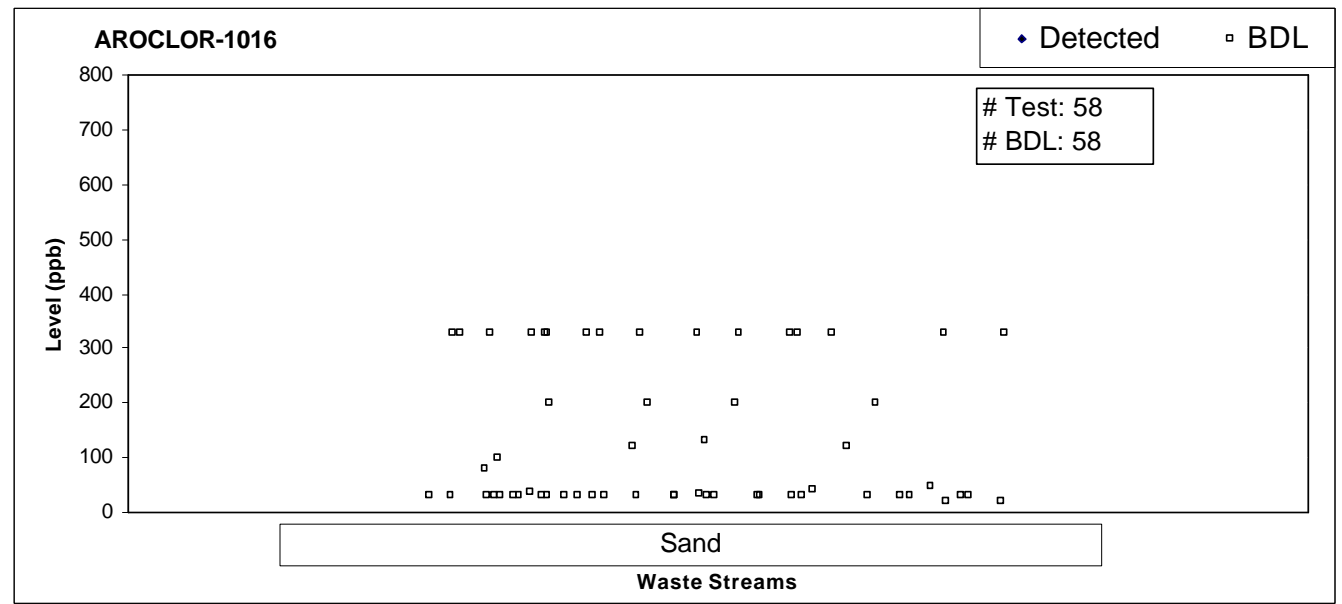

D-16 

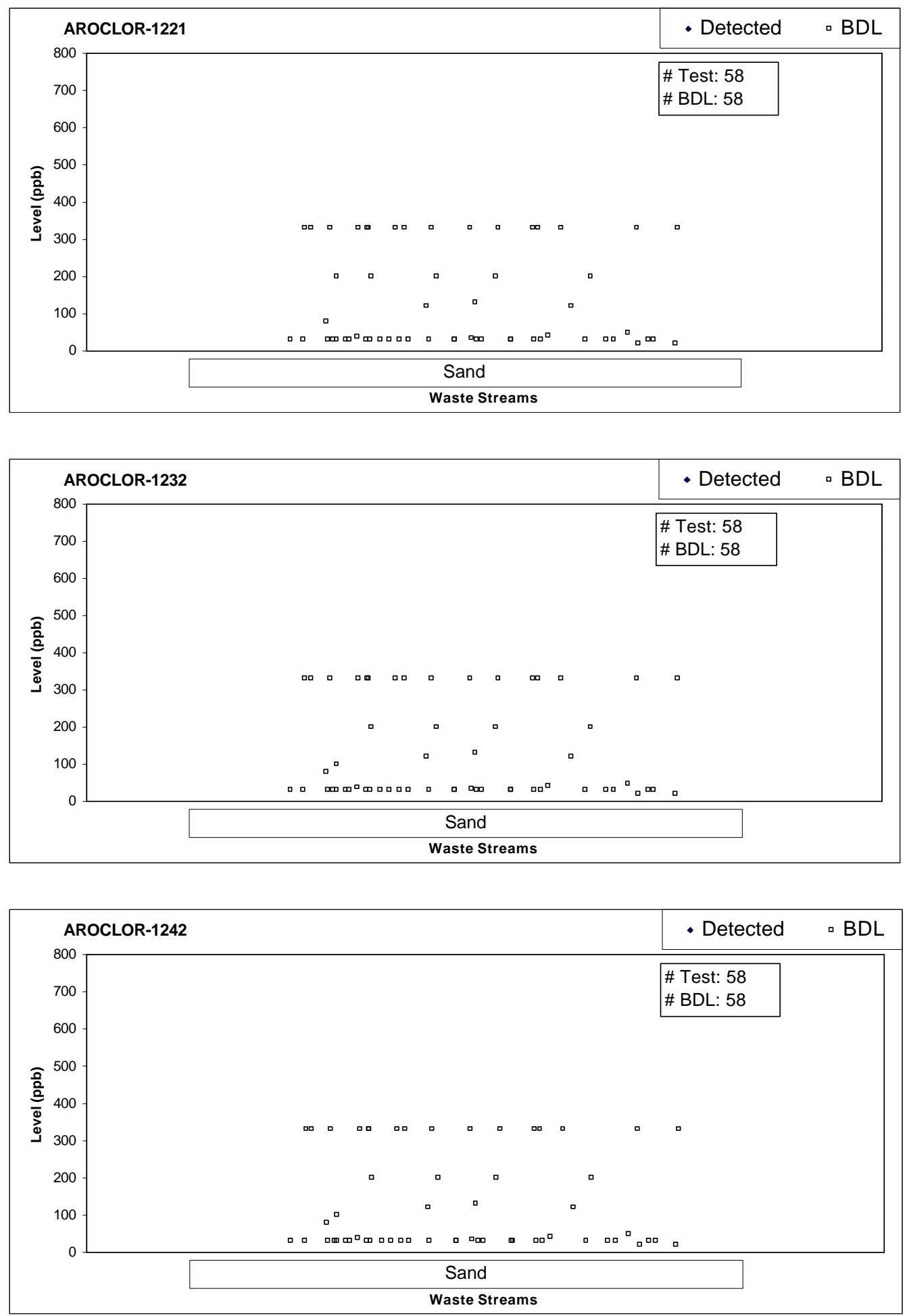

D-17 

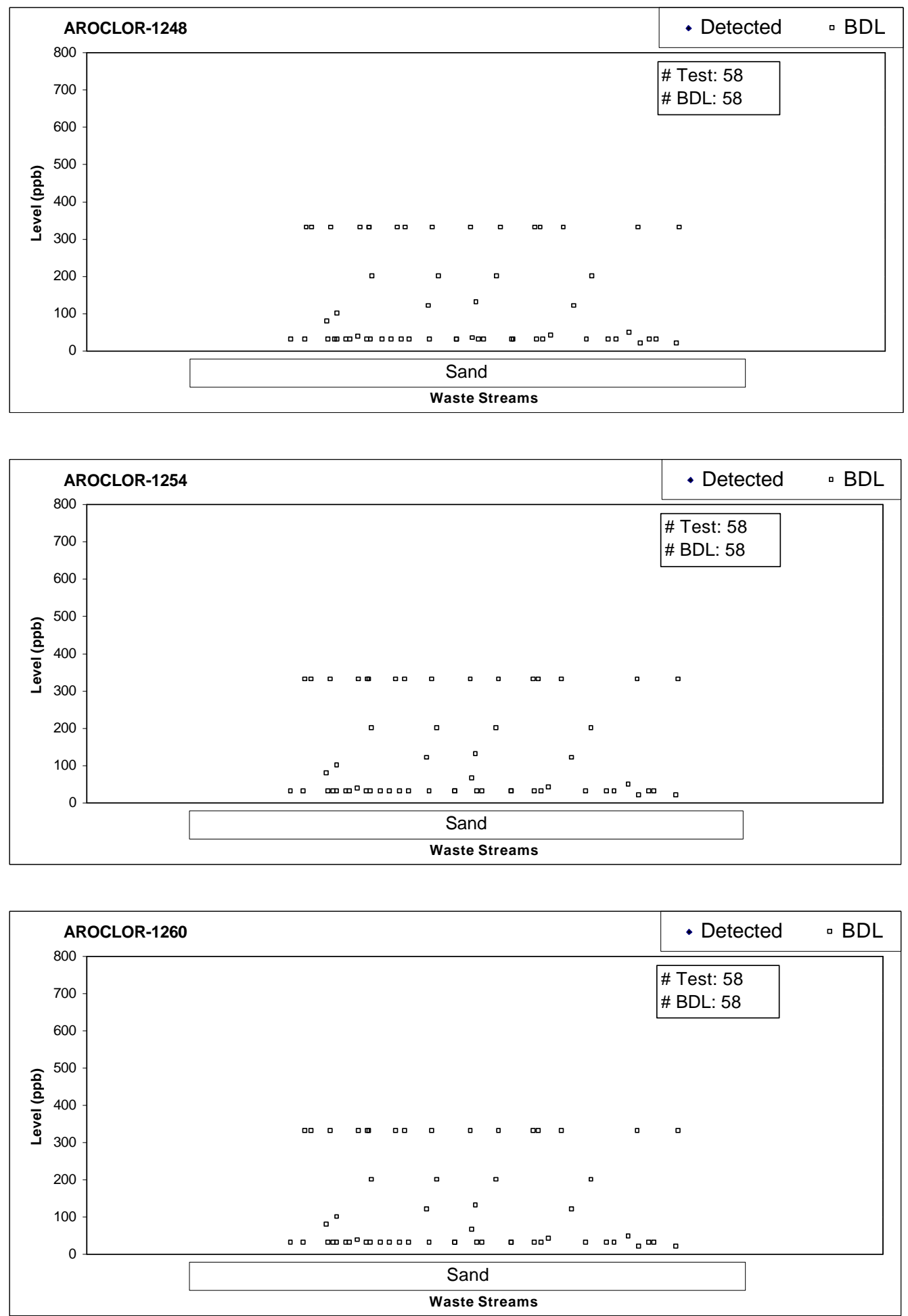

D-18 

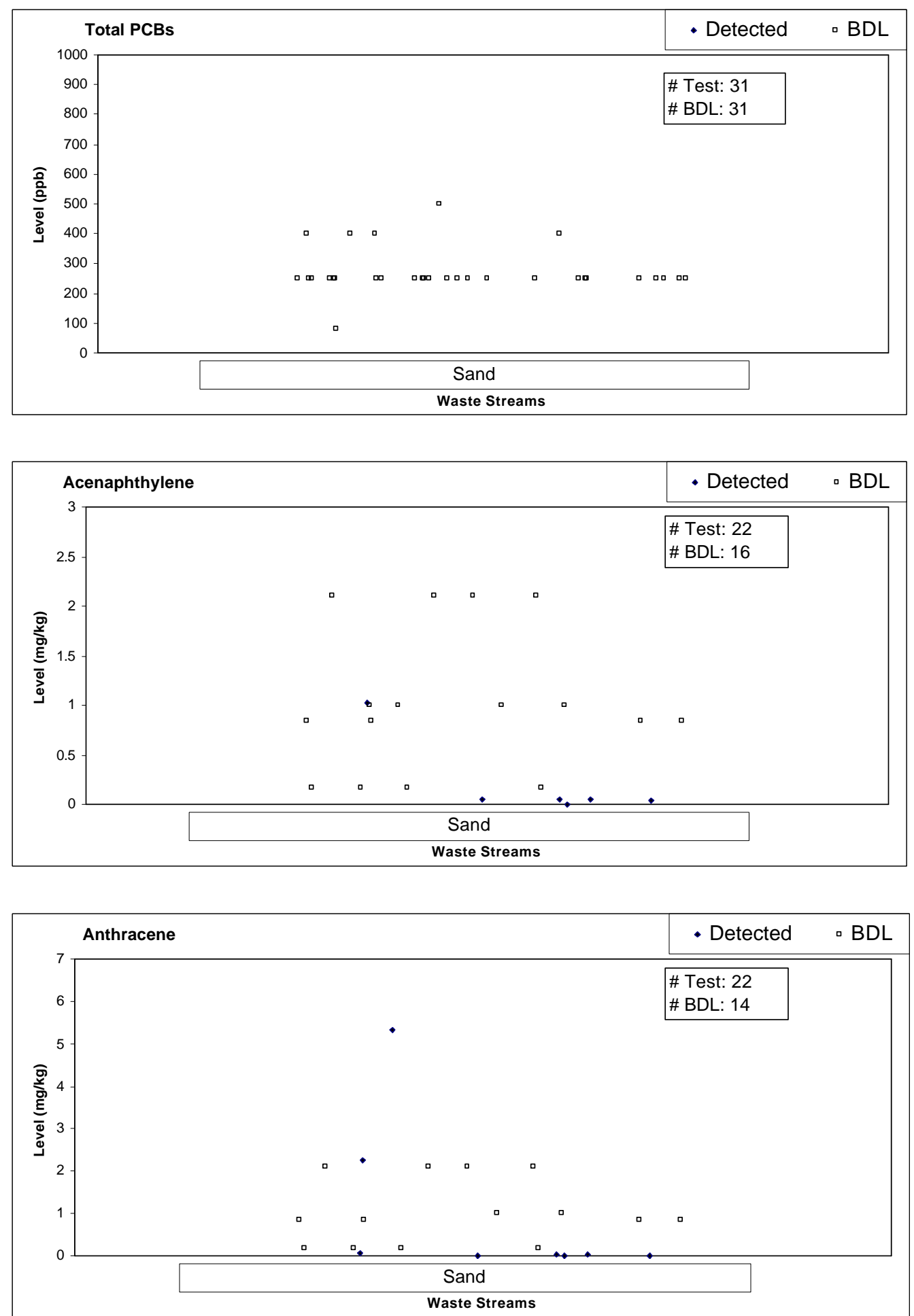

D-19 

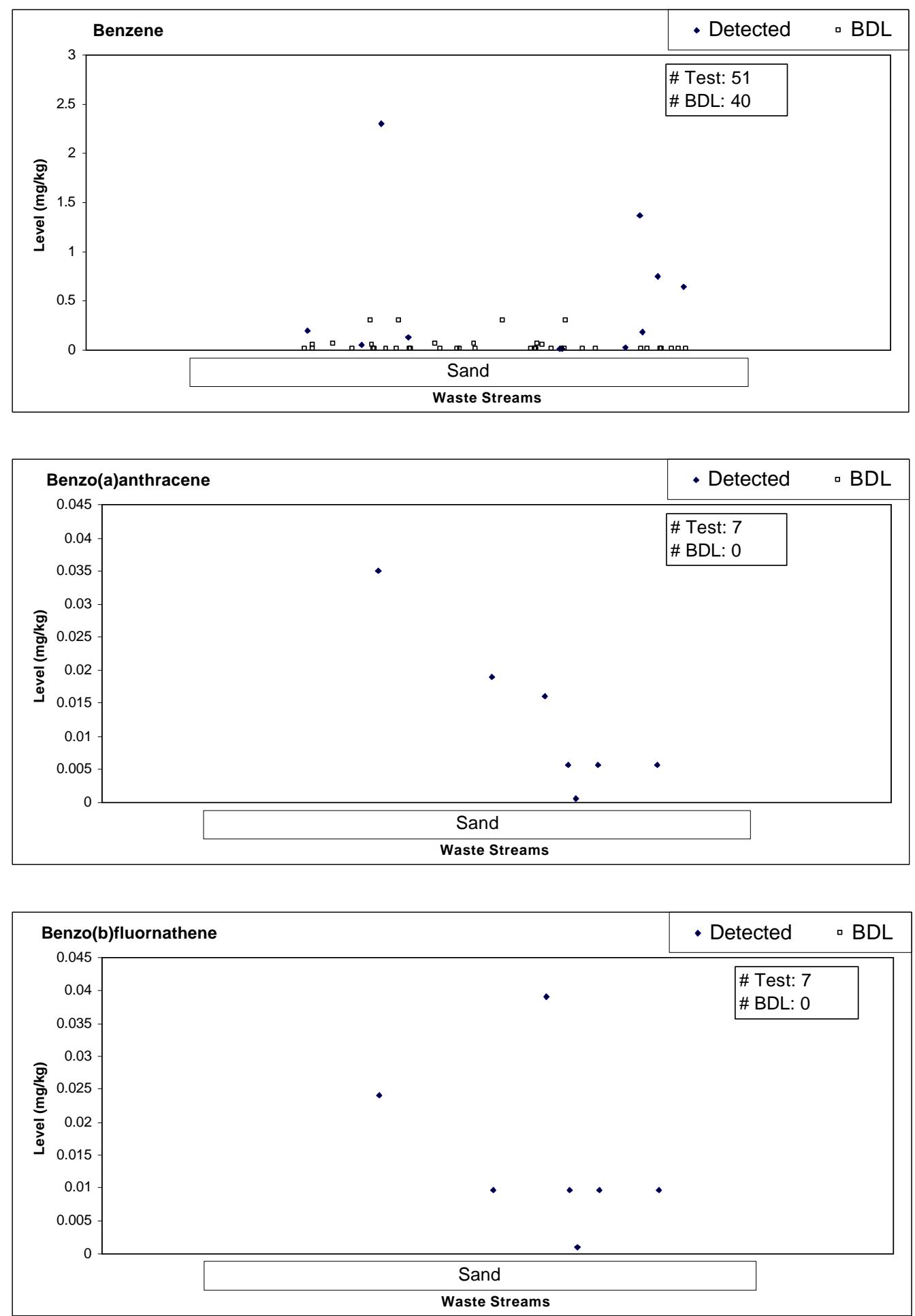

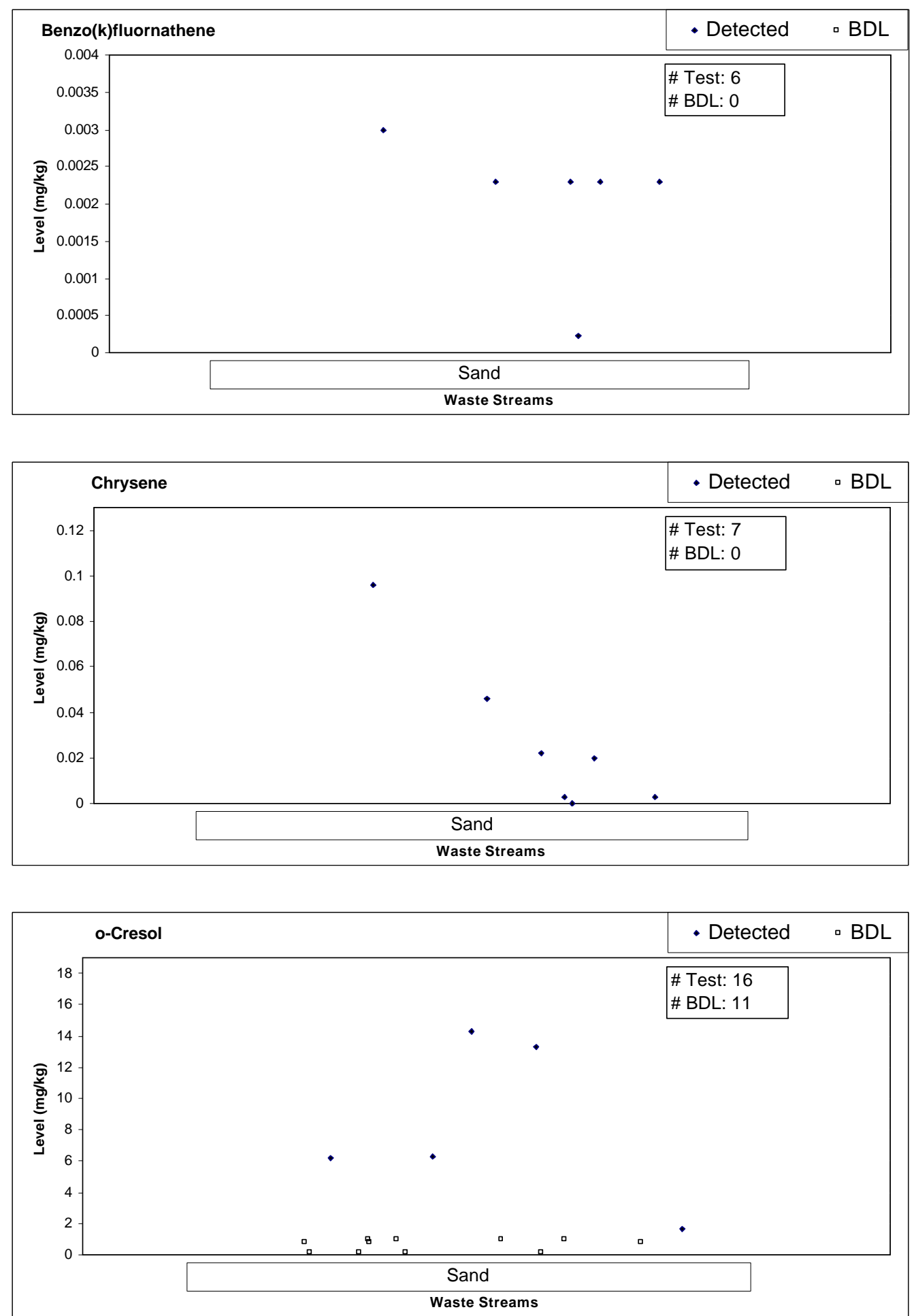

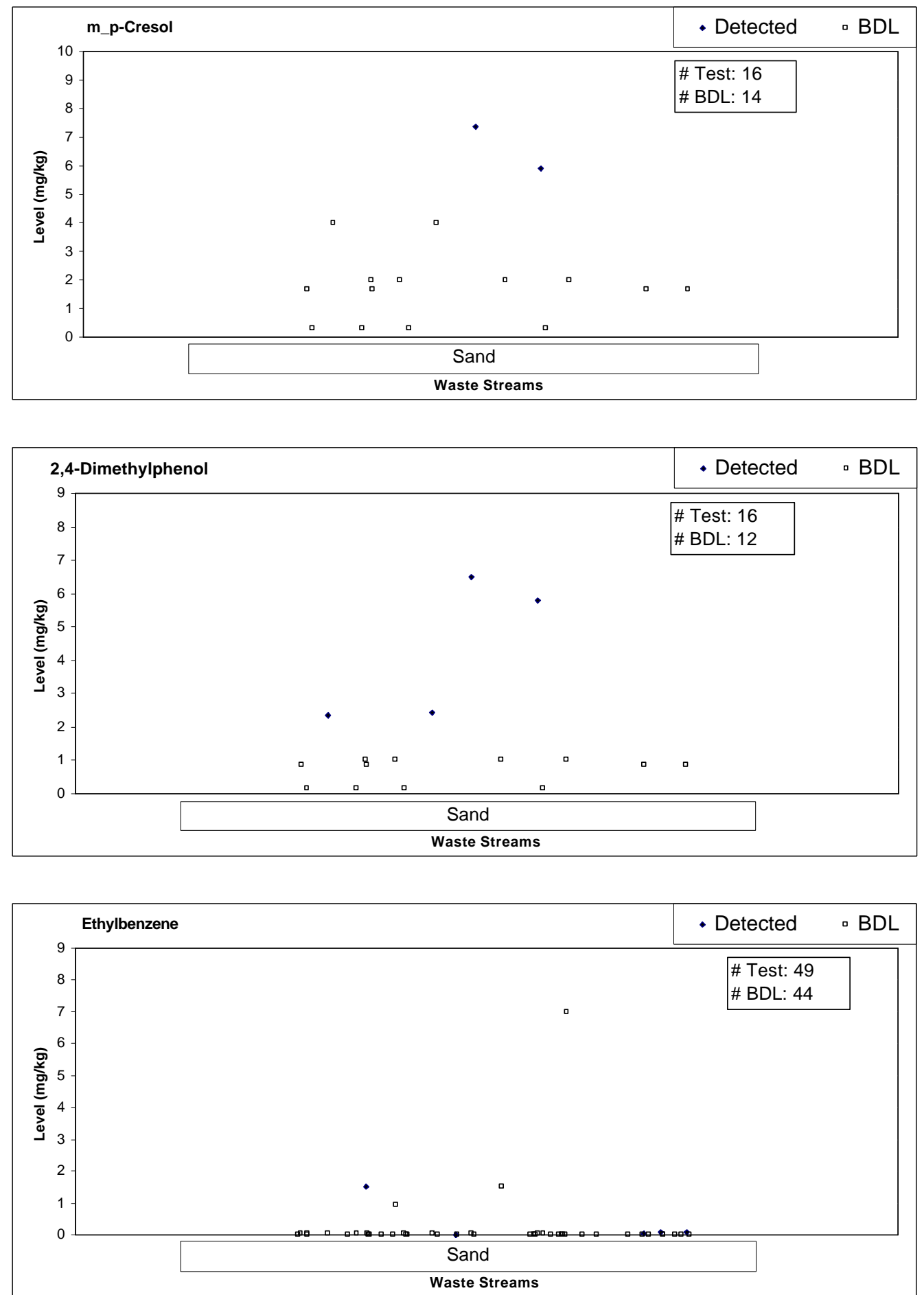

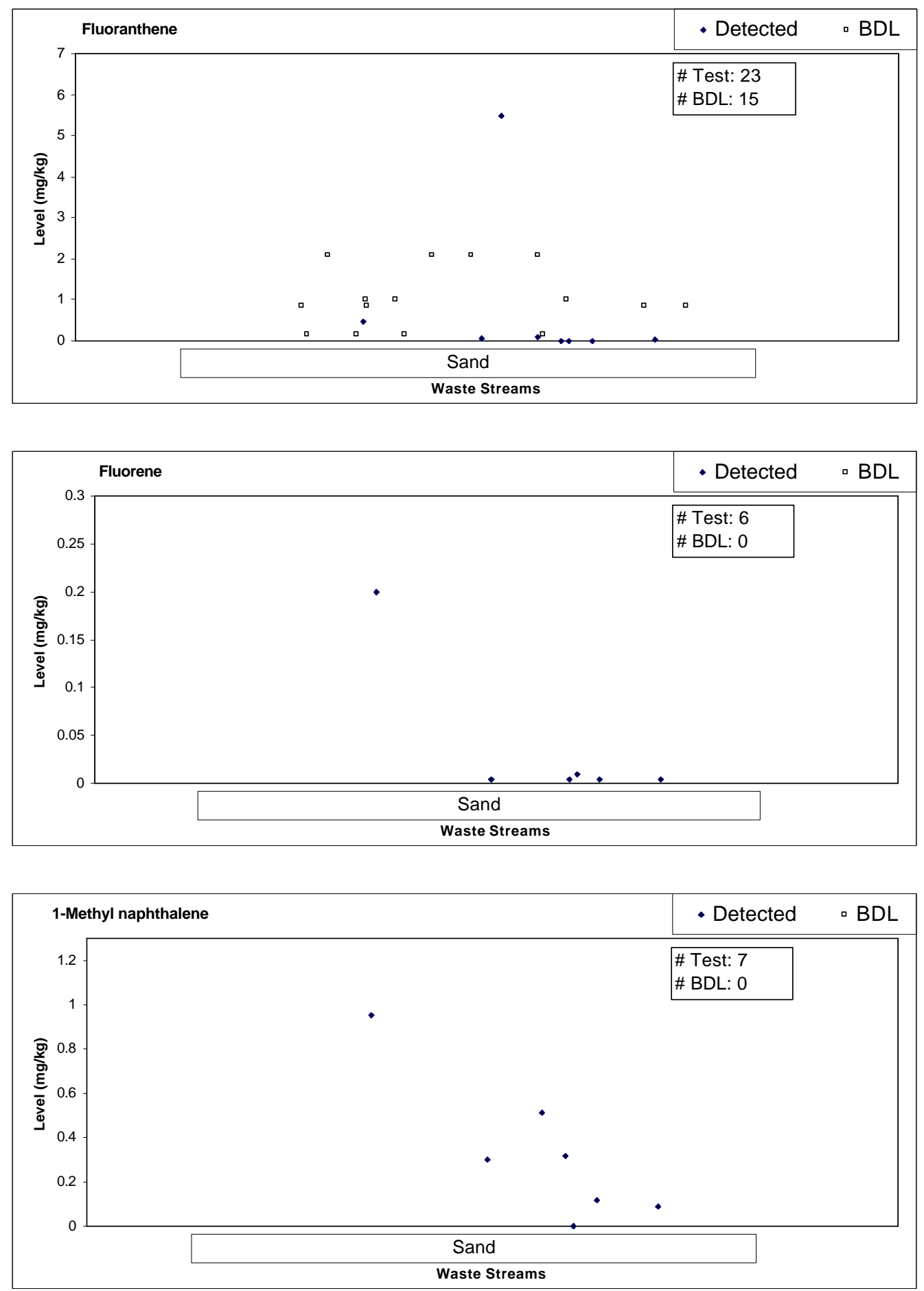

D-23 

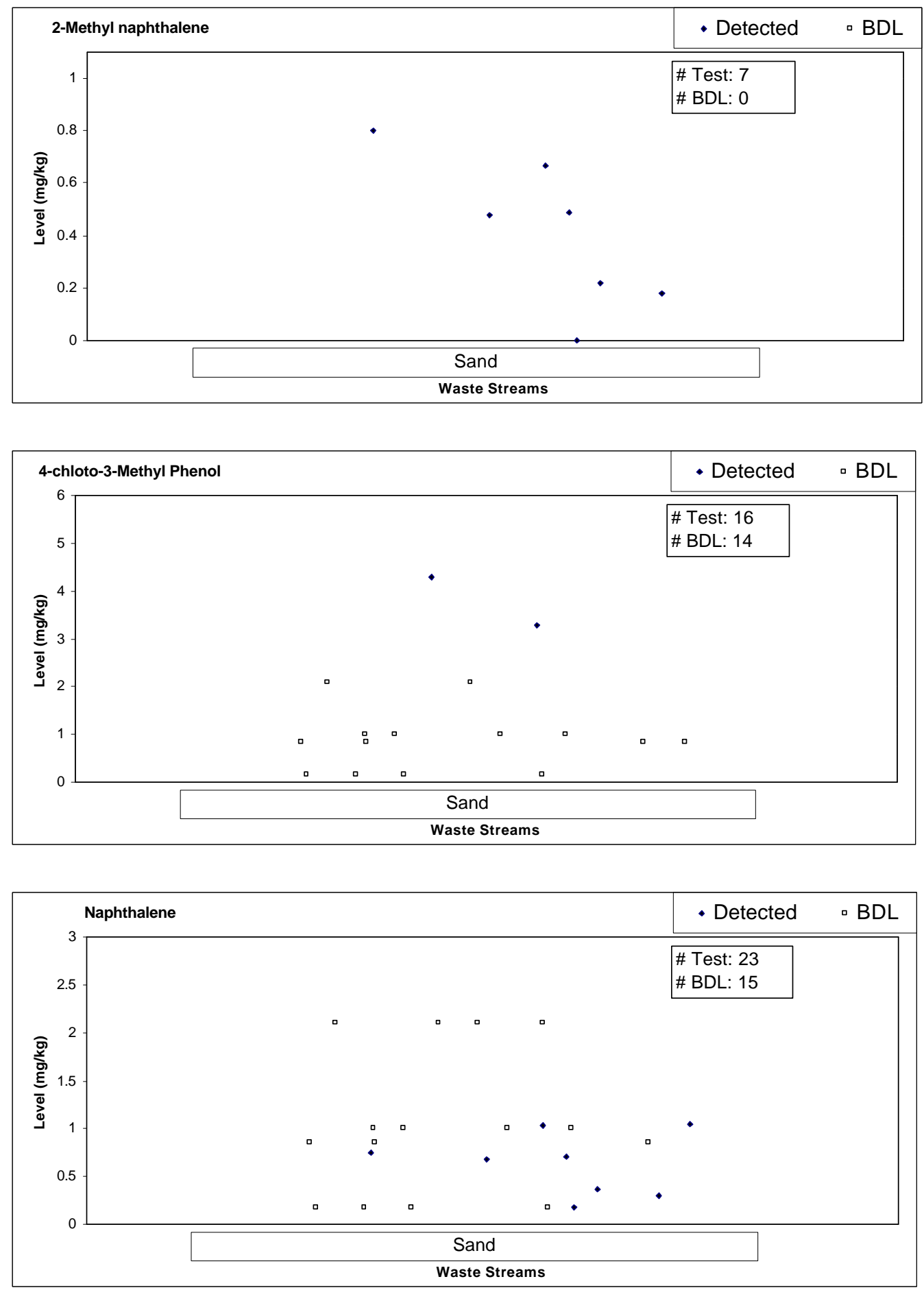

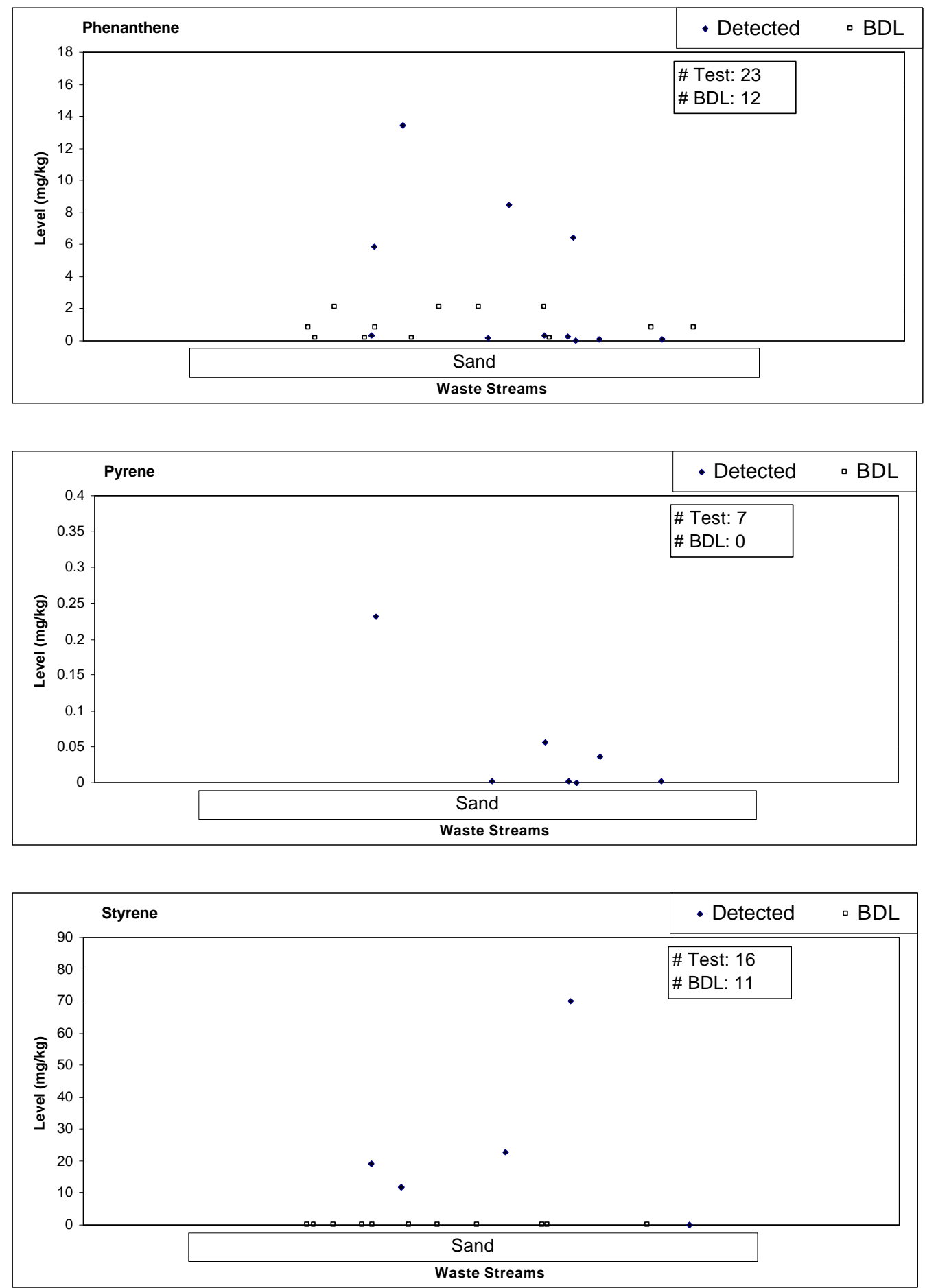

\section{D-25}



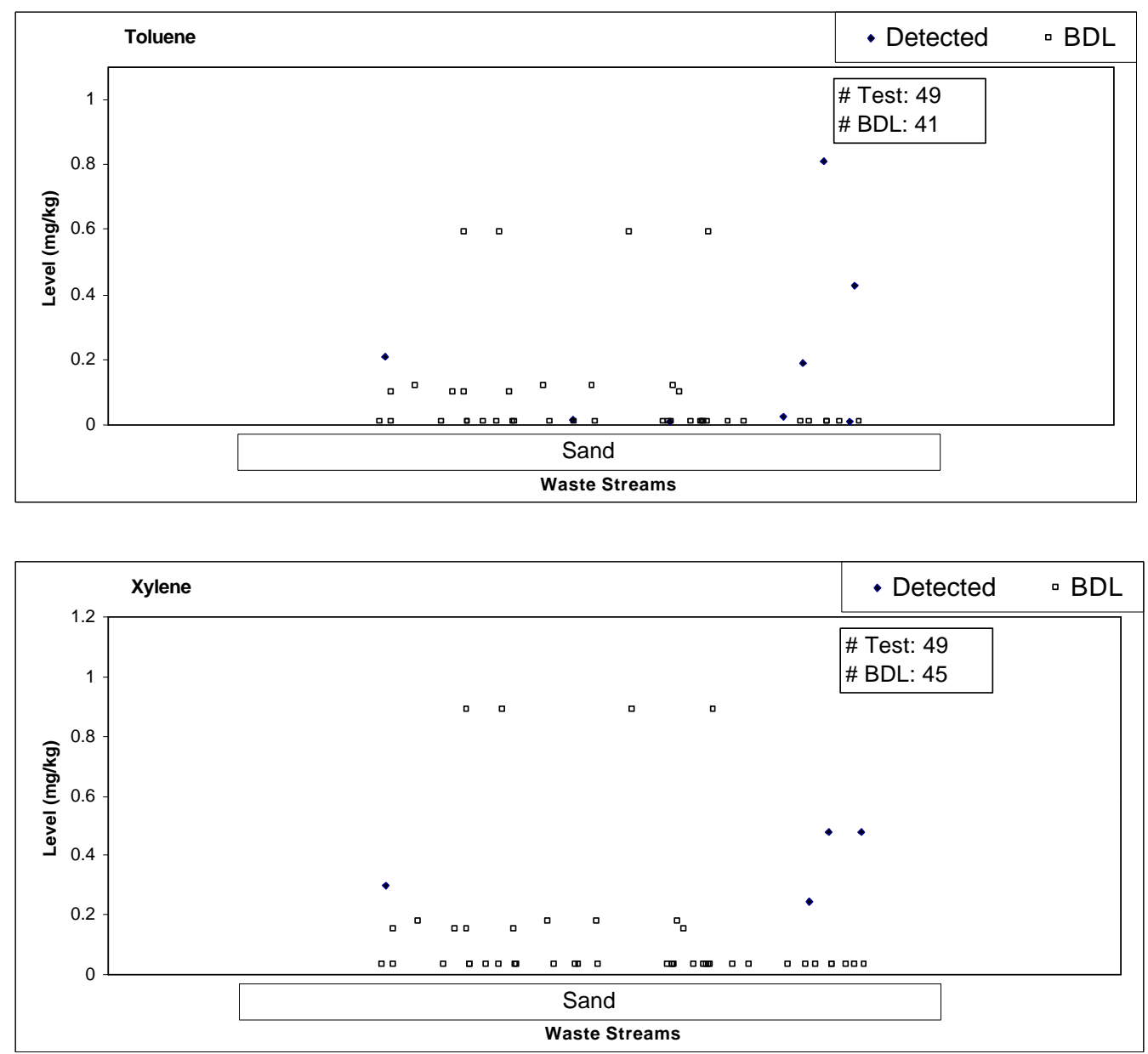

Figure D-3: Bulk analysis of organic compounds (30 charts) 


\section{Appendix E}

\section{Composition of Leachate Extracted from Sand Stream}

In this appendix, the composition of leachate extracted from foundry sand streams is shown element by element, including 23 metallic elements, 21 general chemical parameters, and 37 organic compounds at trace level. The matrix of the composition is essentially leachate extracted from the waste streams, not as-received waste streams. The waste stream-based analysis is presented in Appendix D.

The notes to individual charts are defined as follows:

- The number following “\# Tests" represents total observations for corresponding leachates;

- The number following "\# BDL" represents total censored observations for corresponding leachates;

- The solid diamond legend " " represents an uncensored observation;

- The hollow square legend "o" represents a censored observation;

- The horizontal dashed line represents TCLP threshold. 


\section{E.1 Metallic Elements}

The following series of 23 charts, grouped into Figure E-1, presents the concentration distribution for 23 trace metallic elements in sand leachates per leaching protocol.
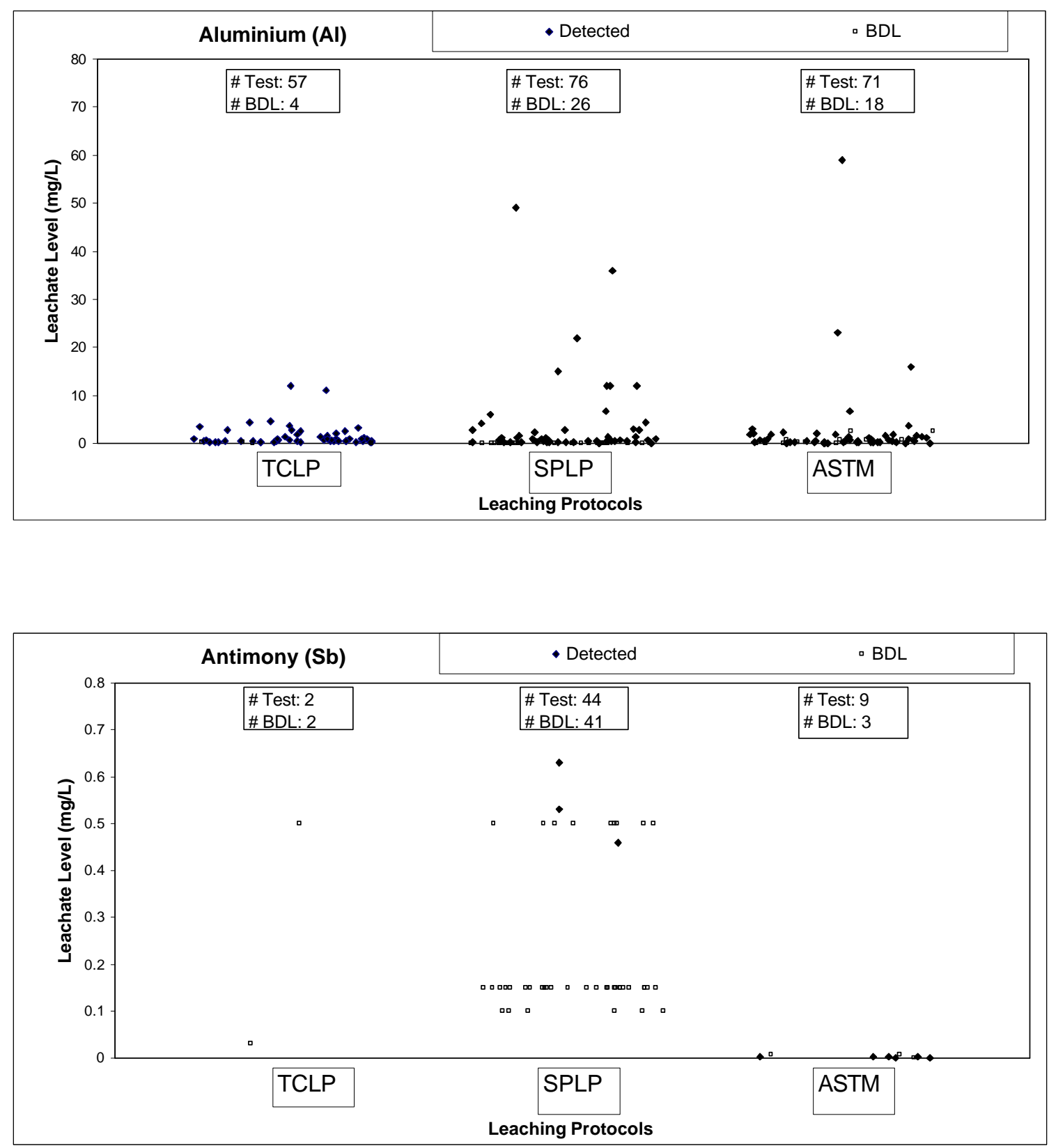

E-2 

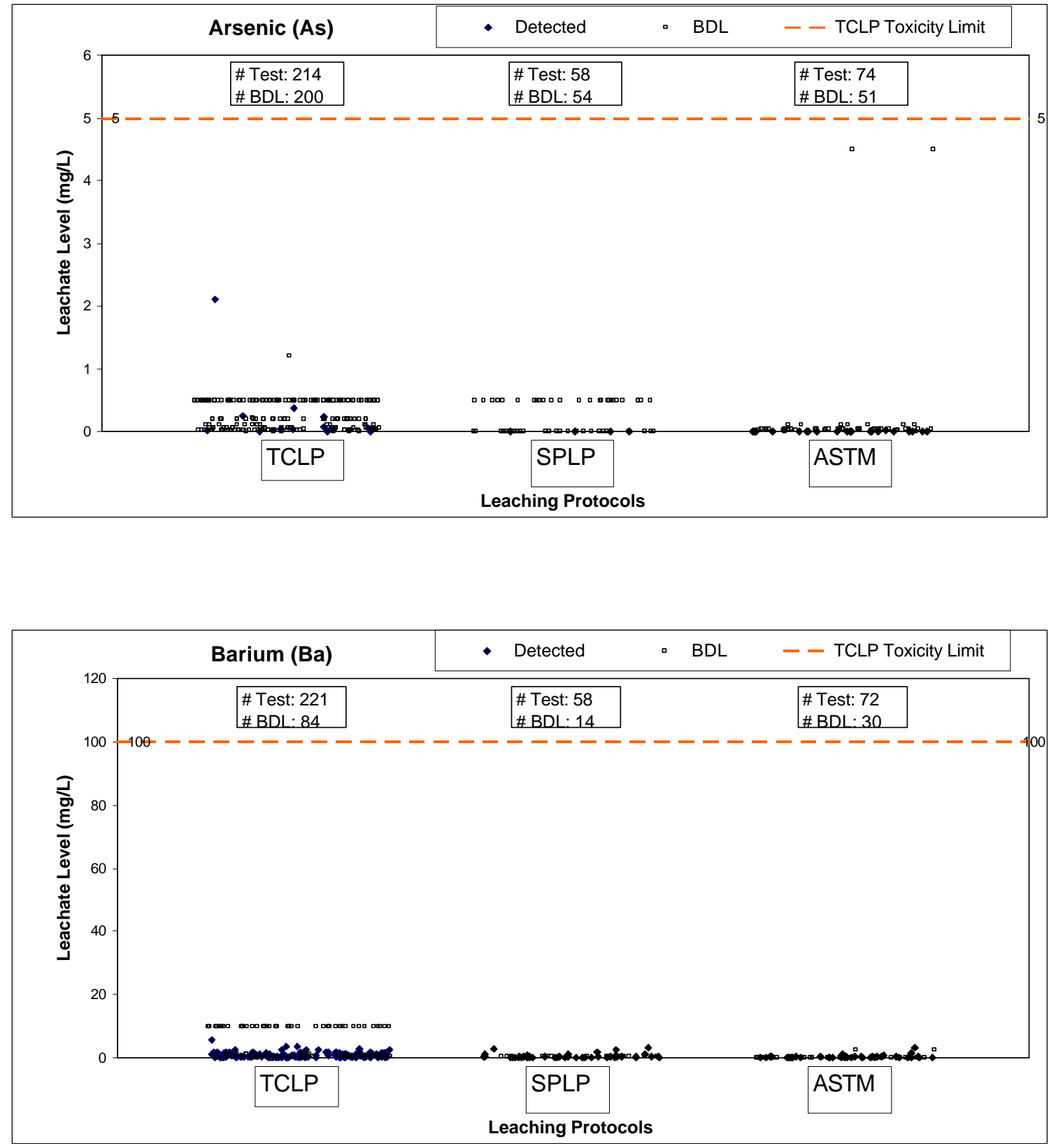

E-3 

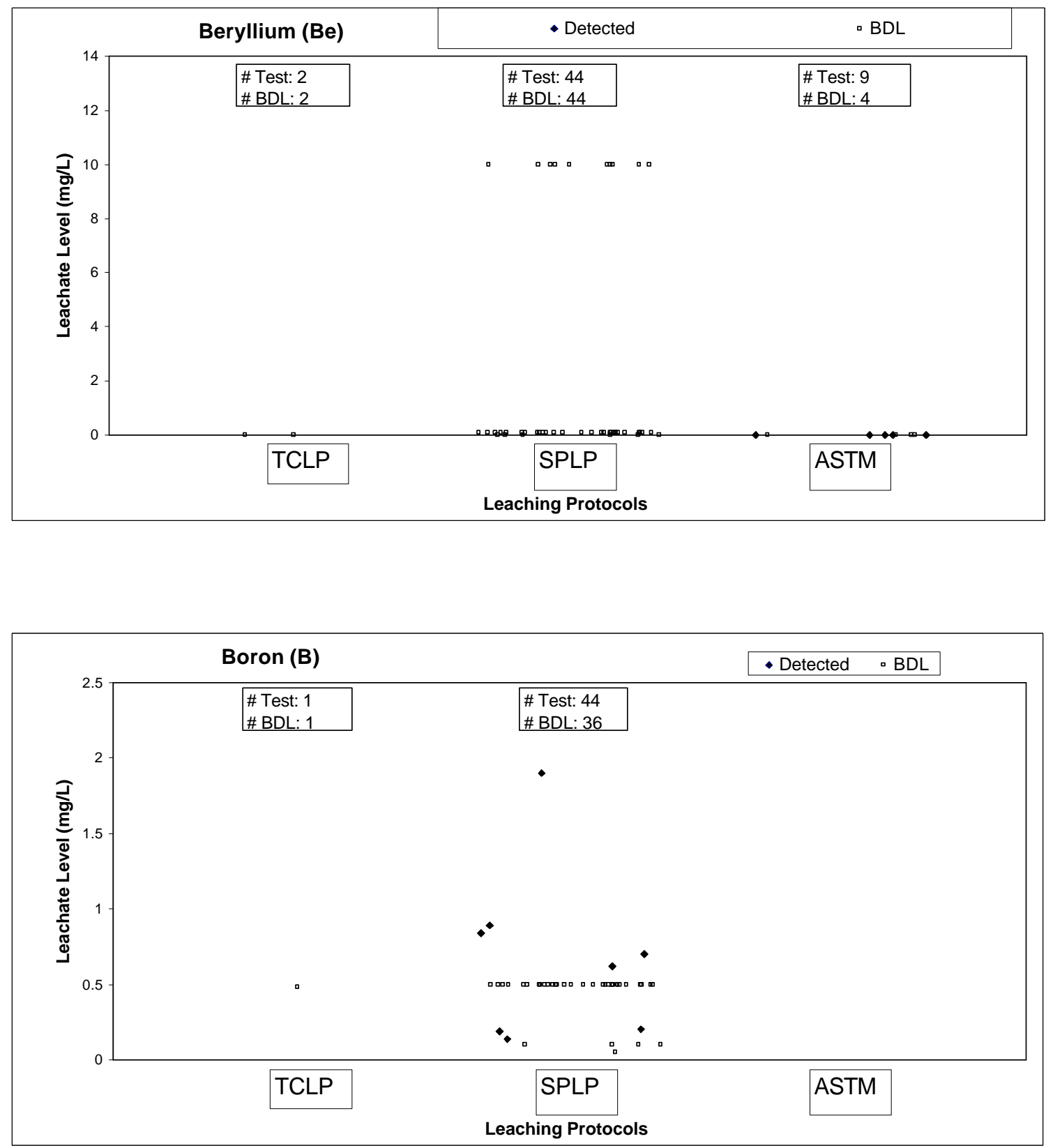

E-4 

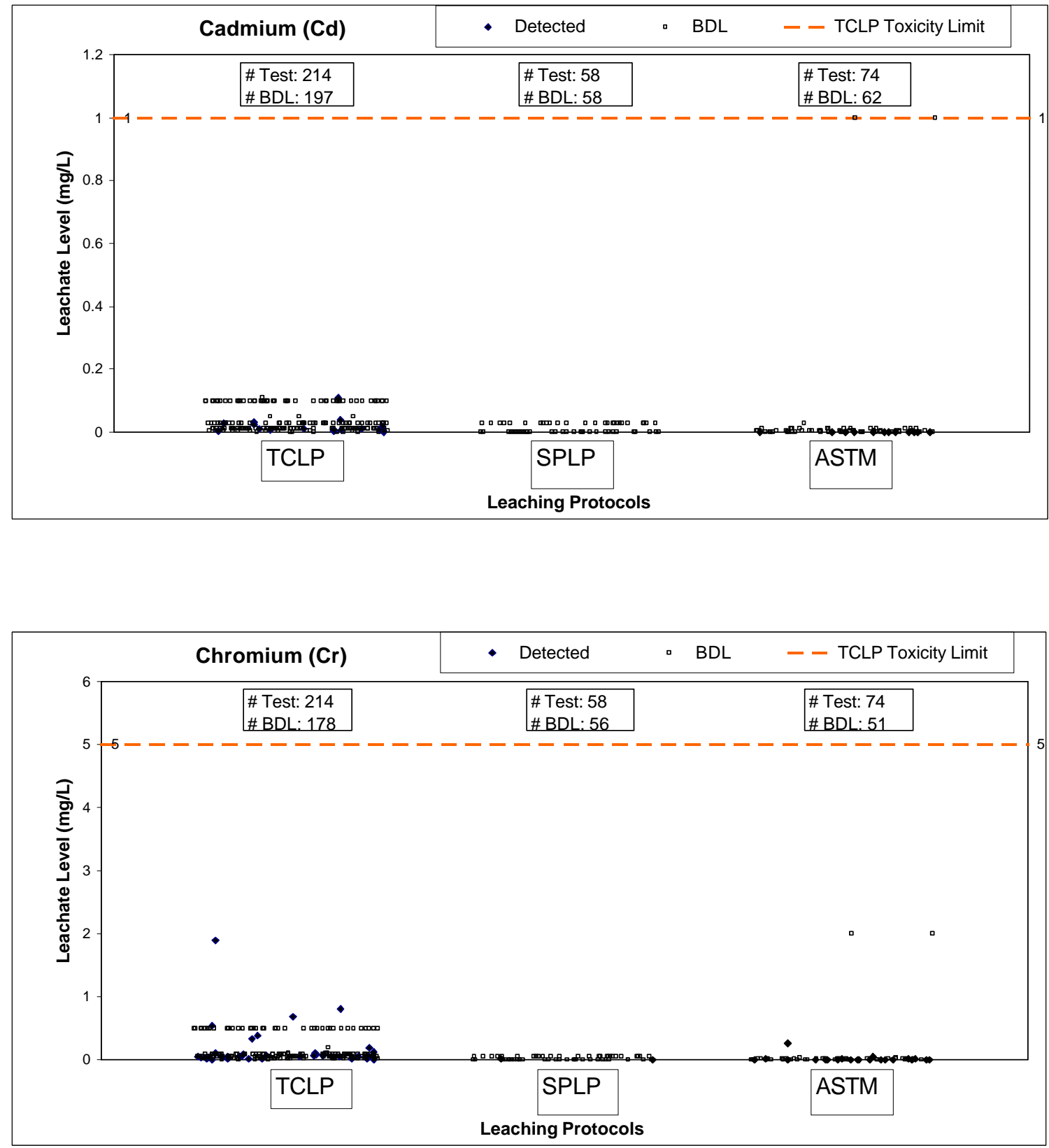

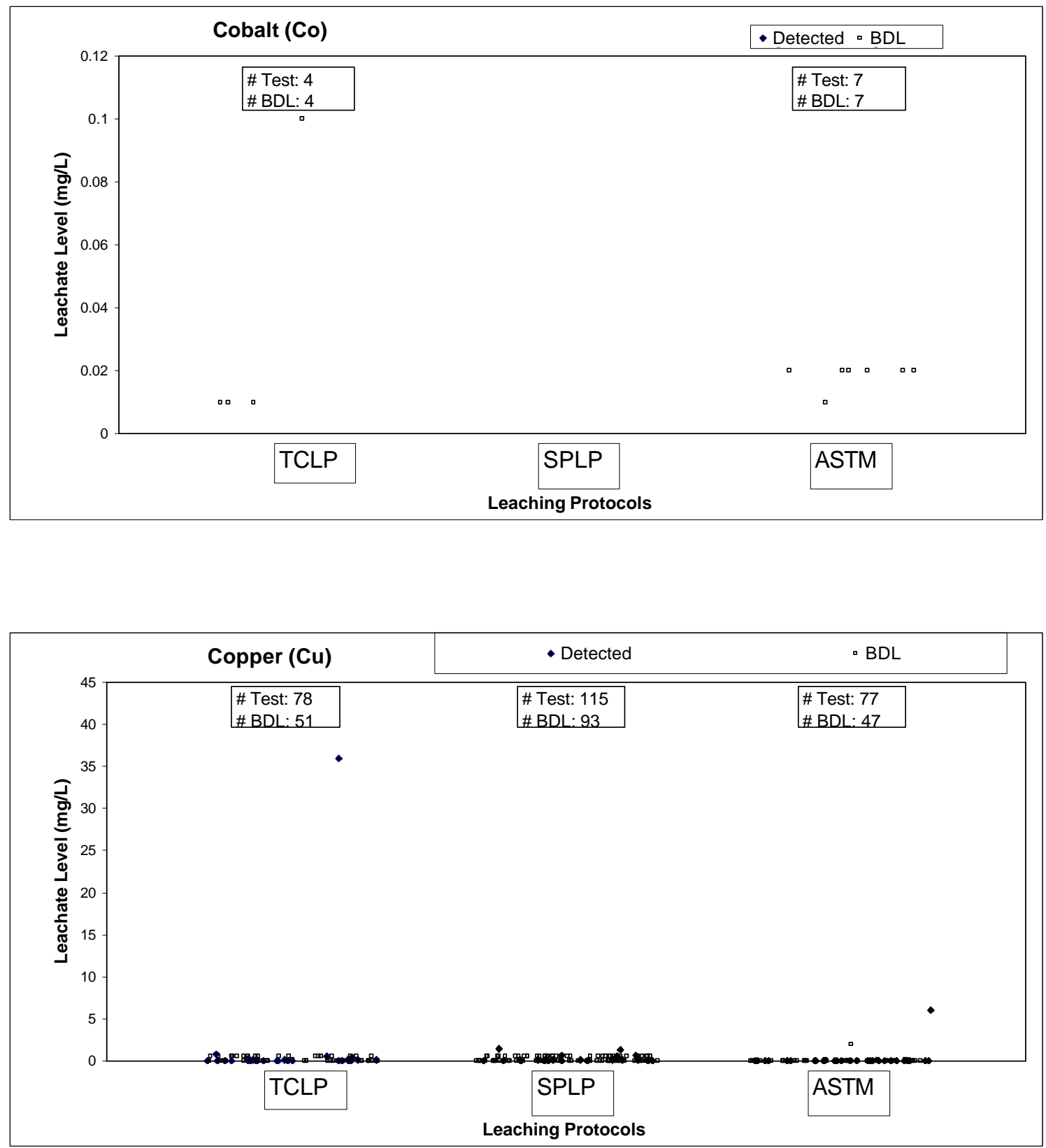

E-6 

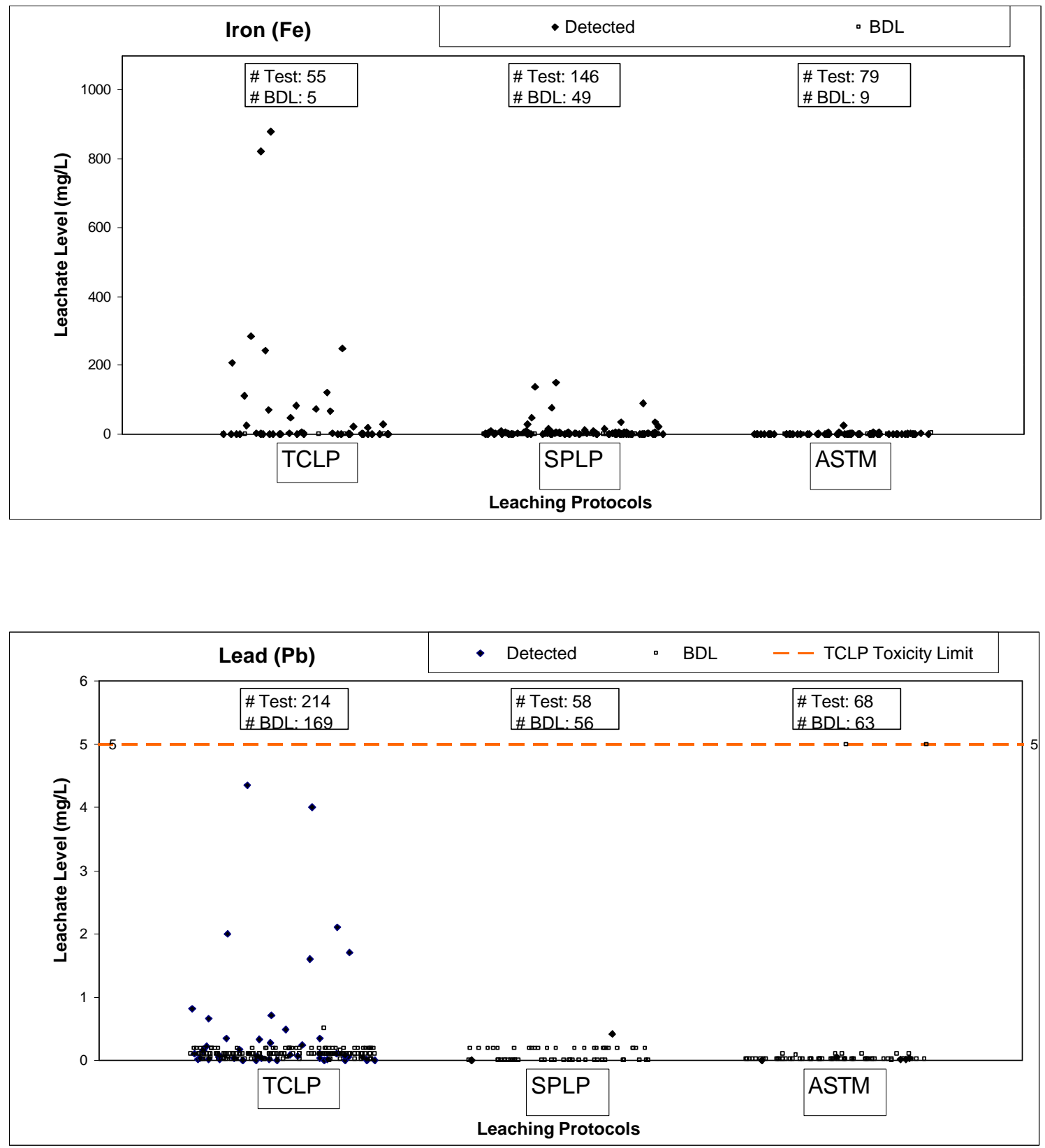

E-7 

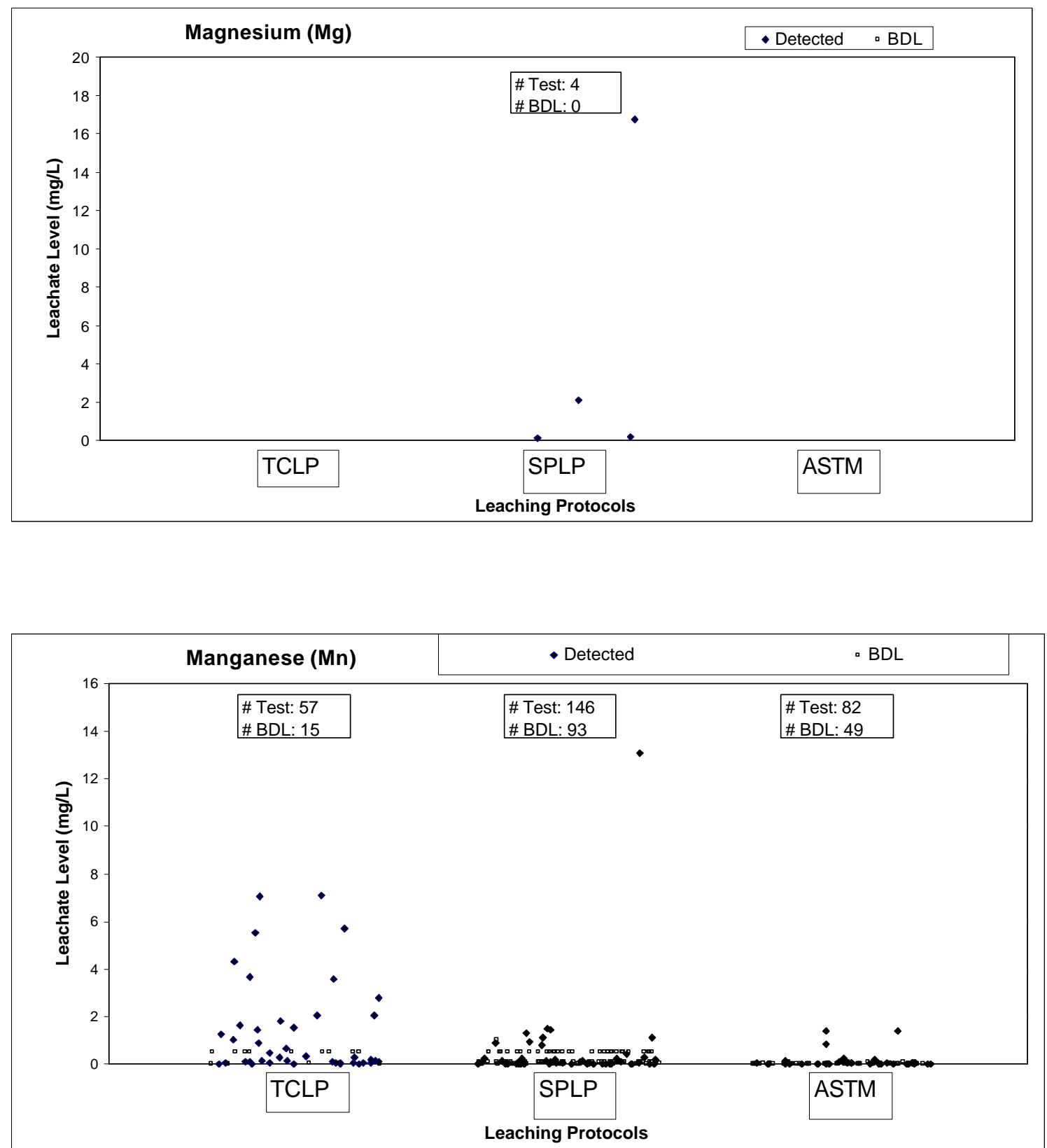

E-8 

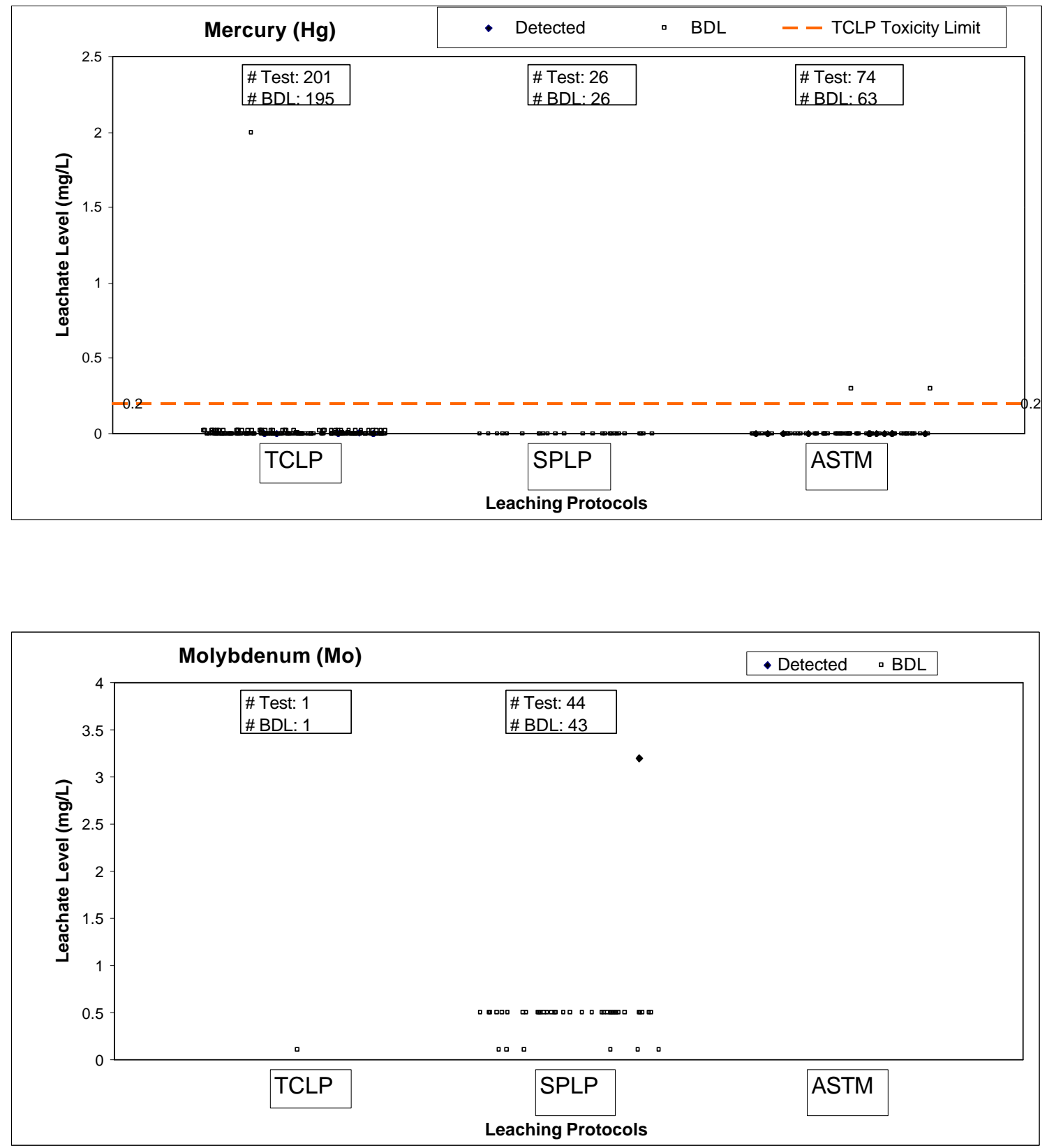

E-9 

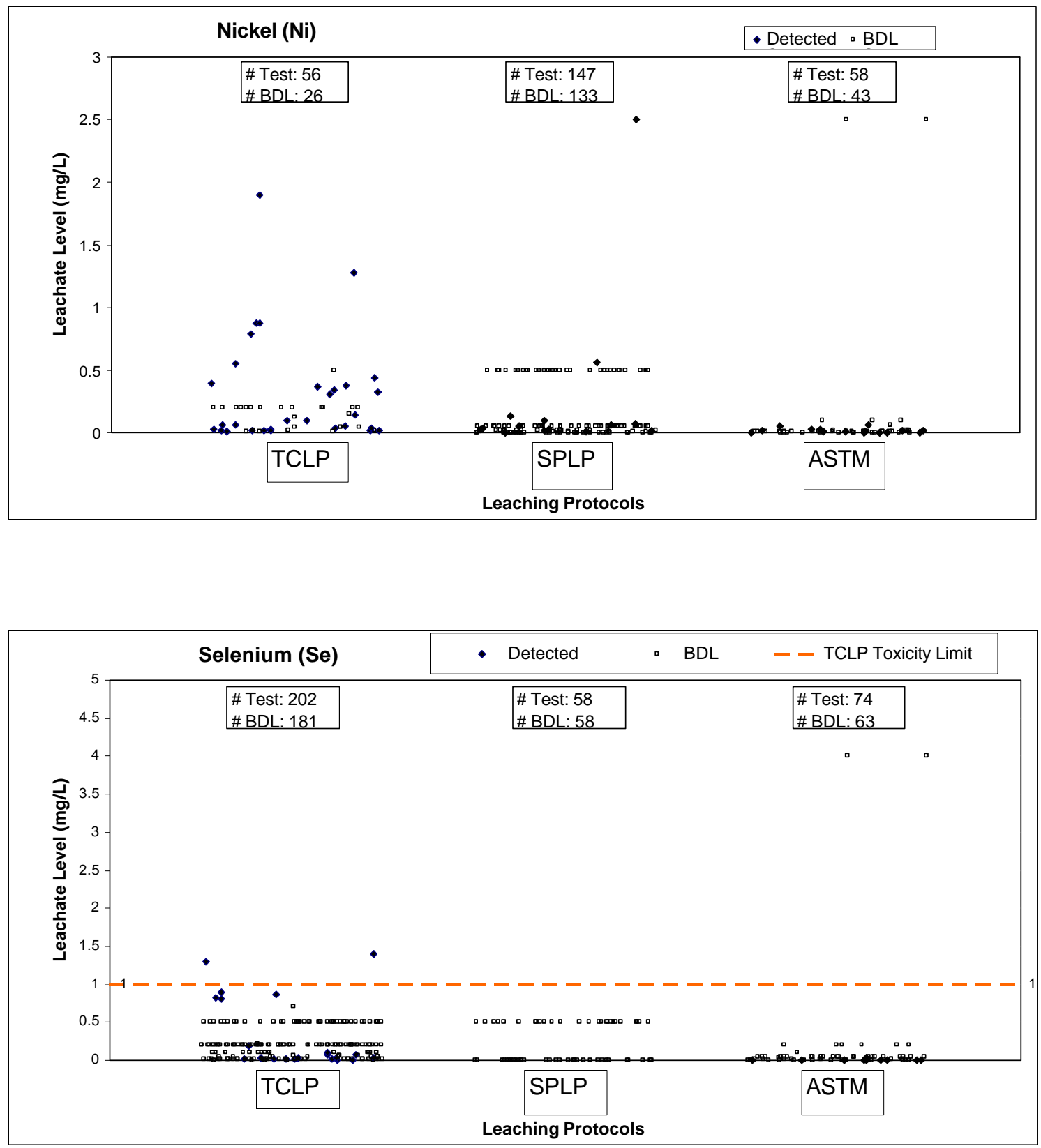

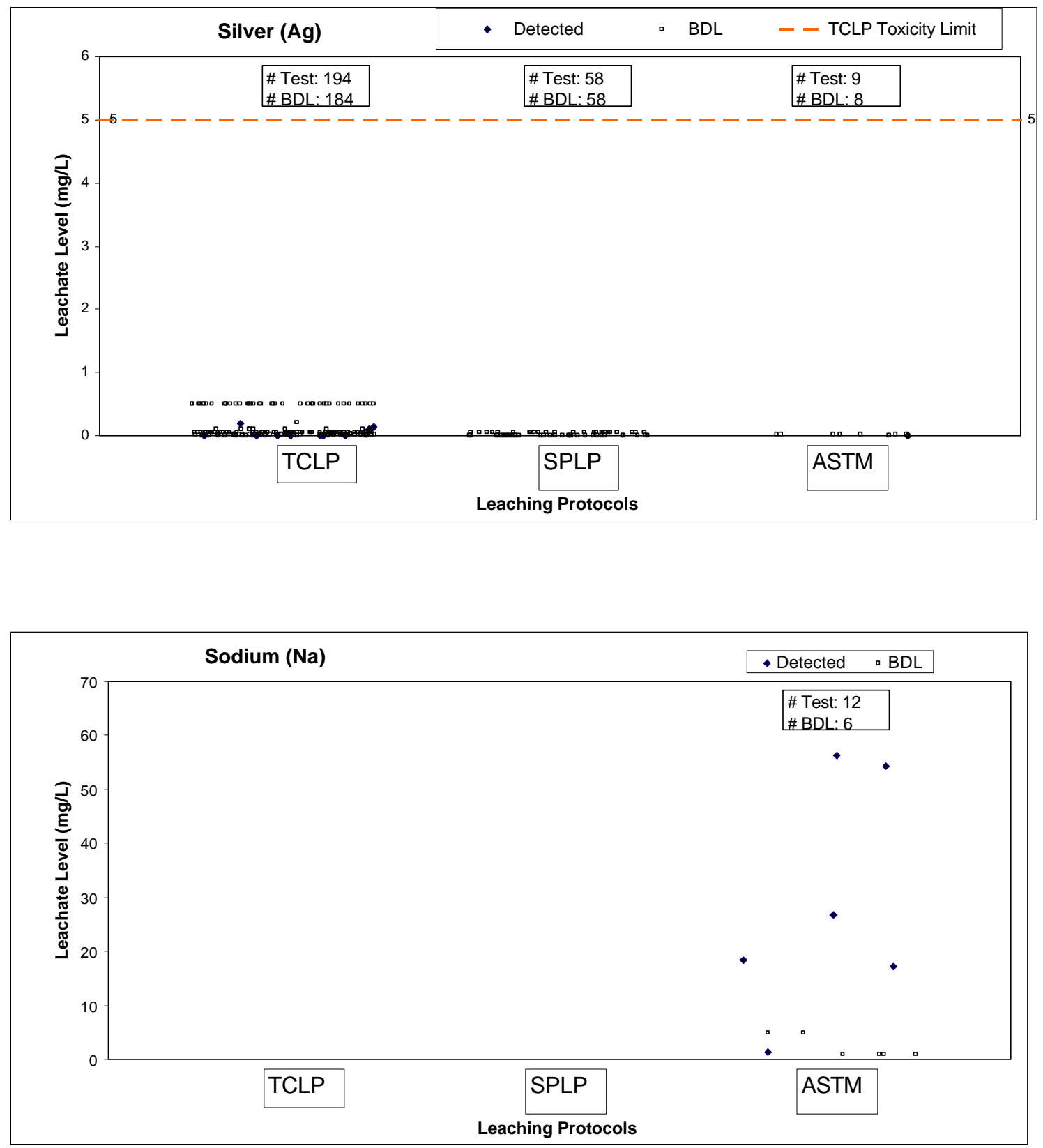

E-11 

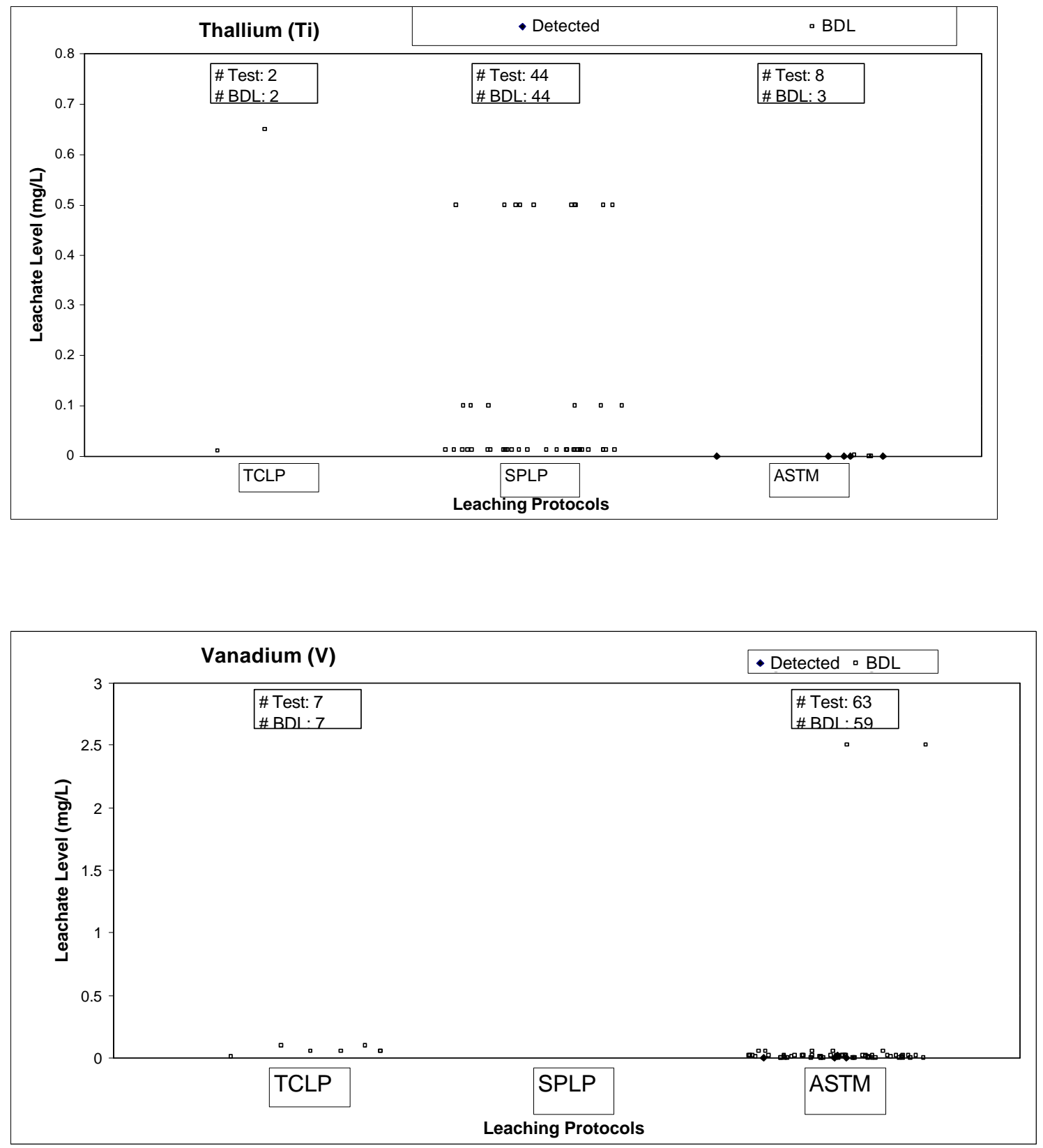


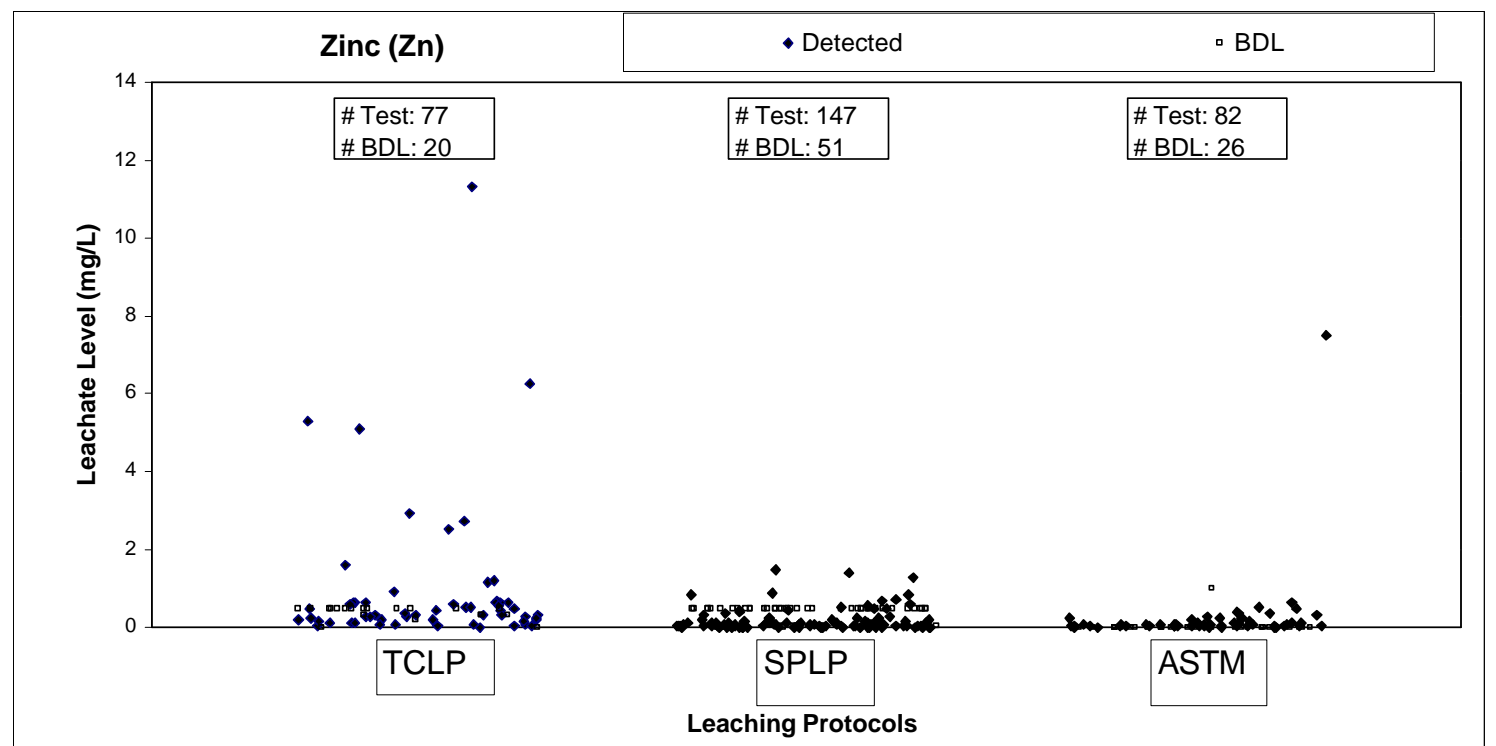

Figure E-1: Leachate concentration of metallic elements per leaching protocol (23 charts)

\section{E.2 General Chemical Parameters}

Following a series of 21 charts, grouped into Figure E-2, present the concentration distribution for 21 general chemical parameters in sand leachate per leaching protocol. 

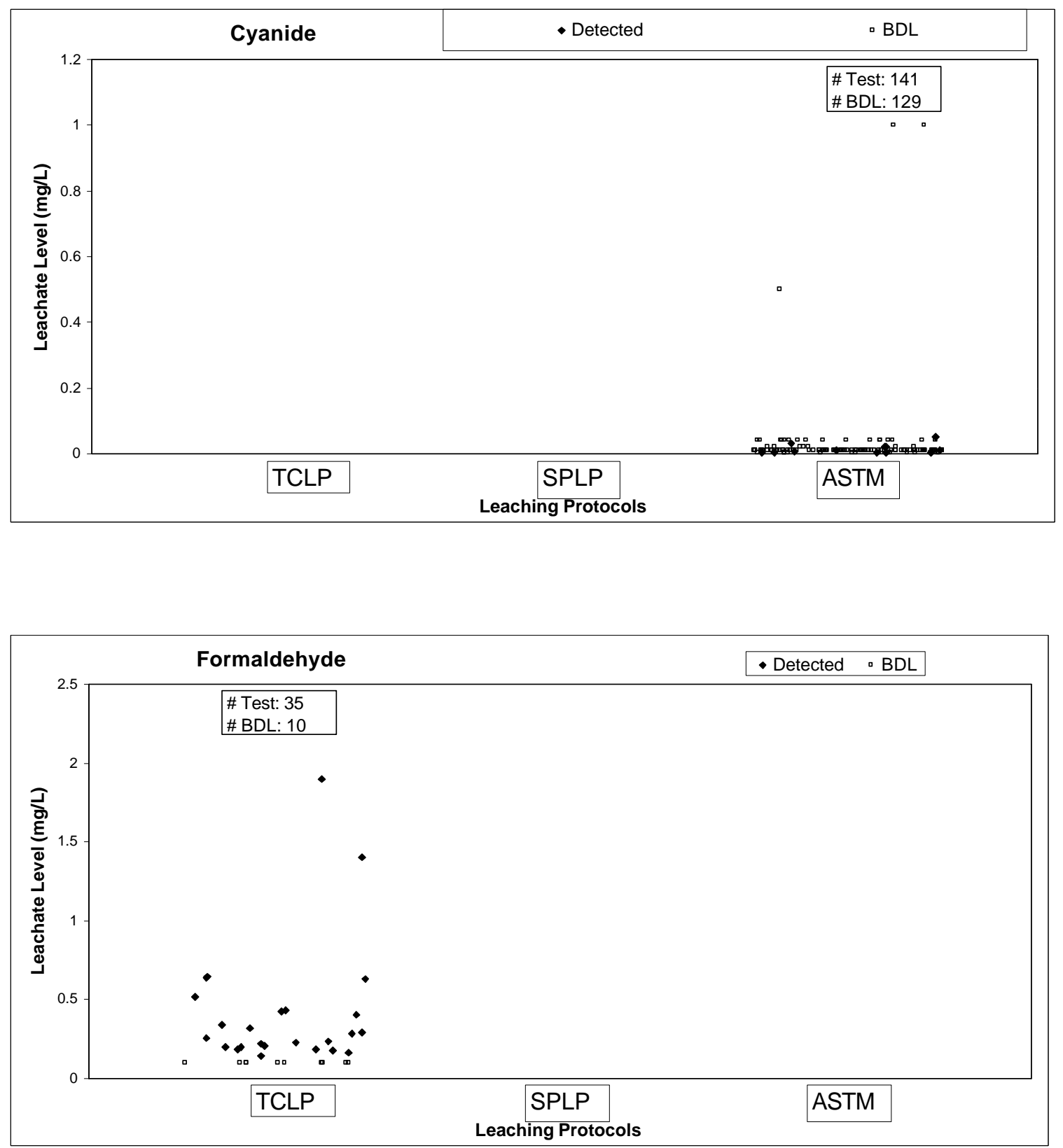

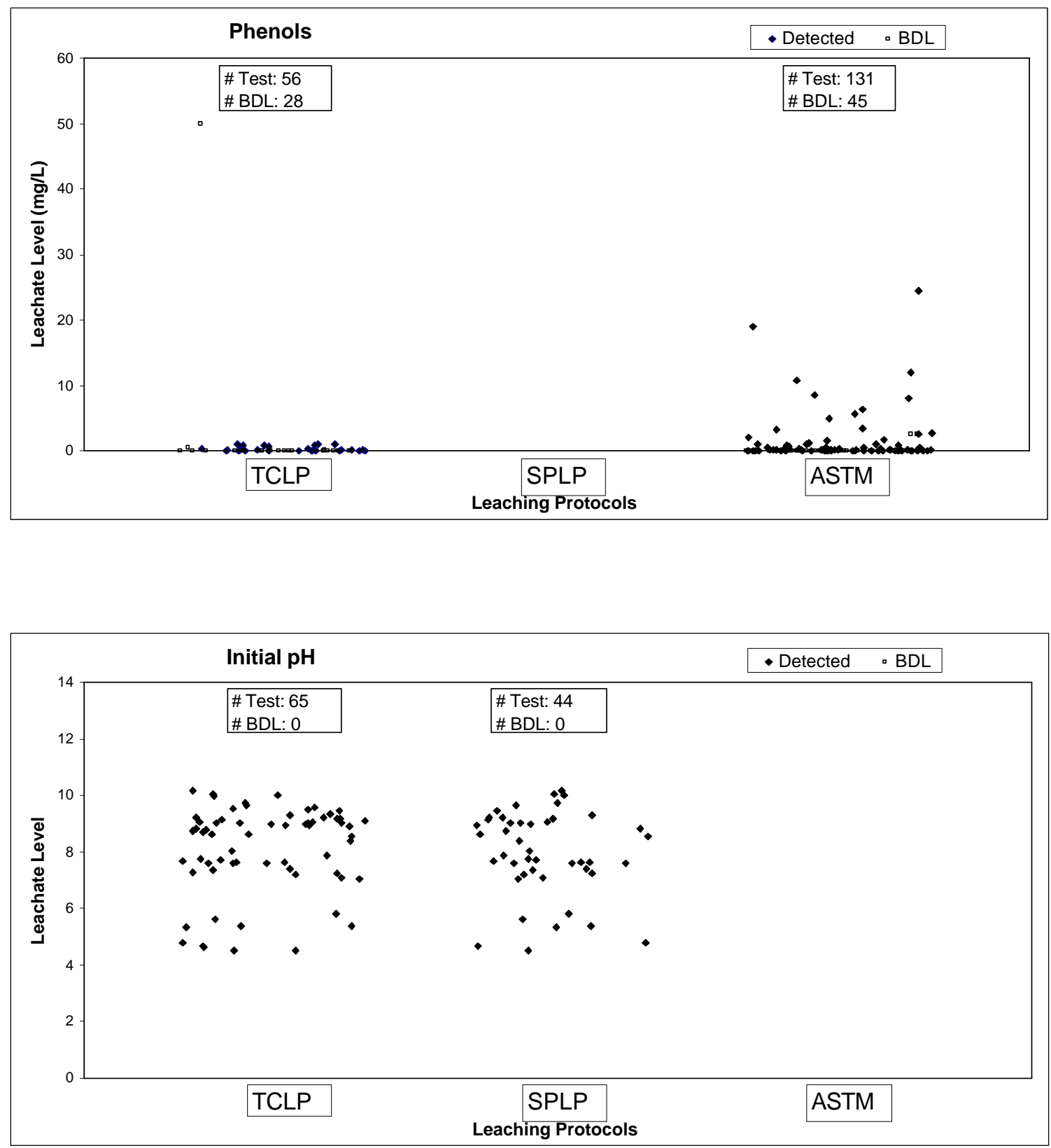


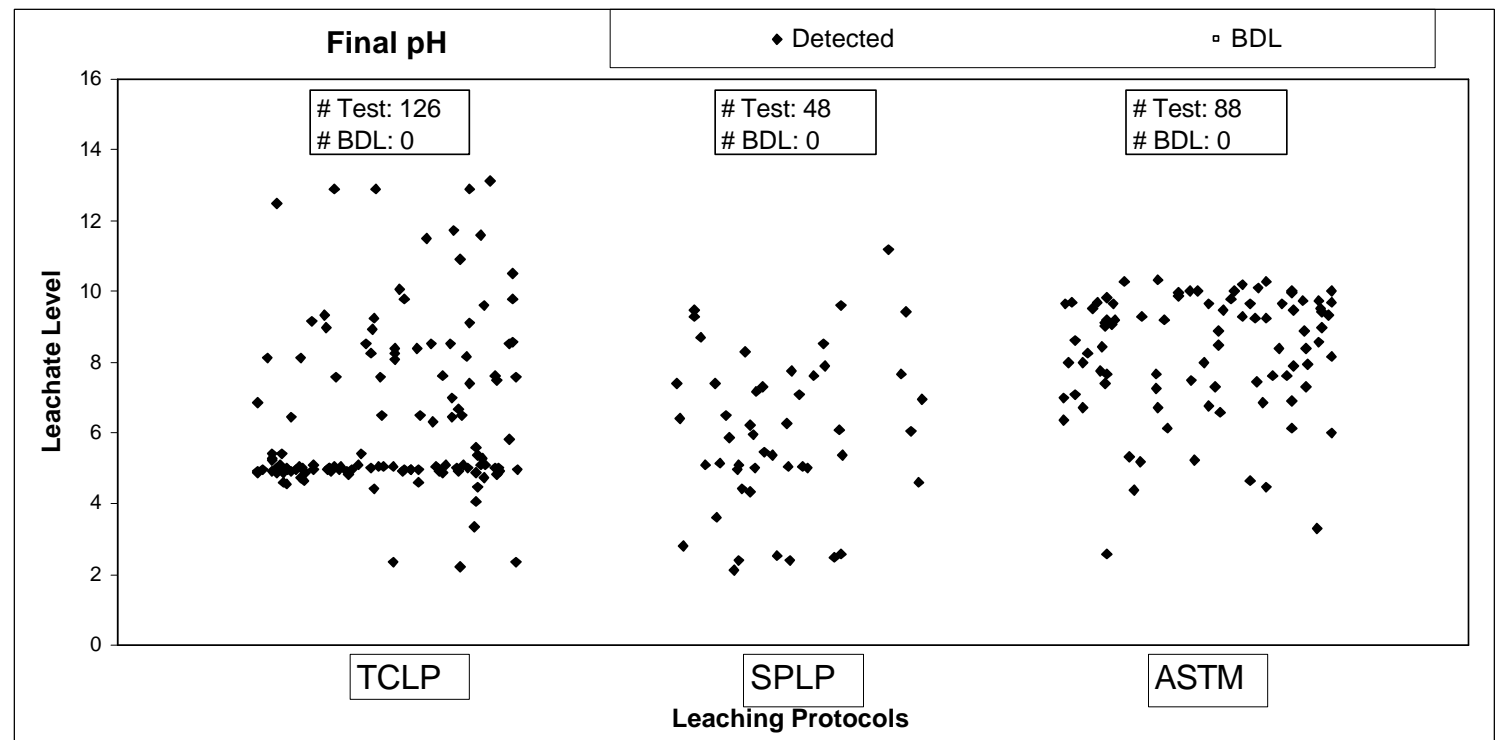

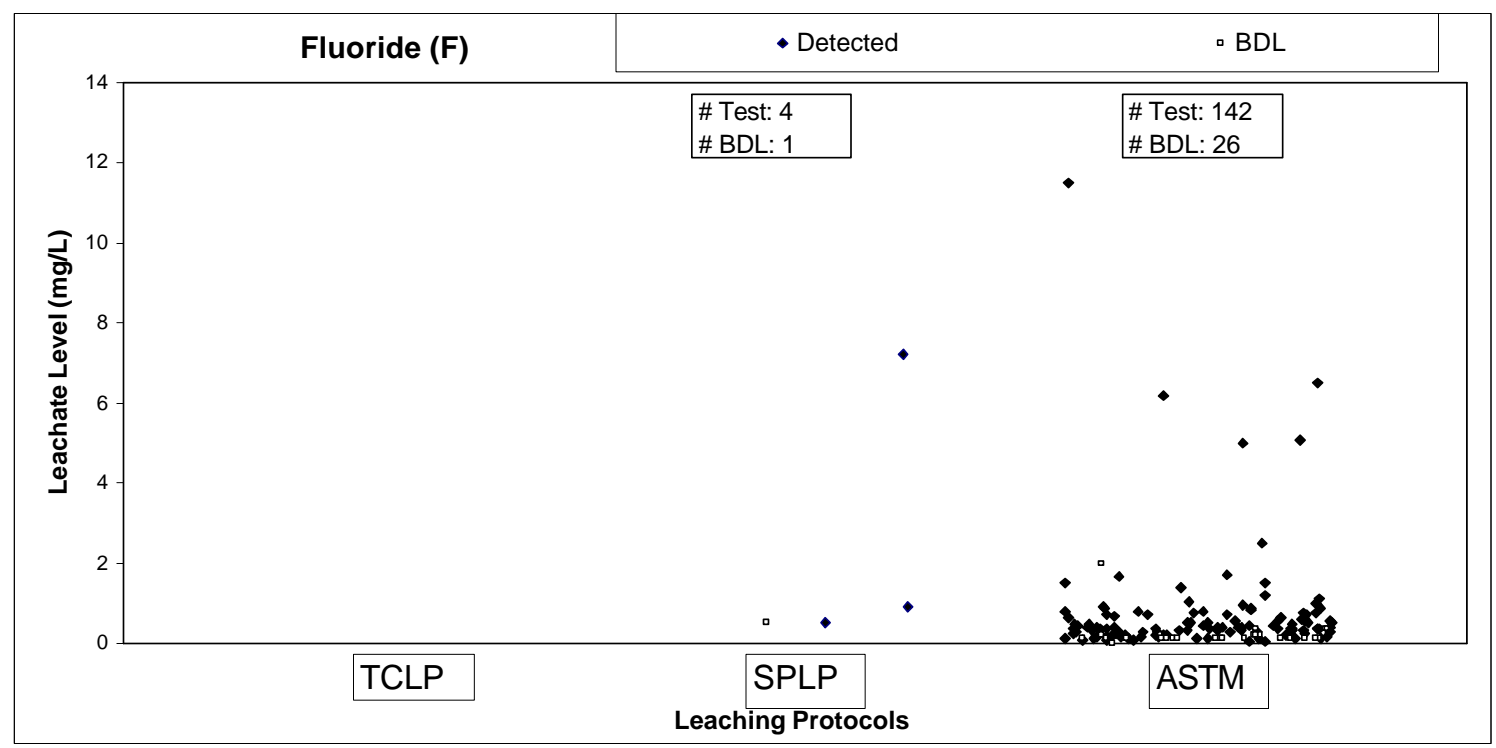



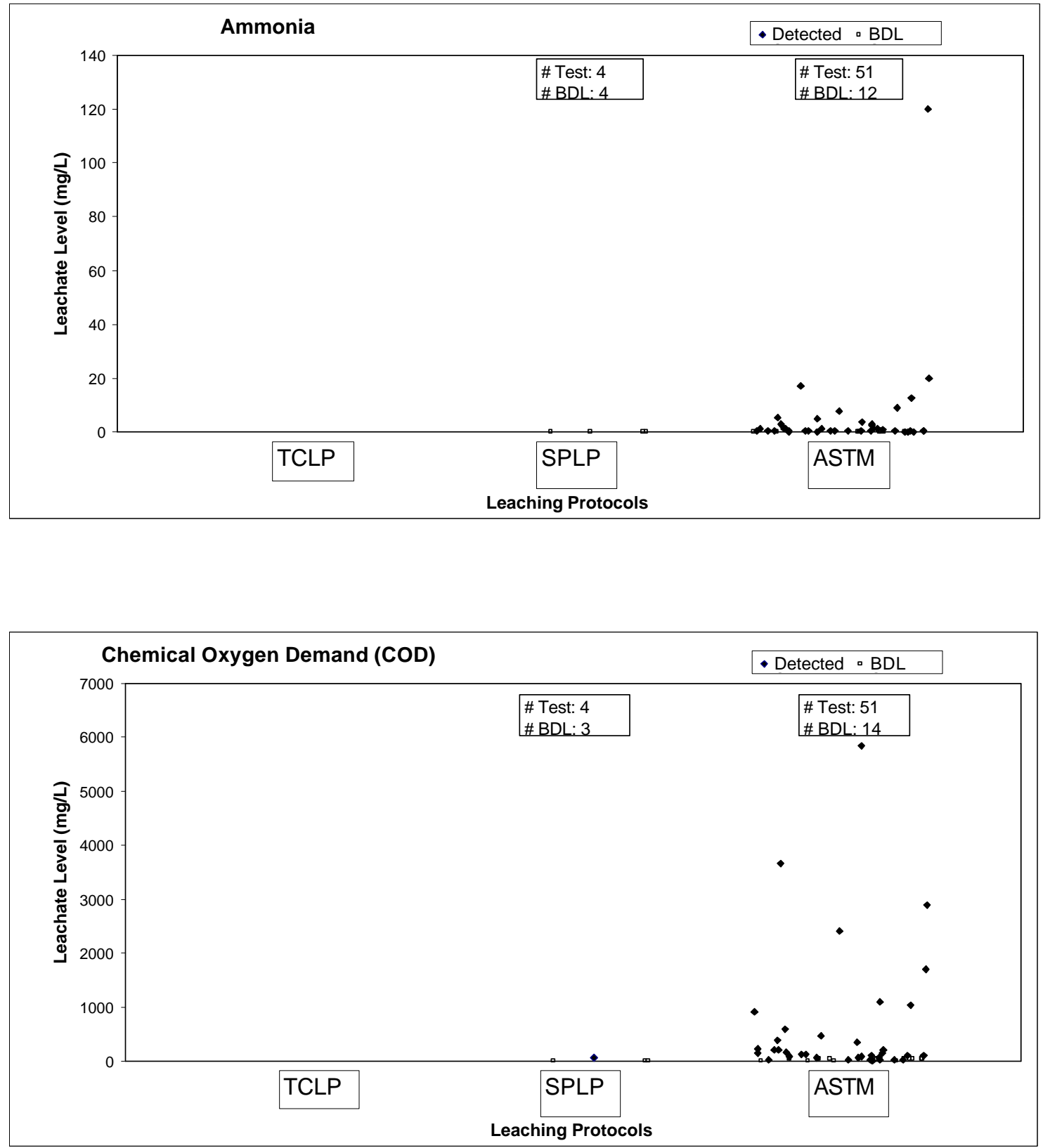

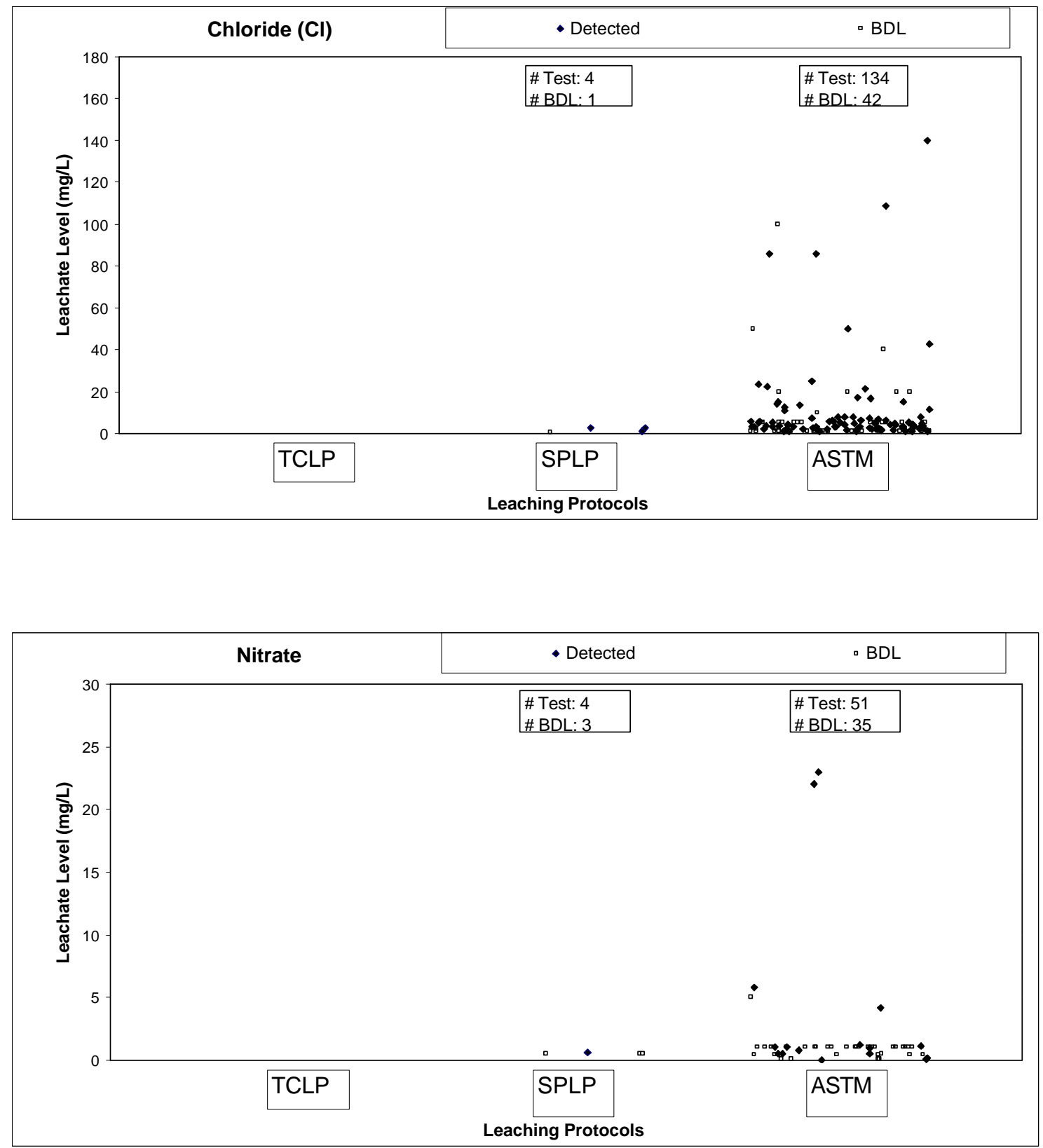

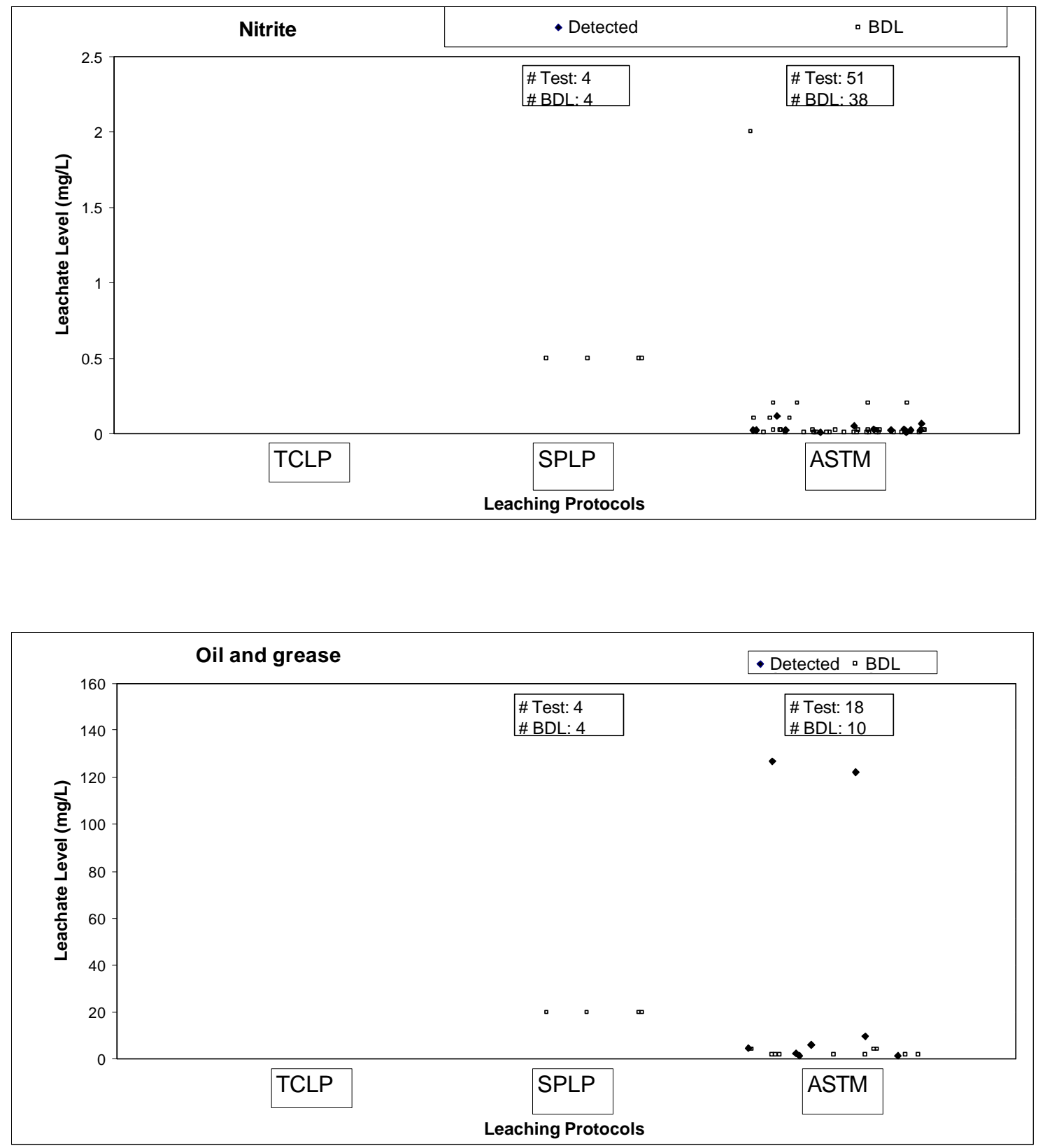

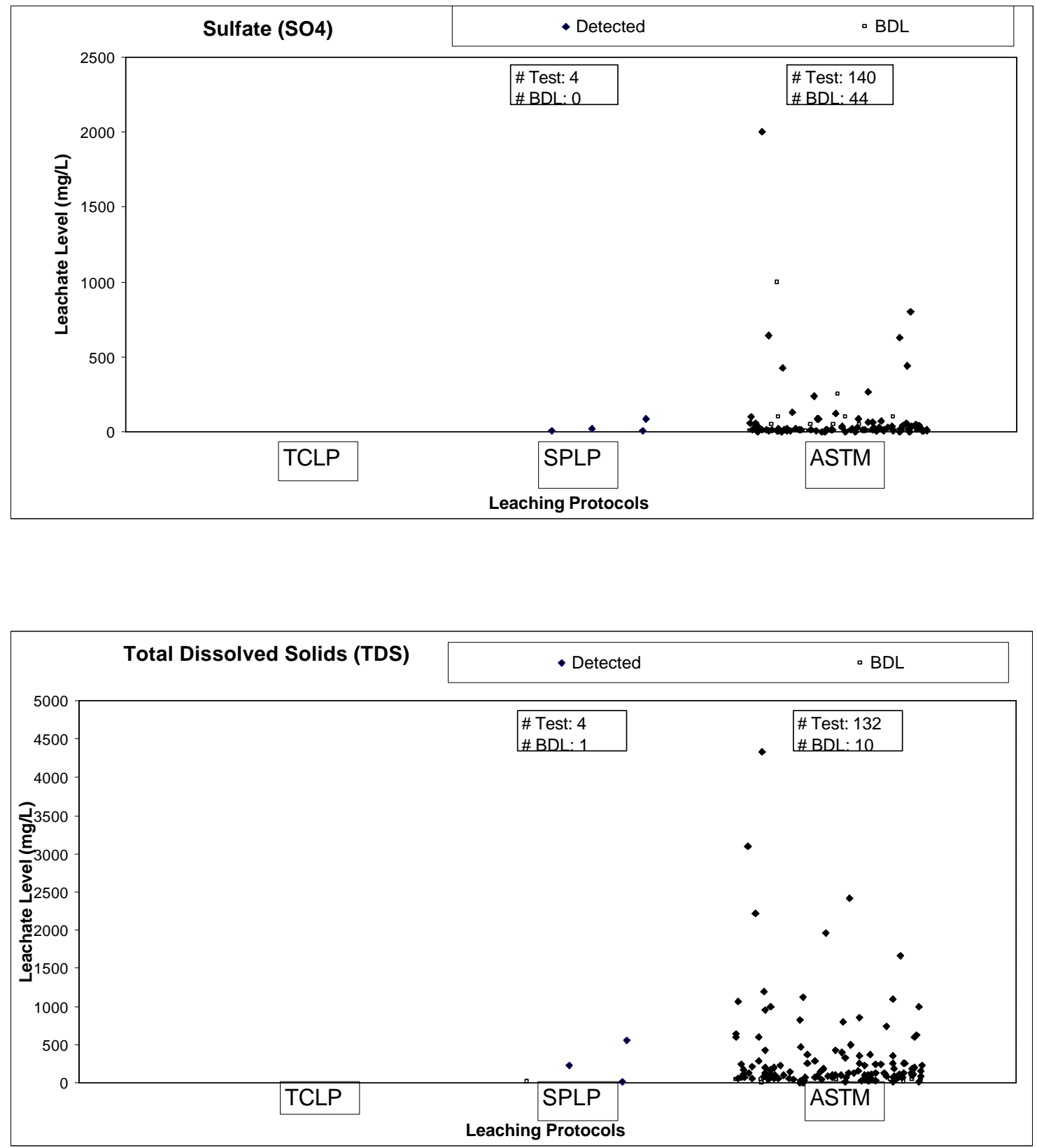

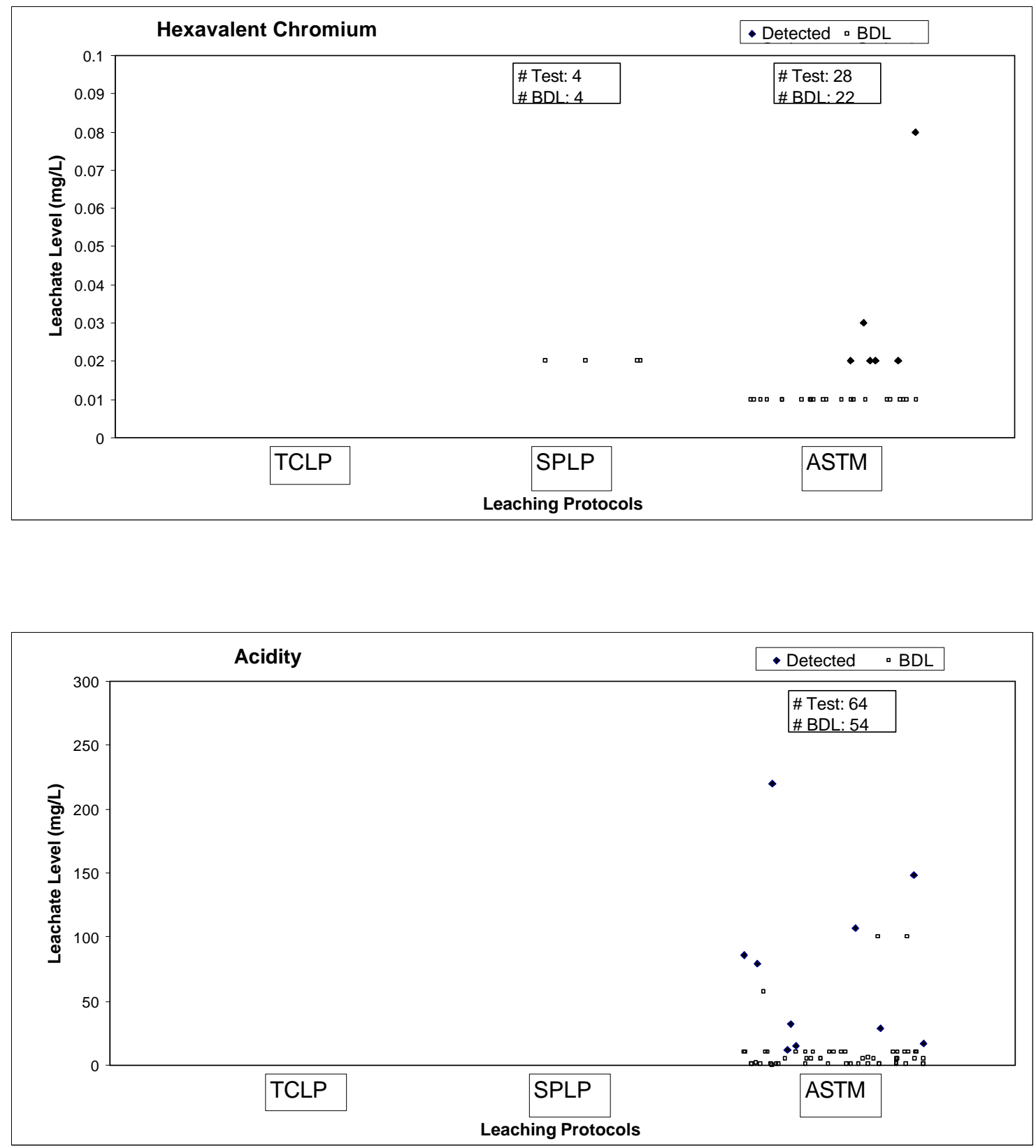

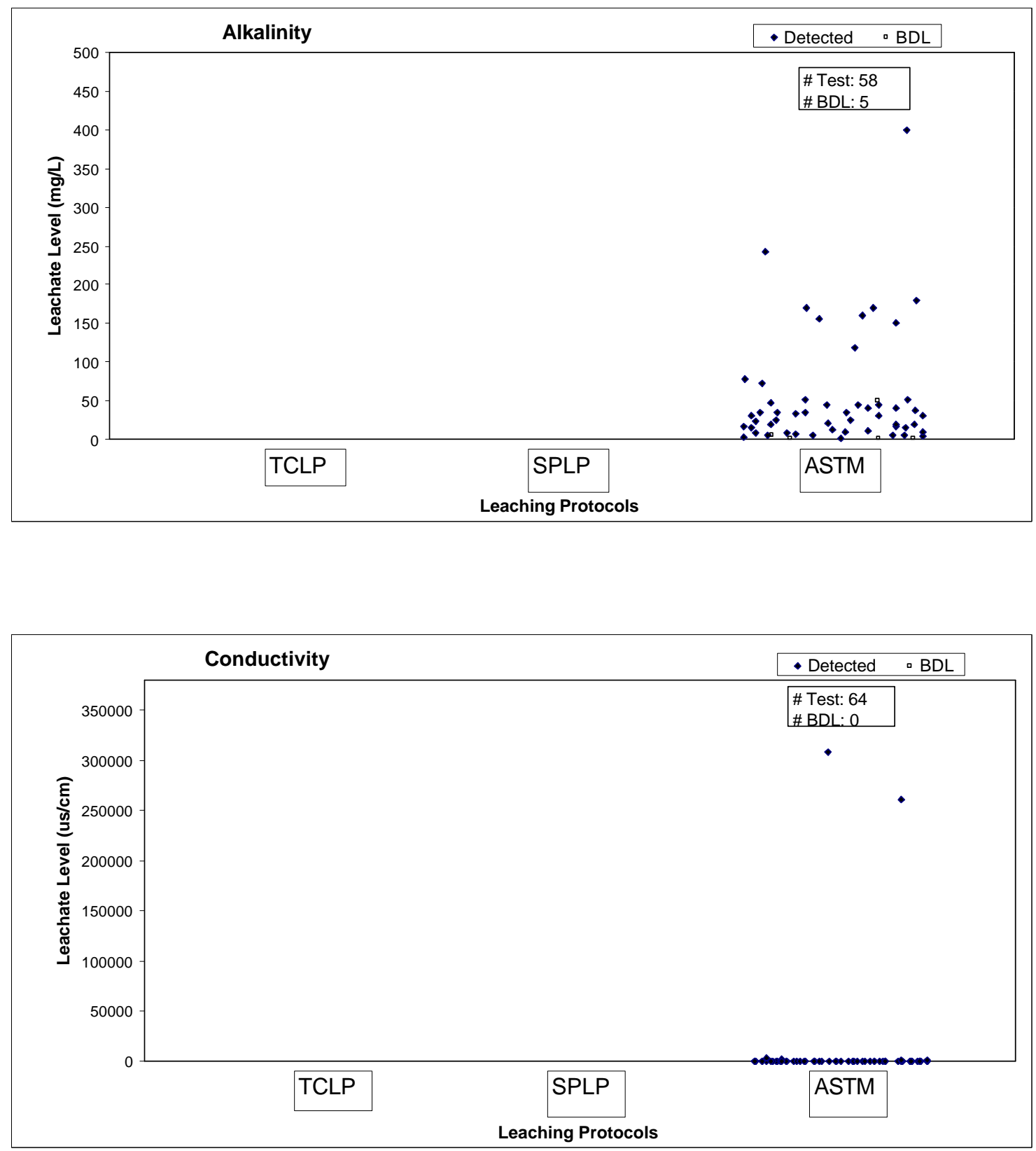

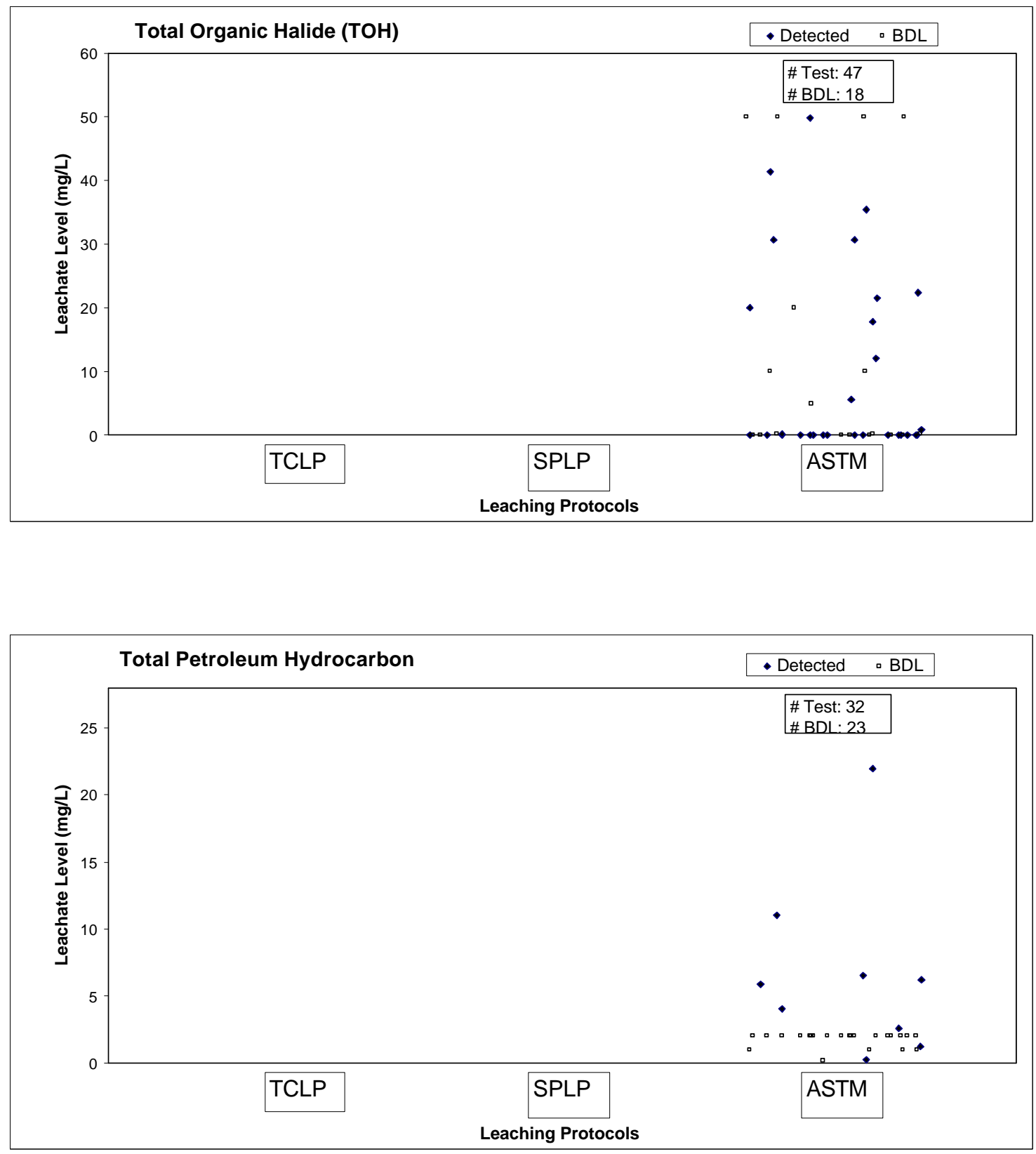


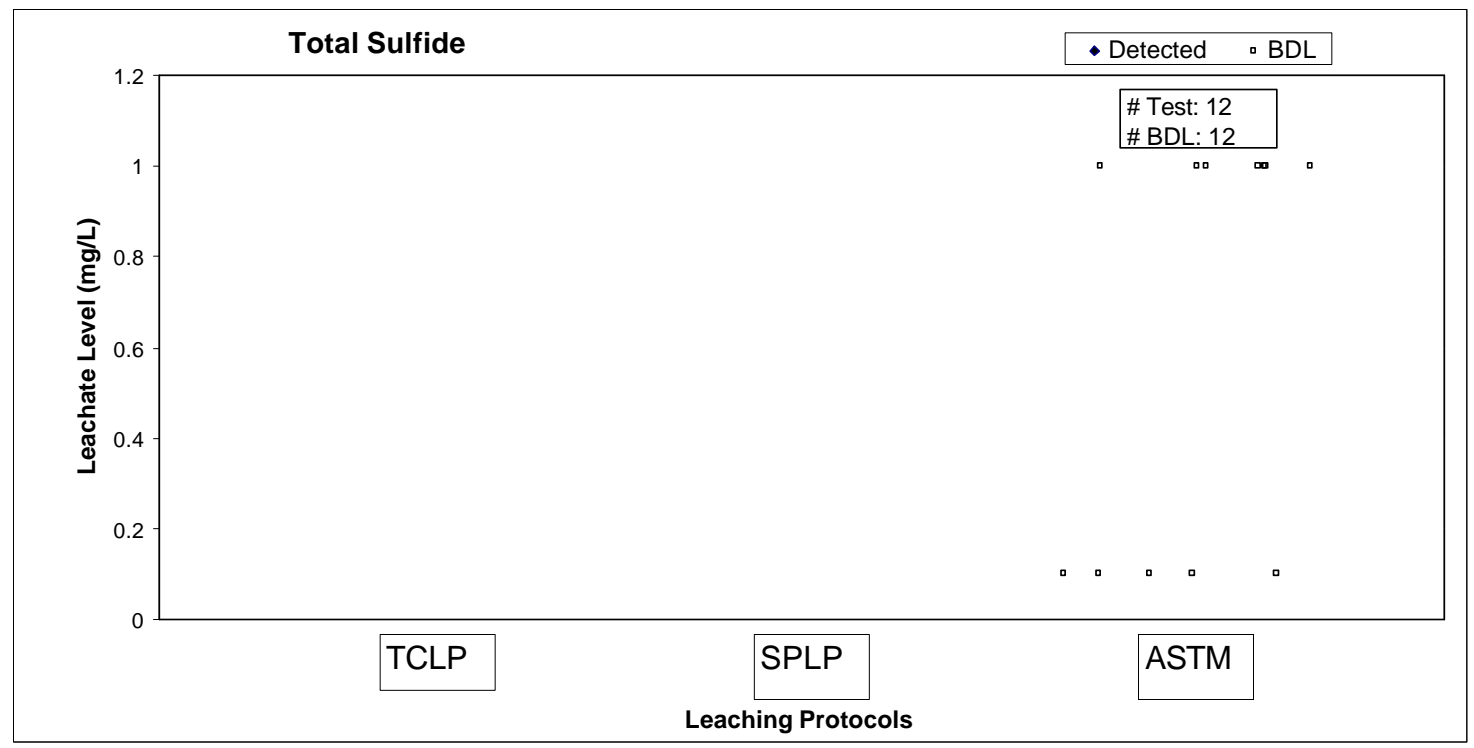

Figure E-2: Leachate concentration of general chemical parameters (21 charts)

\section{E.3 Organic Compounds}

Following a series of 37 charts, grouped into Figure E-3, present the concentration distribution for 37 organic compounds in TCLP leachate extracted from sand stream.

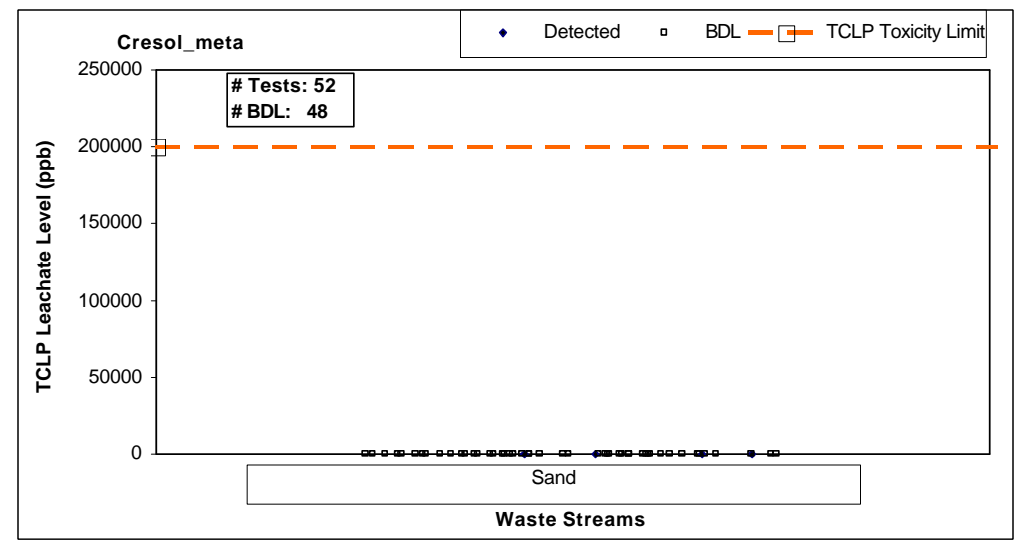



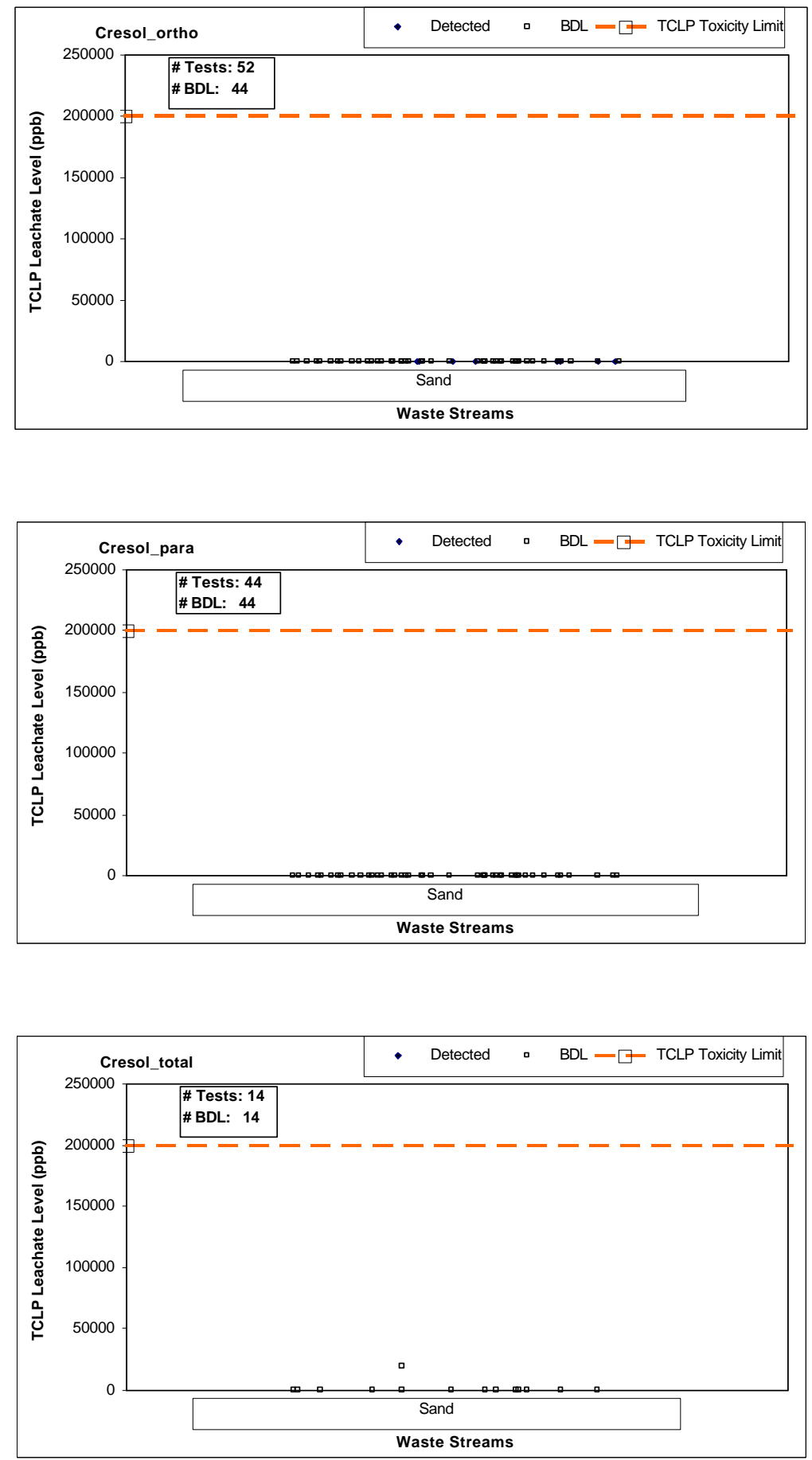

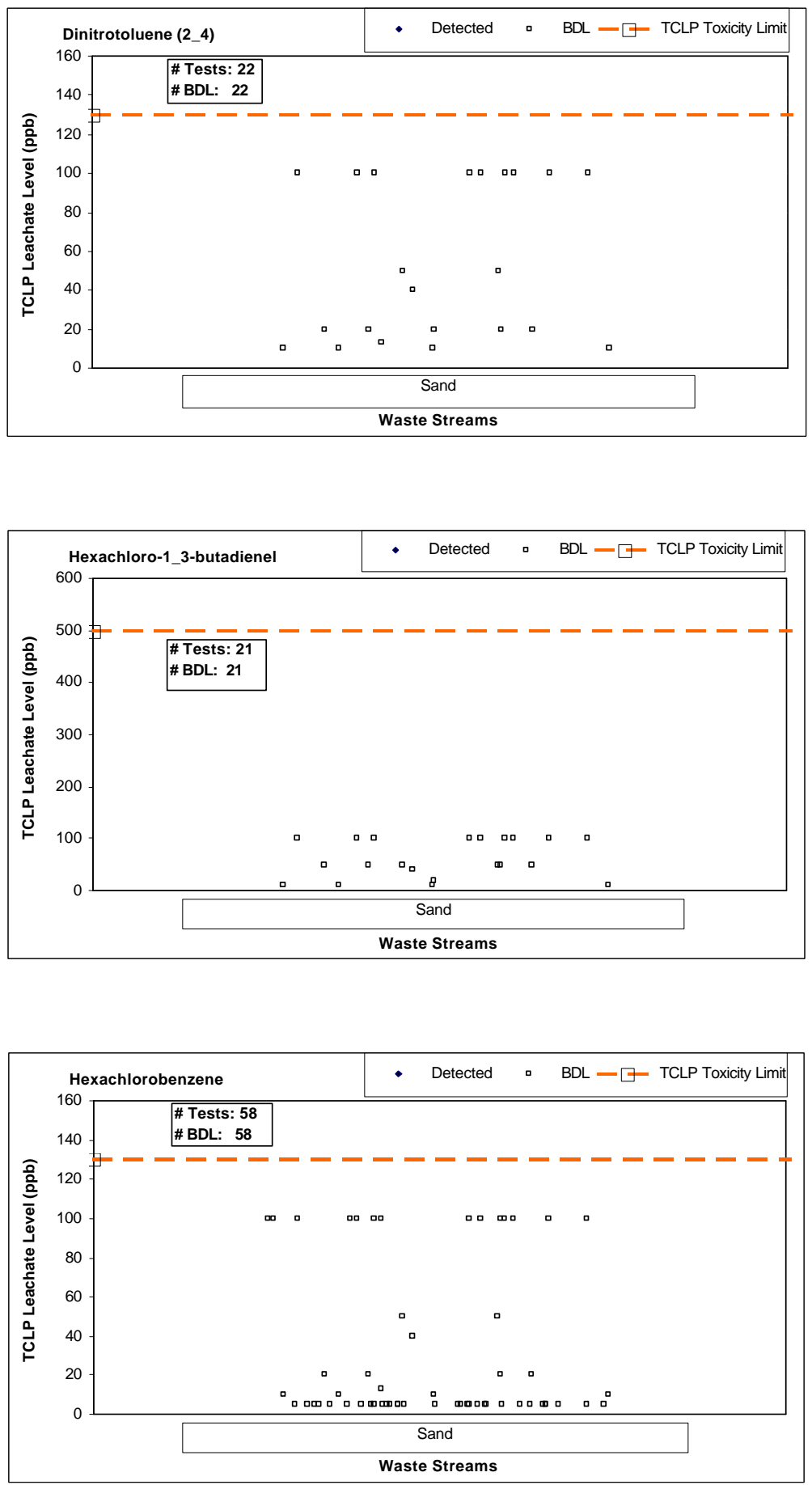

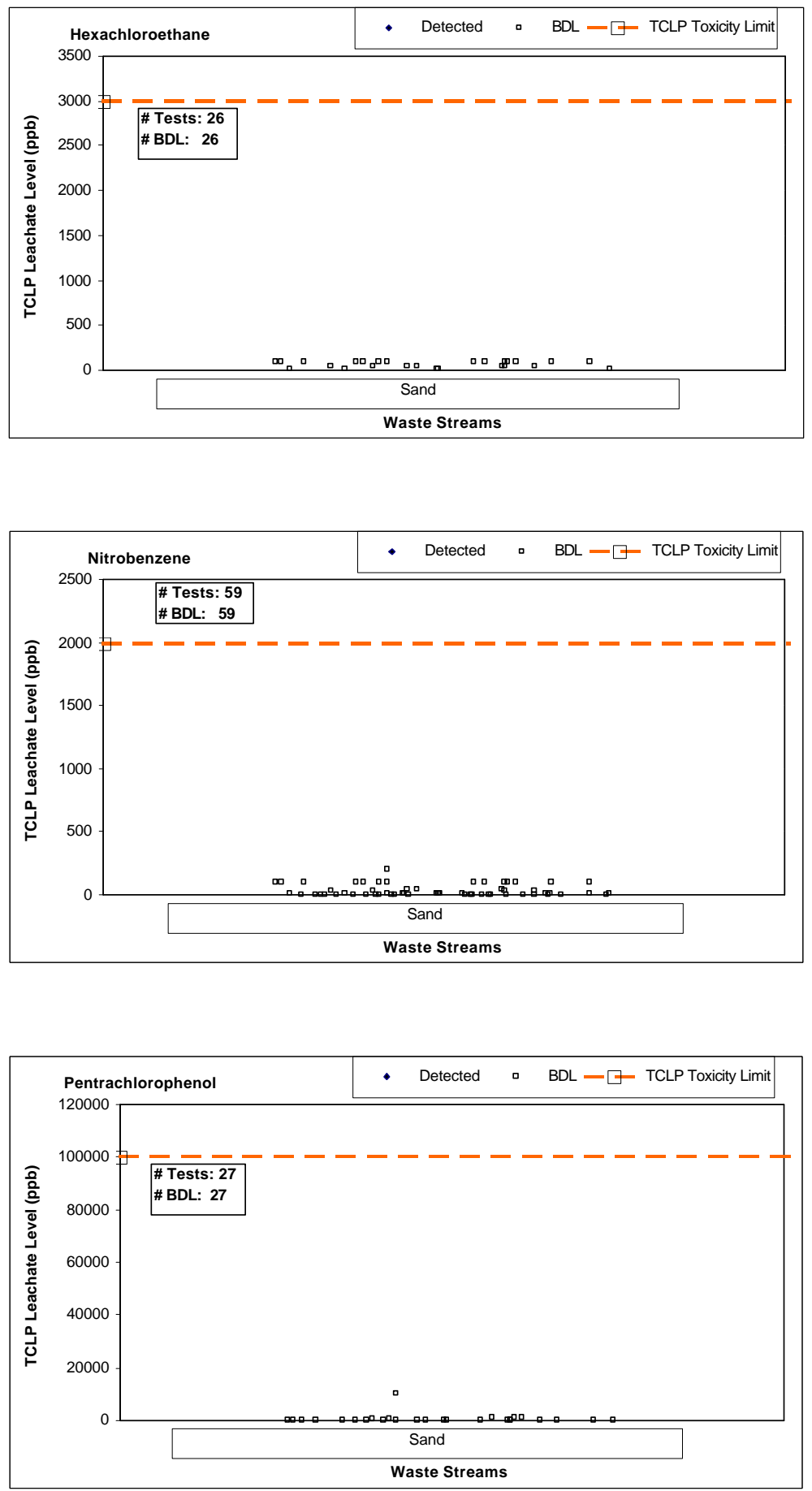

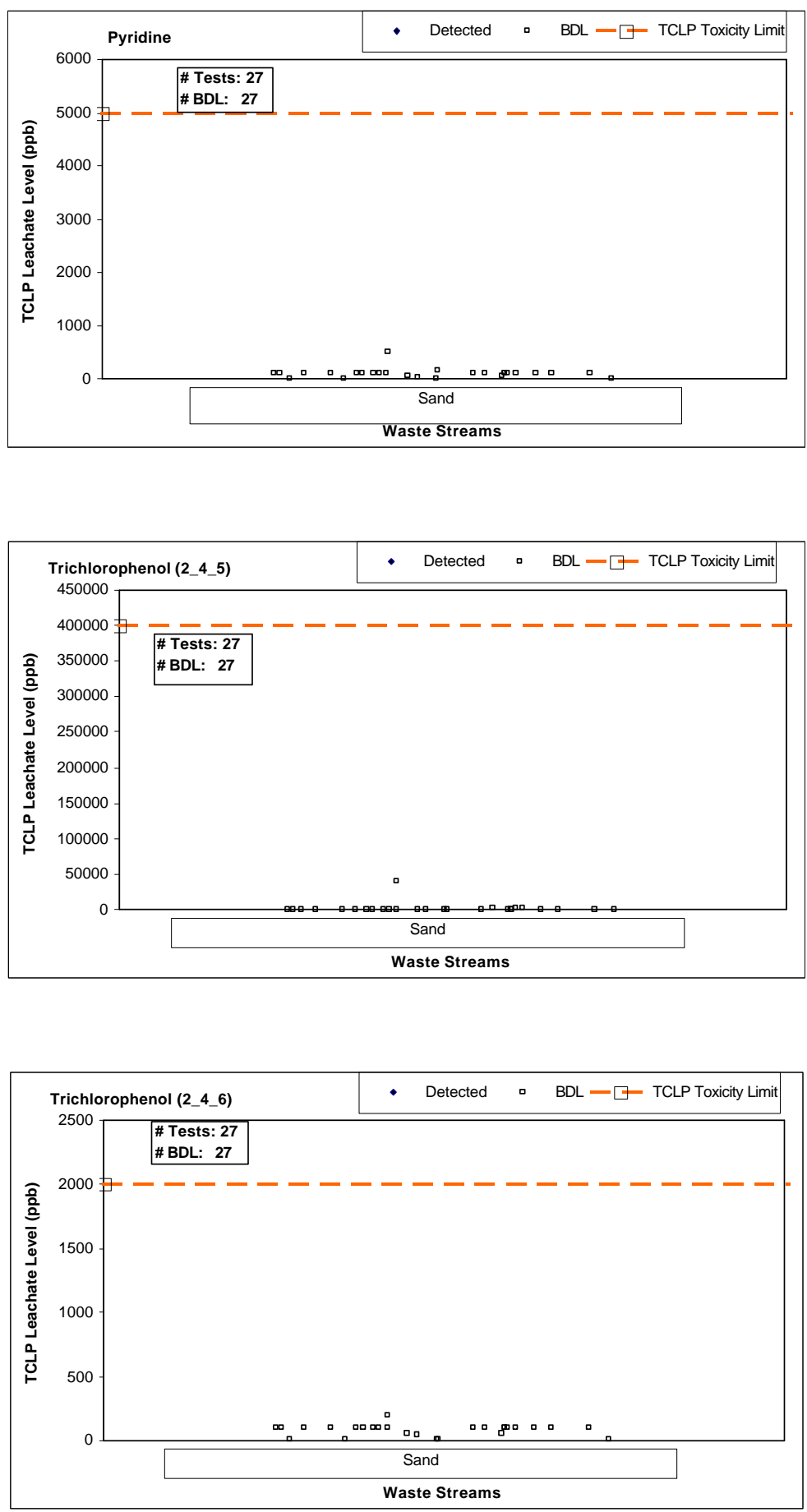

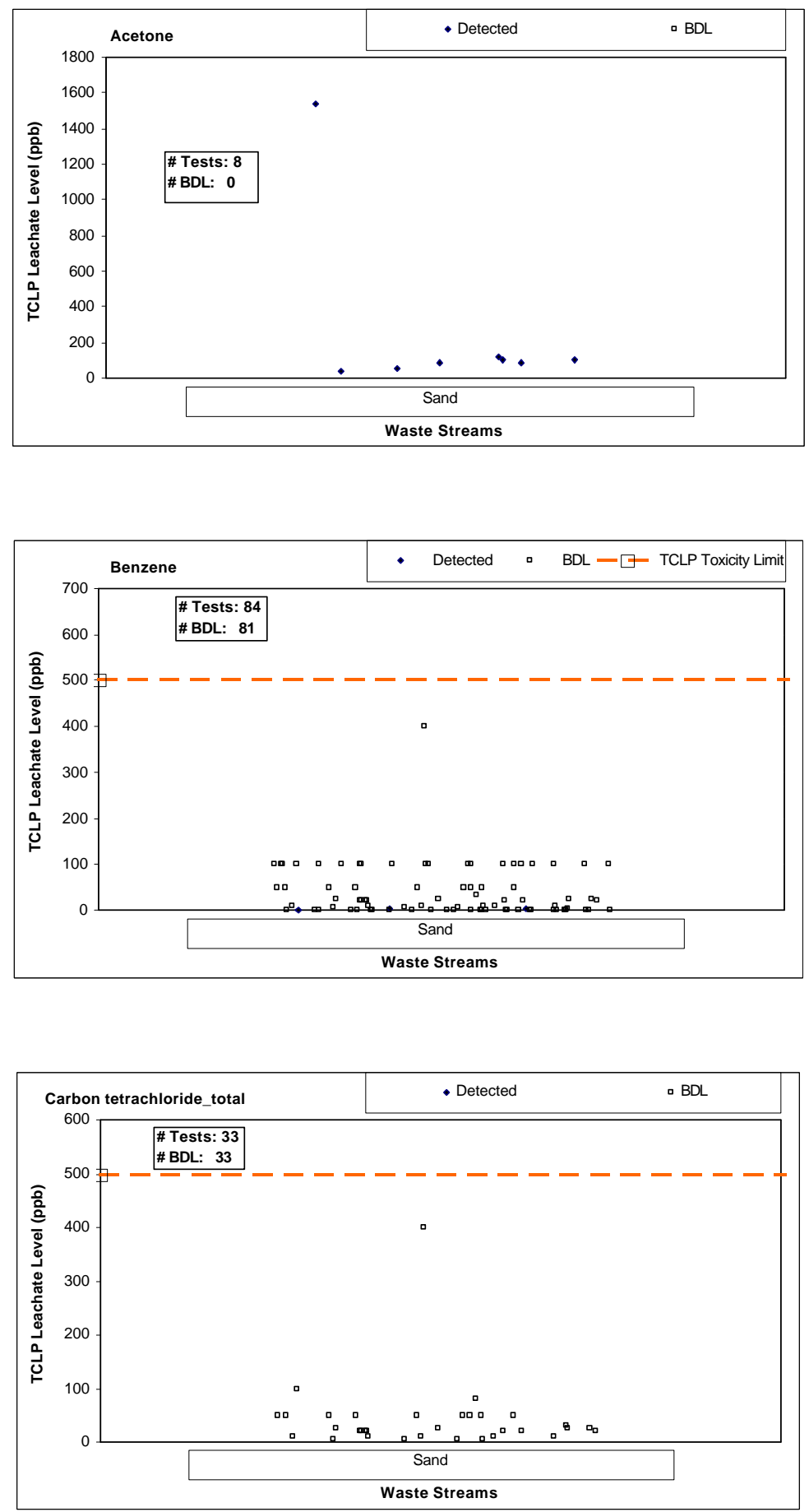

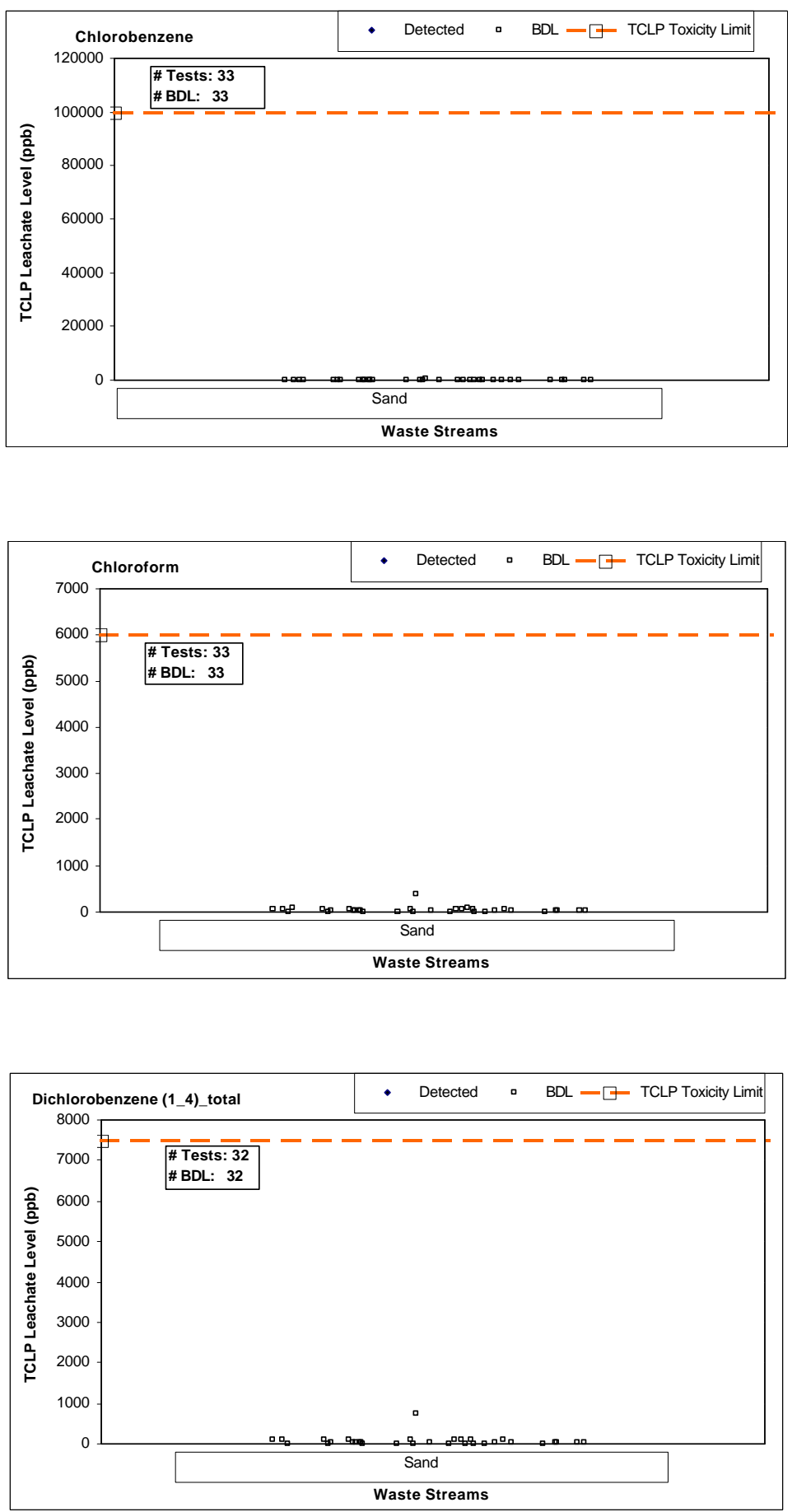

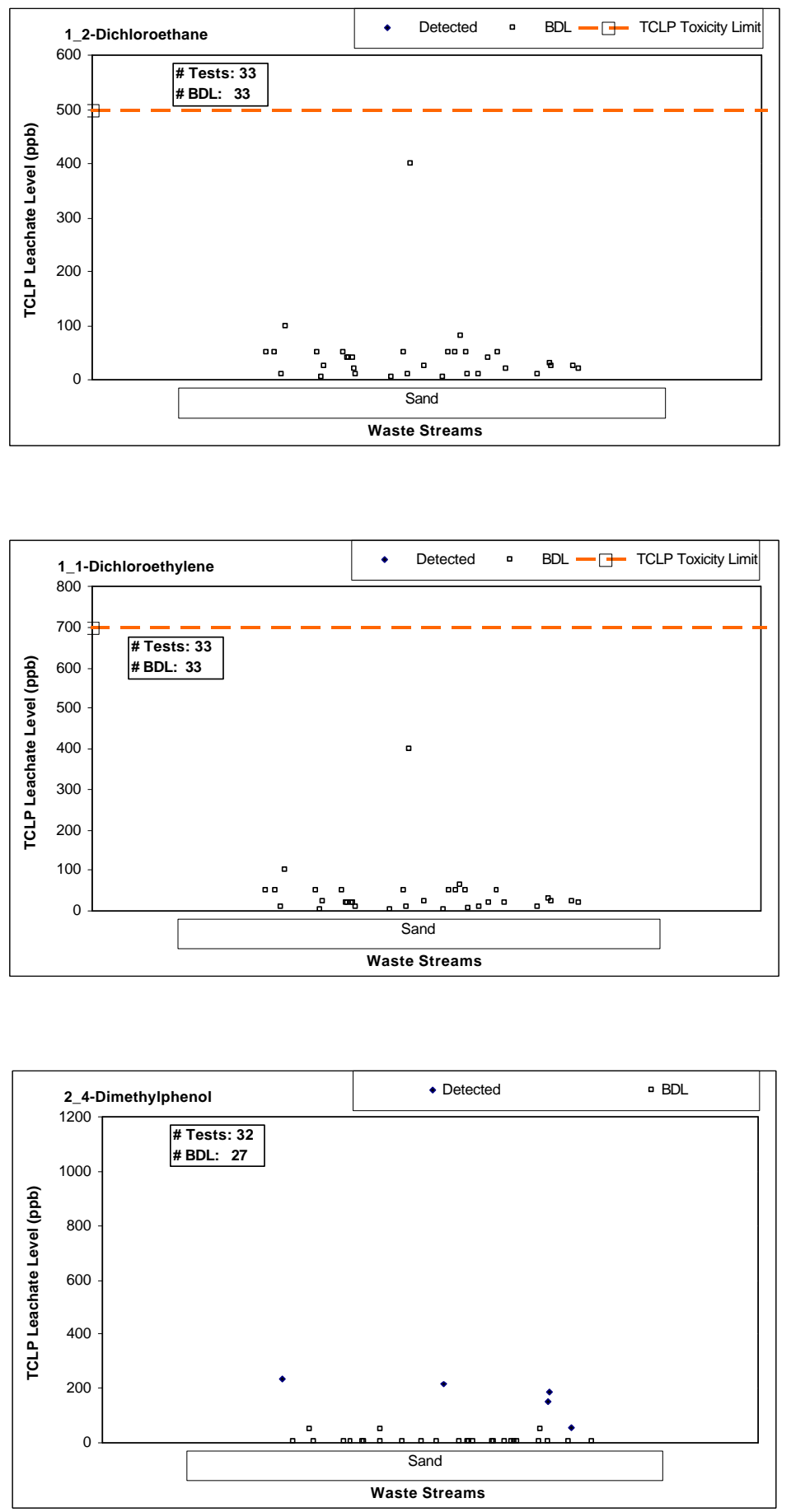

E-31 

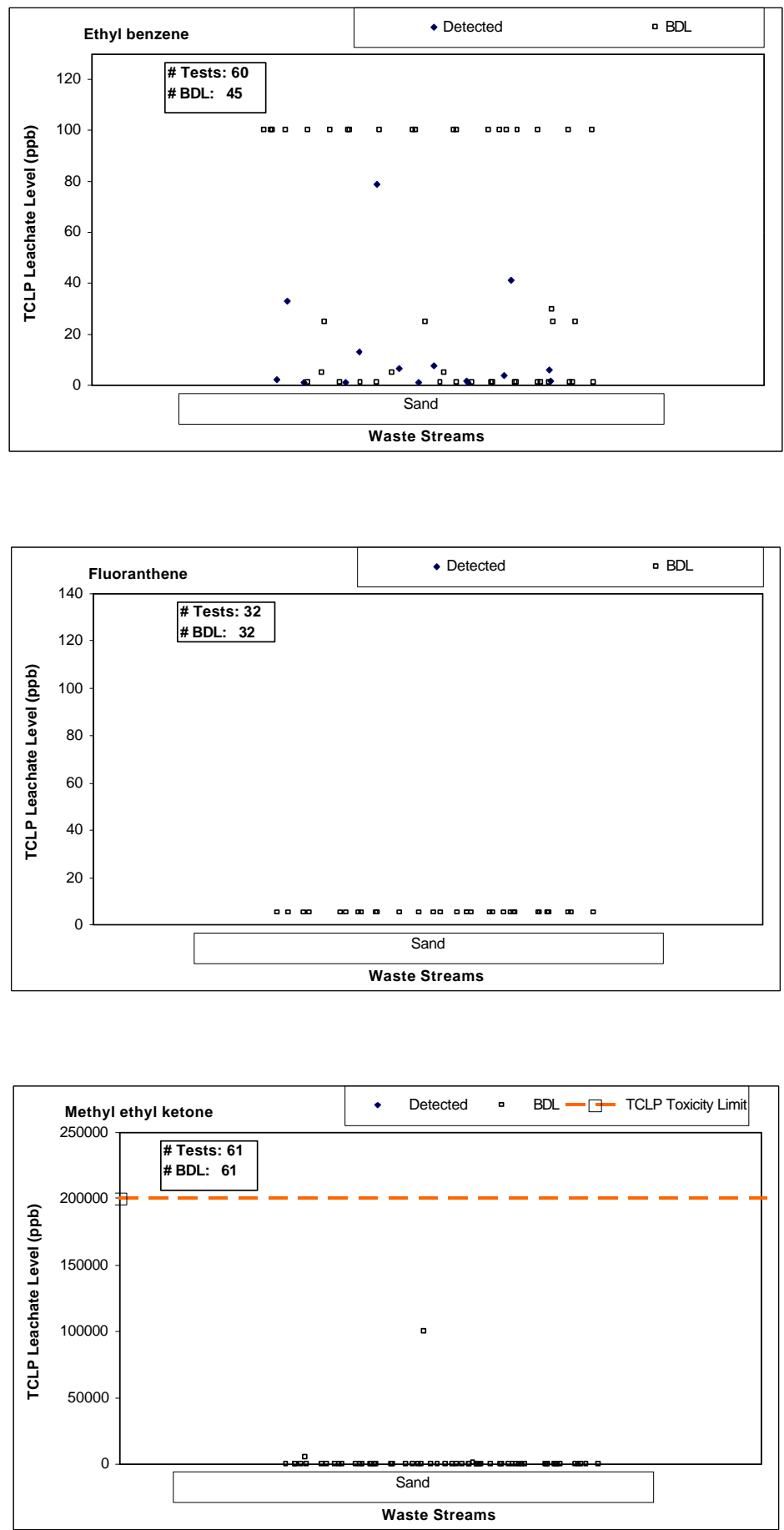

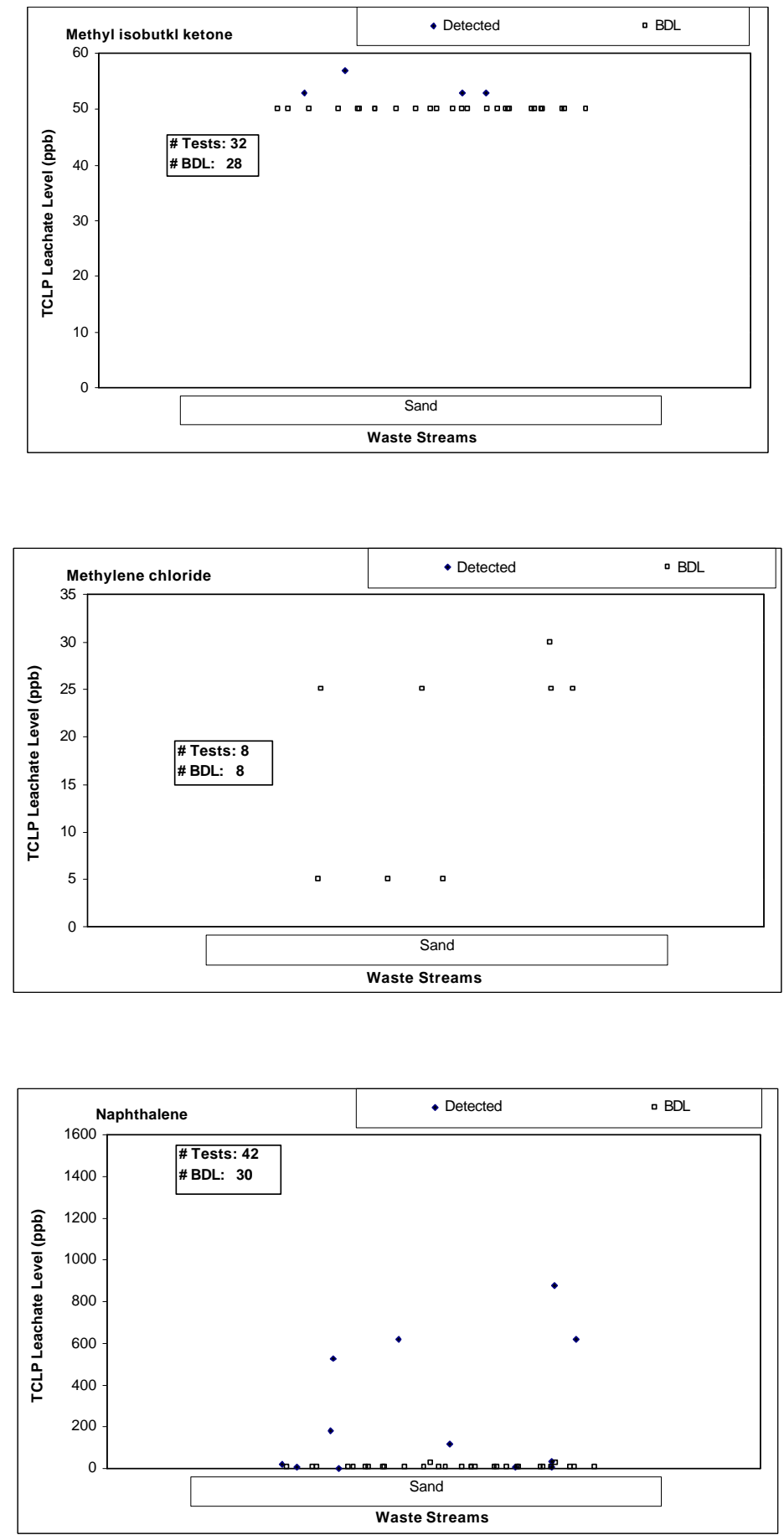

E-33 

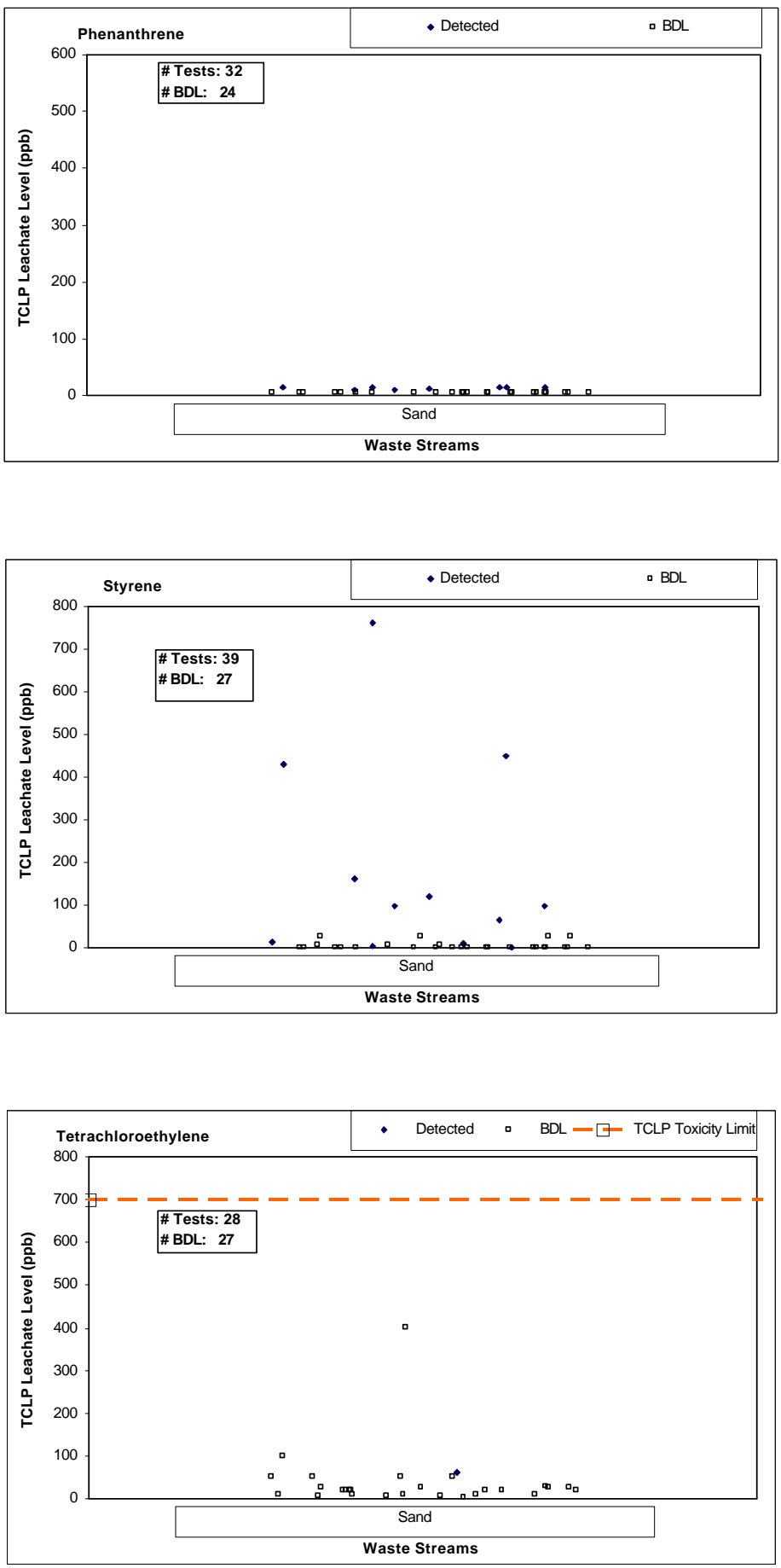

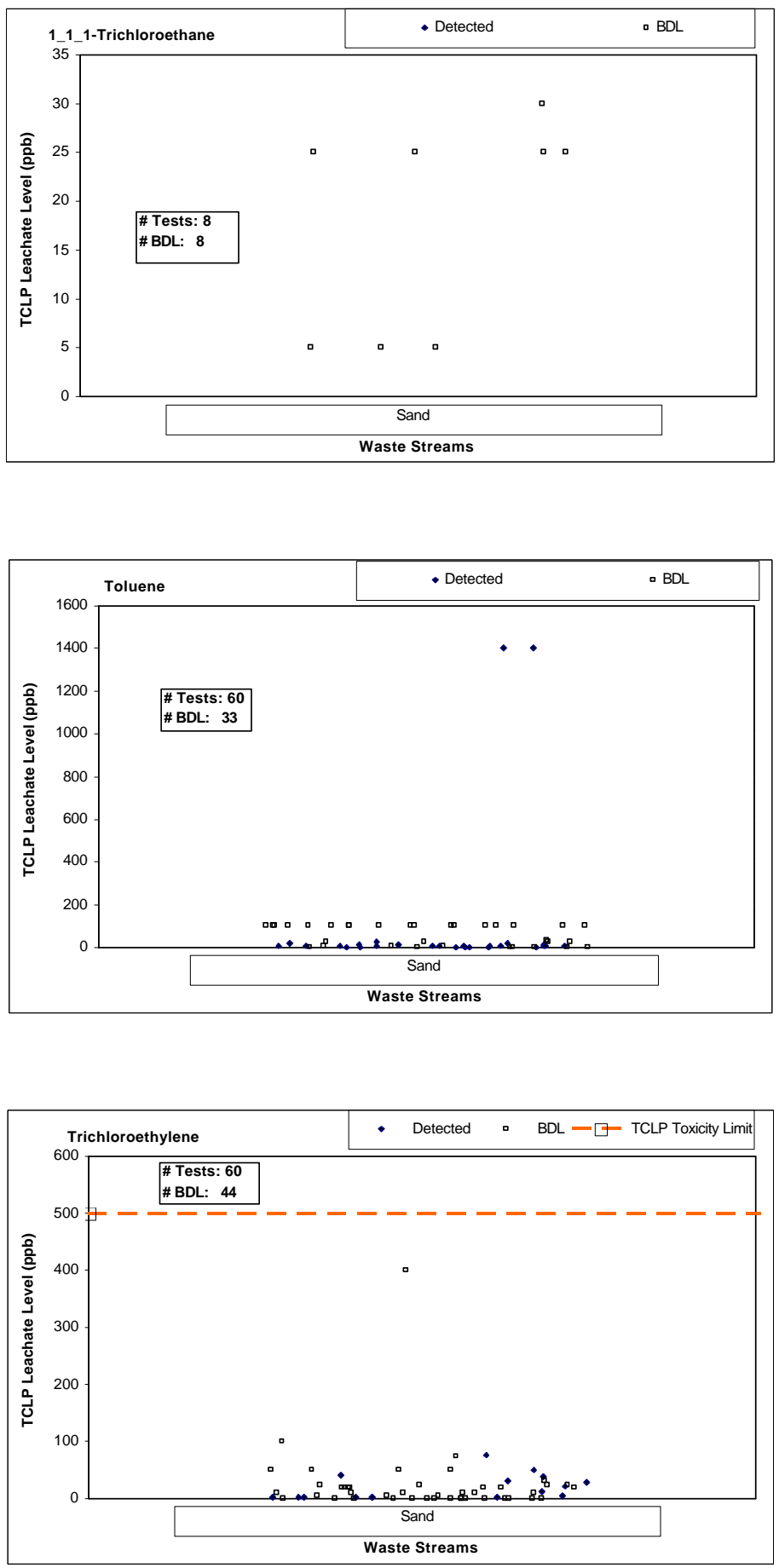

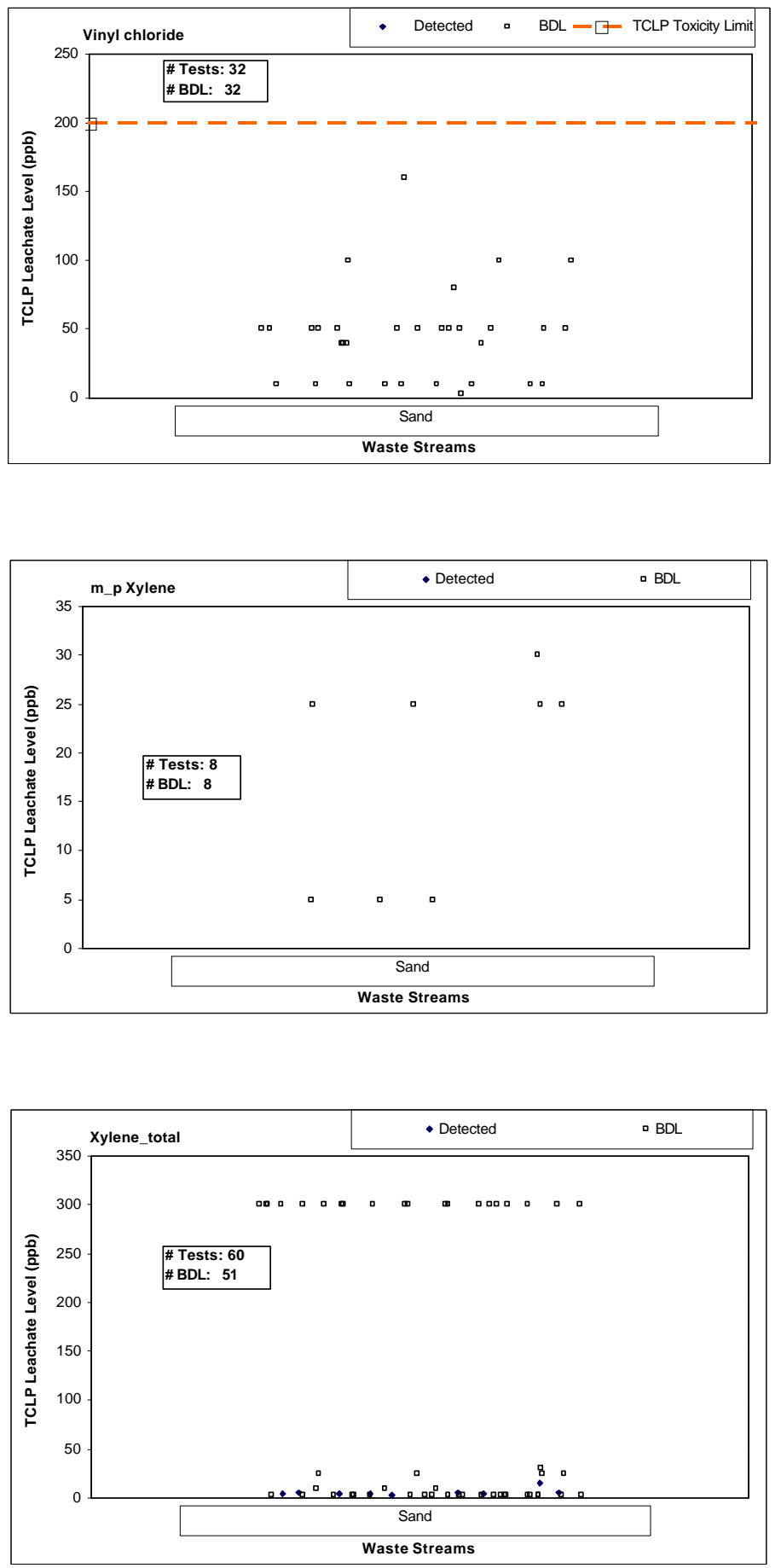

Figure E-3: Leachate concentration of organic compounds (37 charts) 


\section{Appendix F}

\section{Survival Plot and Estimated Mean}

Precise quantification of chemical characterization of excess foundry sand is important for the marketability of the excess foundry sand. In this appendix, survival plot is used to demonstrate the survival probabilities for up to 27 general chemical parameters, up to 24 metallic elements, and up to 55 organic compounds in both as-received condition and leachate (Table 3-3 to Table 3-8), and to present the effects of two factors: leaching protocol and metal cast. Treatments of leaching protocol include TCLP, SPLP, and ASTM D 3987. Treatments of metal cast include iron-based facility, steel-based facility, aluminum-based facility, and copper-based facility. Estimated mean corresponding to the individual survival plot is presented after each individual legend. Further discussion is presented in Sections 5.1 through 5.7 of the body of the report. Due to the occurrences that data for a parameter may be censored completely, the survival plot for that parameter is not available.

\section{F.1 Bulk Characterization of Excess Foundry Sand}

In this section, a series of 55 charts present the bulk characterization of excess foundry sand. The first 11 charts, grouped into Figure F-1, are for general chemical parameters. The second 23 charts, grouped into Figure F-2, are for metallic elements. The remaining 21 charts, grouped into Figure F-3, are for organic compounds. 


\section{F.1.1 General Chemical Parameters}

Nonparametric Survival Plot for Silica (Mean=77.2\%)

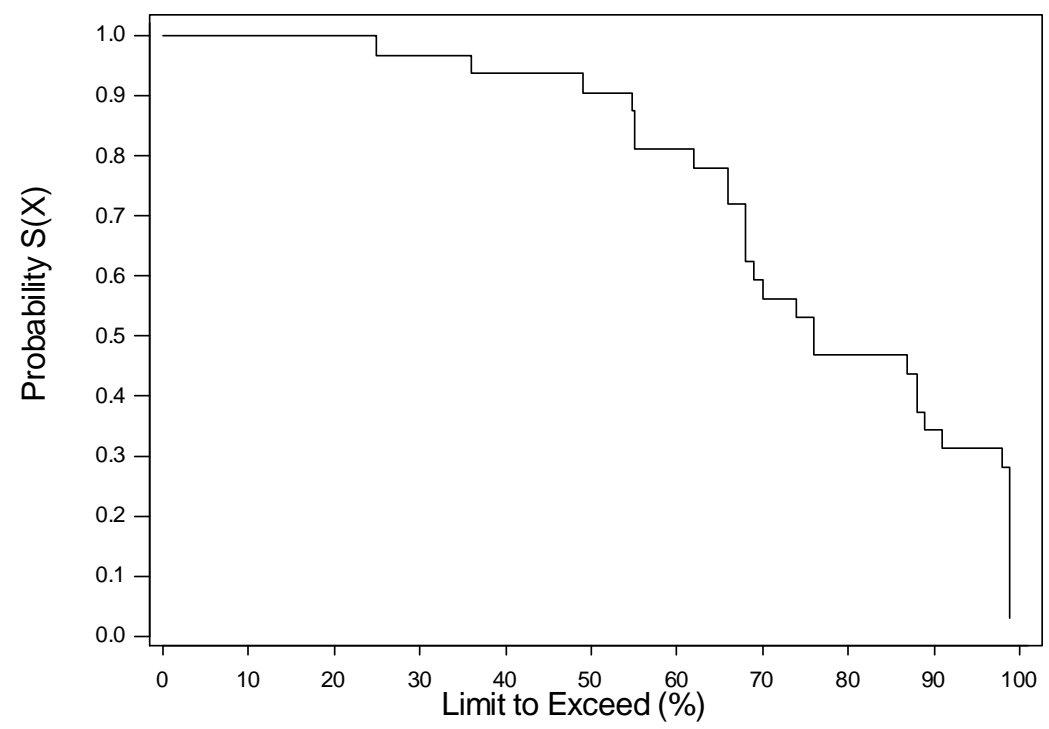

Nonparametric Survival Plot for Loss on ignition (Mean=2.54\%)

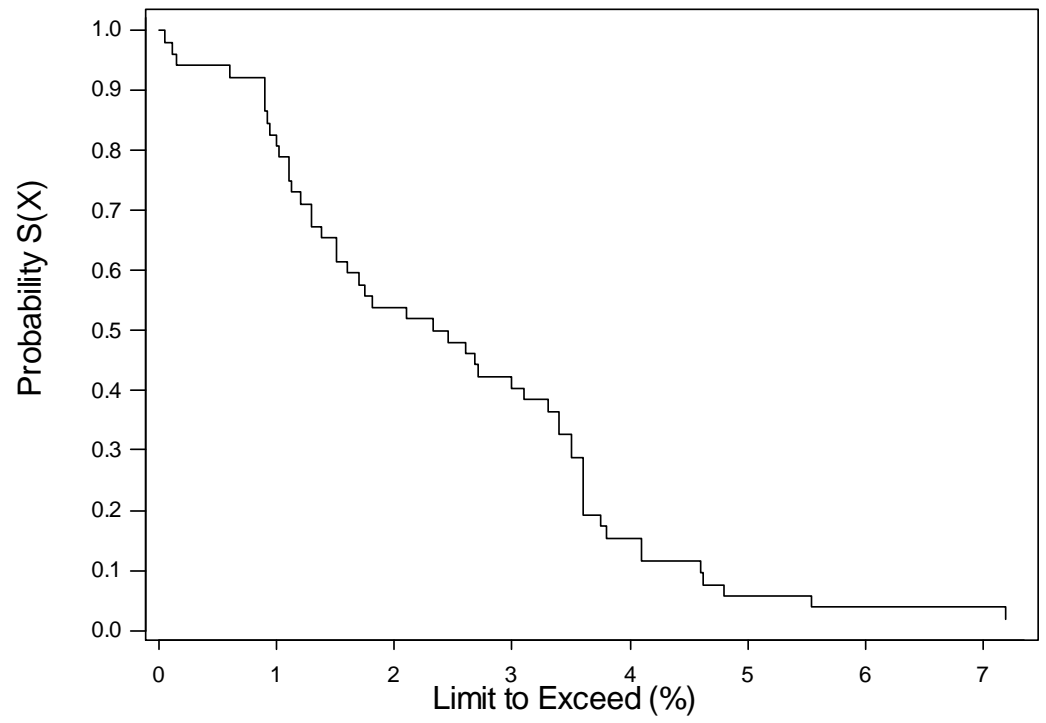

F - 2 
Nonparametric Survival Plot for Total moisture (Mean=1.27\%)

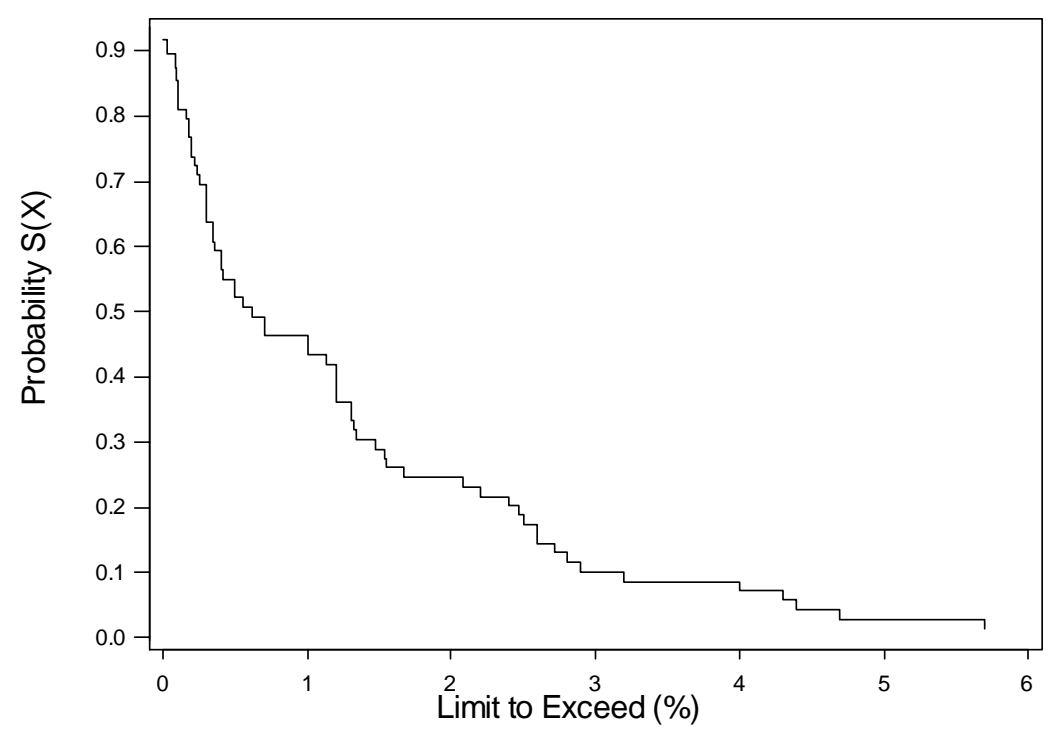

Nonparametric Survival Plot for Oil and grease (Mean=1774 ppm)

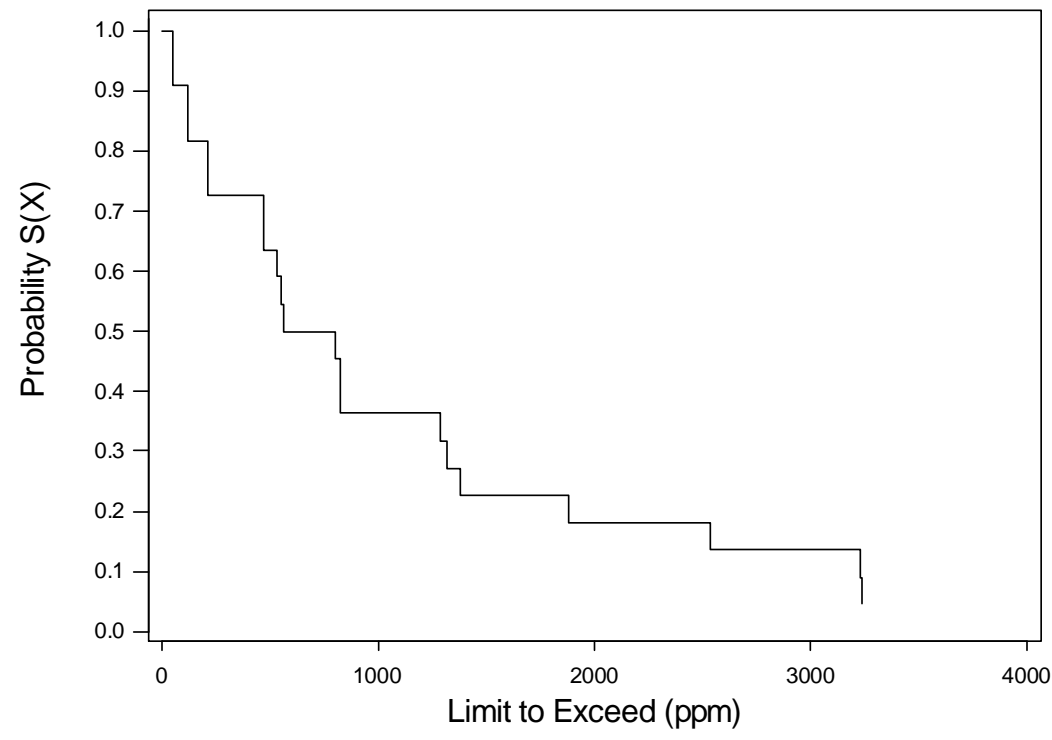

F - 3 
Nonparametric Survival Plot for Total Petroleum

(Mean=223.52 ppm)

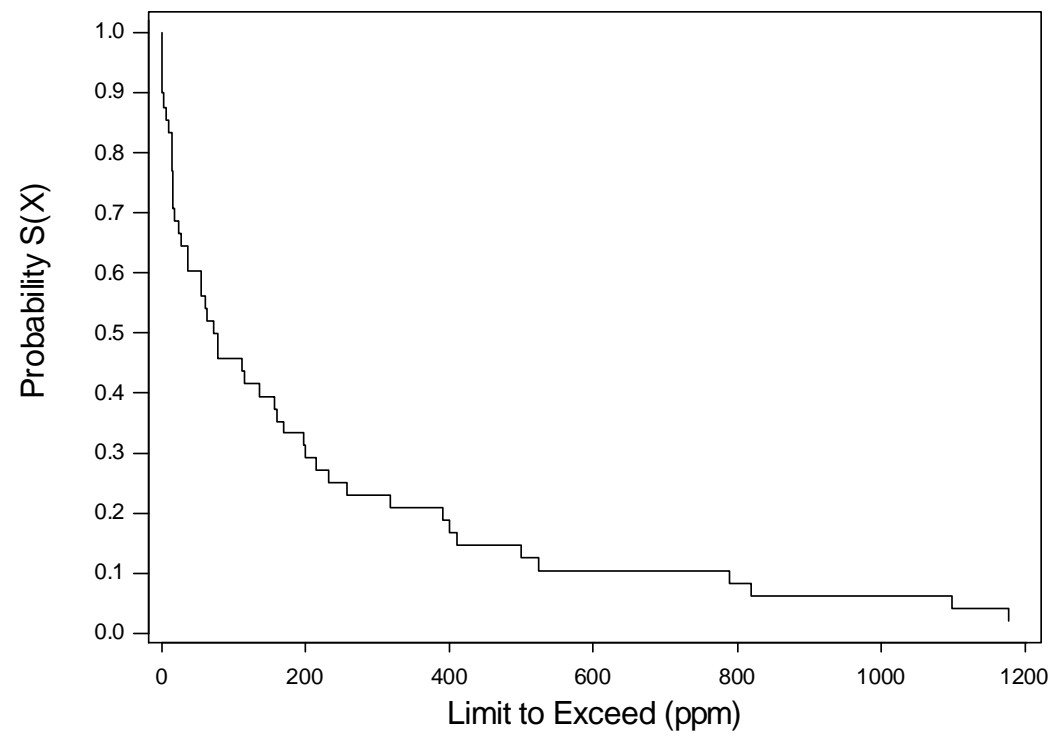

Nonparametric Survival Plot for Formaldehyde (Mean=9.21ppm)

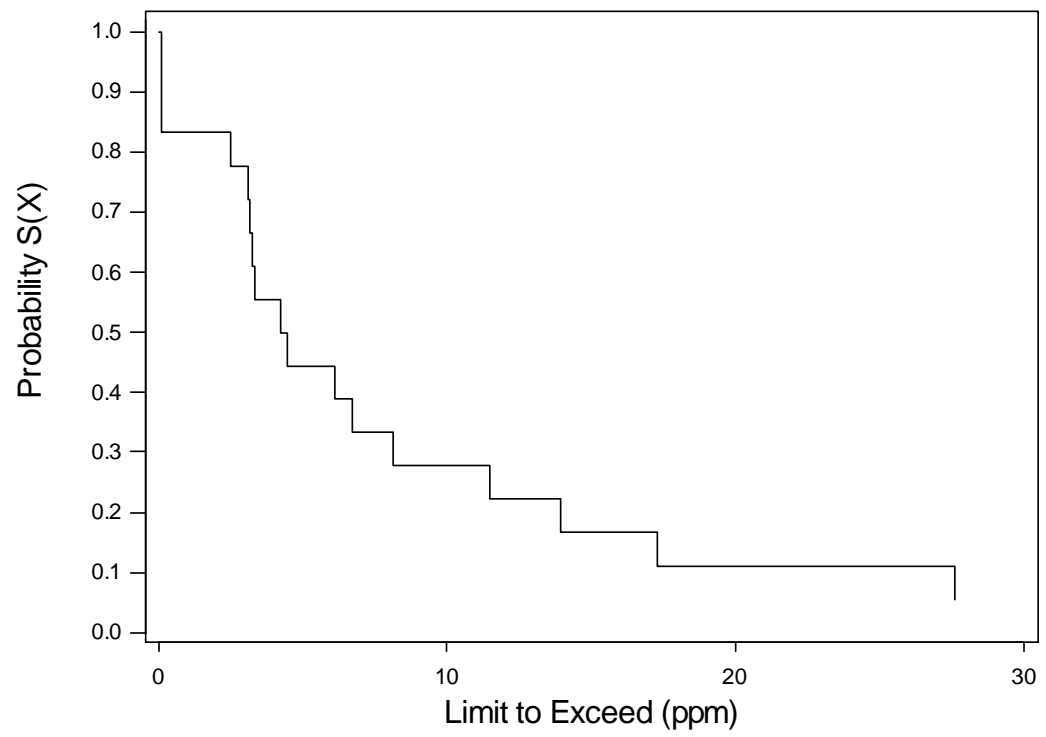

F - 4 
Nonparametric Survival Plot for Phenols Total

$($ Mean $=21.45 \mathrm{ppm})$

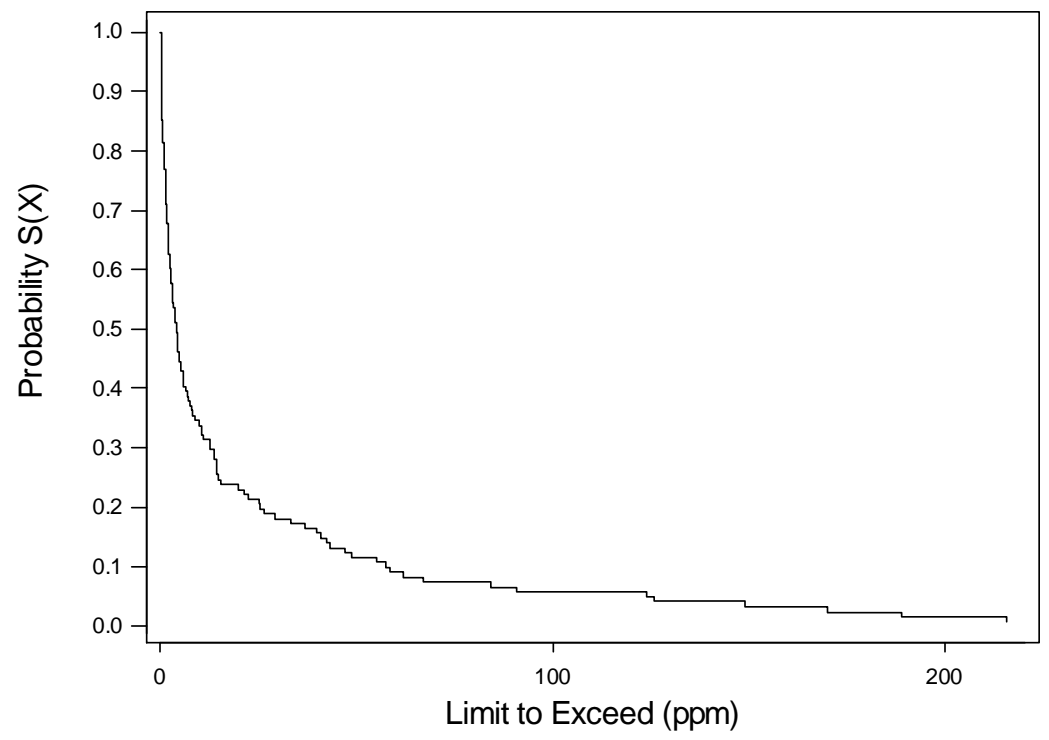

Nonparametric Survival Plot for $\mathrm{PH}$ (Mean=8.11)

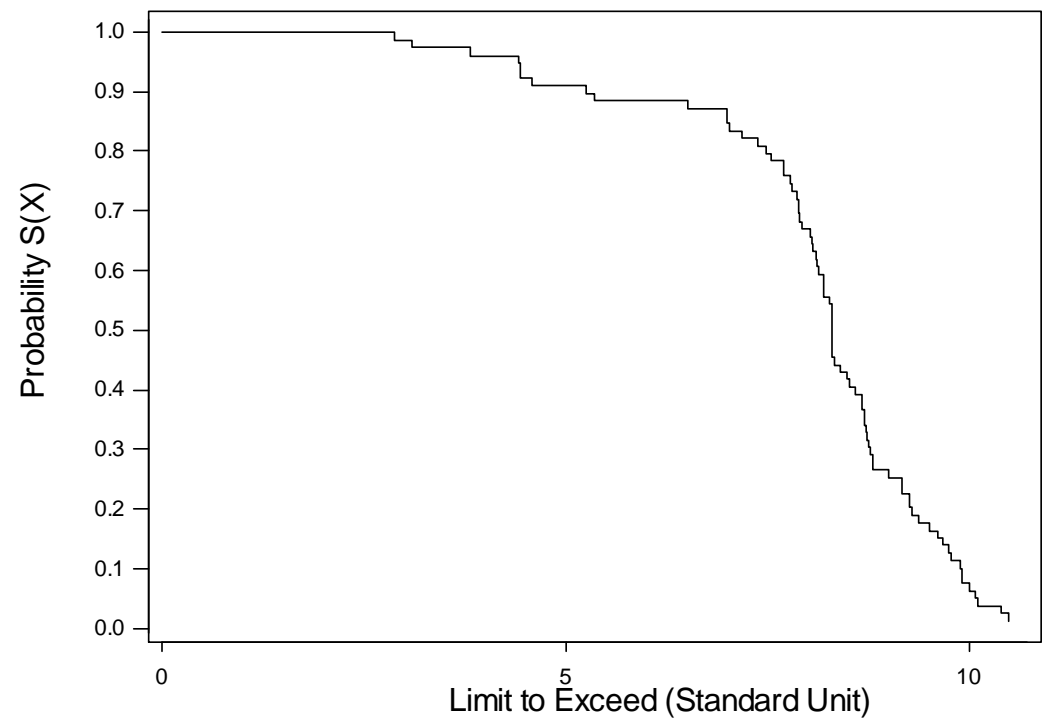

F -5 
Nonparametric Survival Plot for Reactive

Sulfide $($ Mean $=37.02 \mathrm{ppm})$

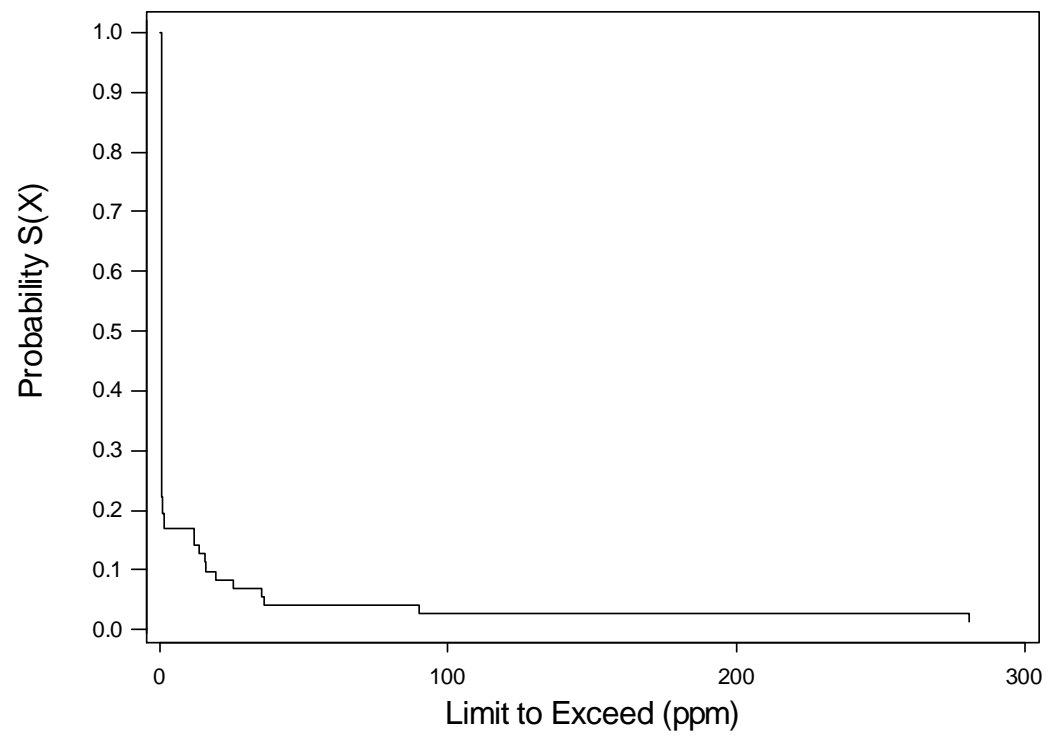

Nonparametric Survival Plot for Total Volatile Residue (Mena=20995 ppm)

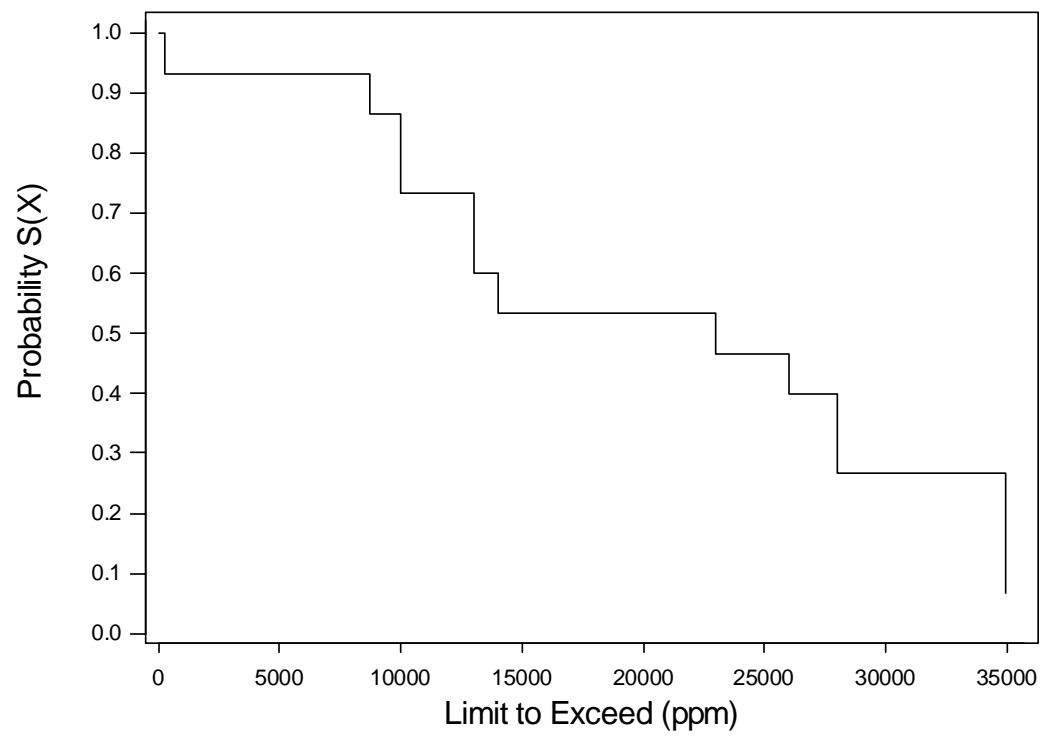

F - 6 
Nonparametric Survival Plot for Total volatile solid (Mean=1.9 ppm)

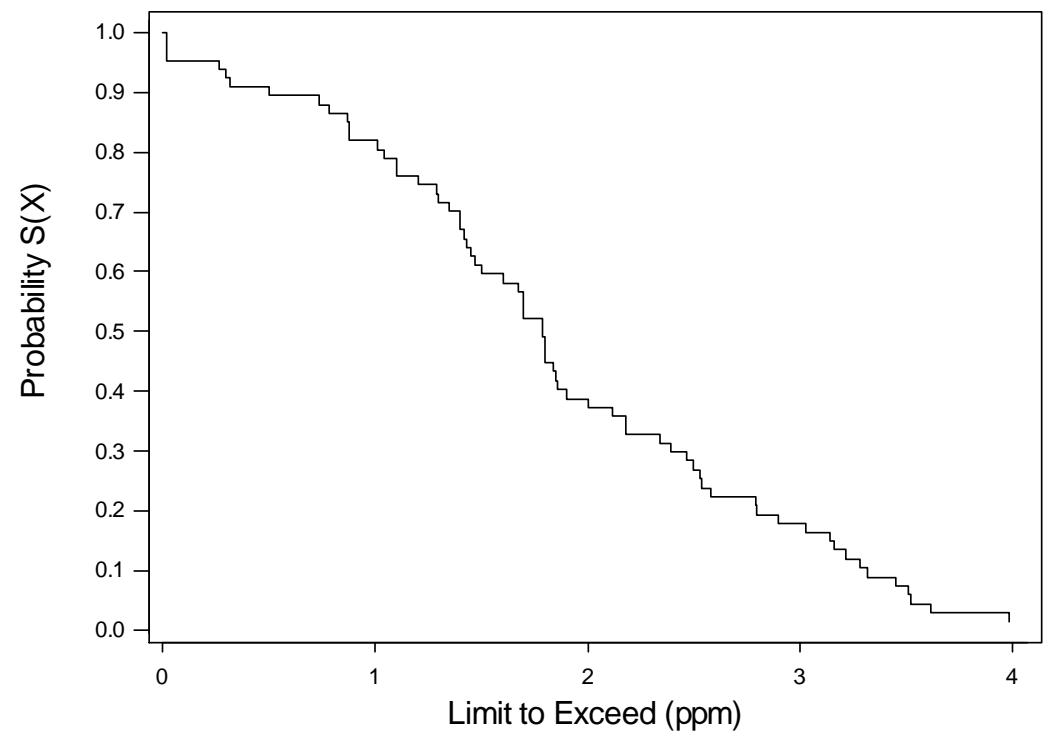

Figure F-1: Survival plots for bulk general chemical parameters (11 charts) 


\section{F.1.2 Metallic Elements}

Nonparametric Survival Plot for Aluminium(Al)

(Mean=3326 ppm)

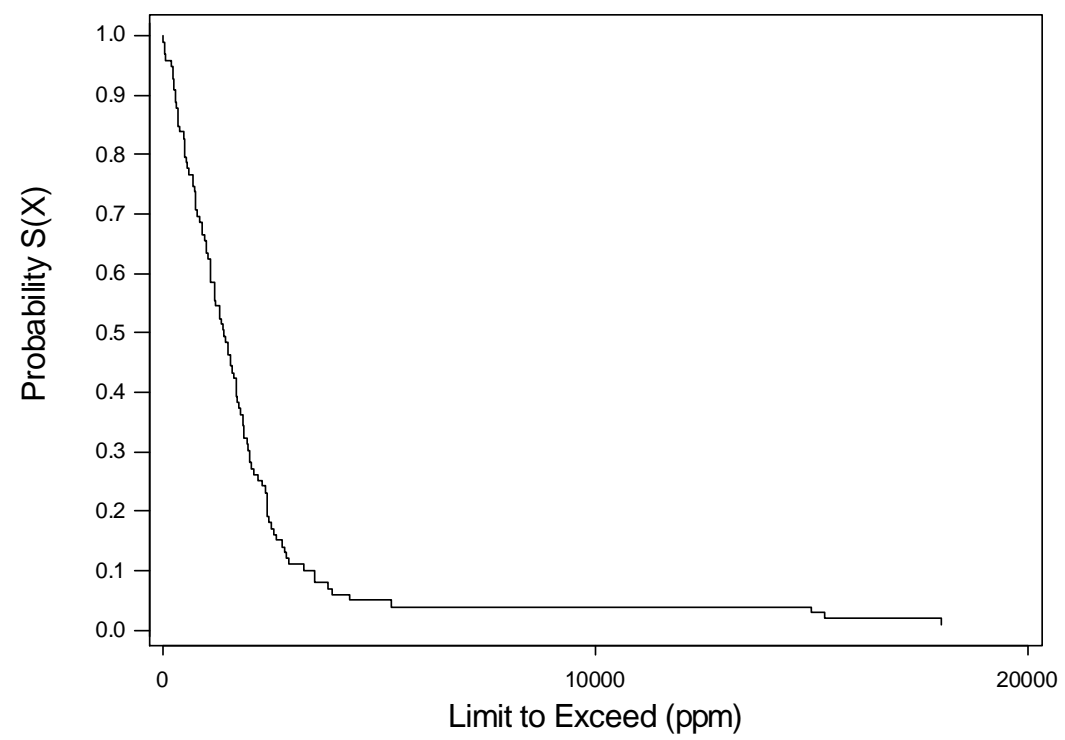

Nonparametric Survival Plot for Antimony(Sb)

(Mena=4.34 ppm)

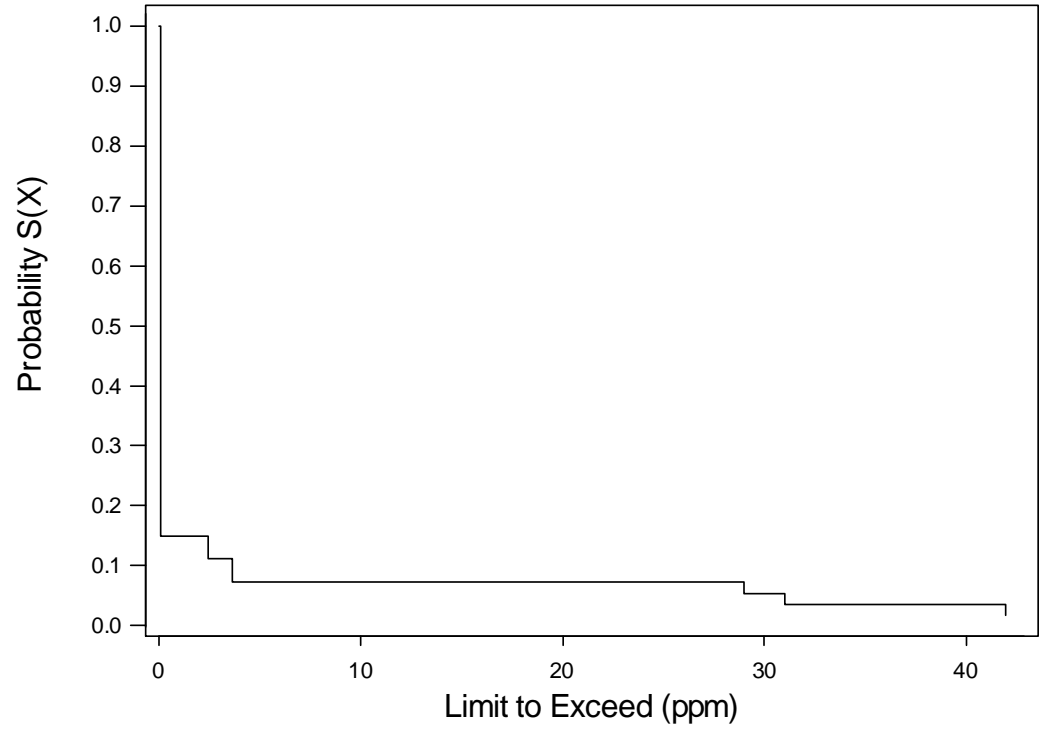

F -8 
Nonparametric Survival Plot for Arsenic(As)

(Mena=0.86 ppm)

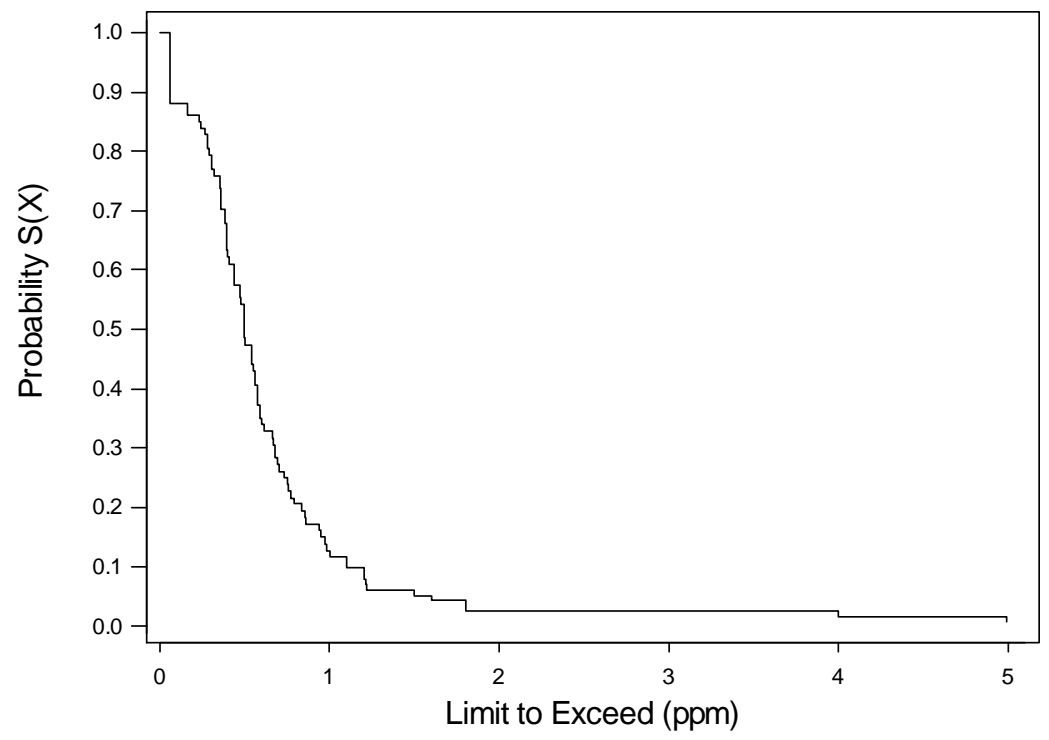

Nonparametric Survival Plot for Barium(Ba)

(Mean=14.95 ppm)

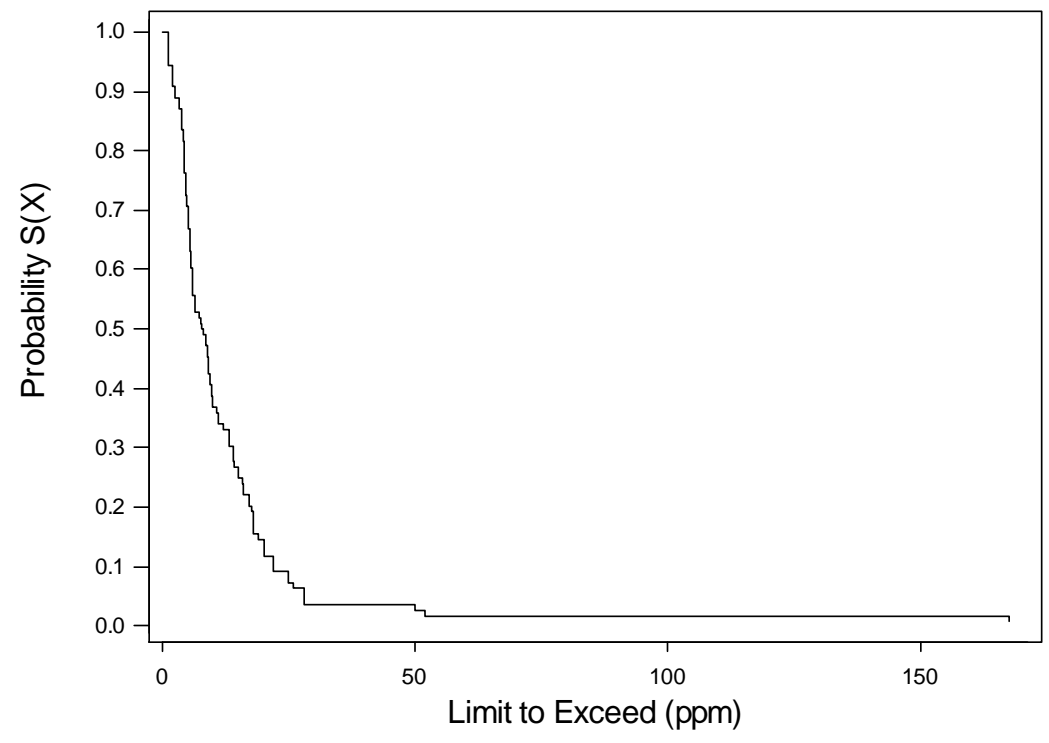

F -9 
Nonparametric Survival Plot for Beryllium (Be)

(Mean $=0.08$ ppm)

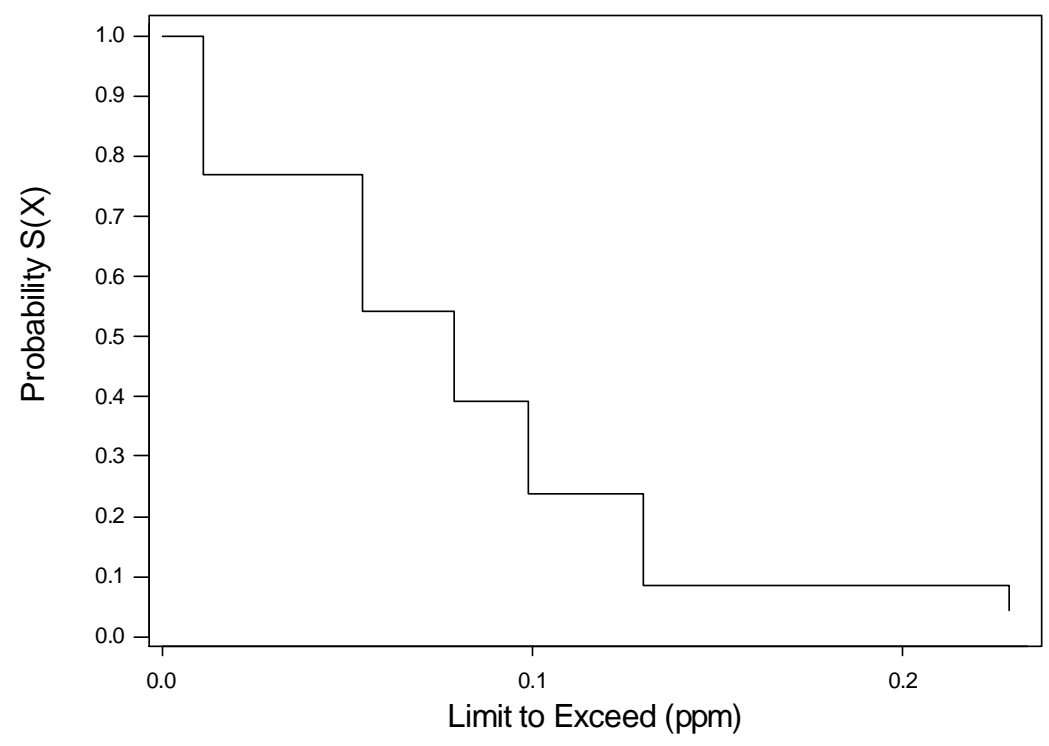

Nonparametric Survival Plot for Boron(B)

(Mean=38.53 ppm)

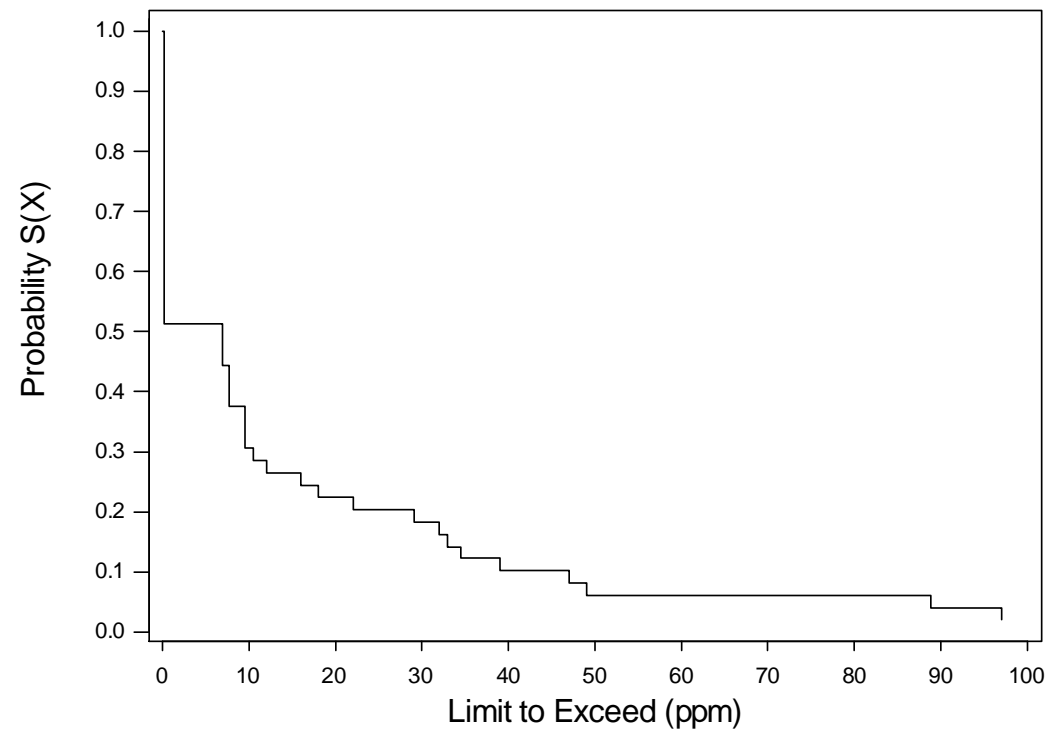


Nonparametric Survival Plot for Cadmium(Cd)

(Mean=0.22 ppm)

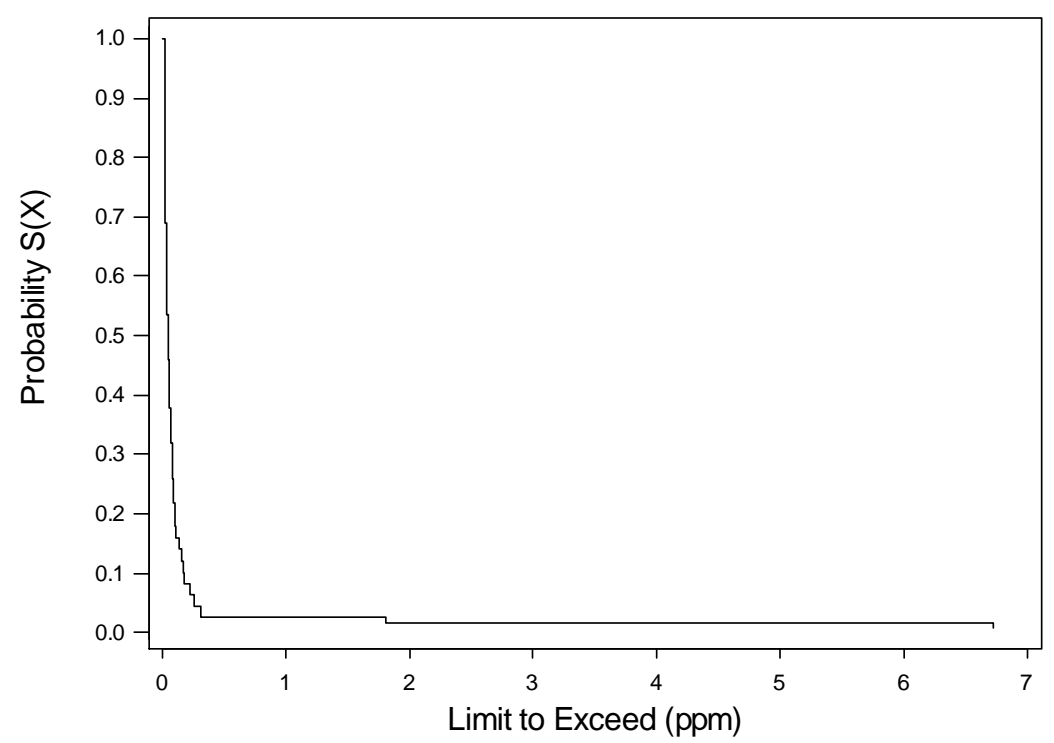

Nonparametric Survival Plot for Calcium (Ca)

(Mean=3309 ppm)

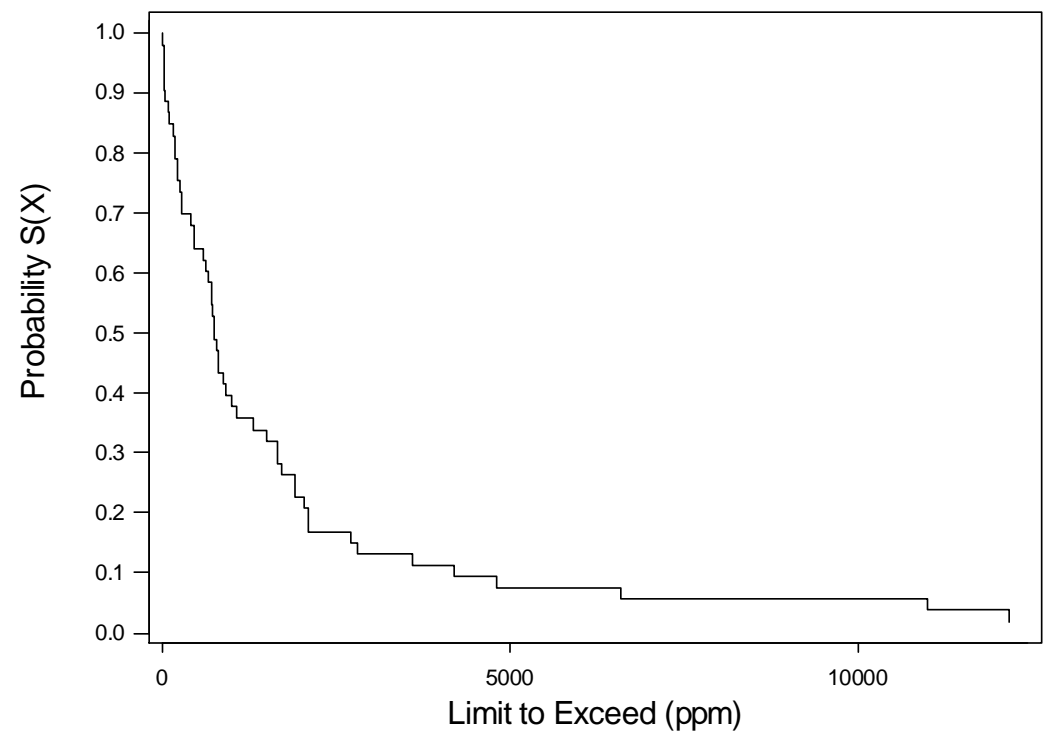

F -11 
Nonparametric Survival Plot for Chromium(Cr)

(Mean=114 ppm)

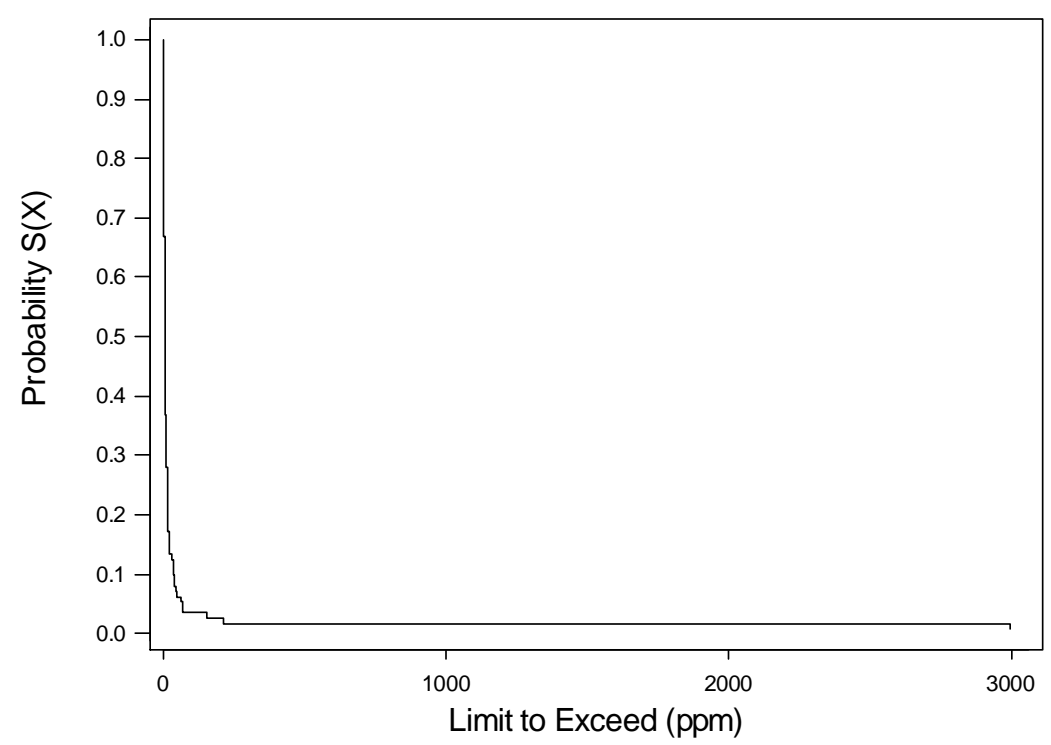

Nonparametric Survival Plot for Hexavalent chromium (Mean=0.08 ppm)

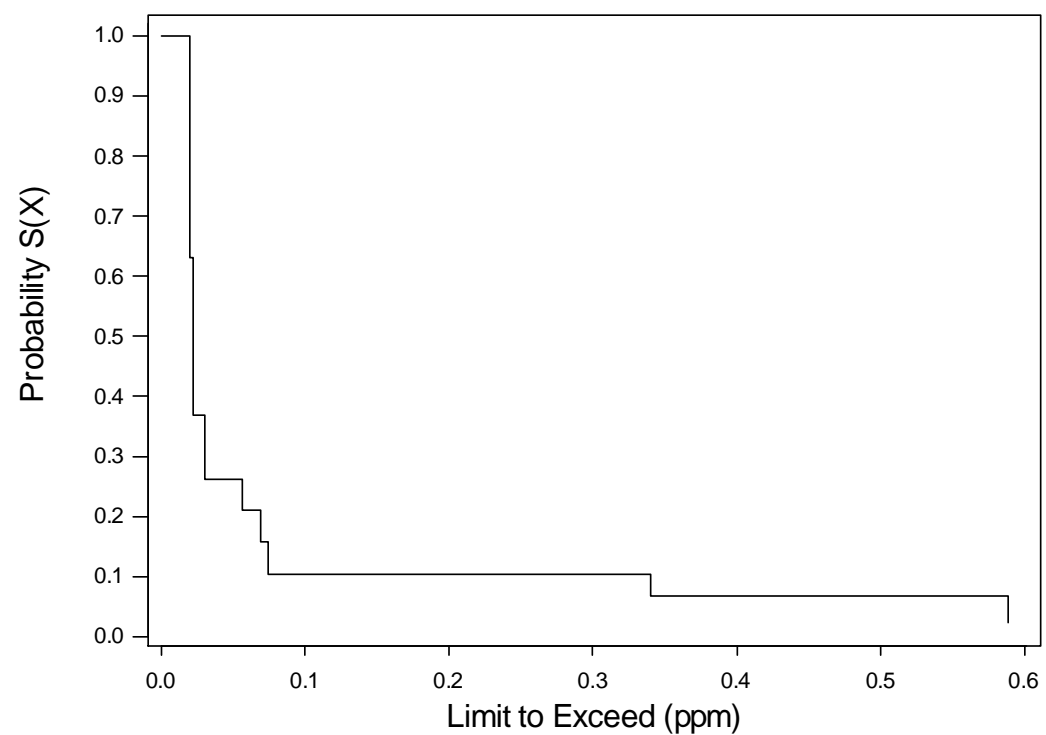


Nonparametric Survival Plot for Copper(Cu)

(Mean=103.6 ppm)

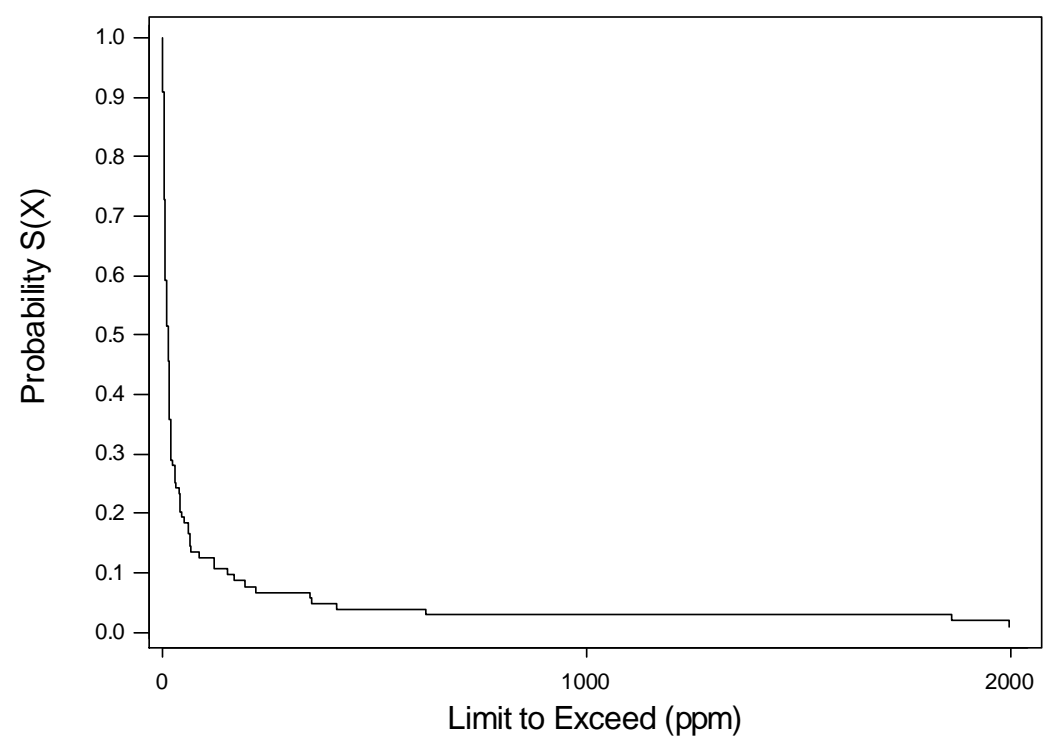

Nonparametric Survival Plot for Iron(Fe)

(Mean=10912 ppm)

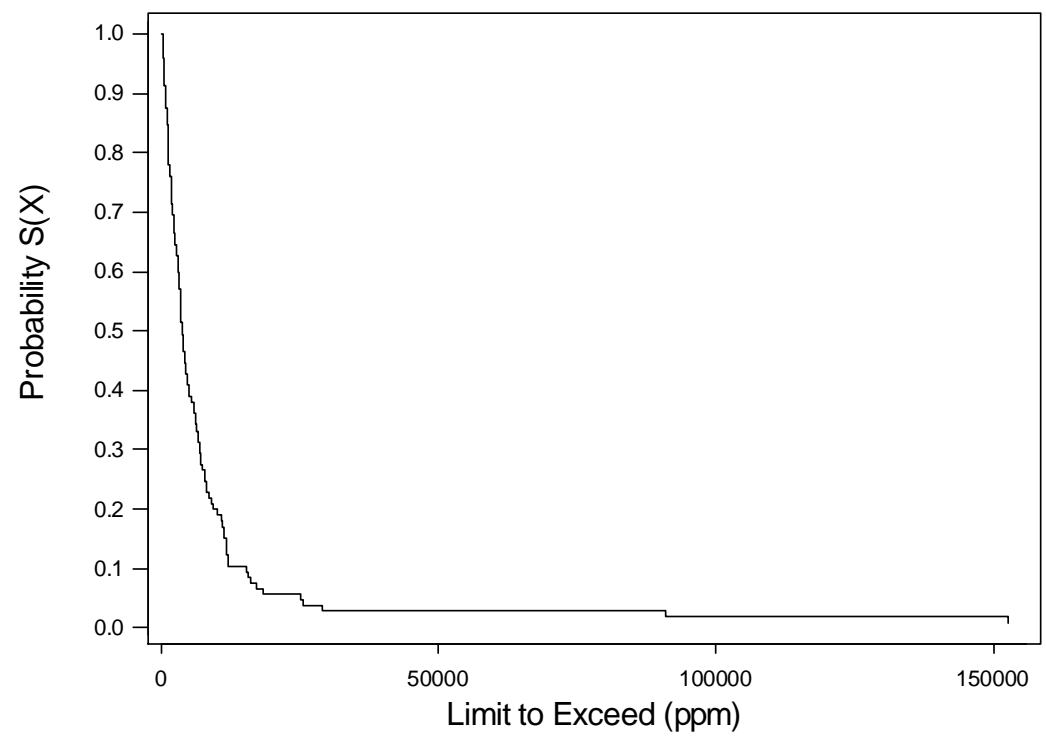

F -13 
Nonparametric Survival Plot for $\operatorname{Lead}(\mathrm{Pb})$

(Mean=15.72 ppm)

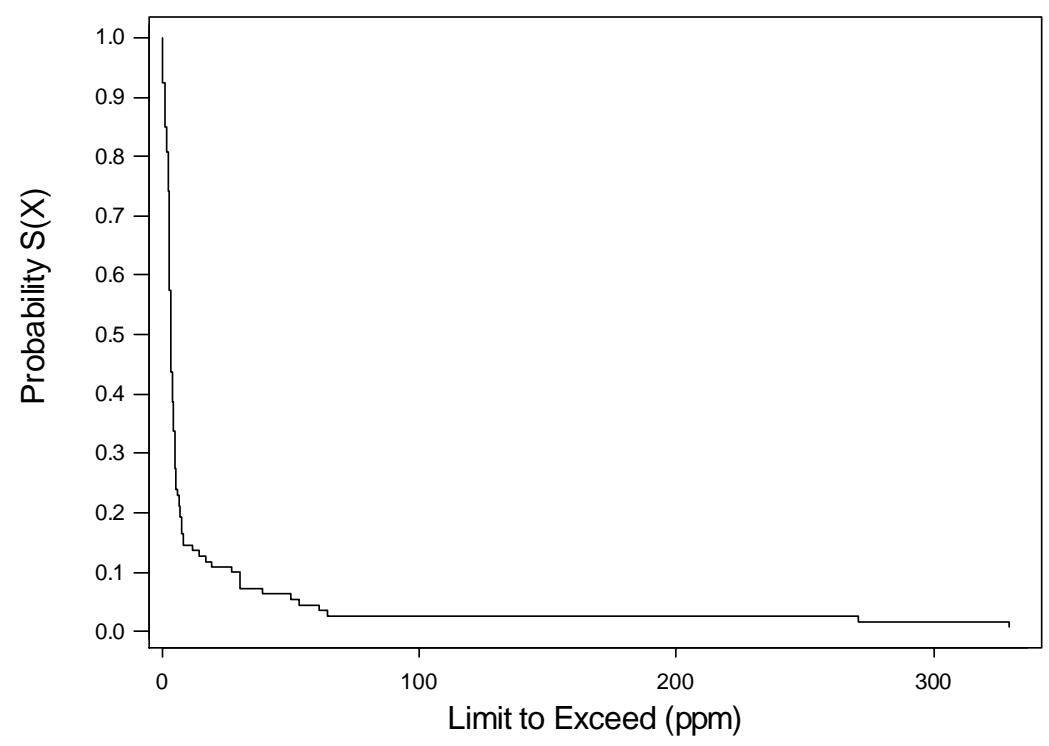

Nonparametric Survival Plot for Magnesium(Mg)

(Mean=1881 ppm)

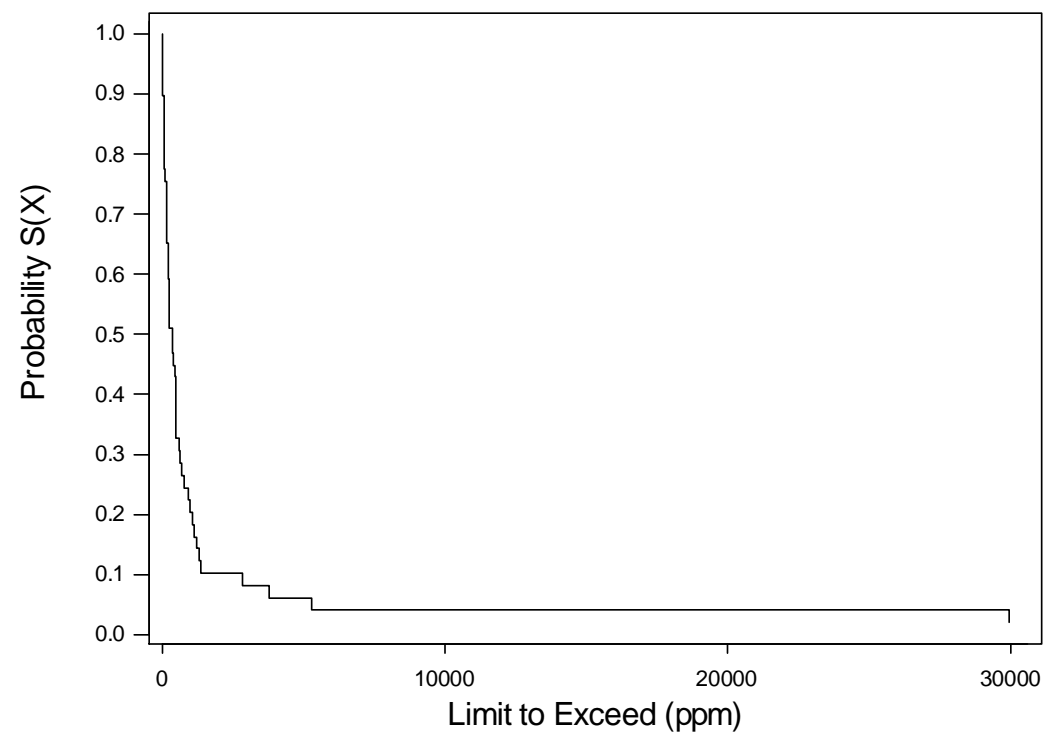

F -14 
Nonparametric Survival Plot for Manganese(Mn)

(Mean=256.9 ppm)

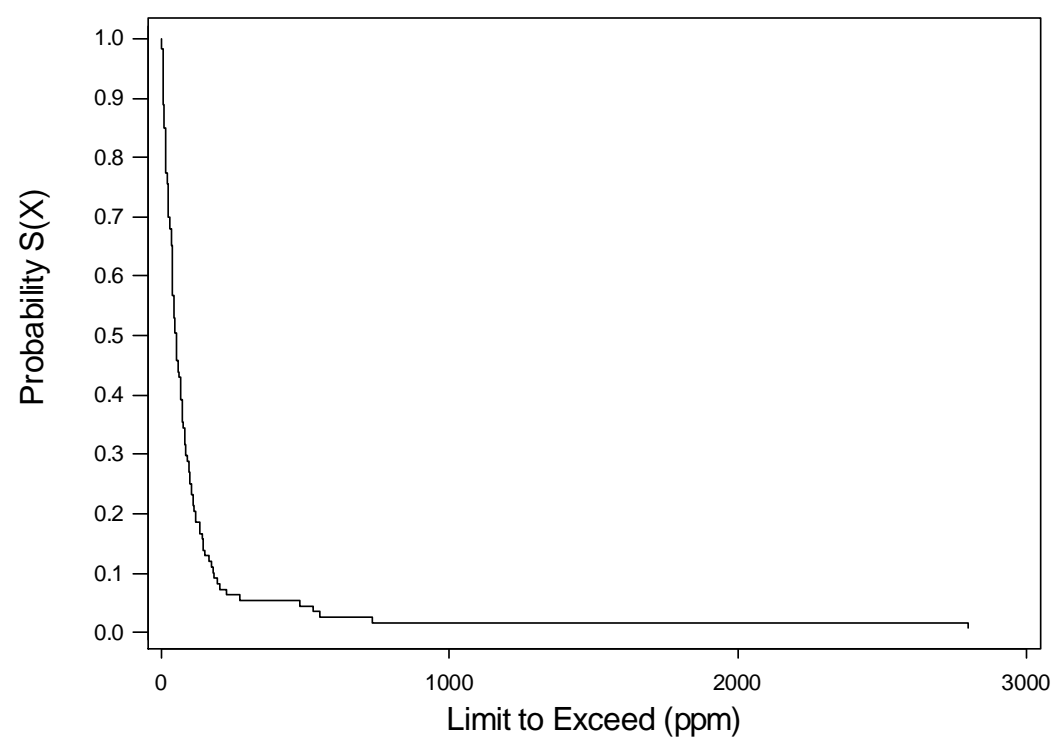

Nonparametric Survival Plot for Mercury $(\mathrm{Hg})$

(Mean=0.04 ppm)

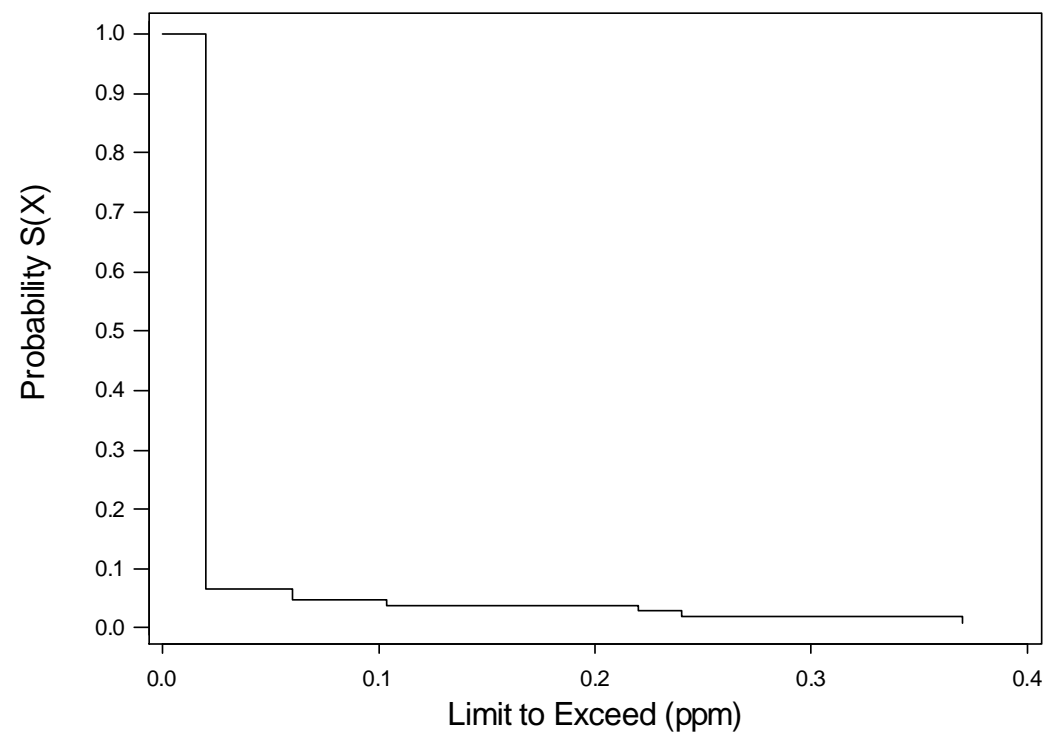

F -15 
Nonparametric Survival Plot for Molybdenum(Mo)

(Mean=38.84 ppm)

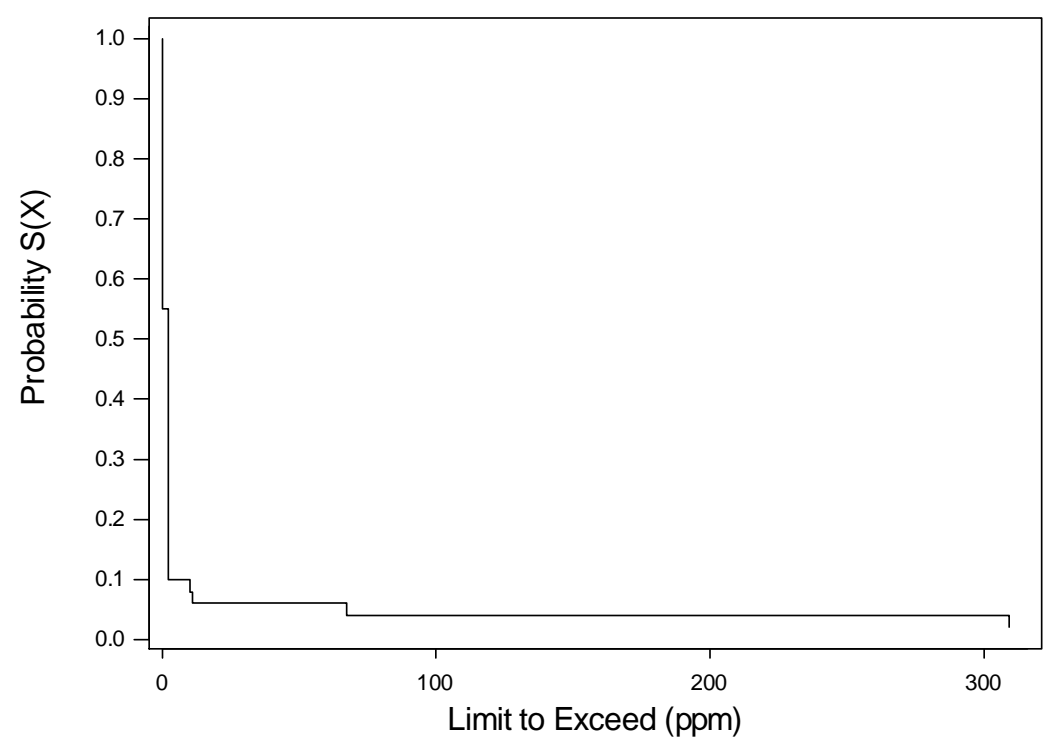

Nonparametric Survival Plot for Nickel(Ni)

(Mean=107.94 ppm)

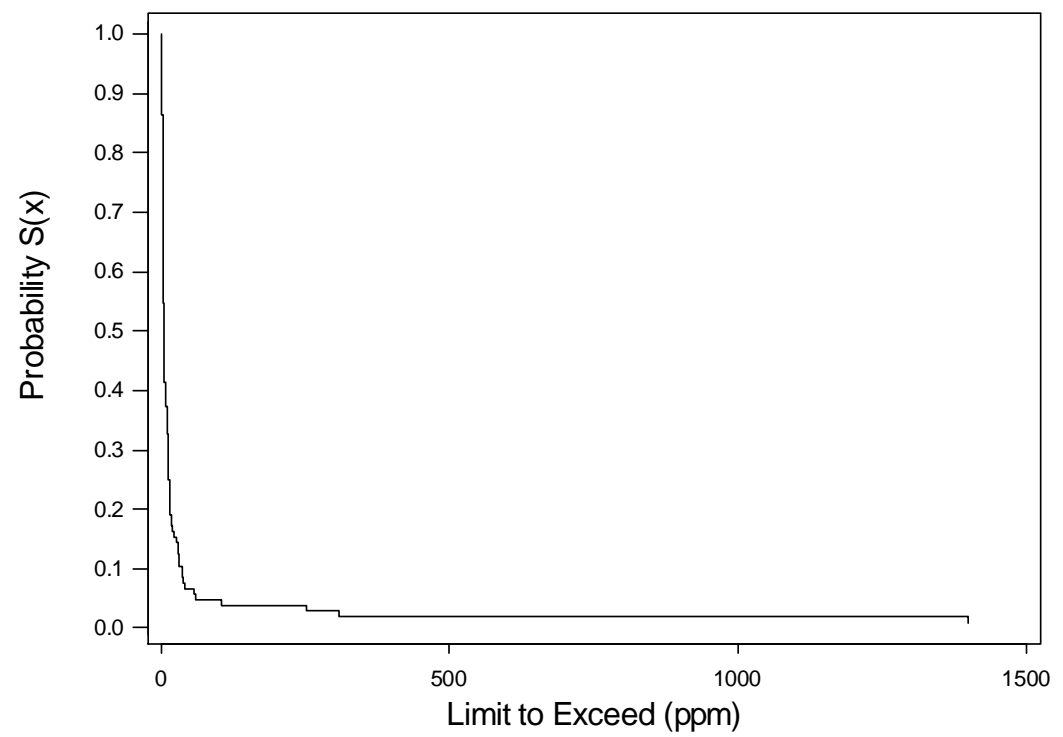

F -16 
Nonparametric Survival Plot for Selenium(Se)

(Mean=0.64 ppm)

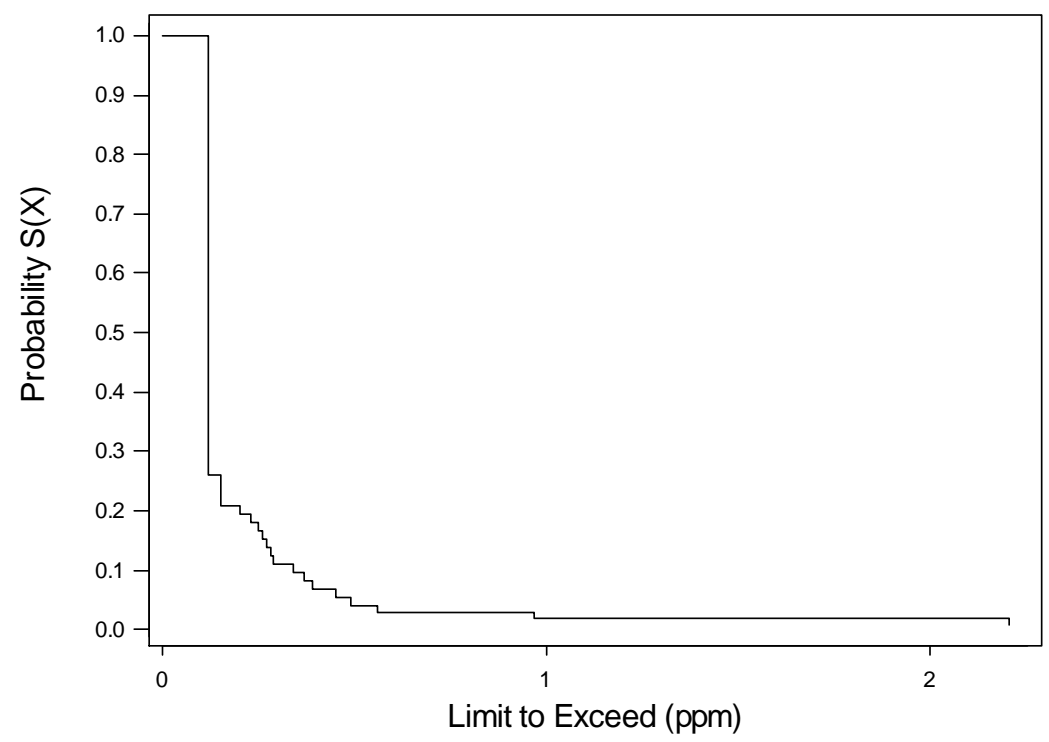

Nonparametric Survival Plot for Silicon(Si)

(Mean=1771.88 ppm)

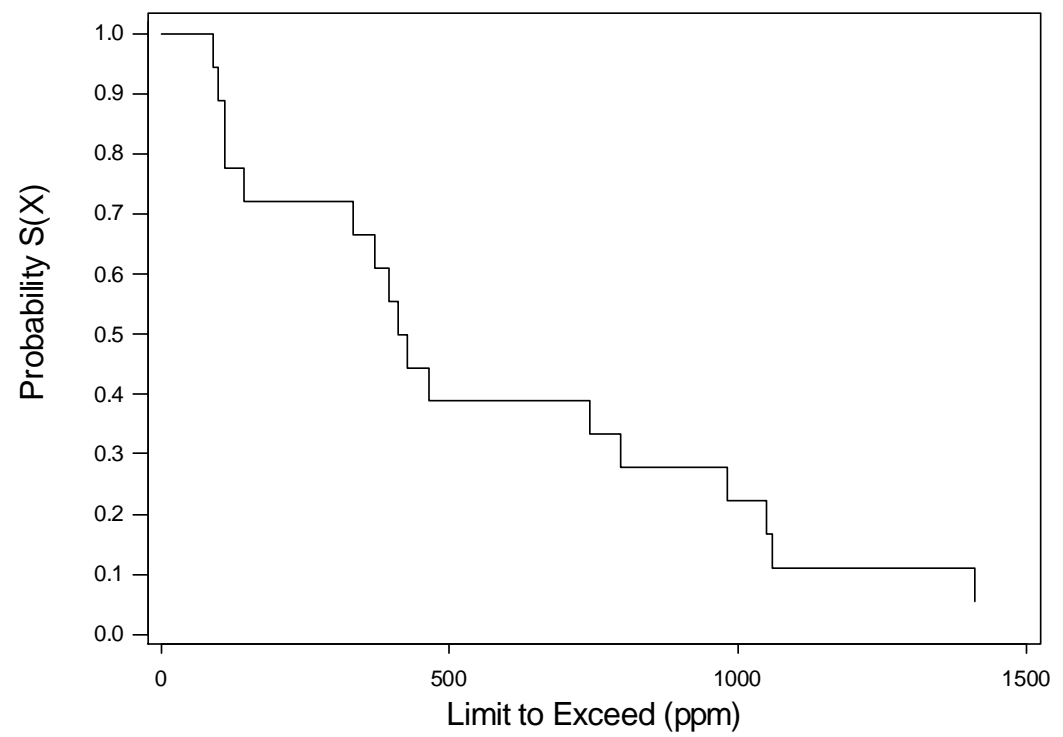


Nonparametric Survival Plot for Silver (Ag)

(Mean=0.47 ppm)

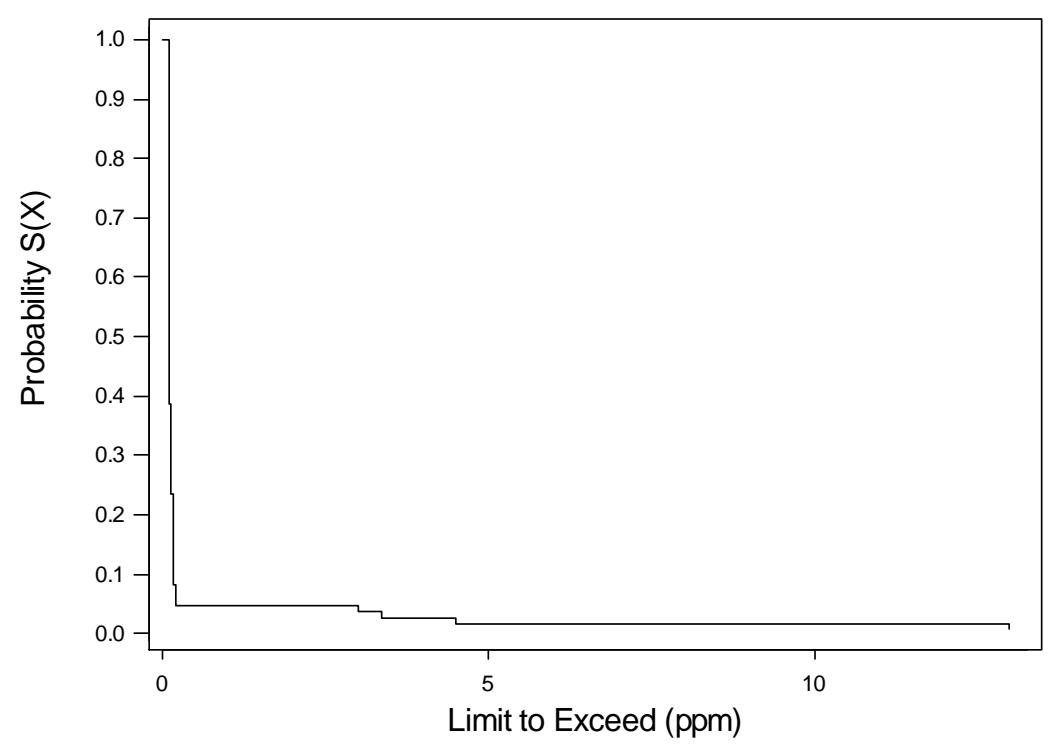

Nonparametric Survival Plot for Thallium (T)

(Mean=0.43 ppm)

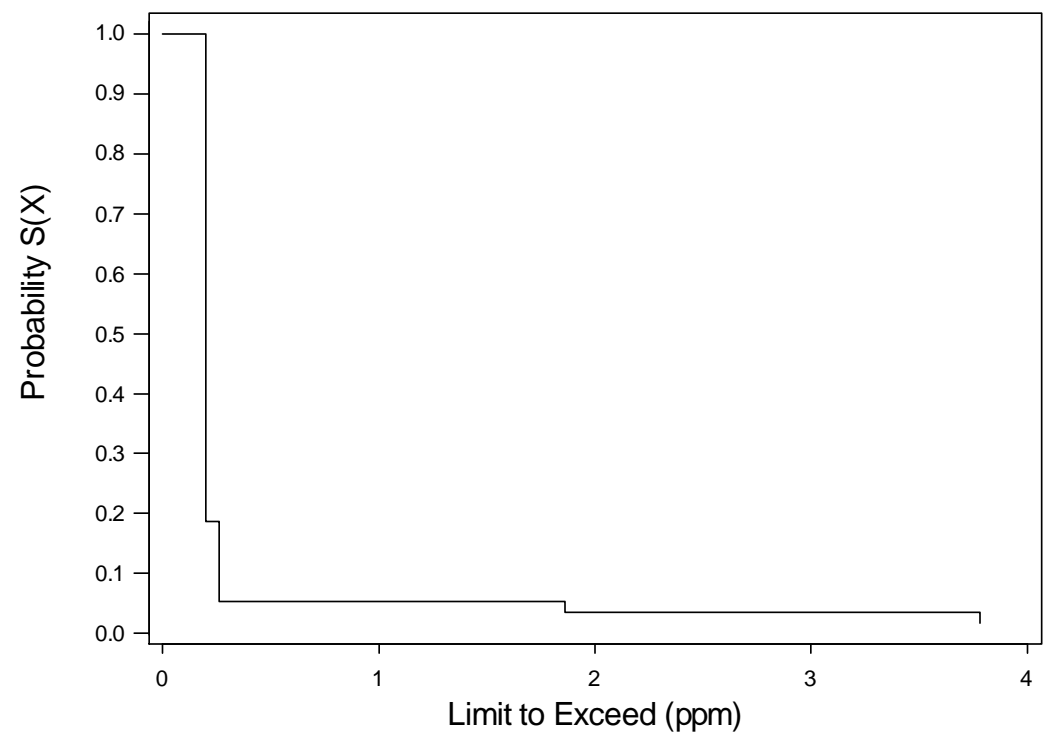


Nonparametric Survival Plot for Zinc(Zn)

(Mean=102.48 ppm)

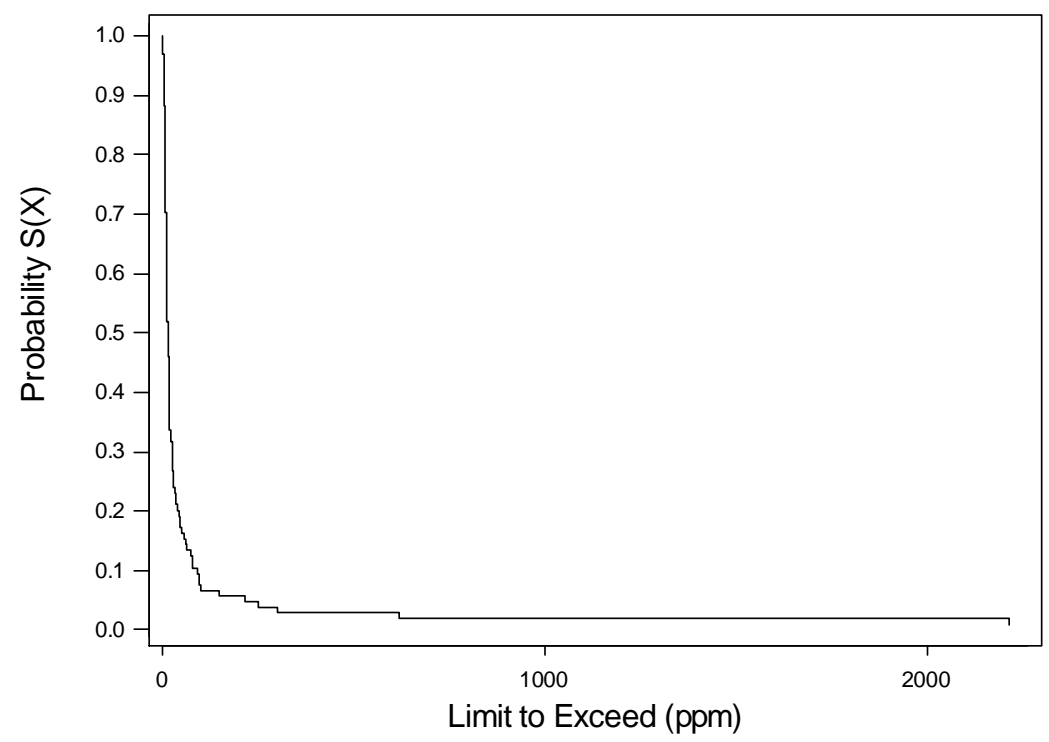

Figure F-2: Survival plots for bulk metallic elements (23 charts) 


\section{F.1.3 Organic Compounds}

Nonparametric Survival Plot for

Acenaphthylene $($ Mean $=0.10 \mathrm{ppm})$

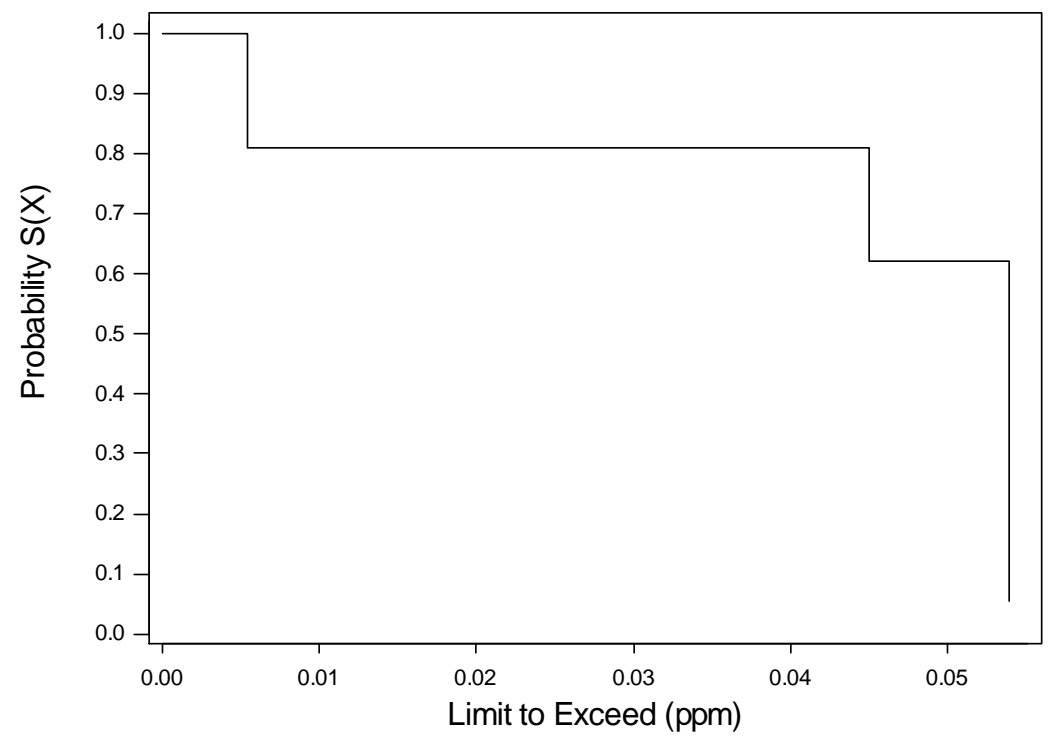

Nonparametric Survival Plot for Anthracene

$($ Mean $=0.37 \mathrm{ppm})$

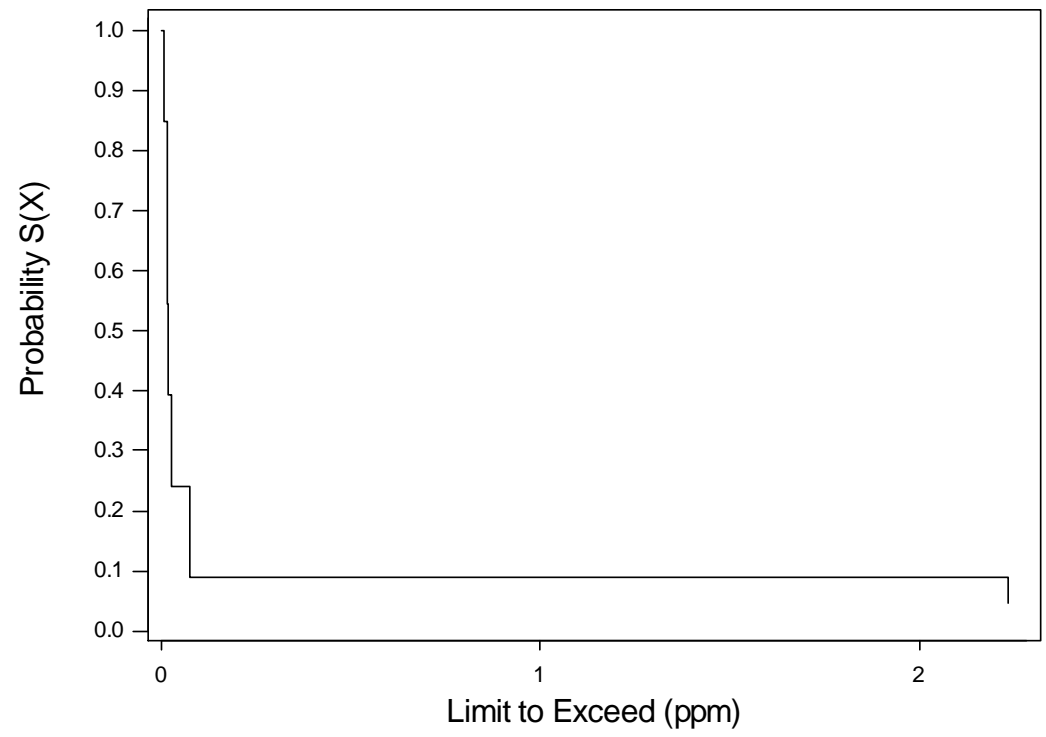


Nonparametric Survival Plot for Benzene

(Mean=0.12 ppm)

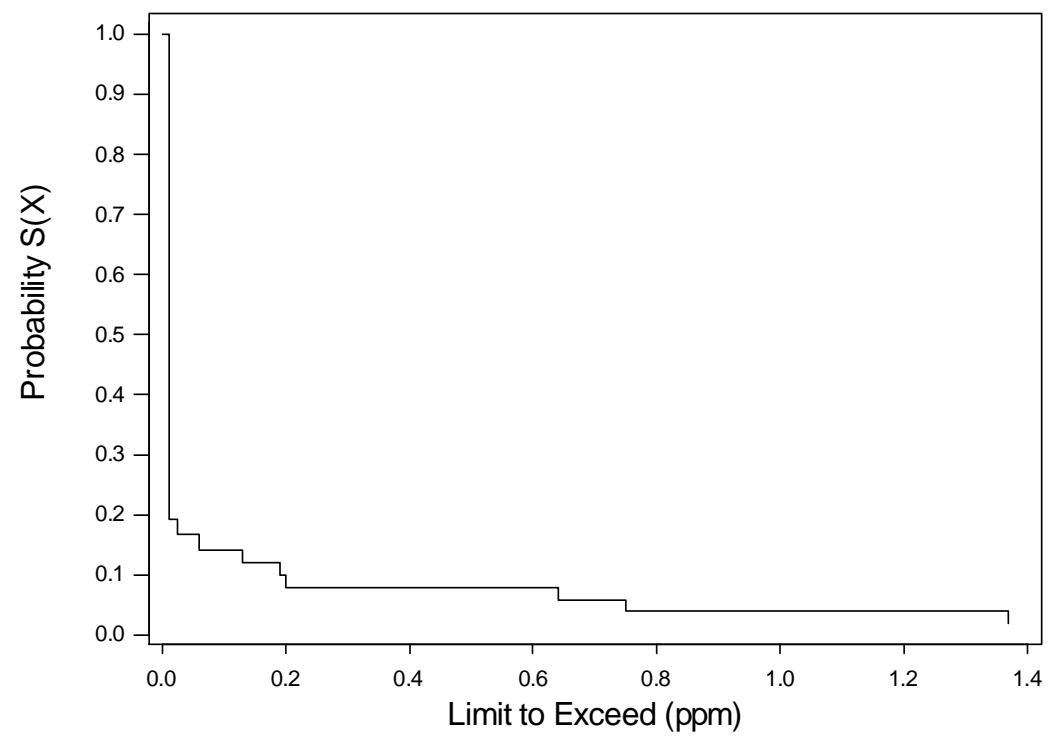

Nonparametric Survival Plot for

Benzo(a)anthrace (Mean = 0.01 ppm)

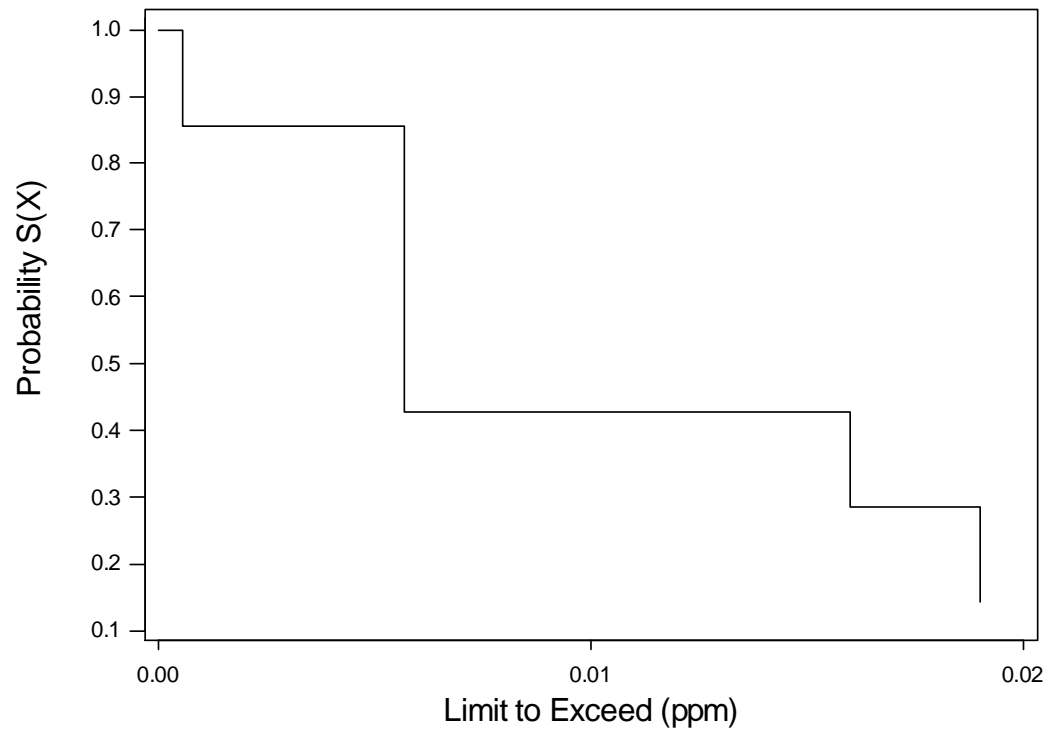


Nonparametric Survival Plot for

Benzo(b)fluornat (Mean $=0.01 \mathrm{ppm}$ )

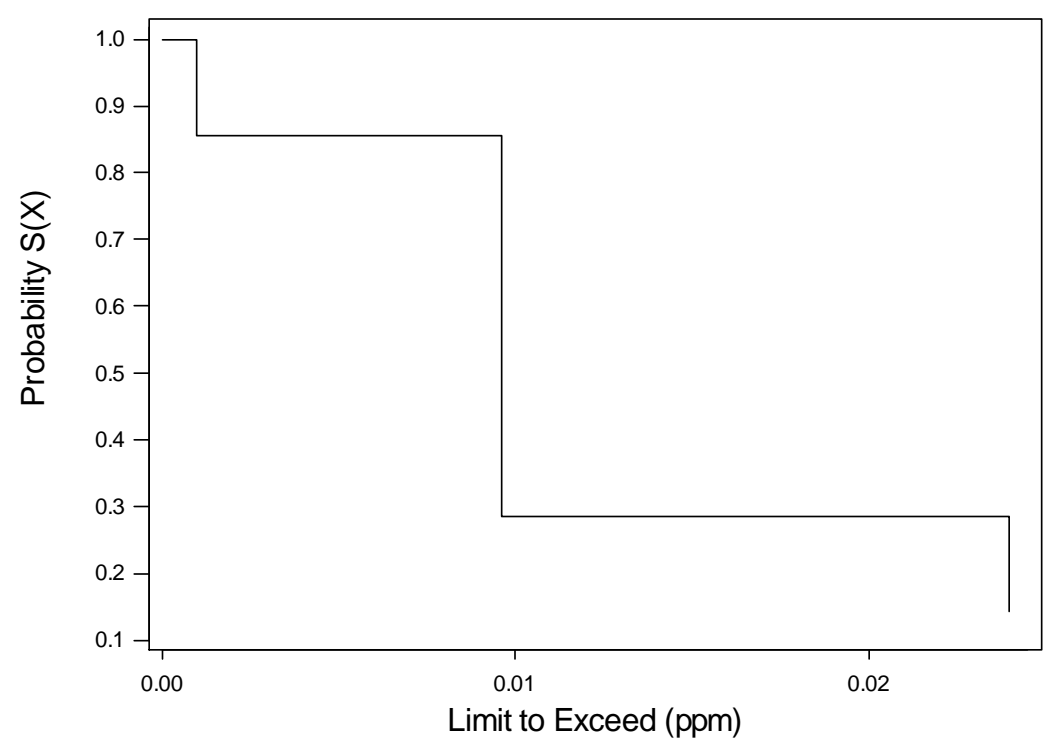

Nonparametric Survival Plot for Benzo(k)fluornat sand_0.002 ppm

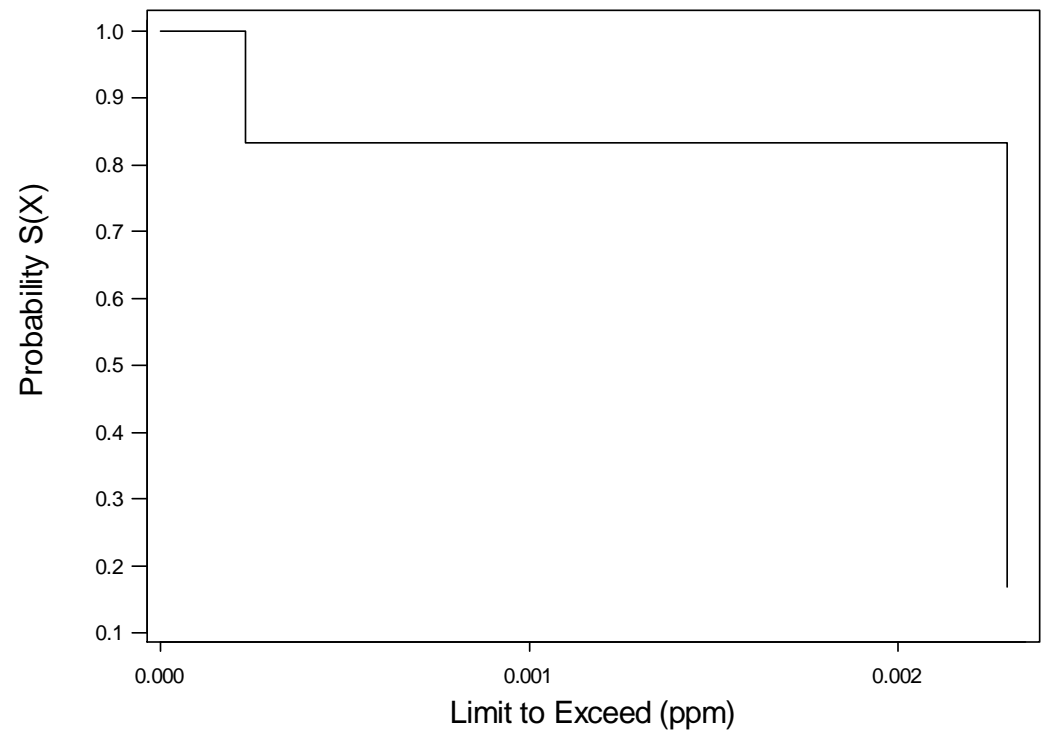


Nonparametric Survival Plot for Chrysene

$($ Mean $=0.027 \mathrm{ppm})$

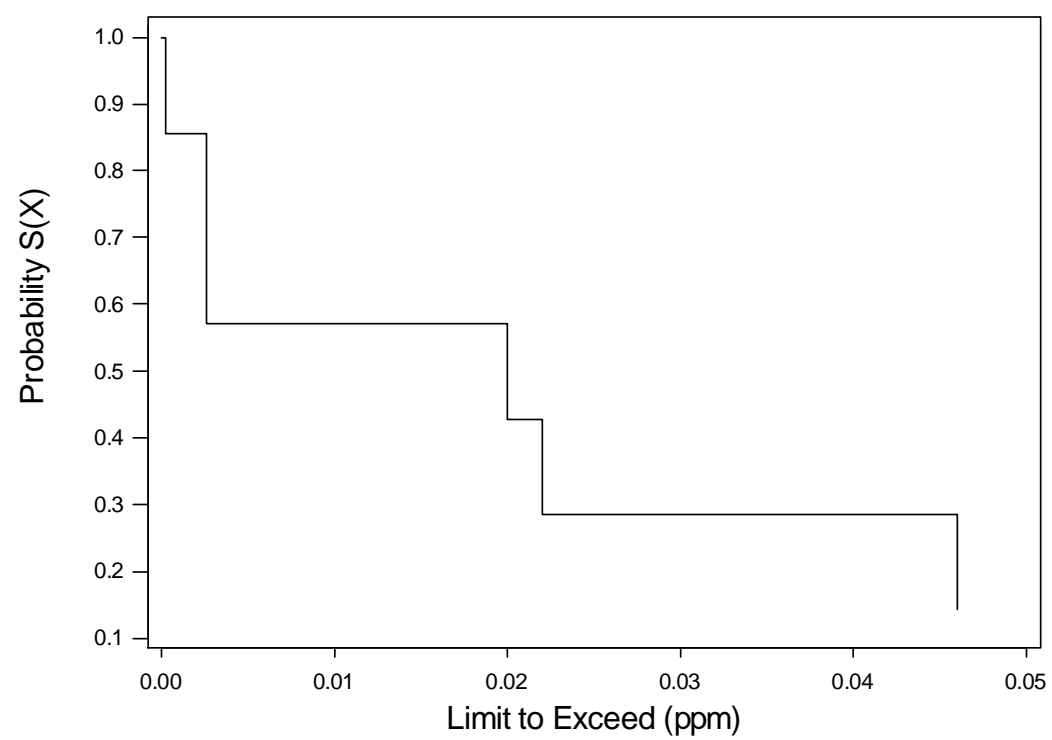

Nonparametric Survival Plot for o-Cresol

(Mean = 2.722 ppm)

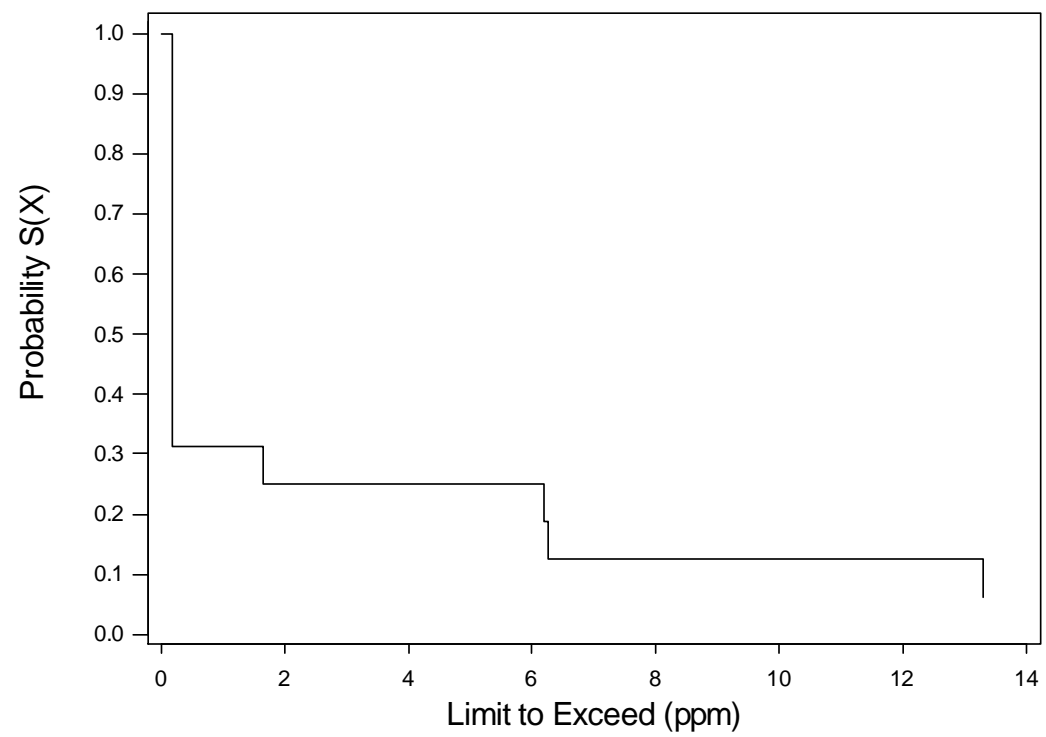


Nonparametric Survival Plot for $m \_p$-Cresol

$($ Mean $=1.119 \mathrm{ppm})$

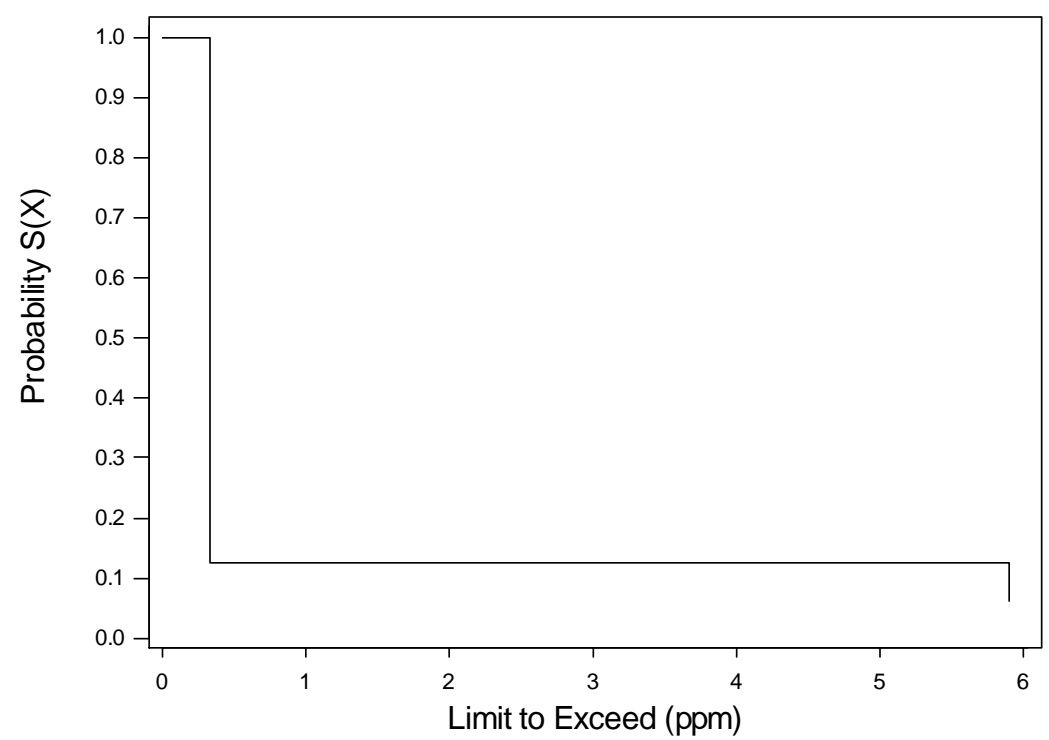

Nonparametric Survival Plot for

2,4-Dimethylphen (Mean = 1.194 ppm)

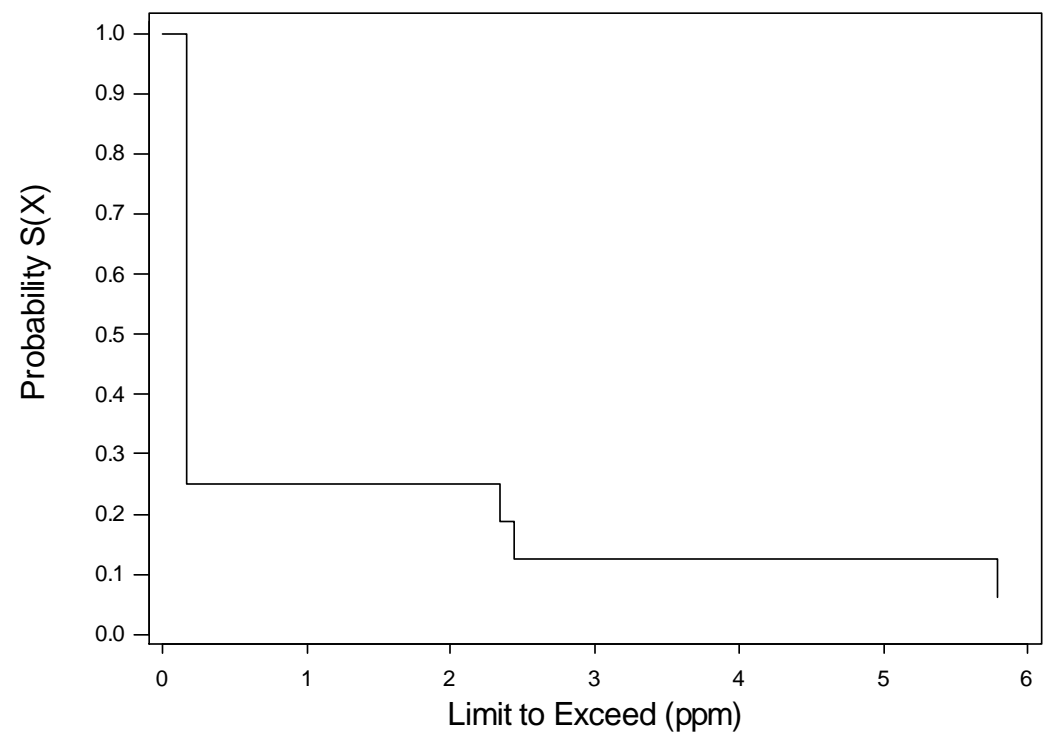


Nonparametric Survival Plot for Ethylbenzene

$($ Mean $=0.046 \mathrm{ppm})$

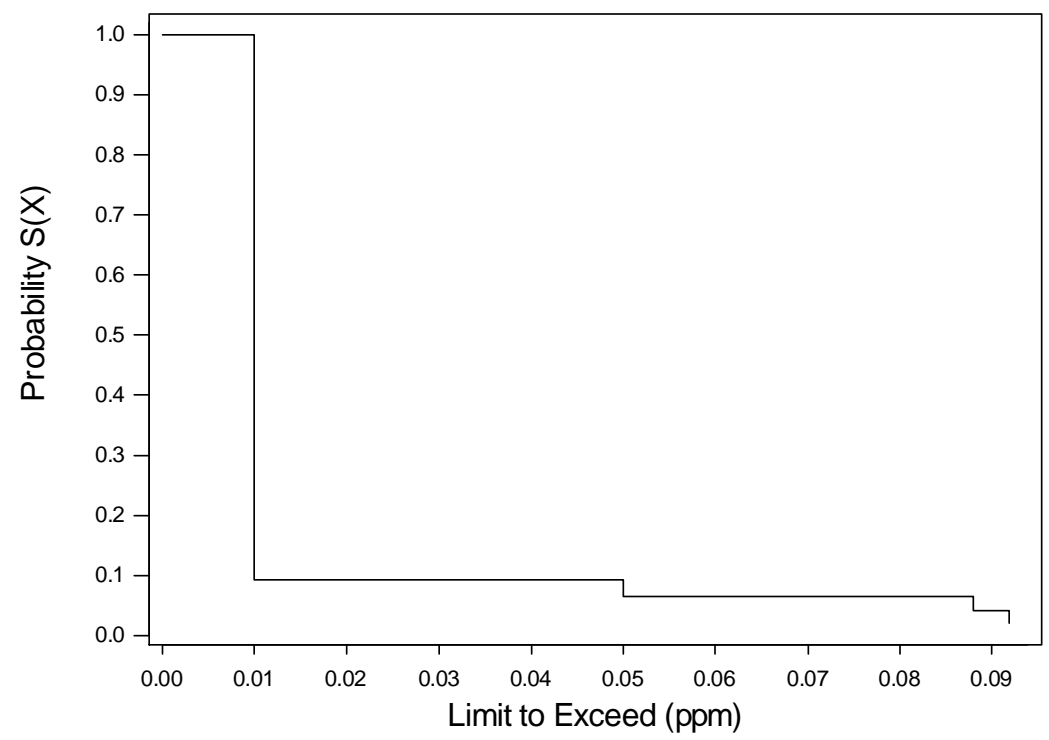

Nonparametric Survival Plot for Fluoranthene

$($ Mean $=0.306 \mathrm{ppm})$

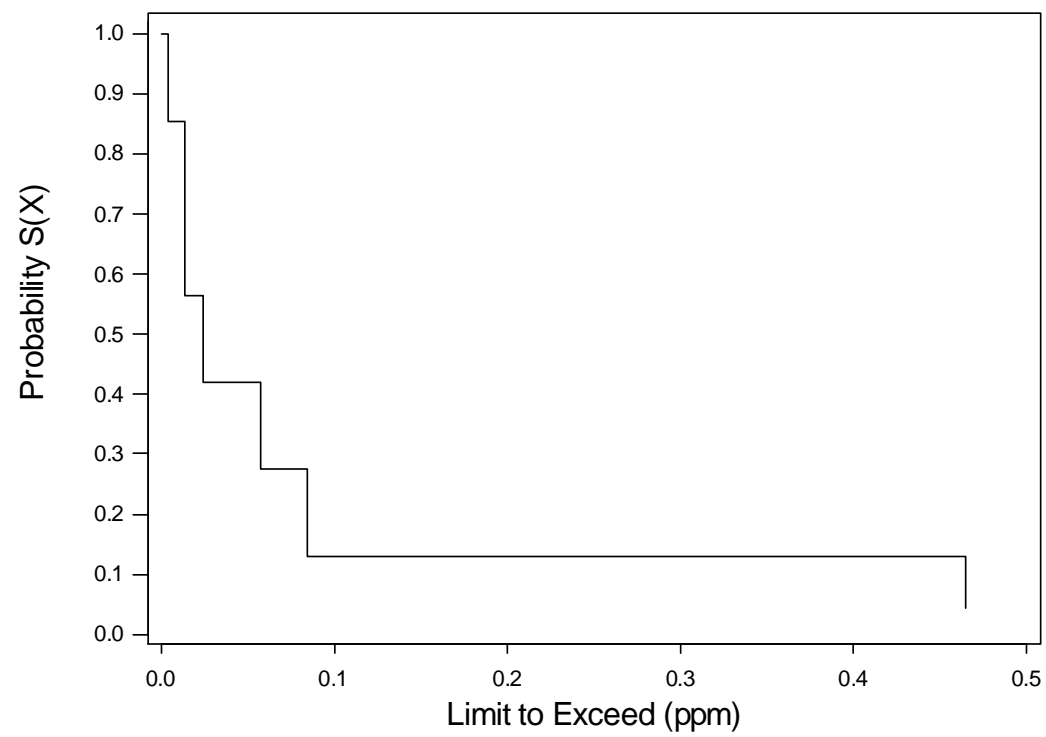


Nonparametric Survival Plot for Fluorene

(Mean=0.037 ppm)

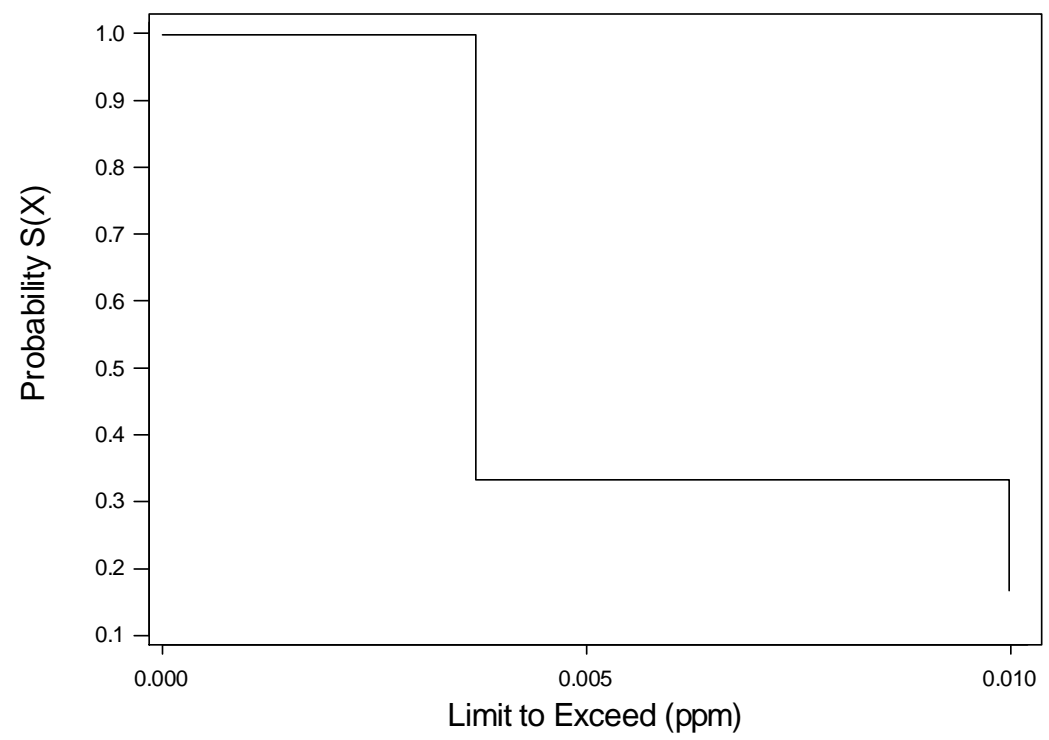

Nonparametric Survival Plot for 1-Methyl naphtha (Mean=0.328 ppm)

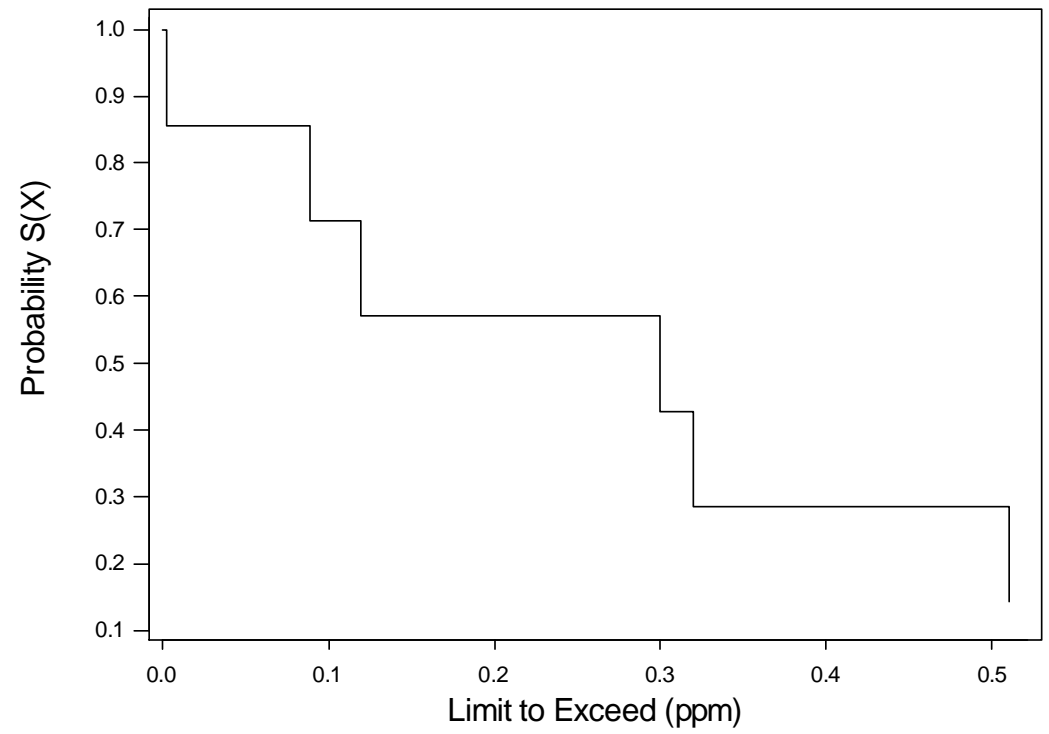


Nonparametric Survival Plot for 2-Methyl

naphtha $($ Mean $=0.406 \mathrm{ppm})$

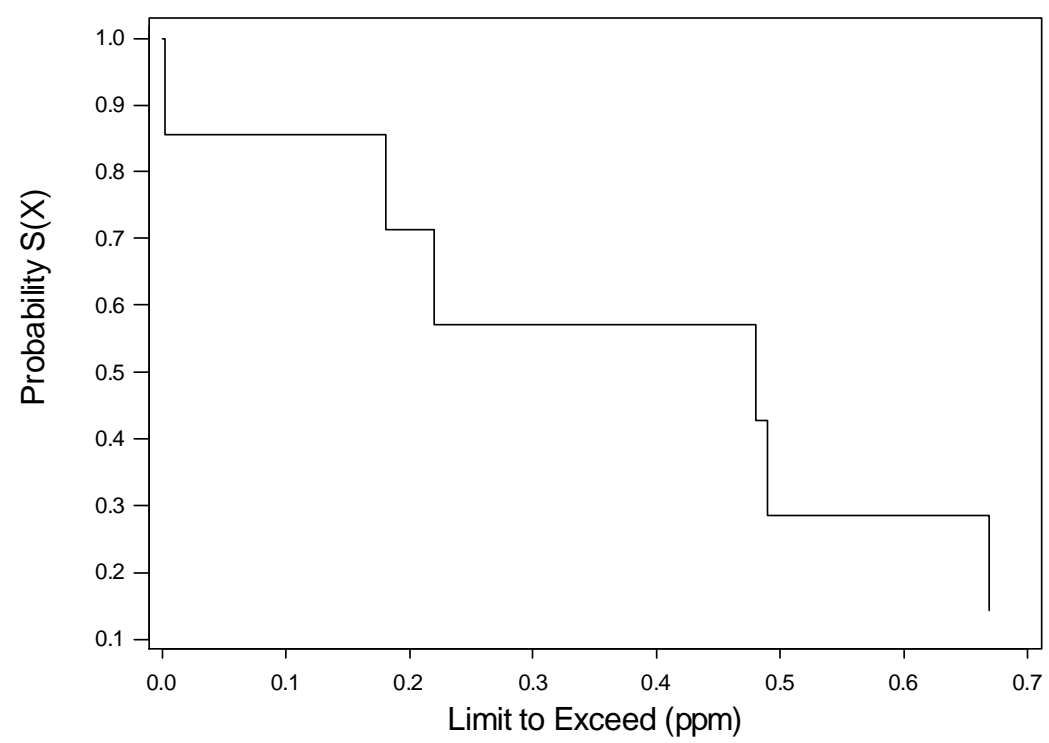

Nonparametric Survival Plot for Naphthalene

$($ Mean $=0.435 \mathrm{ppm})$

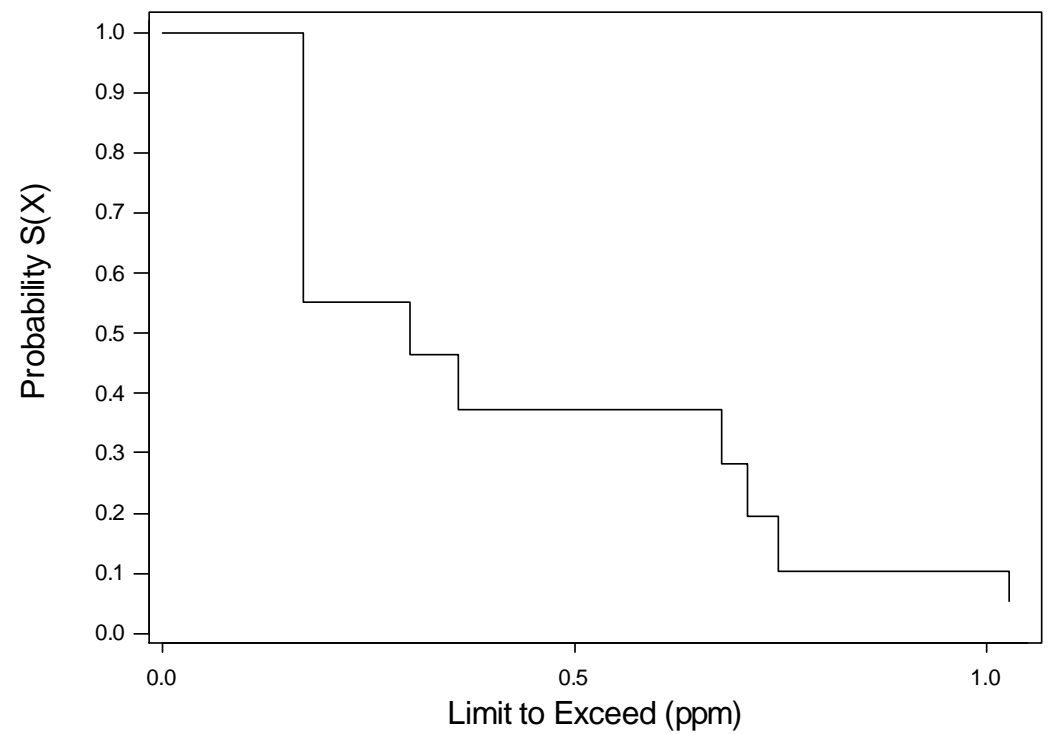


Nonparametric Survival Plot for Phenanthene

$($ Mean $=1.604 \mathrm{ppm})$

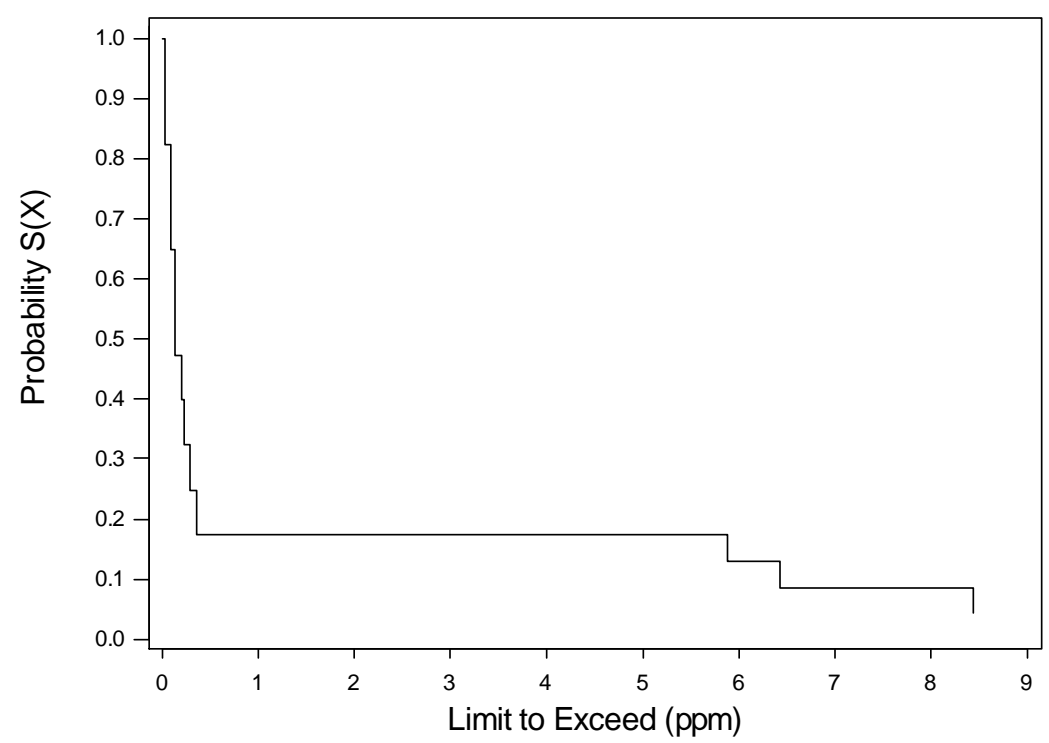

Nonparametric Survival Plot for Pyrene

$($ Mean $=0.047 \mathrm{ppm})$

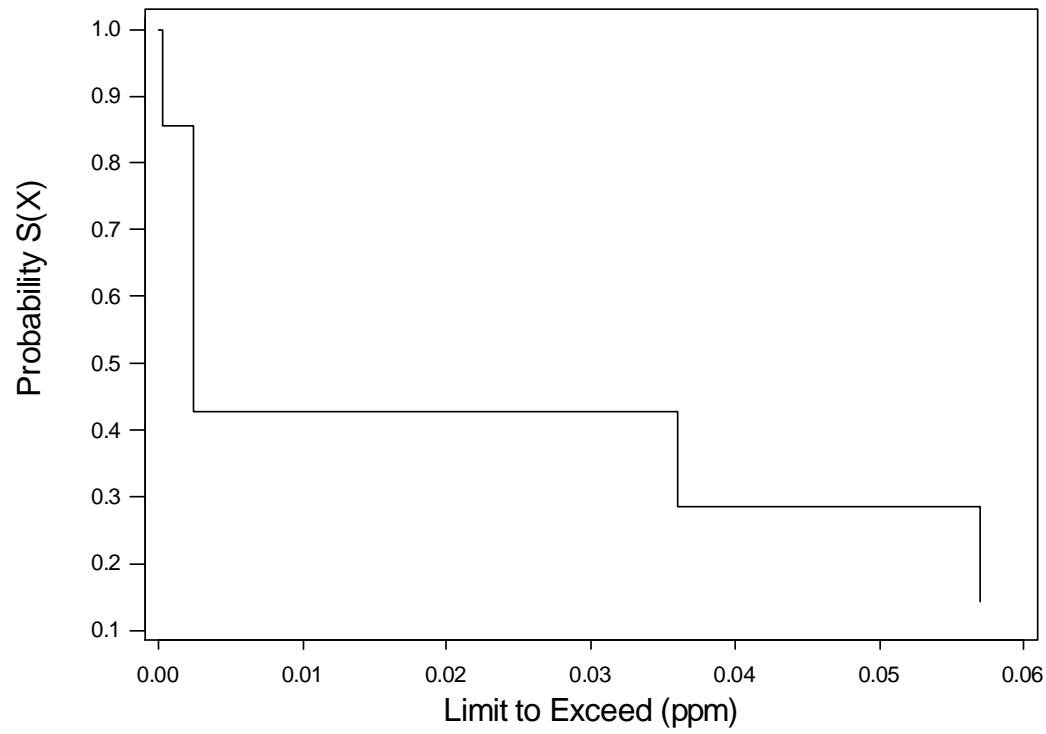


Nonparametric Survival Plot for Styrene

$($ Mean $=7.796$ ppm $)$

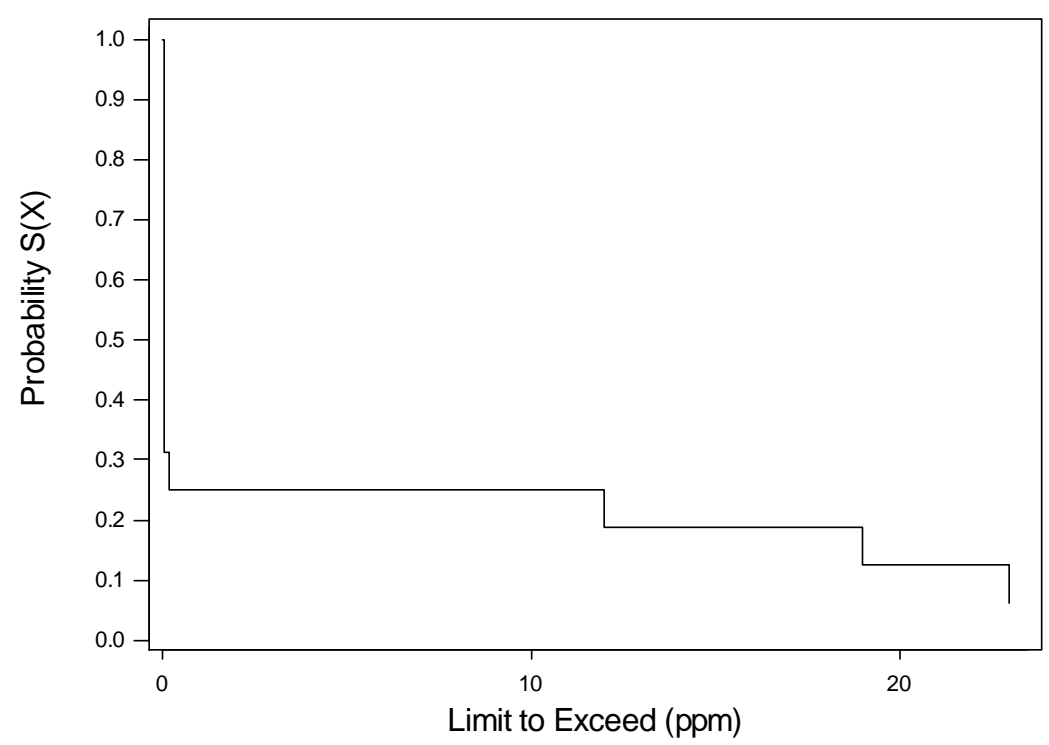

Nonparametric Survival Plot for Toluene

$($ Mean $=0.045 \mathrm{ppm})$

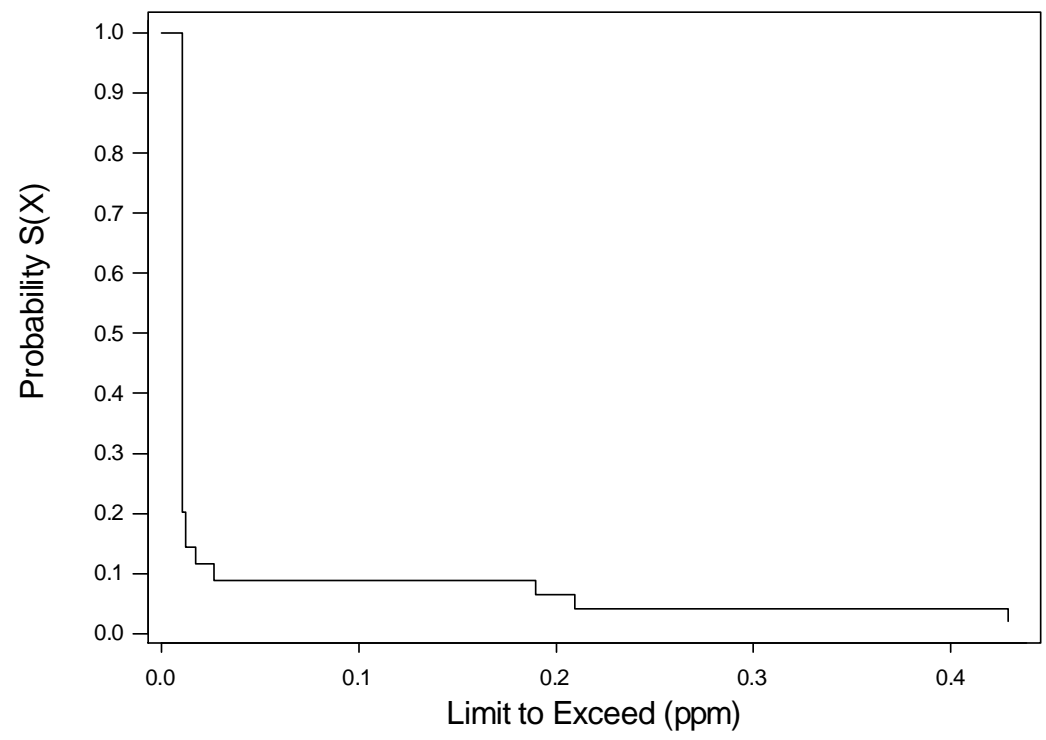


Nonparametric Survival Plot for Xylene

$($ Mean $=0.061 \mathrm{ppm})$

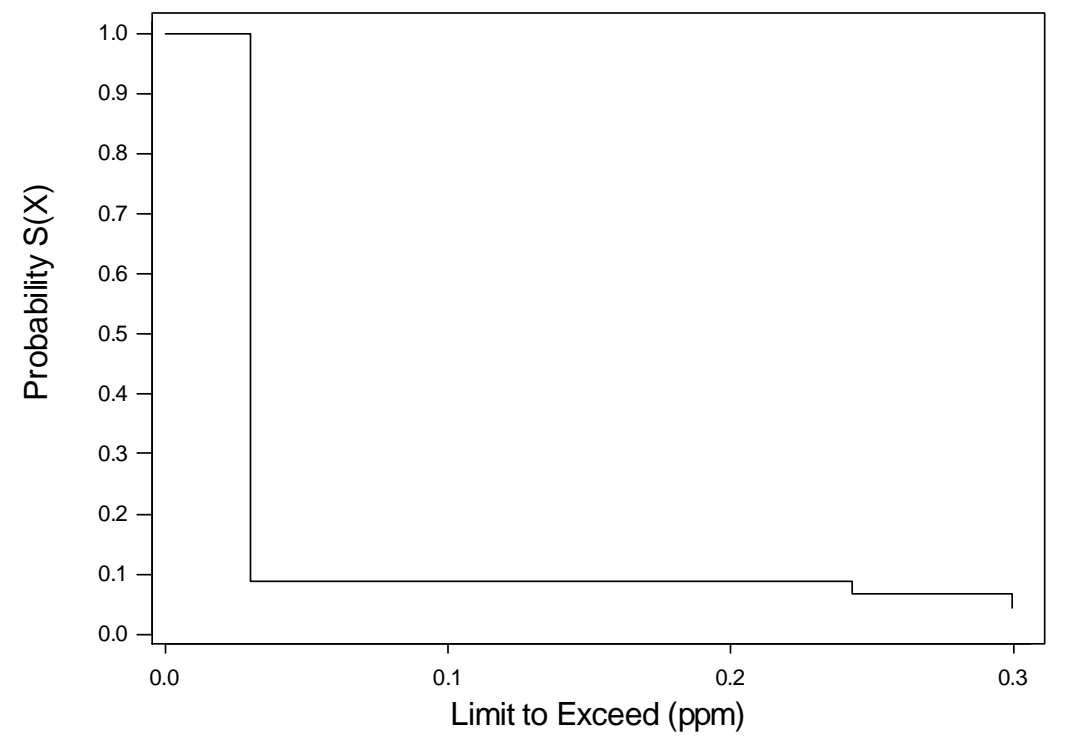

Figure F-3: Survival plots for bulk organic compounds (21 charts)

\section{F.2 Bulk Characterization of Excess Foundry Sand per Metal Cast}

In this section, a series of 12 charts, grouped into Figure F-4, present bulk metallic characterization of excess foundry sand per metal cast. 


\section{F.2.1 Metallic Elements}

\section{Nonparametric Survival Plot for Aluminium (Al)}

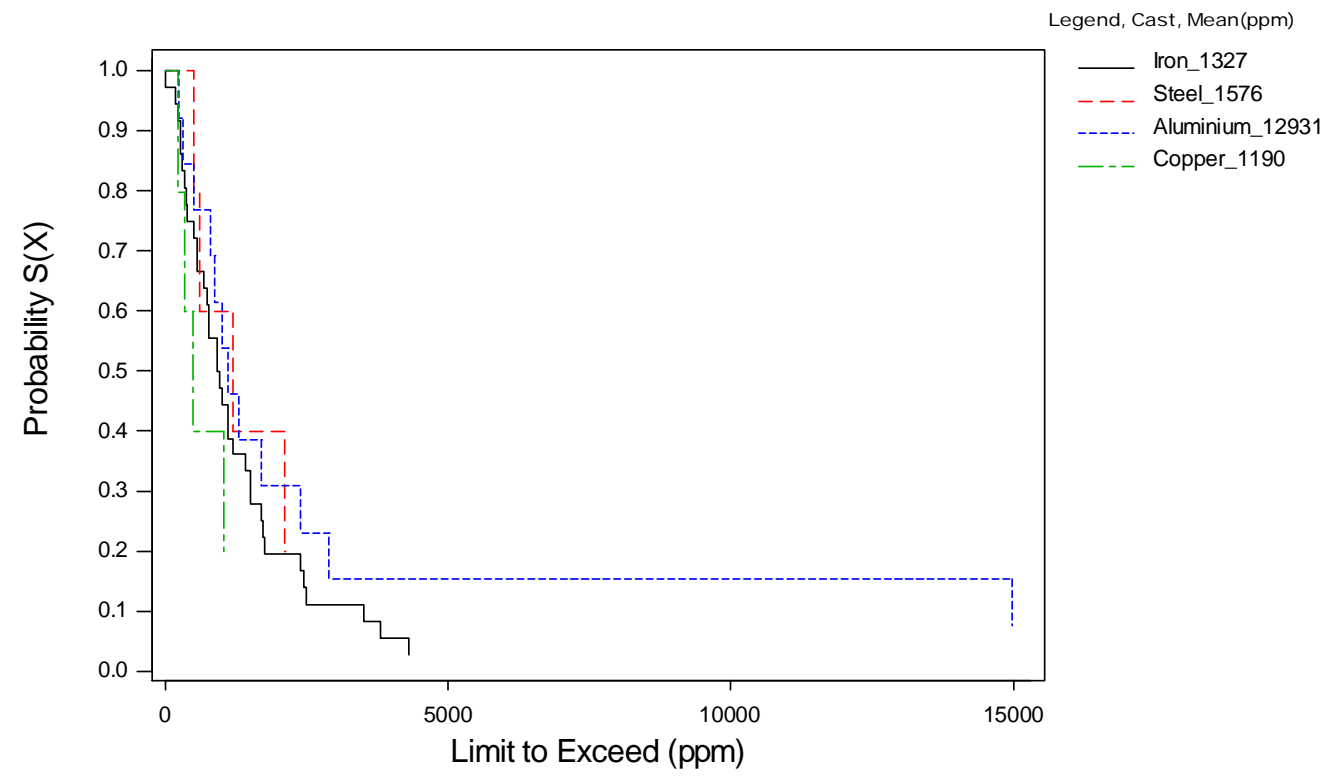

Nonparametric Survival Plot for Arsenic (As)

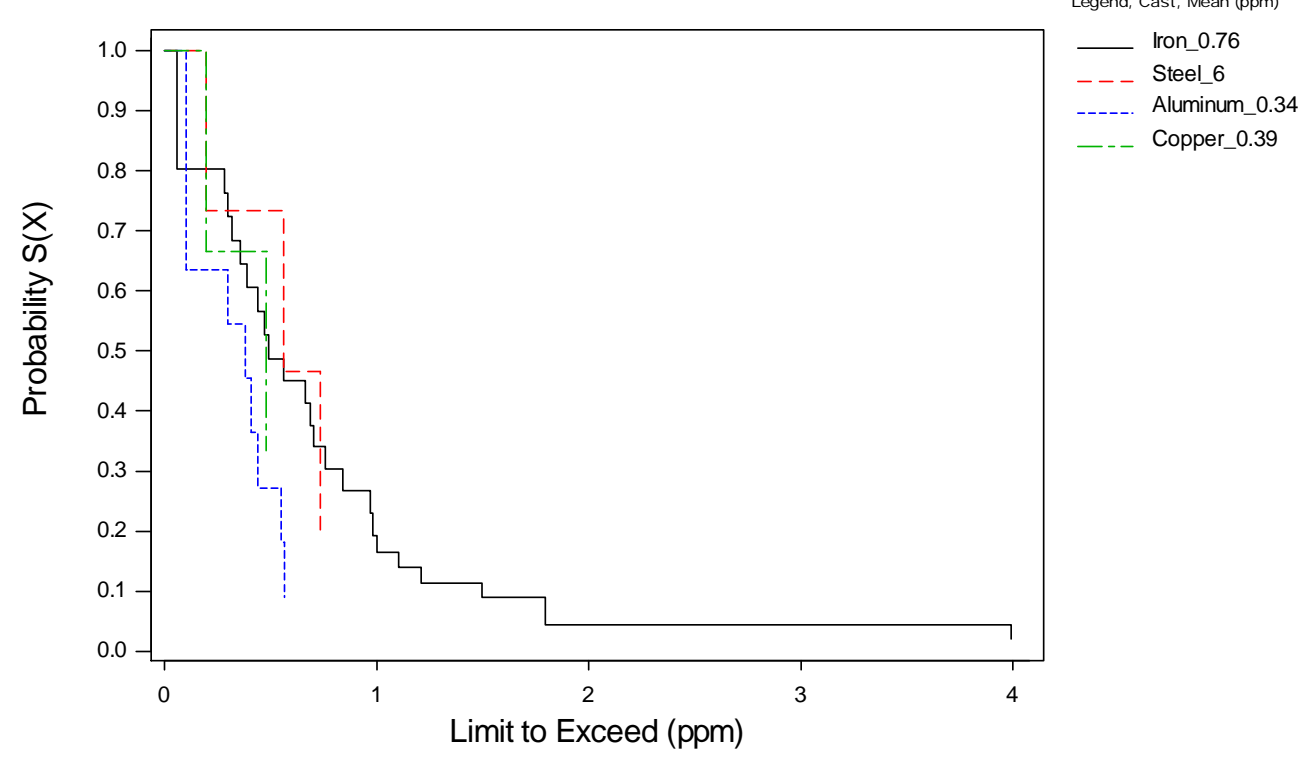


Nonparametric Survival Plot for Barium (Ba)

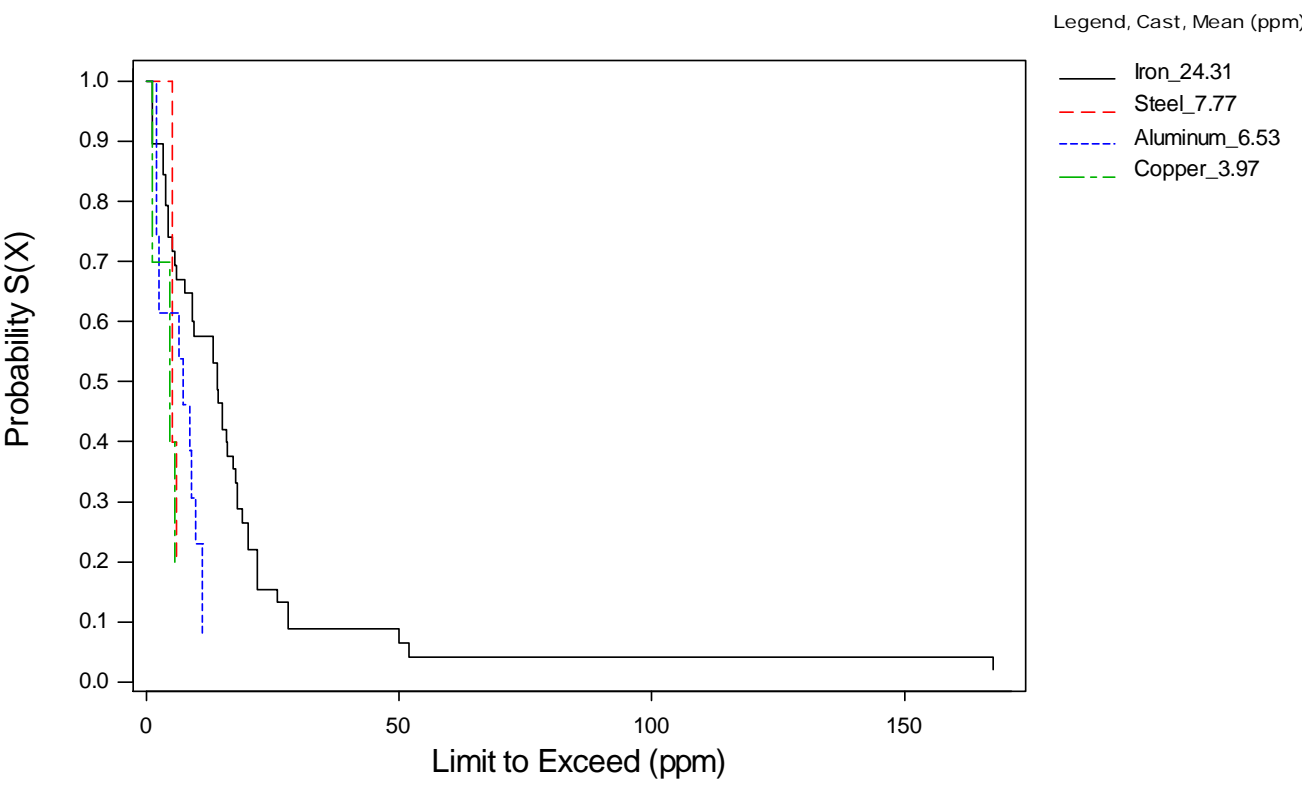

Nonparametric Survival Plot for Boron (B)

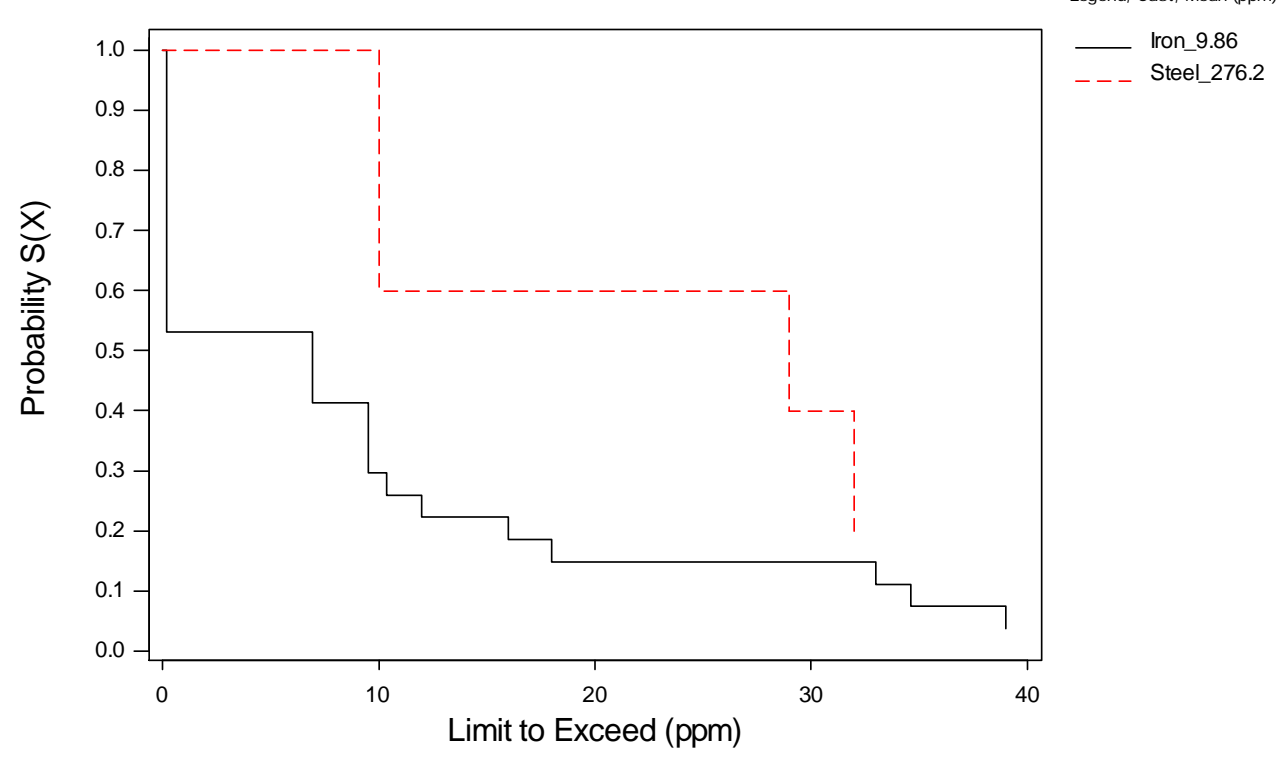


Nonparametric Survival Plot for Calcium (Ca)

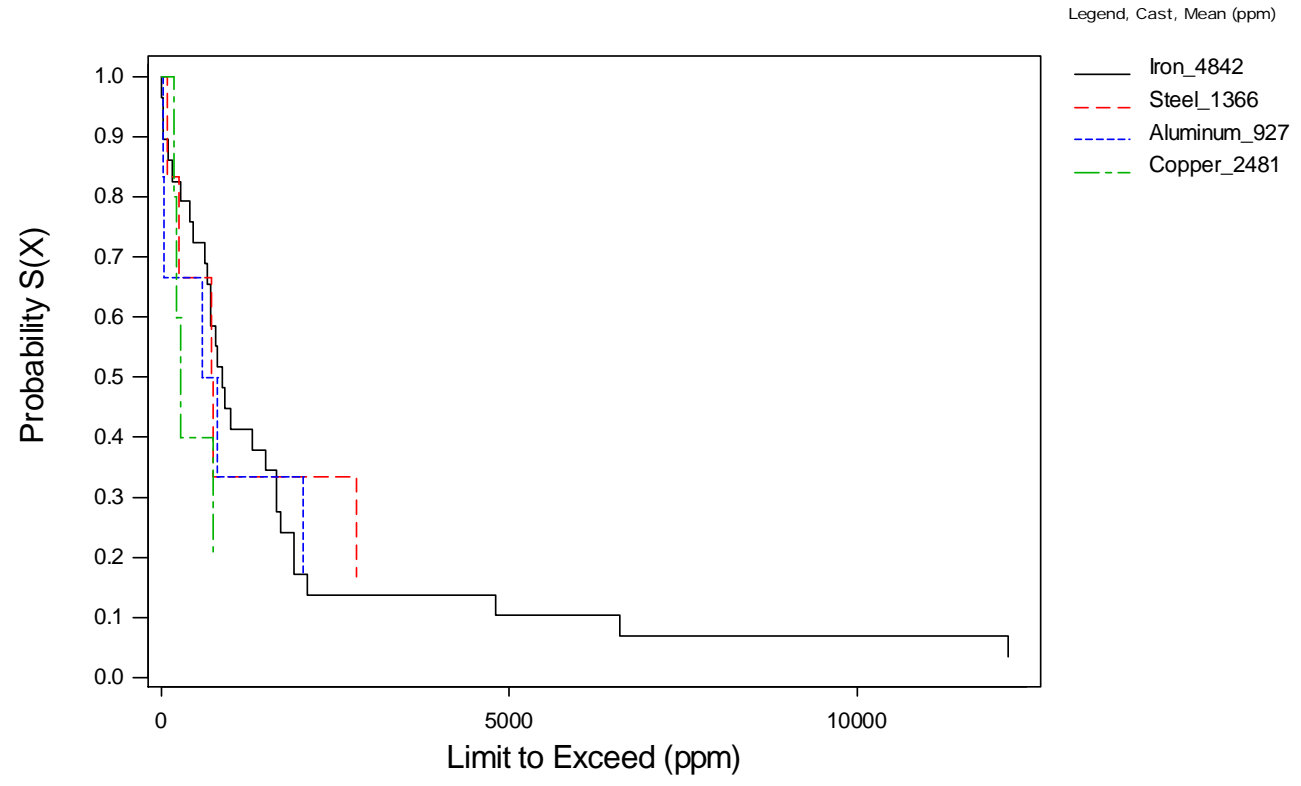

Nonparametric Survival Plot for Chromium (Cr)

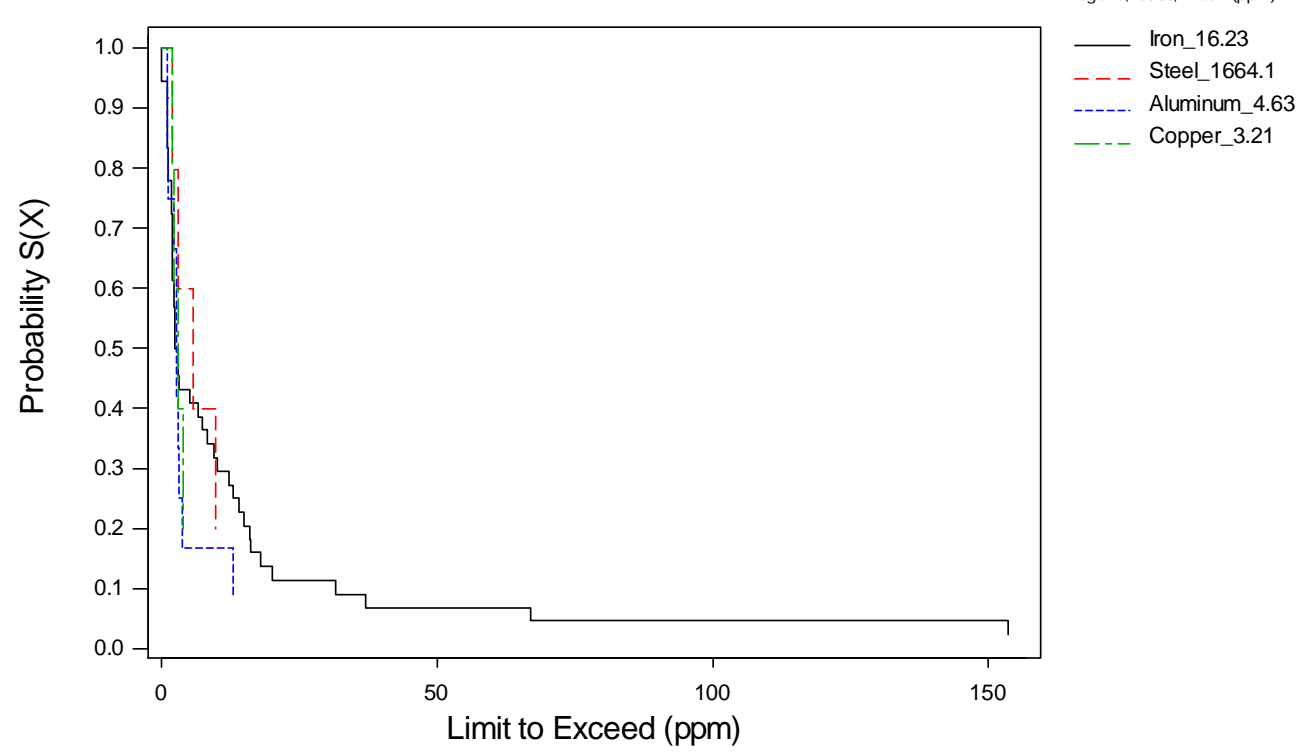


Nonparametric Survival Plot for Copper (Cu)

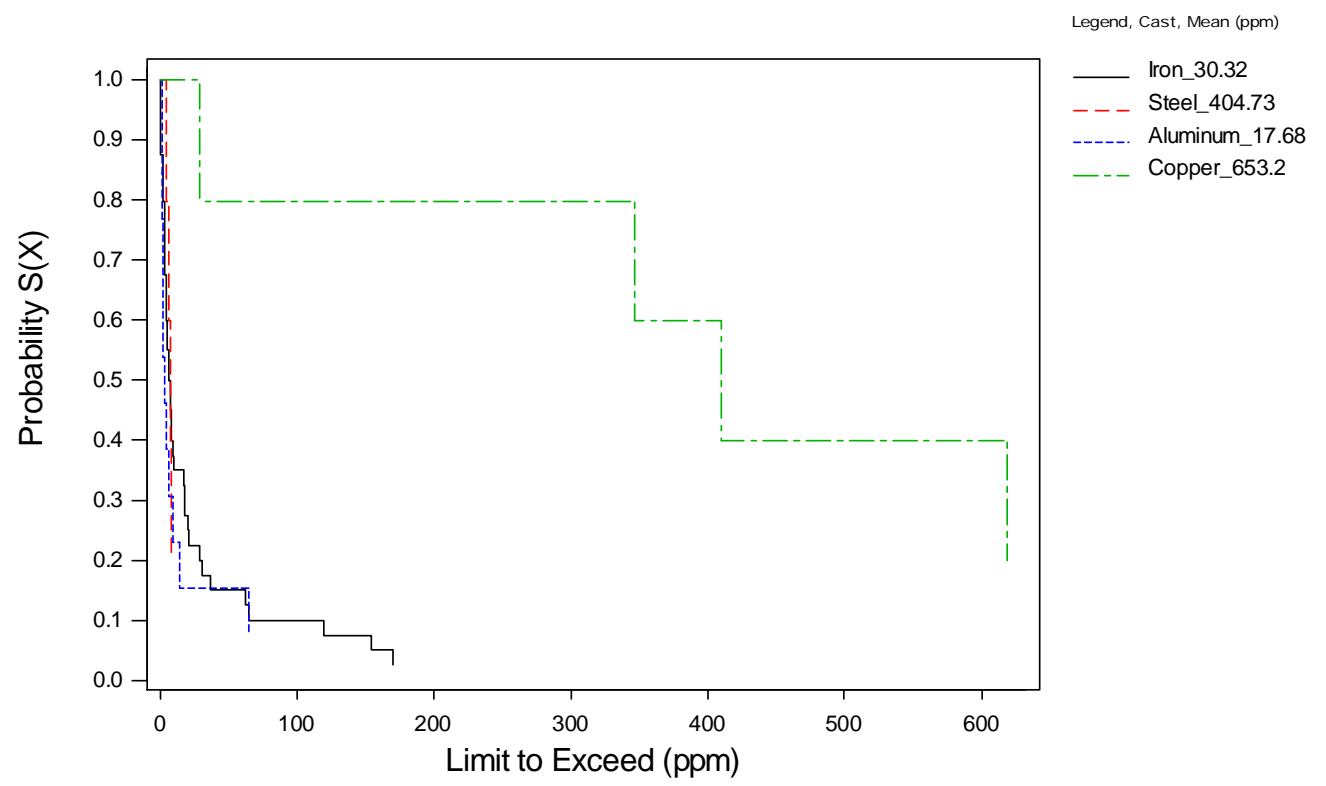

Nonparametric Survival Plot for Iron (Fe)

Legend, Cast, Mean (ppm)

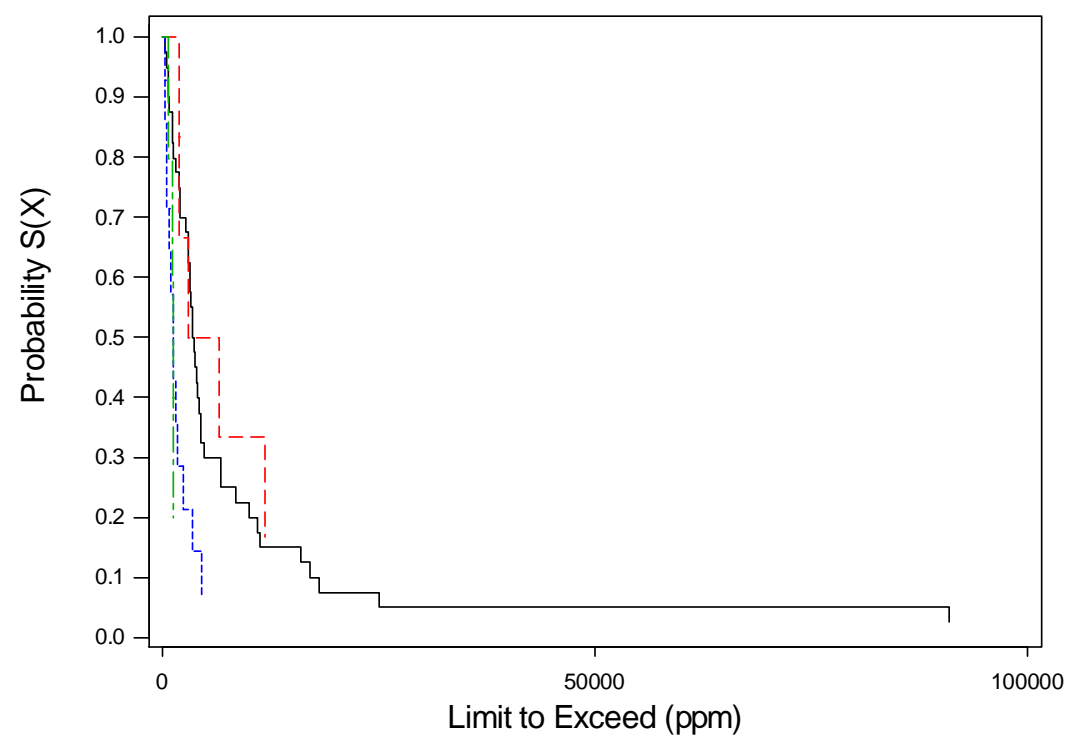

Iron 11163

Steel_60819

Aluminum_1898

Copper_1986 
Nonparametric Survival Plot for Lead (Pb)

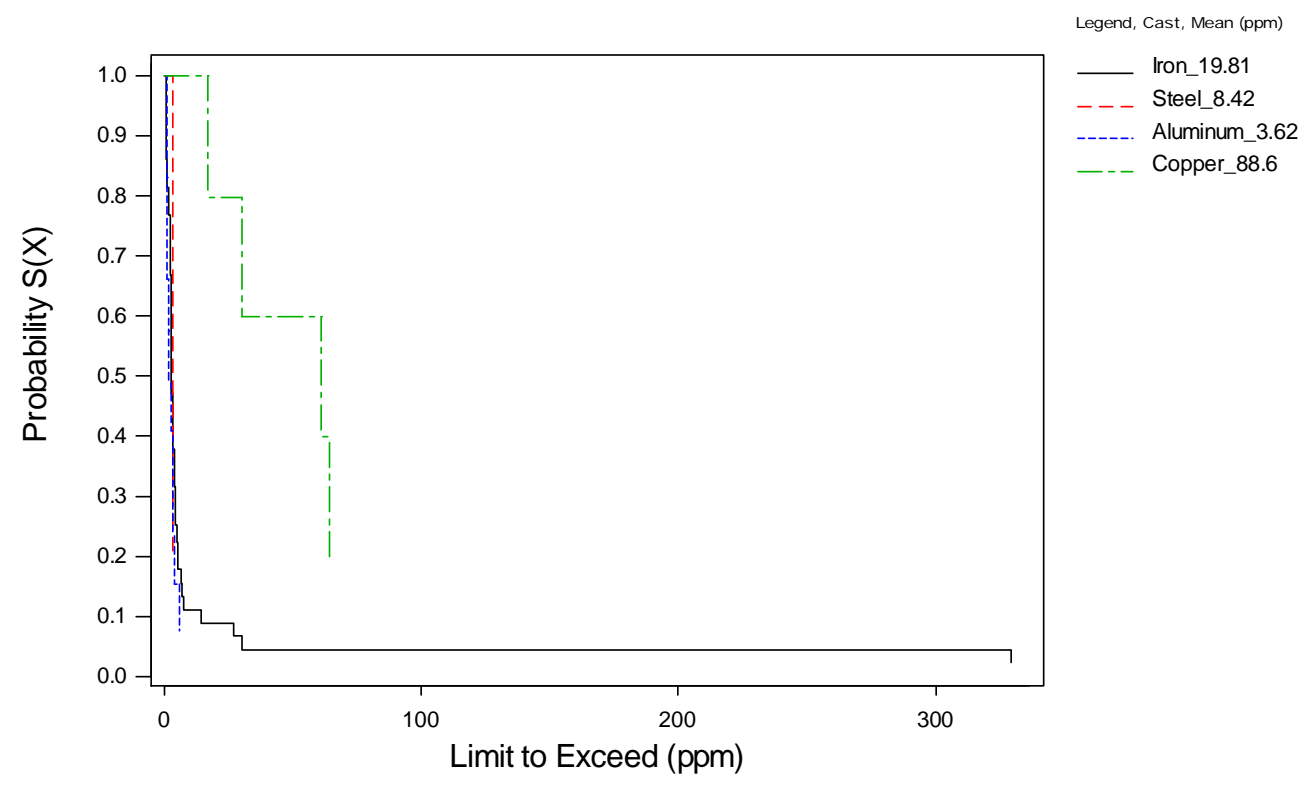

Nonparametric Survival Plot for Manganese (Mn)

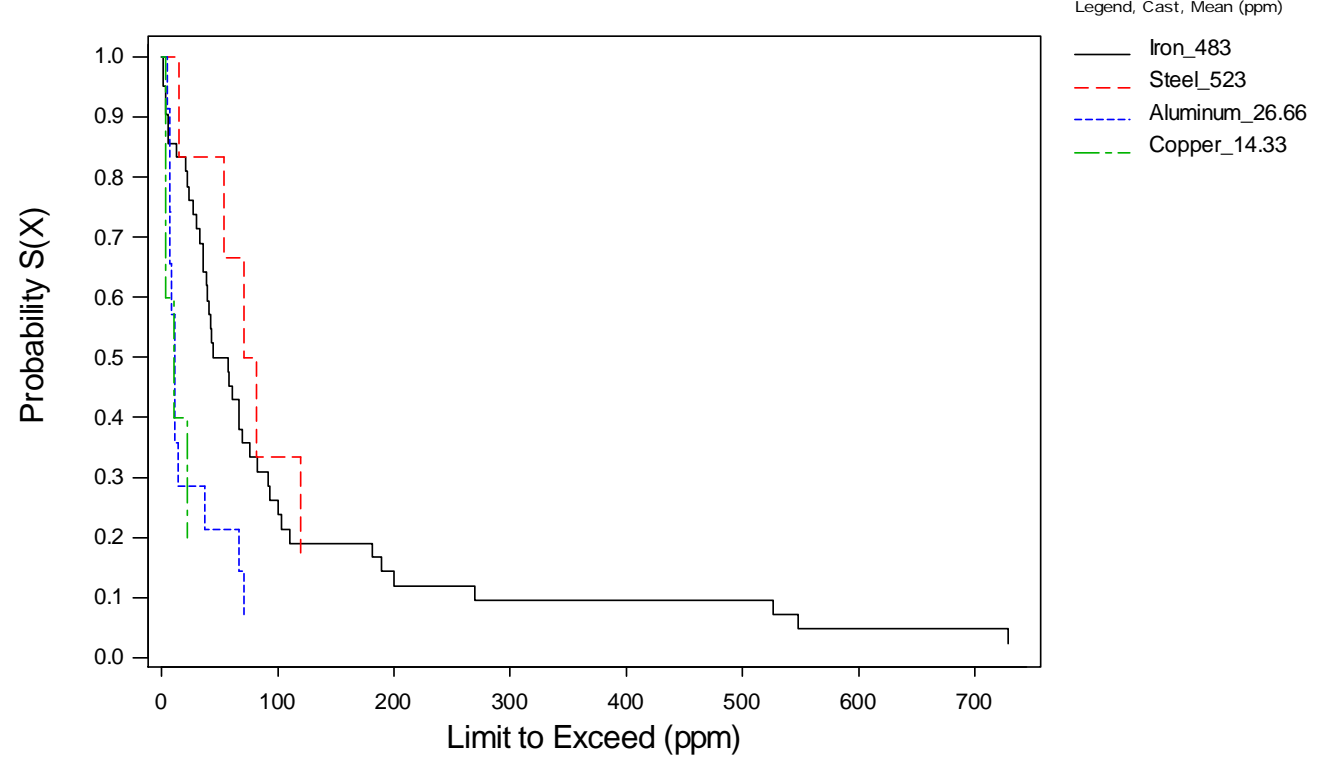


Nonparametric Survival Plot for Nickel (Ni)

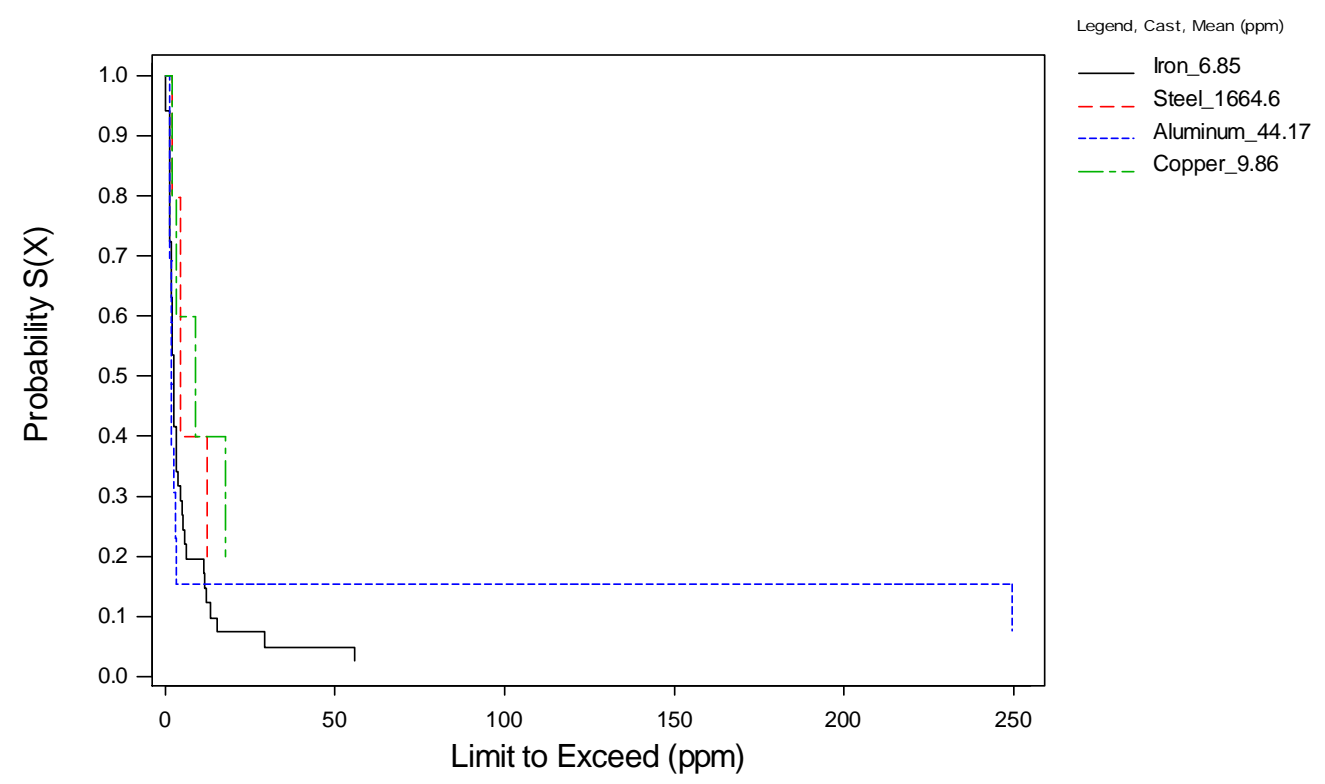

Nonparametric Survival Plot for Zinc (Zn)

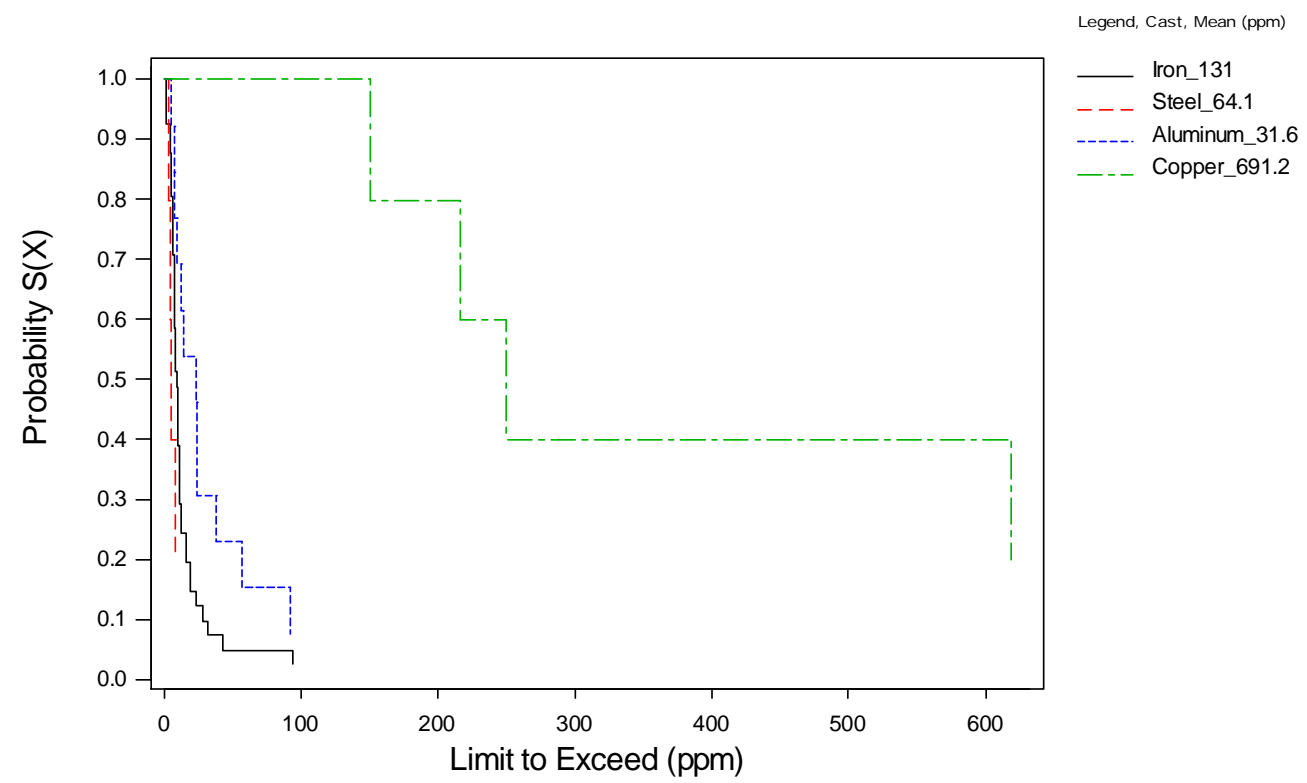

Figure F-4: Survival plots for bulk metallic elements per metal cast (12 charts) 


\section{F.3 Leaching Characterization of Excess Foundry Sand per Leaching Protocols}

In this section, a series of 49 charts present the leaching characterization of excess foundry sand per leaching protocol. The first 16 charts, grouped into Figure F-5, are for general chemical parameters. The second 20 charts, grouped into Figure F-6, are for metallic elements. The remaining 13 charts, grouped into Figure F-7, are for organic compounds exclusively in TCLP leachate.

\section{F.3.1 General Chemical Parameters}

Nonparametric Survival Plot for Acidity

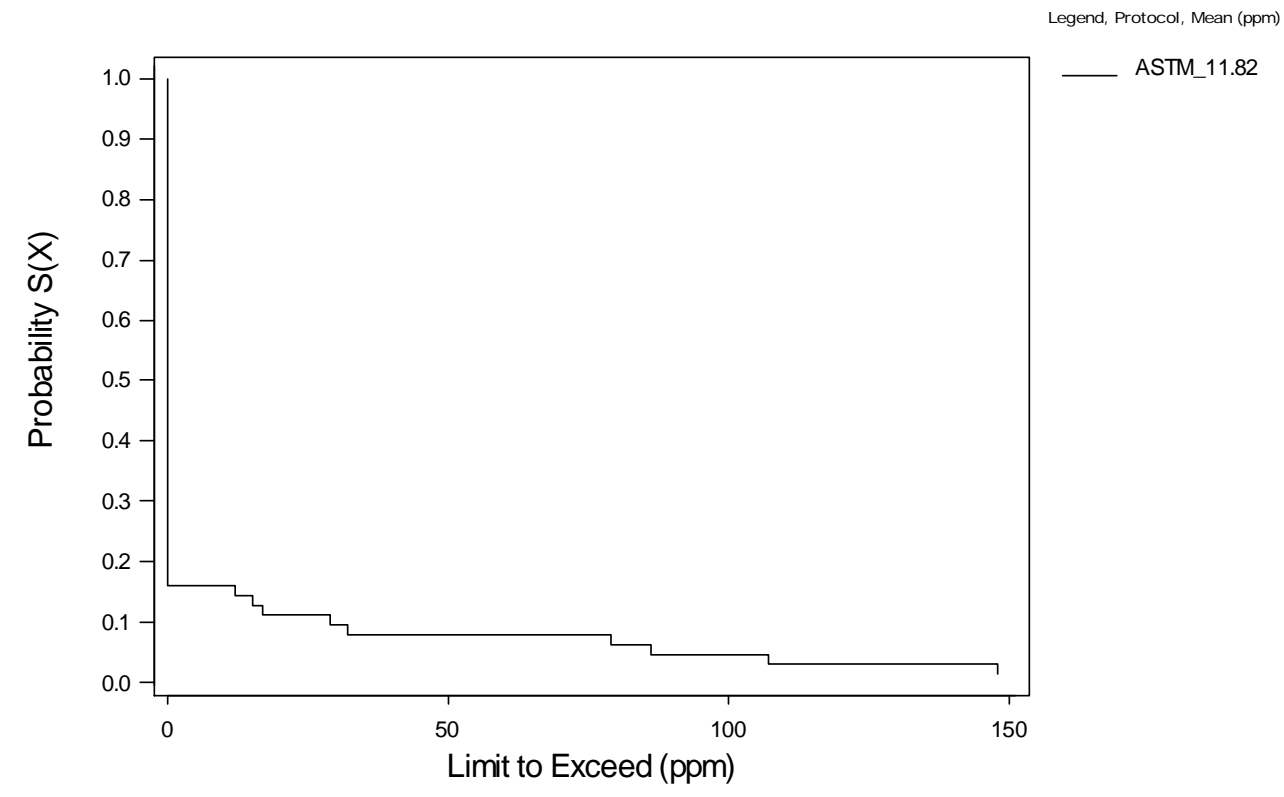


Nonparametric Sunvival Plot for Alkalinity

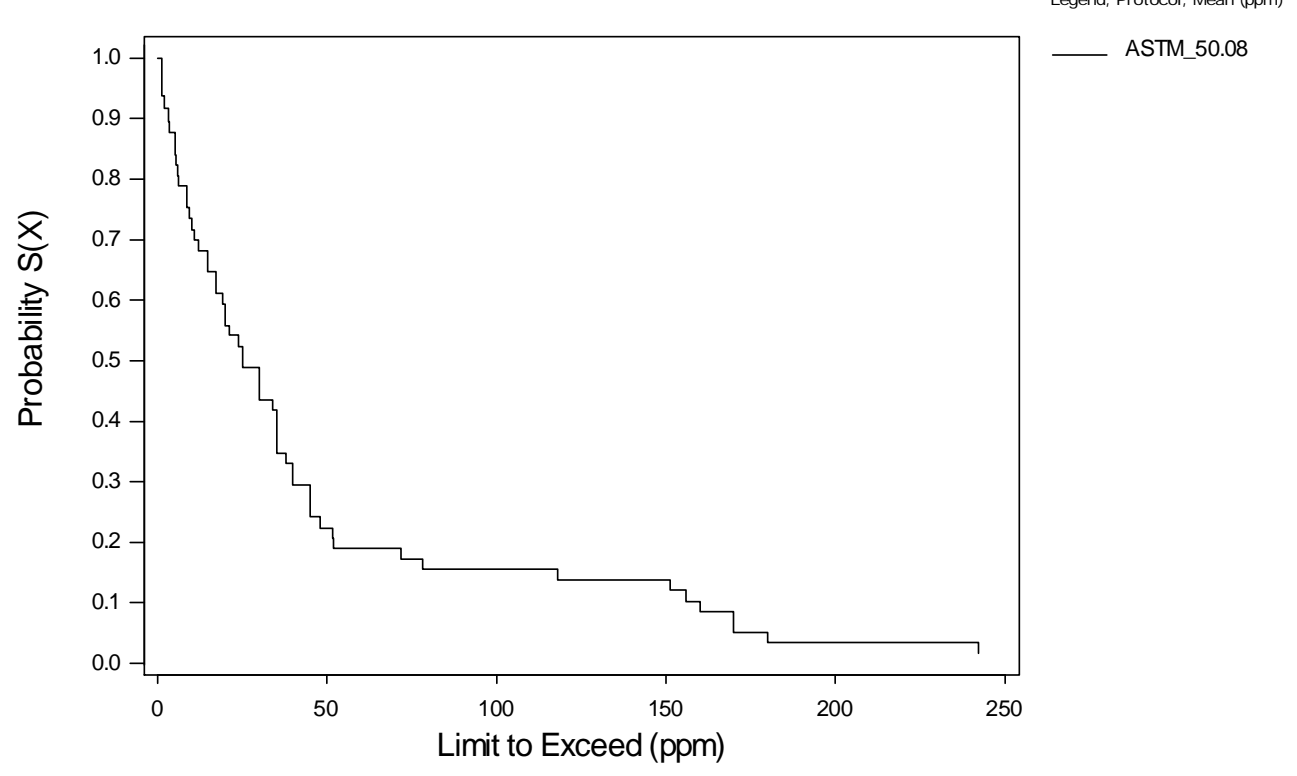

Nonparametric Survival Plot for Ammonia

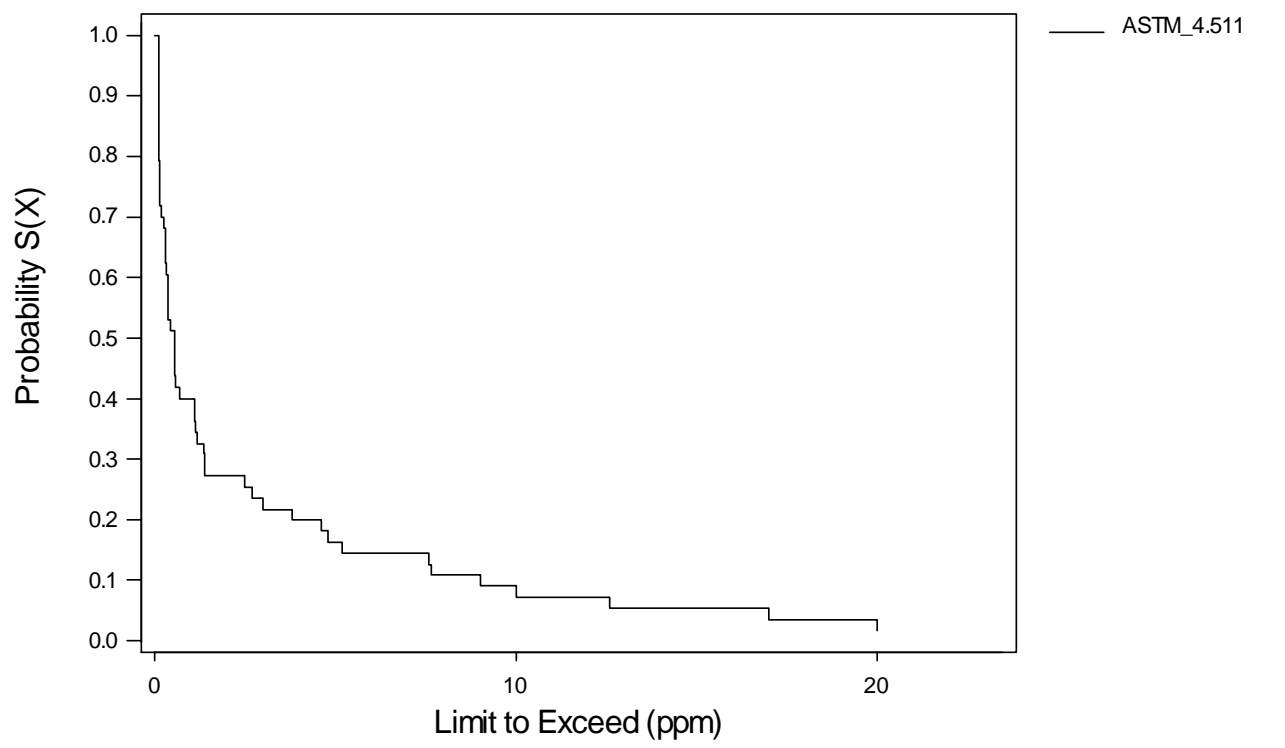


Nonparametric Survival Plot for Chemical Oxygen Demand (COD)

Legend, Protocol, Mean (ppm)

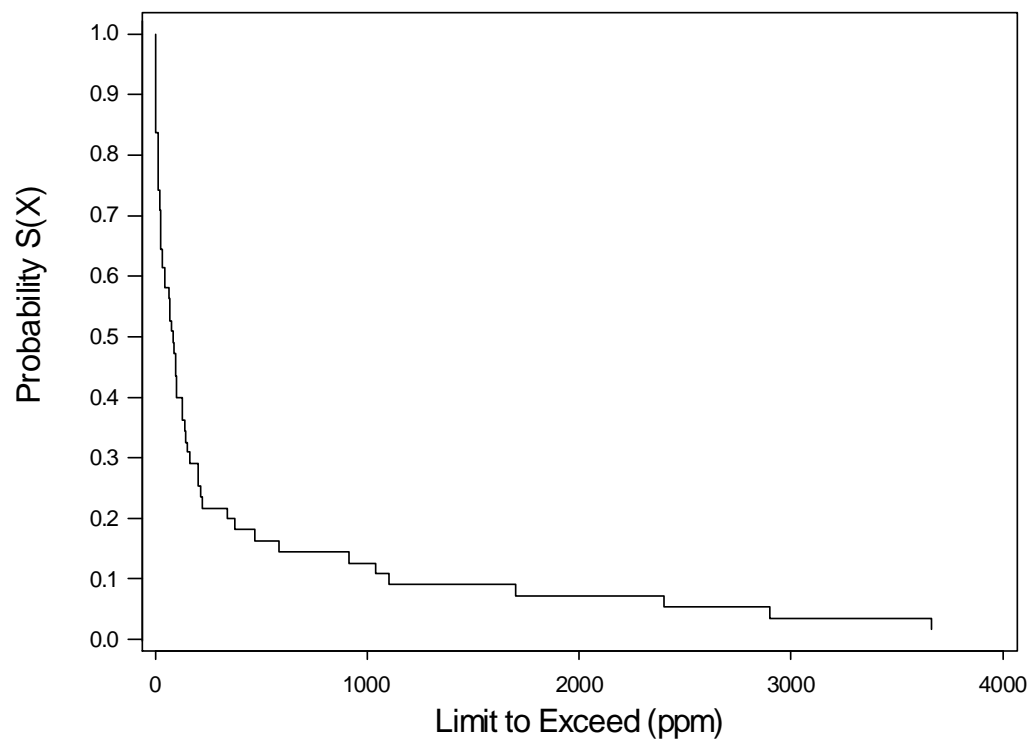

ASTM_439

Nonparametric Sunvival Plot for Chlorides (Cl)

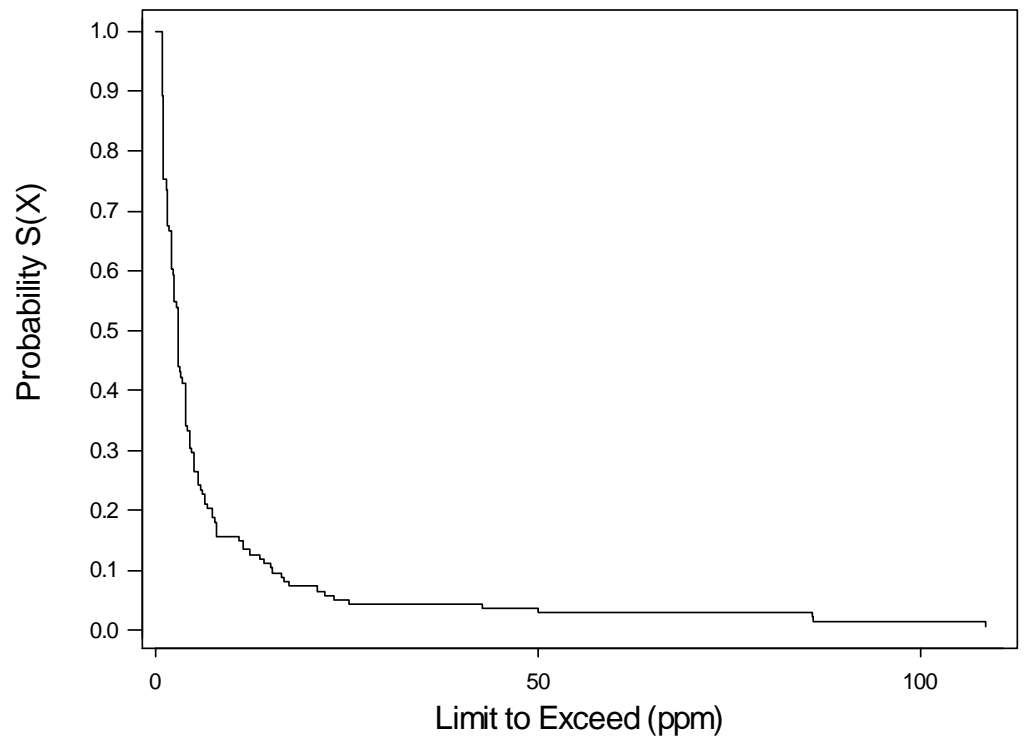

Legend, Protocol, Mean (ppm) ASTM_8 
Nonparametric Survival Plot for Cyanide (Cy)

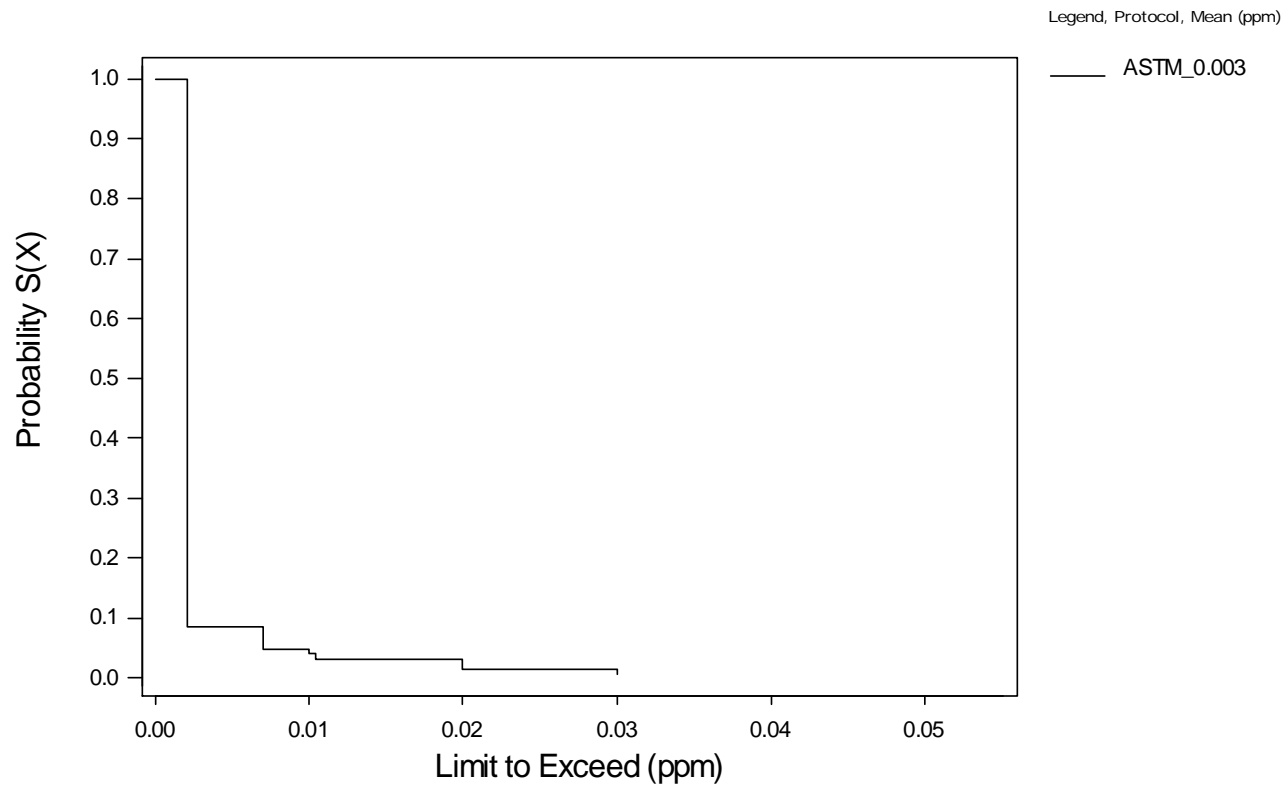

Nonparametric Survival Plot for Fluoride (F)

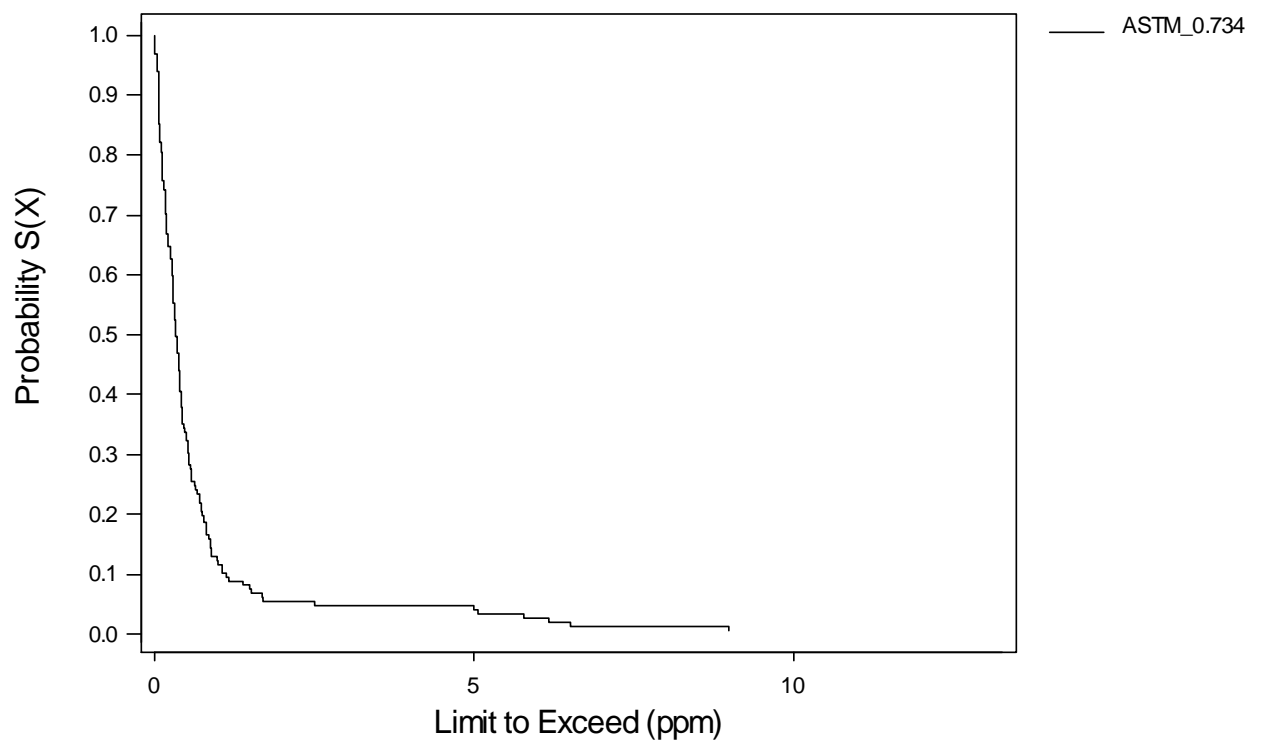




\section{Nonparametric Survival Plot for Formaldehyde}

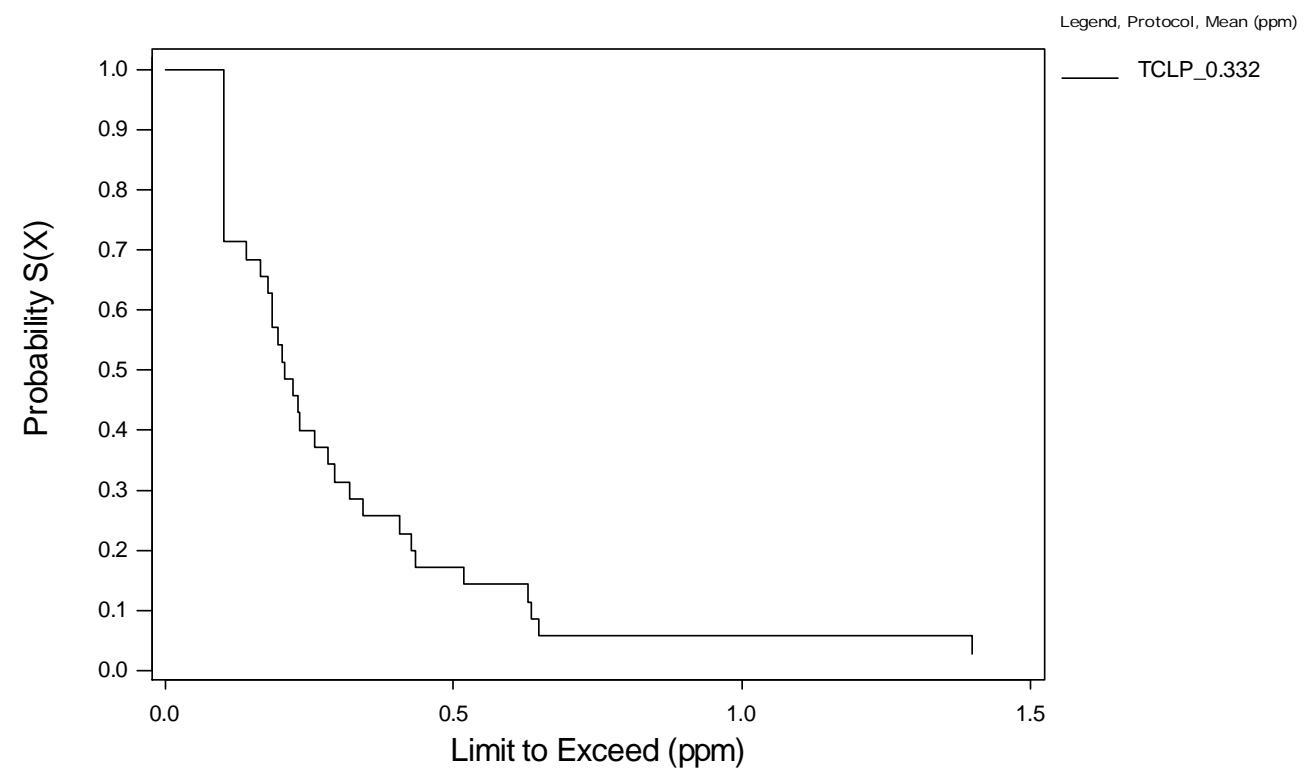

Nonparametric Survival Plot for Nitrate

Legend, Protocol, Mean (ppm)

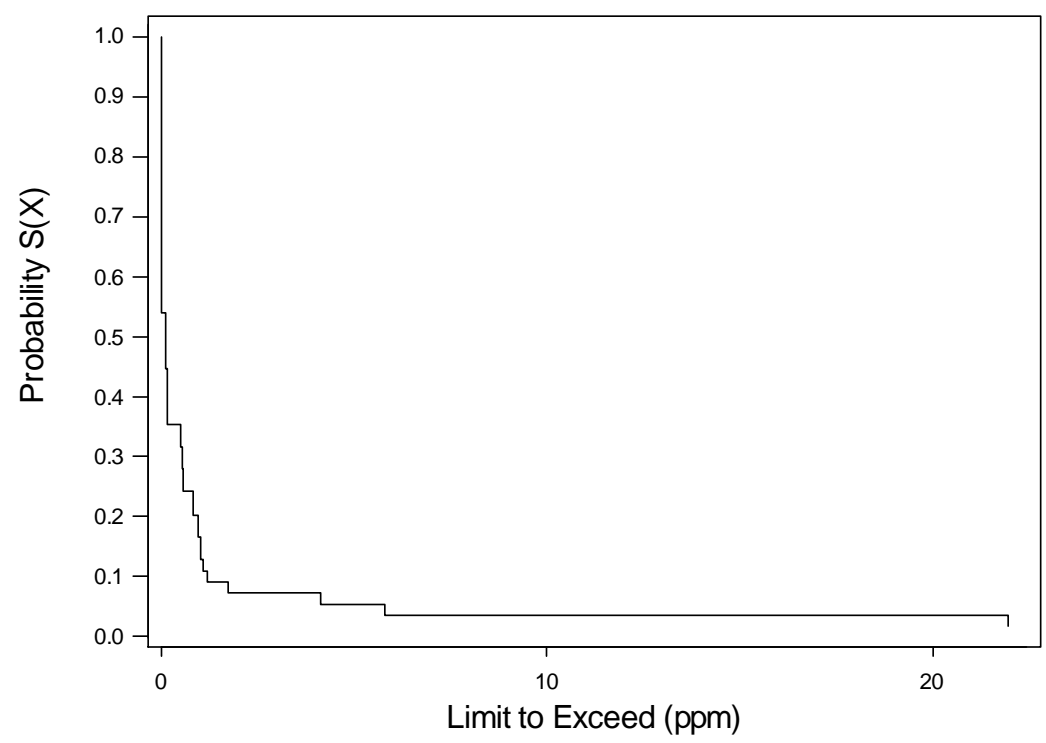

ASTM_1.266 
Nonparametric Survival Plot for Nitrite

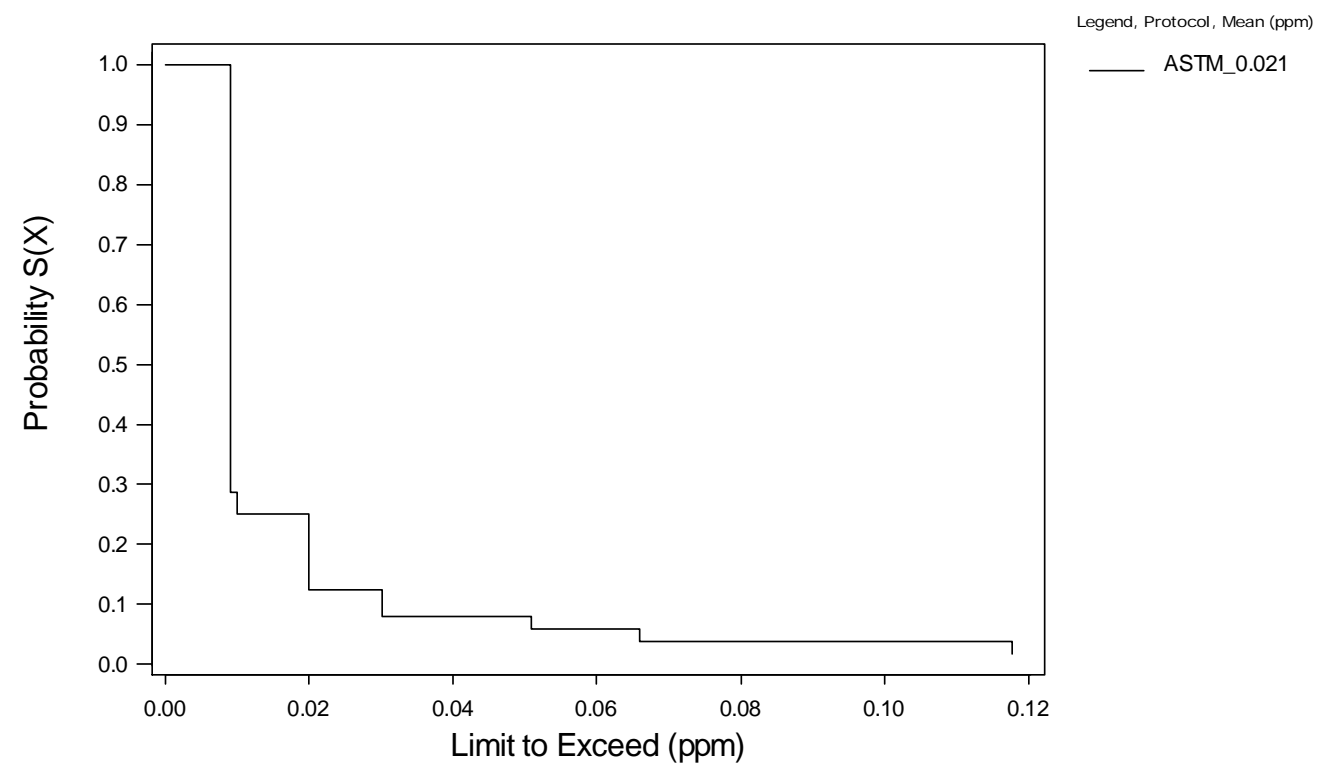

Nonparametric Sunvival Plot for Oil and grease

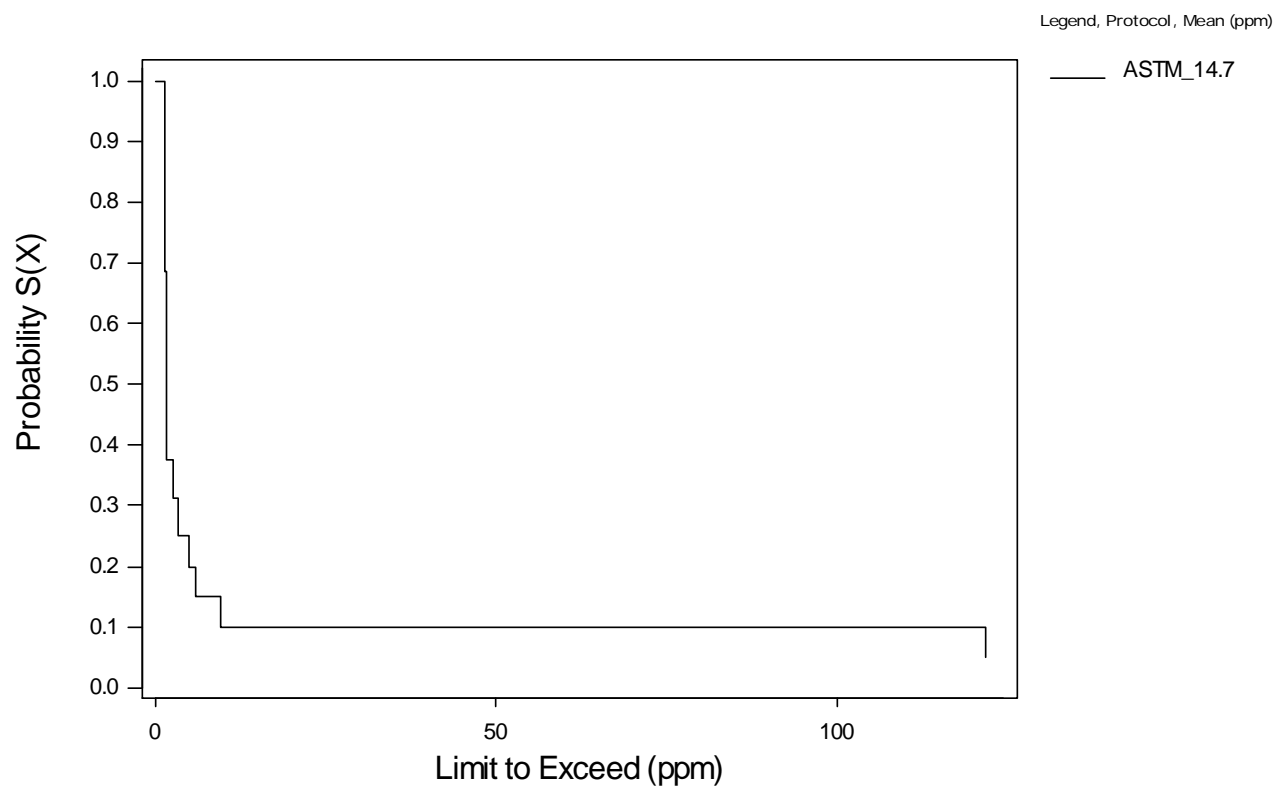




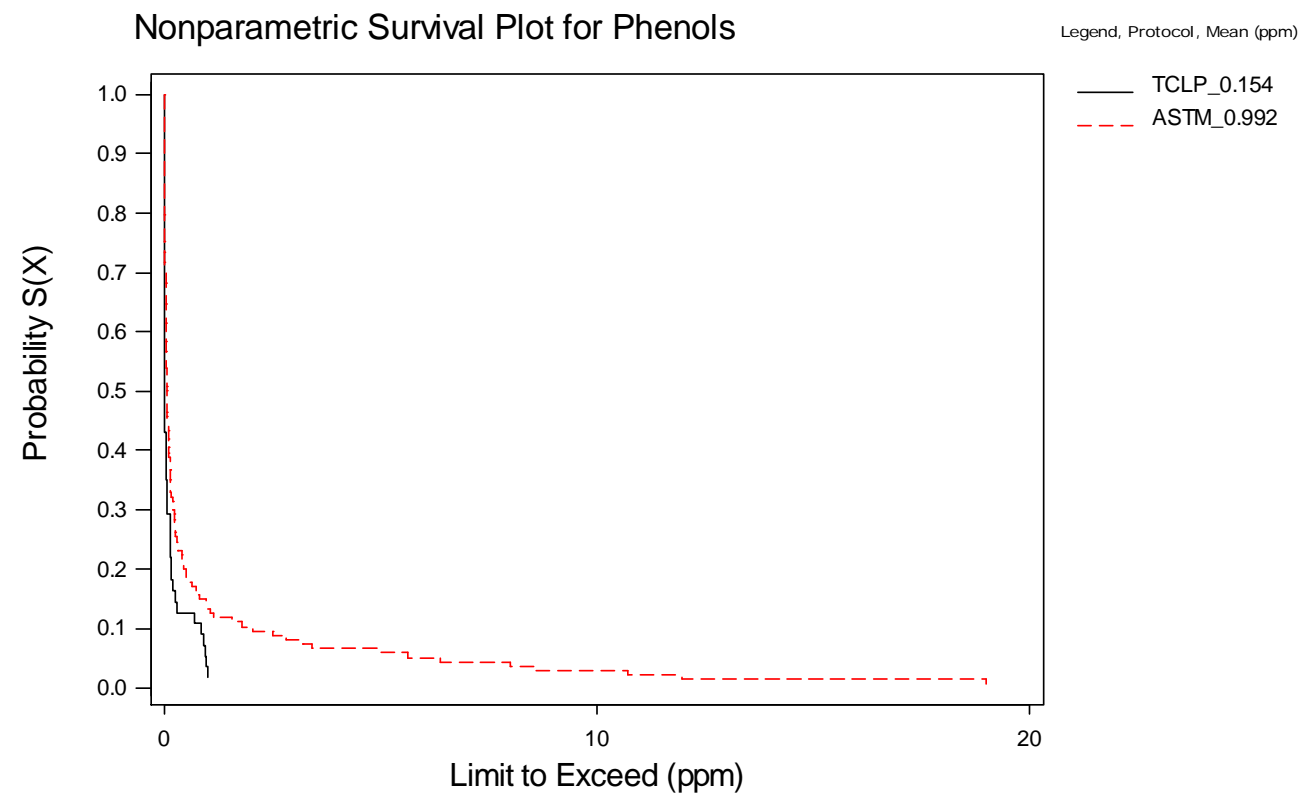

Nonparametric Survival Plot for Sulfate(SO4)

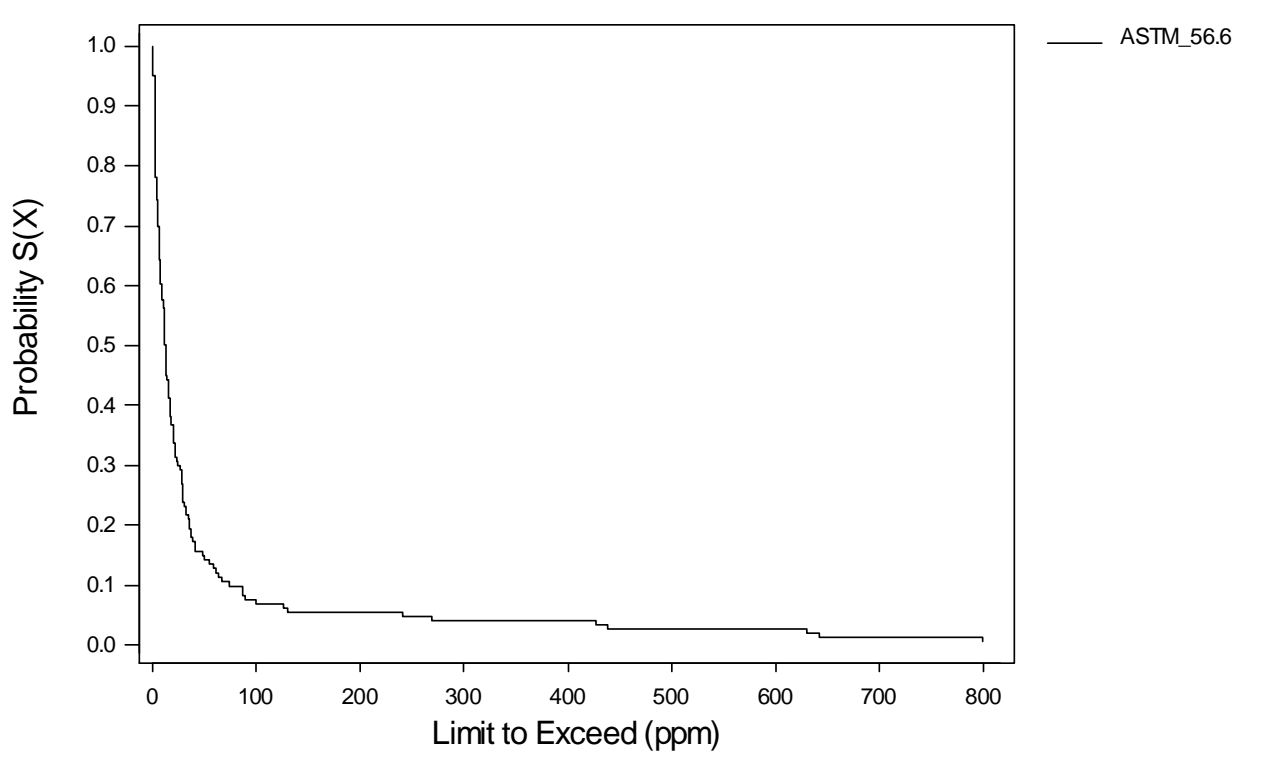


Nonparametric Survival Plot for Total Dissoluble Solid

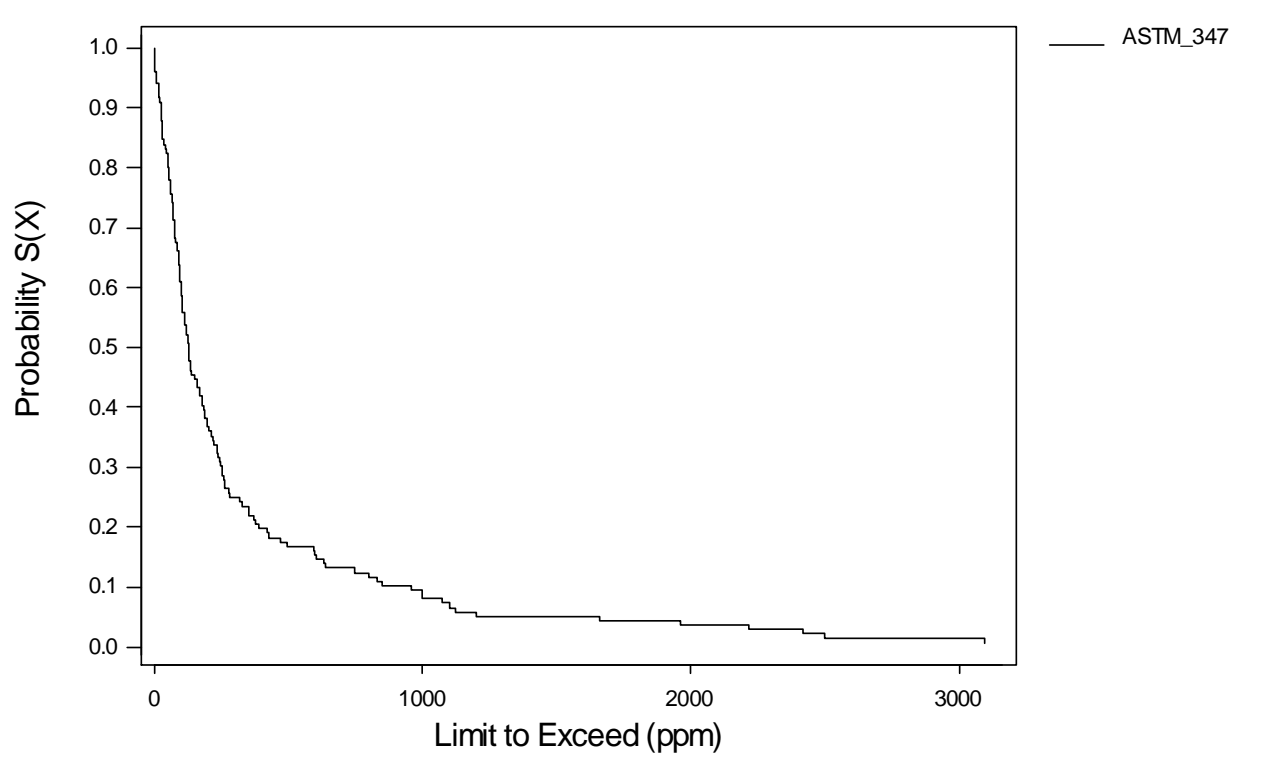

Nonparametric Survival Plot for Total Organic Haligen (TOH)

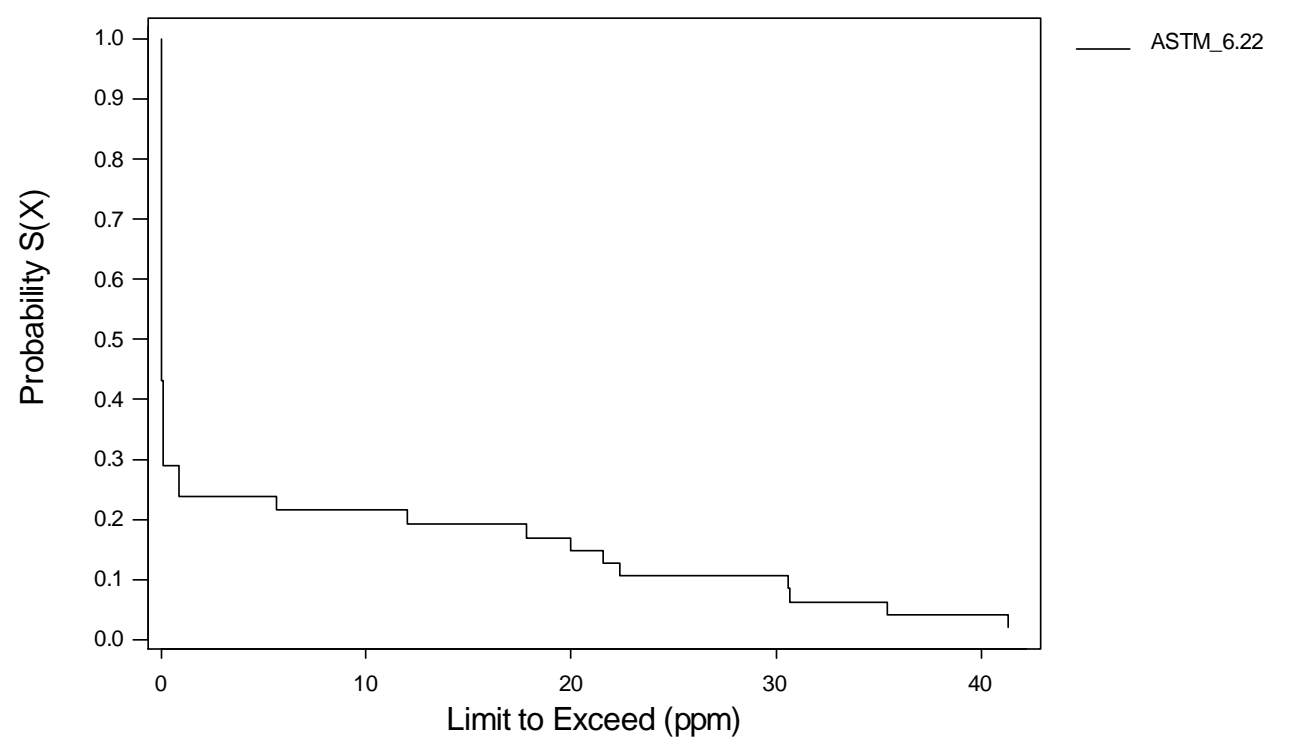


Nonparametric Survival Plot for Total Petroleum Hydrocarbon

Legend, Protocol, Mean (ppm)

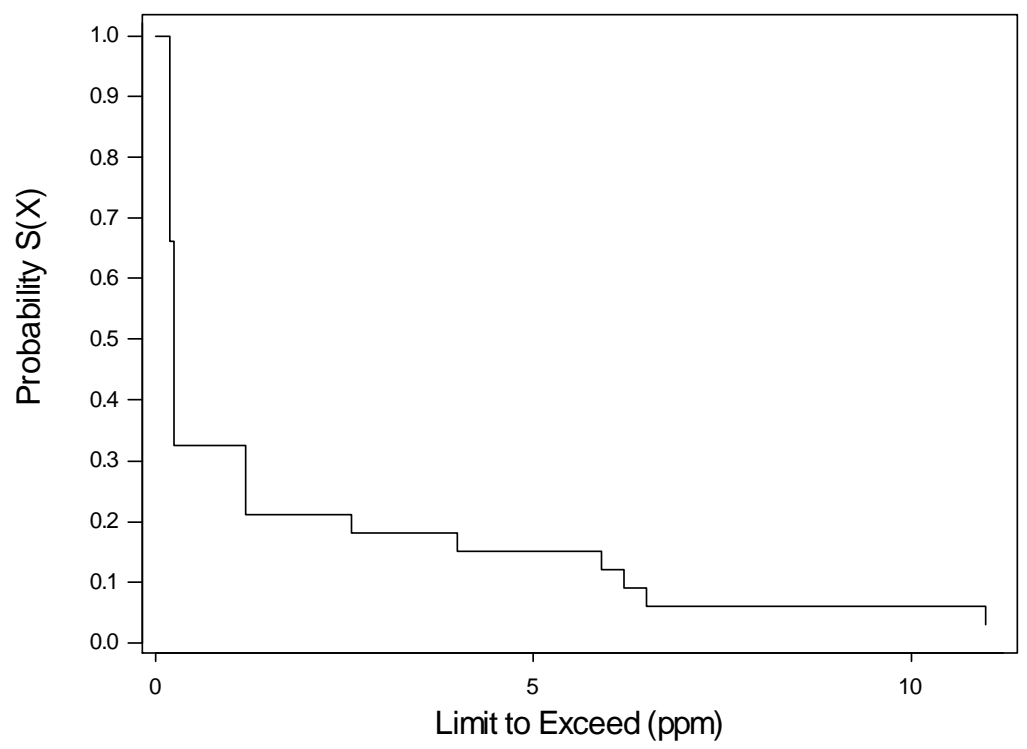

ASTM_2.047

Figure F-5: Survival plots for general chemical parameters in leachates (16 charts) 


\section{F.3.2 Metallic Elements}

Nonparametric Survival Plot for Aluminium (Al)

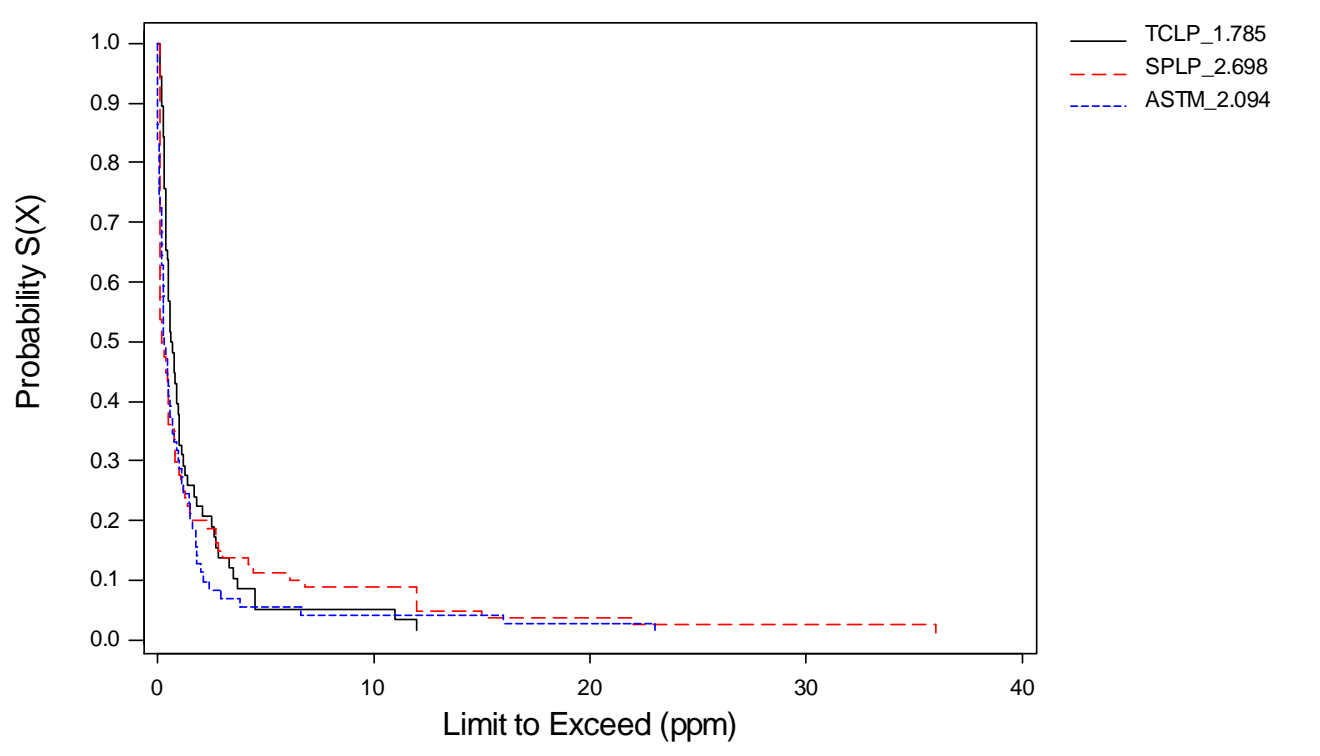

Nonparametric Survival Plot for Antimony (Sb)

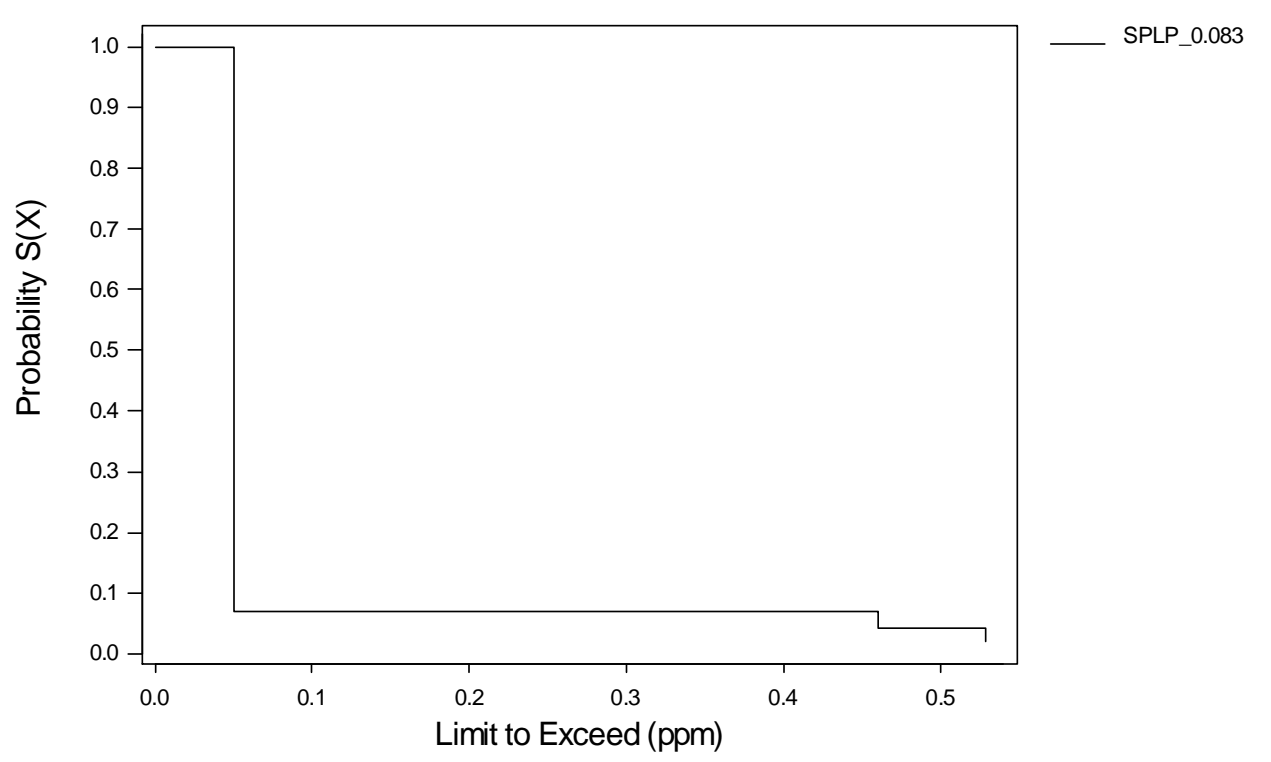


Nonparametric Survival Plot for Arsenic (As)

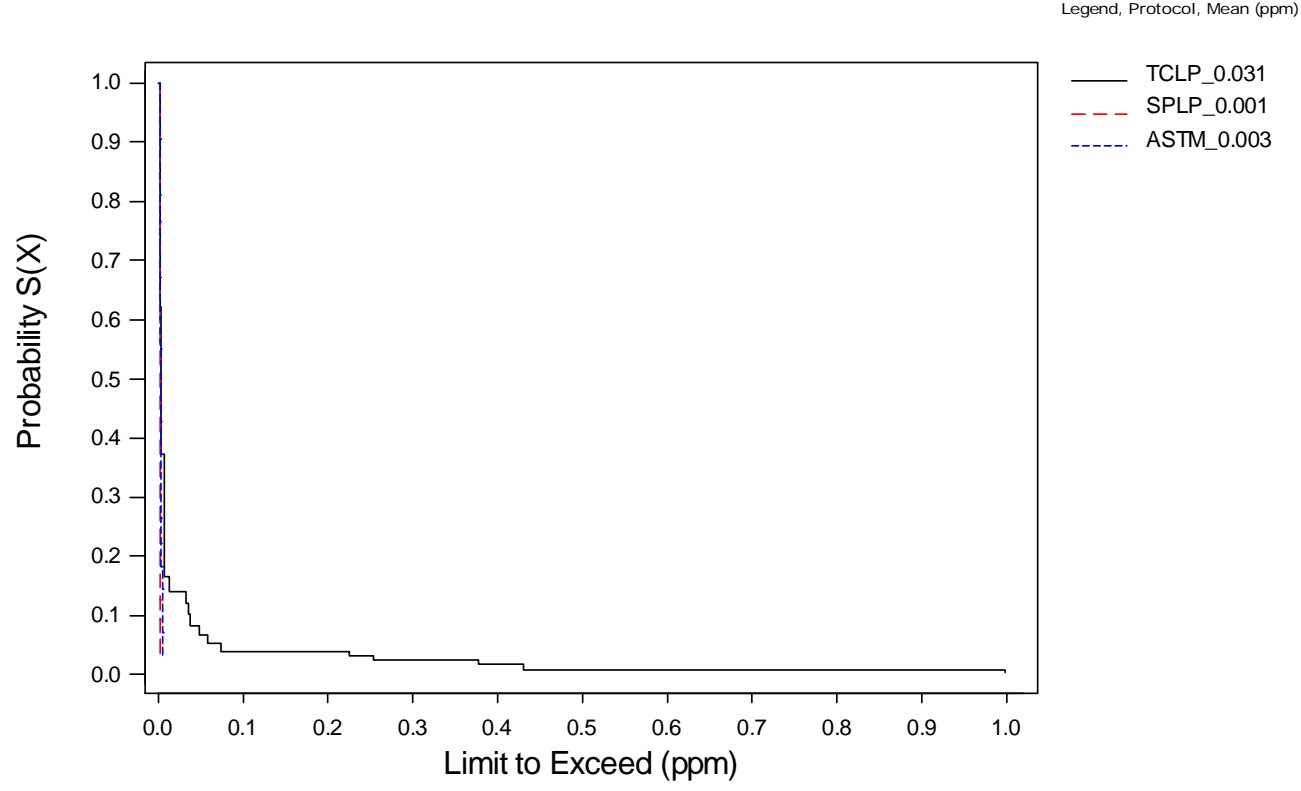

Nonparametric Survival Plot for Barium(Ba)

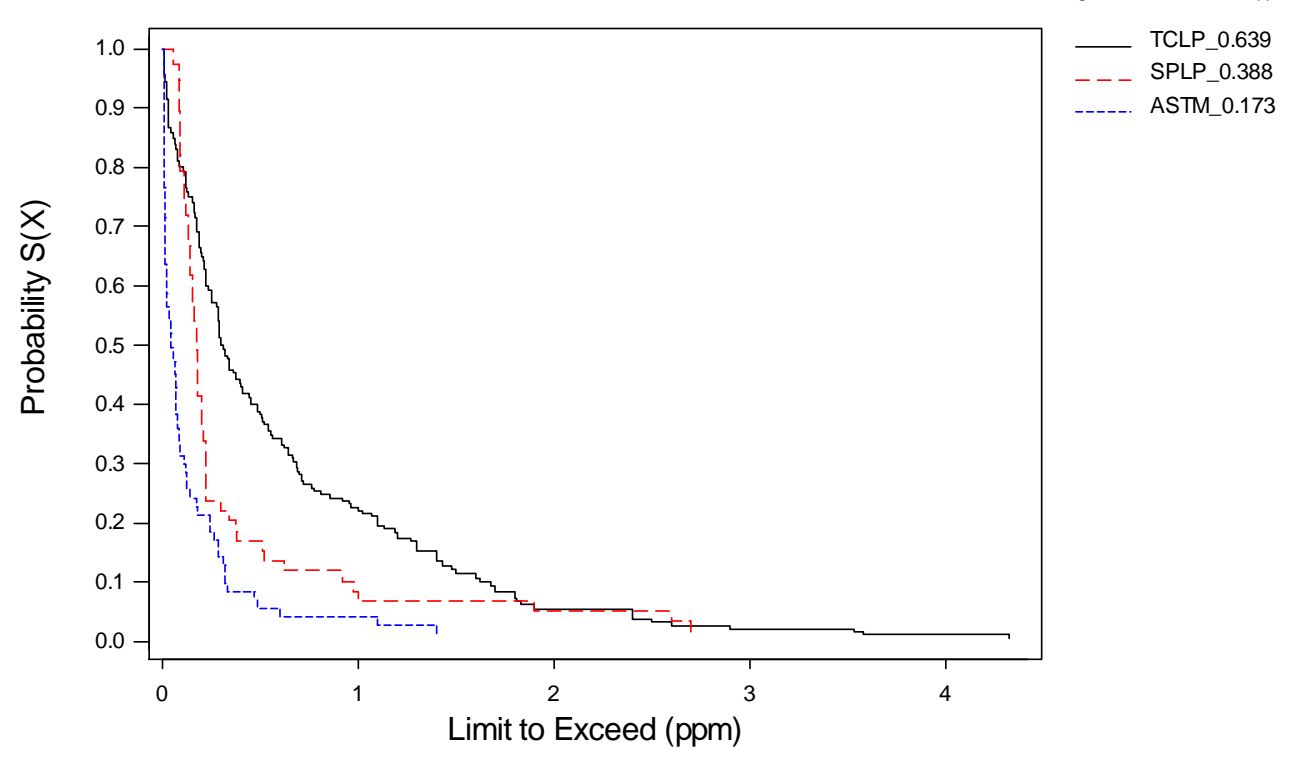


Nonparametric Survival Plot for Boron(B)

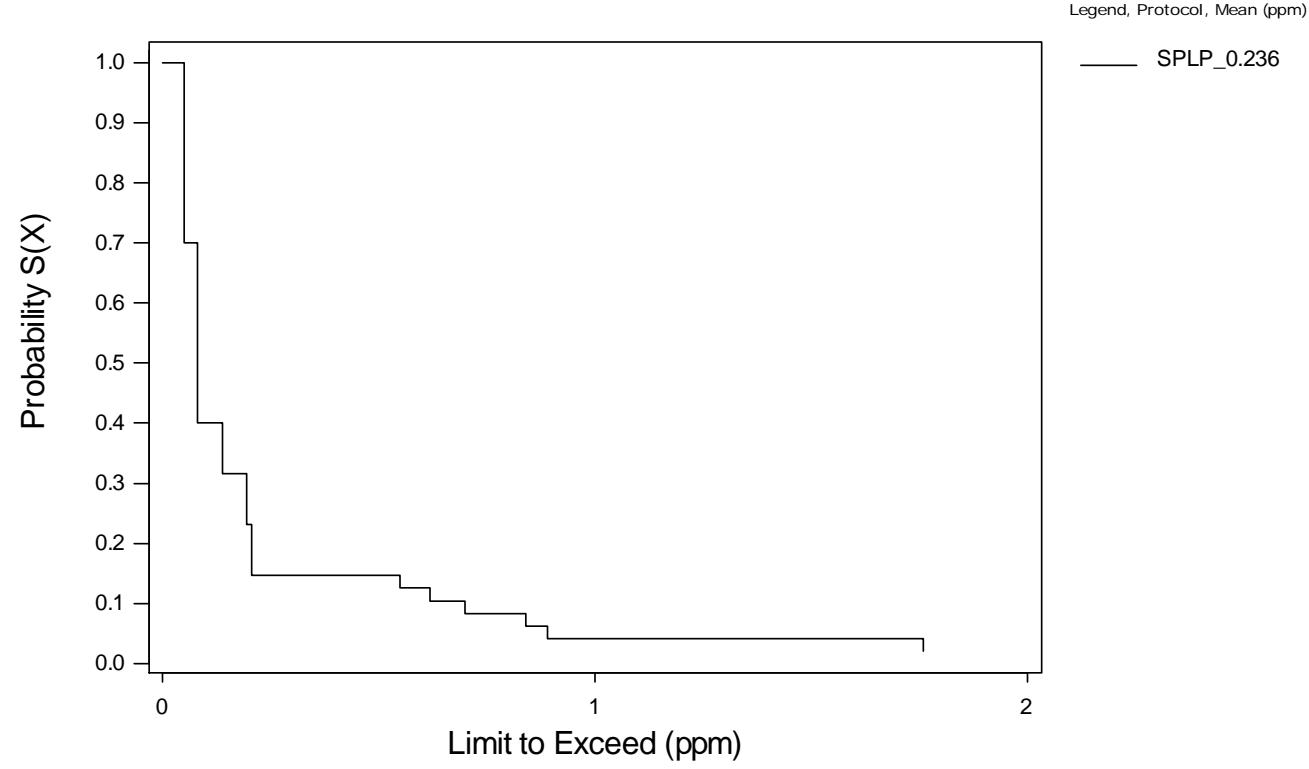

Nonparametric Survival Plot for Cadmium (Cd)

Legend, Protocol, Mean (ppm)

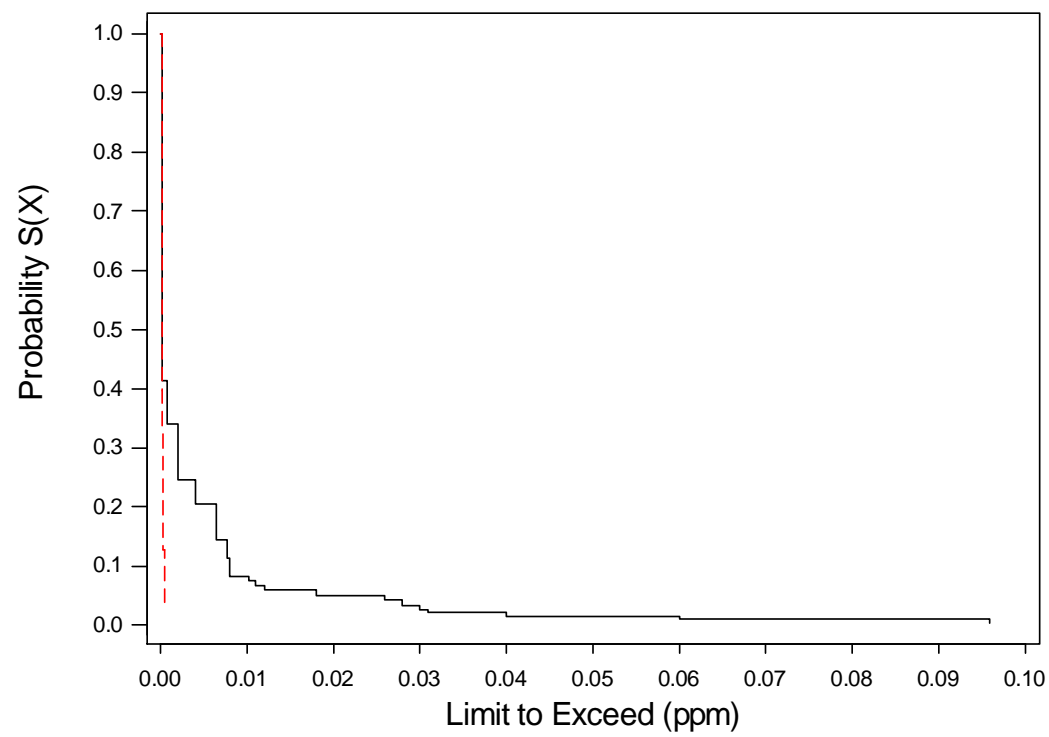

TCLP 0.004

_ _ _ ASTM_0.0003 
Nonparametric Survival Plot for Chromium (Cr)

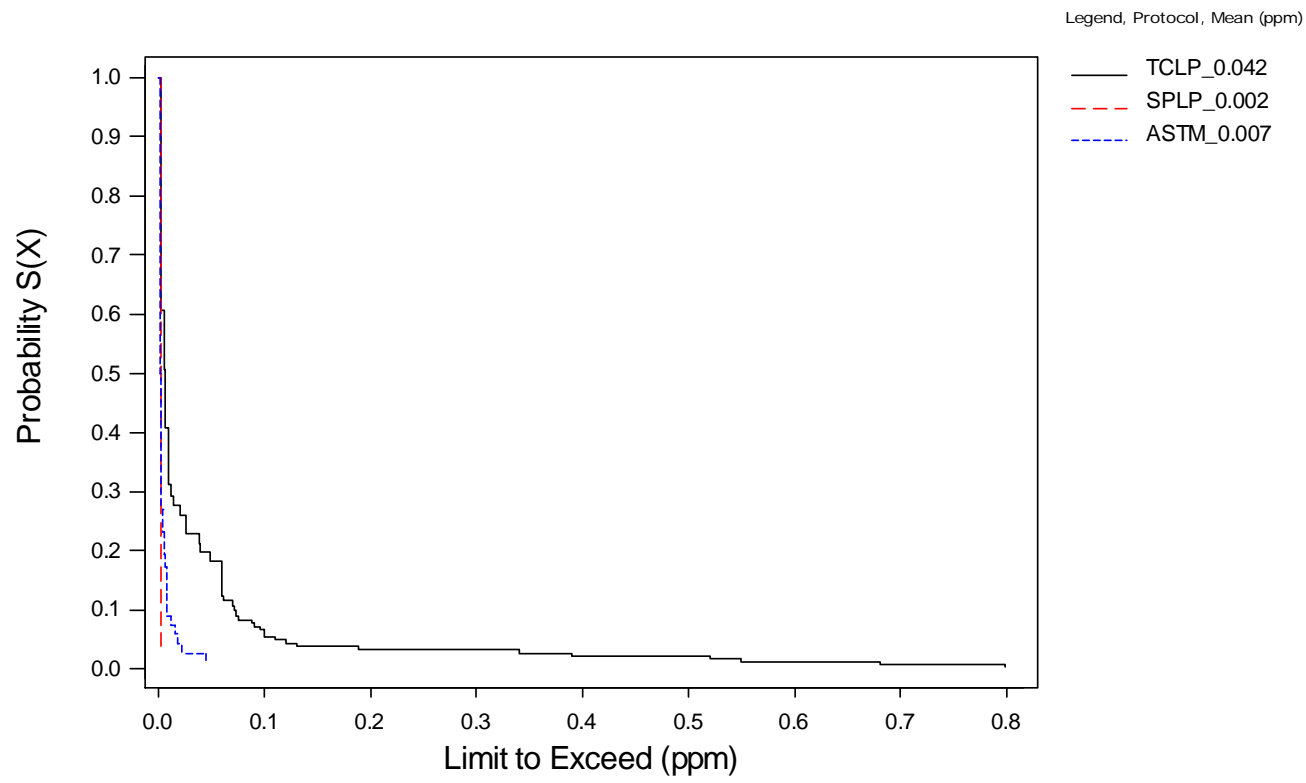

Nonparametric Survival Plot for Hexavalent Chromium

Legend, Protocol, Mean (ppm)

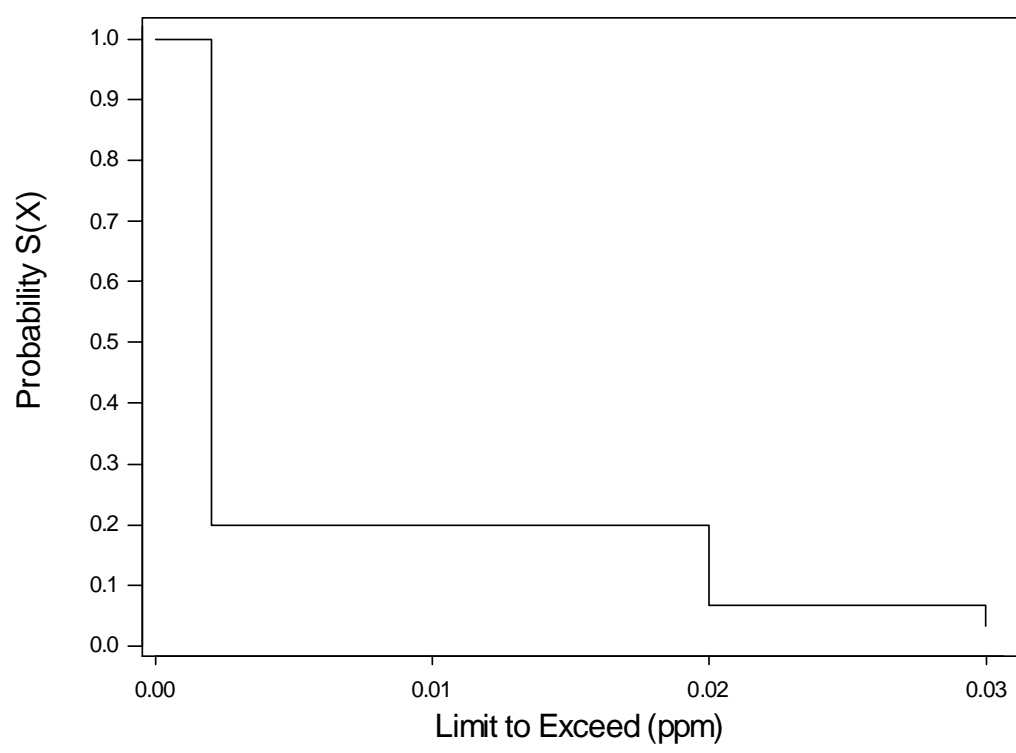

ASTM_0.008 
Nonparametric Survival Plot for Copper (Cu)

Legend, Protocol, Mean (ppm)

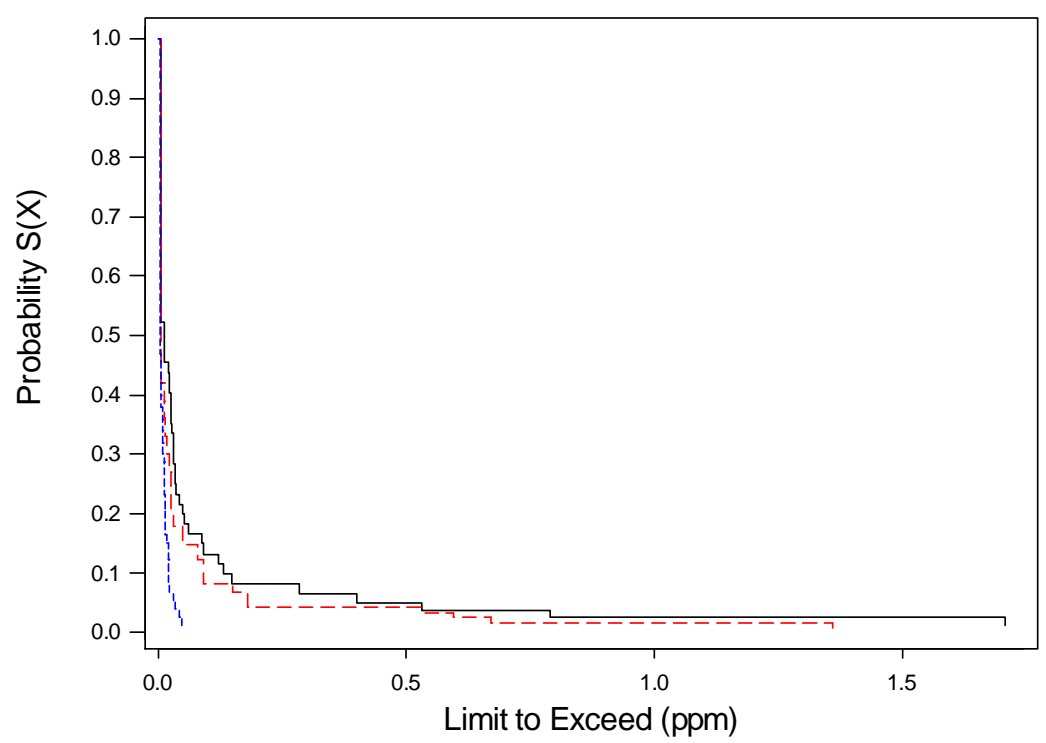

CLP 0.521

- - - SPLP_0.061

ASTM 0.087

Nonparametric Survival Plot for Iron ( $\mathrm{Fe})$

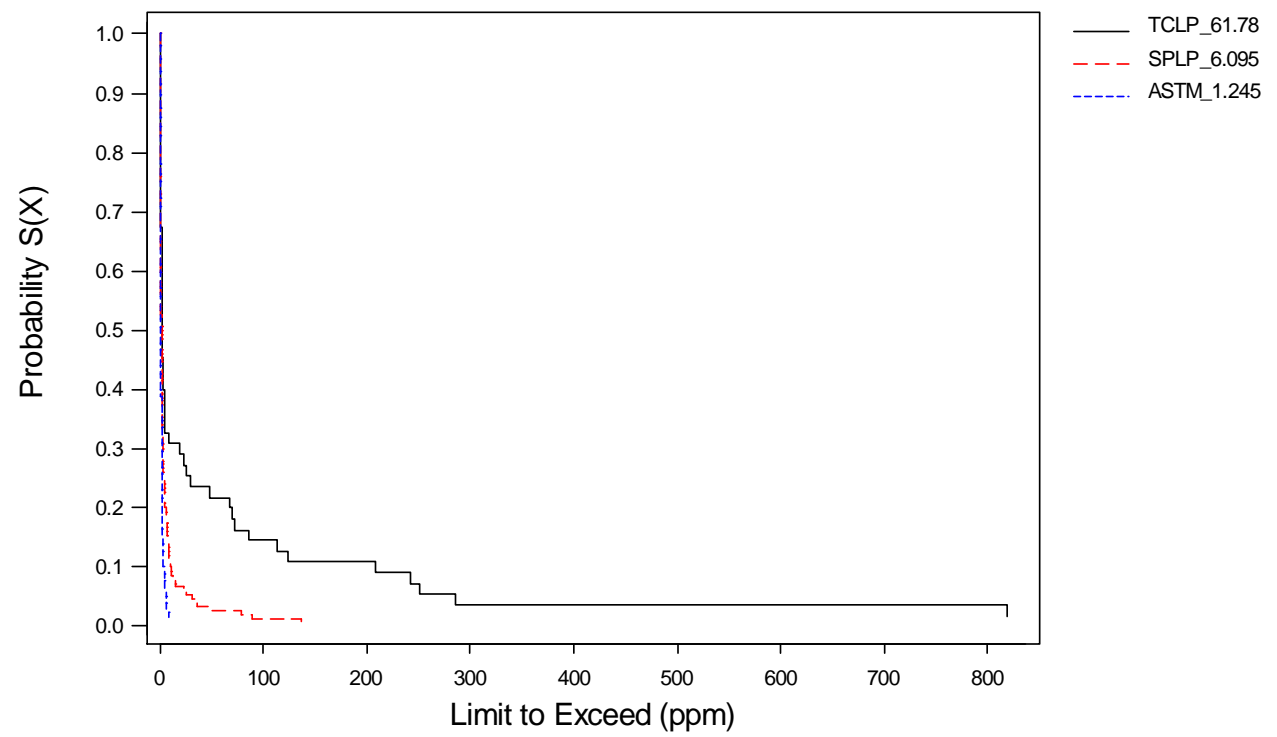


Nonparametric Survival Plot for Lead (Pb)

Legend, Protocol, Mean (ppm)

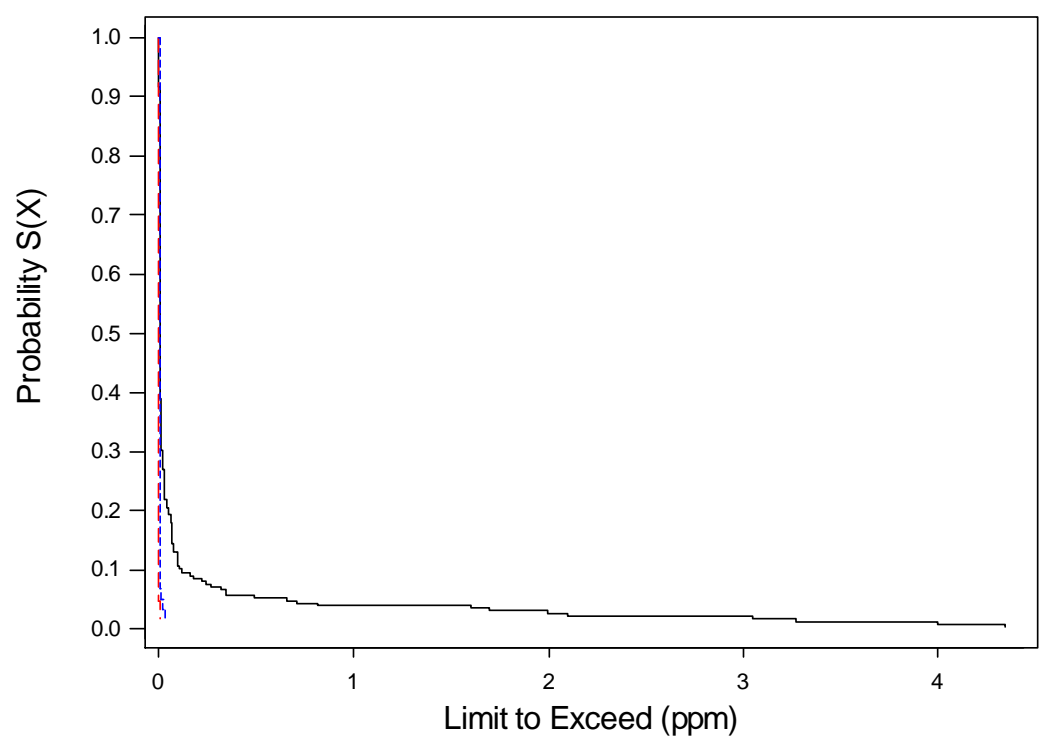

TCLP 0.222

SPLP_0.009

-- SPLP_0.009
ASTM 0.008

Nonparametric Survival Plot for Manganese (Mn)

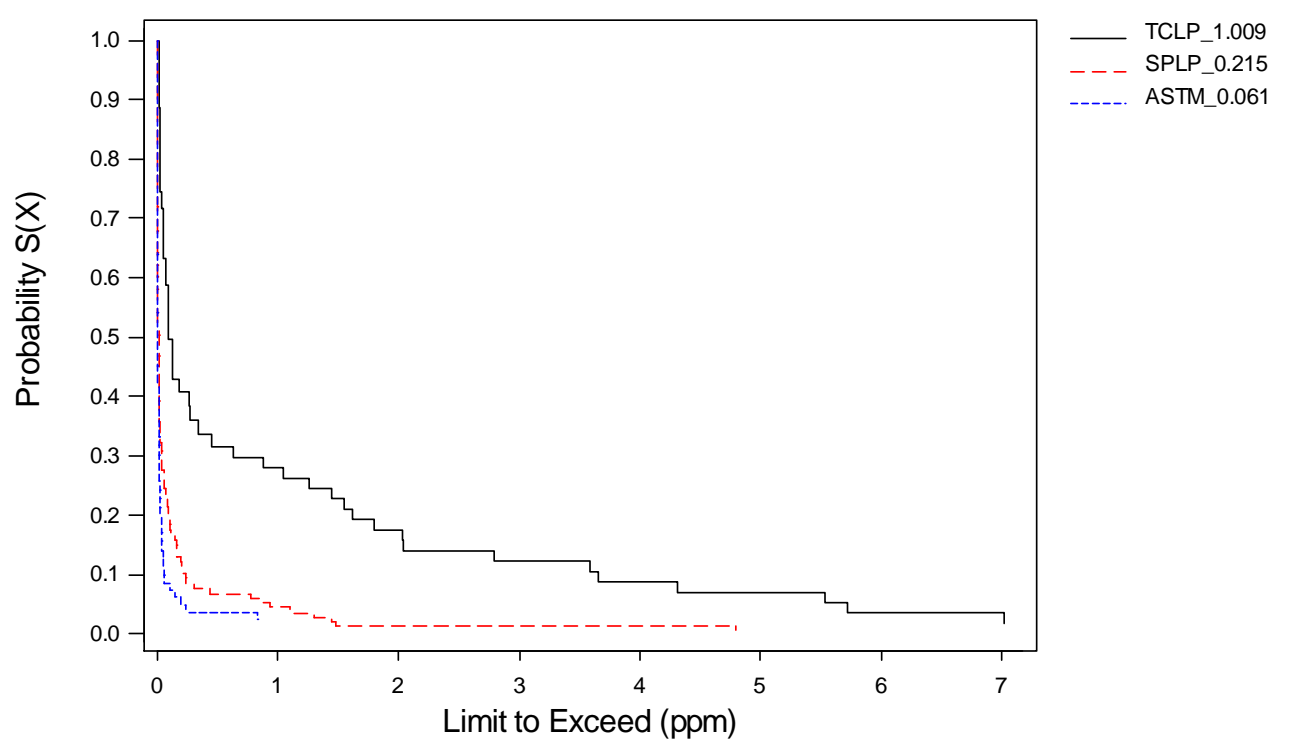


Nonparametric Survival Plot for Mercury $(\mathrm{Hg})$

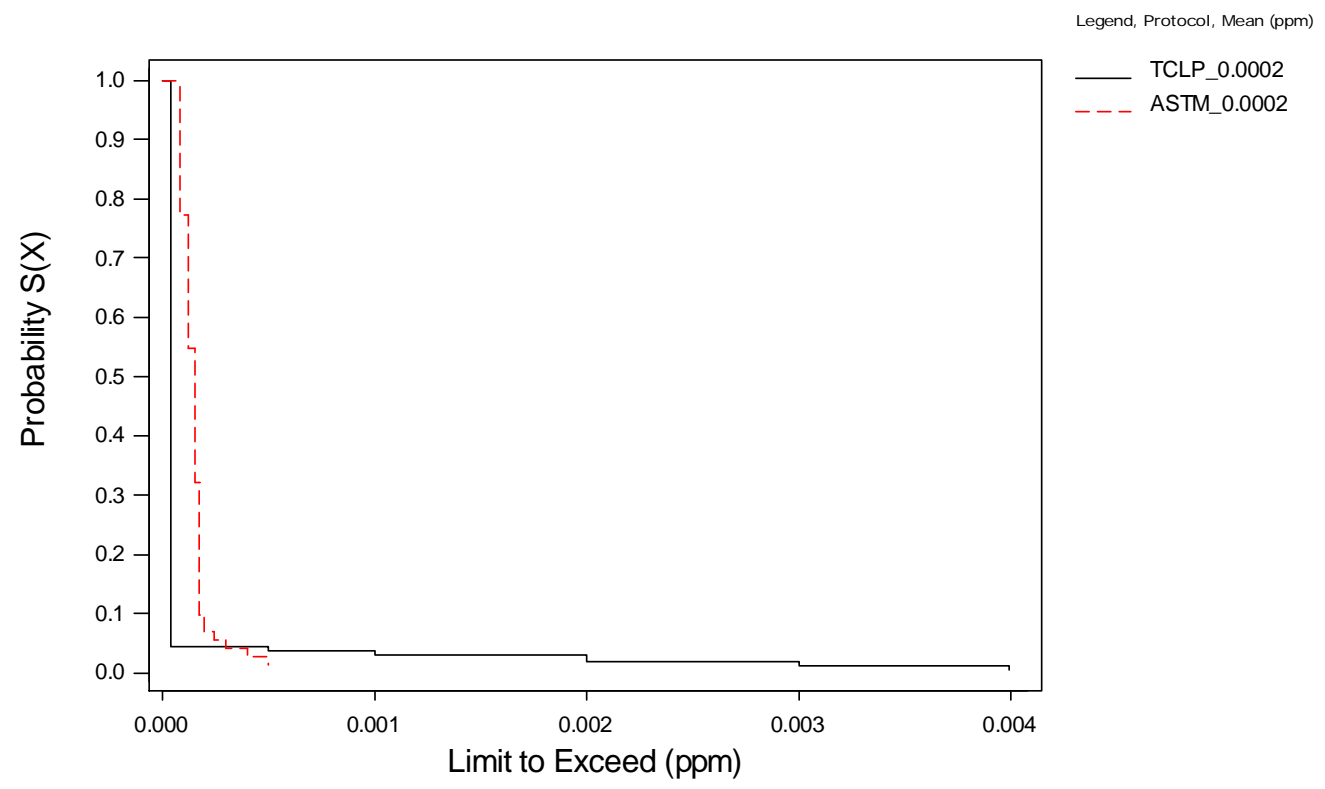

Nonparametric Survival Plot for Molybdenum (Mo)

Legend, Protocol, Mean (ppm)

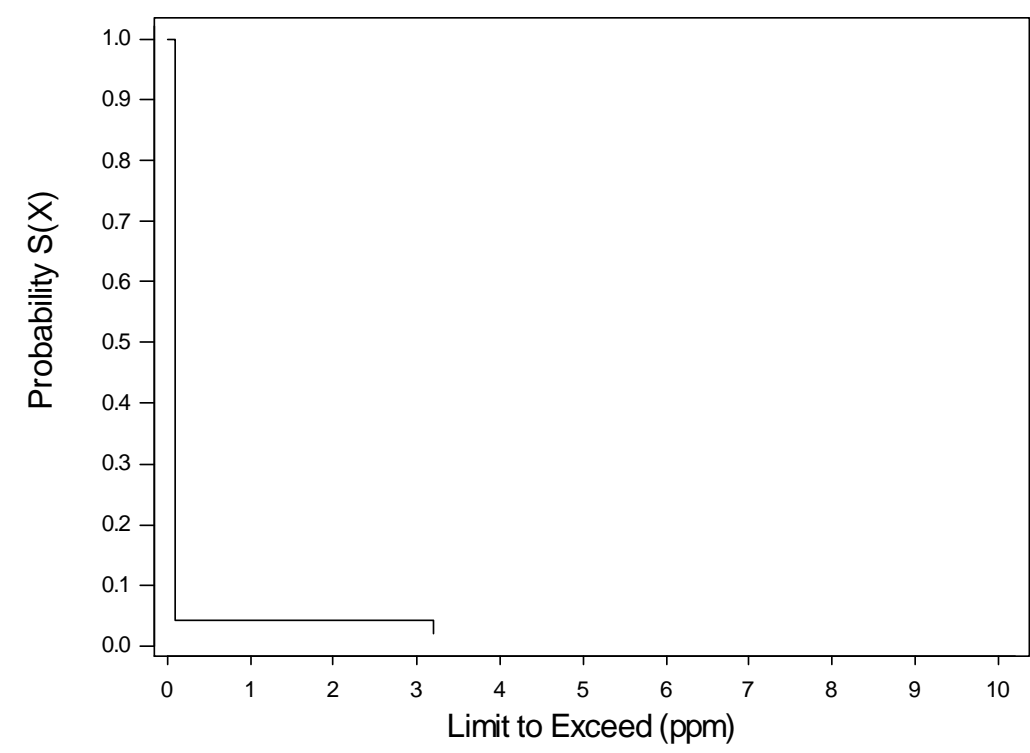

SPLP_0.233 
Nonparametric Survival Plot for Nickel (Ni)

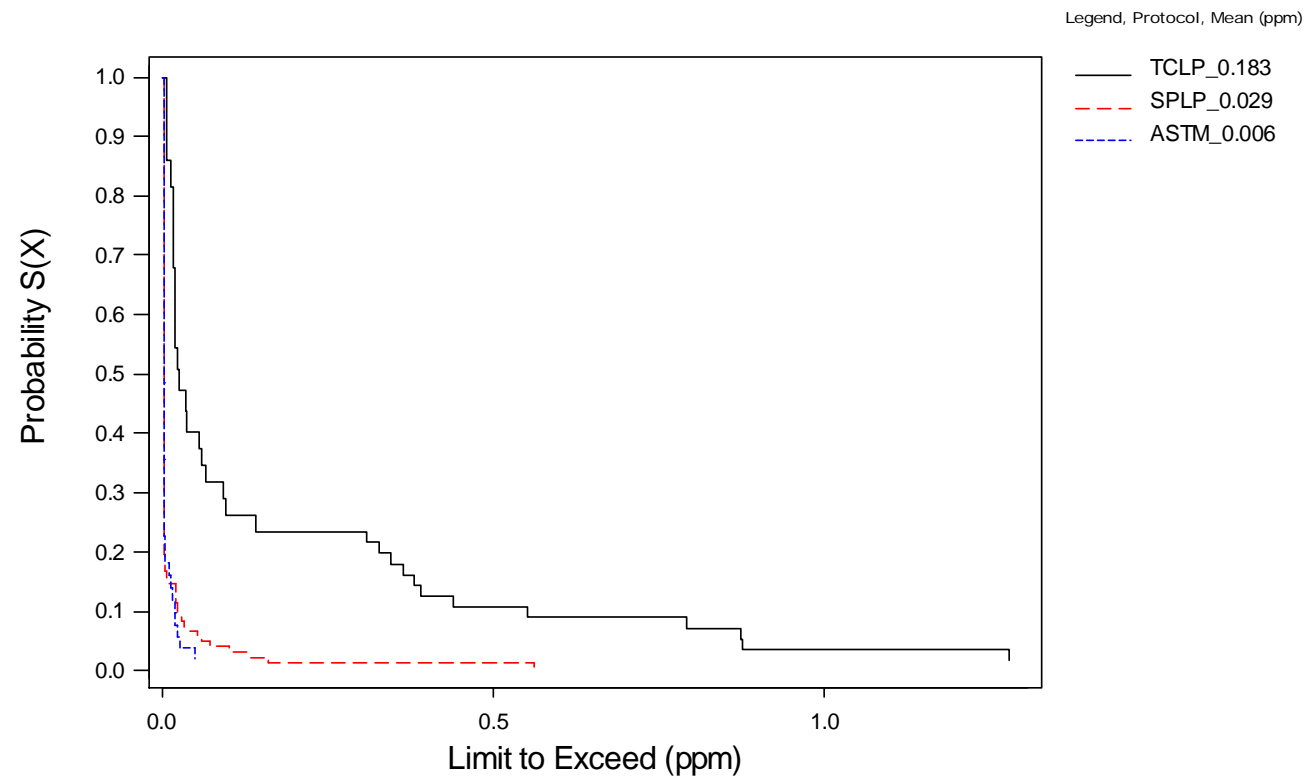

Nonparametric Survival Plot for Selenium (Se)

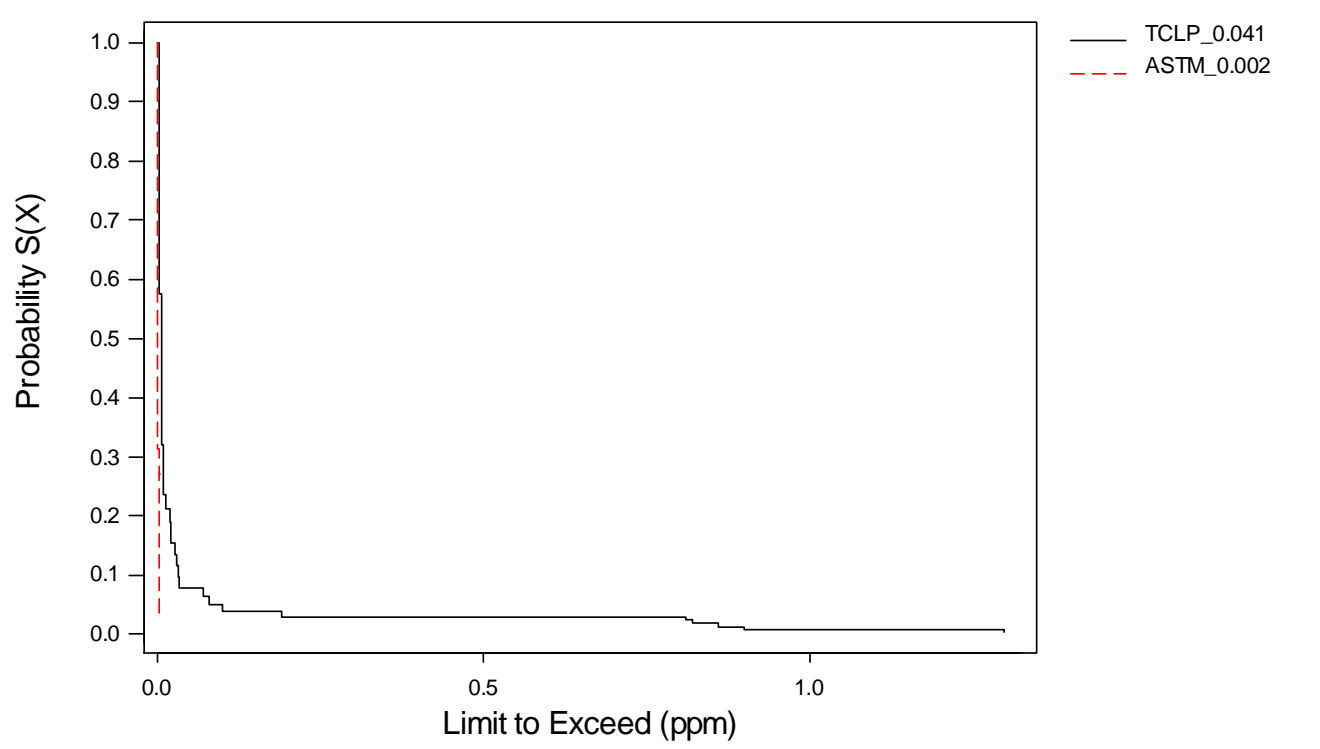


Nonparametric Survival Plot for Silver (Ag)

Legend, Protocol, Mean (ppm)

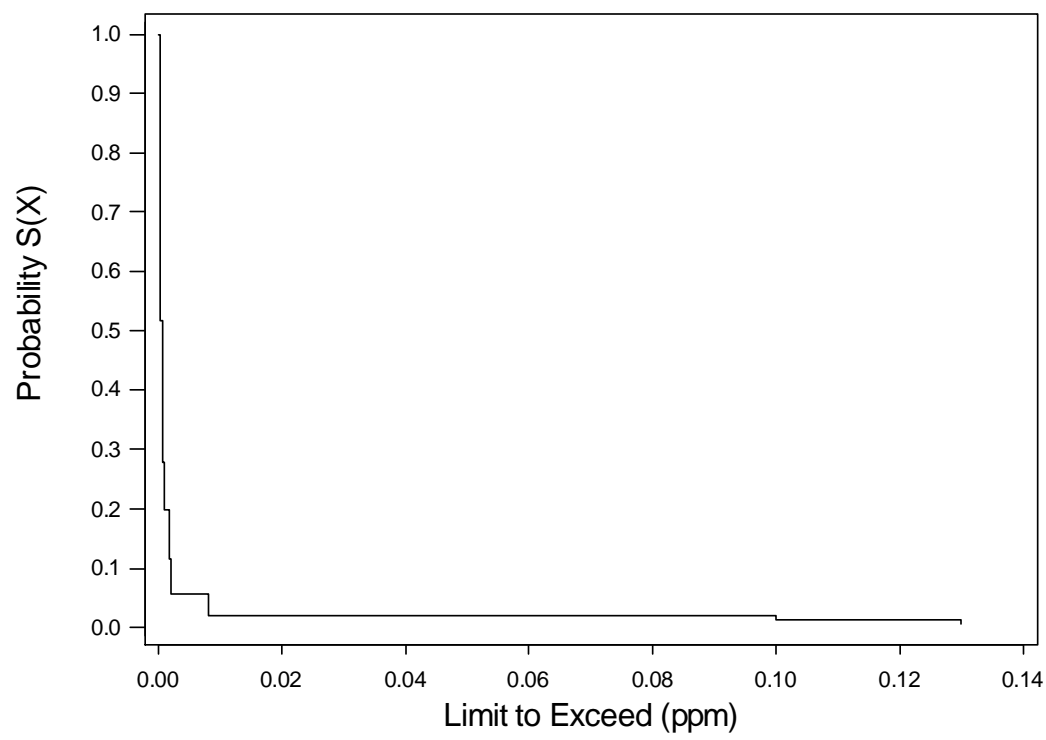

TCLP_0.004

Nonparametric Survival Plot for Sodium (Na)

Legend, Protocol, Mean (ppm)

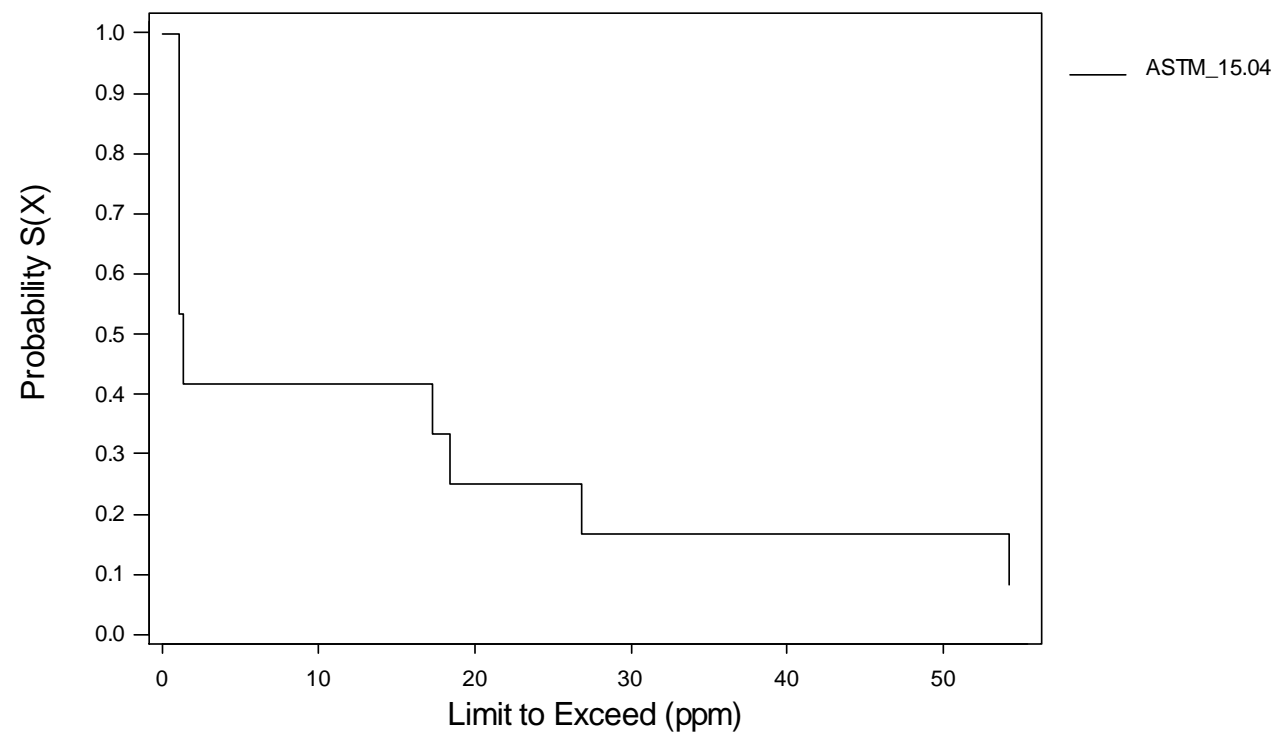


Nonparametric Sunvival Plot for Vanadium (Va)

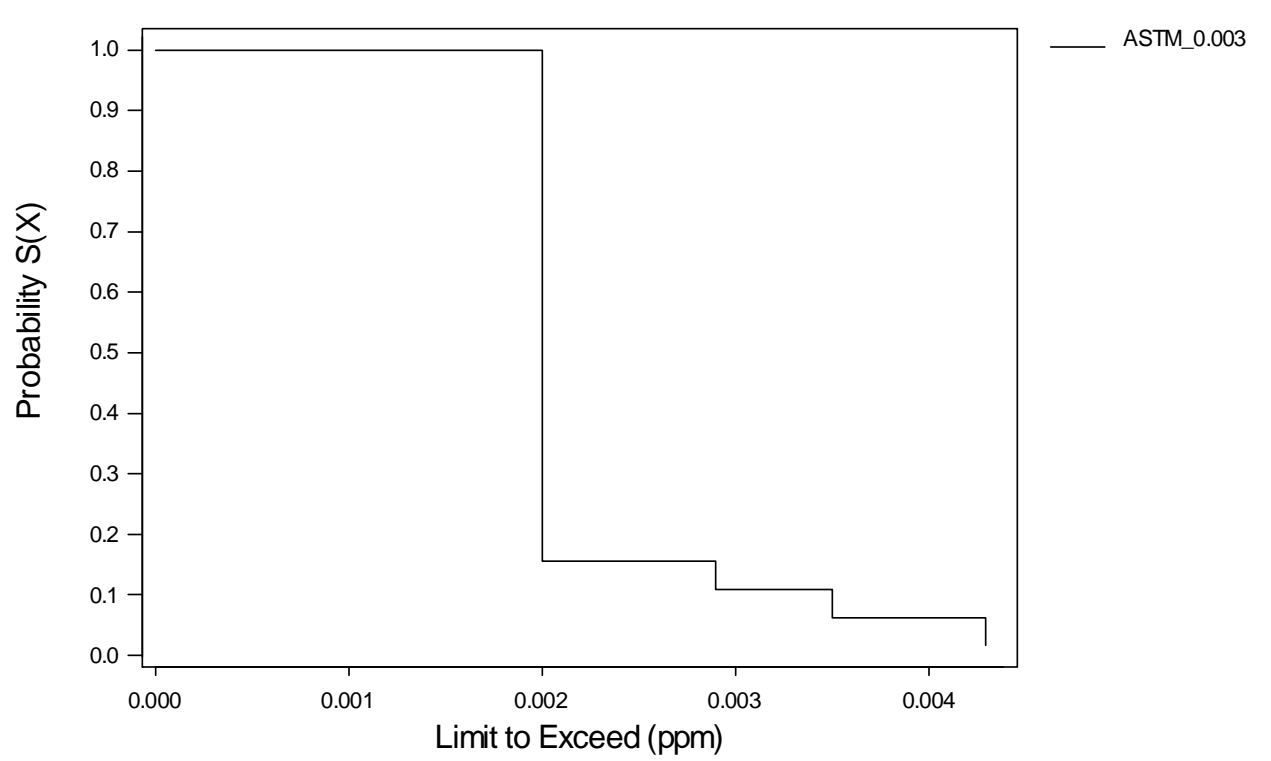

Nonparametric Survival Plot for Zinc (Zn)

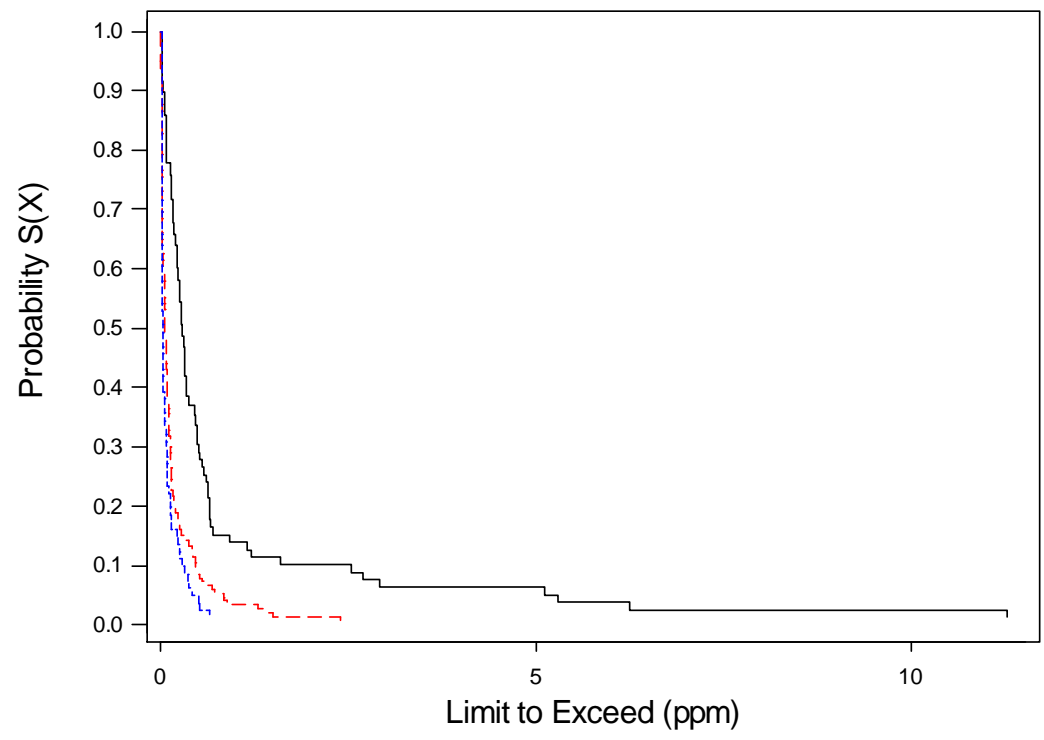

Legend, Protocol, Mean (ppm)

TCLP 1.006

_- - SPLP_0.264

ASTM 0.177

Figure F-6: Survival plots for metallic elements in leachates (20 charts) 


\section{F.3.3 Organic Compounds}

Nonparametric Survival Plot for Cresol_meta

by TCLP $($ Mean=22.33 ppb)

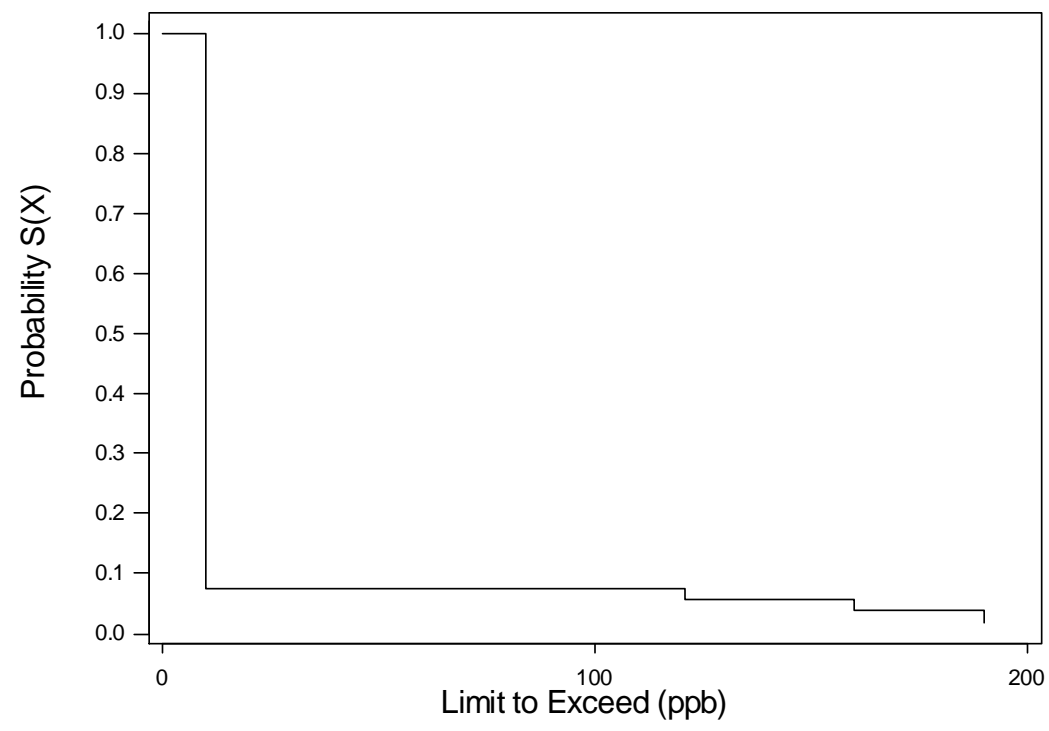

Nonparametric Survival Plot for Cresol_ortho by TCLP $($ Mean $=46.4 \mathrm{ppb})$

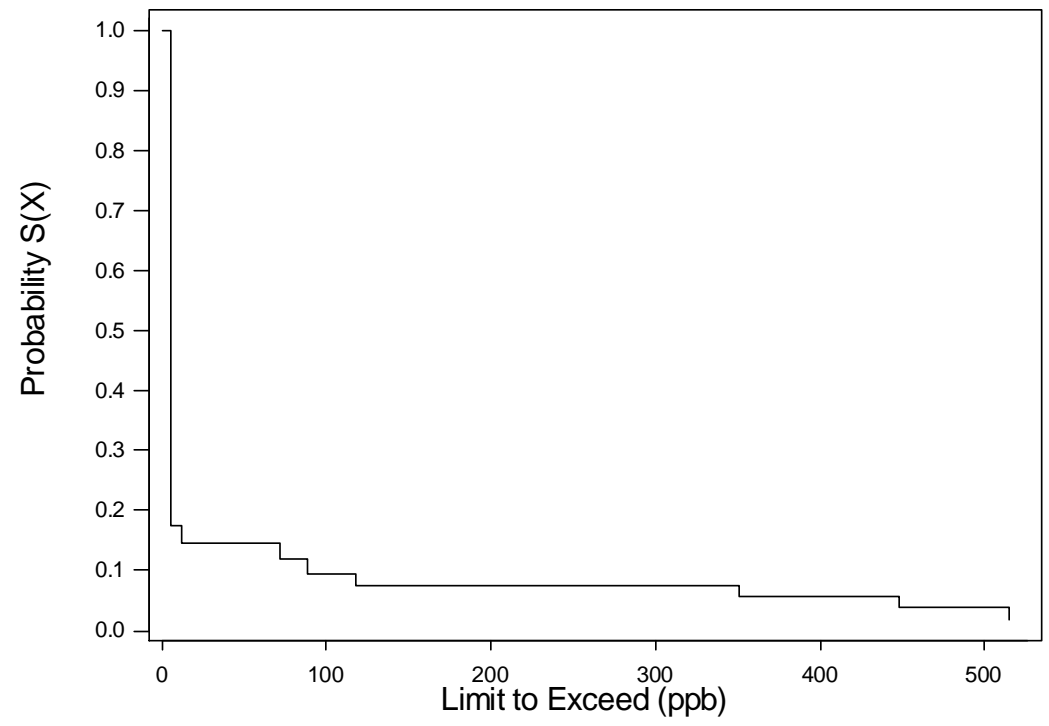


Nonparametric Survival Plot for Acetone

by TCLP (Mean=265.5 ppb)

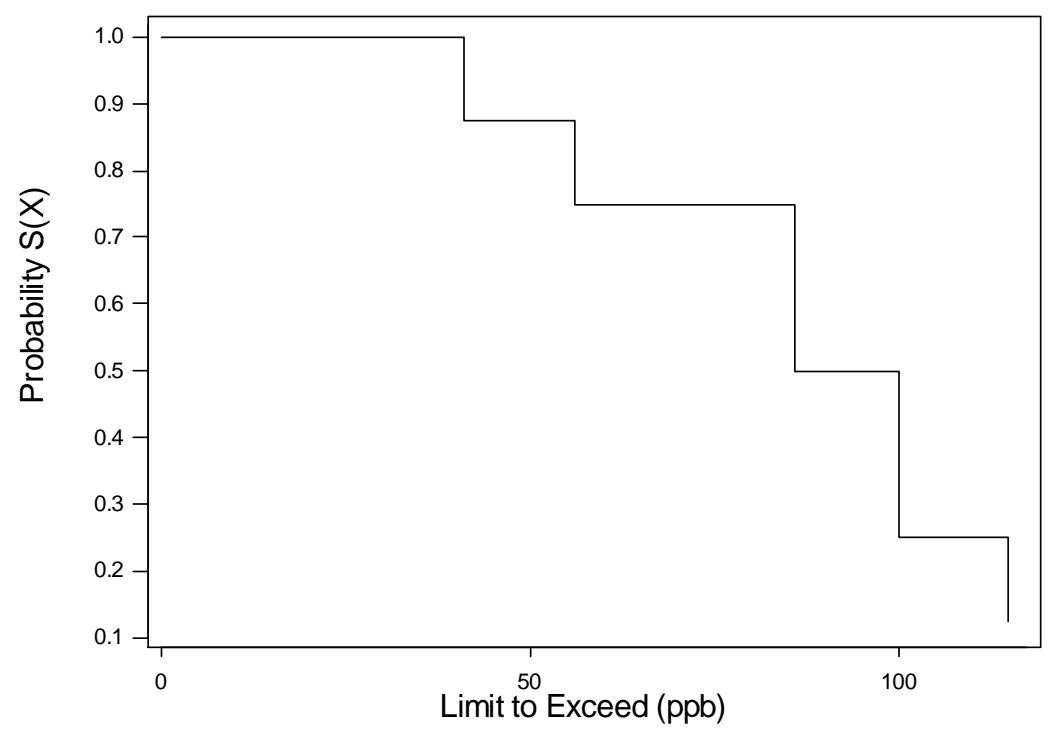

Nonparametric Survival Plot for Benzene

by TCLP $($ Mean=1.46 ppb)

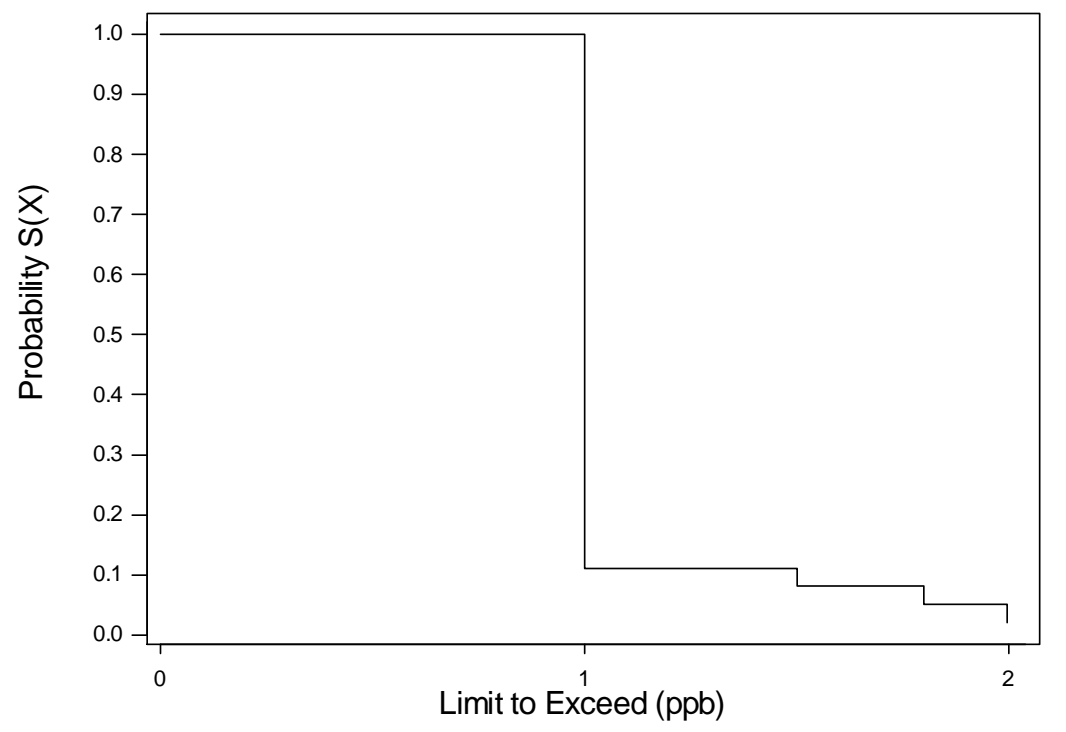


Nonparametric Survival Plot for 2_4-Dimethylphen by TCLP (Mean=30.6 ppb)

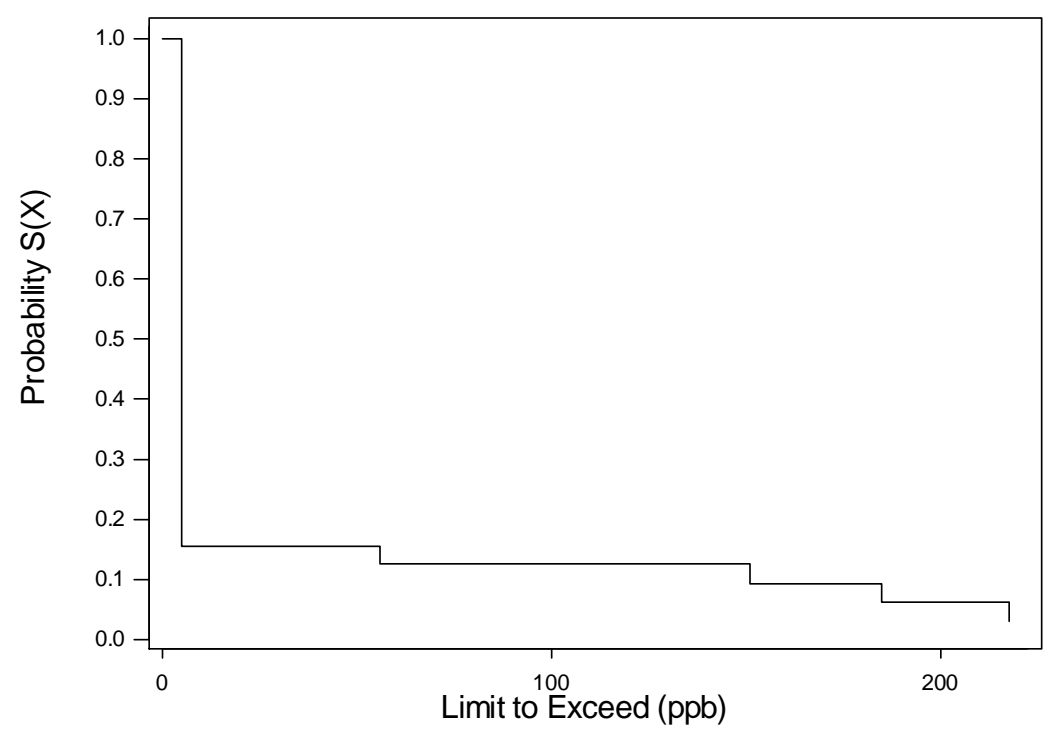

Nonparametric Survival Plot for Ethyl benzene by TCLP (Mean=5.78 ppb)

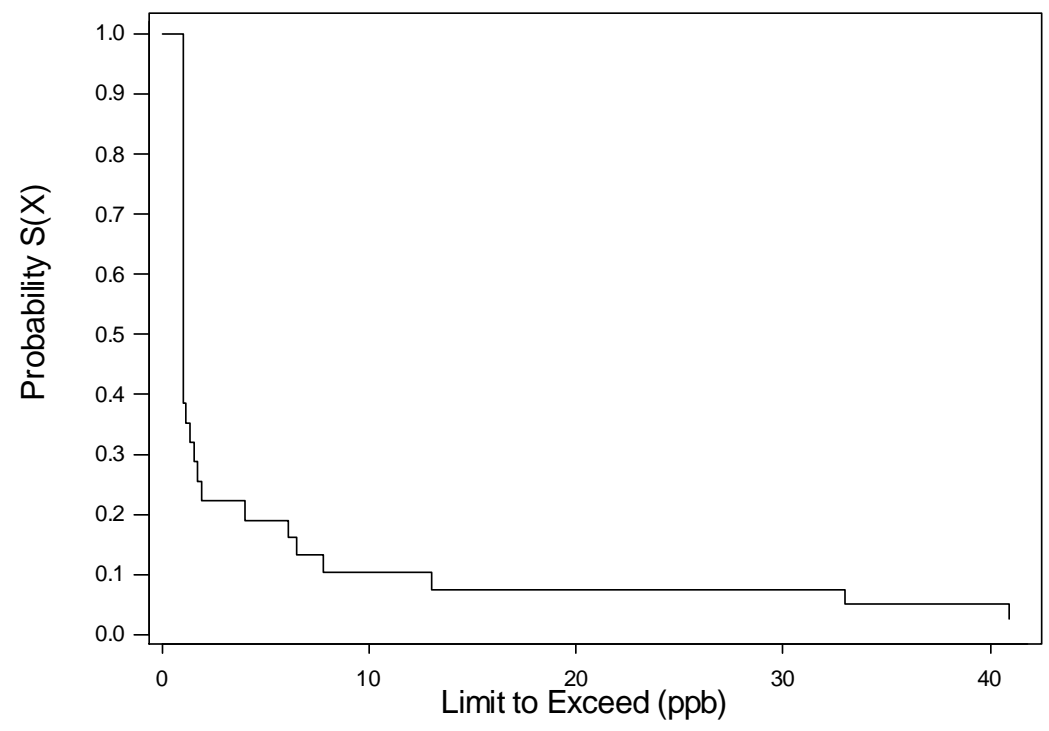


Nonparametric Survival Plot for Naphthalene

by TCLP (Mean=71.69 ppb)

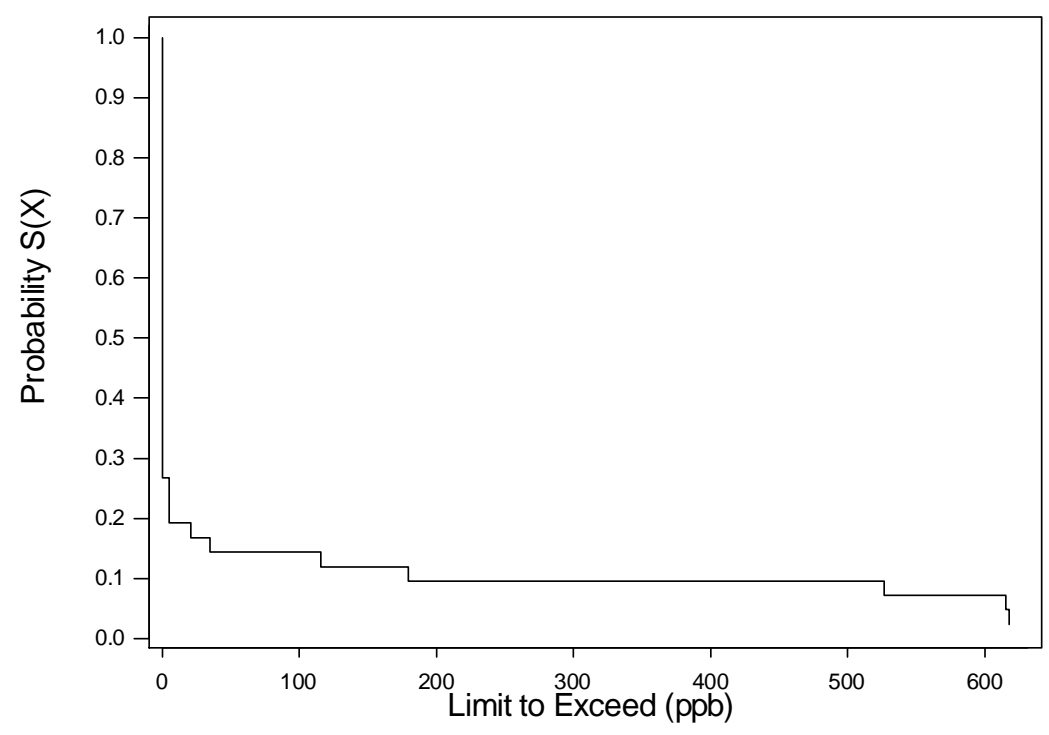

Nonparametric Survival Plot for Phenanthrene by TCLP (Mean=6.99 ppb)

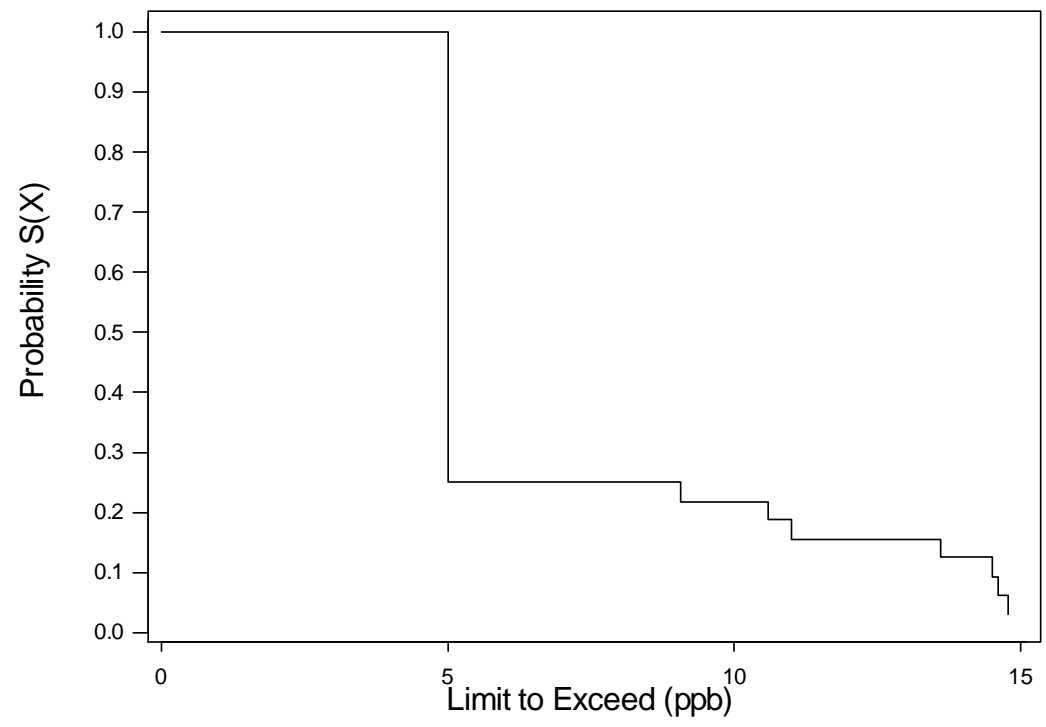


Nonparametric Survival Plot for Styrene

by TCLP (Mean=57.30 ppb)

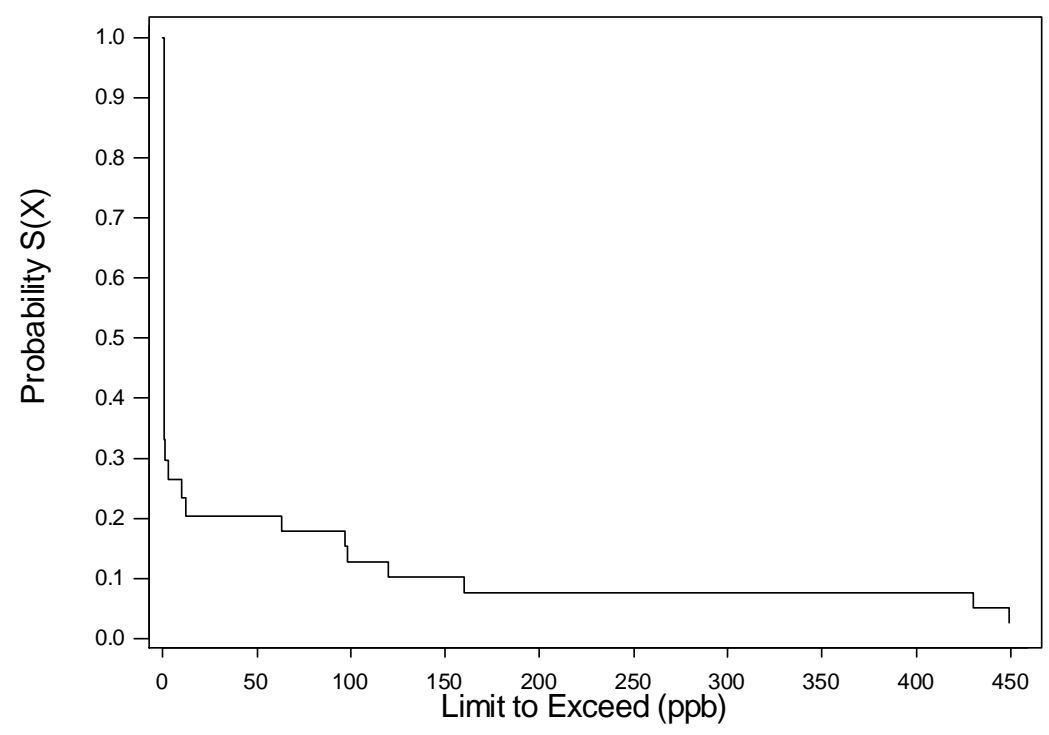

Nonparametric Survival Plot for Toluene by TCLP (Mean=51.92 ppb)

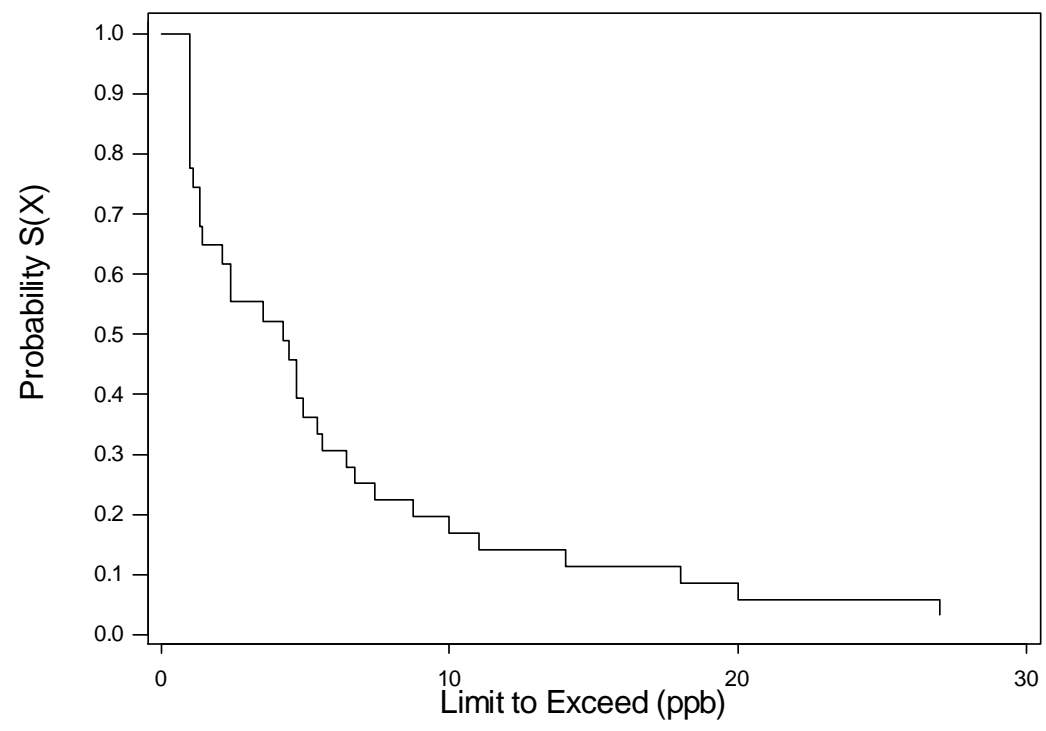


Nonparametric Survival Plot for Trichloroethylen

by TCLP (Mean=7.23 ppb)

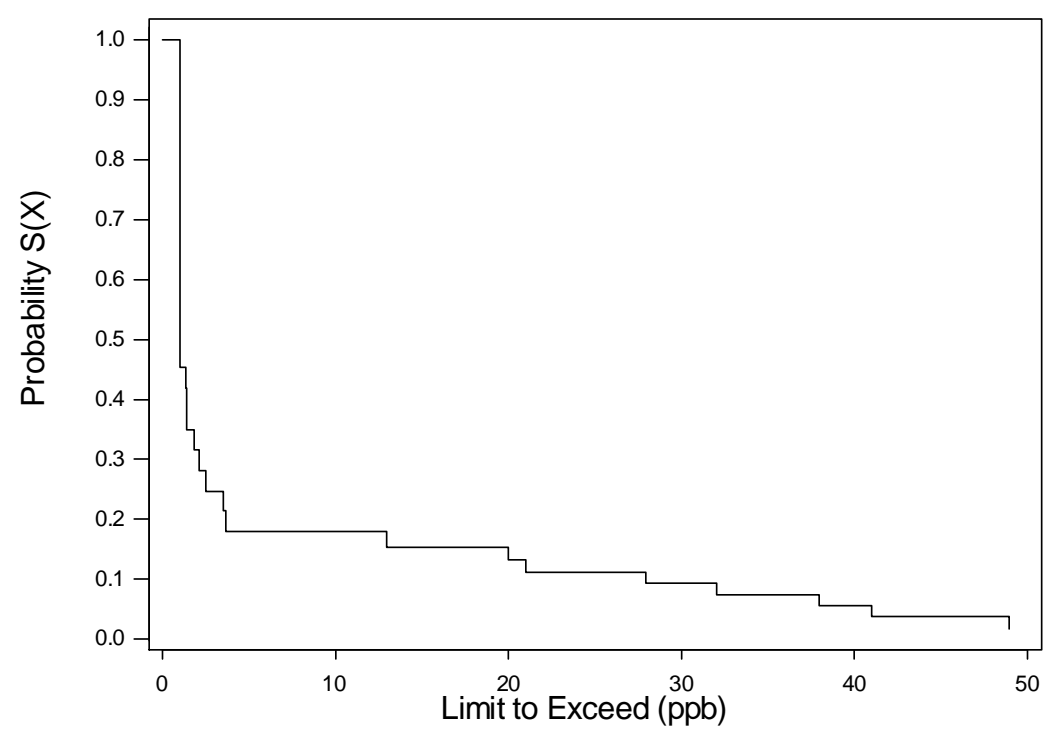

Nonparametric Survival Plot for Xylene_total by TCLP (Mean=3.73 ppb)

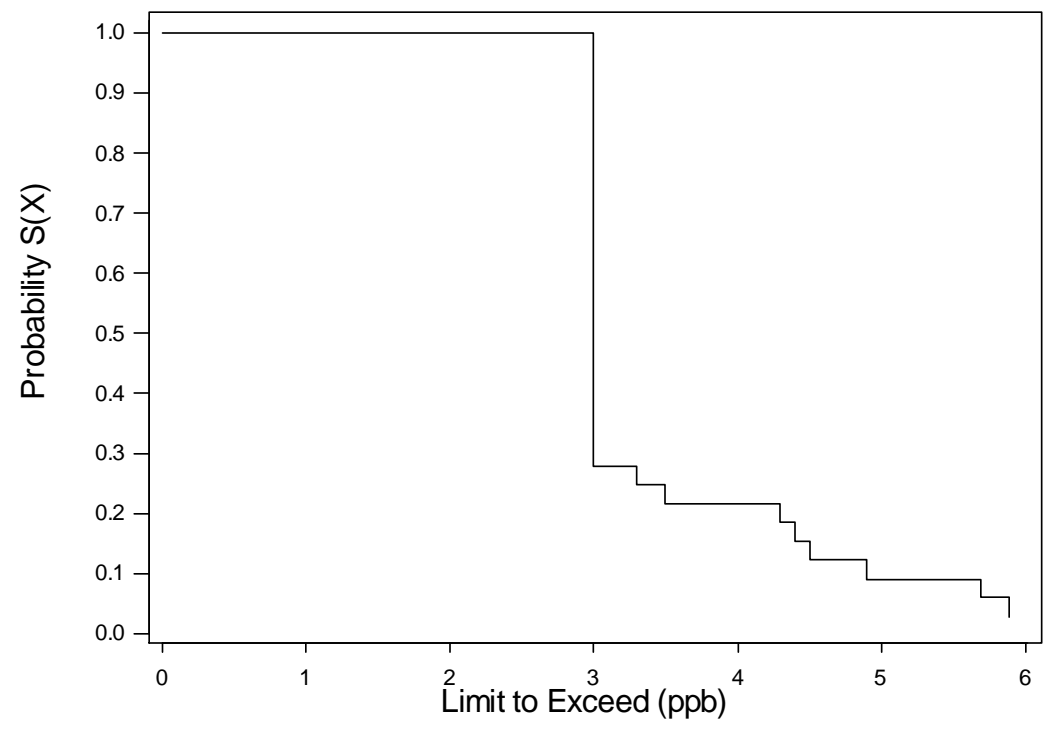


Nonparametric Survival Plot for Methyl isobutkl

by TCLP $($ Mean $=50.5 \mathrm{ppb})$

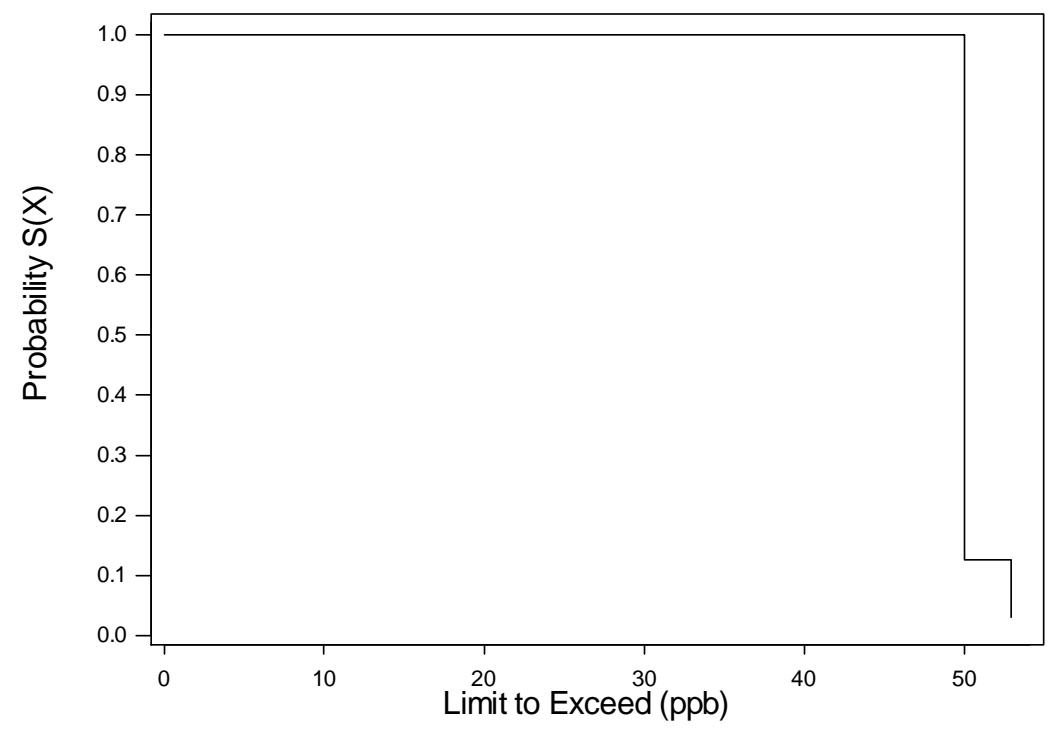

Figure F-7: Survival plots for organic compounds in TCLP leachate (13 charts)

\section{F.4 TCLP Leaching Characterization of Excess Foundry Sand per Metal Cast}

In this section, a series of 11 charts, grouped into Figure F-8, present TCLP leaching characterization of excess foundry sand per metal cast. 


\section{F.4.1 Metallic Elements}

\section{Nonparametric Survival Plot for Aluminium (Al)}

Legend, Cast, Mean (ppm'

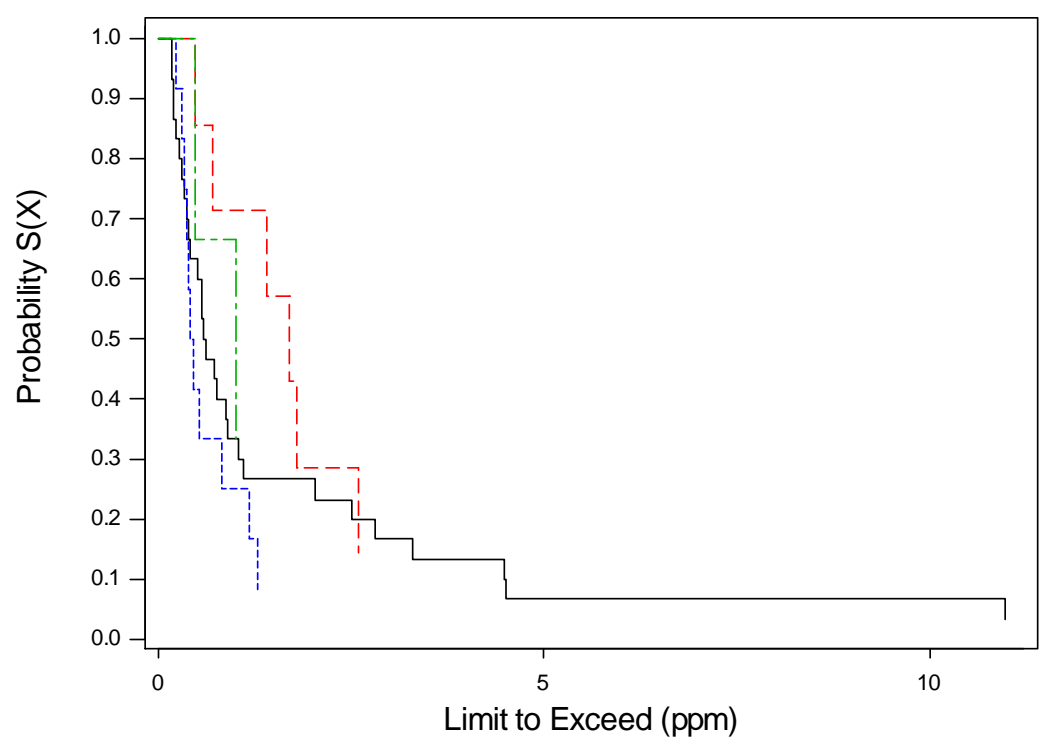

_ Iron_1.793

_ _ _ Steel_4.239

-----. Aluminium_0.816

____ Copper_1.393

Nonparametric Survival Plot for Barium (Ba)

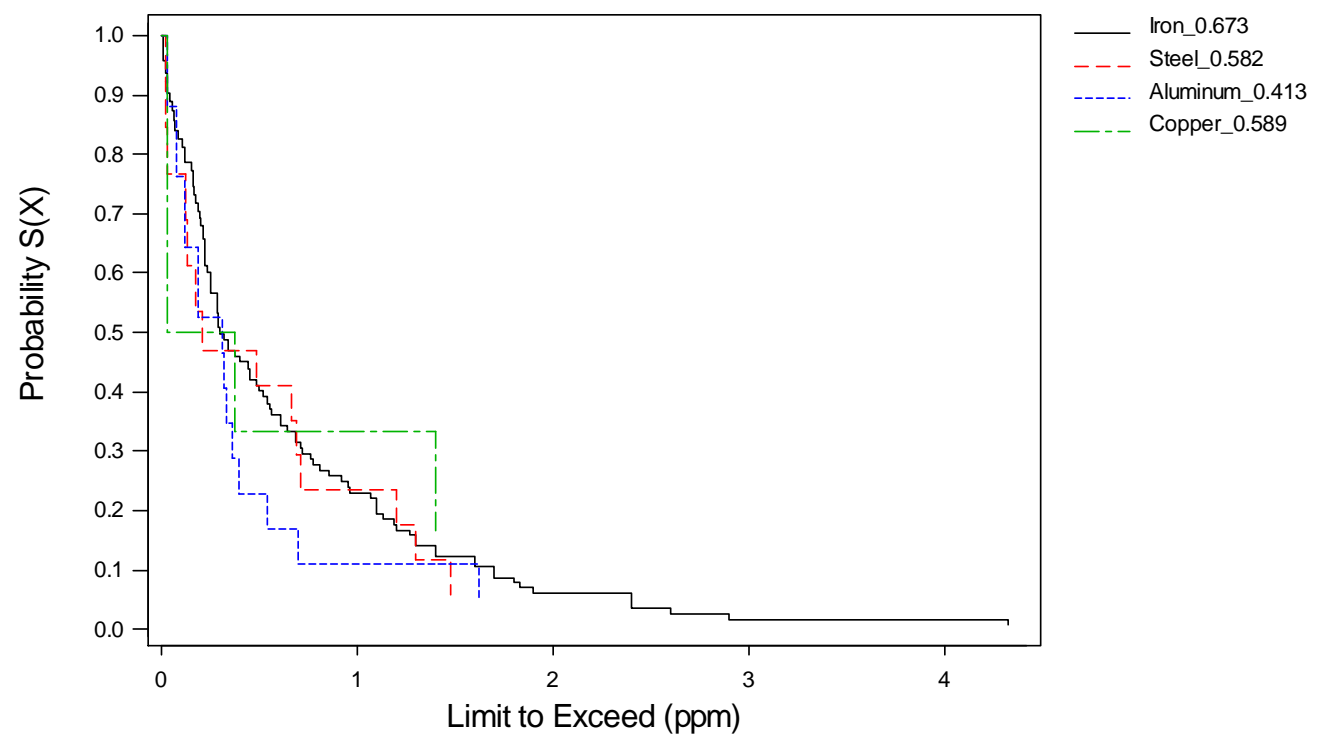


Nonparametric Survival Plot for Cadmium (Cd)

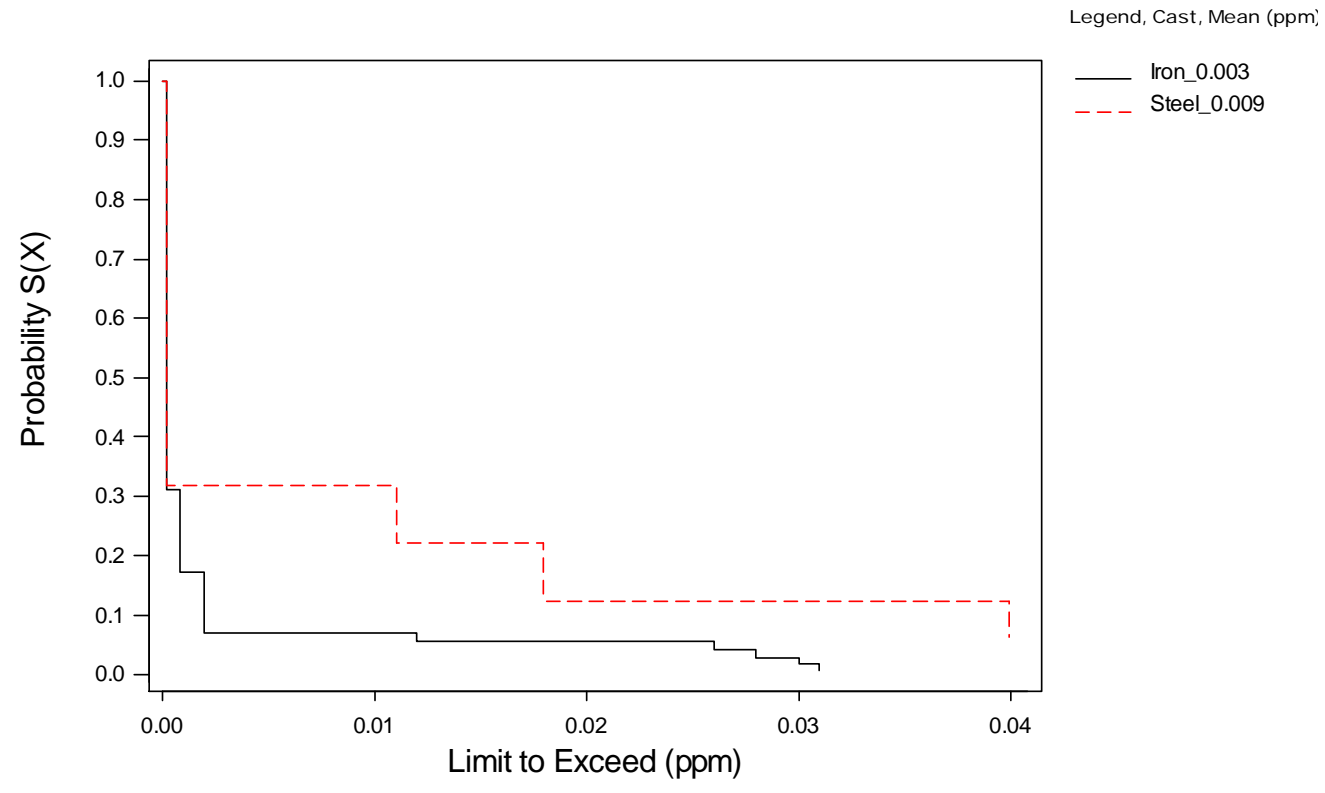

Nonparametric Survival Plot for Chromium (Cr)

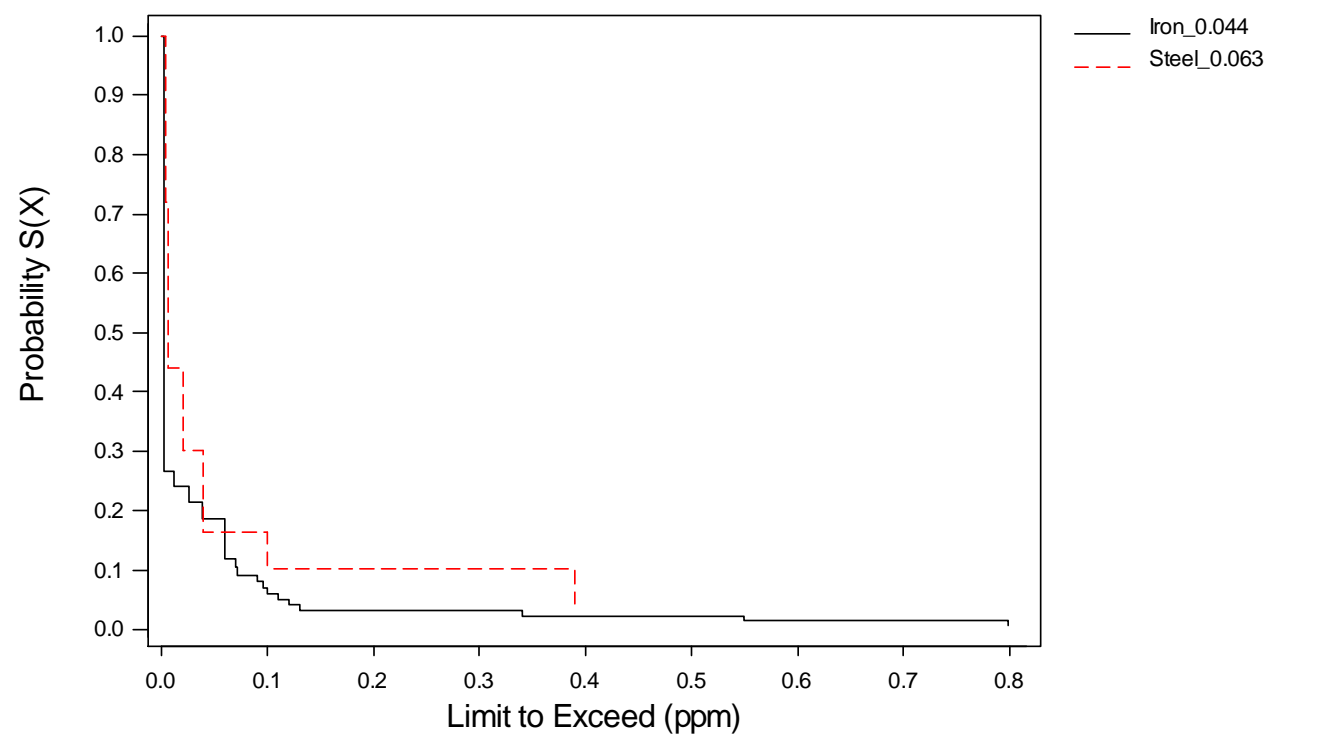


Nonparametric Survival Plot for Iron ( $\mathrm{Fe})$

Legend, Cast, Mean (ppm)

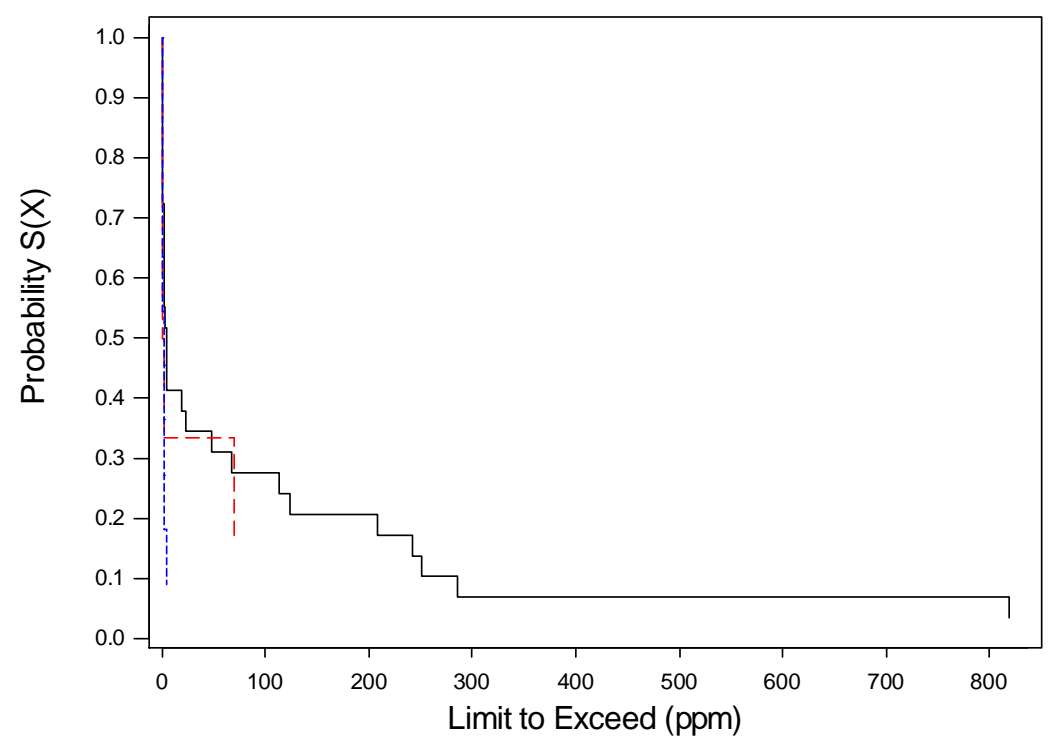

Iron 106.7

- - - Steel_23.8

_._-_. Aluminum 3.44

Nonparametric Survival Plot for Lead $(\mathrm{Pb})$

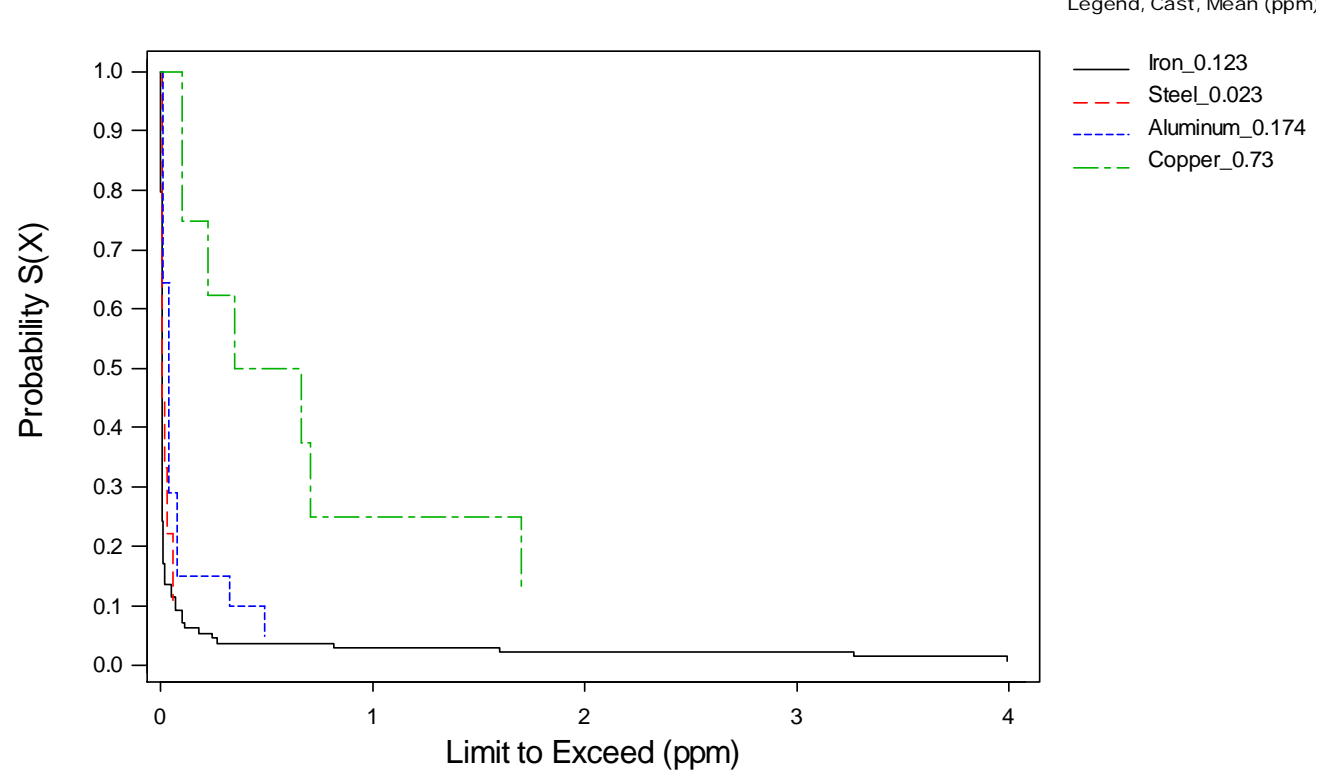


Nonparametric Survival Plot for Manganese (Mn)

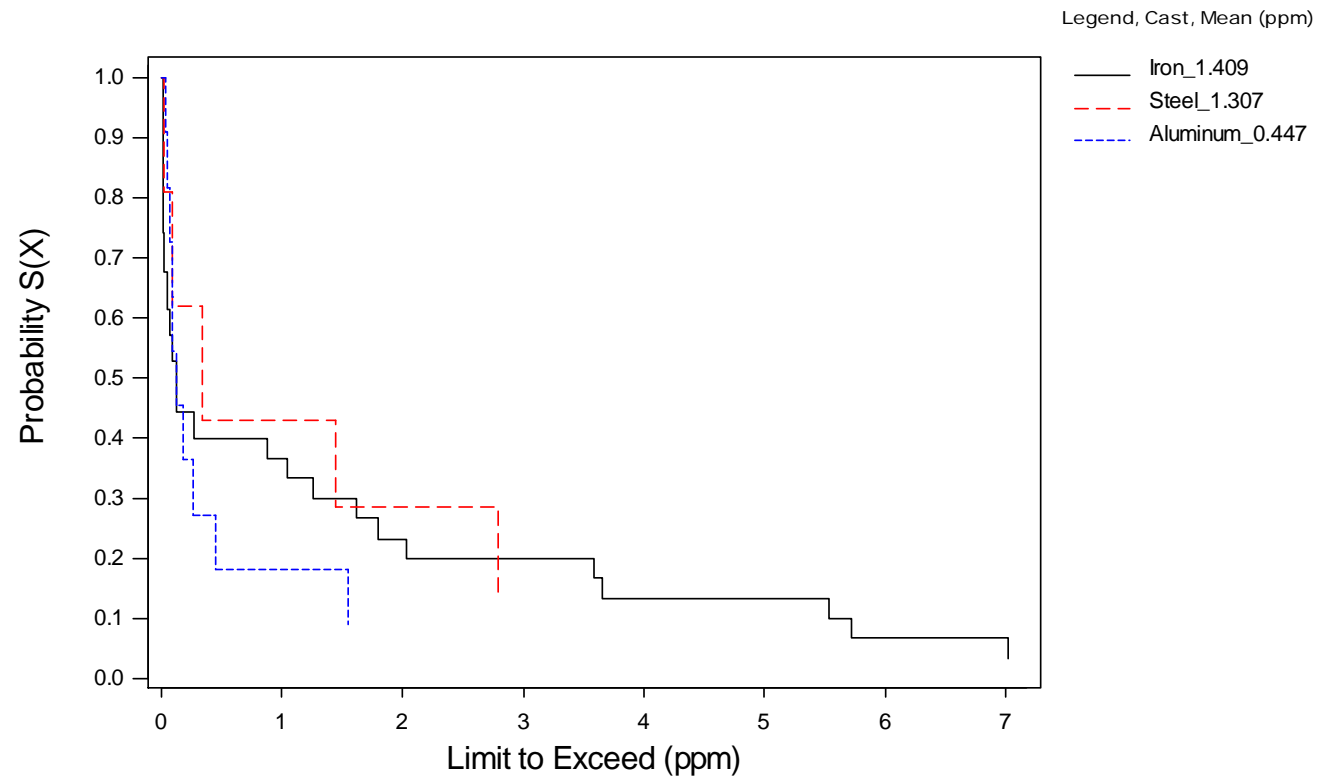

Nonparametric Survival Plot for Mercury $(\mathrm{Hg})$

Legend, Cast, Mean (ppm)

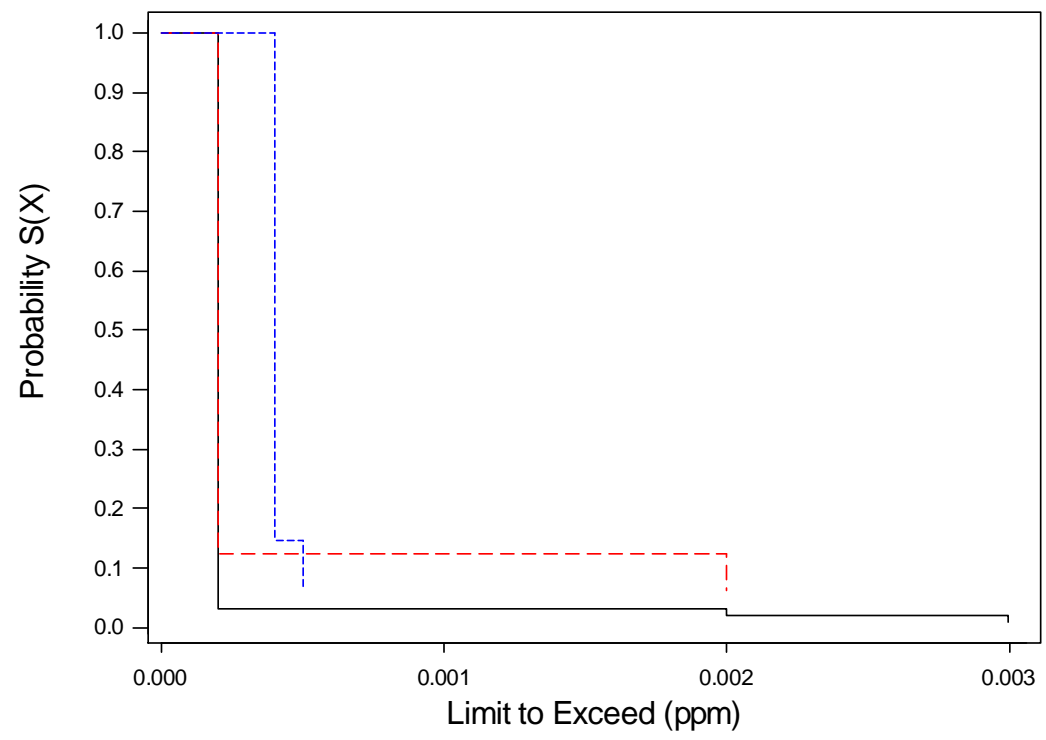

Iron 0.0004

Steel_0.0006

- - - Al_0.0005 
Nonparametric Survival Plot for Nickel (Ni)

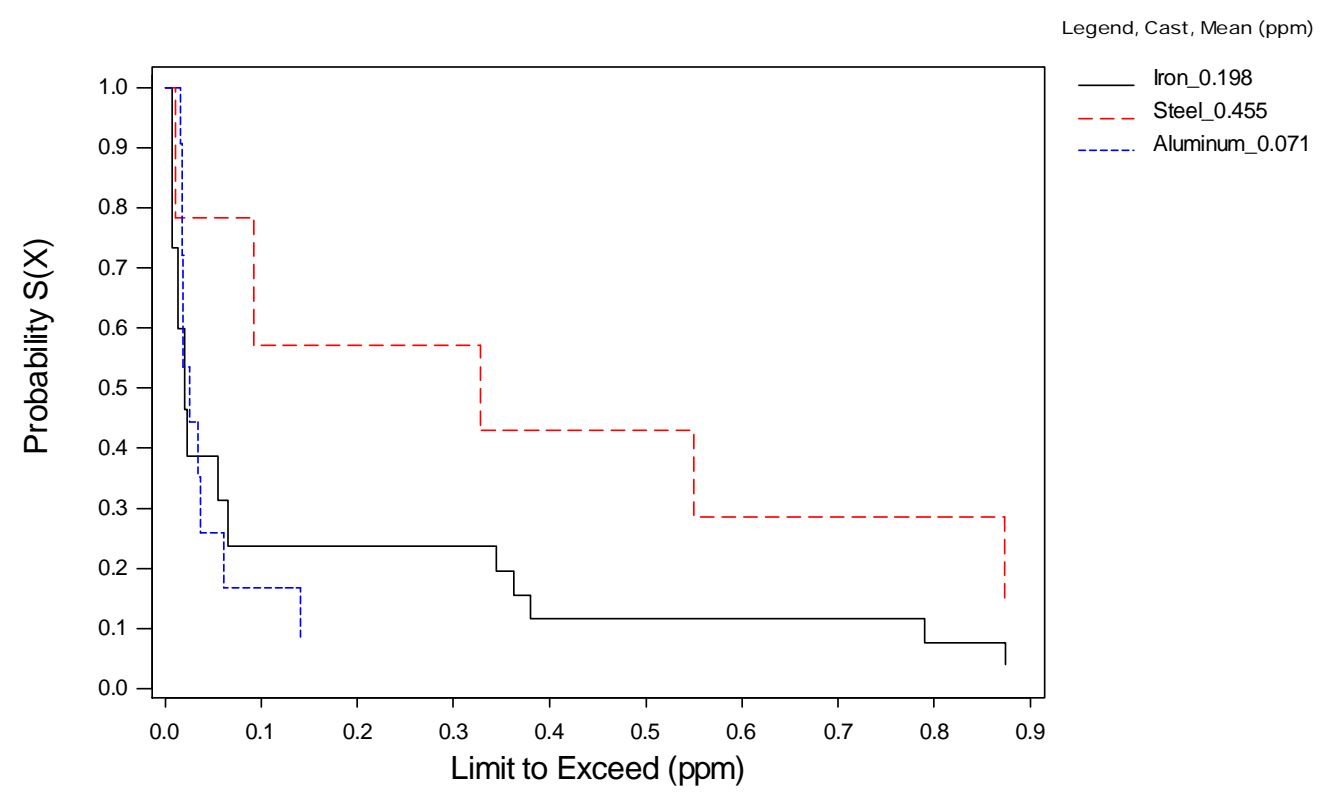

Nonparametric Survival Plot for Selenium (Se)

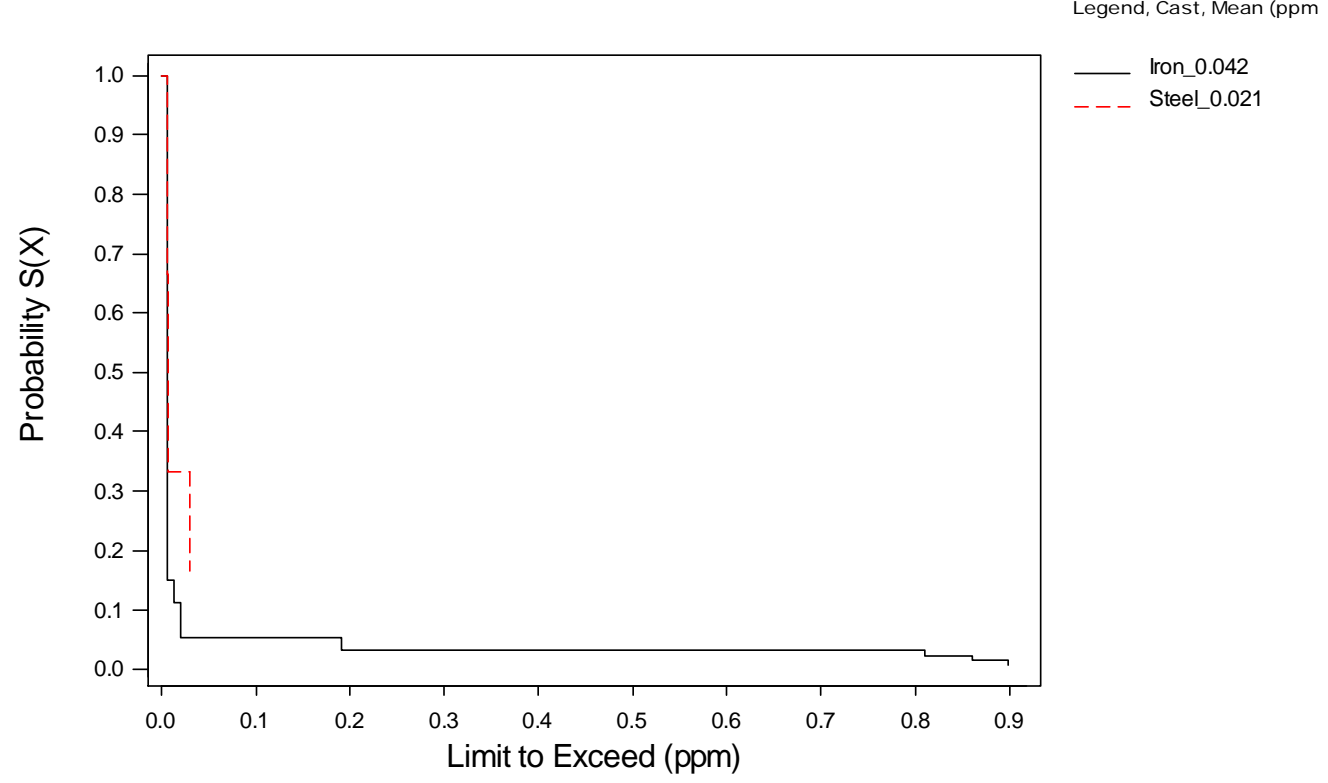


Nonparametric Survival Plot for Zinc (Zn)

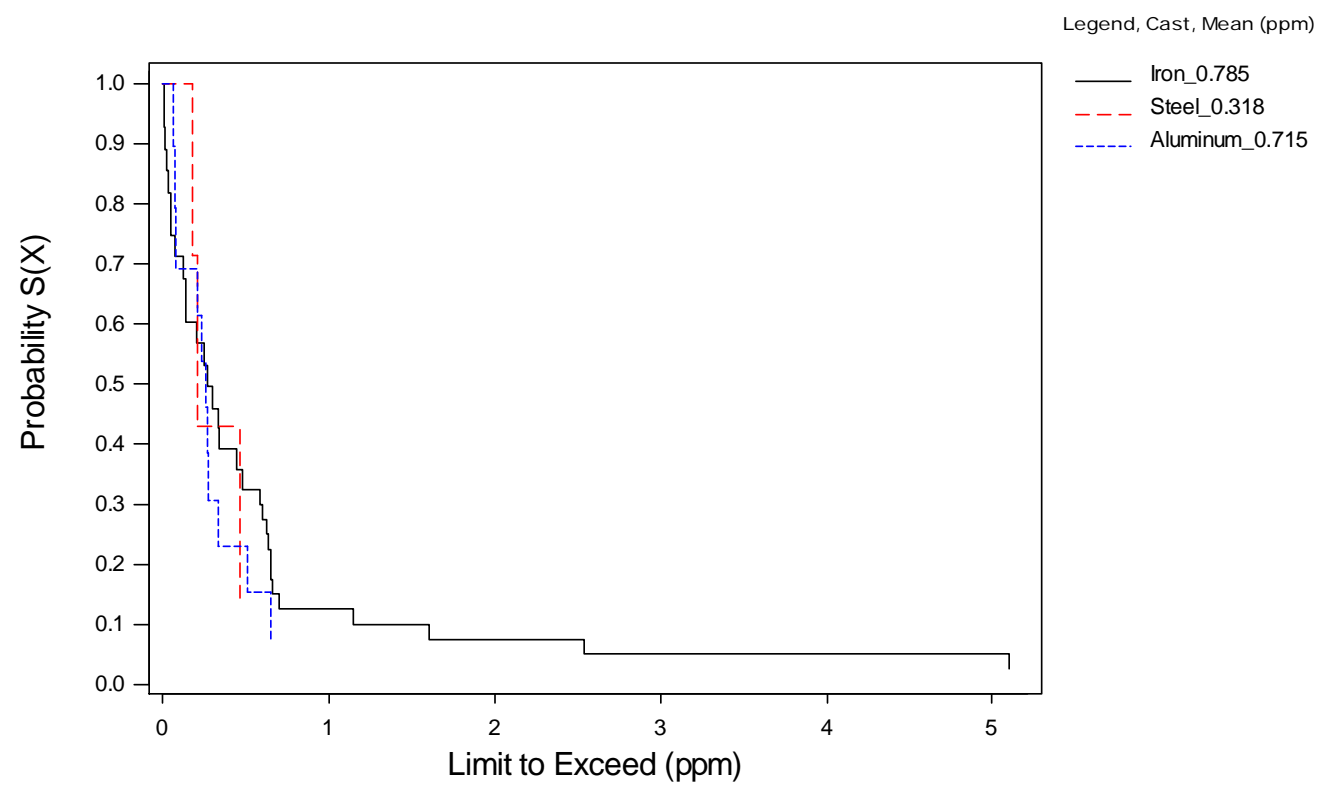

Figure F-8: Survival plots for metallic elements in TCLP leachate per metal cast (11 charts) 


\section{Appendix G}

\section{Gradation and Grain Shape}

\section{G.1 Gradation}

The sieve analysis is conducted on the oven-dry excess foundry sand to investigate its gradation. The results are presented in Table G-1 and Table G-2. 
Table G-1: Summary of oven-dry sieve analysis of excess foundry sand ---percentage passing by weight (\%)

\begin{tabular}{|c|c|c|c|c|c|c|c|c|c|c|c|}
\hline & $\# 6$ & $\# 12$ & \# 20 & $\# 40$ & \# 50 & \# 70 & \# 100 & \# 140 & \# 200 & \# 270 & \# 325 \\
\hline FS02 & 100.00 & 99.95 & 98.77 & 75.88 & 35.34 & --- & 1.97 & --- & 0.08 & --- & 0.03 \\
\hline FS04 & 100.00 & 98.11 & 97.71 & 95.89 & 85.74 & --- & 14.66 & --- & 1.19 & --- & 0.29 \\
\hline FS05 & 100.00 & 99.39 & 98.65 & 85.43 & 55.15 & --- & 8.12 & --- & 0.49 & --- & 0.16 \\
\hline FS06 & 97.24 & 92.94 & 90.24 & 65.32 & 23.75 & --- & 0.97 & --- & 0.00 & --- & 0.00 \\
\hline FS09 & 99.79 & 99.56 & 99.12 & 86.22 & 53.01 & --- & 5.74 & --- & 0.52 & --- & 0.20 \\
\hline FS10 & 99.83 & 99.09 & 98.30 & 90.25 & 44.24 & --- & 3.74 & --- & 1.35 & --- & 0.78 \\
\hline FS11 & 99.99 & 99.86 & 98.53 & 67.09 & 30.81 & --- & 1.64 & --- & 0.08 & --- & 0.01 \\
\hline FS12 & 100.00 & 99.26 & 98.77 & 98.52 & 96.80 & 72.29 & 16.75 & 1.97 & 0.37 & 0.25 & --- \\
\hline FS13 & 100.00 & 100.00 & 100.00 & 84.81 & 45.95 & 23.29 & 8.75 & 2.06 & 0.51 & 0.13 & --- \\
\hline Min. & 97.24 & 92.94 & 90.24 & 65.32 & 23.75 & 12.15 & 0.97 & 0.69 & 0 & 0.13 & 0 \\
\hline Max. & 100 & 100 & 100 & 98.52 & 96.8 & 72.29 & 32.81 & 15.18 & 9.21 & 7.39 & 0.78 \\
\hline$A v g$. & 99.66 & 98.71 & 97.63 & 83.78 & 52.04 & 37.94 & 7.46 & 3.89 & 1.04 & 1.49 & 0.18 \\
\hline$S D$ & 0.74 & 1.78 & 2.42 & 9.21 & 21.05 & 26.44 & 7.85 & 5.55 & 2.14 & 2.89 & 0.22 \\
\hline
\end{tabular}


Table G-2: Summary of oven-dry sieve analysis of foundry sand ---percentage retained by weight (\%)

\begin{tabular}{|c|c|c|c|c|c|c|c|c|c|c|c|c|}
\hline & \# 6 & $\# 12$ & $\# 20$ & $\# 40$ & \# 50 & \# 70 & \# 100 & \# 140 & \# 200 & $\# 270$ & \# 325 & pan \\
\hline FS ID & $3.35 \mathrm{~mm}$ & $1.7 \mathrm{~mm}$ & $0.85 \mathrm{~mm}$ & $0.425 \mathrm{~mm}$ & $0.3 \mathrm{~mm}$ & $0.212 \mathrm{~mm}$ & $0.15 \mathrm{~mm}$ & $0.106 \mathrm{~mm}$ & $0.075 \mathrm{~mm}$ & $0.053 \mathrm{~mm}$ & $0.045 \mathrm{~mm}$ & \\
\hline FS01 & 0.00 & 2.92 & 2.52 & 18.71 & 41.29 & --- & 30.71 & --- & 3.37 & --- & 0.36 & 0.13 \\
\hline FS02 & 0.00 & 0.05 & 1.18 & 22.89 & 40.55 & --- & 33.36 & --- & 1.89 & --- & 0.05 & 0.03 \\
\hline FS03 & 0.00 & 0.57 & 0.27 & 14.03 & 46.84 & --- & 35.97 & --- & 2.14 & --- & 0.10 & 0.07 \\
\hline FS04 & 0.00 & 1.89 & 0.40 & 1.83 & 10.14 & --- & 71.09 & --- & 13.47 & --- & 0.90 & 0.29 \\
\hline FS05 & 0.00 & 0.61 & 0.74 & 13.22 & 30.28 & --- & 47.03 & --- & 7.63 & --- & 0.33 & 0.16 \\
\hline FS06 & 2.76 & 4.30 & 2.71 & 24.92 & 41.57 & --- & 22.78 & --- & 0.97 & --- & 0.00 & 0.00 \\
\hline FS07 & 0.00 & 0.04 & 0.36 & 8.04 & 36.15 & --- & 50.36 & --- & 4.39 & --- & 0.46 & 0.19 \\
\hline FS08 & 0.99 & 2.14 & 1.40 & 11.53 & 35.14 & --- & 43.77 & --- & 4.53 & --- & 0.33 & 0.17 \\
\hline FS09 & 0.21 & 0.23 & 0.43 & 12.91 & 33.21 & --- & 47.27 & --- & 5.22 & --- & 0.32 & 0.20 \\
\hline FS10 & 0.17 & 0.74 & 0.79 & 8.05 & 46.01 & --- & 40.50 & --- & 2.39 & --- & 0.57 & 0.78 \\
\hline FS11 & 0.01 & 0.13 & 1.33 & 31.44 & 36.28 & --- & 29.17 & --- & 1.56 & --- & 0.06 & 0.01 \\
\hline FS12 & 0.00 & 0.74 & 0.49 & 0.25 & 1.72 & 24.51 & 55.54 & 14.78 & 1.60 & 0.12 & --- & 0.25 \\
\hline FS13 & 0.00 & 0.00 & 0.00 & 15.19 & 38.87 & 22.65 & 14.54 & 6.69 & 1.54 & 0.39 & --- & 0.13 \\
\hline FS14 & 0.00 & 0.26 & 2.85 & 3.11 & 4.67 & 19.71 & 36.58 & 17.64 & 5.97 & 1.82 & --- & 7.39 \\
\hline FS15 & 0.00 & 0.07 & 0.07 & 20.58 & 42.27 & 24.86 & 9.39 & 2.07 & 0.28 & 0.14 & --- & 0.28 \\
\hline FS16 & 0.29 & 0.87 & 2.03 & 11.76 & 20.32 & 31.64 & 25.40 & 6.10 & 0.87 & 0.29 & --- & 0.44 \\
\hline FS17 & 1.39 & 0.46 & 0.93 & 16.94 & 34.34 & 28.54 & 12.41 & 3.13 & 0.93 & 0.46 & --- & 0.46 \\
\hline Min. & 0.00 & 0.00 & 0.00 & 0.25 & 1.72 & 19.71 & 9.39 & 2.07 & 0.28 & 0.12 & 0.00 & 0.00 \\
\hline Max. & 2.76 & 4.30 & 2.85 & 31.44 & 46.84 & 31.64 & 71.09 & 17.64 & 13.47 & 1.82 & 0.90 & 7.39 \\
\hline$A v g$. & 0.34 & 0.94 & 1.09 & 13.85 & 31.74 & 25.32 & 35.64 & 8.40 & 3.46 & 0.54 & 0.32 & 0.65 \\
\hline$S D$ & 0.74 & 1.20 & 0.93 & 8.36 & 14.04 & 4.24 & 16.28 & 6.36 & 3.30 & 0.64 & 0.27 & 1.75 \\
\hline
\end{tabular}




\section{G.2 Grain Shape}

A series of 17 charts, corresponding to individual excess foundry sand, is grouped into Figure G-1 to address the grain shape of excess foundry sand partitioned by gradation.
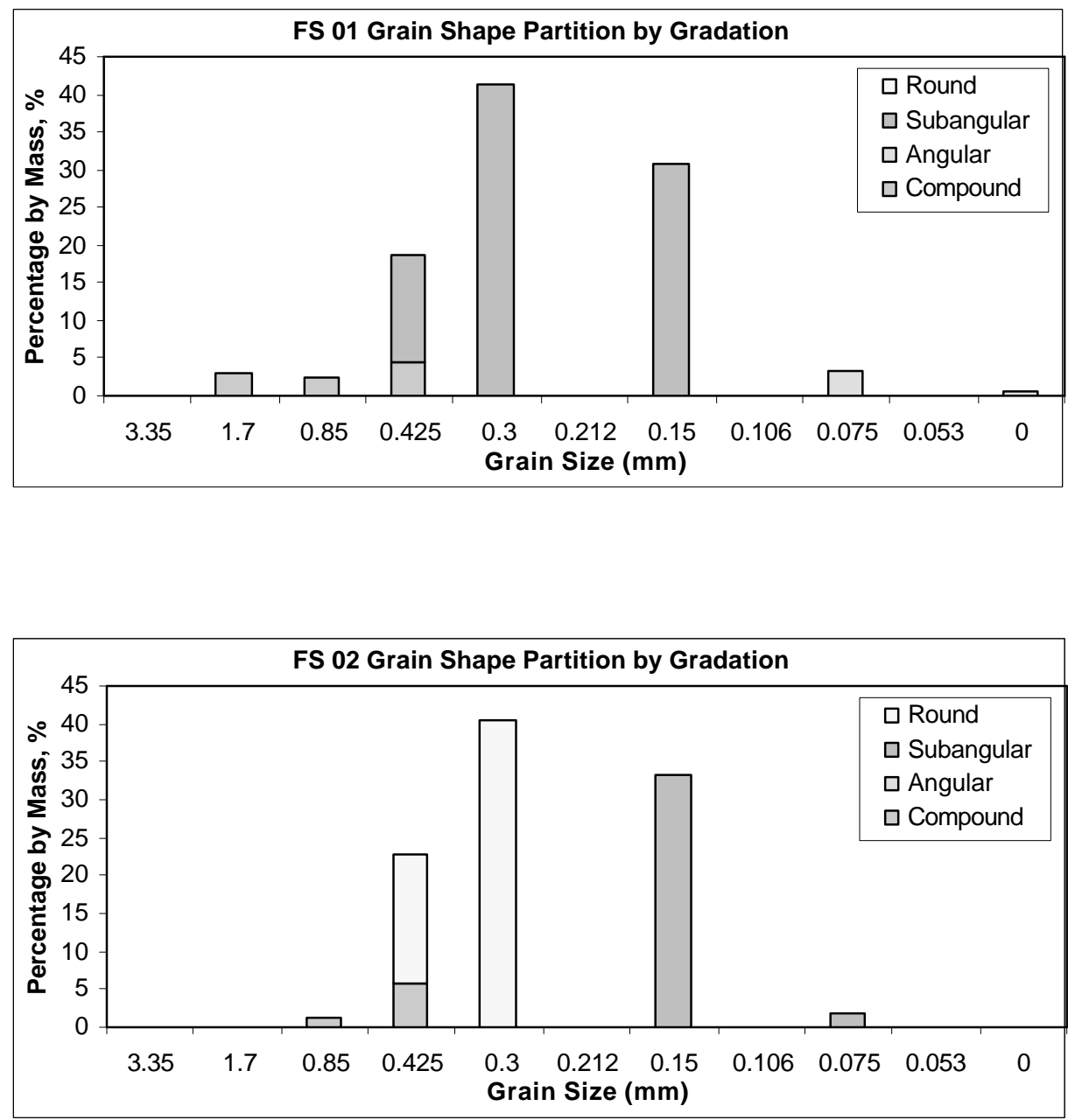

G- 4 

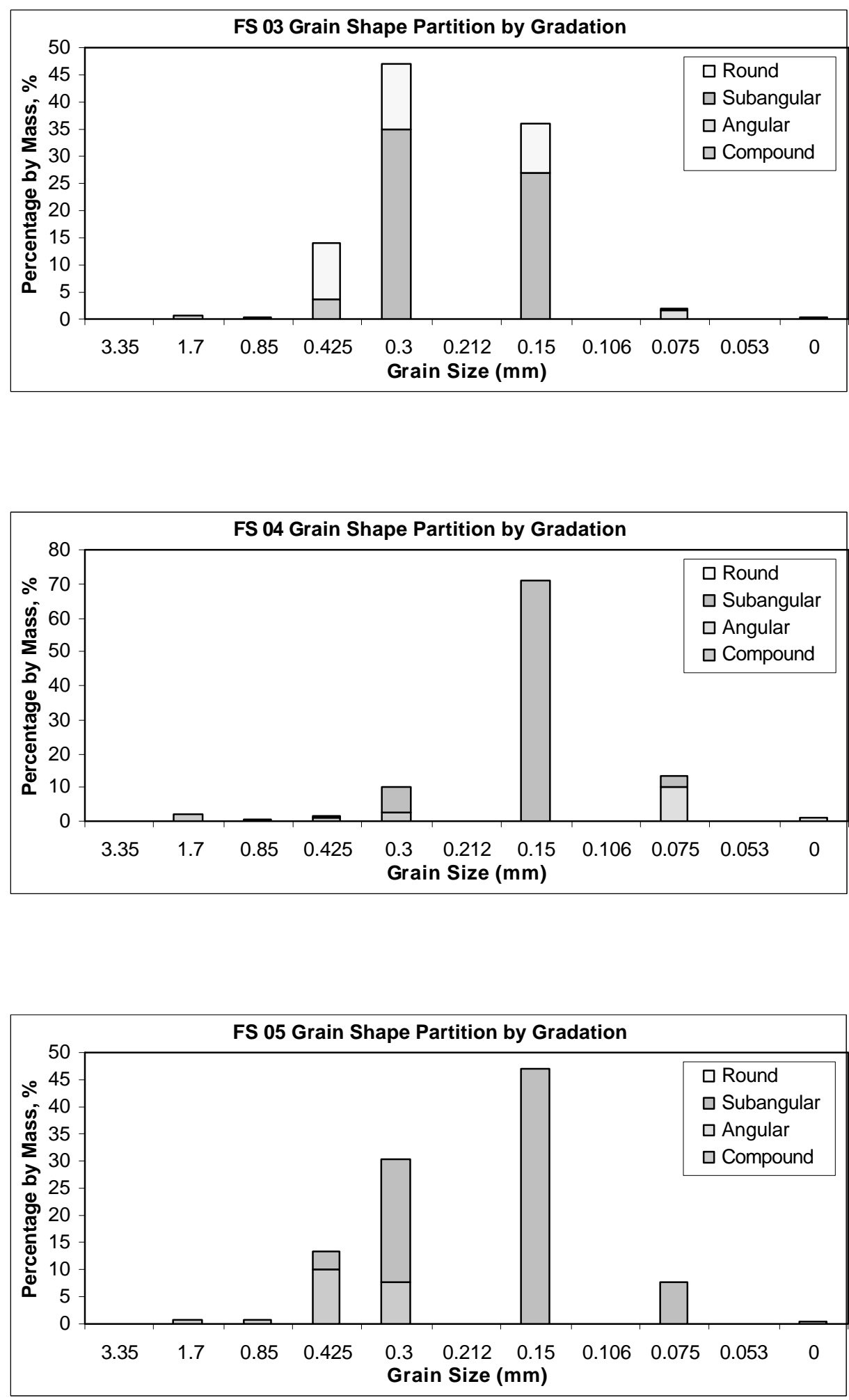

G- 5 

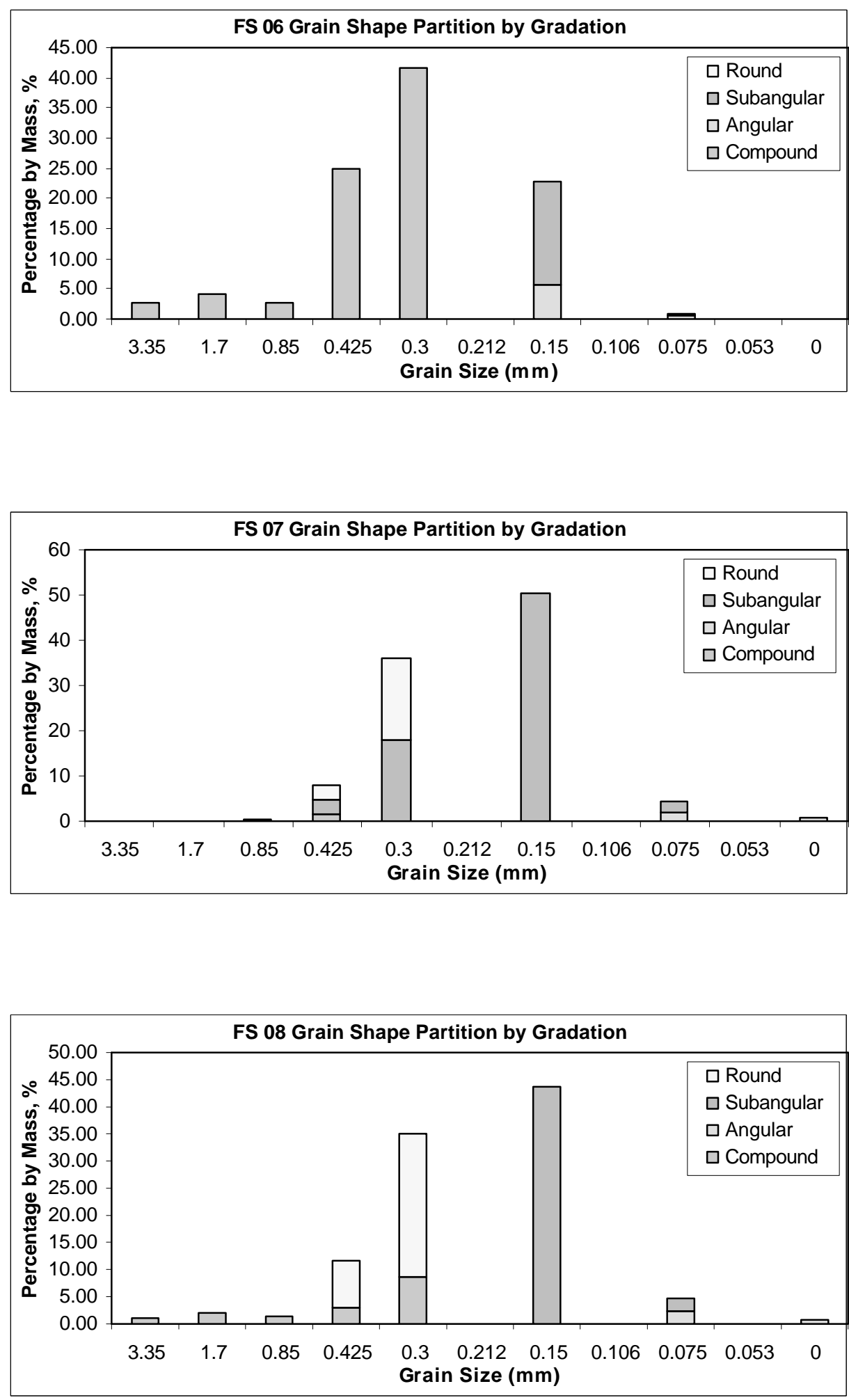

G- 6 

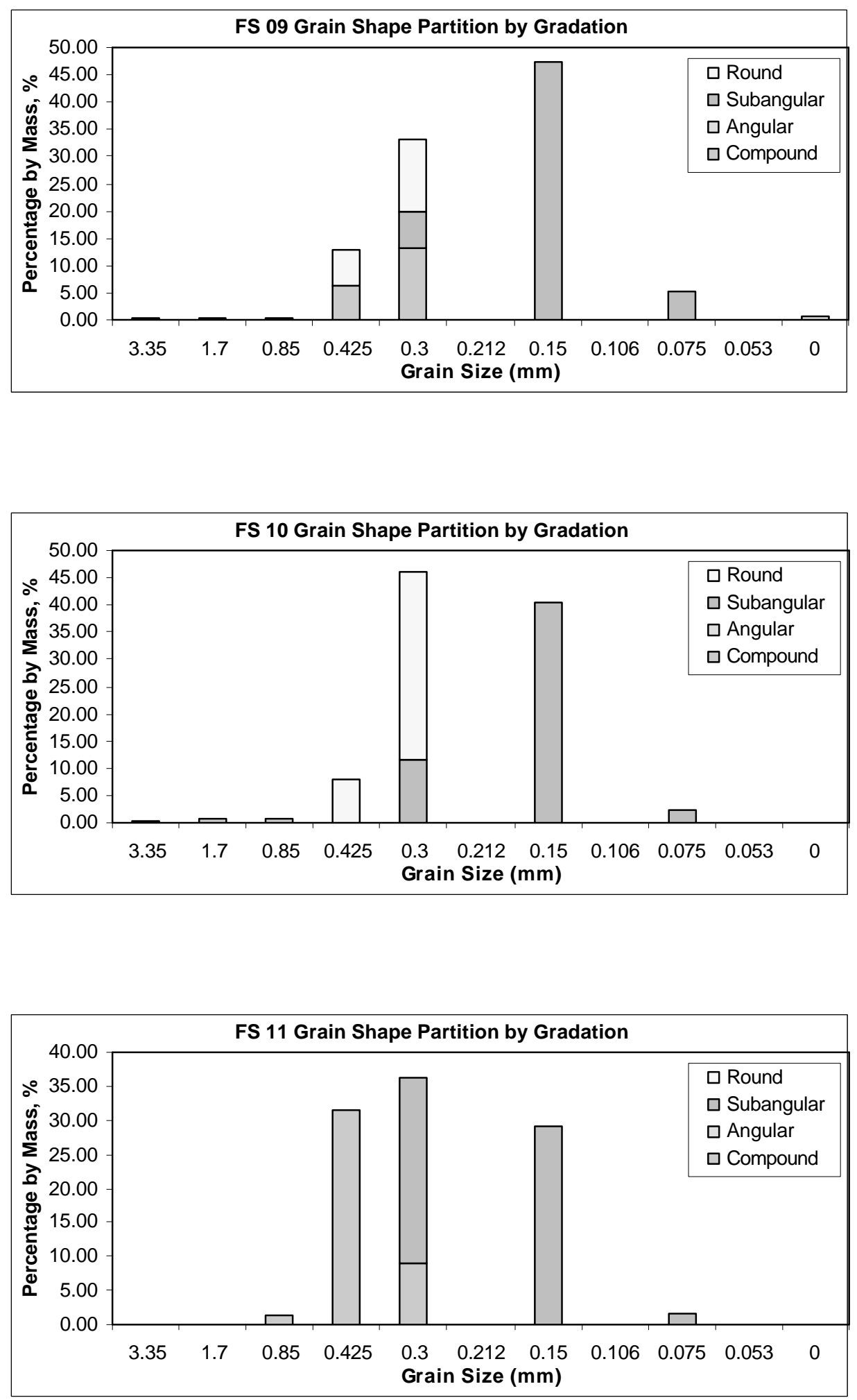

G- 7 

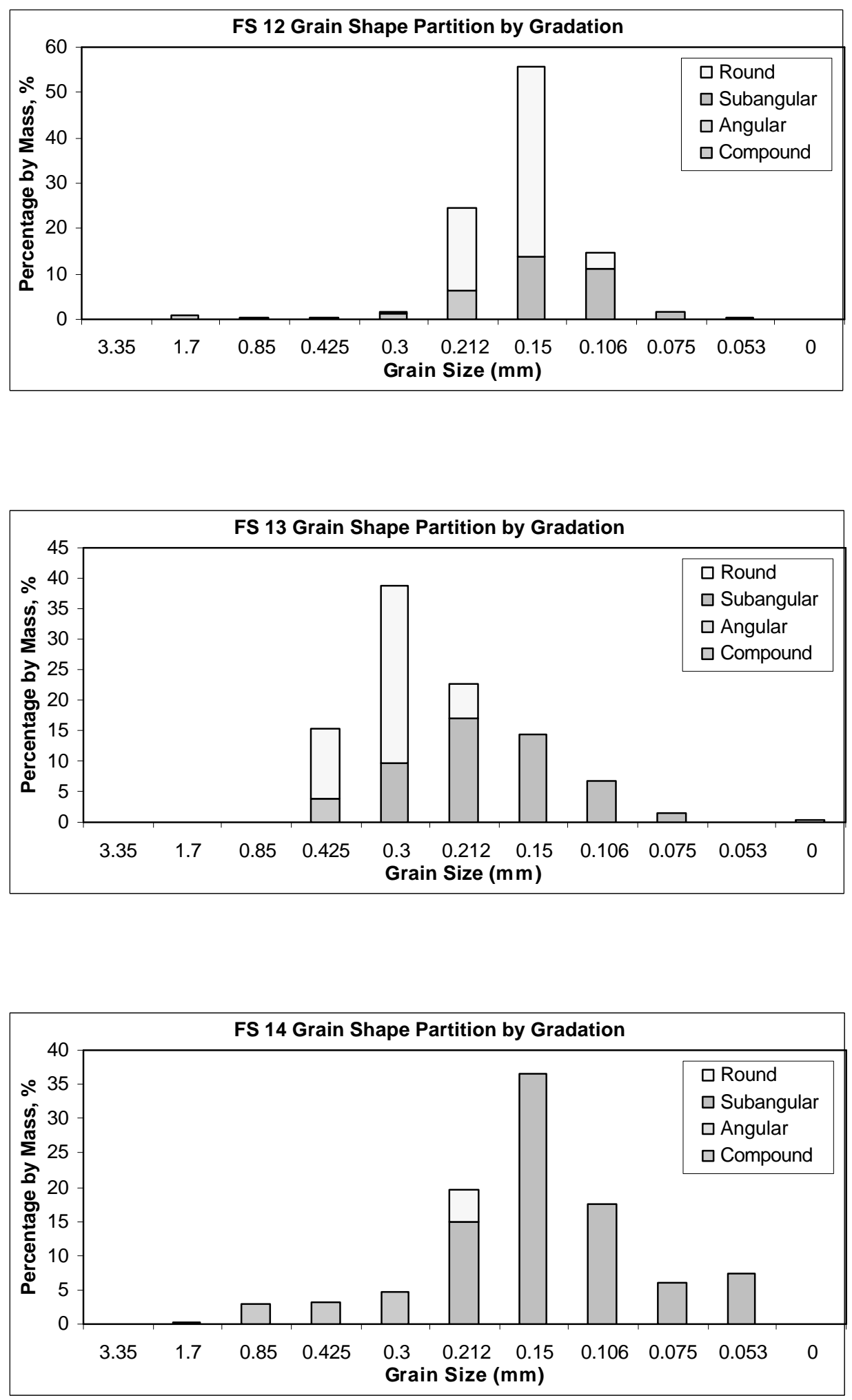

G- 8 

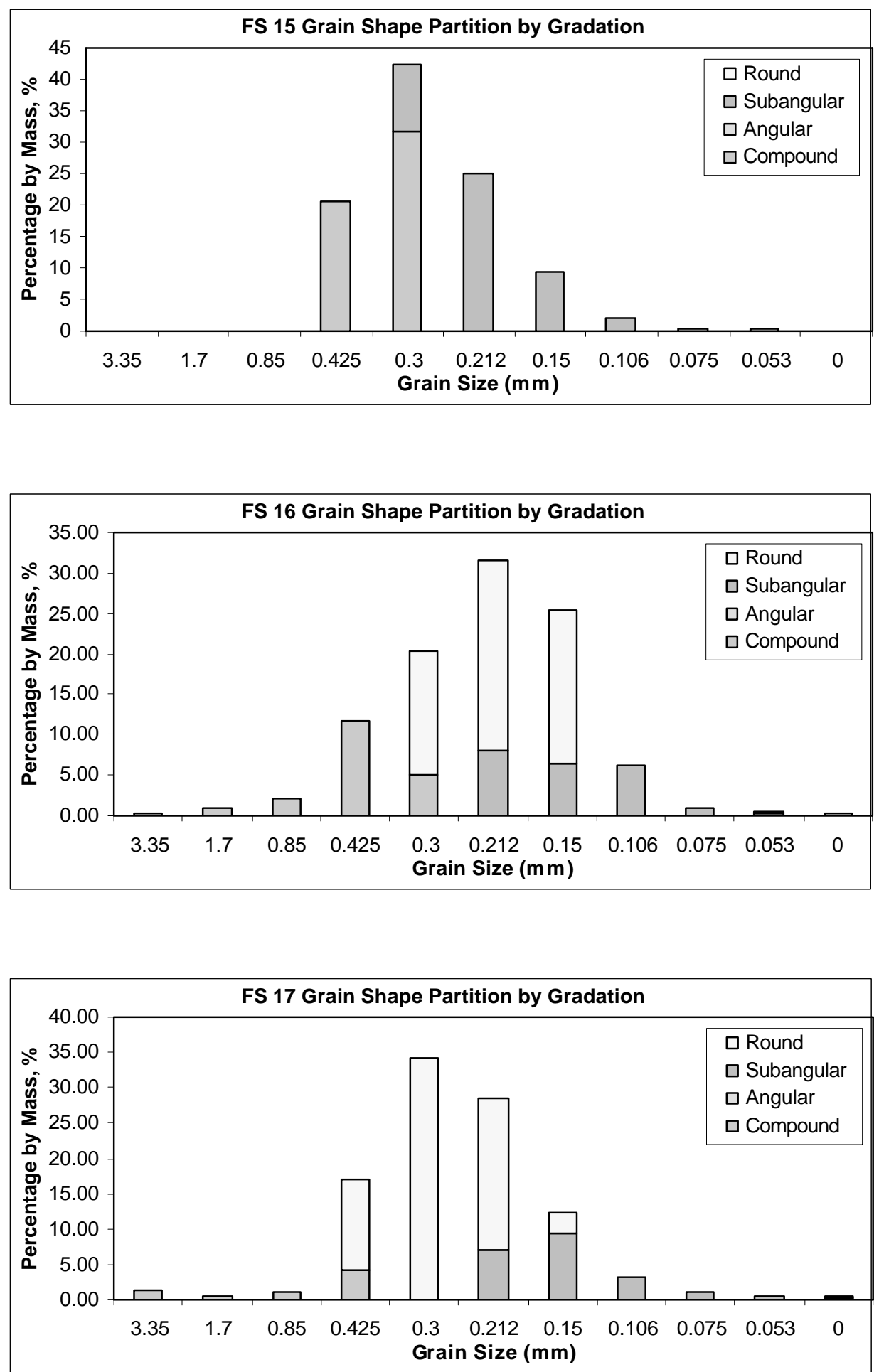

Figure G-1: Grain shape partitioned by gradation (17 charts) 


\section{Appendix H \\ Bulk Chemical Analysis of Excess Foundry Sands in Experiments}

\section{H.1 Organic Compounds}

The bulk analyses of organic compounds for 16 excess foundry sands involved in the experimental investigation are presented in Table H-1. The analyses were performed by a third-party professional chemical laboratory using methods presented in EPA SW846, "Test Methods For Evaluating Solid Waste Physical Chemical Methods."

\section{H.2 Oxides}

The bulk analyses of oxides for 16 excess foundry sands involved in the experimental investigation are presented in Table $\mathrm{H}-2$. The analyses were performed by a third-party professional chemical laboratory using X-ray fluoresce method. 
Table H-1: Organic compounds of excess foundry sands

\begin{tabular}{|c|c|c|c|c|c|c|c|c|c|c|c|c|c|c|c|c|c|}
\hline & & FS01 & FSO2 & FS03 & FS04 & FS05 & FS06 & FS07 & FS08 & FS09 & FS10 & FS11 & FS12 & FS13 & FS14 & FS15 & FS16 \\
\hline O-Cresol & \multirow{17}{*}{ 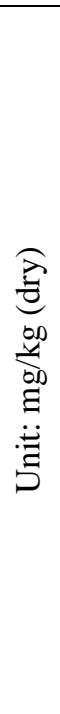 } & .829 & $<.341$ & .716 & 1.27 & 26.8 & $<.363$ & .986 & 1.47 & .514 & $<.357$ & 3.20 & 1.97 & .672 & $<.324$ & .366 & 2.64 \\
\hline M,P-Cresol & & .550 & $<.341$ & $<.392$ & 1.33 & 15.6 & $<.363$ & .509 & .666 & $<.368$ & $<.357$ & 1.05 & .828 & $<.342$ & $<.324$ & $<.355$ & 1.46 \\
\hline Cresol-Total & & 1.38 & $<.341$ & .716 & 2.60 & 42.4 & $<.363$ & 1.50 & 2.14 & .514 & $<.357$ & 4.25 & 2.80 & .672 & $<.324$ & .366 & 4.10 \\
\hline 2,4-Dinitrotoluene & & $<.358$ & $<.341$ & $<.392$ & $<.334$ & $<.342$ & $<.363$ & $<.332$ & $<.354$ & $<.368$ & $<.357$ & $<.346$ & $<.328$ & $<.342$ & $<.324$ & $<.355$ & $<.354$ \\
\hline 1,3-Hexachlorobutadiene & & $<.358$ & $<.341$ & $<.392$ & $<.334$ & $<.342$ & $<.363$ & $<.332$ & $<.354$ & $<.368$ & $<.357$ & $<.346$ & $<.328$ & $<.342$ & $<.324$ & $<.355$ & $<.354$ \\
\hline Hexachlorobenzene & & $<.358$ & $<.341$ & $<.392$ & $<.334$ & $<.342$ & $<.363$ & $<.332$ & $<.354$ & $<.368$ & $<.357$ & $<.346$ & $<.328$ & $<.342$ & $<.324$ & $<.355$ & $<.354$ \\
\hline Hexachloroethane & & $<.358$ & $<.341$ & $<.392$ & $<.334$ & $<.342$ & $<.363$ & $<.332$ & $<.354$ & $<.368$ & $<.357$ & $<.346$ & $<.328$ & $<.342$ & $<.324$ & $<.355$ & $<.354$ \\
\hline Nitrobenzene & & $<.358$ & $<.341$ & $<.392$ & $<.334$ & $<.342$ & $<.363$ & $<.332$ & $<.354$ & $<.368$ & $<.357$ & $<.346$ & $<.328$ & $<.342$ & $<.324$ & $<.355$ & $<.354$ \\
\hline Pentachlorophenol & & $<.358$ & $<.341$ & $<.392$ & $<.334$ & $<.342$ & $<.363$ & $<.332$ & $<.354$ & $<.368$ & $<.357$ & $<.346$ & $<.328$ & $<.342$ & $<.324$ & $<.355$ & $<.354$ \\
\hline Pyridine & & $<.358$ & $<.341$ & $<.392$ & $<.334$ & $<.342$ & $<.363$ & $<.332$ & $<.354$ & $<.368$ & $<.357$ & $<.346$ & $<.328$ & $<.342$ & $<.324$ & $<.355$ & $<.354$ \\
\hline 2,4,5-trichlorophenol & & $<.358$ & $<.341$ & $<.392$ & $<.334$ & $<.342$ & $<.363$ & $<.332$ & $<.354$ & $<.368$ & $<.357$ & $<.346$ & $<.328$ & $<.342$ & $<.324$ & $<.355$ & $<.354$ \\
\hline 2,4,6-trichlorophenol & & $<.358$ & $<.341$ & $<.392$ & $<.334$ & $<.342$ & $<.363$ & $<.332$ & $<.354$ & $<.368$ & $<.357$ & $<.346$ & $<.328$ & $<.342$ & $<.324$ & $<.355$ & $<.354$ \\
\hline Benzo(a)anthracene & & $<.358$ & $<.341$ & $<.392$ & $<.334$ & $<.342$ & $<.363$ & $<.332$ & $<.354$ & $<.368$ & $<.357$ & $<.346$ & $<.328$ & $<.342$ & $<.324$ & $<.355$ & $<.354$ \\
\hline Chrysene & & $<.358$ & $<.341$ & $<.392$ & $<.334$ & $<.342$ & $<.363$ & $<.332$ & $<.354$ & $<.368$ & $<.357$ & $<.346$ & $<.328$ & $<.342$ & $<.324$ & $<.355$ & $<.354$ \\
\hline 2,4-Dimethylphenol & & $<.358$ & $<.341$ & $<.392$ & .602 & 8.81 & $<.363$ & $<.332$ & $<.354$ & $<.368$ & $<.357$ & 1.89 & $<.328$ & 4.14 & $<.324$ & $<.355$ & $<.354$ \\
\hline Fluoranthene & & $<.358$ & $<.341$ & $<.392$ & .619 & $<.342$ & $<.363$ & $<.332$ & $<.354$ & $<.368$ & $<.357$ & $<.346$ & $<.328$ & $<.342$ & $<.324$ & $<.355$ & $<.354$ \\
\hline Phenanthrene & & 0.358 & $<.341$ & .596 & 2.11 & 1.29 & $<.363$ & .556 & .686 & .500 & $<.357$ & $<.346$ & $<.328$ & $<.342$ & $<.324$ & .399 & $<.354$ \\
\hline Acetone & \multirow{20}{*}{ 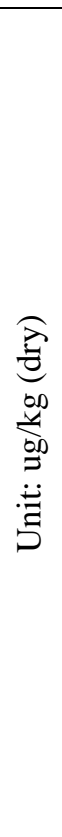 } & 33 & 49 & $<10$ & 81 & 37 & 12 & 242 & 23 & 15 & $<10$ & 16 & 247 & 11 & 57 & $\mathbf{1 0}$ & 740 \\
\hline Benzene & & $<5$ & 5 & 5 & 13 & 9 & $<5$ & 9 & $<4$ & $<4$ & $<5$ & $<5$ & $<5$ & $<4$ & $<5$ & $<5$ & 32 \\
\hline Carbon Tetrachloride & & $<5$ & $<5$ & $<5$ & $<5$ & $<5$ & $<5$ & $<4$ & $<4$ & $<4$ & $<5$ & $<5$ & $<5$ & $<4$ & $<5$ & $<5$ & $<5$ \\
\hline Chlorobenzene & & $<5$ & $<5$ & $<5$ & $<5$ & $<5$ & $<5$ & $<4$ & $<4$ & $<4$ & $<5$ & $<5$ & $<5$ & $<4$ & $<5$ & $<5$ & $<5$ \\
\hline Chloroform & & $<5$ & $<5$ & $<5$ & $<5$ & $<5$ & $<5$ & $<4$ & $<4$ & $<4$ & $<5$ & $<5$ & $<5$ & $<4$ & $<5$ & $<5$ & $<5$ \\
\hline 1,4-Dichlorobenzene & & $<5$ & $<5$ & $<5$ & $<5$ & $<5$ & $<5$ & $<4$ & $<4$ & $<4$ & $<5$ & $<5$ & $<5$ & $<4$ & $<5$ & $<5$ & $<5$ \\
\hline 1,2-Dichloroethane & & $<5$ & $<5$ & $<5$ & $<5$ & $<5$ & $<5$ & $<4$ & $<4$ & $<4$ & $<5$ & $<5$ & $<5$ & $<4$ & $<5$ & $<5$ & $<5$ \\
\hline 1,1-Dichloroethene & & $<5$ & $<5$ & $<5$ & $<5$ & $<5$ & $<5$ & $<4$ & $<4$ & $<4$ & $<5$ & $<5$ & $<5$ & $<4$ & $<5$ & $<5$ & $<5$ \\
\hline Ethyl benzene & & $<5$ & $<5$ & $<5$ & $<5$ & $<5$ & $<5$ & $<4$ & $<4$ & $<4$ & $<5$ & $<5$ & $<5$ & $<4$ & $<5$ & $<5$ & $<5$ \\
\hline Methyl ethyl ketone & & $<10$ & $<10$ & $<10$ & 19 & $<9$ & $<10$ & 31 & $<9$ & $<9$ & $<10$ & $<10$ & $<9$ & $<8$ & $<10$ & $<10$ & 146 \\
\hline Methylene chloride & & $<5$ & $<5$ & $<5$ & 7 & $<5$ & $<5$ & $<4$ & $<4$ & 5 & $<5$ & 12 & $<5$ & $<4$ & 16 & $<5$ & 11 \\
\hline Naphthalene & & $<10$ & $<10$ & $<10$ & 10 & $<9$ & $<10$ & $<9$ & $<9$ & $<9$ & $<10$ & $<10$ & 89600 & 37700 & $<10$ & $<10$ & 13 \\
\hline Styrene & & $<5$ & $<5$ & $<5$ & $<5$ & $<5$ & $<5$ & $<4$ & $<4$ & $<4$ & $<5$ & $<5$ & $<5$ & $<4$ & $<5$ & $<5$ & $<5$ \\
\hline Tetrachloroethene & & $<5$ & $<5$ & $<5$ & $<5$ & $<5$ & $<5$ & $<4$ & $<4$ & $<4$ & $<5$ & $<5$ & $<5$ & $<4$ & $<5$ & $<5$ & $<5$ \\
\hline Toluene & & $<5$ & $<5$ & $<5$ & 11 & $<5$ & $<5$ & 4 & $<4$ & $<4$ & $<5$ & $<5$ & $<5$ & $<4$ & $<5$ & $<5$ & 19 \\
\hline 1,1,1-Trichloroethane & & $<5$ & $<5$ & $<5$ & $<5$ & $<5$ & $<5$ & $<4$ & $<4$ & $<4$ & $<5$ & $<5$ & $<5$ & $<4$ & $<5$ & $<5$ & $<5$ \\
\hline Trichloroethene & & $<5$ & $<5$ & $<5$ & $<5$ & $<5$ & $<5$ & $<4$ & $<4$ & $<4$ & $<5$ & $<5$ & $<5$ & $<4$ & $<5$ & $<5$ & $<5$ \\
\hline Vinyl chloride & & $<10$ & $<10$ & $<10$ & $<9$ & $<9$ & $<10$ & $<9$ & $<9$ & $<9$ & $<10$ & $<10$ & $<9$ & $<8$ & $<10$ & $<10$ & $<10$ \\
\hline M, P-Xylene & & $<5$ & $<5$ & $<5$ & 6 & $<5$ & $<5$ & $<4$ & $<4$ & $<4$ & $<5$ & $<5$ & $<5$ & $<4$ & $<5$ & $<5$ & 8 \\
\hline Xylene-total & & $<5$ & $<5$ & $<5$ & 6 & $<5$ & $<5$ & $<4$ & $<4$ & $<4$ & $<5$ & $<5$ & $<5$ & $<4$ & $<5$ & $<5$ & 8 \\
\hline
\end{tabular}


Table H-2: Oxides of excess foundry sands

\begin{tabular}{|c|c|c|c|c|c|c|c|c|c|c|c|c|c|c|c|c|c|}
\hline & & FS01 & FS02 & FS03 & FS04 & FS05 & FS06 & FS07 & FS08 & FS09 & FS10 & FS11 & FS12 & FS13 & FS14 & FS15 & FS16 \\
\hline $\mathrm{Na}_{2} \mathrm{O}$ & \multirow{16}{*}{ 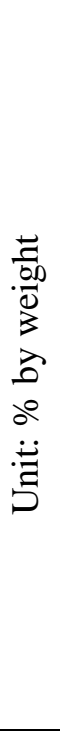 } & .28 & $<.009$ & $<.009$ & .28 & $<.009$ & 2.11 & .19 & .14 & .21 & $<.009$ & .05 & $<.009$ & $<.009$ & .20 & .01 & .32 \\
\hline $\mathrm{MgO}$ & & .89 & .09 & .38 & .49 & .52 & $<.003$ & .60 & .91 & $<.003$ & 2.32 & .33 & $<.003$ & $<.003$ & 2.08 & .50 & .62 \\
\hline $\mathrm{Al}_{2} \mathrm{O}_{3}$ & & 6.54 & 1.19 & 5.30 & 6.41 & 4.17 & 4.28 & 5.22 & 6.96 & 3.08 & .17 & 3.80 & .30 & .68 & 3.25 & 5.05 & 5.88 \\
\hline $\mathrm{SiO}_{2}$ & & 84.1 & 92.0 & 86.5 & 85.0 & 88.9 & 87.0 & 88.4 & 83.1 & 92.1 & 90.5 & 91.9 & 98.0 & 96.9 & 64.8 & 83.1 & 86.0 \\
\hline $\mathrm{P}_{2} \mathrm{O}_{5}$ & & .03 & .02 & .03 & .06 & .03 & .04 & .04 & .04 & .04 & .01 & .06 & .02 & .03 & $<.001$ & .04 & .05 \\
\hline $\mathrm{SO}_{3}$ & & .23 & .57 & .22 & .19 & .19 & $<.001$ & .21 & .28 & .06 & 3.00 & .11 & $<.001$ & $<.001$ & .89 & .33 & .10 \\
\hline $\mathrm{K}_{2} \mathrm{O}$ & & .84 & .17 & .24 & .86 & .14 & 2.13 & .93 & .83 & 1.18 & $<.001$ & 1.02 & $<.001$ & .54 & .30 & .18 & .23 \\
\hline $\mathrm{CaO}$ & & .91 & $<.001$ & .17 & .49 & .35 & .74 & .52 & .55 & .38 & 2.72 & .41 & $<.001$ & $<.001$ & 21.8 & .32 & .73 \\
\hline $\mathrm{TiO}_{2}$ & & .07 & .05 & .07 & .13 & .06 & .11 & .04 & .07 & .04 & $<.001$ & .04 & $<.001$ & .08 & .12 & 1.61 & .04 \\
\hline $\mathrm{Cr}_{2} \mathrm{O}_{3}$ & & .02 & 1.99 & .02 & .02 & .02 & .02 & .02 & .02 & .02 & .03 & .02 & .02 & .02 & .02 & .02 & .06 \\
\hline $\mathrm{MnO}$ & & .02 & .04 & .02 & .03 & .02 & .03 & .02 & .04 & .02 & .01 & .02 & .01 & .02 & .04 & .03 & .03 \\
\hline $\mathrm{Fe}_{2} \mathrm{O}_{3}$ & & 2.01 & 1.61 & 1.15 & 2.01 & 1.08 & 1.24 & 1.06 & 1.95 & 1.00 & .37 & .87 & .29 & 0.50 & 1.22 & 1.82 & 3.12 \\
\hline $\mathrm{C}$ & & 4 & 2.2 & 5.9 & 4.0 & 4.5 & 2.3 & 2.7 & 5.1 & 1.8 & .9 & 1.4 & 1.4 & 1.2 & 2.2 & 7.0 & 2.8 \\
\hline $\mathrm{CuO}$ & & --- & --- & --- & --- & --- & --- & --- & --- & --- & --- & --- & --- & --- & .36 & --- & --- \\
\hline $\mathrm{ZnO}$ & & --- & --- & --- & --- & --- & --- & --- & --- & --- & --- & --- & --- & --- & 2.44 & --- & --- \\
\hline $\mathrm{PbO}$ & & --- & --- & --- & --- & --- & --- & --- & --- & --- & --- & --- & --- & --- & .24 & --- & --- \\
\hline
\end{tabular}




\section{Appendix I}

\section{Setting Time and Penetration Resistance for CLSM}

\section{I.1 Excavatable CLSM}

The charts of setting time versus penetration resistance for excavatable CLSM phase I and II are presented in Figure I-1 (4 charts) and Figure I-2 (2 charts), respectively.

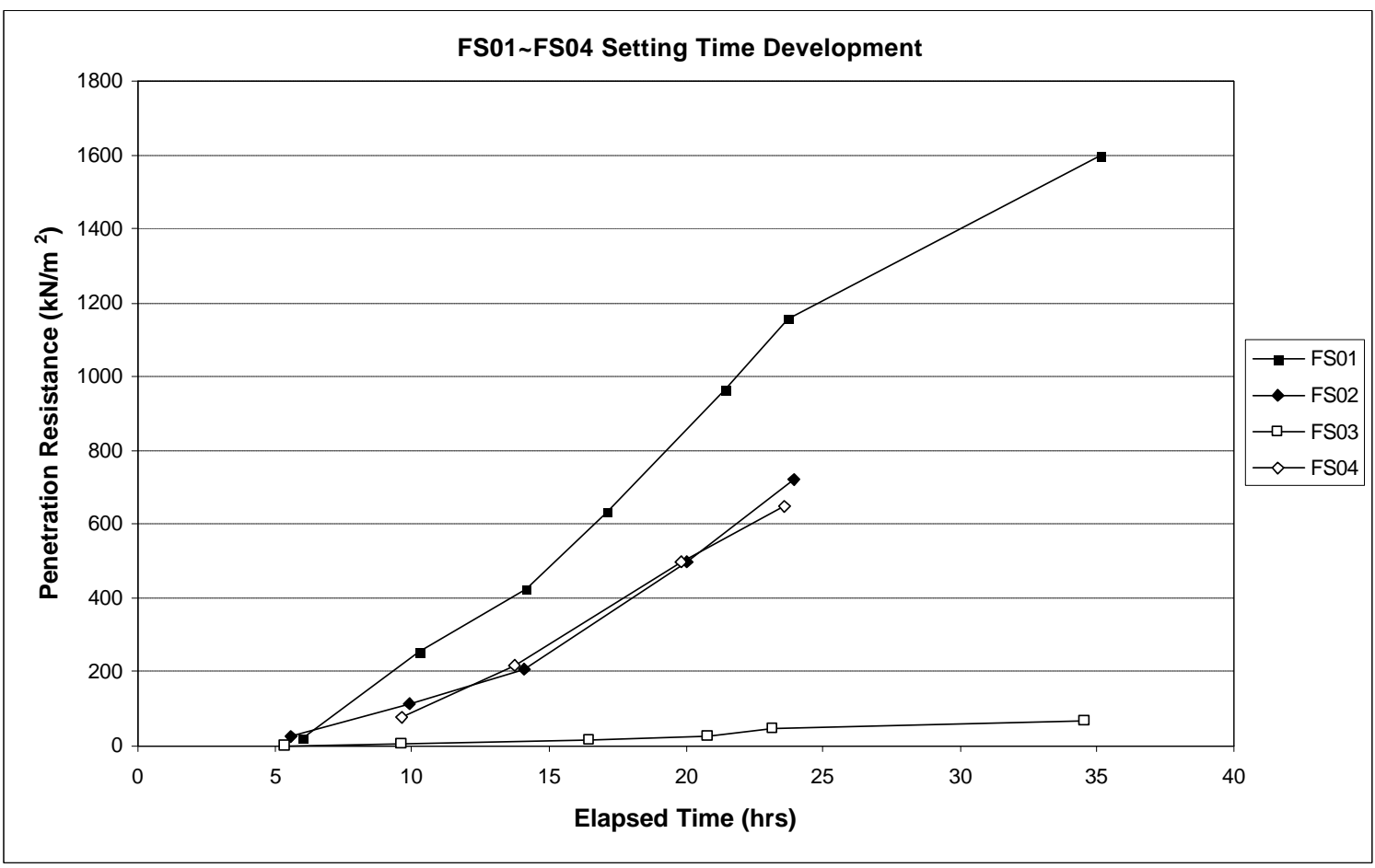



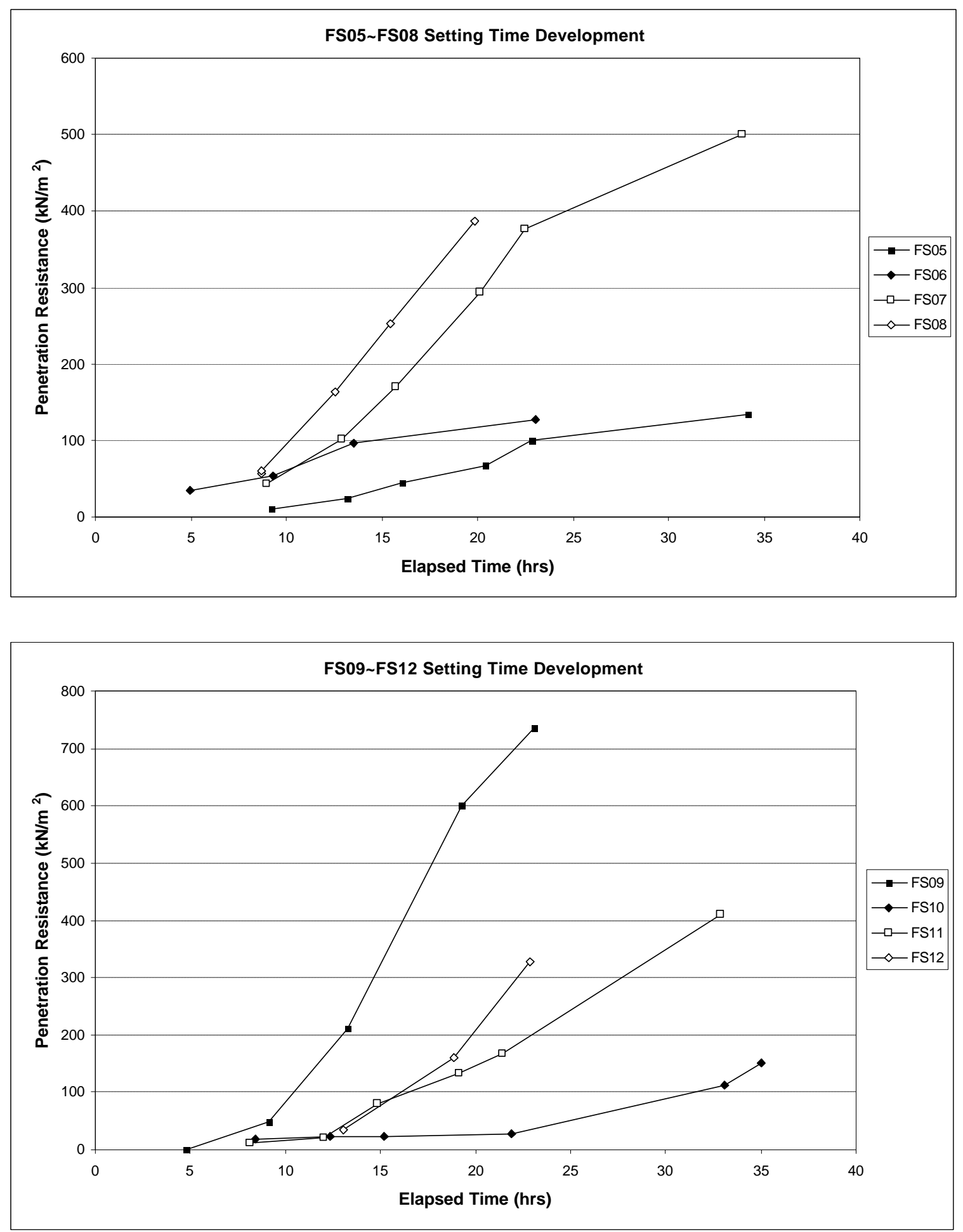


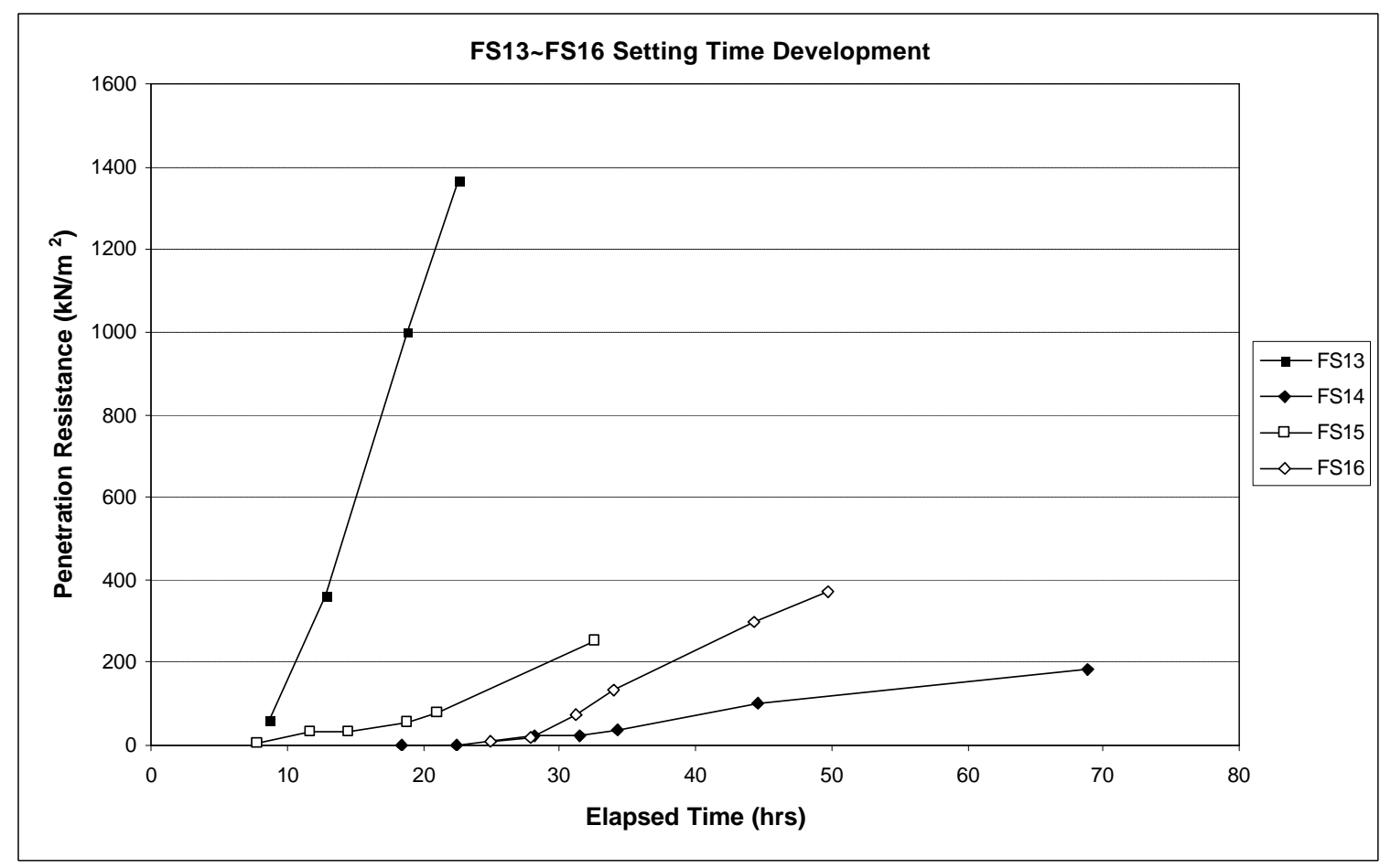

Figure I-1: Setting time of excavatable CLSM phase I (4 charts)

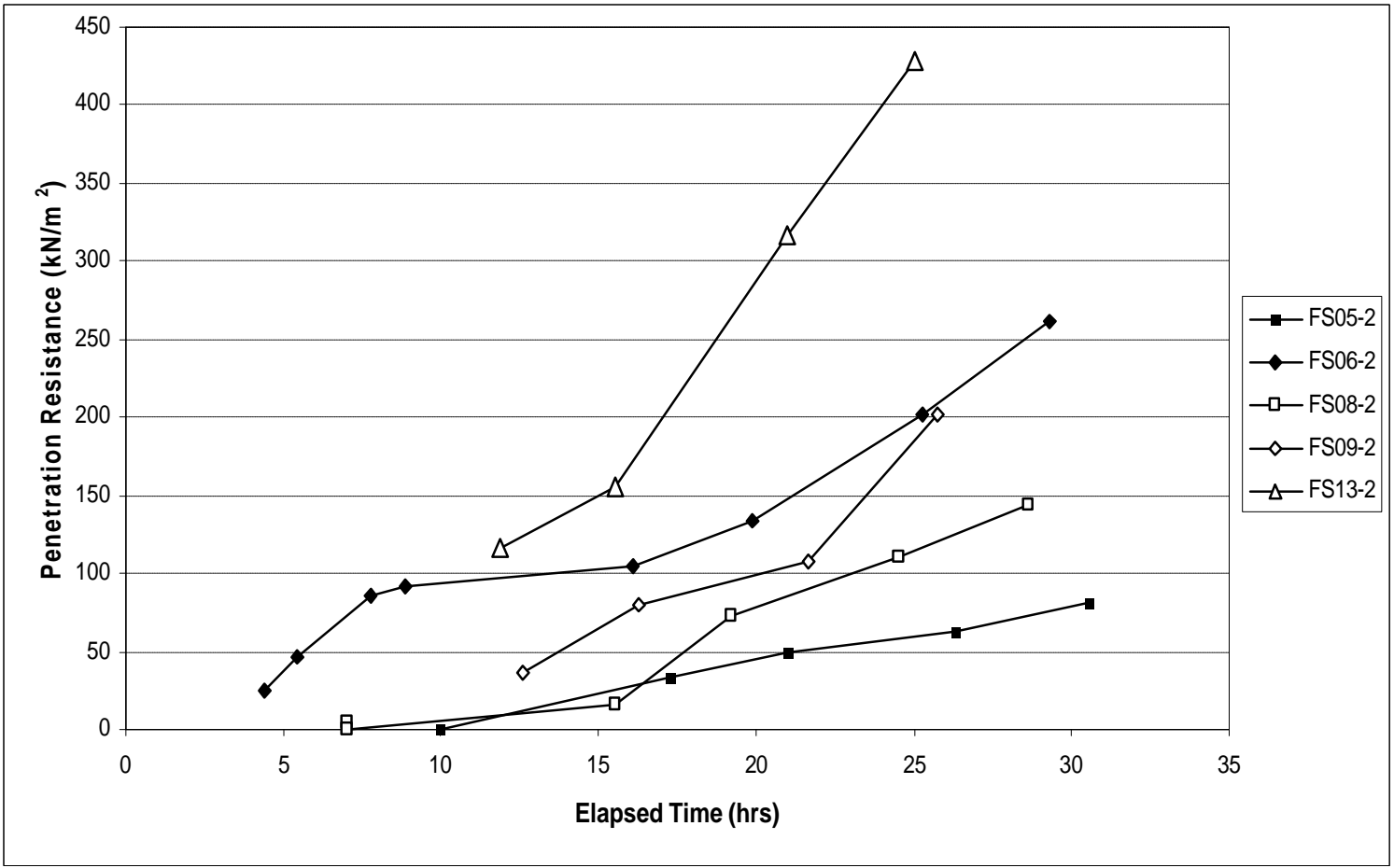




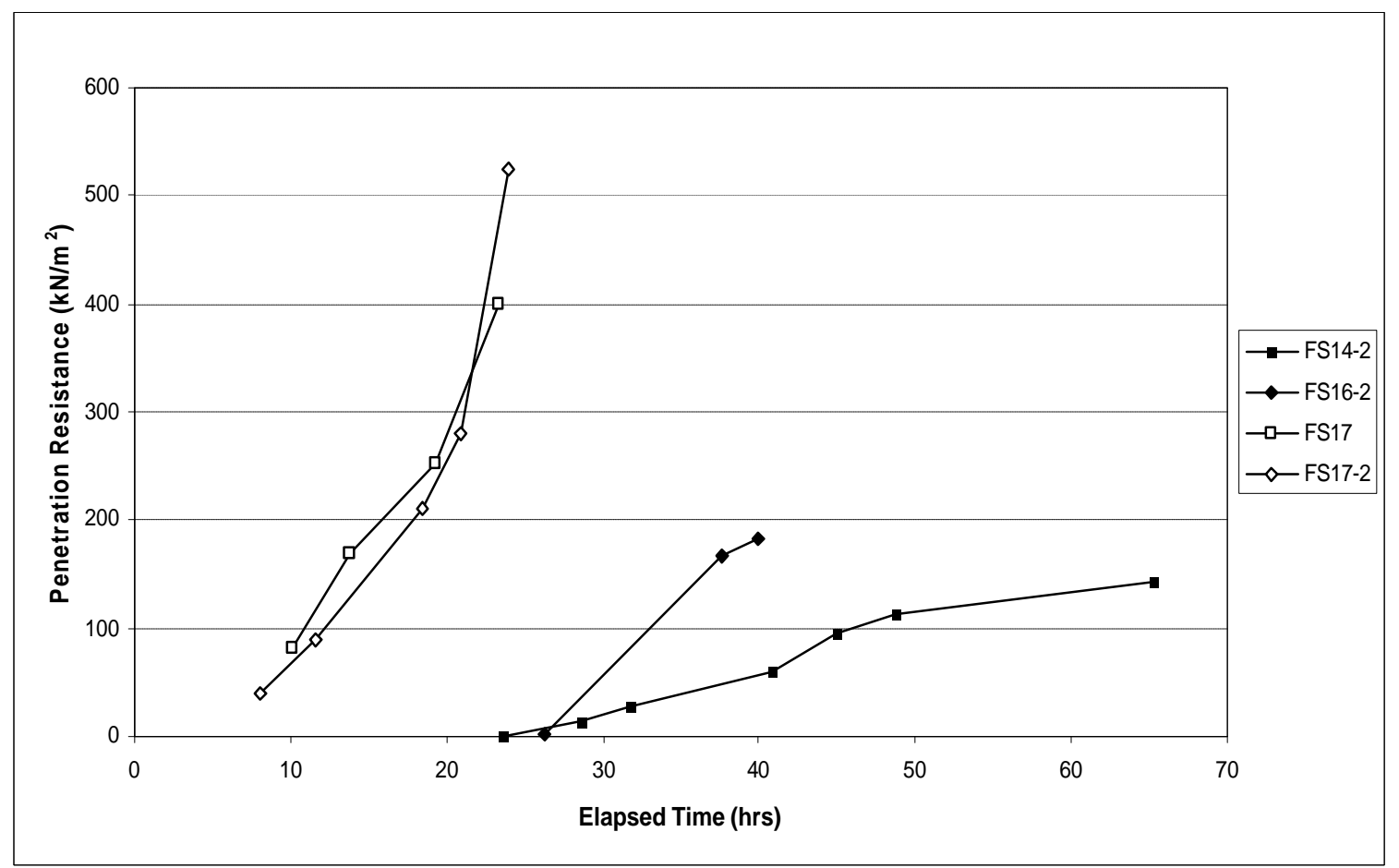

Figure I-2: Setting time of excavatable CLSM phase II (2 charts)

\section{I.2 Structural CLSM}

The charts of setting time versus penetration resistance for structural CLSM are presented in Figure I-3 (3 charts). 

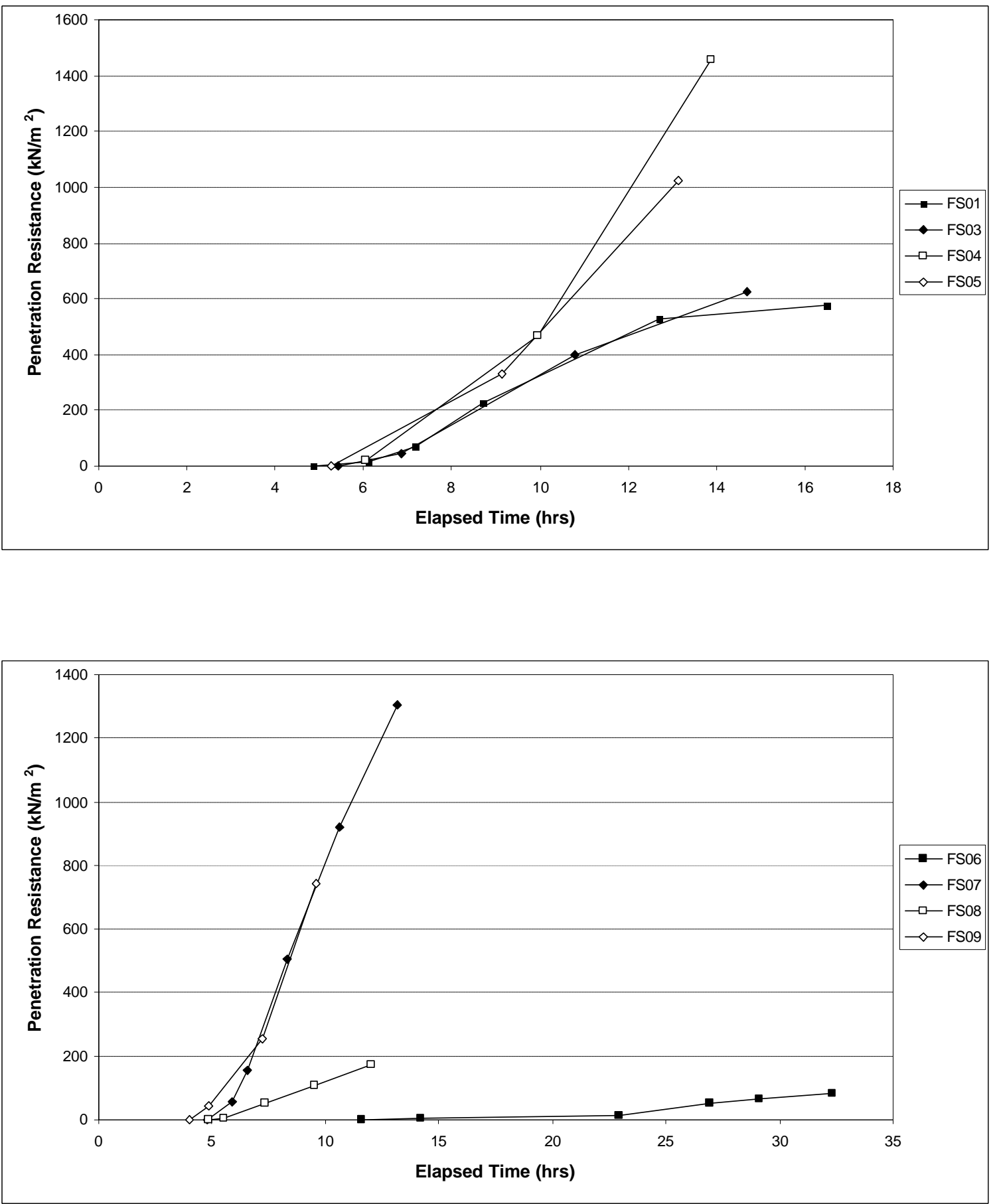


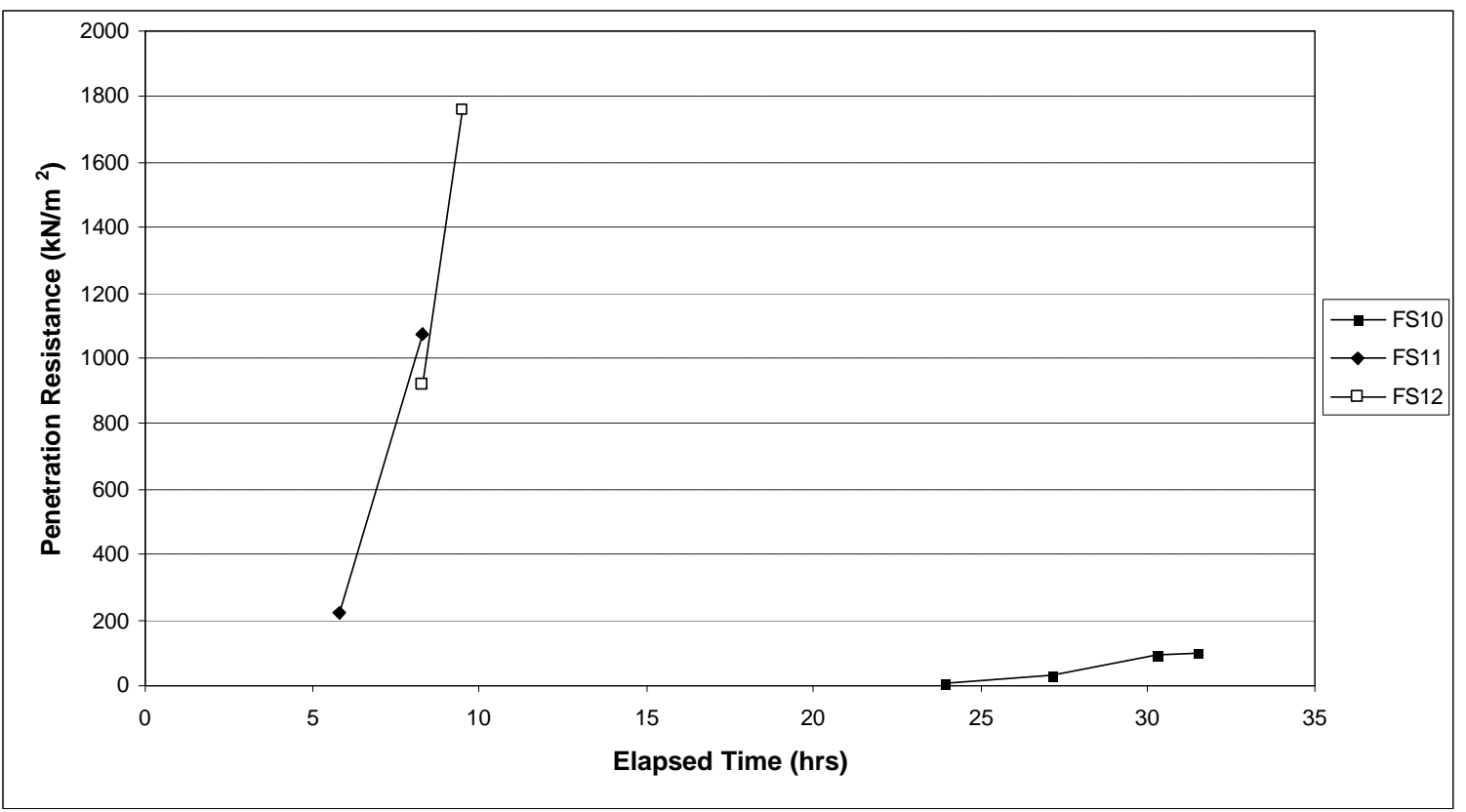

Figure I-3: Setting time of structural CLSM (3 charts) 


\section{Appendix J}

\section{Proof of Mean}

\section{J.1 Proof of Mean}

In this appendix, the proof that the mean of a nonnegative random variable is equal to the area under the corresponding survival function is presented. Visualized aid of demonstrated survival function is presented in Figure J-1.
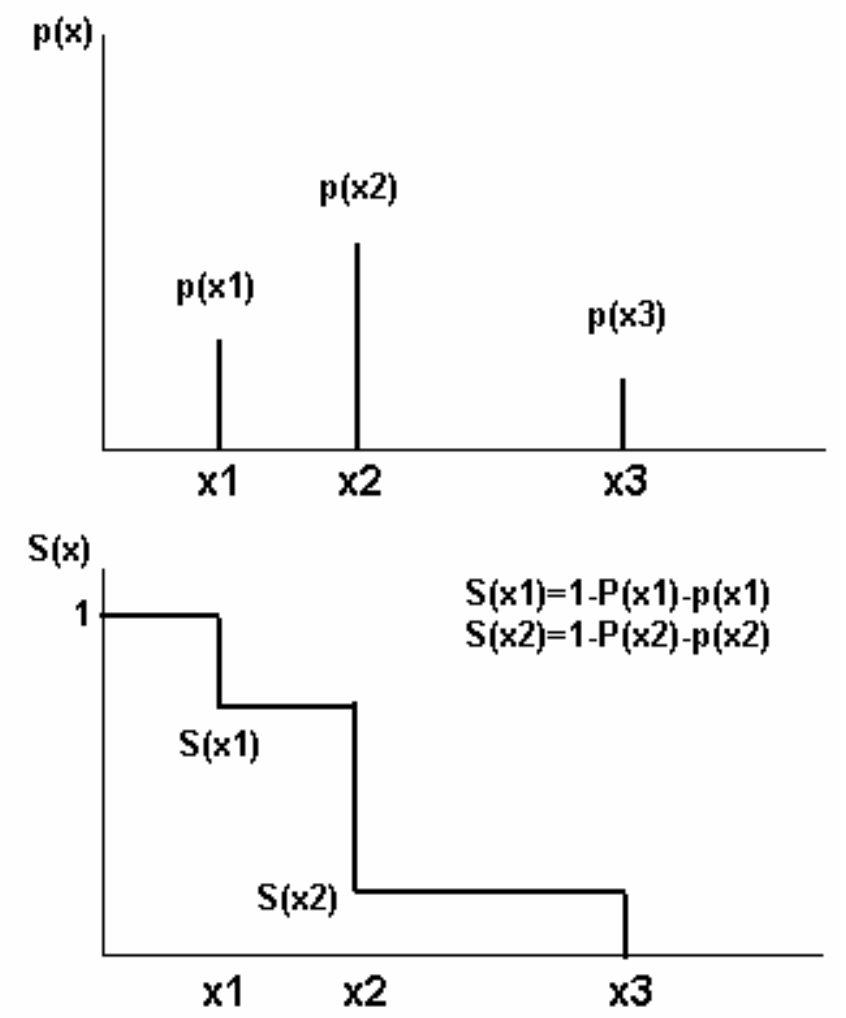

Figure J-1: Demonstration of probability function and survival function 


$$
\begin{aligned}
& \int_{0}^{x_{n}} S(\mathrm{x}) \mathrm{dx}=\int_{0}^{x_{1}} S(\mathrm{x}) \mathrm{dx}+\int_{x_{1}}^{x_{2}} S(\mathrm{x}) \mathrm{dx}+\int_{x_{2}}^{x_{3}} S(\mathrm{x}) \mathrm{dx}+\ldots \ldots+\int_{x_{n-2}}^{x_{n-1}} S(\mathrm{x}) \mathrm{dx}+\int_{x_{n-1}}^{x_{n}} S(\mathrm{x}) \mathrm{d} \mathrm{x} \\
& =\int_{0}^{x_{1}} 1 \mathrm{dx}+\int_{x_{1}}^{x_{2}} S\left(\mathrm{x}_{1}\right) \mathrm{dx}+\int_{x_{2}}^{x_{3}} S\left(\mathrm{x}_{2}\right) \mathrm{d} \mathrm{x}+\ldots . .+\int_{x_{n-2}}^{x_{n-1}} S\left(\mathrm{x}_{\mathrm{n}-2}\right) \mathrm{d} \mathrm{x}+\int_{x_{n-1}}^{x_{n}} S\left(\mathrm{x}_{\mathrm{n}-1}\right) \mathrm{dx} \\
& =\mathrm{x}_{1}+S\left(\mathrm{x}_{1}\right)\left(\mathrm{x}_{2}-\mathrm{x}_{1}\right)+S\left(\mathrm{x}_{2}\right)\left(\mathrm{x}_{3}-\mathrm{x}_{2}\right)+\ldots \ldots \\
& +S\left(\mathrm{x}_{\mathrm{n}-2}\right)\left(\mathrm{x}_{\mathrm{n}-2}-\mathrm{x}_{\mathrm{n}-1}\right)+S\left(\mathrm{x}_{\mathrm{n}-1}\right)\left(\mathrm{x}_{\mathrm{n}}-\mathrm{x}_{\mathrm{n}-1}\right) \\
& =\mathrm{x}_{1}+\left[1-P\left(\mathrm{x}_{1}\right)\right]\left(\mathrm{x}_{2}-\mathrm{x}_{1}\right)+\left[1-P\left(\mathrm{x}_{2}\right)\right]\left(\mathrm{x}_{3}-\mathrm{x}_{2}\right)+\ldots \ldots \\
& +\left[1-P\left(\mathrm{x}_{\mathrm{n}-2}\right)\right]\left(\mathrm{x}_{\mathrm{n}-1}-\mathrm{x}_{\mathrm{n}-2}\right)+\left[1-P\left(\mathrm{x}_{\mathrm{n}-1}\right)\right]\left(\mathrm{x}_{\mathrm{n}}-\mathrm{x}_{\mathrm{n}-1}\right) \\
& =\mathrm{x}_{1}+\left[\mathrm{x}_{2}-\mathrm{x}_{1}-P\left(\mathrm{x}_{1}\right)\left(\mathrm{x}_{2}\right)+P\left(\mathrm{x}_{1}\right)\left(\mathrm{x}_{1}\right)\right]+\left[\mathrm{x}_{3}-\mathrm{x}_{2}-P\left(\mathrm{x}_{2}\right)\left(\mathrm{x}_{3}\right)+P\left(\mathrm{x}_{2}\right)\left(\mathrm{x}_{2}\right)\right] \\
& +\ldots \ldots\left[\mathrm{x}_{\mathrm{n}-1}-\mathrm{x}_{\mathrm{n}-2}-P\left(\mathrm{x}_{\mathrm{n}-2}\right)\left(\mathrm{x}_{\mathrm{n}-1}\right)+P\left(\mathrm{x}_{\mathrm{n}-2}\right)\left(\mathrm{x}_{\mathrm{n}-2}\right)\right]+ \\
& {\left[\mathrm{x}_{\mathrm{n}}-\mathrm{x}_{\mathrm{n}-1}-P\left(\mathrm{x}_{\mathrm{n}-1}\right)\left(\mathrm{x}_{\mathrm{n}}\right)+P\left(\mathrm{x}_{\mathrm{n}-1}\right)\left(\mathrm{x}_{\mathrm{n}-1}\right)\right]} \\
& =p\left(\mathrm{x}_{1}\right)\left(\mathrm{x}_{1}\right)+p\left(\mathrm{x}_{2}\right)\left(\mathrm{x}_{2}\right)+\ldots \ldots+p\left(\mathrm{x}_{\mathrm{n}-1}\right)\left(\mathrm{x}_{\mathrm{n}-1}\right)+\mathrm{x}_{\mathrm{n}}-S\left(\mathrm{x}_{\mathrm{n}-1}\right)\left(\mathrm{x}_{\mathrm{n}}\right) \\
& =p\left(\mathrm{x}_{1}\right)\left(\mathrm{x}_{1}\right)+p\left(\mathrm{x}_{2}\right)\left(\mathrm{x}_{2}\right)+\ldots \ldots+p\left(\mathrm{x}_{\mathrm{n}-1}\right)\left(\mathrm{x}_{\mathrm{n}-1}\right)+p\left(\mathrm{x}_{\mathrm{n}}\right) \mathrm{x}_{\mathrm{n}} \\
& =\hat{\mu}
\end{aligned}
$$

where, $\mathrm{x}_{1}, \mathrm{x}_{2}, \ldots \ldots, \mathrm{x}_{\mathrm{n}}=$ observations

$S(\mathrm{x})=$ survival function

$P(\mathrm{x})=$ cumulative distribution function

$p(\mathrm{x})=$ probabilit $\mathrm{y}$ function

$\hat{\mu}=$ estimated population mean 


\section{Appendix K \\ Flow Chart of Procedure for CLSM}

\section{K.1 Characterization of Foundry Waste Streams}

The flow chart for characterization of foundry waste streams is presented in Figure K-1.

\section{K.2 Experimental Investigation of CLSM}

The flow chart for experimental investigation of CLSM containing excess foundry sand is presented in Figure K-2. 


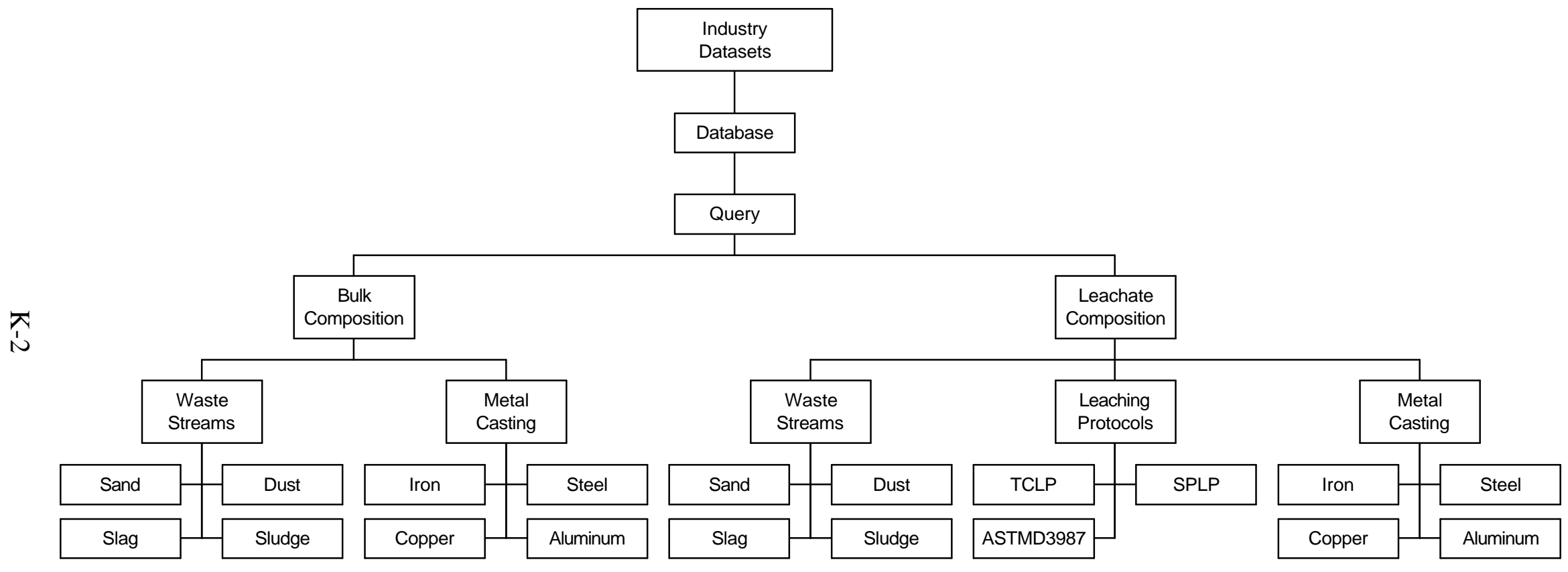

Figure K-1: Flow chart for characterization of foundry waste streams 


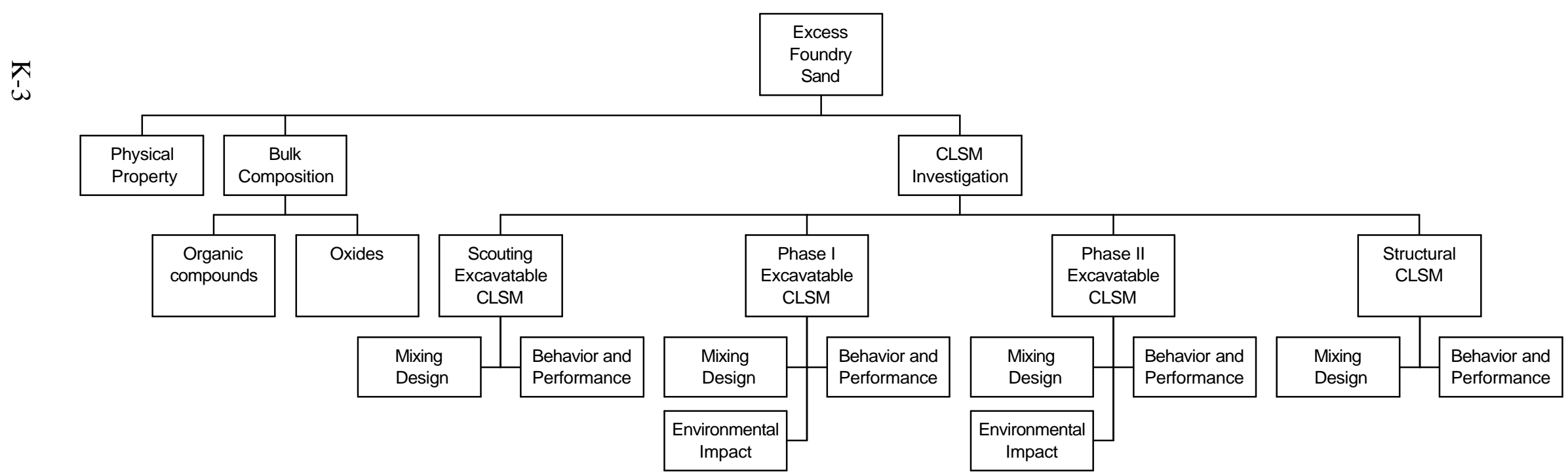

Figure K-2: Flow chart for experimental investigation of CLSM 


\section{Appendix L \\ Standard Specification for Excess Foundry Sand for Use in Controlled Low- Strength Material (CLSM)}

\section{Scope}

1.1 This specification covers the requirements for excess foundry sand used as a fine aggregate in controlled low-strength material (CLSM).

1.2 The specification is for use by contractor, excess foundry sand or CLSM supplier, or other purchaser as part of the purchase document describing the material to be furnished. Those responsible for selecting the proportions for the CLSM mixture shall have the responsibility of determining the proportions of excess foundry sand as a fine aggregate.

1.3 The values stated in SI units are to be regarded as the standard. The values given in parentheses are for information only.

1.4 The text of this standard references notes and footnotes which provide explanatory information. These notes and footnotes (excluding those in tables and figures) shall not be considered as requirements of this standard.

\section{Referenced Documents}

$2.1 \quad$ ASTM Standards

C 88 Test Method for Soundness of Aggregates by Use of Sodium Sulfate or Magnesium Sulfate ${ }^{1}$

C 117 Standard Test Method for Materials Finer than 75- $\mu$ m (No. 200) Sieve in Mineral Aggregates by Washing ${ }^{2}$

C 125 Standard Terminology Relating to Concrete and Concrete Aggregates ${ }^{2}$

\footnotetext{
${ }^{1}$ Annual Book of ASTM Standards, Vol 04.02, available from the ASTM, 100 Barr Harbor Drive, West Conshocken, PA 19428-2959
} 
C 128 Standard Test Method for Density, Relative Density (Specific Gravity), and Absorption of Fine Aggregate ${ }^{2}$

C 566 Standard Test Method for Total Evaporable Moisture Content of Aggregate by Drying $^{3}$

D 75 Standard Practice for Sampling Aggregates ${ }^{3}$

D 653 Standard Terminology Relating to Soil, Rock, and Contained Fluids ${ }^{3}$

D 3665 Standard Practice for Random Sampling of Construction Materials ${ }^{4}$

\subsection{Federal Registrar}

SW846 1312 EPA Test Method, Synthetic Precipitation Leaching Procedure (SPLP) ${ }^{4}$

SW846 7.3.3 EPA Test Method, Interim Guidance For Reactive Cyanide ${ }^{5}$

SW846 7.3.4 EPA Test Method, Interim Guidance For Reactive Sulfide ${ }^{5}$

SW846 9045 EPA Test Method, Soil And Waste $\mathrm{pH}^{5}$

2.3 AFS Mold \& Core Test Handbook:

AFS 1105-00-S Sieve Analysis (Particle Size Determination of Sand) ${ }^{5}$

AFS 1106-00-S Grain Fineness Number (GFN) ${ }^{6}$

AFS 1107-00-S Grain Shape Classfication ${ }^{6}$

AFS 1116-00-S Specific Gravity of Sand ${ }^{6}$

\section{Terminology}

3.1 foundry sand - a fine aggregate to produce mold or core in foundry facilities, which is used to cast ferrous and non-ferrous metals, consisting mostly silica sand, sometime lake sand, olivine sand, and zircon sand.

2 Annual Book of ASTM Standards, Vol 04.02, available from the ASTM, 100 Barr Harbor Drive, West Conshocken, PA 19428-2959

${ }^{3}$ Annual Book of ASTM Standards, Vol 04.03, available from the ASTM, 100 Barr Harbor Drive, West Conshocken, PA 19428-2959

${ }^{4}$ EPA SW-846: Test Methods for Evaluating Solid Wastes-Physical/Chemical Methods, available from the Department of Commerce, National Technical Information Center, 5285 Port Royal Road, Springfield, VA 22161. Tel: (800) 553-NTIS (553-6847)

5 AFS Mold \& Core Test Handbook, available from American Foundry Society, 505 State Street Des Plaines, Illinois 60016-8399 
3.2 excess foundry sand - excess foundry sand is disposed after several cycles of reclamation when a typical foundry facility wants to maintain the casting quality and removes those less servable foundry sand.

3.3 environmental stability - foundry sand shall be evaluated for environmental consideration (air quality, water quality, and storage) using the required local, state, and federal test methods in effect at the time of use.

3.4 Other definitions of terms used in this standard, refer to ASTM C 125, ASTM D 653, AFS Mold \& Core Test Handbook, and EPA SW-846: Test Methods for Evaluating Solid Wastes-Physical/Chemical Methods.

\section{Ordering Information}

4.1 Order for the material under this specification shall include the following information:

4.1.1 Quantity, in metric tons,

4.1.2 Whether the soundness of the material in 6.7.2 is required. If it is applied, which salt is to be used. If none is stated, either sodium sulfate or magnesium sulfate shall be used,

4.1.4 Any exceptions or additions to this specification.

\section{General Characteristics}

5.1 The foundry sand shall consist of hard, tough, durable pieces of fine aggregates. The aggregates shall be processed, as necessary to meet the requirements of this specification, by crushing and or screening, and magnetic separation for the removal of metallics.

5.2 The foundry sand shall be free of potential waste streams in foundry facilities: slag, dust, sludge, and other waste streams.

5.3 At the time of delivery, the foundry sand shall be free of injurious amounts of foreign materials such as clay, loams, wood, tramp metal, hard cores and other mill wastes. 


\section{Physical Requirements}

6.1 The grading of the foundry sand shall conform to the requirements for grading.

Sieve Percentage Passing by Weight

3.35-mm (No. 6) 97 to $100^{\mathrm{A}}$

1.7-mm (No. 12) 92 to 100

850- m (No. 20) 90 to 100

425- m (No. 40) 65 to 99

300-m (No. 50) 23 to 97

212- m (No. 70) 12 to 73

150- m (No. 100) 1 to 33

106- m (No. 140) $\quad 0.5$ to 15

75- $\mathrm{m}$ (No. 200) $\quad 0$ to $2^{\mathrm{B}}$

53- m (No. 270) 0 to 0.5

Grain Fineness Number ${ }^{\mathrm{C}}$ : 45 - 75

Note: ${ }^{A}$ Exercise care to avoid sand agglomerations, hard core, and other mill wastes.

${ }^{B}$ Too much clay or dust content interferes the strength development of CLSM.

${ }^{\mathrm{C}}$ Grain fineness number indicates the grain size distribution is fine moderately to enhance the flowability and self-compaction of CLSM.

6.2 The maximum percentage by weight of particle finer than 75- $\mathrm{m}$ (No. 200) sieve by washing is $15 \%$.

6.3 The major (>50\%) grain shape of foundry sand particles is subangular to round to facilitate the flowability and self-compaction of CLSM.

6.4 The bulk specific gravity of foundry sand applied to CLSM is recommended between 2.4 and 2.7. Too low or too high specific gravity interferes the flowability and strength controlling of CLSM.

6.5 Foundry sand failing to meet 6.1-6.4 shall meet the requirements of relevant properties provided that the supplier can demonstrate to the purchaser or specifier that CLSM of the class specified, made with foundry sand under consideration, will have relevant properties at least equal to those of CLSM made with the same ingredients, with the exception that the reference foundry sand shall be selected from a source having an acceptable performance record in similar CLSM construction.

6.6 The maximum evaporable moisture content of the foundry sand shall be $5 \%$ to ensure the uniformly blending of raw materials prior to water addition.

6.7 Optional physical requirements. 
6.7.1 The optional physical requirements apply to non-excavatable CLSM or are applied at the request of the purchaser.

6.7.2 Soundness: except as provided in 6.7.3 and 6.7.4, foundry sand subject to five cycles of the soundness test shall have a weighted average loss not greater than $15 \%$ when sodium sulfate is used or $20 \%$ when magnesium sulfate is used.

6.7.3 Foundry sand failing to meet the requirements of 6.7.2 shall be regarded as meeting the requirements of soundness provided that the supplier demonstrates to the

purchaser or specifier that CLSM of comparable properties, made from similar foundry sand from the same source, has given satisfactory service when exposed to weathering similar to that to be encountered.

6.7.4 Foundry sand not having a demonstrable service record and failing to meet the requirements of 6.7.2 shall be regarded as meeting the requirements of soundness provided that the supplier demonstrates to the purchaser or specifier it gives satisfactory results in CLSM subjected to freezing and thawing tests.

\section{Chemical Composition Requirements}

7.1 Excess foundry sand contains less than $3 \% \mathrm{CaO}$ by its bulk composition.

\section{Environmental Requirements}

8.1 Excess foundry sand from bronze/brass foundry is not recommended for the application of CLSM.

8.2 The foundry sand shall meet all applicable local, state, and federal environmental requirements in effect following testing program in 8.3 and 8.4.

8.2.1 Toxicity: the characteristics of leachate from the foundry sand shall be tested using the SPLP or appropriate test method as approved by the purchaser. Results shall indicate that all areas tested (inorganic chemicals and organic chemicals) are below regulatory limits.

8.2.2 Reactivity: the amount of reactive cyanide and reactive sulfide in the foundry sand shall be tested using Interim Guidance For Reactive Cyanide (EPA Method SW846 7.3.3) and Interim Guidance For Reactive Sulfide (EPA Method SW846 7.3.4) 
respectively. Results shall be below regulatory limits: $250 \mathrm{mg} \mathrm{HCN} / \mathrm{kg}$ waste, and 500 $\mathrm{mg} \mathrm{HS} / \mathrm{kg}$ waste.

8.2.3 Corrosivity: the $\mathrm{pH}$ of foundry sand shall be tested using Soil And Waste $\mathrm{pH}$ (EPA Method SW846 9045) or appropriate test method as approved by the purchaser. Result shall indicate that the $\mathrm{pH}$ is between 2 and 12.5 .

8.3 For an individual excess sand source, only if its metals cast, binder systems employed, and waste streams are not changed, hazardous evaluation testing is applied each month for three months, and annually thereafter.

8.4 In each testing, no less than 3 tests out of every $1000 \mathrm{yd}^{3}$ per sand source are required. If the yield is less than $1000 \mathrm{yd}^{3}$ per sand source, no less than 3 tests are required per sand source.

\section{Sampling and Testing Methods}

9.1 Sample and test the foundry sand in accordance with the following methods, except as otherwise provided in this specification. It is not intended to prohibit use of separated sizes from the sieve analysis for preparation of samples for soundness test. For determination of all other testes and for evaluation of potential alkali reactivity where required, use independent test specimens.

9.2 Sampling — ASTM D75 and ASTM D 3665.

9.3 Grading and GFN - AFS 1105-00-S and AFS 1106-00-S

9.4 Particles Finer Than 75 - m (No. 200) Sieve by Washing - ASTM C 117

9.5 Grain Shape - AFS 1107-00-S

9.6 Evaporable Moisture Content — ASTM C566

9.7 Specific Gravity — AFS 1116-00-S or ASTM C 128

9.8 Soundness - ASTM C 88

9.9 Reactivity - ASTM G 57

9.10 Toxicity — EPA Test method SW846 1312

9.11 Reactivity - EPA Test method SW846 7.3.3 and 7.3.4

9.12 Corrosivity — EPA Test Method SW846 9045 


\section{Rejection and Rehearing}

10.1 The purchaser has the right to reject material that fails to conform to the requirements of this specification. Rejection shall be reported to the producer or supplier promptly and in writing. In case of dissatisfaction with the results of the tests, the producer or supplier is not prohibited from making a claim for retesting.

\section{Certification}

11.1 When specified in the purchase order or contract, the purchaser shall be furnished certification that samples representing each lot have been tested as directed in this specification and the specified requirements have been met. When specified in the purchase order or contract, a report of the test results shall be furnished.

\section{Manufacture's Statement}

12.1 At the request of the purchaser, the manufacture shall state in writing the nature of the foundry sand, including metal cast, the material mineral type, the cast processing type (mold or core), the binder materials and the binding methods. The amount and any processing to foundry sand shall be furnished as the request of purchaser.

\section{Package Marking and Shipping Information}

13.1 When the foundry sand is delivered in package, the name and the brand of the manufacturer, the weight of the foundry sand contained therein shall be plainly marked on each package. Similar information shall be provided in the shipping invoices accompanying the shipment of packaged or bulk foundry sand. All packages shall be in good condition at the time of inspection.

\section{Keywords}

14.1 CLSM; environmental stability; fine aggregate; foundry sand 